\title{
Property-selected Asymmetric Azobenzenes for Photoswitchable Ligands ${ }^{\dagger}$
}

\author{
Florian Küllmer, ${ }^{a, b}$ Lucas Gregor, ${ }^{a}$ and Hans-Dieter Arndt ${ }^{\star, a}$
}

a Institute for Organic Chemistry and Macromolecular Chemistry, Friedrich-Schiller-University, Humboldtstr. 10, 07743 Jena, Germany; Email: hd.arndt@uni-jena.de

${ }^{b}$ New address: Institute of Chemistry, Leiden University, Einsteinweg 55, 2333 CC Leiden, The Netherlands

${ }^{\dagger}$ Electronic Supplementary Information (ESI) available: See DOI: xx.xxxx/xxxx00000x

\begin{abstract}
Suitable designed photoswitches based on azobenzenes are essential structural features for photopharmacological compounds. Optimized azobenzenes are important for serving as building blocks in „azo extension“ strategies, and for designing photodrugs with tailored properties. Herein we present the synthesis and characterization of a variety of asymmetric azobenzenes by addressing selected structural features of the diazene core, such as polarity, steric demand, and electronical properties. Systematic exploration led to photoswitches with a relaxation half-life of seconds, minutes, hours, or days. Furthermore, the influence of different substitution pattern on the photophysical properties was charted. For analysis of all switches, robust characterization as well as examination under near-to physiological conditions was established, in order to assist with photoswitch choice for specific biological applications.
\end{abstract}




\section{INTRODUCTION}

Influencing biological systems by light is non-invasive and offers high temporal and spatial resolution, which can be used for studying living organisms in functional detail. In photopharmacology ${ }^{1}$ the reported application possibilities grow constantly including the manipulation of peptides and proteins ${ }^{2}$, ion channels ${ }^{3}$, enzymes, ${ }^{1}$ or the cytoskeleton. ${ }^{4,5}$ Photoswitching biologically active molecules can be realized

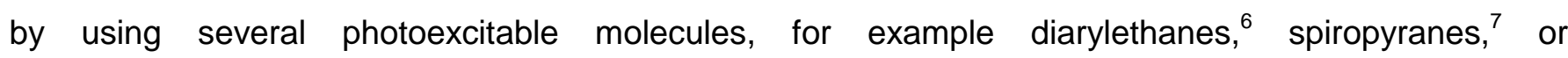
hemithioindigo, ${ }^{8}$ all of which offer distinct property profiles. ${ }^{9}$ However, due to ease of synthesis, favorable absorption properties, and useful structural reorganization upon photoswitching, "azobenzenes" (i. e. diaryldiazenes) are most frequently applied. ${ }^{1-3,10}$ Their photophysical properties, such as quantum yield, bleaching propensity, or thermal relaxation rate, have been reported to respond to changes in chemical structure. ${ }^{10-14}$

Azobenzenes can serve as a photoswitches, because the diazene bond is configurationally bistable. Its two possible configurational isomers differ in shape and in their dipole moments (Figure 1A). The $E$ isomer (trans) has an extended, linear shape, and co-planar aryl substituents, compared to the $Z$-isomer (cis), which adopts a curled, bent shape, with twisted aryl rings. ${ }^{14}$ Isomerization can be triggered by excitation of the strong $\pi \rightarrow \pi^{*}$ transition of the $E$-isomer, leading to configurational relaxation to the metastable $Z$-isomer. ${ }^{15,16}$ The $\pi \rightarrow \pi^{*}$ transition of the $Z$-isomer that induces back-isomerization to $E$ configuration is typically weaker and more blue shifted, but both absorption spectra often overlap considerably. Hence, a so called photostationary state (PSS) is reached upon irradiation, i. e. a constant ratio of both forms is established by simultaneously exciting both $E$ - and $Z$-isomers at the same time with a ratio depending on the absorption band intensity at the particular wavelength. ${ }^{15,17}$ Back isomerization to the thermodynamically favored $E$-isomer also takes place spontaneously, leading to an exclusive recovery of the $E$-isomer over time in the dark. ${ }^{11}$ Alternatively, excitation of the less favoured $n \rightarrow \pi^{*}$ transition, which is stronger in the nonplanar $Z$-isomer, might lead to a PSS with excess of $E$-isomer (Figure 1B), depending on excitation wavelength and intensity. ${ }^{18}$

The maximum of the $\pi \rightarrow \pi^{*}$ and $n \rightarrow \pi^{*}$ transitions, photoswitch geometry, as well as the thermal relaxation rate are important factors for tuning the activity and function of a biological active photoswitch. In small molecules, azobenzene moieties may serve as isosteric motifs ("azalogs") like in VUF $\mathbf{1 4 7 3 8}^{19}$ or in the photostatins. ${ }^{4}$ Alternatively, attached switches like in optojasp-1 may render bioactive ligands susceptible to light. ${ }^{5,20}$ Furthermore, asymmetric azobenzenes may be utilized for switching chain geometry $\left(\text { L-MAG- } \mathbf{0}_{460}\right)^{21}$ or sidechain presentation $(\mathbf{9 c - 8 a z o})^{22}$ in larger biomolecules or ligands (Figure 1C). Finally, they may connect peptide chains or other polymers mechanically and making them reactive to light-induced conformational switching. ${ }^{10,23,24}$ 
For any of these molecular designs, the unimolecular thermal reversion rate of the switch is a structural property of high importance for applications, as biological response may occur on variable time scales, depending on the particular application (ms - days). ${ }^{1}$ However, with the exception of "azalogs", the majority of azobenzene switches for biological are based on simple monosubstituted azobenzenes. ${ }^{1,25-27}$ These contain push-pull substitution, frequently amines or anilines, displaying either very fast $(<1 \mathrm{~s})$ or very slow ( hours) thermal kinetics. ${ }^{28,29}$ Structural variations such as ortho substitutions were mainly investigated for addressing the activation wavelength, and for state control. ${ }^{2,10,29-32}$ In order to optimize properties of the optojasp actin photoswitches, we have conducted a systematic study which led to a photoswitch design with thermal relaxation properties fitting to the practically important sec-mins range.

A

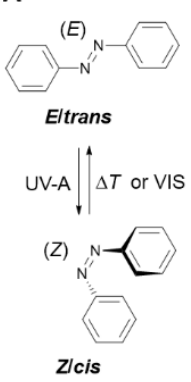

C
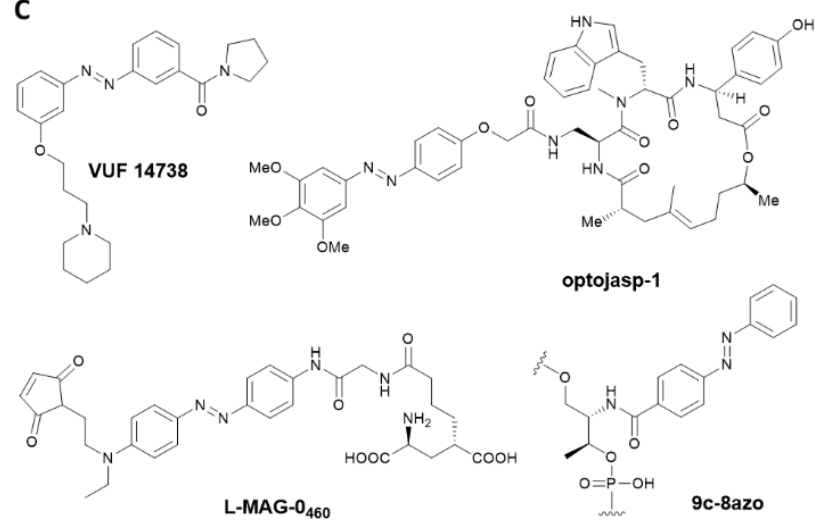

Figure 1. A: E-Z-isomerization and resulting structural change of azobenzene photoswitches. B: Absorption spectra of azobenzene depending on configuration. C: Azobenzene-based photoswitches applied in biologically active ligands. 


\section{RESULTS AND DISCUSSION}

\section{Research Design}

We aimed for structures where any ligand of interest could be possibly attached to the structural ring "A", while the exposed "B"-ring, that would change its relative orientation upon photoswitching, allows for suitable modifications or elongations (Figure 2). This asymmetric switch design is also inspired by structural biology data $\mathrm{we}^{20}$ and others ${ }^{33}$ have recently been able to obtain on target-bound azobenzene photoswitches. To generate asymmetric azobenzenes straightforwardly, an azo coupling of diazonium salt intermediates to phenols may be used. ${ }^{34}$ The phenolic hydroxyl group enables further functionalization or could become later part of either the "A"-, or the "B"-ring substructure. Thereby 4,4'substituted azobenzenes with electron donating groups are obtained, related to the photostatins and the optojasps. $^{4,5}$

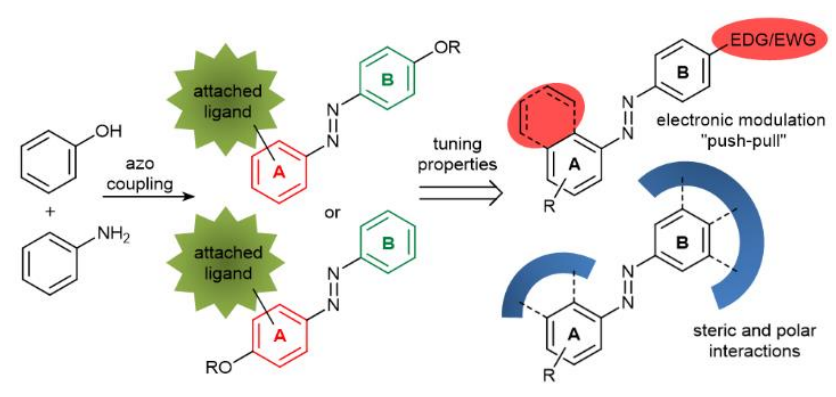

Figure 2. Asymmetric azobenzene design for biological applications.

\section{Synthesis}

To systematically study structural-function relationship a panel of differently shaped azobenzenes was prepared. This collection featured azobenzenes with phenol being either part of the " $A$ "- or the " $B$ "-ring, and being connected to a potential ligand in para- or ortho- position of the diazene (Scheme $1-3)$. A carboxylic acid should enable ligand attachment or coupling to biomolecules of interest.

A diazo coupling was used to form phenolic azobenzenes. 4-Methoxyanilin (1a), 4-bromoanilin (1b), 3,5dimethoxyanilin (2), 3,4,5-trimethoxyanilin (3), and known ${ }^{35}$ oxazin 4 were efficiently converted into diazonium salts by using isoamyl nitrite. ${ }^{4,36}$ Protected aniline precursors $\mathbf{5}$ and $\mathbf{6}$ were deprotected in situ before forming the diazonium salts. During transformation of oxazin 4 an excess of isoamyl nitrite had to be used because the secondary amine became transiently nitrosylated as well (evident from LC-MS). The following coupling of the diazonium salts was performed with several phenols 7a-c, 8, 9, 10a-e, 8hydroxy-quinoline (11a) and naphthol 11b-d, 12. In general, designs for para attached azobenzenes (Scheme 1 and 3) were coupled in rewarding yields, accessing a broad variety of phenolic azobenzenes 
$(13,14,15,16 a-c, 18,21 a-e, 22 a-d, 23)$. The $N$-nitrosylation of oxazine 4 was removed by treating the crude diazo coupling product with anhydrous $\mathrm{HCl}$ in dioxane/MeOH (Scheme $1 \mathrm{~A}$ ). Basic conditions during diazo couplings with Boc-deprotected aniline $\mathbf{5}$ in $\mathrm{MeOH}$ led to transesterification in some cases (Scheme 1B). Diazo couplings of ortho substituted derivatives 6 and 8 that contained a succinimidyl residue lead to reaction mixtures of imide, methyl ester and carboxylic acid of compounds 17a,b, 19b,c and 20 (Scheme 2). However, the mixture of imid and ester could be used for further steps (combined yields approx. 60\%). Alternatively, the succinimidyl residue was introduced at the end. From Bocprotected hydroxyaniline $\mathbf{9}$ azobenzene $\mathbf{1 8}$ was obtained by azo coupling. After ether formation $(\rightarrow \mathbf{2 4})$ and Boc-deprotection, a succinyl acid sidechain was introduced by using succinic anhydride to obtain azobenzene 25 in good yield (Scheme 2A).
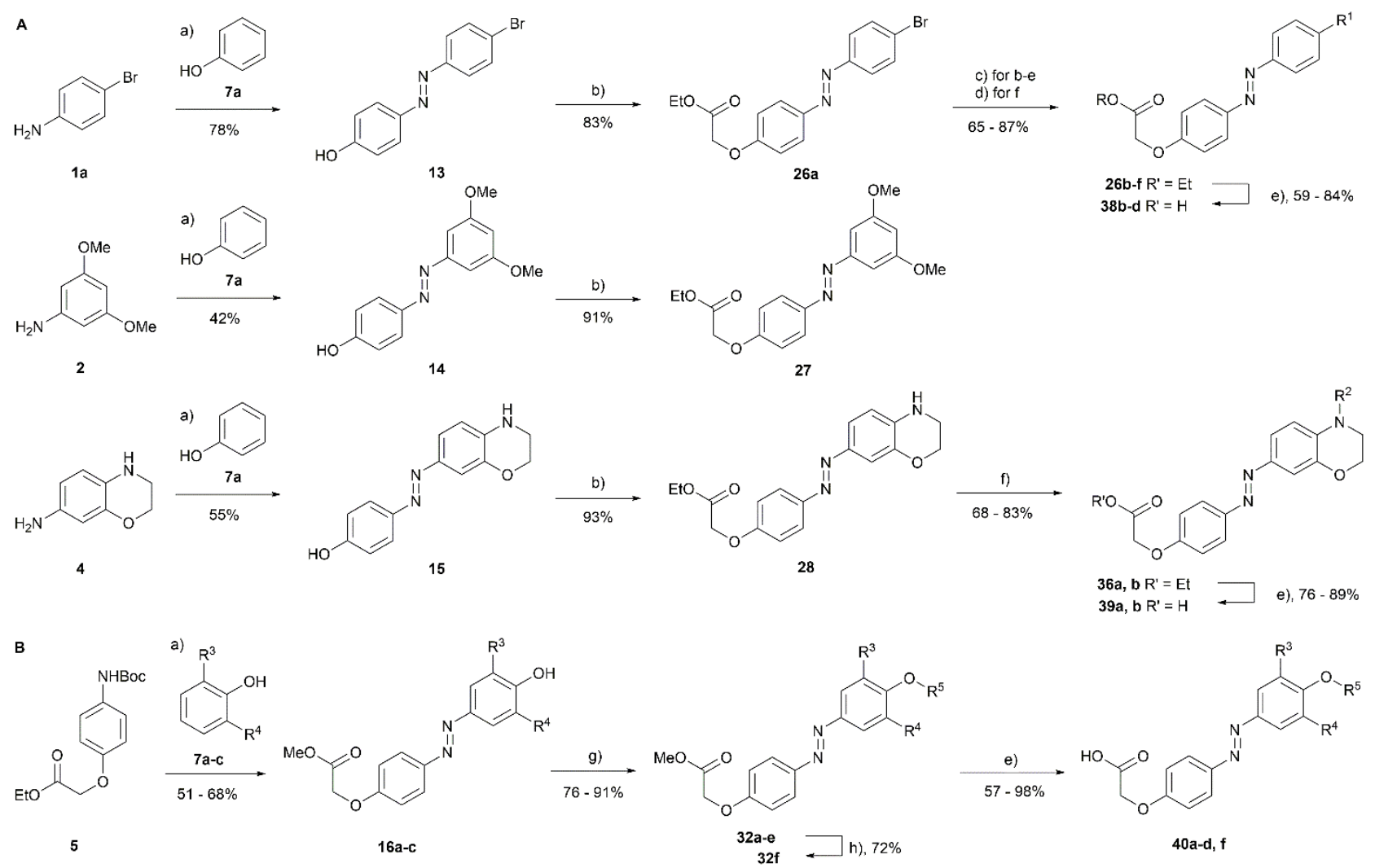

Scheme 1. Synthesis of para attached linkers with "B"-ring modifications. A: Phenol part of the "A"-ring. B: Phenol part of the "B"-ring. Conditions: a) 1. Isoamyl nitrite, $\mathrm{HCl}^{*} \mathrm{aq}, \mathrm{MeOH}, 0{ }^{\circ} \mathrm{C} ; 2$. $\mathrm{NaOH}{ }^{*} \mathrm{aq}, \mathrm{MeOH}$, phenol, $\left.0^{\circ} \mathrm{C} ; \mathrm{b}\right) \mathrm{EtOOCCH}_{2} \mathrm{Br} \mathrm{K}_{2} \mathrm{CO}_{3}$, $\mathrm{Me}_{2} \mathrm{CO}$, rfx; c) amine, $\mathrm{Cs}_{2} \mathrm{CO}_{3}, \mathrm{Pd}(\mathrm{dpa})_{2}$, RuPhos, $\mathrm{CH}_{3} \mathrm{CN}, 100^{\circ} \mathrm{C}$; d) Cul, K-phthalimide, DMF, $160{ }^{\circ} \mathrm{C}$; e) $\mathrm{THF} / \mathrm{aq} .2 \mathrm{M} \mathrm{LiOH}$, r.t.; f) aldehyde, $\mathrm{NaCNBH}_{3}, \mathrm{AcOH} / \mathrm{EtOH} 1: 9 ; \mathrm{g}$ ) alkyl halide, $\mathrm{K}_{2} \mathrm{CO}_{3}, \mathrm{Me}_{2} \mathrm{CO}$, rfx; h) $\mathrm{K}_{2} \mathrm{CO}_{3}, \mathrm{~N}$-methylpiperazine, $\mathrm{CH}_{3} \mathrm{CN}, 140{ }^{\circ} \mathrm{C}$, ))); Substituents: $\mathbf{2 6}, 3 \mathbf{8}$ a: $\mathbf{R}^{1}=\mathrm{Br} ; \mathbf{b}: \mathrm{R}^{1}=\mathrm{NMe}_{2} ; \mathbf{c}: \mathbf{R}^{1}=$ morpholino; $\mathbf{d}: \mathrm{R}^{1}=N$-methylpiperazino; $\mathbf{e}: \mathbf{R}^{1}=\mathrm{NHAc} \mathbf{f}: \mathrm{R}^{1}=\mathrm{NPhth}$; 36, 39 a: $R^{2}=M e ; b: R^{2}=B n ; 7,16,32,40$ a: $R^{3}=H, R^{4}=H, R^{5}=M e ; b: R^{3}=O M e, R^{4}=H, R^{5}=M e ; c: R^{3}=O M e, R^{4}=O M e$, $\mathrm{R}^{5}=\mathrm{Me} ; \mathbf{d}: \mathrm{R}^{3}=\mathrm{OMe}, \mathrm{R}^{4}=\mathrm{OMe}, \mathrm{R}^{5}=2$-hydroxyethoxy; $\mathbf{e}: \mathrm{R}^{3}=\mathrm{OMe}, \mathrm{R}^{4}=\mathrm{OMe}, \mathrm{R}^{5}=2$-bromoethoxy; $\mathbf{f}: \mathrm{R}^{3}=\mathrm{OMe}, \mathrm{R}^{4}=\mathrm{OMe}$, $R^{5}=2$-(4-methylpiperazino)ethoxy. 

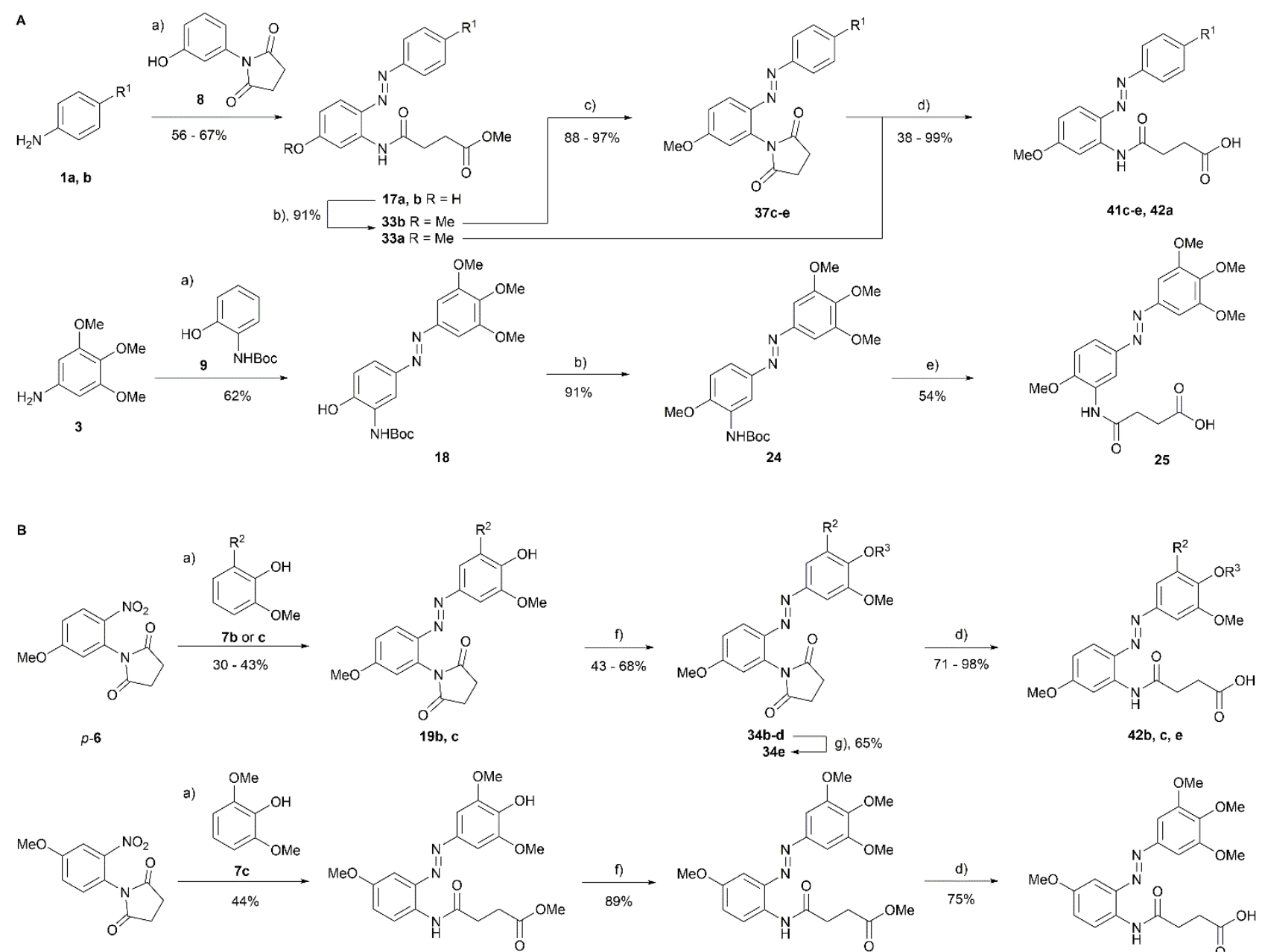

$m-6$
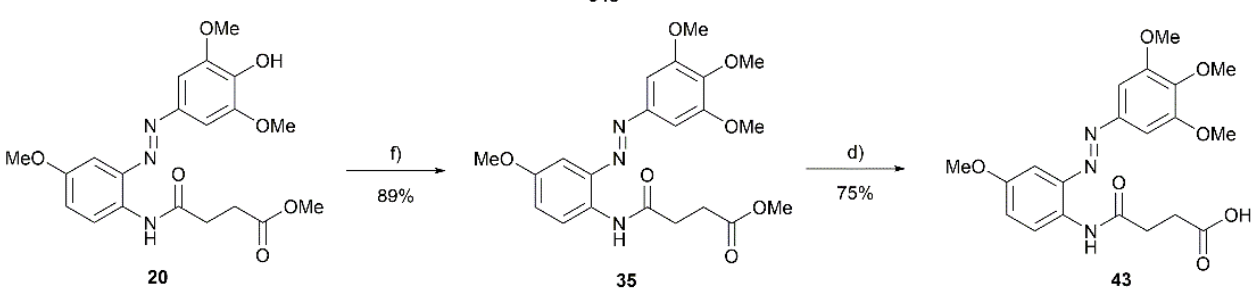

Scheme 2. Synthesis of ortho attached linkers with "B"-ring modifications. A: Phenol part of the "A"-ring. B: Phenol part of the "B"-ring. Conditions: a) 1. $\mathrm{Pd} / \mathrm{C}, \mathrm{H}_{2}$, EtOH/EtOAc/AcOH 2. Isoamyl nitrite, $\mathrm{HCl}{ }^{*} a q, \mathrm{MeOH}, 0{ }^{\circ} \mathrm{C} 3 . \mathrm{NaOH}^{*} \mathrm{aq}, \mathrm{MeOH}$, phenol, $0^{\circ} \mathrm{C}$; b) Mel, $\mathrm{K}_{2} \mathrm{CO}_{3}, \mathrm{Me}_{2} \mathrm{CO}$, rfx; c) Amine, $\mathrm{Cs}_{2} \mathrm{CO}_{3}, \mathrm{Pd}(\mathrm{dba})_{2}$, RuPhos, $\mathrm{CH}_{3} \mathrm{CN}, 100^{\circ} \mathrm{C}$; d) THF/aq., 2M LiOH, r.t.; e) 1. $4 \mathrm{M} \mathrm{HCl}$ in 1,4-dioxane, $\mathrm{MeOH}$ 2. succinic anhydride, $\mathrm{K}_{2} \mathrm{CO}_{3}, \mathrm{DMF}, 70^{\circ} \mathrm{C}$; f) alkyl halide, $\mathrm{K}_{2} \mathrm{CO}_{3}, \mathrm{Me}_{2} \mathrm{CO}$, rfx; g) $\mathrm{K}_{2} \mathrm{CO}_{3}, \mathrm{~N}$ methylpiperazine, $\mathrm{CH}_{3} \mathrm{CN}, 140{ }^{\circ} \mathrm{C}$, ))); Substituents: 1, 17, 33, 37, 41, 42 a: $\mathrm{R}^{1}=\mathrm{OMe} ; \mathbf{b}: \mathrm{R}^{1}=\mathrm{Br} ; \mathbf{c}: \mathrm{R}^{1}=\mathrm{NMe}_{2} ; \mathbf{d}: \mathrm{R}^{1}=$ morpholino; e: $R^{1}=N$-methylpiperazino; 7, 19, 34, 42 b: $R^{2}=H, R^{3}=M e ; c: R^{2}=O M e, R^{3}=M e ; d: R^{2}=O M e, R^{3}=$ 2-bromoethoxy; e: $R^{2}=\mathrm{OMe}, \mathrm{R}^{3}=2$-(4-methylpiperazino)ethoxy.

All azobenzene phenols were next converted to ethers. Para-attached derivatives with the phenol in ring "A" were synthesized with ethyl bromoacetate in dry acetone and $\mathrm{K}_{2} \mathrm{CO}_{3}$, providing ethers 26a, 27, 28, 29a-e, 30a-d and 31 in good to excellent yields (Scheme 1A, Scheme 3). For TBS-protected phenol 21b the ether $29 \mathrm{~b}$ was formed by using sodium hydride in dry THF at $-20^{\circ} \mathrm{C}$ to prevent silyl group cleavage.

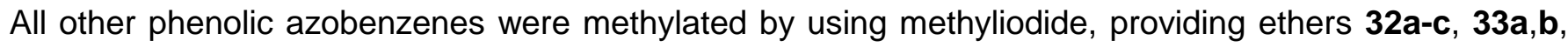
34b,c and 35 in excellent yields (Scheme 1B, Scheme 2). For additional modifications in the "B"-ring phenolic azobenzenes $\mathbf{1 6 c}$ and $\mathbf{1 9 c}$ were alternatively converted by alkylation with 2-bromethanol or 1,2dibromethan to azobenzenes $\mathbf{3 2 d}$,e and $\mathbf{3 4 d}$ in good yields. 
The 4,4'-substituted azobenzenes such obtained were further modified (Scheme 1 - 3). Amine 28 was converted by reductive amination with paraformaldehyde or benzaldehyde to ester $\mathbf{3 6 a}$ and $\mathbf{3 6} \mathbf{b}$ in good yields. The arylbromides $\mathbf{2 6 a}, \mathbf{2 9 d}$ and $\mathbf{3 3 b}$ were derivatized in good to excellent yields by BuchwaldHartwig amination ${ }^{37,38}$ to introduce amino (26b-d, 29f, 37c-e) or amide substituents (26e). To obtain phthalimide 26f, an UlImann-type coupling with potassium phthalimide was performed instead, because Buchwald-Hartwig conditions failed to provide clean material. Alkylbromides $\mathbf{3 2 e}$ and $\mathbf{3 4 d}$ offer a handle for attaching additional groups or to elongate to a chain. As an example, they were converted in a microwave assisted nucleophilic substitution reaction with $N$-methylpiperazine to the polar azobenzenes $\mathbf{3 2 f}$ and $34 \mathrm{e}$ (Scheme 1 and 2). Finally, the free Carboxylic acids were liberated by hydrolysis of ester or succinimidyl residue with aq. LiOH in THF in good to excellent yields (Scheme 1 3), giving carboxylic acids 5b-d, 10a,b, 15a-d,f, 21b-e, 26a-c,e, 31a,c,g and 35a-d for conjugation.

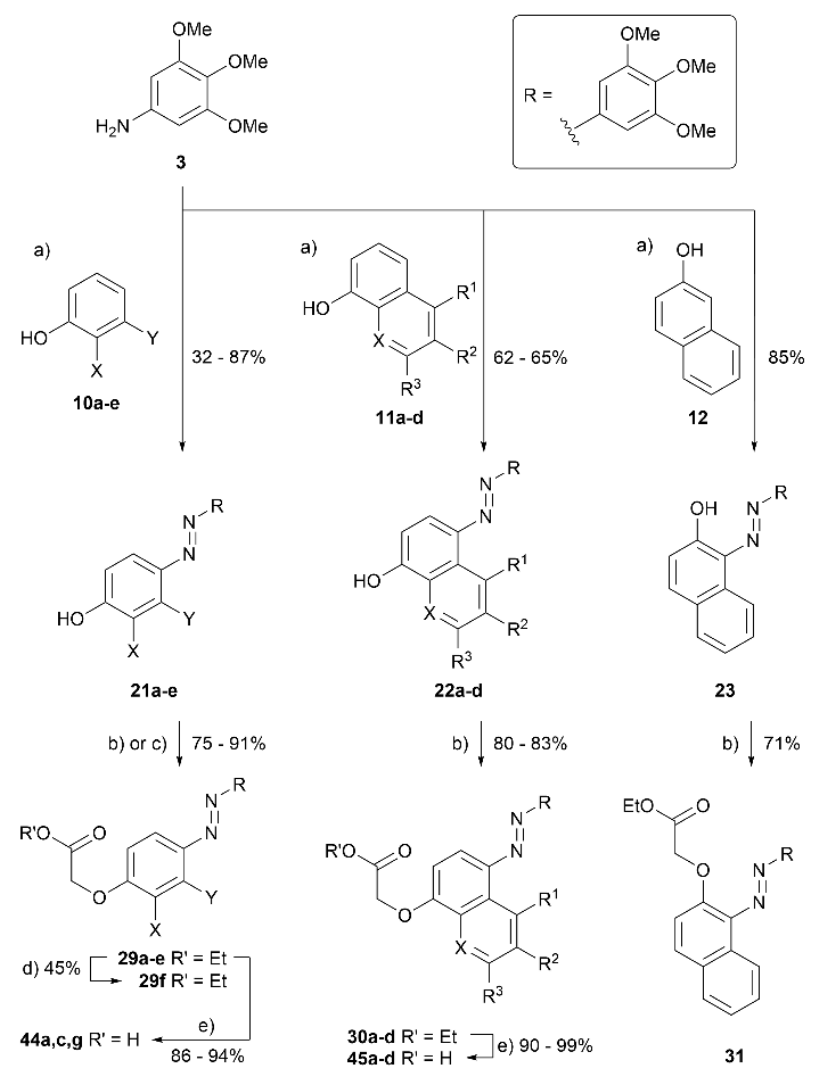

Scheme 3. Modifications at the "A"-ring. Conditions: a) 1. Isoamyl nitrite, $\mathrm{HCl}{ }^{*} a q, \mathrm{MeOH}, 0{ }^{\circ} \mathrm{C} 2 . \mathrm{NaOH}^{*} \mathrm{aq}, \mathrm{MeOH}, \mathrm{phenol}, 0^{\circ} \mathrm{C}$; b) $\mathrm{EtOOCCH}_{2} \mathrm{Br}, \mathrm{K}_{2} \mathrm{CO}_{3}, \mathrm{Me}_{2} \mathrm{CO}$, rfx; c) EtOOCCH${ }_{2} \mathrm{Br}, \mathrm{NaH}, \mathrm{THF},-20^{\circ} \mathrm{C}$; d) amine, $\mathrm{Cs}_{2} \mathrm{CO}_{3}, \mathrm{Pd}(\mathrm{dba})_{2}, \mathrm{RuPhos}, \mathrm{CH}_{3} \mathrm{CN}, 100^{\circ} \mathrm{C}$; e) THF/aq., 2M LiOH, r.t.; substituents: 10, 21, 29, 44 a: $X=H, Y=H$; b: $X=O T B S, Y=H$; $: X=H, Y=O H ; d: X=B r, Y=H$; e: $X=H, Y=B r ; f: X=$ morpholino, $Y=H ; \mathbf{g}: X=O H, Y=H ; 11,22,30,45$ a: $R^{1}=H, R^{2}=H, R^{3}=H, X=N ; b: R^{1}=H, R^{2}=H$, $\mathrm{R}^{3}=\mathrm{H}, \mathrm{X}=\mathrm{CH} ; \mathbf{c}: \mathrm{R}^{1}=\mathrm{OMe}, \mathrm{R}^{2}=\mathrm{H}, \mathrm{R}^{3}=\mathrm{H}, \mathrm{X}=\mathrm{CH} ; \mathbf{d}: \mathrm{R}^{1}=\mathrm{H}, \mathrm{R}^{2}=\mathrm{OMe}, \mathrm{R}^{3}=\mathrm{OMe}, \mathrm{X}=\mathrm{CH}$. 


\section{Photophysical characterization}

Absorption maxima $\left(\lambda_{\max }\right)$ and the thermal $Z \rightarrow E$ relaxation half-life $\left(\tau_{\tau_{2}}\right)$ were determined by using $U V$-Vis spectroscopy. Surprisingly, thermal half-life seemed to vary in pure organic solvent (acetonitrile), and literature data seemed to be variable, too, especially for aniline derivatives. ${ }^{39}$ A brief examination was hence undertaken (Table 1). HPLC grade acetonitrile showed changing relaxation half-life over time after bottle opening (Table 1, entry 1-3), independent of water content (Table 1, entry 4-6). After treatment with activated $\mathrm{Al}_{2} \mathrm{O}_{3}$ a significant change was observed, indicating that traces of protic acid(s) may be involved (Table 1, entry 6-8). Indeed, air purging (20 min) led to variations of $\tau_{1 / 2}$ depending on water content, suggesting carbonic acid to be formed. Indeed, by adding $0.5 \%$ piperidine the relaxation kinetics data became reproducible. The absorption spectrum of the azobenzene $\mathbf{2 6 c}$ did not change under the different conditions, excluding the presence of azonium ions. ${ }^{40}$ This property notwithstanding, azobenzene photoswitch properties may still be dependent on local pH or on its binding environment.

To determine the photostationary state (PSS) ratio at different wavelengths of irradiation, the method developed by Fischer was implemented. ${ }^{41}$ It allows computing the ratio of the isomers in the PSS after irradiation at two different wavelengths when the absorption spectrum of the pure $E$-isomer is known. The spectrum of the pure Z-isomer can additionally be computed from these data. This method is fast and reliable for azobenzenes with a half-life long enough to complete the measurement. When comparing it to NMR methods (Figure 3), the PSS ratio is easily extracted at several wavelengths and its determination is not compromised by potentially overlapping NMR signals.

We then studied the influence of the solvent. Solvent polarity did not strongly affect the absorption spectra of the investigated azobenzenes. The absorption maxima of the $\Pi \rightarrow \pi^{*}$ transition remained constant (Table 2). Depending on the solvent, the $n \rightarrow \pi^{*}$ transition was found somewhat modulated. Under aqueous conditions the absorption was broader, resulting in measurable changes of the PSS between $420 \mathrm{~nm}$ and $470 \mathrm{~nm}$ (Table 2, Figure S1) especially for aniline derivatives. In general, the maximal achievable PSS for PBS-buffer compared to acetonitrile was not significantly different.

Table 1 Thermal relaxation influence of solvent on azobenzene $\mathbf{2 6 c}$.

\begin{tabular}{ccccc}
\hline Entry & Solvent & \multicolumn{3}{c}{$\mathbf{t}_{1 / 2}$ [min] at $37^{\circ} \mathbf{C}$} \\
& Pure & $\begin{array}{c}\text { Air } \\
\text { purge }\end{array}$ & $\begin{array}{c}+5 \% \\
\text { piperidine }\end{array}$ \\
\hline $\mathbf{1}$ & $\mathrm{CH}_{3} \mathrm{CN}$ (new bottle) & 29.4 & - & 30.4 \\
$\mathbf{2}$ & $\mathrm{CH}_{3} \mathrm{CN}(2$ months old) & 24.4 & - & - \\
$\mathbf{3}$ & $\mathrm{CH}_{3} \mathrm{CN}(5$ months old) & 13.6 & - & 31.2 \\
$\mathbf{4}$ & $\mathrm{CH}_{3} \mathrm{CN}\left(0.0001 \% \mathrm{H}_{2} \mathrm{O}\right)$ & 28.1 & 29.1 & 29.8 \\
$\mathbf{5}$ & $\mathrm{CH}_{3} \mathrm{CN}\left(0.01 \% \mathrm{H}_{2} \mathrm{O}\right)$ & 29.7 & - & - \\
$\mathbf{6}$ & $\mathrm{CH}_{3} \mathrm{CN}\left(1.0 \% \mathrm{H}_{2} \mathrm{O}\right)$ & 29.1 & 12.9 & 31.5 \\
$\mathbf{7}$ & $\mathrm{CH}_{3} \mathrm{CN}\left(\right.$ basic $\left.\mathrm{Al}_{2} \mathrm{O}_{3}\right)$ & 28.8 & - & 32.1 \\
$\mathbf{8}$ & $\mathrm{CH}_{3} \mathrm{CN}\left(\right.$ neutral $\left.\mathrm{Al}_{2} \mathrm{O}_{3}\right)$ & 26.3 & - & 32.0 \\
$\mathbf{9}$ & $\mathrm{CH}_{3} \mathrm{CN}\left(\right.$ acidic $\left.\mathrm{Al}_{2} \mathrm{O}_{3}\right)$ & sec. & - & 29.9
\end{tabular}




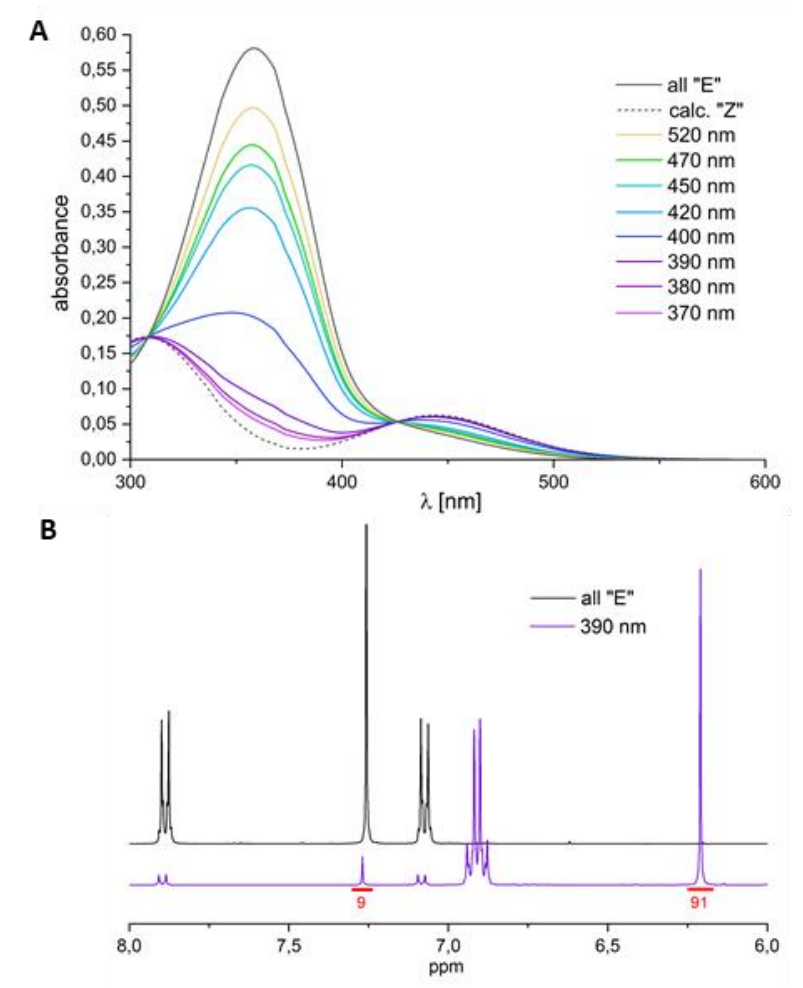

Figure 3. E- to Z-isomer ratio determination in the PSS of azobenzene 32c in methanol. A: UV-Vis spectra by using the method of E. Fischer ${ }^{41}$ indicate an $E$ - to Z-isomer ratio of $90 \%$ Z-isomer at $390 \mathrm{~nm}$ irradiation. B: $400 \mathrm{MHz}{ }^{1} \mathrm{H}-\mathrm{NMR}$ of dark (all "E') and irradiated (390 nm) azobenzene 32c in $\mathrm{MeOH}-\mathrm{d}_{4}$, indicating $91 \%$ Z-isomer at $390 \mathrm{~nm}$ irradiation.

Table 2 PSS of 32c in different solvents.

\begin{tabular}{|c|c|c|c|c|c|c|c|c|c|c|}
\hline \multirow{2}{*}{ Solvent } & \multicolumn{8}{|c|}{ PSS [\%] ${ }^{[a]}$} & \multirow{2}{*}{$\begin{array}{c}\lambda_{\max , \mathrm{E}} \\
{[\mathrm{nm}]}\end{array}$} & \multirow{2}{*}{$\begin{array}{c}\lambda_{\max , \mathrm{z}} \\
{[\mathrm{nm}]}\end{array}$} \\
\hline & $370 \mathrm{~nm}$ & $380 \mathrm{~nm}$ & $390 \mathrm{~nm}$ & $400 \mathrm{~nm}$ & $420 \mathrm{~nm}$ & $450 \mathrm{~nm}$ & $470 \mathrm{~nm}$ & $520 \mathrm{~nm}$ & & \\
\hline PBS $^{[b]}$ & 96 & 94 & 90 & 71 & 49 & 36 & 30 & 18 & 360 & 439 \\
\hline $\mathrm{CH}_{3} \mathrm{CN}$ & 97 & 95 & 91 & 72 & 44 & 33 & 29 & 18 & 360 & 442 \\
\hline $\mathrm{CH}_{3} \mathrm{CN}^{[\mathrm{c}]}$ & 97 & 95 & 91 & 72 & 45 & 34 & 28 & 18 & 360 & 442 \\
\hline $\mathrm{CHCl}_{3}$ & 95 & 94 & 91 & 73 & 48 & 35 & 29 & 18 & 361 & 447 \\
\hline $\mathrm{MeOH}$ & 96 & 94 & 90 & 69 & 41 & 30 & 25 & 15 & 358 & 443 \\
\hline toluene & 96 & 96 & 92 & 73 & 44 & 31 & 25 & 14 & 362 & 448 \\
\hline
\end{tabular}

[a] amount of $Z$-isomer after irradiation at referred wavelength. [b] $1 x P B S$ buffer $/ \mathrm{CH}_{3} \mathrm{CN}, 2: 1$. [c] $\mathrm{CH}_{3} \mathrm{CN}+0.5 \%$ piperidine.

Acetonitrile was used to compare the PSS ratio of all synthesized azobenzenes overall, as thermal relaxation of some azobenzenes was very fast in PBS-buffer (Table 3, PSS in PBS: Table S1). The absorption maxima $\left(\lambda_{\max }\right.$ ) belonging to the strong $\pi \rightarrow \pi^{*}$ band are given for the solvent in which the PSS is measured. Absorption spectra of all azobenzenes are depicted in the ESI. 
Table 3 PSS and photophysical data of azobenzenes.

\begin{tabular}{|c|c|c|c|c|c|c|c|c|c|c|c|c|}
\hline \multirow{2}{*}{ Entry } & \multicolumn{8}{|c|}{ PSS [\%] $]^{[a]}$} & \multirow{2}{*}{$\begin{array}{l}\lambda_{\max } \\
{[\mathrm{nm}]}\end{array}$} & \multirow{2}{*}[\mathrm{l}\cdot\mathrm{mol}^{-1}\cdot\mathrm{cm}^{-1}]{} & \multirow{2}{*}{$\begin{array}{c}\mathbf{t}_{1 / 2}^{[\mathrm{b}]} \text { in } \\
\mathrm{CH}_{3} \mathrm{CN}^{[\mathrm{c}]} \\
{[\mathrm{h}]}\end{array}$} & \multirow{2}{*}{$\begin{array}{c}\mathbf{t}_{1 / 2}{ }^{[\mathrm{b}]} \text { in } \\
\mathrm{PBS}^{[\mathrm{d}]} \\
{[\mathrm{min}]} \\
\end{array}$} \\
\hline & $370 \mathrm{~nm}$ & $380 \mathrm{~nm}$ & $390 \mathrm{~nm}$ & $400 \mathrm{~nm}$ & $420 \mathrm{~nm}$ & $450 \mathrm{~nm}$ & $470 \mathrm{~nm}$ & $520 \mathrm{~nm}$ & & & & \\
\hline 13 & - & - & - & - & - & - & - & - & 350 & 23800 & fast & fast \\
\hline 14 & - & - & - & - & - & - & - & - & 355 & 21900 & fast & fast \\
\hline 15 & - & - & - & - & - & - & - & - & 406 & 24000 & fast & fast \\
\hline $16 a$ & - & - & - & - & - & - & - & - & 359 & 16500 & fast & fast \\
\hline $16 b$ & - & - & - & - & - & - & - & - & 376 & 19000 & fast & fast \\
\hline $16 c$ & - & - & - & - & - & - & - & - & 384 & 20000 & fast & fast \\
\hline $17 a$ & - & - & - & - & - & - & - & - & 390 & 23000 & fast & fast \\
\hline $17 b$ & - & - & - & - & - & - & - & - & 464 & 19800 & fast & fast \\
\hline 18 & - & - & - & - & - & - & - & - & 473 & 27300 & fast & fast \\
\hline $19 b$ & - & - & - & - & - & - & - & - & 394 & 16500 & fast & fast \\
\hline $19 c$ & - & - & - & - & - & - & - & - & 401 & 24100 & fast & fast \\
\hline 20 & - & - & - & - & - & - & - & - & 515 & 19100 & fast & fast \\
\hline $21 a$ & - & - & - & - & - & - & - & - & 362 & 24600 & fast & fast \\
\hline $21 b$ & - & - & - & - & - & - & - & - & 375 & 17300 & fast & fast \\
\hline $21 c$ & - & - & - & - & - & - & - & - & 350 & 23800 & fast & fast \\
\hline 21d & - & - & - & - & - & - & - & - & 458 & 28600 & fast & fast \\
\hline $21 e$ & - & - & - & - & - & - & - & - & 458 & 28300 & fast & fast \\
\hline $22 a$ & - & - & - & - & - & - & - & - & 494 & 17400 & fast & fast \\
\hline $22 b$ & - & - & - & - & - & - & - & - & 517 & 18000 & fast & fast \\
\hline $22 c$ & - & - & - & - & - & - & - & - & 519 & 25200 & fast & fast \\
\hline 22d & - & - & - & - & - & - & - & - & 517 & 23000 & fast & fast \\
\hline 23 & - & - & - & - & - & - & - & - & 469 & 26300 & fast & fast \\
\hline 24 & 95 & 96 & 95 & 84 & 59 & 41 & 33 & 19 & 372 & 22100 & 21.7 & 132 \\
\hline 25 & 96 & 97 & 96 & 85 & 62 & 42 & 32 & 18 & 373 & 22600 & 16.3 & 204 \\
\hline $26 a$ & - & - & - & - & - & - & - & - & 470 & 20900 & fast & fast \\
\hline $26 b$ & 68 & 84 & - & 93 & 87 & 77 & 66 & 19 & 409 & 24700 & 2.36 & fast \\
\hline $26 c$ & 86 & 92 & 94 & 91 & 79 & 63 & 57 & 15 & 392 & 26200 & 4.05 & 0.065 \\
\hline $26 d$ & 82 & 90 & 93 & 92 & 83 & 68 & 62 & 22 & 396 & 27300 & 3.58 & 0.046 \\
\hline $26 e$ & 97 & 96 & 93 & 71 & 41 & 31 & 27 & 17 & 361 & 25700 & 2.15 & 144 \\
\hline $26 f$ & 97 & 95 & 87 & 55 & 32 & 28 & 28 & 24 & 351 & 25300 & 12.5 & 834 \\
\hline 27 & 96 & 89 & 78 & 44 & 30 & 29 & 31 & 28 & 345 & 20100 & 17,03 & slow \\
\hline 28 & - & - & - & - & - & - & - & - & 408 & 24000 & fast & fast \\
\hline $29 a$ & 97 & 95 & 91 & 72 & 45 & 34 & 28 & 18 & 360 & 22100 & 37.0 & 313 \\
\hline $29 b$ & 96 & 95 & - & 77 & 52 & 35 & 29 & 18 & 369 & 21300 & 43.1 & 314 \\
\hline $29 c$ & - & - & - & - & - & - & - & - & 390 & 27300 & fast & fast \\
\hline 29d & 96 & 95 & 93 & 77 & 44 & 29 & 24 & 13 & 364 & 25700 & 37.3 & 216 \\
\hline $29 e$ & 94 & 95 & 93 & 81 & 48 & 35 & 33 & 31 & 369 & 23000 & 43.0 & 330 \\
\hline $29 f$ & 91 & 92 & 91 & 82 & 62 & 44 & 34 & 18 & 368 & 22000 & 24.7 & 245 \\
\hline $30 a$ & 88 & 91 & 92 & 87 & 71 & 50 & 38 & 25 & 389 & 20900 & 0.63 & 9.45 \\
\hline $30 b$ & 86 & 92 & 93 & 90 & 78 & 62 & 49 & 32 & 397 & 20100 & 0.71 & 8.46 \\
\hline $30 c$ & 78 & 77 & 75 & 59 & 49 & 44 & 43 & 44 & 388 & 20300 & 5.53 & 256 \\
\hline $30 \mathrm{~d}$ & 84 & 90 & 92 & 91 & 84 & 72 & 59 & 40 & 408 & 21300 & 0.63 & 6.71 \\
\hline 31 & 77 & 82 & 80 & 61 & 41 & 35 & 40 & 55 & 370 & 26800 & slow & slow \\
\hline $32 a$ & 99 & 96 & - & 68 & 37 & 30 & 25 & 15 & 354 & 26000 & 33.3 & 231 \\
\hline $32 b$ & 98 & 98 & - & 81 & 51 & 34 & 26 & 16 & 368 & 20100 & 35.4 & 126 \\
\hline $32 \mathrm{c}$ & 97 & 95 & 91 & 72 & 45 & 34 & 28 & 18 & 360 & 22100 & 37.0 & 313 \\
\hline 32d & 97 & 95 & - & 69 & 44 & 34 & 30 & 20 & 360 & 19800 & 76.5 & 116 \\
\hline $32 e$ & 97 & 95 & - & 72 & 43 & 33 & 30 & 23 & 360 & 27200 & 53.2 & 322 \\
\hline $32 f$ & 97 & 95 & - & 72 & 49 & 36 & 31 & 21 & 362 & 21600 & 35.3 & 17.7 \\
\hline $33 a$ & 94 & 96 & 96 & 89 & 72 & 50 & 30 & 5 & 384 & 24500 & 0.46 & 17.2 \\
\hline $33 b$ & 90 & 93 & 93 & 86 & 71 & 51 & 38 & 19 & 383 & 19100 & 0.56 & 26.8 \\
\hline
\end{tabular}

[a] amount of $Z$-isomer after irradiation at referred wavelength. [b] "fast" means $\mathrm{t}_{1 / 2}<1 \mathrm{~s}$, "slow" means $\mathrm{t}_{1 / 2}>72 \mathrm{~h}$. [c] measured at $20{ }^{\circ} \mathrm{C}$ in $\mathrm{CH} \mathrm{H}_{3} \mathrm{CN}+$ $0.5 \%$ piperidine. [d] measured at $37^{\circ} \mathrm{C}$ in PBS-buffer/ $\mathrm{CH}_{3} \mathrm{CN}(2: 1)$ mixture. 
Table 3 continued

\begin{tabular}{|c|c|c|c|c|c|c|c|c|c|c|c|c|}
\hline \multirow{2}{*}{ Entry } & \multicolumn{8}{|c|}{ PSS [\%] ${ }^{[a]}$} & \multirow{2}{*}{$\begin{array}{l}\lambda_{\max } \\
{[\mathrm{nm}]}\end{array}$} & \multirow{2}{*}[\mathrm{l}\cdot\mathrm{mol}^{-1}\cdot\mathrm{cm}^{-1}]{} & \multirow{2}{*}{$\begin{array}{c}\mathbf{t}_{1 / 2}^{[\mathrm{b}]} \text { in } \\
\mathrm{CH}_{3} \mathrm{CN}^{[\mathrm{c}]} \\
{[\mathrm{h}]}\end{array}$} & \multirow{2}{*}{$\begin{array}{l}\mathbf{t}_{1 / 2}{ }^{[\mathrm{b}]} \text { in } \\
\mathrm{PBS}^{[\mathrm{d}]} \\
{[\mathrm{min}]}\end{array}$} \\
\hline & $370 \mathrm{~nm}$ & $380 \mathrm{~nm}$ & $390 \mathrm{~nm}$ & $400 \mathrm{~nm}$ & $420 \mathrm{~nm}$ & $450 \mathrm{~nm}$ & $470 \mathrm{~nm}$ & $520 \mathrm{~nm}$ & & & & \\
\hline $34 c$ & 94 & 95 & - & 84 & 58 & 38 & 27 & 13 & 374 & 20600 & 6.25 & 60.2 \\
\hline 34d & 97 & 97 & 94 & 76 & 48 & 31 & 27 & 19 & 368 & 24200 & 6.78 & 82.2 \\
\hline $34 \mathrm{e}$ & 93 & 93 & - & 79 & 54 & 35 & 28 & 18 & 371 & 26400 & 9.81 & 23.1 \\
\hline 35 & 85 & 87 & 87 & 81 & 74 & 68 & 64 & 46 & 361 & 17100 & 1.74 & 86.7 \\
\hline $36 a$ & 61 & 71 & - & 86 & 85 & 78 & 67 & 26 & 419 & 26800 & 1.12 & fast \\
\hline $36 b$ & 56 & 68 & - & 85 & 84 & 76 & 65 & 27 & 420 & 25300 & 1.23 & fast \\
\hline $37 c$ & 55 & 67 & - & 89 & 86 & 78 & 66 & 26 & 421 & 22400 & 1.25 & fast \\
\hline $37 d$ & 72 & 84 & - & 90 & 81 & 66 & 49 & 10 & 404 & 24300 & 3.24 & 0.108 \\
\hline $37 e$ & 72 & 84 & - & 92 & 87 & 74 & 52 & 16 & 409 & 21000 & 2.31 & 0.061 \\
\hline $38 b$ & 71 & 86 & - & 93 & 88 & 78 & 61 & 17 & 408 & 24400 & 1.24 & fast \\
\hline $38 \mathrm{c}$ & 89 & 94 & 94 & 92 & 82 & 65 & 46 & 22 & 392 & 26200 & 3.10 & 0.069 \\
\hline $38 d$ & 84 & 92 & 94 & 92 & 84 & 66 & 50 & 26 & 395 & 26200 & 2.28 & 0.042 \\
\hline $39 a$ & 66 & 75 & - & 89 & 86 & 77 & 65 & 25 & 417 & 26600 & 0.70 & fast \\
\hline $39 b$ & 63 & 74 & - & 88 & 86 & 78 & 65 & 22 & 418 & 22700 & 0.74 & fast \\
\hline $40 a$ & 99 & 98 & - & 71 & 43 & 30 & 29 & 15 & 360 & 25000 & 19.9 & 171 \\
\hline $40 \mathrm{~b}$ & 97 & 97 & 93 & 85 & 60 & 39 & 30 & 13 & 372 & 25300 & 33.7 & 78.6 \\
\hline $40 c$ & 97 & 97 & 95 & 81 & 53 & 37 & 30 & 16 & 368 & 20800 & 17.1 & 193 \\
\hline $40 d$ & 99 & 98 & - & 80 & 56 & 40 & 34 & 26 & 369 & 22000 & 25.8 & 148 \\
\hline $40 f$ & 97 & 97 & - & 81 & 56 & 39 & 31 & 17 & 368 & 22900 & 25.8 & 12.4 \\
\hline 41c & 39 & 55 & - & 82 & 80 & 76 & 69 & 24 & 450 & 21300 & 0.11 & fast \\
\hline 41d & 69 & 80 & - & 90 & 86 & 77 & 67 & 31 & 414 & 28000 & 0.23 & 0.088 \\
\hline $41 e$ & 62 & 77 & - & 90 & 87 & 80 & 71 & 33 & 417 & 19300 & 0.22 & 0.062 \\
\hline $42 a$ & 93 & 97 & 97 & 90 & 75 & 51 & 32 & 7 & 386 & 21400 & 1.58 & 10.7 \\
\hline $42 b$ & 89 & 93 & 95 & 91 & 82 & 63 & 41 & 8 & 395 & 24700 & 0.91 & 8.10 \\
\hline $42 c$ & 92 & 95 & 95 & 91 & 78 & 62 & 46 & 24 & 393 & 20600 & 1.34 & 10.6 \\
\hline $42 e$ & 89 & 93 & 94 & 90 & 76 & 59 & 42 & 22 & 393 & 24100 & 1.03 & 3.51 \\
\hline 43 & 83 & 86 & 85 & 81 & 75 & 70 & 68 & 58 & 360 & 19800 & 4.86 & 85.3 \\
\hline $44 a$ & 97 & 97 & 95 & 81 & 53 & 37 & 30 & 16 & 368 & 20800 & 17.1 & 193 \\
\hline $44 c$ & - & - & - & - & - & - & - & - & 394 & 19300 & fast & fast \\
\hline $44 \mathrm{~g}$ & 90 & 89 & - & 70 & 50 & 35 & 30 & 21 & 367 & 21100 & 25.2 & 318 \\
\hline $45 a$ & 79 & 89 & 91 & 87 & 74 & 53 & 40 & 24 & 393 & 25500 & 0.50 & 4.75 \\
\hline $45 b$ & 68 & 86 & 90 & 90 & 83 & 70 & 57 & 31 & 409 & 23300 & 0.32 & 4.17 \\
\hline $45 c$ & 78 & 84 & 82 & 71 & 60 & 54 & 53 & 44 & 398 & 22000 & slow & 200 \\
\hline $45 d$ & 69 & 83 & 88 & 91 & 87 & 77 & 67 & 34 & 419 & 26000 & 0.52 & 3.49 \\
\hline $44 a$ & 97 & 97 & 95 & 81 & 53 & 37 & 30 & 16 & 368 & 20800 & 17.1 & 193 \\
\hline $44 c$ & - & - & - & - & - & - & - & - & 394 & 19300 & fast & fast \\
\hline $44 \mathrm{~g}$ & 90 & 89 & - & 70 & 50 & 35 & 30 & 21 & 367 & 21100 & 25.2 & 318 \\
\hline $45 a$ & 79 & 89 & 91 & 87 & 74 & 53 & 40 & 24 & 393 & 25500 & 0.50 & 4.75 \\
\hline $45 b$ & 68 & 86 & 90 & 90 & 83 & 70 & 57 & 31 & 409 & 23300 & 0.32 & 4.17 \\
\hline $45 c$ & 78 & 84 & 82 & 71 & 60 & 54 & 53 & 44 & 398 & 22000 & slow & 200 \\
\hline $45 d$ & 69 & 83 & 88 & 91 & 87 & 77 & 67 & 34 & 419 & 26000 & 0.52 & 3.49 \\
\hline
\end{tabular}

[a] amount of $Z$-isomer after irradiation at referred wavelength. [b] "fast" means $\mathrm{t}_{1 / 2}<1 \mathrm{~s}$, "slow" means $\mathrm{t}_{1 / 2}>72 \mathrm{~h}$. [c] measured at $20{ }^{\circ} \mathrm{C}$ in $\mathrm{CH}_{3} \mathrm{CN}+$ $0.5 \%$ piperidine. [d] measured at $37^{\circ} \mathrm{C}$ in PBS-buffer/ $\mathrm{CH}_{3} \mathrm{CN}(2: 1)$ mixture. 
Interesting trends are obtained for tuning photoswitch properties. In general, a more delocalized diazene provides a more redshifted $\pi \rightarrow \pi^{*}$ transition and faster thermal relaxation kinetics under physiological conditions. To investigate this property, a first set of compounds with para attached linkers was tuned by changing electron density in the "B"-ring. Electron withdrawing groups (EWGs) such as bromine ( $\rightarrow$ 26a) result in a push-pull system with red shifted absorption and short half-life $(<1 \mathrm{~s})$, compared to azobenzene. In contrast, substituents with electron donating groups (EDGs) like in the anilines 26b-d and $36 \mathbf{a}, \mathbf{b}$ only show a polarization of the diazene bond. A thermal relaxation half-life in the range of several seconds was observed for them. As expected, ${ }^{2,10,29}$ this measure led to a weaker redshift of the absorption maximum as for derivative 26a. By attaching functional groups with decreasing electron donating properties the thermal relaxation gets retarded, as illustrated by methylether $32 \mathrm{a}$ and amide 26e $\left(\tau_{3 / 2}=2-3 h\right)$ and imide $26 f\left(\tau_{1 / 2}=12-14 h\right)$. Thermal relaxation of derivative 27, without an EDG, occurs in the range of several days. Along with prolonged thermal relaxation rate, the absorption maximum is shifted to shorter wavelengths.

Hence, azobenzenes with very fast or very slow relaxation are easily accessed. However, obtaining azobenzenes with thermal relaxation in the minutes range in aqueous environment, as necessary for cell biology and photopharmacology, would be highly desirable. By implementing quinoline as "A"-ring motif (30a) a half-life of around 10 minutes was achieved, accompanied by a significant bathochromic shift of nearly $30 \mathrm{~nm}$ compared to the benzyl derivative 29a. Increasing the electron density even more by using a naphthalene core $(\mathbf{3 0 b})$ and by electron donating substituents (OMe, $\mathbf{3 0 d}$ ) this effect becomes more prominent. For derivative $30 \mathrm{c}$ the methoxy group in 8-position seemed to stabilize the $Z$-isomer. The resulting blueshift of the transitions however leads to an unfavorable PSS for this compound.

With an EDG in the ortho position (e. g. methoxy, amines), the Z-isomer gets destabilized. ${ }^{2,29,30}$ By introducing an amide in ortho position of 4,4'-substituted ethers, switches with relaxation rate in the minute range were obtained $(\mathbf{4 2 a}-\mathbf{c}, \mathbf{e})$. Thereby, the absorbance of the E-conjugated form was shifted to longer wavelengths by approximately $25 \mathrm{~nm}$. In addition, the amide structure was used to introduce an attachment point for potential ligands in ortho position. The general trends of the photophysical properties for ortho attached linkers by modifications at the "B"-ring were identical compared to the para attached linkers, like anilines $\mathbf{4 1 c}$-e compared to $\mathbf{3 8 b - d}$, or the methoxy substituted compounds $\mathbf{4 2 a - c , e}$ compared to 40 a-f. Hydrogen bond donors in the ortho position (e.g. compounds 23 or $29 \mathrm{c}$ ) destabilize the Z-configured diazene bond even more, resulting in a very short thermal half-life. ${ }^{42}$ Interestingly, multiple ortho substitutions were reported to interfere with bond, slowing down isomerization kinetics. ${ }^{2}$ Retarding thermal relaxation rate excessively creates persistent switches, approaching properties of a caged compound. In this set this was observed for weakly polarized diazene bonds obtained from removing one para substituent, shown for compounds 27 and $\mathbf{3 1 .}$ 
The design of any photoswitch needs to additionally consider interactions of the switch with the ligand and/or the target. ${ }^{20}$ In order to study such variations, e. g. in optojasps, ${ }^{5}$ derivatives with different steric demands and polarity are required, but with absorption and thermal relaxation properties unchanged. In this regard, a methoxy substitution in meta position to the diazene was having only minor influence on photophysical properties, as seen for monomethoxy compound 32a compared to trimethoxy compound 32c. Asymmetric substitution patterns in the " $B$ "-ring, such shown by compounds $\mathbf{3 2 b}$ or $\mathbf{3 6 a}$, have a twofold reduced thermal relaxation rate and a absorption maximum redshifted by $10 \mathrm{~nm}$, compared to their symmetric analogues $\mathbf{3 2 a}$ or $\mathbf{2 6 b}$. This applies also to meta substituents in the "A"-ring $(\mathbf{2 9 b}, \mathbf{d}, \mathbf{f})$.

The residue introduced by reductive amination to aniline $\mathbf{2 8}$ does not modify the absorption spectra significantly, allowing to modify this position $(36,39)$. Similarly, extension of the para-methoxy group also did not affect photophysical properties, as shown by compounds $\mathbf{3 2} \mathbf{d}$ and $\mathbf{3 2 f}$. However, depending on the polarity of the residue, the thermal relaxation time was modified $(\rightarrow \mathbf{3 2 f})$. The same was true for the free acid analogues compared to their corresponding esters, which generally relax faster. Furthermore, attachment of large ligands may also modify this property. For example, a significant increase in the thermal relaxation kinetics was observed for the switches attached to jasplakinolide analogues. ${ }^{5}$

A 4,4'-substitution with an EDG was an important factor for advantageous PSS ratios, because the band overlap of the $E$ - and Z-isomers UV spectra gets reduced. As a result, high ratios of over $95 \%$ of $Z$ isomer could be achieved for most derivatives through exposure to blue or violet light. For amines, the ratio in the PSS drops to around $90 \%$ of $Z$-isomer because of more strongly overlapping $\pi \rightarrow \pi^{*}$ and $\mathrm{n} \rightarrow \pi^{*}$ transitions. A similar trend is observed for ortho attachment, compared to the para isomers. In general, the achieved PSS ratios are sufficient for applications as photoswitches. ${ }^{26}$ Without a 4,4'substitution pattern, the ratio in the PSS dropped (e. g. 31). In general, the PSS observed for meta substituted compounds in the "B"-ring was minimal decreased. This applied also to meta modifications in the "A"-ring (29b-f) or to the attachment of a linker in meta position (25). Extension of the residues with additional groups or linkers did not influence the PSS (e. g. 32f).

\section{CONCLUSIONS}

A set of $>50$ azobenzenes with a wide range of thermal relaxation kinetics, steric demand, and varying polarity was synthesized. The influence of various symmetrical and asymmetrical substitutions at different positions on the photophysical properties was investigated, extending the knowledge of azobenzene photoswitches. ${ }^{2,29,30}$ This was achieved by establishing a reproducible UV-Vis characterization for thermal relaxation kinetics and by uniform determination of photostationary state ratios. Different electron donating groups in para position were found to polarize the diazene bond and 
lower the energy of the $\pi \rightarrow \pi^{*}$ transition, resulting in shortened relaxation rates and a red shifted absorption maximum. This polarization can be modulated by varying the electron density in the two aromatic rings providing half-life from several hours to milliseconds (Figure 4). Polar interactions from ortho substituents with the diazene bond reduce the half-life and strongly influence kinetics. On the other hand, modifications in the meta position only show marginal modulation on the photophysical properties. Furthermore, asymmetric substitution of the aromatic rings induces a slight bathochromic shift, to go along with a reduced half-life. In general, photophysical properties are not affected by extension of the residues, but may depend on polarity and compound size (Figure 4).

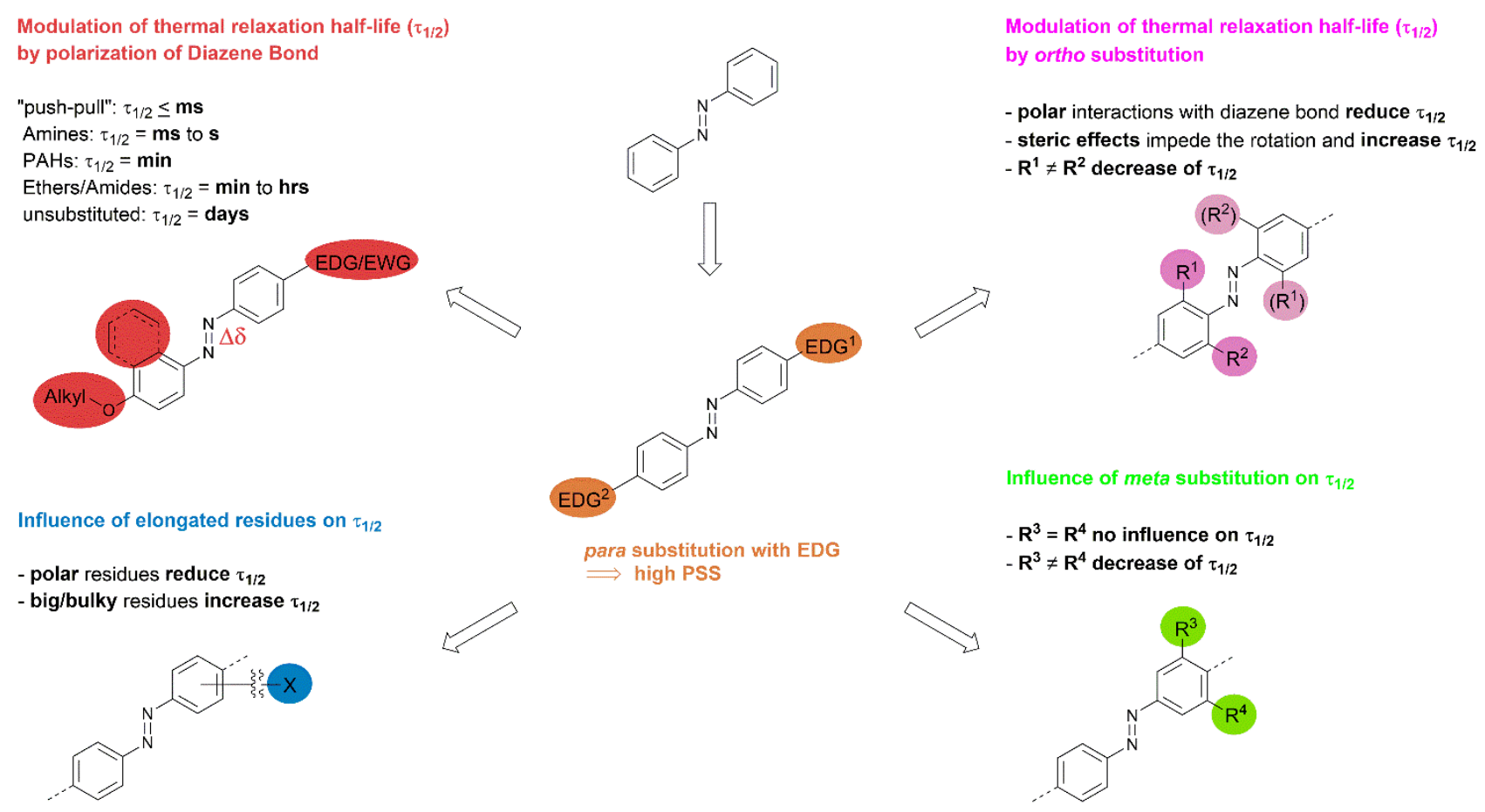

Figure 4. General influence of azobenzene substitution patterns on thermal relaxation half-life.

Individual photoswitch design is certainly depending on the individual activities of the $Z$ - and $E$-isomers, as well as their target engagement and pharmacokinetics. The extensive range of the thermal half-life that is now available for azobenzene photoswitches offers diverse application possibilities in biology and pharmacology on different time scales (sub-second to days), fitting to neuronal and cellular signaling, cell division, or tissue development. Positions to be modified without substantial property change were identified. Therefore, this panel of compounds may provide cues and structural leads for future "azo extension" strategies of ligands, or for linking designs in proteins, peptides, or other biomolecules. The molecular structures also allow further modifications while maintaining excellent photophysical properties. These data expand the understanding of the molecular structure of azobenzenes and allow the improvement of azobenzene-based systems in biological applications. 


\section{Conflicts of interest}

There are no conflicts to declare.

\section{Acknowledgement}

We thank Dr. Oliver Thorn-Seshold (LMU Munich) for discussions and Dr. Peter Bellstedt (NMR Platform Jena) for excellent support. This work benefitted in part from an equipment grant of the DFG (INST 275/442-1 FUGG) that is kindly acknowledged.

\section{Notes and references}

1 K. Hüll, J. Morstein and D. Trauner, Chem. Rev., 2018, 118, 10710.

2 R. J. Mart and R. K. Allemann, Chem. Commun., 2016, 52, 12262.

P. Paoletti, G. C. R. Ellis-Davies and A. Mourot, Nat. Rev. Neurosci., 2019, 20, 514.

M. Borowiak, W. Nahaboo, M. Reynders, K. Nekolla, P. Jalinot, J. Hasserodt, M. Rehberg, M.

Delattre, S. Zahler, A. Vollmar, D. Trauner and O. Thorn-Seshold, Cell, 2015, 162, 403.

M. Borowiak, F. Küllmer, F. Gegenfurtner, S. Peil, V. Nasufovic, S. Zahler, O. Thorn-Seshold, D. Trauner and H.-D. Arndt, J. Am. Chem. Soc., 2020, 142, 9240.

6 C. Falenczyk, M. Schiedel, B. Karaman, T. Rumpf, N. Kuzmanovic, M. Grøtli, W. Sippl, M. Jung and B. König, Chem. Sci., 2014, 5, 4794.

7 C. Brieke and A. Heckel, Chem. Eur. J., 2013, 19, 15726.

8 S. Herre, T. Schadendorf, I. Ivanov, C. Herrberger, W. Steinle, K. Rück-Braun, R. Preissner and H. Kuhn, ChemBioChem, 2006, 7, 1089.

9 J. Volarić, W. Szymanski, N. A. Simeth and B. L. Feringa, Chem. Soc. Rev., 2021, 50, 12377.

10 A. A. Beharry and G. A. Woolley, Chem. Soc. Rev., 2011, 40, 4422.

H. Rau, Angew. Chem., 1973, 85, 248; Angew. Chem. Int. Ed., 1973, $12,224$.

H. Fliegl, A. Köhn, C. Hättig and R. Ahlrichs, J. Am. Chem. Soc., 2003, 125, 9821.

A. R. Dias, M. E. Minas Da Piedade, J. A. Martinho Simões, J. A. Simoni, C. Teixeira, H. P.

Diogo, Y. Meng-Yan and G. Pilcher, J. Chem. Thermodyn., 1992, 24, 439.

F. Hamon, F. Djedaini-Pilard, F. Barbot and C. Len, Tetrahedron, 2009, 65, 10105.

J. Griffiths, Chem. Soc. Rev., 1972, 1, 481.

E. Merino and M. Ribagorda, Beilstein J Org. Chem., 2012, 8, 1071.

H. D. Bandara and S. C. Burdette, Chem. Soc. Rev., 2012, 41, 1809.

I. Conti, M. Garavelli and G. Orlandi, J. Am. Chem. Soc., 2008, 130, 5216.

N. J. Hauwert, T. A. M. Mocking, D. Da Costa Pereira, A. J. Kooistra, L. M. Wijnen, G. C. M. Vreeker, E. W. E. Verweij, A. H. De Boer, M. J. Smit, C. De Graaf, H. F. Vischer, I. J. P. de Esch, M. Wijtmans and R. Leurs, J. Am. Chem. Soc., 2018, 140, 4232.

S. Pospich, F. Küllmer, V. Nasufovic, J. Funk, A. Belyy, P. Bieling, H.-D. Arndt, S. Raunser, Angew. Chem., 2021, 133, 8760; Angew. Chem. Int. Ed., 2021, 60, 8678.

M. A. Kienzler, A. Reiner, E. Trautman, S. Yoo, D. Trauner and E. Y. Isacoff, J. Am. Chem. Soc., 2013, 135, 17683.

Y. Kim, J. A. Phillips, H. Liu, H. Kang and W. Tan, Proc. Natl. Acad. Sci. U.S.A., 2009, 106, 6489.

L. Albert and O. Vázquez, Chem. Commun., 2019, 55, 10192.

W. Szymański, J. M. Beierle, H. A. Kistemaker, W. A. Velema and B. L. Feringa, Chem. Rev., 2013, 113, 6114.

25 P. Leippe and J. A. Frank, Curr. Opin. Struct. Biol., 2019, 57, 23.

28 J. García-Amorós and D. Velasco, Beilstein J. Org. Chem., 2012, 8, 1003. 
D. Cameron and S. Eisler, J. Phys. Org. Chem., 2018, 31, e3858.

30 M. Dong, A. Babalhavaeji, S. Samanta, A. A. Beharry and G. A. Woolley, Acc. Chem. Res., 2015, 48, 2662.

31 D. Bléger and S. Hecht, Angew. Chem. 2015, 127, 11494; Angew. Chem. Int. Ed. 2015, 54, 11338.

32 S. Samanta, A. A. Beharry, O. Sadovski, T. M. McCormick, A. Babalhavaeji, V. Tropepe and G. A. Woolley, J. Am. Chem. Soc., 2013, 135, 9777.

33 V. Arkhipova, H. Fu, M. W. H. Hoorens, G. Trinco, L. N. Lameijer, E. Marin, B. L. Feringa, G. J. Poelarends, W. Szymanski, D. J. Slotboom and A. Guskov, J. Am. Chem. Soc., 2021, 143, 1513.

34 E. Merino, Chem. Soc. Rev., 2011, 40, 3835.

35 E. M. Doherty, C. Fotsch, Y. Bo, P. P. Chakrabarti, N. Chen, N. Gavva, N. Han, M. G. Kelly, J. Kincaid, L. Klionsky, Q. Liu, V. I. Ognyanov, R. Tamir, X. Wang, J. Zhu, M. H. Norman and J. J. S. Treanor, J. Med. Chem., 2005, 48, 71. M. J. Crookes and D. L. H. Williams, J. Chem. Soc. Perkin Trans. 2, 1989, 1319. F. Paul, J. Patt and J. F. Hartwig, J. Am. Chem. Soc., 1994, 116, 5969. A. S. Guram and S. L. Buchwald, J. Am. Chem. Soc., 1994, 116, 7901. Z. Ahmed, A. Siiskonen, M. Virkki and A. Priimagi, Chem. Commun., 2017, 53, 12520. M. Dong, A. Babalhavaeji, M. J. Hansen, L. Kálmán and G. A. Woolley, Chem. Commun., 2015, 51, 12981.

41 E. Fischer, J. Phys. Chem., 1967, 71, 3704.

42 D. Zheng, Y. Gu, X. Li, L. Zhang, W. Zhao and J. Ma, J. Chem. Inf. Model., 2019, 59, 2110. 
Property-selected Asymmetric Azobenzenes for Photoswitchable Ligands ${ }^{\dagger}$ Florian Küllmer, Lucas Gregor and Hans-Dieter Arndt

- Supplemental Information - 


\section{TABLE OF CONTENTS}

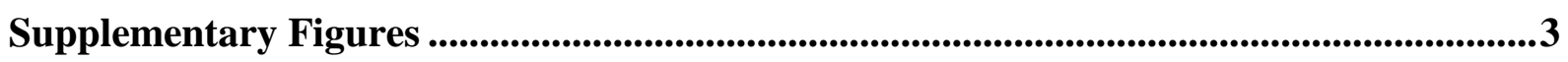

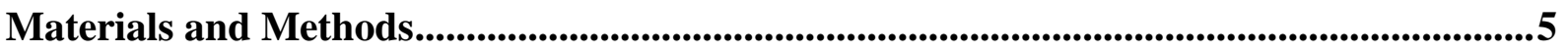

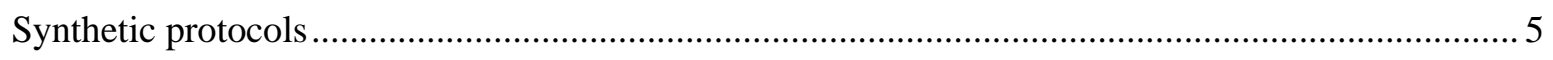

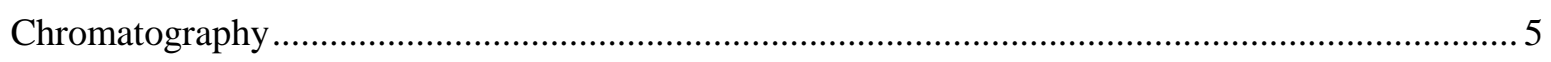

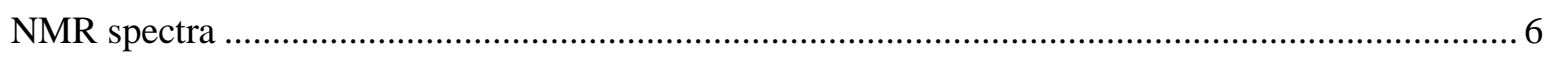

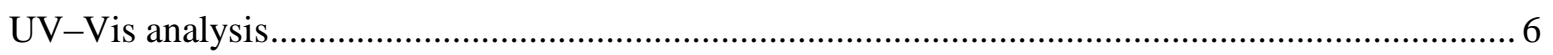

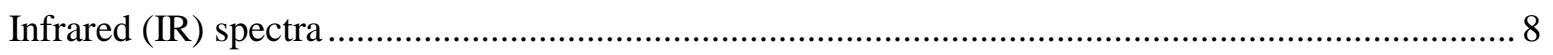

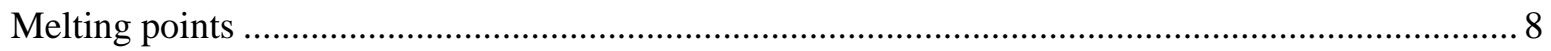

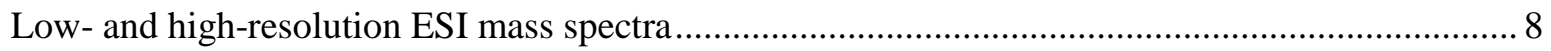

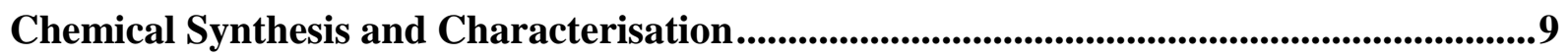

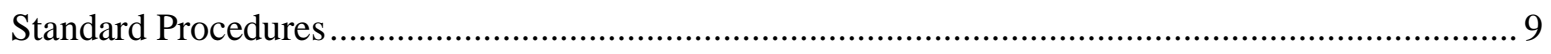

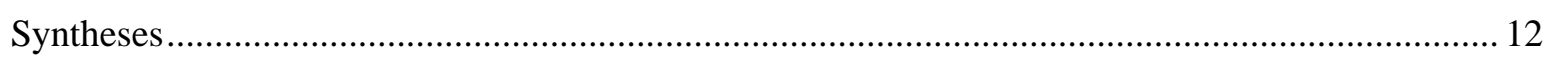

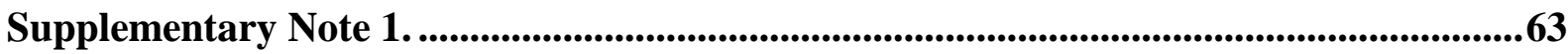

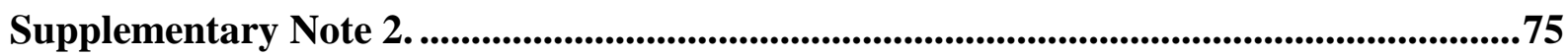

Supplemental References 


\section{Supplementary Figures}
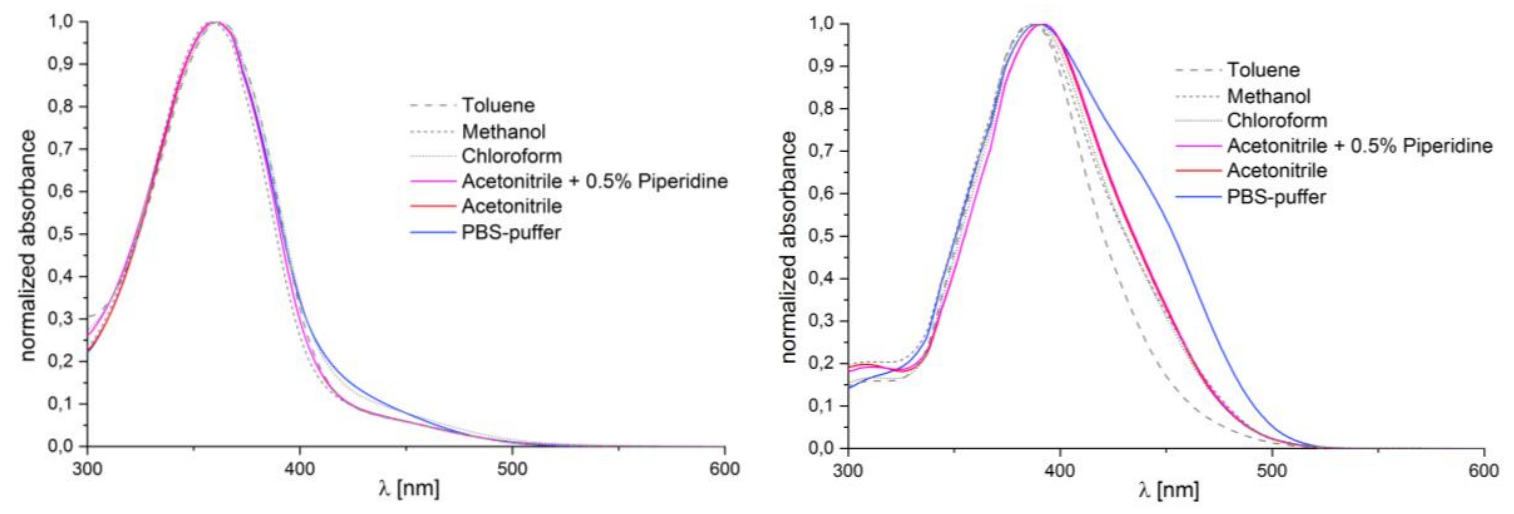

Figure S1. UV-Vis spectra of 32c (left) and 26c (right) in different solvents. 
Table S1. PSS of azobenzenes in PBS-buffer. ${ }^{[a]}$

\begin{tabular}{|c|c|c|c|c|c|c|c|c|c|}
\hline \multirow{2}{*}{ Entry } & \multicolumn{8}{|c|}{ PSS [\%] $]^{[b]}$} & \multirow{2}{*}{$\begin{array}{l}\lambda_{\max } \\
{[\mathrm{nm}]}\end{array}$} \\
\hline & $370 \mathrm{~nm}$ & $380 \mathrm{~nm}$ & $390 \mathrm{~nm}$ & $400 \mathrm{~nm}$ & $420 \mathrm{~nm}$ & $450 \mathrm{~nm}$ & $470 \mathrm{~nm}$ & $520 \mathrm{~nm}$ & \\
\hline 24 & 93 & 93 & 91 & 80 & 62 & 46 & 35 & 16 & 373 \\
\hline 25 & 96 & 94 & 88 & 67 & 44 & 32 & 26 & 15 & 370 \\
\hline $26 \mathrm{e}$ & 96 & 94 & 88 & 67 & 44 & 32 & 26 & 15 & 360 \\
\hline $26 f$ & 93 & 87 & 79 & 46 & 32 & 28 & 26 & 21 & 352 \\
\hline $29 a$ & 96 & 94 & 90 & 71 & 49 & 36 & 30 & 18 & 360 \\
\hline $29 b$ & 95 & 96 & - & 79 & 55 & 37 & 28 & 13 & 369 \\
\hline $29 d$ & 95 & 94 & 89 & 71 & 48 & 34 & 27 & 15 & 363 \\
\hline $29 \mathrm{e}$ & 93 & 92 & 88 & 72 & 50 & 40 & 37 & 33 & 368 \\
\hline $29 f$ & 81 & 86 & 83 & 71 & 54 & 41 & 32 & 16 & 368 \\
\hline $30 a$ & 86 & 90 & 90 & 82 & 66 & 49 & 38 & 24 & 388 \\
\hline $30 b$ & 85 & 90 & 92 & 90 & 80 & 66 & 55 & 32 & 400 \\
\hline $30 c$ & 77 & 78 & 75 & 64 & 54 & 51 & 50 & 45 & 388 \\
\hline $30 d$ & 83 & 88 & 90 & 88 & 81 & 70 & 59 & 30 & 407 \\
\hline $32 a$ & 97 & 94 & - & 60 & 41 & 29 & 22 & 10 & 356 \\
\hline $32 b$ & 94 & 95 & - & 81 & 61 & 42 & 29 & 13 & 368 \\
\hline $32 c$ & 96 & 94 & 90 & 71 & 49 & 36 & 30 & 18 & 360 \\
\hline $32 d$ & 93 & 91 & - & 69 & 46 & 34 & 27 & 14 & 361 \\
\hline $32 e$ & 95 & 94 & - & 72 & 47 & 34 & 27 & 14 & 360 \\
\hline $32 f$ & 94 & 92 & - & 70 & 49 & 36 & 28 & 16 & 361 \\
\hline $33 b$ & 89 & 89 & 85 & 74 & 57 & 42 & 35 & 20 & 368 \\
\hline $33 a$ & 96 & 96 & 94 & 86 & 71 & 53 & 39 & 14 & 380 \\
\hline $34 b$ & 91 & 94 & 94 & 84 & 67 & 50 & 36 & 15 & 381 \\
\hline $34 d$ & 94 & 94 & 91 & 74 & 48 & 32 & 25 & 16 & 368 \\
\hline $34 \mathrm{e}$ & 92 & 93 & - & 74 & 54 & 39 & 31 & 18 & 373 \\
\hline 35 & 82 & 82 & 78 & 64 & 47 & 39 & 38 & 34 & 360 \\
\hline $40 a$ & 99 & 97 & - & 74 & 51 & 35 & 25 & 10 & 361 \\
\hline $40 \mathrm{~b}$ & 93 & 93 & 91 & 79 & 61 & 40 & 26 & 8 & 370 \\
\hline $40 c$ & 94 & 95 & 91 & 75 & 55 & 40 & 30 & 15 & 365 \\
\hline $40 \mathrm{~d}$ & 96 & 96 & - & 81 & 62 & 46 & 35 & 18 & 368 \\
\hline $40 f$ & 96 & 95 & - & 76 & 57 & 42 & 33 & 17 & 366 \\
\hline $42 a$ & 94 & 95 & 93 & 84 & 69 & 51 & 36 & 12 & 380 \\
\hline $42 b$ & 90 & 93 & 93 & 87 & 75 & 61 & 45 & 15 & 390 \\
\hline $42 c$ & 92 & 94 & 93 & 86 & 72 & 57 & 44 & 20 & 384 \\
\hline $42 e$ & 91 & 93 & 92 & 83 & 70 & 56 & 43 & 17 & 384 \\
\hline 43 & 81 & 80 & 77 & 67 & 55 & 49 & 48 & 40 & 360 \\
\hline $44 a$ & 94 & 95 & 91 & 75 & 55 & 40 & 30 & 15 & 365 \\
\hline $44 \mathrm{~g}$ & 90 & 91 & - & 80 & 60 & 41 & 28 & 7 & 373 \\
\hline $45 a$ & 82 & 88 & 89 & 83 & 68 & 52 & 41 & 24 & 392 \\
\hline $45 b$ & 78 & 85 & 88 & 87 & 79 & 67 & 58 & 32 & 406 \\
\hline $45 c$ & 68 & 76 & 74 & 66 & 57 & 53 & 53 & 45 & 394 \\
\hline $45 d$ & 75 & 83 & 87 & 88 & 83 & 73 & 64 & 32 & 415 \\
\hline
\end{tabular}

[a] measured at $37^{\circ} \mathrm{C}$ in PBS-buffer/ $\mathrm{CH}_{3} \mathrm{CN}(2: 1)$ mixture. [b] amount of $Z$-isomer after irradiation at referred wavelength. 


\section{Materials and Methods}

\section{Synthetic protocols}

The Boc-protected anilines $\mathbf{5}$ and 9, mono protected catechol 7b and phenol $\mathbf{8}$ were synthesized using standard procedures. ${ }^{1}$ TBS-protected catechol 10b was synthesised following literature procedure of Stein. ${ }^{2}$ Naphtols $\mathbf{1 1 c}$ and $\mathbf{1 1 d}$ were synthesized according to literature procedures of Deyris ${ }^{3}$ and Ramkumar. ${ }^{4}$ Oxazine 4 was synthesised according to literature procedures of Mofford ${ }^{5}$ and Doherty. ${ }^{6}$ All other reagents and solvents were purchased from vendors (Acros, Alfa Aesar, ChemPur, Fluka, Fluorochem, Sigma-Aldrich, Strem Chemicals, TCI Europe N.V., VWR) and were used without further purification unless noted otherwise.

All solvents, when not purchased in suitable purity or dryness, were distilled using standard methods, ${ }^{7}$ or suitable dehydration procedures: tetrahydrofuran (THF) was distilled under a $\mathrm{N}_{2}$ atmosphere from $\mathrm{Na}$ /benzophenone before use; dichloromethane $\left(\mathrm{CH}_{2} \mathrm{Cl}_{2}\right)$ was distilled under a $\mathrm{N}_{2}$ atmosphere from $\mathrm{CaH}_{2}$ before use. Other anhydrous solvents such as toluene and dimethylformamide (DMF) were obtained in HPLC quality and passed through a solvent purification system equipped with $\mathrm{Al}_{2} \mathrm{O}_{3}$ (toluene) or molecular sieves $3 \AA$ (DMF, Pure Solv, Innovative Technology, Inc., USA) by applying $\mathrm{N}_{2}$ overpressure immediately before use. Commercial-grade distilled ( $\mathrm{Pr})_{2} \mathrm{NEt}(99 \%)$ was used without additional distillation. The petroleum ether used had a boiling range of $40-60^{\circ} \mathrm{C}$. Phosphate buffer $(\mathrm{pH}=7)$ was prepared by dissolving $\mathrm{Na}_{3} \mathrm{PO}_{4} \times 12 \mathrm{H}_{2} \mathrm{O}(54.8 \mathrm{~g}, 0.14 \mathrm{~mol})$ und $\mathrm{NaH}_{2} \mathrm{PO}_{4}(42.7 \mathrm{~g}, 0.36 \mathrm{~mol})$ in water $(1.0 \mathrm{~L})$. Deionized water was used for all experiments.

\section{Chromatography}

Reaction progress was monitored by TLC on precoated, Merck Silica gel 60 F254 aluminabacked plates. TLC chromatograms were first visualized by UV irradiation at $254 \mathrm{~nm}$ or 320 $\mathrm{nm}$, followed by staining with aqueous $\mathrm{KMnO}_{4}\left(2 \mathrm{~g} \mathrm{KMnO}_{4}, 13.2 \mathrm{~g} \mathrm{~K}_{2} \mathrm{CO}_{3}, 165 \mathrm{mg} \mathrm{NaOH}\right.$, $200 \mathrm{~mL} \mathrm{H} 2 \mathrm{O}$ ) or ceric ammonium molybdate solution $\left(0.5 \mathrm{~g}\right.$ of $\mathrm{Ce}\left(\mathrm{NH}_{4}\right)_{4}\left(\mathrm{SO}_{4}\right)_{4} \times 2 \mathrm{H}_{2} \mathrm{O}, 12 \mathrm{~g}$ $\left(\mathrm{NH}_{4}\right)_{6} \mathrm{Mo}_{7} \mathrm{O}_{24} \times 4 \mathrm{H}_{2} \mathrm{O}, 15 \mathrm{~mL} \mathrm{H}_{2} \mathrm{SO}_{4}, 235 \mathrm{~mL} \mathrm{H} \mathrm{O}_{2} \mathrm{O}$ ) followed by gentle heating on air for detection. Primary and secondary amines were detected with ninhydrin (6\% in EtOH). 
Flash chromatography was performed using silica gel $\left(\mathrm{SiO}_{2}\right.$, particle size $\left.40-63 \mu \mathrm{m}\right)$ purchased from Macherey \& Nagel, Düren (Germany) under a pressure of $0.3-0.5$ bar. Preparative HPLC purification was performed on a Varian system consisting of a ProStar 215 (pump), ProStar 340 (UV/VIS-detector) and a ProStar 701 (collector). A VP250/21 Nucleodur C18 Gravity $5 \mu \mathrm{m}$ column was used. A gradient of Water and Acetonitrile was applied as mobile phase.

\section{NMR spectra}

${ }^{1} \mathrm{H}$ - and ${ }^{13} \mathrm{C}-\mathrm{NMR}$ spectra were recorded on Bruker Avance I $250\left(250 \mathrm{MHz}\left({ }^{1} \mathrm{H}\right)\right.$ and $63 \mathrm{~Hz}$ $\left.\left({ }^{13} \mathrm{C}\right)\right)$, Bruker Fourier $300\left(300 \mathrm{MHz}\left({ }^{1} \mathrm{H}\right)\right.$ and $\left.75 \mathrm{~Hz}\left({ }^{13} \mathrm{C}\right)\right)$, Bruker Avance III $400(400 \mathrm{MHz}$ $\left({ }^{1} \mathrm{H}\right)$ and $\left.100 \mathrm{MHz}\left({ }^{13} \mathrm{C}\right)\right)$, Bruker Avance III HD $500\left(500 \mathrm{MHz}\left({ }^{1} \mathrm{H}\right)\right.$ and $\left.100 \mathrm{MHz}\left({ }^{13} \mathrm{C}\right)\right)$, and Bruker AC $600\left(600 \mathrm{MHz}\left({ }^{1} \mathrm{H}\right)\right.$ and $\left.150 \mathrm{MHz}\left({ }^{13} \mathrm{C}\right)\right)$ spectrometers. Chemical shifts are expressed in parts per million (ppm). The spectra were calibrated to residual solvent signals of $\mathrm{CHCl}_{3}\left(7.26 \mathrm{ppm}\left({ }^{1} \mathrm{H}\right)\right.$ and $\left.77.0 \mathrm{ppm}\left({ }^{13} \mathrm{C}\right)\right)$, DMSO- $d_{5}\left(2.50 \mathrm{ppm}\left({ }^{1} \mathrm{H}\right)\right.$ and $\left.39.43 \mathrm{ppm}\left({ }^{13} \mathrm{C}\right)\right)$, $\mathrm{CHD}_{2} \mathrm{OD}\left(3.31 \mathrm{ppm}\left({ }^{1} \mathrm{H}\right)\right.$ and $\left.49.0 \mathrm{ppm}\left({ }^{13} \mathrm{C}\right)\right)$, DHO $\left(4.79 \mathrm{ppm}\left({ }^{1} \mathrm{H}\right)\right)$, respectively. Coupling constants are given in Hertz $(\mathrm{Hz})$ and the following notations indicate the multiplicity of the signals: s (singlet), d (doublet), t (triplet), q (quartet), m (multiplet), br (broad signal). Owing to the $E / Z$ equilibrium of some compounds containing an azobenzene functionality, more signals were observed in the ${ }^{1} \mathrm{H}$ and ${ }^{13} \mathrm{C}$ spectra than would be expected for a single isomer. Signals for the major $E$ - isomer are reported. Signal identity and peak assignments were verified by 2D-NMR experiments (COSY, TOCSY, HSQC and HMBC) whenever necessary. NMR spectra are displayed in Supplementary Note 2.

\section{UV-Vis analysis}

UV-Vis spectra were recorded using a JASCO V-730 UV-Visible Spectrophotometer with Helma SUPRASIL precision cuvettes (5 $\mathrm{mm}$ light path). All compounds were dissolved as 10$30 \mathrm{mM}$ stock solutions in DMF and diluted to the given concentrations using cosolvents and buffers as indicated. Switching was achieved by irradiating the cuvettes at 0.2 to $0.5 \mathrm{~cm}$ within the spectrometer for 10 to $30 \mathrm{~s}$. For irradiation high power single chip SMD LEDs from Roithner Lasertechnik GmbH with a current of $350 \mathrm{~mA}$ were used. The LEDs show their 
main intensity at the specified wavelength with a width of $\pm 5 \mathrm{~nm}$. UV-spectra of all compounds are reproduced in Supplementary Note 1.

\begin{tabular}{lll} 
Table S2. LEDs from Roithner Lasertechnik \\
GmbH used in this paper \\
\hline Entry & $\boldsymbol{\lambda}[\mathrm{nm}]$ & LED code \\
& & \\
\hline $\mathbf{1}$ & 370 & VL370-5050 \\
$\mathbf{2}$ & 380 & VL380-5050 \\
$\mathbf{3}$ & 390 & VL390-5050 \\
$\mathbf{4}$ & 400 & VL400-5050 \\
$\mathbf{5}$ & 420 & SMB1N-420H \\
$\mathbf{6}$ & 450 & SMB1N-D450 \\
$\mathbf{7}$ & 470 & SMD1N-D470 \\
$\mathbf{8}$ & 520 & SMD1N-D520
\end{tabular}

Relaxation rates were evaluated assigning first order kinetics of the $E$ - $Z$-isomerization reaction. Samples were measured under dark $(E)$ conditions at their absorption maximum, irradiated $(Z$ ) to reach photostationary equilibrium, followed by measuring the changing absorbance over time according to first order kinetics. Data were evaluated by fitting the rate equation $[\mathrm{A}]=[\mathrm{A}]_{0} \mathrm{e}^{\wedge}(-k \mathrm{t})$ using the Excel program. Half-lives were obtained by: $t_{1 / 2}=$ $\ln (2) / k$.

The determine the ratio $\alpha$ of the two isomers in the PSS Fischer's method was applied. ${ }^{8}$ The absorbance $(A)$ of the pure $E$ - isomer (dark) and mixed spectra of $E$ - and $Z$ - isomer after irradiation with two different wavelengths $\left(\lambda_{1}\right.$ and $\left.\lambda_{2}\right)$ are required. According to Fischer's formula:

$$
\alpha_{\lambda 1}=\frac{\frac{\Delta \mathrm{A}_{\lambda 1}}{\mathrm{~A}_{\lambda 1, \lambda 1}}-\frac{\Delta \mathrm{A}_{\lambda 2}}{\mathrm{~A}_{\lambda 2, \lambda 2}}}{1+\frac{\Delta \mathrm{A}_{\lambda 1}}{\mathrm{~A}_{\lambda 1, \lambda 1}}-n\left(1+\frac{\Delta \mathrm{A}_{\lambda 2}}{\mathrm{~A}_{\lambda 2, \lambda 2}}\right)} \quad \text { with } \quad n=\frac{\mathrm{A}_{\text {dark }, \lambda 1}-\mathrm{A}_{\lambda 1, \lambda 1}}{\mathrm{~A}_{\text {dark }, \lambda 1}-\mathrm{A}_{\lambda 2, \lambda 1}}
$$

the PSS (PSS $=\alpha * 100$ ) at the irradiated wavelengths can be determined. In this work, irradiation was carried out at seven to eight different wavelengths. To determine the PSS, the wavelength that provided the largest percentage of $(Z$ isomers was chosen as the basis and the ratio $\alpha$ was determined with the other wavelengths. For this wavelength, the PSS was determined from the mean value of all received $\alpha$. The other wavelengths were specified relative to it. The determined $\alpha$ values generally had a standard deviation of \pm 0.01 -0.03 , for some aniline derivatives the deviation was up to \pm 0.05 . Based on the obtained ratio $\alpha$, the absorbance of the pure $(Z)$ isomer can be calculated using the formula:

$$
\mathrm{A}_{Z-\text { Isomer }, \lambda}=\mathrm{A}_{\text {dark }, \lambda}+\frac{\mathrm{A}_{\lambda 1, \lambda}-\mathrm{A}_{\text {darkl, }}}{\alpha_{\lambda 1}}
$$




\section{Infrared (IR) spectra}

Fourier transform infrared spectroscopy (FT-IR) spectra were obtained by using an IRAffinity-1 from Shimadzu (ATR, neat or as a thin film). Wave numbers are reported in $\mathrm{cm}^{-1}$.

\section{Microwave reactor}

A Biotage Initiator Sixty microwave reactor with sealed glass vessels was used for the irradiation with microwaves. The temperature was measured using an IR sensor (accuracy $\pm 2 \%$ ). Reaction times indicate how long the mixture was stirred at the specified temperature, not how long the mixture was irradiated overall.

\section{Melting points}

The melting points were measured on a Stuart Melting Point SMP 3 device.

\section{Low- and high-resolution ESI mass spectra}

Low- and high-resolution ESI mass spectra were obtained on Thermo-Finnigan LCQ (LR) and Bruker Maxis Impact (HR) spectrometers operating in either positive or negative ionization modes, respectively, fitted to Shimadzu AL-10 (LR-ESI-MS) or Dionex Ultima 3000 HPLC systems (HR-ESI-MS). 


\section{Chemical Synthesis and Characterisation}

\section{Standard Procedures}

For Standard Procedures, the conditions and ratios of reactants/reagents employed were kept constant. Solvent volumes refer to 1.0 equiv. In general, the $E$ - and $Z$ - isomers of the azobenzenes were separable by silica-gel chromatography and on TLC when the thermal relaxation half-life time was in the range of minutes or longer. For improving separation, yields and purity of the desired product the crude materials were kept in the dark at room temperature overnight after isolation and protected from UV light during chromatographic separation (i.e. work under red light, wrapped/shielded column wherever possible).

Standard Procedure A: Diazo Coupling using isoamyl nitrite

To a solution of the aniline (1.0 equiv.) in $\mathrm{MeOH}(5.0 \mathrm{~mL} / \mathrm{mmol}$ ) conc. $\mathrm{HCl}$ (6.0 equiv.) was added and cooled to $0{ }^{\circ} \mathrm{C}$ (icebath). In case of Boc-protected anilines the amount of $\mathrm{HCl}$ was doubled and the solution was stirred at room temperature until TLC showed full deprotection before cooling down (approx. 2 h). A solution of isoamyl nitrite (1.02 equiv.) in methanol $(1.0 \mathrm{~mL} / \mathrm{mmol})$ was added slowly and the mixture was stirred for $30 \mathrm{~min}$ in the cold.

A1: A cold solution of the phenol (1.05 equiv.) in methanol $(2.0 \mathrm{~mL} / \mathrm{mmol})$ was added followed by addition of $\mathrm{NaOH}(2.0 \mathrm{M})$ until $\mathrm{pH}=11$ was reached (characteristic color change from yellow to deep red).

A2: A solution of the phenol (1.05 equiv.) in methanol $(2.0 \mathrm{~mL} / \mathrm{mmol})$ was basified with $\mathrm{NaOH}$ (2.0 M, 7.2 equiv.) and cooled in an icebath. To it was added the solution of the diazonium salt prepared above dropwise over 1 minute.

After 30 minutes stirring at $0{ }^{\circ} \mathrm{C}$, the $\mathrm{pH}$ was adjusted to 7 with phosphate buffer (30 mL/mmol) and $\mathrm{CHCl}_{3}(30 \mathrm{~mL} / \mathrm{mmol}$ ) was added. The organic layer was separated followed by extraction of the aqueous layer with $\mathrm{CHCl}_{3}(3-5 \times 20 \mathrm{~mL} / \mathrm{mmol})$. The combined organic extracts were dried with $\mathrm{Na}_{2} \mathrm{SO}_{4}$, filtered, and concentrated under reduced pressure. Purification by silica-gel chromatography or recrystallization provided the pure azobenzene. 
Standard Procedure B: Phenol etherification in acetone

To a solution of the phenol (1.0 equiv.) and $\mathrm{K}_{2} \mathrm{CO}_{3}$ (4.0 equiv.) in anhydrous acetone $(10 \mathrm{~mL} / \mathrm{mmol})$ the alkylhalogenide (1.0 - 10.0 equiv.) was added and stirred at reflux for 2 $18 \mathrm{~h}$ until TLC indicated satisfactory conversion. The reaction mixture was concentrated under reduced pressure. The residue was dissolved in EtOAc $(20 \mathrm{~mL} / \mathrm{mmol})$ and phosphate buffer $(\mathrm{pH}=7,30 \mathrm{~mL} / \mathrm{mmol})$. The organic layer was separated followed by extraction of the aqueous layer with EtOAc $(3 \times 20 \mathrm{~mL} / \mathrm{mmol})$. The combined organic extracts were dried with $\mathrm{Na}_{2} \mathrm{SO}_{4}$, filtered, and concentrated under reduced pressure. Purification by silica-gel chromatography or recrystallization provided the arylether.

Standard Procedure C: Ester or Imine cleavage with $\mathrm{LiOH}$

Ester or Imine (1.0 equiv.) was dissolved in THF $(20 \mathrm{~mL} / \mathrm{mmol})$, treated with aqueous $\mathrm{LiOH}$ (2 $\mathrm{M}, 10.0$ equiv.), and stirred for $2 \mathrm{~h}$ at room temperature. The solution was neutralized with aqueous $\mathrm{HCl}(1 \mathrm{M})$. Most of the THF was removed under reduced pressure and $\mathrm{CHCl}_{3}$ $(20 \mathrm{~mL} / \mathrm{mmol})$ was added. The organic layer was separated followed by extraction of the aqueous layer with $\mathrm{CHCl}_{3}$ or EtOAc $(3-12 \times 20 \mathrm{~mL} / \mathrm{mmol})$. The combined organic extracts were dried with $\mathrm{Na}_{2} \mathrm{SO}_{4}$, filtered, and concentrated under reduced pressure. If necessary, the residue was purified by silica-gel chromatography or prep. HPLC to obtain the carboxylic acid.

Standard Procedure D: Buchwald - Hartwig amination

A solution of bromoaryl azobenzene (1.0 equiv.) and $\mathrm{CS}_{2} \mathrm{CO}_{3}$ (4.0 equiv.) in anhydrous acetonitrile $(25 \mathrm{~mL} / \mathrm{mmol})$ was purged with $\mathrm{N}_{2}$ in a microwave reaction vial over 20 minutes. Amine (2.0 equiv.), $\mathrm{Pd}(\mathrm{dba})_{2}$ ( 0.1 equiv.) and RuPhos ( 0.2 equiv.) were added and the vial was capped under nitrogen flow. The resulting suspension was heated with stirring to $100{ }^{\circ} \mathrm{C}$ and kept at this temperature until TLC indicated satisfactory conversion (typically $16 \mathrm{~h}$ ). The mixture was cooled to RT, filtered, and concentrated under reduced pressure. Purification by silica-gel chromatography provided the aminated azobenzene.

Standard Procedure E: Reductive amination 
To a solution of amine (1.0 equiv.) in a mixture of $\mathrm{AcOH}$ and $\mathrm{EtOH}(1: 9,25 \mathrm{~mL} / \mathrm{mmol})$ aldehyde (3.0 equiv.) was added and stirred for 2 hours at room temperature. The mixture was cooled to $0{ }^{\circ} \mathrm{C}$ (icebath) and $\mathrm{NaCNBH}_{3}$ (4.5 equiv.) was added. The icebath was removed and stirring was continued until TLC indicated satisfactory conversion (16 - $38 \mathrm{~h})$. The solution was neutralized with aqueous $\mathrm{NaOH}(2 \mathrm{M})$ and most of the $\mathrm{EtOH}$ was removed under reduced pressure followed by extraction of the aqueous phase with $\mathrm{CHCl}_{3}(3 \times 20$ $\mathrm{mL} / \mathrm{mmol}$ ). The combined organic extracts were dried with $\mathrm{Na}_{2} \mathrm{SO}_{4}$, filtered, and concentrated under reduced pressure. Purification by silica-gel chromatography provided the pure azobenzene.

Standard Procedure F: Reduction of nitroaryl compounds

Nitrobenzene (1.0 equiv.) was dissolved in a mixture of EtOH, EtOAc and $\mathrm{AcOH}(5: 10: 1,50 \mathrm{~mL} / \mathrm{mmol})$. $\mathrm{Pd} / \mathrm{C}(10 \mathrm{~m} \%, 55 \mathrm{mg} / \mathrm{mmol})$ was added and $\mathrm{H}_{2}$ was bubbled trough the solution until TLC indicates satisfactory conversion $(6-24 \mathrm{~h})$. The mixture was filtered through a pad of celite and the filter cake was washed with EtOH ( $\sim 100 \mathrm{~mL} / \mathrm{mmol})$. Toluene $(5 \mathrm{~mL} / \mathrm{mmol})$ was added and the mixture was concentrated under reduced pressure. The resulting crude product was dried under vacuum and used without further purification. 


\section{Syntheses}

$N$-(5'-Methoxy-2'-nitrophenyl)succinimide $\boldsymbol{p}-6$<smiles>COc1ccc([N+](=O)[O-])c(N2C(=O)CCC2=O)c1</smiles>

Under an atmosphere of nitrogen 5-methoxy-2-nitroaniline (2.00 g, 11.9 mmol, 1.0 equiv.) and $\mathrm{K}_{2} \mathrm{CO}_{3}$ (4.11 g, $29.7 \mathrm{mmol}, 2.5$ equiv.) were suspended in dry THF $(160 \mathrm{~mL})$. Succinylchloride (1.31 mL, $11.9 \mathrm{mmol}, 1.0$ equiv.) was added dropwise and the reaction mixture was heated to $45^{\circ} \mathrm{C}$ for $16 \mathrm{~h}$. The reaction mixture was concentrated under reduced pressure. The residue was dissolved in EtOAc $(100 \mathrm{~mL})$ and washed with water $(2 \times 30 \mathrm{~mL})$. The combined extracts were dried with $\mathrm{MgSO}_{4}$, filtered, and concentrated under reduced pressure. Purification by silica-gel chromatography (EtOAc/petroleum ether, 1:1) provided succinimide $\boldsymbol{p}-\mathbf{6}(1.95 \mathrm{~g}, 66 \%)$ as a yellow solid. $R_{f}=0.34$ (EtOAc/petroleum ether, 1:1); m.p. $176{ }^{\circ} \mathrm{C} ;{ }^{1} \mathrm{H}-\mathrm{NMR}\left(250 \mathrm{MHz}, \mathrm{CDCl}_{3}\right): \delta=8.27(\mathrm{~d}, J=9.3 \mathrm{~Hz}, 1 \mathrm{H}), 7.07(\mathrm{dd}, J=2.7,9.1 \mathrm{~Hz}, 1 \mathrm{H})$, $6.83(\mathrm{~d}, J=2.6 \mathrm{~Hz}, 1 \mathrm{H}), 3.94(\mathrm{~s}, 3 \mathrm{H}), 3.16-2.82(\mathrm{~m}, 4 \mathrm{H}) ;{ }^{13} \mathrm{C}-\mathrm{NMR}\left(63 \mathrm{MHz}, \mathrm{CDCl}_{3}\right): \delta=176.3$, 164.9, 139.0, 129.3, 129.2, 116.9, 115.7, 57.2, 29.8; IR: $\tilde{v}=3356,2839,1786,1701,1585,1489,1280$, 1173, 1092, 825; HRMS (ESI): $m / z$ calcd for $\mathrm{C}_{11} \mathrm{H}_{11} \mathrm{~N}_{2} \mathrm{O}_{5}: 251.0662[\mathrm{M}+\mathrm{H}]^{+}$; found: 251.0666 .

$N$-(4'-Methoxy-2'-nitrophenyl)succinimide $\boldsymbol{m}-\mathbf{6}$<smiles>COc1ccc(N2C(=O)CCC2=O)c([N+](=O)[O-])c1</smiles>

Under an atmosphere of nitrogen 5-methoxy-2-nitroaniline (2.00 g, 11.9 mmol, 1.0 equiv.) and $\mathrm{K}_{2} \mathrm{CO}_{3}$ (4.11 g, $29.7 \mathrm{mmol}, 2.5$ equiv.) were suspended in dry THF $(160 \mathrm{~mL})$. Succinylchloride (1.31 mL, $11.9 \mathrm{mmol}, 1.0$ equiv.) was added dropwise and the reaction mixture was heated to $45^{\circ} \mathrm{C}$ for $16 \mathrm{~h}$. The reaction mixture was concentrated under reduced pressure. The residue was dissolved in EtOAc $(100 \mathrm{~mL})$ and washed with water $(2 \times 30 \mathrm{~mL})$. The combined organic extracts were dried with $\mathrm{MgSO}_{4}$, filtered, and concentrated under reduced pressure. Purification by silica-gel chromatography (EtOAc/petroleum ether, 1:1) provided succinimide $\boldsymbol{m} \mathbf{- 6}(1.94 \mathrm{~g}, 65 \%)$ as a yellow solid. $R_{f}=0.32$ (EtOAc/petroleum ether, 1:1); m.p. $176{ }^{\circ} \mathrm{C} ;{ }^{1} \mathrm{H}-\mathrm{NMR}\left(300 \mathrm{MHz}, \mathrm{CDCl}_{3}\right): \delta=7.70(\mathrm{dd}, J=1.2,1.8 \mathrm{~Hz}, 1 \mathrm{H}), 7.28-7.25(\mathrm{~m}, 2 \mathrm{H}), 3.92$ $(\mathrm{s}, 3 \mathrm{H}), 2.94(\mathrm{~d}, J=5.2 \mathrm{~Hz}, 4 \mathrm{H}) ;{ }^{13} \mathrm{C}-\mathrm{NMR}\left(75 \mathrm{MHz}, \mathrm{CDCl}_{3}\right): \delta=175.7,160.3,145.7,131.3,120.3$, 
118.3, 110.9, 56.2, 28.7; IR: $\tilde{v}=3348,3089,2939,1774,1705,1539,1357,1280,1171,1030,887,664$; HRMS (ESI): $m / z$ calcd for $\mathrm{C}_{11} \mathrm{H}_{11} \mathrm{~N}_{2} \mathrm{O}_{5}: 251.0662[\mathrm{M}+\mathrm{H}]^{+}$; found: 251.0666 .

4-((4’-bromophenyl)diazenyl)phenol 13

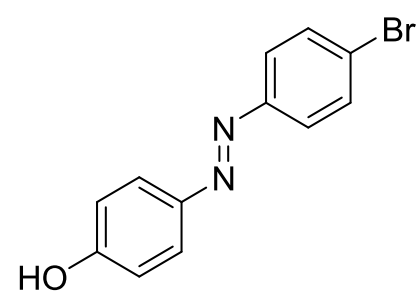

Standard Procedure A1 with p-bromaniline (5.00 g, $29.1 \mathrm{mmol}, 1.0$ equiv.) and phenol (2.87 g, $30.5 \mathrm{mmol}, 1.05$ equiv.) gave azobenzene $13(6.28 \mathrm{~g}, 78 \%)$ as an ocher solid after recryztalisation (toluene/petroleum ether, 1:3). $R_{f}=0.40\left(\right.$ EtOAc/petroleum ether, 1:4); m.p. $160{ }^{\circ} \mathrm{C} ;{ }^{1} \mathrm{H}-\mathrm{NMR}(300 \mathrm{MHz}$, $\left.\mathrm{CDCl}_{3}\right): \delta=7.88(\mathrm{~d}, J=8.8 \mathrm{~Hz}, 2 \mathrm{H}), 7.77(\mathrm{~d}, J=8.8 \mathrm{~Hz}, 2 \mathrm{H}), 7.63(\mathrm{~d}, J=8.8 \mathrm{~Hz}, 2 \mathrm{H}), 6.96(\mathrm{~d}, J=8.8$ $\mathrm{Hz}, 2 \mathrm{H}), 5.19$ (br s, $1 \mathrm{H}$ ); ${ }^{13} \mathrm{C}-\mathrm{NMR}\left(75 \mathrm{MHz}, \mathrm{CDCl}_{3}\right): \delta=158.4,151.4,147.0,132.3,125.1,124.7$, 124.1, 115.9; IR: $\tilde{v}=3159,2951,2854,1739,1601,1462,1366,1253,837,671$; UV-VIS $\left(\mathrm{CH}_{3} \mathrm{CN}+\right.$ $0.5 \%$ piperidine): $\lambda_{\max }(\varepsilon)=350 \mathrm{~nm}\left(238001 \cdot \mathrm{mol}^{-1} \cdot \mathrm{cm}^{-1}\right)$; HRMS (ESI): $\mathrm{m} / z$ calcd for $\mathrm{C}_{12} \mathrm{H}_{10} \mathrm{BrN}_{2} \mathrm{O}$ : 276.9971/278.9951 [M+H] $]^{+}$; found: 276.9975/278.9954.

4-((3',5'-dimethoxyphenyl)diazenyl)phenol 14<smiles>COc1cc(/N=N/c2ccc(O)cc2)cc(OC)c1</smiles>

Standard Procedure A1 with 3,5-trimethoxyaniline (307 mg, $2.0 \mathrm{mmol}, 1.0$ equiv.) and phenol (198 mg, $2.1 \mathrm{mmol}, 1.05$ equiv.) gave azobenzene $14(215 \mathrm{mg}, 42 \%)$ as an orange solid after purification by silicagel chromatography (EtOAc/petroleum ether, 1:4). $R_{f}=0.38$ (EtOAc/petroleum ether, 1:2); m.p. $129{ }^{\circ} \mathrm{C}$; ${ }^{1} \mathrm{H}-\mathrm{NMR}\left(300 \mathrm{MHz}, \mathrm{CDCl}_{3}\right): \delta=7.89(\mathrm{~d}, J=8.8 \mathrm{~Hz}, 2 \mathrm{H}), 7.10(\mathrm{~d}, J=2.2 \mathrm{~Hz}, 2 \mathrm{H}), 6.95(\mathrm{~d}, J=8.8 \mathrm{~Hz}$, $2 \mathrm{H}), 6.59(\mathrm{t}, J=2.2 \mathrm{~Hz}, 1 \mathrm{H}), 3.88(\mathrm{~s}, 6 \mathrm{H}) ;{ }^{13} \mathrm{C}-\mathrm{NMR}\left(101 \mathrm{MHz}, \mathrm{CDCl}_{3}\right): \delta=161.1,158.4,154.5$, 147.0, 125.1, 115.8, 103.4, 100.7, 55.6; IR: $\tilde{v}=3062$, 2935, 1585, 1416, 1281, 1207, 1151, 1053, 942, 667; UV-VIS $\left(\mathrm{CH}_{3} \mathrm{CN}+0.5 \%\right.$ piperidine): $\lambda_{\max }(\varepsilon)=355 \mathrm{~nm}\left(219001 \cdot \mathrm{mol}^{-1} \cdot \mathrm{cm}^{-1}\right) ; \mathrm{HRMS}(\mathrm{ESI}): \mathrm{m} / \mathrm{z}$ calcd for $\mathrm{C}_{14} \mathrm{H}_{15} \mathrm{~N}_{2} \mathrm{O}_{3}: 259.1077[\mathrm{M}+\mathrm{H}]^{+}$; found: 259.1082 . 
4-((3',4'-dihydro-2H-benzo[b][1',4']oxazin-7'-yl)diazenyl)phenol 15

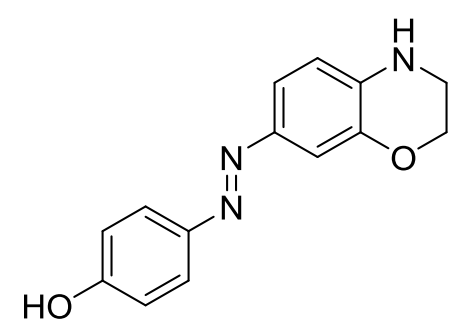

Standard Procedure A1 with aniline 4 (156 mg, 1.04 mmol, 1.0 equiv.), phenol (103 mg, 1.09 mmol, 1.05 equiv.), and isoamyl nitrite (256 mg, $2.18 \mathrm{mmol}, 2.1$ equiv.) were converted to give a yellow solid $(229 \mathrm{mg})$ that was dissolved in dry $\mathrm{MeOH}(12 \mathrm{~mL})$, and $\mathrm{HCl}(4 \mathrm{M}$ in dioxane), and stirred at room temperature for $18 \mathrm{~h}$. All solvents were removed under reduced pressure, and aq. $\mathrm{NaHCO}_{3}(15 \mathrm{~mL})$, $\mathrm{CHCl}_{3}(20 \mathrm{~mL})$, and acetone $(2 \mathrm{~mL})$ were added. The organic layer was separated, and the aqueous phase was extracted with $\mathrm{CHCl}_{3}(3 \times 20 \mathrm{~mL})$. The combined organic extracts were dried with $\mathrm{Na}_{2} \mathrm{SO}_{4}$, filtered, and concentrated under reduced pressure. Azobenzene 15 (145 mg, $55 \%)$ was isolated as a brown oil after purification by silica-gel chromatography (EtOAc/petroleum ether, 1:4 $\rightarrow 1: 1$ ). $R_{f}=0.45$ (EtOAc/petroleum ether, 1:1); ${ }^{1} \mathrm{H}-\mathrm{NMR}\left(400 \mathrm{MHz}, \mathrm{DMSO}-d_{6}\right): \delta=10.00$ (br s, $\left.1 \mathrm{H}\right), 7.65(\mathrm{~d}, J=8.8 \mathrm{~Hz}$, $2 \mathrm{H}), 7.30(\mathrm{dd}, J=2.0,8.5 \mathrm{~Hz}, 1 \mathrm{H}), 7.15(\mathrm{~d}, J=2.3 \mathrm{~Hz}, 1 \mathrm{H}), 6.88(\mathrm{~d}, J=9.1 \mathrm{~Hz}, 2 \mathrm{H}), 6.66(\mathrm{~d}, J=8.5$ $\mathrm{Hz}, 1 \mathrm{H}), 4.15(\mathrm{t}, J=4.4 \mathrm{~Hz}, 2 \mathrm{H}) ;{ }^{13} \mathrm{C}-\mathrm{NMR}\left(101 \mathrm{MHz}, \mathrm{DMSO}-d_{6}\right): \delta=159.8,145.9,143.6,143.2$, 138.7, 124.2, 120.1, 116.2, 113.8, 108.2, 64.6, 40.1; IR: $\tilde{v}=3360,2920,2851,1740,1582,1501,1211$, 840, 810; UV-VIS $\left(\mathrm{CH}_{3} \mathrm{CN}+0.5 \%\right.$ piperidine $): \lambda_{\max }(\varepsilon)=406 \mathrm{~nm}\left(240001 \cdot \mathrm{mol}^{-1} \cdot \mathrm{cm}^{-1}\right)$; HRMS (ESI): $m / z$ calcd for $\mathrm{C}_{14} \mathrm{H}_{14} \mathrm{~N}_{3} \mathrm{O}_{2}: 256.1081[\mathrm{M}+\mathrm{H}]^{+}$; found: 256.1081 .

2-(4’-((4"'-hydroxyphenyl)diazenyl)phenoxy)acetic acid 16a<smiles>O=C(O)COc1ccc(/N=N/c2ccc(O)cc2)cc1</smiles>

Standard Procedure A1 with Boc-protected aniline 5 (100 mg, $0.34 \mathrm{mmol}, 1.0$ equiv.) and phenol (33.5 mg, $0.35 \mathrm{mmol}, 1.05$ equiv.) gave azobenzene $\mathbf{1 6 a}(47 \mathrm{mg}, 51 \%)$ as an orange solid after silica-gel chromatography $\left(\mathrm{CH}_{2} \mathrm{Cl}_{2} / \mathrm{MeOH}, 98: 2+1.0 \%\right.$ formic acid). $R_{f}=0.17\left(\mathrm{CH}_{2} \mathrm{Cl}_{2} / \mathrm{MeOH}, 98: 2+1.0 \%\right.$ 
formic acid); m.p. $186{ }^{\circ} \mathrm{C}$; ${ }^{1} \mathrm{H}-\mathrm{NMR}\left(300 \mathrm{MHz}, \mathrm{CD}_{3} \mathrm{OD}\right): \delta=7.83(\mathrm{~d}, J=8.9 \mathrm{~Hz}, 2 \mathrm{H}), 7.77(\mathrm{~d}, J=8.8$ $\mathrm{Hz}, 2 \mathrm{H}), 7.06(\mathrm{~d}, J=8.9 \mathrm{~Hz}, 2 \mathrm{H}), 6.90(\mathrm{~d}, J=8.8 \mathrm{~Hz}, 2 \mathrm{H}), 4.75(\mathrm{~s}, 2 \mathrm{H}) ;{ }^{13} \mathrm{C}-\mathrm{NMR}(101 \mathrm{MHz}$, $\left.\mathrm{CD}_{3} \mathrm{OD}\right): \delta=171.0,160.2,159.9,147.4,146.1,124.2,123.7,115.3,114.6,64.7$; IR: $\tilde{v}=3406,2924$, $1728,1585,1497,1435,1215,1146,1080,837$; UV-VIS $\left(\mathrm{CH}_{3} \mathrm{CN}+0.5 \%\right.$ piperidine $): \lambda_{\max }(\varepsilon)=359 \mathrm{~nm}$ (16500 $1 \cdot \mathrm{mol}^{-1} \cdot \mathrm{cm}^{-1}$ ); HRMS (ESI): $\mathrm{m} / z$ calcd for $\mathrm{C}_{14} \mathrm{H}_{13} \mathrm{~N}_{2} \mathrm{O}_{4}: 273.0870[\mathrm{M}+\mathrm{H}]^{+}$; found: 273.0874 .

Methyl-2-(4'-((4"'-hydroxy-3"'-methoxyphenyl)diazenyl)phenoxy)acetate $\mathbf{1 6 b}$<smiles>COC(=O)COc1ccc(/N=N/c2ccc(O)c(OC)c2)cc1</smiles>

Standard Procedure A1 with Boc-protected aniline 5 (200 mg, $0.68 \mathrm{mmol}$, 1.0 equiv.) and 2-methoxyphenol (88.3 mg, $0.71 \mathrm{mmol}, 1.05$ equiv.) gave after extraction, and evaporation of the solvent a residue that was redissolved in anhydrous $\mathrm{MeOH}(20 \mathrm{~mL})$. Thionylchloride $(1 \mathrm{~mL})$ was added, and the mixture was stirred at room temperature for $2 \mathrm{~h}$. The $\mathrm{pH}$ was adjusted to 7 with phosphate buffer, and $\mathrm{CHCl}_{3}(30 \mathrm{~mL})$ was added. The organic layer was separated followed by extraction of the aqueous layer with $\mathrm{CHCl}_{3}(3 \times 20 \mathrm{~mL})$. The combined organic extracts were dried with $\mathrm{Na}_{2} \mathrm{SO}_{4}$, filtered, and concentrated under reduced pressure. Purification by silica-gel chromatography $\left(\mathrm{CH}_{2} \mathrm{Cl}_{2} / \mathrm{MeOH}, 98: 2\right)$ provided azobenzene $\mathbf{1 6 b}(146 \mathrm{mg}, 68 \%)$ as an ocher solid. $R_{f}=0.47\left(\mathrm{CH}_{2} \mathrm{Cl}_{2} / \mathrm{MeOH}, 98: 2+1.0 \%\right.$ formic acid); m.p. $133{ }^{\circ} \mathrm{C}$; ${ }^{1} \mathrm{H}-\mathrm{NMR}\left(300 \mathrm{MHz}, \mathrm{CDCl}_{3}\right): \delta=7.99(\mathrm{~d}, J=8.7 \mathrm{~Hz}, 2 \mathrm{H}), 7.67(\mathrm{dd}, J=2.1$, $8.4 \mathrm{~Hz}, 1 \mathrm{H}), 7.60(\mathrm{~d}, J=2.1 \mathrm{~Hz}, 1 \mathrm{H}), 7.16(\mathrm{~d}, J=8.6 \mathrm{~Hz}, 1 \mathrm{H}), 7.13(\mathrm{~d}, J=9.1 \mathrm{~Hz}, 2 \mathrm{H}), 6.06$ (br s, 1 $\mathrm{H}), 4.83(\mathrm{~s}, 2 \mathrm{H}), 4.10(\mathrm{~s}, 3 \mathrm{H}), 3.95(\mathrm{~s}, 3 \mathrm{H}) ;{ }^{13} \mathrm{C}-\mathrm{NMR}\left(101 \mathrm{MHz}, \mathrm{CDCl}_{3}\right): \delta=169.0,159.5,148.5$, 147.6, 147.1, 146.7, 124.3, 121.0, 114.9, 114.2, 101.8, 65.4, 56.1, 52.4; IR: $\tilde{v}=3190,2916,2851,1771$, 1582, 1501, 1207, 1022, 837; UV-VIS $\left(\mathrm{CH}_{3} \mathrm{CN}+0.5 \%\right.$ piperidine $): \lambda_{\max }(\varepsilon)=376 \mathrm{~nm}\left(190001 \cdot \mathrm{mol}^{-1} \cdot \mathrm{cm}^{-}\right.$ ${ }^{1}$ ); HRMS (ESI): $m / z$ calcd for $\mathrm{C}_{16} \mathrm{H}_{17} \mathrm{~N}_{2} \mathrm{O}_{5}: 317.1132[\mathrm{M}+\mathrm{H}]^{+}$; found: 317.1135 .

Methyl-2-(4'-((4"'-hydroxy-3"',5"'-dimethoxyphenyl)diazenyl)phenoxy)acetate 16c 
<smiles>COC(=O)COc1ccc(/N=N/c2cc(OC)c(O)c(OC)c2)cc1</smiles>

Standard Procedure A1 with Boc-protected aniline 5 (500 mg, $1.69 \mathrm{mmol}, 1.0$ equiv.) and 2,6dimethoxyphenol (274 mg, $1.78 \mathrm{mmol}, 1.05$ equiv.) gave after extraction and evaporation of the solvent a residue that was redissolved in dry $\mathrm{MeOH}(20 \mathrm{~mL})$. Thionylchloride $(1 \mathrm{~mL})$ was added and the mixture was stirred at room temperature for $2 \mathrm{~h}$. The $\mathrm{pH}$ was adjusted to 7 with phosphate buffer, and $\mathrm{CHCl}_{3}$ $(30 \mathrm{~mL})$ was added. The organic layer was separated followed by extraction of the aqueous layer with $\mathrm{CHCl}_{3}(3 \times 20 \mathrm{~mL})$. The combined organic extracts were dried with $\mathrm{Na}_{2} \mathrm{SO}_{4}$, filtered, and concentrated under reduced pressure. Purification by silica-gel chromatography (EtOAc/petroleum ether, 1:1) provided azobenzene $16 \mathrm{c}\left(397 \mathrm{mg}, 68 \%\right.$ ) as an ocher solid. $R_{f}=0.53$ (EtOAc/petroleum ether, 1:1); m.p. $122{ }^{\circ} \mathrm{C}$; ${ }^{1} \mathrm{H}-\mathrm{NMR}\left(300 \mathrm{MHz}, \mathrm{CDCl}_{3}\right): \delta=7.89(\mathrm{~d}, J=8.9 \mathrm{~Hz}, 2 \mathrm{H}), 7.28(\mathrm{~s}, 2 \mathrm{H}), 7.03(\mathrm{~d}, J=8.9 \mathrm{~Hz}, 2 \mathrm{H}), 5.82$ $(\mathrm{s}, 1 \mathrm{H}), 4.73(\mathrm{~s}, 2 \mathrm{H}), 4.01(\mathrm{~s}, 6 \mathrm{H}), 3.84(\mathrm{~s}, 3 \mathrm{H}) ;{ }^{13} \mathrm{C}-\mathrm{NMR}\left(63 \mathrm{MHz}, \mathrm{CDCl}_{3}\right): \delta=169.0,159.6,147.5$, $147.2,145.5,137.5,124.3,114.9,100.2,65.4,56.4,52.4 ;$ IR: $\tilde{v}=3453,2951,1751,1585,1497,1315$, 1200, 1107, 841; UV-VIS $\left(\mathrm{CH}_{3} \mathrm{CN}+0.5 \%\right.$ piperidine $): \lambda_{\max }(\varepsilon)=384 \mathrm{~nm}\left(20000 \mathrm{l} \cdot \mathrm{mol}^{-1} \cdot \mathrm{cm}^{-1}\right) ; \mathrm{HRMS}$ (ESI): $m / z$ calcd for $\mathrm{C}_{17} \mathrm{H}_{19} \mathrm{~N}_{2} \mathrm{O}_{6}: 347.1238[\mathrm{M}+\mathrm{H}]^{+}$; found: 347.1242 .

Methyl- $N$-(5'-hydroxy-2'-((4"'-methoxyphenyl)diazenyl)phenyl)succinamate $\mathbf{1 7 a}$<smiles>COC(=O)CCC(=O)Nc1cc(O)ccc1/N=N/c1ccc(OC)cc1</smiles>

Standard Procedure A1 with $p$-anisidine (123 mg, $1.00 \mathrm{mmol}, 1.0$ equiv.) and phenol 8 (201 mg, $1.05 \mathrm{mmol}, 1.05$ equiv.) gave after extraction and evaporation of the solvent a residue that was redissolved in anhydrous $\mathrm{MeOH}(20 \mathrm{~mL})$. Thionylchloride $(1 \mathrm{~mL})$ was added and the mixture was stirred at room temperature for $2 \mathrm{~h}$. The $\mathrm{pH}$ was adjusted to 7 with phosphate buffer and EtOAc (30 mL) was added. The organic layer was separated followed by extraction of the aqueous layer with EtOAc $(3 \times$ $20 \mathrm{~mL}$ ). The combined organic extracts were dried with $\mathrm{Na}_{2} \mathrm{SO}_{4}$, filtered, and concentrated under reduced 
pressure. Purification by silica-gel chromatography (EtOAc/petroleum ether, 1:2) provided azobenzene 17a (201 mg, $56 \%$ ) as a yellow solid. $R_{f}=0.21$ (EtOAc/petroleum ether, 1:2); m.p. $189{ }^{\circ} \mathrm{C}$; ${ }^{1} \mathrm{H}-\mathrm{NMR}\left(300 \mathrm{MHz}, \mathrm{DMSO}-d_{6}\right): \delta=10.28$ (br s, $\left.1 \mathrm{H}\right), 10.19$ (s, $\left.1 \mathrm{H}\right), 7.95$ (d, $\left.J=8.9 \mathrm{~Hz}, 2 \mathrm{H}\right), 7.86$ (d, $J$ $=2.5 \mathrm{~Hz}, 1 \mathrm{H}), 7.64(\mathrm{~d}, J=8.9 \mathrm{~Hz}, 1 \mathrm{H}), 7.10(\mathrm{~d}, J=9.0 \mathrm{~Hz}, 2 \mathrm{H}), 6.57(\mathrm{dd}, J=2.6,8.9 \mathrm{~Hz}, 1 \mathrm{H}), 3.85(\mathrm{~s}$, $3 \mathrm{H}), 3.59(\mathrm{~s}, 3 \mathrm{H}), 2.80(\mathrm{t}, J=6.5 \mathrm{~Hz}, 2 \mathrm{H}), 2.63(\mathrm{t}, J=6.9 \mathrm{~Hz}, 2 \mathrm{H}) ;{ }^{13} \mathrm{C}-\mathrm{NMR}\left(126 \mathrm{MHz}, \mathrm{DMSO}-d_{6}\right): \delta$ $=173.4,170.8,161.7,161.7,146.9,138.6,134.0,125.0,119.6,114.9,111.8,107.6,56.0,51.9,31.9$, 29.1; IR: $\tilde{v}=3113,2951,2839,1720,1663,1597,1454,1242,1172,1111,841$; UV-VIS $\left(\mathrm{CH}_{3} \mathrm{CN}+\right.$ $0.5 \%$ piperidine): $\lambda_{\max }(\varepsilon)=390 \mathrm{~nm}(230001 \cdot \mathrm{mol}-1 \cdot \mathrm{cm}-1)$; HRMS (ESI): $m / z$ calcd for $\mathrm{C}_{18} \mathrm{H}_{20} \mathrm{~N}_{3} \mathrm{O}_{5}$ : $358.1397[\mathrm{M}+\mathrm{H}]^{+}$; found: 358.1401 .

Methyl- $N$-(2’-((4"'-bromophenyl)diazenyl)-5'-hydroxyphenyl)succinamate $\mathbf{1 7 b}$<smiles>COC(=O)CCC(=O)Nc1cc(O)ccc1/N=N/c1ccc(Br)cc1</smiles>

Standard Procedure A1 with p-bromaniline (400 mg, $2.33 \mathrm{mmol}, 1.0$ equiv.), and phenol 8 (467 mg, $2.44 \mathrm{mmol}, 1.05$ equiv.) gave azobenzene $17 \mathrm{~b}(651 \mathrm{mg}, 67 \%)$ as an orange solid after recrystallization (toluene/petroleum ether, 3:1). $R_{f}=0.30\left(\mathrm{CH}_{2} \mathrm{Cl}_{2} / \mathrm{MeOH}, 95: 5\right)$; m.p. $192{ }^{\circ} \mathrm{C} ;{ }^{1} \mathrm{H}-\mathrm{NMR}(400 \mathrm{MHz}$, DMSO- $\left.d_{6}\right): \delta=10.55$ (br s, $\left.1 \mathrm{H}\right), 10.24(\mathrm{~s}, 1 \mathrm{H}), 7.92$ (d, $\left.J=8.8 \mathrm{~Hz}, 2 \mathrm{H}\right), 7.89$ (d, J=2.6 Hz, $\left.1 \mathrm{H}\right), 7.77$ $(\mathrm{d}, J=8.5 \mathrm{~Hz}, 2 \mathrm{H}), 7.70(\mathrm{~d}, J=8.8 \mathrm{~Hz}, 1 \mathrm{H}), 6.61(\mathrm{dd}, J=2.5,8.9 \mathrm{~Hz}, 1 \mathrm{H}), 3.61(\mathrm{~s}, 3 \mathrm{H}), 2.82(\mathrm{t}, J=$ $6.7 \mathrm{~Hz}, 2 \mathrm{H}), 2.64(\mathrm{t}, J=6.4 \mathrm{~Hz}, 2 \mathrm{H}) ;{ }^{13} \mathrm{C}-\mathrm{NMR}\left(101 \mathrm{MHz}, \mathrm{DMSO}-d_{6}\right): \delta=173.4,170.9,162.9,151.6$, 139.5, 134.1, 132.7, 125.0, 124.1, 120.0, 112.1, 107.7, 51.9, 31.9, 29.0; IR: $\tilde{v}=3012,2951,2322,1736$, 1458, 1366, 1207, 833; UV-VIS $\left(\mathrm{CH}_{3} \mathrm{CN}+0.5 \%\right.$ piperidine $): \lambda_{\max }(\varepsilon)=464 \mathrm{~nm}\left(198001 \cdot \mathrm{mol}^{-1} \cdot \mathrm{cm}^{-1}\right)$; HRMS (ESI): $m / z$ calcd for $\mathrm{C}_{17} \mathrm{H}_{17} \mathrm{BrN}_{3} \mathrm{O}_{4}$ : 406.0397/408.0379 [M+H] $]^{+}$; found: 406.0393/408.0375.

tert-Butyl-2-hydroxy-5-((3',4’,5'-trimethoxyphenyl)diazenyl)phenylcarbamate 18 


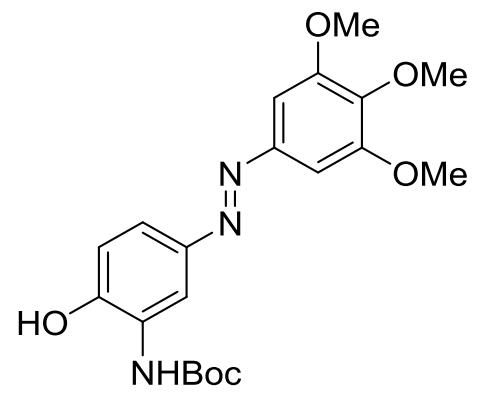

Standard Procedure A2 with 3,4,5-trimethoxyaniline (1.0 g, 5.46 mmol, 1.0 equiv.) and phenol 9 (1.20 g, $5.73 \mathrm{mmol}, 1.05$ equiv.) gave azobenzene $18(1.36 \mathrm{~g}, 62 \%)$ as red solid after purification by silica-gel chromatography (EtOAc/petroleum ether, 1:4). $R_{f}=0.52$ (EtOAc/petroleum ether, 1:4); m.p. $80{ }^{\circ} \mathrm{C} ;{ }^{1} \mathrm{H}-$ NMR (300 MHz, $\left.\mathrm{CDCl}_{3}\right): \delta=8.73($ br s, $1 \mathrm{H}), 7.76(\mathrm{~d}, J=1.6 \mathrm{~Hz}, 1 \mathrm{H}), 7.68(\mathrm{dd}, J=2.0,8.7 \mathrm{~Hz}, 1 \mathrm{H})$, $7.20(\mathrm{~s}, 2 \mathrm{H}), 7.08(\mathrm{~d}, J=8.8 \mathrm{~Hz}, 1 \mathrm{H}), 6.79(\mathrm{br} \mathrm{s}, 1 \mathrm{H}), 3.96(\mathrm{~s}, 6 \mathrm{H}), 3.94(\mathrm{~s}, 3 \mathrm{H}), 1.56(\mathrm{~s}, 9 \mathrm{H}) ;{ }^{13} \mathrm{C}-$ NMR (101 MHz, $\left.\mathrm{CDCl}_{3}\right): \delta=154.8,153.6,148.4,140.6,129.9,126.8,122.1,118.8,112.6,105.2,100.2$, 82.4, 61.1, 56.3, 28.2; IR: $\tilde{v}=3260,2970,2361,1736,1597,1493,1366,1219,1123$, 999; UV-VIS $\left(\mathrm{CH}_{3} \mathrm{CN}+0.5 \%\right.$ piperidine $): \lambda_{\max }(\varepsilon)=473 \mathrm{~nm}\left(273001 \cdot \mathrm{mol}^{-1} \cdot \mathrm{cm}^{-1}\right) ; \mathrm{HRMS}(\mathrm{ESI}): \mathrm{m} / z$ calcd for $\mathrm{C}_{20} \mathrm{H}_{26} \mathrm{~N}_{3} \mathrm{O}_{6}: 404.1816[\mathrm{M}+\mathrm{H}]^{+}$; found: 404.1824 .

Methyl- $N-\left(2^{\prime}-\left(\left(4^{\prime \prime}-\right.\right.\right.$ hydroxy-3"'-methoxyphenyl)diazenyl)-5'-methoxyphenyl) succinamate 19b<smiles>COC(=O)CCC(=O)Nc1cc(OC)ccc1/N=N/c1ccc(O)c(OC)c1</smiles>

Nitrobenzene $\boldsymbol{p}$-6 (250 mg, $1.14 \mathrm{mmol}, 1.0$ equiv.) was reduced to the corresponding aniline (quant.) following Standard Procedure F. The crude material was converted according to Standard Procedure A1 with 2-methoxyphenol (176 mg, $1.42 \mathrm{mmol}, 1.05$ equiv.) to give azobenzene 19b (128 mg, $30 \%)$ as chartreuse solid after purification by silica-gel chromatography (EtOAc/petroleum ether, 7:3 $\rightarrow$ EtOAc). $R_{f}=0.39$ (EtOAc/petroleum ether, 7:3); m.p. $104{ }^{\circ} \mathrm{C} ;{ }^{1} \mathrm{H}-\mathrm{NMR}\left(300 \mathrm{MHz}, \mathrm{CDCl}_{3}\right): \delta=10.85(\mathrm{~s}, 1 \mathrm{H})$, $8.31(\mathrm{~d}, J=2.8 \mathrm{~Hz}, 1 \mathrm{H}), 7.81(\mathrm{~d}, J=9.0 \mathrm{~Hz}, 1 \mathrm{H}), 7.52-7.44(\mathrm{~m}, 2 \mathrm{H}), 7.06(\mathrm{~d}, J=8.9 \mathrm{~Hz}, 1 \mathrm{H}), 6.71$ $(\mathrm{dd}, J=2.7,9.0 \mathrm{~Hz}, 1 \mathrm{H}), 4.01(\mathrm{~s}, 3 \mathrm{H}), 3.90(\mathrm{~s}, 3 \mathrm{H}), 3.71(\mathrm{~s}, 3 \mathrm{H}), 2.80(\mathrm{~s}, 4 \mathrm{H}) ;{ }^{13} \mathrm{C}-\mathrm{NMR}(63 \mathrm{MHz}$, $\left.\mathrm{CDCl}_{3}\right): \delta=174.1,171.2,163.6,149.3,148.1,147.3,137.5,134.2,125.5,120.6,115.3,111.7,104.6$, 103.0, 57.0, 56.6, 52.9, 33.8, 30.1; IR: $\tilde{v}=3348,2940,2322,1728,1585,1431,1200,1157,1111,1022$, 
848, 806; UV-VIS $\left(\mathrm{CH}_{3} \mathrm{CN}+0.5 \%\right.$ piperidine $): \lambda_{\max }(\varepsilon)=401 \mathrm{~nm}\left(241001 \cdot \mathrm{mol}^{-1} \cdot \mathrm{cm}^{-1}\right)$; HRMS (ESI): $m / z$ calcd for $\mathrm{C}_{19} \mathrm{H}_{21} \mathrm{~N}_{3} \mathrm{O}_{6}: 388.1503[\mathrm{M}+\mathrm{H}]^{+}$; found: 388.1511 .

$N-\left(2^{\prime}-\left(\left(4^{\prime \prime}-H y d r o x y-3 "{ }^{\prime \prime}, 5^{\prime \prime}-\right.\right.\right.$ dimethoxyphenyl)diazenyl)-5'-methoxyphenyl)succinimide 19c<smiles>COc1ccc(N=Nc2cc(OC)c(O)c(OC)c2)c(N2C(=O)CCC2=O)c1</smiles>

Nitrobenzene $\boldsymbol{p}$-6 (751 mg, $3.00 \mathrm{mmol}, 1.0$ equiv.) was reduced to the corresponding aniline (quant.) following Standard Procedure F. The crude material was converted according to Standard Procedure A1 with 2,6-dimethoxyphenol (486 mg, $3.15 \mathrm{mmol}, 1.05$ equiv.) to give azobenzene 19c (495 $\mathrm{mg}, 43 \%$ ) as a red solid after purification by silica-gel chromatography $\left(\mathrm{CH}_{2} \mathrm{Cl}_{2} / \mathrm{MeOH}, 98: 2 \rightarrow 95: 5\right) . R_{f}=0.48$ $\left(\mathrm{CH}_{2} \mathrm{Cl}_{2} / \mathrm{MeOH}, 97: 3\right)$; m.p. $76{ }^{\circ} \mathrm{C} ;{ }^{1} \mathrm{H}-\mathrm{NMR}\left(300 \mathrm{MHz}, \mathrm{CDCl}_{3}\right): \delta=7.88(\mathrm{~d}, J=9.0 \mathrm{~Hz}, 1 \mathrm{H}), 7.09$ (s, 2 H), $7.06(\mathrm{dd}, J=2.6,9.1 \mathrm{~Hz}, 1 \mathrm{H}), 6.85(\mathrm{~d}, J=2.6 \mathrm{~Hz}, 1 \mathrm{H}), 3.95(\mathrm{~s}, 6 \mathrm{H}), 3.90$ (s, $3 \mathrm{H}), 3.02-2.87$ (m, 4 $\mathrm{H}) ;{ }^{13} \mathrm{C}-\mathrm{NMR}\left(101 \mathrm{MHz}, \mathrm{CDCl}_{3}\right): \delta=176.1,162.0,147.1,146.0,141.1,137.8,132.3,118.8,116.3$, 113.5, 100.3, 56.2, 55.8, 28.8; IR: $\tilde{v}=2940,2839,1705,1604,1508,1377,1234,1176,1026,818$; UVVIS $\left(\mathrm{CH}_{3} \mathrm{CN}+0.5 \%\right.$ piperidine $): \lambda_{\max }(\varepsilon)=394 \mathrm{~nm}\left(165001 \cdot \mathrm{mol}^{-1} \cdot \mathrm{cm}^{-1}\right) ; \mathrm{HRMS}(\mathrm{ESI}): \mathrm{m} / z$ calcd for $\mathrm{C}_{19} \mathrm{H}_{20} \mathrm{~N}_{3} \mathrm{O}_{6}: 386.1347[\mathrm{M}+\mathrm{H}]^{+}$; found: 386.1351 .

Methyl-N-(2'-((4"'-hydroxy-3"',5"-dimethoxyphenyl)diazenyl)-4'-methoxyphenyl) succinamate 20 
<smiles>COC(=O)CCC(=O)Nc1ccc(OC)cc1/N=N/c1cc(OC)c(O)c(OC)c1</smiles>

Nitrobenzene $\boldsymbol{m}-6$ ( $1.14 \mathrm{~g}, 4.56 \mathrm{mmol}, 1.0$ equiv.) was reduced to the corresponding aniline (quant.) following Standard Procedure F. The crude material was further converted according to Standard Procedure A1 with 2,6-dimethoxyphenol (738 mg, $4.80 \mathrm{mmol}, 1.05$ equiv.). The resulting crude material was dissolved in anhydrous $\mathrm{MeOH}(20 \mathrm{~mL})$. Thionylchloride $(1 \mathrm{~mL})$, was added and the mixture was stirred at room temperature for $2 \mathrm{~h}$. The $\mathrm{pH}$ was adjusted to 7 with phosphate buffer, and $\mathrm{CHCl}_{3}(40 \mathrm{~mL})$ was added. The organic layer was separated followed by extraction of the aqueous layer with $\mathrm{CHCl}_{3}(3 \times$ $30 \mathrm{~mL}$ ). The combined organic extracts were dried with $\mathrm{Na}_{2} \mathrm{SO}_{4}$, filtered, and concentrated under reduced pressure. Purification by silica-gel chromatography (EtOAc/petroleum ether, 2:1 $\rightarrow$ EtOAc) provided azobenzene 20 (829 mg, $44 \%)$ as an ocher solid. $R_{f}=0.21$ (EtOAc/petroleum ether, 1:2); m.p. $159{ }^{\circ} \mathrm{C}$; ${ }^{1} \mathrm{H}-\mathrm{NMR}\left(300 \mathrm{MHz}, \mathrm{CDCl}_{3}\right): \delta=9.67$ (br s, $\left.1 \mathrm{H}\right), 8.54(\mathrm{~d}, J=9.1 \mathrm{~Hz}, 1 \mathrm{H}), 7.32(\mathrm{~s}, 2 \mathrm{H}), 7.27(\mathrm{~s}, 1 \mathrm{H})$, $7.03(\mathrm{dd}, J=3.0,9.1 \mathrm{~Hz}, 1 \mathrm{H}), 5.98(\mathrm{~s}, 1 \mathrm{H}), 4.03(\mathrm{~s}, 6 \mathrm{H}), 3.86(\mathrm{~s}, 3 \mathrm{H}), 3.67(\mathrm{~s}, 3 \mathrm{H}), 2.84-2.72(\mathrm{~m}, 4$ $\mathrm{H}) ;{ }^{13} \mathrm{C}-\mathrm{NMR}\left(75 \mathrm{MHz}, \mathrm{CDCl}_{3}\right): \delta=173.4,169.3,155.8,147.3,145.5,140.0,138.4,130.4,121.6,119.3$, 101.4, 100.7, 56.5, 55.6, 52.0, 32.8, 29.5; IR: $\tilde{v}=3360,2947,2361,1732,1609,1504,1462,1308,1107$, 1033, 814; UV-VIS $\left(\mathrm{CH}_{3} \mathrm{CN}+0.5 \%\right.$ piperidine $): \lambda_{\max }(\varepsilon)=515 \mathrm{~nm}\left(191001 \cdot \mathrm{mol}^{-1} \cdot \mathrm{cm}^{-1}\right)$; HRMS (ESI): $m / z$ calcd for $\mathrm{C}_{20} \mathrm{H}_{24} \mathrm{~N}_{3} \mathrm{O}_{7}$ : $418.1609[\mathrm{M}+\mathrm{H}]^{+}$; found: 418.1622 .

4-((3',4',5'-Trimethoxyphenyl)diazenyl)phenol 21a<smiles>COc1cc(/N=N/c2ccc(O)cc2)cc(OC)c1OC</smiles>

Standard Procedure A1 with 3,4,5-trimethoxyaniline (1.09 g, $5.0 \mathrm{mmol}, 1.0$ equiv.) and phenol (500 mg, $5.25 \mathrm{mmol}, 1.05$ equiv.) gave azobenzene $21 \mathrm{a}(1.25 \mathrm{~g}, 87 \%)$ as an ocher solid after purification by silicagel chromatography (EtOAc/petroleum ether, 1:2). $R_{f}=0.32$ (EtOAc/petroleum ether, 1:2); m.p. $165^{\circ} \mathrm{C}$; ${ }^{1} \mathrm{H}-\mathrm{NMR}\left(300 \mathrm{MHz}, \mathrm{CDCl}_{3}\right): \delta=7.87(\mathrm{~d}, J=8.8 \mathrm{~Hz}, 2 \mathrm{H}), 7.22(\mathrm{~s}, 2 \mathrm{H}), 6.96(\mathrm{~d}, J=8.9 \mathrm{~Hz}, 2 \mathrm{H}), 5.38$ 
(br s, $1 \mathrm{H}), 3.97(\mathrm{~s}, 6 \mathrm{H}), 3.94(\mathrm{~s}, 3 \mathrm{H}) ;{ }^{13} \mathrm{C}-\mathrm{NMR}\left(63 \mathrm{MHz}, \mathrm{CDCl}_{3}\right): \delta=158.3,153.4,148.6,146.9$, 140.0, 124.8, 115.8, 100.0, 61.0, 56.2; IR: $\tilde{v}=3283$, 2947, 2839, 1740, 1585, 1227, 1126, 991, 845; UVVIS $\left(\mathrm{CH}_{3} \mathrm{CN}+0.5 \%\right.$ piperidine $): \lambda_{\max }(\varepsilon)=362 \mathrm{~nm}(246001 \cdot \mathrm{mol}-1 \cdot \mathrm{cm}-1) ; \mathrm{HRMS}(\mathrm{ESI}): \mathrm{m} / \mathrm{z}$ calcd for $\mathrm{C}_{15} \mathrm{H}_{17} \mathrm{~N}_{2} \mathrm{O}_{4}: 289.1183[\mathrm{M}+\mathrm{H}]^{+}$; found: 289.1184 .

2-((tert-Butyldimethylsilyl)oxy)-4-((3',4’,5’-trimethoxyphenyl)diazenyl)phenol 21b<smiles>COc1cc(/N=N/c2ccc(O)c(OC(C)(C)C)c2)cc(OC)c1OC</smiles>

Standard Procedure A1 with 3,4,5-trimethoxyaniline (366 mg, $2.0 \mathrm{mmol}, 1.0$ equiv.) and phenol 10b (471 mg, $2.1 \mathrm{mmol}, 1.05$ equiv.) gave azobenzene $\mathbf{2 1 b}$ (264 mg, $32 \%$ ) as a red oil after purification by silica-gel chromatography (EtOAc/petroleum ether, 1:4). $R_{f}=0.44$ (EtOAc/petroleum ether, 1:4); ${ }^{1} \mathrm{H}-$ NMR $\left(250 \mathrm{MHz}, \mathrm{CDCl}_{3}\right): \delta=7.57(\mathrm{dd}, J=2.2,8.6 \mathrm{~Hz}, 1 \mathrm{H}), 7.45(\mathrm{~d}, J=2.2 \mathrm{~Hz}, 1 \mathrm{H}), 7.20(\mathrm{~s}, 2 \mathrm{H}), 7.06$ $(\mathrm{d}, J=8.6 \mathrm{~Hz}, 1 \mathrm{H}), 5.81(\mathrm{~s}, 1 \mathrm{H}), 3.98(\mathrm{~s}, 6 \mathrm{H}), 3.93(\mathrm{~s}, 3 \mathrm{H}), 1.06(\mathrm{~s}, 9 \mathrm{H}), 0.36(\mathrm{~s}, 6 \mathrm{H}) ;{ }^{13} \mathrm{C}-\mathrm{NMR}$ $\left(63 \mathrm{MHz}, \mathrm{CDCl}_{3}\right): \delta=153.5,150.3,148.5,147.8,146.5,142.8,119.6,117.5,110.7,100.1,61.0,56.2$, 25.7, 18.2, -4.3; IR: $\tilde{v}=2932,2859,2361,1740,1593,1493,1258,1219,1126,829,783$; UV-VIS $\left(\mathrm{CH}_{3} \mathrm{CN}+0.5 \%\right.$ piperidine $): \lambda_{\max }(\varepsilon)=375 \mathrm{~nm}\left(173001 \cdot \mathrm{mol}^{-1} \cdot \mathrm{cm}^{-1}\right) ; \mathrm{HRMS}(\mathrm{ESI}): \mathrm{m} / z$ calcd for $\mathrm{C}_{21} \mathrm{H}_{31} \mathrm{~N}_{2} \mathrm{O}_{5} \mathrm{Si}: 419.1997[\mathrm{M}+\mathrm{H}]^{+}$; found: 419.2004.

4-((3',4',5'-Trimethoxyphenyl)diazenyl)resorcin 21c 
<smiles>COc1cc(/N=N/c2ccc(O)cc2O)cc(OC)c1OC</smiles>

Standard Procedure A1 with 3,4,5-trimethoxyaniline (366 mg, $2.0 \mathrm{mmol}, 1.0$ equiv.) and resorcin (231 mg, $2.1 \mathrm{mmol}, 1.05$ equiv.) gave azobenzene 21c (347 mg, $57 \%$ ) as a red solid after purification by silica-gel chromatography (EtOAc/petroleum ether, 1:4 $\rightarrow$ 1:2). $R_{f}=0.42$ (EtOAc/petroleum ether, 1:2); m.p. $95{ }^{\circ} \mathrm{C} ;{ }^{1} \mathrm{H}-\mathrm{NMR}\left(400 \mathrm{MHz}, \mathrm{CDCl}_{3}\right): \delta=13.83$ (br s, $\left.1 \mathrm{H}\right), 7.76(\mathrm{~d}, J=8.8 \mathrm{~Hz}, 1 \mathrm{H}), 7.10(\mathrm{~s}, 2 \mathrm{H})$, $6.58(\mathrm{dd}, J=2.5,8.6 \mathrm{~Hz}, 1 \mathrm{H}), 6.46(\mathrm{~d}, J=2.6 \mathrm{~Hz}, 1 \mathrm{H}), 3.97(\mathrm{~s}, 6 \mathrm{H}), 3.95(\mathrm{~s}, 3 \mathrm{H}) ;{ }^{13} \mathrm{C}-\mathrm{NMR}$ $\left(101 \mathrm{MHz}, \mathrm{CDCl}_{3}\right): \delta=160.7,156.6,153.7,145.9,139.7,135.1,132.9,108.9,103.8,99.0,61.1,56.3$; IR: $\tilde{v}=3360,2940,2832,1724,1597,1215,1115,1006,826,783 ;$ UV-VIS $\left(\mathrm{CH}_{3} \mathrm{CN}+0.5 \%\right.$ piperidine): $\lambda_{\max }(\varepsilon)=350 \mathrm{~nm}\left(238001 \cdot \mathrm{mol}^{-1} \cdot \mathrm{cm}^{-1}\right) ; \mathrm{HRMS}(\mathrm{ESI}): \mathrm{m} / z$ calcd for $\mathrm{C}_{15} \mathrm{H}_{17} \mathrm{~N}_{2} \mathrm{O}_{5}: 305.1132$ $[\mathrm{M}+\mathrm{H}]^{+}$; found: 305.1133 .

2-Bromo-4-((3',4',5'-trimethoxyphenyl)diazenyl)phenol 21d<smiles>COc1cc(/N=N/c2ccc(O)c(Br)c2)cc(OC)c1OC</smiles>

Standard Procedure A1 with 3,4,5-trimethoxyaniline (366 mg, $2.0 \mathrm{mmol}, 1.0$ equiv.) and 2-bromphenol (363 mg, $2.1 \mathrm{mmol}, 1.05$ equiv.) gave azobenzene 21d (402 mg, $55 \%$ ) as an ocher solid after purification by silica-gel chromatography $\left(\mathrm{CH}_{2} \mathrm{Cl}_{2} / \mathrm{MeOH}, 95: 5+1.0 \%\right.$ formic acid $) . R_{f}=0.45\left(\mathrm{CH}_{2} \mathrm{Cl}_{2} / \mathrm{MeOH}, 95: 5\right.$ $+1.0 \%$ formic acid); m.p. $161{ }^{\circ} \mathrm{C} ;{ }^{1} \mathrm{H}-\mathrm{NMR}\left(250 \mathrm{MHz}, \mathrm{CDCl}_{3}\right): \delta=8.11(\mathrm{~d}, J=2.2 \mathrm{~Hz}, 1 \mathrm{H}), 7.88(\mathrm{dd}, J$ = 2.3, 8.7 Hz, 1 H), 7.23 (s, $2 \mathrm{H}), 7.17(\mathrm{~d}, J=8.6 \mathrm{~Hz}, 1 \mathrm{H}), 5.83(\mathrm{~s}, 1 \mathrm{H}), 3.97(\mathrm{~s}, 6 \mathrm{H}), 3.95(\mathrm{~s}, 3 \mathrm{H}) ;{ }^{13} \mathrm{C}-$ NMR (101 MHz, $\left.\mathrm{CDCl}_{3}\right): \delta=154.4,153.5,148.2,147.2,140.6,125.5,116.1,111.1,100.3,61.1,56.2$; IR: $\tilde{v}=3325,2955,1740,1597,1334,1207,1130,991 ; \mathrm{UV}-\mathrm{VIS}\left(\mathrm{CH}_{3} \mathrm{CN}+0.5 \%\right.$ piperidine $): \lambda_{\max }(\varepsilon)=$ $458 \mathrm{~nm}\left(28600 \mathrm{l} \cdot \mathrm{mol}^{-1} \cdot \mathrm{cm}^{-1}\right)$; HRMS (ESI): $\mathrm{m} / z$ calcd for $\mathrm{C}_{15} \mathrm{H}_{16} \mathrm{BrN}_{2} \mathrm{O}_{4}: 367.0288 / 369.0267[\mathrm{M}+\mathrm{H}]^{+}$; found: $367.0296 / 369.0275$.

3-Bromo-4-((3',4',5'-trimethoxyphenyl)diazenyl)phenol $21 e$ 
<smiles>COc1cc(/N=N/c2ccc(O)cc2Br)cc(OC)c1OC</smiles>

Standard Procedure A1 with 3,4,5-trimethoxyaniline (366 mg, $2.0 \mathrm{mmol}, 1.0$ equiv.) and 3-bromphenol (363 mg, $2.1 \mathrm{mmol}, 1.05$ equiv.) gave azobenzene 21e $(516 \mathrm{mg}, 70 \%$ ) as a dark green solid after purification by silica-gel chromatography $\left(\mathrm{CH}_{2} \mathrm{Cl}_{2} / \mathrm{MeOH}, 95: 5+1.0 \%\right.$ formic acid $) . R_{f}=0.45$ $\left(\mathrm{CH}_{2} \mathrm{Cl}_{2} / \mathrm{MeOH}, 95: 5+1.0 \%\right.$ formic acid); m.p. $221^{\circ} \mathrm{C} ;{ }^{1} \mathrm{H}-\mathrm{NMR}\left(300 \mathrm{MHz}, \mathrm{DMSO}-d_{6}\right): \delta=7.64(\mathrm{~d}, J=$ $8.9 \mathrm{~Hz}, 1 \mathrm{H}), 7.21(\mathrm{~s}, 2 \mathrm{H}), 7.21(\mathrm{~d}, J=2.5 \mathrm{~Hz}, 1 \mathrm{H}), 6.88(\mathrm{dd}, J=2.5,8.9 \mathrm{~Hz}, 1 \mathrm{H}), 3.86$ (s, $6 \mathrm{H}), 3.75$ (s, $3 \mathrm{H}) ;{ }^{13} \mathrm{C}-\mathrm{NMR}$ (75 MHz, DMSO- $\left.d_{6}\right): \delta=162.1,153.8,148.5,141.8,140.5,127.8,120.0,119.1,116.4$, 100.6, 60.7, 56.4; IR: $\tilde{v}=3237,2940,1740,1593,1458,1227,1123,984,856$; UV-VIS $\left(\mathrm{CH}_{3} \mathrm{CN}+0.5 \%\right.$ piperidine): $\lambda_{\max }(\varepsilon)=458 \mathrm{~nm}\left(283001 \cdot \mathrm{mol}^{-1} \cdot \mathrm{cm}^{-1}\right)$; HRMS (ESI): $m / z$ calcd for $\mathrm{C}_{15} \mathrm{H}_{16} \mathrm{BrN}_{2} \mathrm{O}_{4}$ : 367.0288/369.0267 [M+H] $]^{+}$; found: 367.0295/369.0274.

8-Hydroxy-5-((3',4',5'-trimethoxyphenyl)diazenyl)quinolin 22a<smiles>COc1cc(/N=N/c2ccc(O)c3ncccc23)cc(OC)c1OC</smiles>

Standard Procedure A1 with 3,4,5-trimethoxyaniline (366 mg, $2.0 \mathrm{mmol}, 1.0$ equiv.) and 8-hydroxyquinoline (305 mg, $2.1 \mathrm{mmol}, 1.05$ equiv.) gave azobenzene 22a (419 mg, $62 \%$ ) as a dark green solid after purification by silica-gel chromatography $\left(\mathrm{CH}_{2} \mathrm{Cl}_{2} / \mathrm{MeOH}, 95: 5+1.0 \%\right.$ formic acid $)$. $R_{f}$ $=0.46\left(\mathrm{CH}_{2} \mathrm{Cl}_{2} / \mathrm{MeOH}, 95: 5+1.0 \%\right.$ formic acid $) ;$ m.p. $180{ }^{\circ} \mathrm{C} ;{ }^{1} \mathrm{H}-\mathrm{NMR}\left(300 \mathrm{MHz}, \mathrm{CDCl}_{3}\right): \delta=9.22$ $(\mathrm{dd}, J=1.6,8.5 \mathrm{~Hz}, 1 \mathrm{H}), 8.84(\mathrm{dd}, J=1.5,4.1 \mathrm{~Hz}, 1 \mathrm{H}), 7.98(\mathrm{~d}, J=8.5 \mathrm{~Hz}, 1 \mathrm{H}), 7.59$ (dd, $J=4.2,8.6$ $\mathrm{Hz}, 1 \mathrm{H}), 7.23$ (d, $J=8.5 \mathrm{~Hz}, 1 \mathrm{H}), 7.22$ (s, $2 \mathrm{H}), 3.96(\mathrm{~s}, 6 \mathrm{H}), 3.91$ (s, $3 \mathrm{H}) ;{ }^{13} \mathrm{C}-\mathrm{NMR}(101 \mathrm{MHz}$, $\left.\mathrm{CDCl}_{3}\right): \delta=155.2,153.6,149.1,148.5,140.5,139.9,137.8,132.8,127.0,122.8,115.6,110.0,100.4$, 61.1, 56.3; IR: $\tilde{v}=2940,2322,1740,1574,1493,1458,1312,1215,1126,999,787$; UV-VIS $\left(\mathrm{CH}_{3} \mathrm{CN}+\right.$ $0.5 \%$ piperidine): $\lambda_{\max }(\varepsilon)=494 \mathrm{~nm}\left(174001 \cdot \mathrm{mol}^{-1} \cdot \mathrm{cm}^{-1}\right) ; \mathrm{HRMS}(\mathrm{ESI}): \mathrm{m} / z$ calcd for $\mathrm{C}_{18} \mathrm{H}_{18} \mathrm{~N}_{3} \mathrm{O}_{4}$ : $340.1292[\mathrm{M}+\mathrm{H}]^{+}$; found: 340.1298 . 
4-((3',4',5'-Trimethoxyphenyl)diazenyl)naphth-1-ol 22b<smiles>COc1cc(/N=N/c2ccc(O)c3ccccc23)cc(OC)c1OC</smiles>

Standard Procedure A1 with 3,4,5-trimethoxyaniline (366 mg, $2.0 \mathrm{mmol}, 1.0$ equiv.) and 1-naphthol (302 mg, $2.1 \mathrm{mmol}, 1.05$ equiv.) gave azobenzene 22b (422 mg, 62\%) as an ocher solid after purification by silica-gel chromatography (EtOAc/petroleum ether, $1: 4 \rightarrow 1: 2$ ). $R_{f}=0.59$ (EtOAc/petroleum ether, 1:4); m.p. $185{ }^{\circ} \mathrm{C} ;{ }^{1} \mathrm{H}-\mathrm{NMR}$ (300 MHz, DMSO- $\left.d_{6}\right): \delta=8.89(\mathrm{~d}, J=8.4 \mathrm{~Hz}, 1$ H), $8.23(\mathrm{~d}, J=8.2 \mathrm{~Hz}, 1 \mathrm{H}), 7.87(\mathrm{~d}, J=8.5 \mathrm{~Hz}, 1 \mathrm{H}), 7.71(\mathrm{ddd}, J=1.3,6.9,8.3 \mathrm{~Hz}, 1 \mathrm{H}), 7.59$ (ddd, $J$ $=1.1,7.0,8.2 \mathrm{~Hz}, 1 \mathrm{H}), 7.30(\mathrm{~s}, 2 \mathrm{H}), 7.00(\mathrm{~d}, J=8.6 \mathrm{~Hz}, 1 \mathrm{H}), 3.93(\mathrm{~s}, 6 \mathrm{H}), 3.76(\mathrm{~s}, 3 \mathrm{H}) ;{ }^{13} \mathrm{C}-\mathrm{NMR}$ $\left(75 \mathrm{MHz}, \mathrm{DMSO}-d_{6}\right): \delta=157.8,153.4,148.3,139.3,132.6,127.9,125.5,124.6,122.7,122.5,114.4$, 108.9, 99.7, 79.2, 60.2, 56.0; IR: $\tilde{v}=3244,2997,2322,1740,1593,1551,1477,1188,1123,1014,814$, 752; UV-VIS $\left(\mathrm{CH}_{3} \mathrm{CN}+0.5 \%\right.$ piperidine): $\lambda_{\max }(\varepsilon)=517 \mathrm{~nm}\left(180001 \cdot \mathrm{mol}^{-1} \cdot \mathrm{cm}^{-1}\right) ;$ HRMS $(\mathrm{ESI}): \mathrm{m} / \mathrm{z}$ calcd for $\mathrm{C}_{19} \mathrm{H}_{19} \mathrm{~N}_{2} \mathrm{O}_{4}: 339.1339[\mathrm{M}+\mathrm{H}]^{+}$; found: 339.1342 .

4-((3',4',5'-Trimethoxyphenyl)diazenyl)-6,7-dimethoxynaphth-1-ol 22d<smiles>COc1cc2c(O)ccc(N=Nc3cc(OC)c(OC)c(OC)c3)c2cc1OC</smiles>

Standard Procedure A1 with 3,4,5-trimethoxyaniline (366 mg, $2.0 \mathrm{mmol}, 1.0$ equiv.) and naphthol 11d (429 mg, $2.1 \mathrm{mmol}, 1.05$ equiv.) gave azobenzene 22d (488 mg, 62\%) as a dark red solid after purification by silica-gel chromatography (EtOAc/petroleum ether, 1:2 $\rightarrow 1: 1$ ). $R_{f}=0.45$ (EtOAc/petroleum ether, 1:2); m.p. $191{ }^{\circ} \mathrm{C} ;{ }^{1} \mathrm{H}-\mathrm{NMR}$ (600 MHz, DMSO- $d_{6}$ ): $\delta=8.22$ (br s, $1 \mathrm{H}$ ), $7.73(\mathrm{~d}$, 
$J=8.3 \mathrm{~Hz}, 1 \mathrm{H}), 7.49(\mathrm{~s}, 1 \mathrm{H}), 7.28(\mathrm{br} \mathrm{s}, 2 \mathrm{H}), 6.86(\mathrm{~d}, J=8.4 \mathrm{~Hz}, 1 \mathrm{H}), 3.98(\mathrm{~s}, 3 \mathrm{H}), 3.92(\mathrm{~s}, 3 \mathrm{H}), 3.91$ $(\mathrm{s}, 6 \mathrm{H}), 3.75$ (s, $3 \mathrm{H}) ;{ }^{13} \mathrm{C}-\mathrm{NMR}\left(151 \mathrm{MHz}, \mathrm{DMSO}-d_{6}\right): \delta=157.0,153.8,150.9,149.3,139.6,138.7$, 129.7, 120.2, 112.8, 108.0, 102.1, 101.8, 100.1, 79.6, 60.7, 56.3, 55.8, 55.6; IR: $\tilde{v}=2940,2839,1736$, 1593, 1543, 1458, 1373, 1226, 1122, 1003, 779; UV-VIS $\left(\mathrm{CH}_{3} \mathrm{CN}+0.5 \%\right.$ piperidine $): \lambda_{\max }(\varepsilon)=517 \mathrm{~nm}$ (23000 $1 \cdot \mathrm{mol}^{-1} \cdot \mathrm{cm}^{-1}$ ); HRMS (ESI): $\mathrm{m} / z$ calcd for $\mathrm{C}_{21} \mathrm{H}_{23} \mathrm{~N}_{2} \mathrm{O}_{6}: 399.1551[\mathrm{M}+\mathrm{H}]^{+}$; found: 399.1561 .

1-((3',4',5'-trimethoxyphenyl)diazenyl)naphth-2-ol 23<smiles>COc1cc(/N=N/c2c(O)ccc3ccccc23)cc(OC)c1OC</smiles>

Standard Procedure A1 with 3,4,5-trimethoxyaniline (366 mg, $2.0 \mathrm{mmol}, 1.0$ equiv.) and 2-naphthol (302 mg, $2.1 \mathrm{mmol}, 1.05$ equiv.) gave azobenzene 23 (574 $\mathrm{mg}, 85 \%$ ) as a red solid after purification by silica-gel chromatography (EtOAc/petroleum ether, 1:4 $\rightarrow$ 1:2). $R_{f}=0.41$ (EtOAc/petroleum ether, 1:4); m.p. $114{ }^{\circ} \mathrm{C} ;{ }^{1} \mathrm{H}-\mathrm{NMR}\left(600 \mathrm{MHz}, \mathrm{CDCl}_{3}\right): \delta=8.58(\mathrm{~d}, J=8.3 \mathrm{~Hz}, 1 \mathrm{H}), 7.74(\mathrm{~d}, J=9.4 \mathrm{~Hz}, 1 \mathrm{H}), 7.65$ $(\mathrm{d}, J=7.9 \mathrm{~Hz}, 1 \mathrm{H}), 7.58(\mathrm{ddd}, J=1.1,7.2,8.3 \mathrm{~Hz}, 1 \mathrm{H}), 7.41$ (ddd, $J=0.9,7.2,7.9 \mathrm{~Hz}, 1 \mathrm{H}), 7.04(\mathrm{~s}, 2$ $\mathrm{H}), 6.96(\mathrm{~d}, J=9.4 \mathrm{~Hz}, 1 \mathrm{H}), 3.98(\mathrm{~s}, 6 \mathrm{H}), 3.93(\mathrm{~s}, 3 \mathrm{H}) ;{ }^{13} \mathrm{C}-\mathrm{NMR}\left(151 \mathrm{MHz}, \mathrm{CDCl}_{3}\right): \delta=166.7,154.0$, 142.2, 138.6, 138.5, 133.3, 129.8, 128.6, 128.1, 125.4, 123.5, 121.5, 96.9, 61.1, 56.3; IR: $\tilde{v}=2970,2820$, 1748, 1597, 1450, 1215, 1123, 991, 818, 752; UV-VIS $\left(\mathrm{CH}_{3} \mathrm{CN}+0.5 \%\right.$ piperidine $): \lambda_{\max }(\varepsilon)=469 \mathrm{~nm}$ (26300 $1 \cdot \mathrm{mol}^{-1} \cdot \mathrm{cm}^{-1}$ ); HRMS (ESI): $\mathrm{m} / z$ calcd for $\mathrm{C}_{19} \mathrm{H}_{19} \mathrm{~N}_{2} \mathrm{O}_{4}: 339.1339[\mathrm{M}+\mathrm{H}]^{+}$; found: 339.1344 .

tert-Butyl-2-methoxy-5-((3',4',5'-trimethoxyphenyl)diazenyl)phenylcarbamate 24 


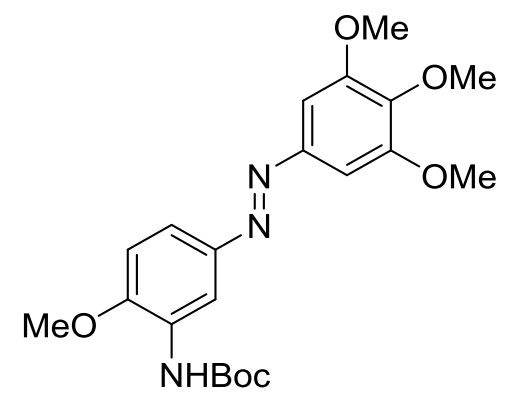

Standard Procedure B with azobenzene 18 (1.25 g, $3.10 \mathrm{mmol}, 1.0$ equiv.) and MeI (386 $\mu \mathrm{L}, 6.20 \mathrm{mmol}$, 2.0 equiv.) gave azobenzene $24(1.18 \mathrm{~g}, 91 \%)$ as a yellow solid after purification by silica-gel chromatography (EtOAc/petroleum ether, 1:4). $R_{f}=0.27$ (EtOAc/petroleum ether, 1:4); m.p. $132{ }^{\circ} \mathrm{C} ;{ }^{1} \mathrm{H}-$ NMR (300 MHz, $\left.\mathrm{CDCl}_{3}\right): \delta=8.72(\mathrm{~s}, 1 \mathrm{H}), 7.62(\mathrm{dd}, J=2.4,8.6 \mathrm{~Hz}, 1 \mathrm{H}), 7.26(\mathrm{~s}, 2 \mathrm{H}), 7.17(\mathrm{~s}, 1 \mathrm{H})$, $6.98(\mathrm{~d}, J=8.7 \mathrm{~Hz}, 1 \mathrm{H}), 3.97(\mathrm{~s}, 9 \mathrm{H}), 3.93(\mathrm{~s}, 3 \mathrm{H}), 1.57(\mathrm{~s}, 9 \mathrm{H}) ;{ }^{13} \mathrm{C}-\mathrm{NMR}\left(75 \mathrm{MHz}, \mathrm{CDCl}_{3}\right): \delta=$ 153.4, 152.5, 149.7, 148.6, 146.9, 139.9, 128.7, 119.0, 111.1, 109.6, 100.1, 80.6, 61.0, 56.2, 56.0, 28.3; IR: $\tilde{v}=2970,2836,2361,1720,1593,1520,1366,1219,1119,992 ; \mathrm{UV}-\mathrm{VIS}\left(\mathrm{CH}_{3} \mathrm{CN}+0.5 \%\right.$ piperidine): $\lambda_{\max }(\varepsilon)=372 \mathrm{~nm}\left(22100 \mathrm{l} \cdot \mathrm{mol}^{-1} \cdot \mathrm{cm}^{-1}\right) ; \mathrm{HRMS}(\mathrm{ESI}): \mathrm{m} / z$ calcd for $\mathrm{C}_{21} \mathrm{H}_{28} \mathrm{~N}_{3} \mathrm{O}_{6}: 418.1973$ $[\mathrm{M}+\mathrm{H}]^{+}$; found: 418.1978 .

$N$-(2-Methoxy-5-((3',4',5'-trimethoxyphenyl)diazenyl)phenyl)succinamic acid 25<smiles>COc1ccc(/N=N/c2cc(OC)c(OC)c(OC)c2)cc1NC(=O)CCC(=O)O</smiles>

Azobenzene 24 (585 mg, $1.40 \mathrm{mmol}, 1.0$ equiv.) dissolved in a mixture of 1,4-dioxane and methanol $(1: 1,10 \mathrm{~mL})$ was treated with $\mathrm{HCl}(8.0 \mathrm{~mL}, 4 \mathrm{M}$ in dioxan) and stirred at room temperature for $4 \mathrm{~h}$. After evaporation of all solvents, $\mathrm{K}_{2} \mathrm{CO}_{3}(2.17 \mathrm{~g}, 15.7 \mathrm{mmol}, 11.2$ equiv.) and succinic anhydride (631 mg, 6.31 mmol, 4.5 equiv.) were added followed by DMF $(8.0 \mathrm{~mL})$. The resulting mixture was stirred for $16 \mathrm{~h}$ at $85{ }^{\circ} \mathrm{C}$. After cooling water $(30 \mathrm{~mL})$ and EtOAc $(20 \mathrm{~mL})$ were added and the $\mathrm{pH}$ was adjusted to 10 with sat. $\mathrm{NaHCO}_{3}$. The organic layer was discarded. The $\mathrm{pH}$ of the aqueous phase was adjusted to 3 with $\mathrm{HCl}$ $(1 \mathrm{M})$, followed by extraction of the aqueous layer with EtOAc $(3 \times 150 \mathrm{~mL})$. The combined organic extracts were dried $\left(\mathrm{Na}_{2} \mathrm{SO}_{4}\right)$ and concentrated under reduced pressure. The residue was purified by silica-gel chromatography (EtOAc/petroleum ether, 3:7 $+0.5 \%$ formic acid) to provide azobenzene 25 
(317 mg, $54 \%$ ) as an orange solid. $R_{f}=0.20$ (EtOAc/petroleum ether, 3:7 + 0.5\% formic acid); m.p. 88 ${ }^{\circ} \mathrm{C} ;{ }^{1} \mathrm{H}-\mathrm{NMR}\left(300 \mathrm{MHz}, \mathrm{DMSO}-d_{6}\right): \delta=12.12$ (br s, $\left.1 \mathrm{H}\right), 9.36(\mathrm{~s}, 1 \mathrm{H}), 8.62$ (d, $\left.J=2.1 \mathrm{~Hz}, 1 \mathrm{H}\right), 7.71$ $(\mathrm{dd}, J=2.4,8.7 \mathrm{~Hz}, 1 \mathrm{H}), 7.24(\mathrm{~d}, J=8.9 \mathrm{~Hz}, 1 \mathrm{H}), 7.22$ (s, $2 \mathrm{H}), 3.95$ (s, $3 \mathrm{H}), 3.89$ (s, $6 \mathrm{H}), 3.75$ (s, 3 H), $2.69(\mathrm{t}, J=6.9 \mathrm{~Hz}, 2 \mathrm{H}), 2.57-2.52(\mathrm{~m}, 2 \mathrm{H}) ;{ }^{13} \mathrm{C}-\mathrm{NMR}\left(101 \mathrm{MHz}, \mathrm{DMSO}-d_{6}\right): \delta=174.4,171.2$, 153.8, 152.2, 148.3, 145.9, 140.2, 128.7, 122.1, 113.6, 111.5, 100.3, 60.7, 56.6, 56.4, 31.4, 29.3; IR: $\tilde{v}=$ 3329, 2920, 2851, 1740, 1705, 1593, 1531, 1408, 1250, 1123, 991; UV-VIS ( $\mathrm{CH}_{3} \mathrm{CN}+0.5 \%$ piperidine): $\lambda_{\text {max }}(\varepsilon)=373 \mathrm{~nm}\left(226001 \cdot \mathrm{mol}^{-1} \cdot \mathrm{cm}^{-1}\right)$; HRMS (ESI): $\mathrm{m} / z$ calcd for $\mathrm{C}_{20} \mathrm{H}_{24} \mathrm{~N}_{3} \mathrm{O}_{7}: 418.1609[\mathrm{M}+\mathrm{H}]^{+}$; found: 418.1610 .

Ethyl-2-(4'-((4"'-bromophenyl)diazenyl)phenoxy)acetate 26a<smiles>CCOC(=O)COc1ccc(/N=N/c2ccc(Br)cc2)cc1</smiles>

Standard Procedure B with azobenzene 3 (1.10 g, $4.0 \mathrm{mmol}, 1.0$ equiv.) and ethyl bromoacetate (1.45 mL, 13.1 mmol, 3,3 equiv.) gave azobenzene 26a (1.20 g, $83 \%)$ as a yellow solid after recrystallization (toluene/petroleum ether, 1:4). $R_{f}=0.55$ (EtOAc/petroleum ether, 1:4); m.p. $203{ }^{\circ} \mathrm{C} ;{ }^{1} \mathrm{H}-\mathrm{NMR}(300 \mathrm{MHz}$, $\left.\mathrm{CDCl}_{3}\right): \delta=7.92(\mathrm{~d}, J=9.0 \mathrm{~Hz}, 2 \mathrm{H}), 7.77(\mathrm{~d}, J=8.6 \mathrm{~Hz}, 2 \mathrm{H}), 7.64(\mathrm{~d}, J=8.5 \mathrm{~Hz}, 2 \mathrm{H}), 7.03(\mathrm{~d}, J=9.0$ $\mathrm{Hz}, 2 \mathrm{H}), 4.71(\mathrm{~s}, 2 \mathrm{H}), 4.31(\mathrm{q}, J=7.1 \mathrm{~Hz}, 2 \mathrm{H}), 1.32(\mathrm{t}, J=7.1 \mathrm{~Hz}, 3 \mathrm{H}) ;{ }^{13} \mathrm{C}-\mathrm{NMR}\left(75 \mathrm{MHz}, \mathrm{CDCl}_{3}\right): \delta$ $=168.4,160.3,151.4,147.4,132.2,124.9,124.8,124.1,115.0,65.5,61.6,14.2 ;$ IR: $\tilde{v}=3167,3024$, 2816, 1740, 1570, 1473, 1409, 1219, 1060, 834; UV-VIS $\left(\mathrm{CH}_{3} \mathrm{CN}+0.5 \%\right.$ piperidine $): \lambda_{\max }(\varepsilon)=464 \mathrm{~nm}$ (19800 $1 \cdot \mathrm{mol}^{-1} \cdot \mathrm{cm}^{-1}$ ); HRMS (ESI): $\mathrm{m} / \mathrm{z}$ calcd for $\mathrm{C}_{16} \mathrm{H}_{16} \mathrm{BrN}_{2} \mathrm{O}_{3}: 363.0339 / 365.0320[\mathrm{M}+\mathrm{H}]^{+}$; found: $363.0344 / 365.0327$.

Ethyl-2-(4'-((4"-(dimethylamino)phenyl)diazenyl)phenoxy)acetate $\mathbf{2 6 \mathbf { b }}$ 


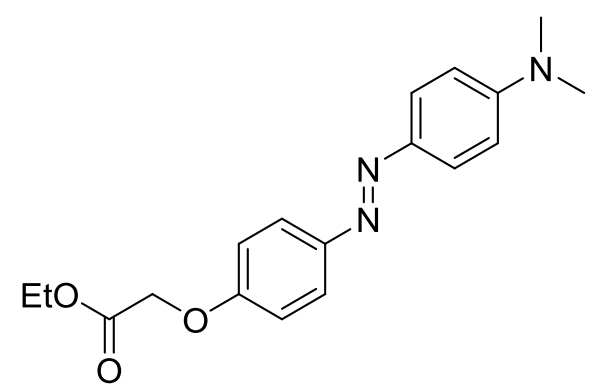

Standard Procedure D with azobenzene 26a (50.0 mg, $0.14 \mathrm{mmol}, 1.0$ equiv.), dimethylamine hydrochloride (22.5 mg, $0.28 \mathrm{mmol}, 2.0$ equiv.) and $\mathrm{Cs}_{2} \mathrm{CO}_{3}$ (269 mg, $0.83 \mathrm{mmol}$, 6.0 equiv.) gave azobenzene $\mathbf{2 6 b}(39.1 \mathrm{mg}, 87 \%)$ as a scarlet solid after silica-gel chromatography $\left(\mathrm{CH}_{2} \mathrm{Cl}_{2} / \mathrm{MeOH}, 20: 1\right)$. $R_{f}=0.26\left(\mathrm{CH}_{2} \mathrm{Cl}_{2} / \mathrm{MeOH}, 20: 1\right)$; m.p. $142{ }^{\circ} \mathrm{C} ;{ }^{1} \mathrm{H}-\mathrm{NMR}\left(300 \mathrm{MHz}, \mathrm{CDCl}_{3}\right): \delta=7.86(\mathrm{~d}, J=3.3 \mathrm{~Hz}, 2 \mathrm{H})$, $7.83(\mathrm{~d}, J=3.1 \mathrm{~Hz}, 2 \mathrm{H}), 7.00(\mathrm{~d}, J=8.9 \mathrm{~Hz}, 2 \mathrm{H}), 6.77(\mathrm{~d}, J=9.1 \mathrm{~Hz}, 2 \mathrm{H}), 4.69(\mathrm{~s}, 2 \mathrm{H}), 4.30(\mathrm{q}, J=$ $7.2 \mathrm{~Hz}, 2 \mathrm{H}), 3.09$ (s, $6 \mathrm{H}), 1.32(\mathrm{t}, J=7.1 \mathrm{~Hz}, 3 \mathrm{H}) ;{ }^{13} \mathrm{C}-\mathrm{NMR}\left(101 \mathrm{MHz}, \mathrm{CDCl}_{3}\right): \delta=168.7,158.9$, 152.1, 148.1, 143.6, 124.6, 123.3, 114.8, 111.5, 65.6, 61.5, 40.3, 14.2; IR: $\tilde{v}=3460,3082,2961,2820$, 1738, 1597, 1366, 1207, 1083, 838; UV-VIS $\left(\mathrm{CH}_{3} \mathrm{CN}+0.5 \%\right.$ piperidine $): \lambda_{\max }(\varepsilon)=409 \mathrm{~nm}(24700$ $1 \cdot \mathrm{mol}^{-1} \cdot \mathrm{cm}^{-1}$ ); HRMS (ESI): $\mathrm{m} / z$ calcd for $\mathrm{C}_{18} \mathrm{H}_{22} \mathrm{~N}_{3} \mathrm{O}_{3}: 328.1656[\mathrm{M}+\mathrm{H}]^{+}$; found: 328.1658 .

Ethyl-2-(4'-((4"'-morpholinophenyl)diazenyl)phenoxy)acetate 26c<smiles>CCOC(=O)COc1ccc(/N=N/c2ccc(N3CCOCC3)cc2)cc1</smiles>

Standard Procedure D with azobenzene 26a (50.0 mg, $0.14 \mathrm{mmol}, 1.0$ equiv.) and morpholine (24.0 mg, $0.28 \mathrm{mmol}, 2.0$ equiv.) gave azobenzene $26 \mathrm{c}(40.2 \mathrm{mg}, 79 \%)$ as an orange solid after silica-gel chromatography (EtOAc/petroleum ether, 1:4 $\rightarrow$ 1:2). $R_{f}=0.28$ (EtOAc/petroleum ether, 1:4); m.p. 160 ${ }^{\circ} \mathrm{C} ;{ }^{1} \mathrm{H}-\mathrm{NMR}\left(300 \mathrm{MHz}, \mathrm{CDCl}_{3}\right): \delta=7.87(\mathrm{~d}, J=9.0 \mathrm{~Hz}, 2 \mathrm{H}), 7.87(\mathrm{~d}, J=9.0 \mathrm{~Hz}, 2 \mathrm{H}), 7.01(\mathrm{~d}, J=9.1$ Hz, 2 H), 6.98 (d, J = 9.1 Hz, 2 H), 4.70 (s, 2 H), 4.30 (q, J = 7.2 Hz, 2 H), 3.95 - 3.82 (m, 4 H), 3.35 $3.28(\mathrm{~m}, 4 \mathrm{H}), 1.32(\mathrm{t}, J=7.2 \mathrm{~Hz}, 3 \mathrm{H}) ;{ }^{13} \mathrm{C}-\mathrm{NMR}\left(75 \mathrm{MHz}, \mathrm{CDCl}_{3}\right): \delta=168.6,159.3,152.8,147.9$, $145.8,124.3,124.1,114.9,114.5,66.7,65.5,61.5,48.2,14.2 ;$ IR: $\tilde{v}=3464,2963,2843,1759,1578$, 1366, 1246, 1153, 921, 841; UV-VIS $\left(\mathrm{CH}_{3} \mathrm{CN}+0.5 \%\right.$ piperidine $): \lambda_{\max }(\varepsilon)=392 \mathrm{~nm}\left(262001 \cdot \mathrm{mol}^{-1} \cdot \mathrm{cm}^{-}\right.$ ${ }^{1}$ ); HRMS (ESI): $m / z$ calcd for $\mathrm{C}_{20} \mathrm{H}_{24} \mathrm{~N}_{3} \mathrm{O}_{4}: 370.1761[\mathrm{M}+\mathrm{H}]^{+}$; found: 370.1766 . 
Ethyl-2-(4'-((4"'-(4"'”-methylpiperazin-1-yl)phenyl)diazenyl)phenoxy)acetate 26d<smiles>CCOC(=O)COc1ccc(/N=N/c2ccc(N3CCN(C)CC3)cc2)cc1</smiles>

Standard Procedure D with azobenzene 26a (100.0 mg, $0.28 \mathrm{mmol}, 1.0$ equiv.) and 1-methylpiperazine (55.2 mg, $0.55 \mathrm{mmol}, 2.0$ equiv.) gave azobenzene $\mathbf{2 6 d}(89.0 \mathrm{mg}, 85 \%)$ as a yellow solid after silica-gel chromatography $\left(\mathrm{CH}_{2} \mathrm{Cl}_{2} / \mathrm{MeOH}, 80: 1 \rightarrow 20: 1\right) . R_{f}=0.25\left(\mathrm{CH}_{2} \mathrm{Cl}_{2} / \mathrm{MeOH}, 20: 1\right)$; m.p. $150{ }^{\circ} \mathrm{C} ;{ }^{1} \mathrm{H}-\mathrm{NMR}$ $\left(300 \mathrm{MHz}, \mathrm{CDCl}_{3}\right): \delta=7.87(\mathrm{~d}, J=3.4 \mathrm{~Hz}, 2 \mathrm{H}), 7.84(\mathrm{~d}, J=3.4 \mathrm{~Hz}, 2 \mathrm{H}), 7.01(\mathrm{~d}, J=8.2 \mathrm{~Hz}, 2 \mathrm{H}), 6.98$ (d, $J=8.2 \mathrm{~Hz}, 2 \mathrm{H}), 4.70(\mathrm{~s}, 2 \mathrm{H}), 4.30$ (q, $J=7.2 \mathrm{~Hz}, 2 \mathrm{H}), 3.43$ - 3.34 (m, $4 \mathrm{H}), 2.64$ - $2.55(\mathrm{~m}, 4 \mathrm{H})$, $2.38(\mathrm{~s}, 3 \mathrm{H}), 1.32(\mathrm{t}, J=7.1 \mathrm{~Hz}, 3 \mathrm{H}) ;{ }^{13} \mathrm{C}-\mathrm{NMR}\left(75 \mathrm{MHz}, \mathrm{CDCl}_{3}\right): \delta=168.6,159.2,152.8,148.0$, 145.4, 124.3, 124.1, 114.8, 114.7, 65.5, 61.5, 54.9, 47.9, 46.1, 14.2; IR: $\tilde{v}=3460,2931,2839,2793$, 1751, 1736, 1581, 1497, 1443, 1377, 1220, 834; UV-VIS $\left(\mathrm{CH}_{3} \mathrm{CN}+0.5 \%\right.$ piperidine $): \lambda_{\max }(\varepsilon)=396 \mathrm{~nm}$ (27300 $\left.1 \cdot \mathrm{mol}^{-1} \cdot \mathrm{cm}^{-1}\right)$; HRMS (ESI): $\mathrm{m} / z$ calcd for $\mathrm{C}_{21} \mathrm{H}_{27} \mathrm{~N}_{4} \mathrm{O}_{3}: 383.2078[\mathrm{M}+\mathrm{H}]^{+}$; found: 383.2084 .

Ethyl-2-(4'-((4"'-acetamidophenyl)diazenyl)phenoxy)acetate 26e<smiles>CCOC(=O)COc1ccc(/N=N/c2ccc(NC(C)=O)cc2)cc1</smiles>

Standard Procedure D with azobenzene 26a (100.0 mg, $0.28 \mathrm{mmol}, 1.0$ equiv.), and acetamide (32.5 mg, $0.55 \mathrm{mmol}, 2.0$ equiv.) gave azobenzene $26 \mathrm{e}(61.2 \mathrm{mg}, 65 \%)$ as an orange solid after silica-gel chromatography $\left(\mathrm{CH}_{2} \mathrm{Cl}_{2} / \mathrm{MeOH}, 9: 1\right) . R_{f}=0.40\left(\mathrm{CH}_{2} \mathrm{Cl}_{2} / \mathrm{MeOH}, 9: 1\right)$; m.p. $192{ }^{\circ} \mathrm{C} ;{ }^{1} \mathrm{H}-\mathrm{NMR}(400 \mathrm{MHz}$, $\left.\mathrm{CDCl}_{3}\right): \delta=7.92(\mathrm{~d}, J=6.7 \mathrm{~Hz}, 2 \mathrm{H}), 7.90(\mathrm{~d}, J=6.7 \mathrm{~Hz}, 2 \mathrm{H}), 7.68(\mathrm{~d}, J=8.5 \mathrm{~Hz}, 2 \mathrm{H}), 7.34(\mathrm{~s}, 1 \mathrm{H})$, $7.04(\mathrm{~d}, J=8.8 \mathrm{~Hz}, 2 \mathrm{H}), 4.72(\mathrm{~s}, 2 \mathrm{H}), 4.32(\mathrm{q}, J=7.2 \mathrm{~Hz}, 2 \mathrm{H}), 2.25(\mathrm{~s}, 3 \mathrm{H}), 1.34$ (t, $J=7.2 \mathrm{~Hz}, 3 \mathrm{H})$; ${ }^{13} \mathrm{C}-\mathrm{NMR}\left(101 \mathrm{MHz}, \mathrm{CDCl}_{3}\right): \delta=168.5,168.2,159.9,149.1,147.7,140.0,124.6,123.7,119.7,114.9$, 
65.5, 61.6, 24.8, 14.2; IR: $\tilde{v}=3005,2913,2322,1717,1601,1504,1377,1203,1076,837,714$; UV-VIS $\left(\mathrm{CH}_{3} \mathrm{CN}+0.5 \%\right.$ piperidine $): \lambda_{\max }(\varepsilon)=361 \mathrm{~nm}\left(257001 \cdot \mathrm{mol}^{-1} \cdot \mathrm{cm}^{-1}\right) ; \mathrm{HRMS}(\mathrm{ESI}): \mathrm{m} / z$ calcd for $\mathrm{C}_{18} \mathrm{H}_{20} \mathrm{~N}_{3} \mathrm{O}_{4}: 342.1448[\mathrm{M}+\mathrm{H}]^{+}$; found: 342.1451 .

Ethyl-2-(4'-((4"'-(1"',3"'”-dioxoisoindolin-2"'-yl)phenyl)diazenyl)phenoxy)acetate 26f

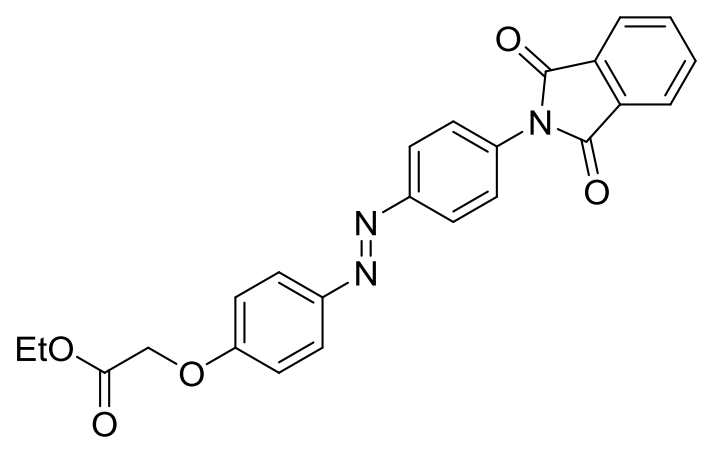

Bromaryl azobenzene 26a (50.0 mg, $0.14 \mathrm{mmol}, 1.0$ equiv.) CuI (39.3 mg, $0.21 \mathrm{mmol}, 1.5 \mathrm{equiv}$ ), and potassium phthalimide $(38.3 \mathrm{mg}, 0.21 \mathrm{mmol}, 1.5$ equiv.) were placed in a microwave reaction vial. The vial was sealed, and the atmosphere replaced by nitrogen. The solids were suspended in dry DMF (4 mL), and the vial was heated for $9 \mathrm{~h}$ to $160^{\circ} \mathrm{C}$. After cooling aqueous $\mathrm{HCl}(1 \mathrm{M}, 3 \mathrm{~mL})$ was added, followed by extraction with $\mathrm{CHCl}_{3}(3 \times 10 \mathrm{~mL})$. The combined organic extracts were washed with $\mathrm{H}_{2} \mathrm{O}(20 \mathrm{~mL})$, and brine $(20 \mathrm{~mL})$, dried with $\mathrm{Na}_{2} \mathrm{SO}_{4}$, filtered, and concentrated under reduced pressure. Purification by silica-gel chromatography (EtOAc/petroleum ether, 1:9 $\rightarrow$ 1:1) provided pure azobenzene 26f (42.0 mg, $71 \%)$ as an ocher solid. $R_{f}=0.39$ (EtOAc/petroleum ether, 1:2); m.p. $189{ }^{\circ} \mathrm{C} ;{ }^{1} \mathrm{H}-\mathrm{NMR}(300 \mathrm{MHz}$, $\left.\mathrm{CDCl}_{3}\right): \delta=8.06-7.92(\mathrm{~m}, 6 \mathrm{H}), 7.84-7.80(\mathrm{~m}, J=3.1,5.5 \mathrm{~Hz}, 2 \mathrm{H}), 7.64(\mathrm{~d}, J=8.8 \mathrm{~Hz}, 2 \mathrm{H}), 7.04(\mathrm{~d}$, $J=9.0 \mathrm{~Hz}, 2 \mathrm{H}), 4.72(\mathrm{~s}, 2 \mathrm{H}), 4.31(\mathrm{q}, J=7.2 \mathrm{~Hz}, 2 \mathrm{H}), 1.33$ (t, $J=7.2 \mathrm{~Hz}, 3 \mathrm{H}) ;{ }^{13} \mathrm{C}-\mathrm{NMR}(75 \mathrm{MHz}$, $\left.\mathrm{CDCl}_{3}\right): \delta=168.4,167.1,160.3,151.5,147.6,134.6,133.5,131.7,126.8,124.9,123.9,123.3,114.9$, 65.5, 61.6, 14.2; IR: $\tilde{v}=3375,2990,2322,1740,1694,1524,1223,1153,1080,841$; UV-VIS $\left(\mathrm{CH}_{3} \mathrm{CN}+\right.$ $0.5 \%$ piperidine): $\lambda_{\max }(\varepsilon)=351 \mathrm{~nm}\left(253001 \cdot \mathrm{mol}^{-1} \cdot \mathrm{cm}^{-1}\right) ; \mathrm{HRMS}(\mathrm{ESI}): \mathrm{m} / z$ calcd for $\mathrm{C}_{24} \mathrm{H}_{20} \mathrm{~N}_{3} \mathrm{O}_{5}$ : $430.1397[\mathrm{M}+\mathrm{H}]^{+}$; found: 430.1410 .

Ethyl-2-(4'-((3"',5"-trimethoxyphenyl)diazenyl)phenoxy)acetate 27 


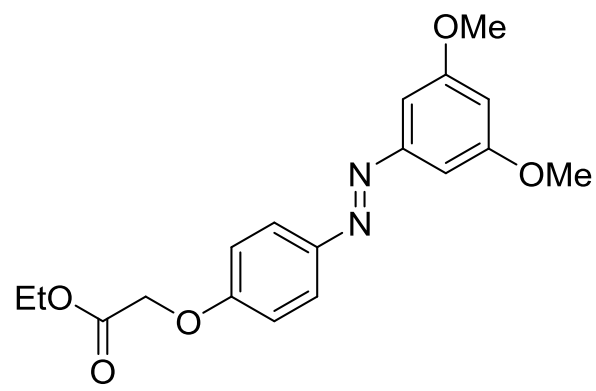

Standard Procedure B with azobenzene 14 (199 mg, $0.77 \mathrm{mmol}, 1.0$ equiv.) and ethyl bromoacetate (282 $\mu \mathrm{L}, 13.4 \mathrm{mmol}, 3.3$ equiv.) gave azobenzene $27(241 \mathrm{mg}, 91 \%)$ as an orange solid after purification by silica-gel chromatography (EtOAc/petroleum ether, 1:4). $R_{f}=0.40$ (EtOAc/petroleum ether, 1:4); m.p. $93{ }^{\circ} \mathrm{C} ;{ }^{1} \mathrm{H}-\mathrm{NMR}\left(400 \mathrm{MHz}, \mathrm{CDCl}_{3}\right): \delta=7.94(\mathrm{~d}, J=9.1 \mathrm{~Hz}, 2 \mathrm{H}), 7.11(\mathrm{~d}, J=2.3 \mathrm{~Hz}, 2 \mathrm{H}), 7.05(\mathrm{~d}, J=$ $9.1 \mathrm{~Hz}, 2 \mathrm{H}), 6.60(\mathrm{t}, J=2.3 \mathrm{~Hz}, 1 \mathrm{H}), 4.72(\mathrm{~s}, 2 \mathrm{H}), 4.32(\mathrm{q}, J=7.0 \mathrm{~Hz}, 2 \mathrm{H}), 3.89(\mathrm{~s}, 6 \mathrm{H}), 1.33(\mathrm{t}, J=$ $7.2 \mathrm{~Hz}, 3 \mathrm{H}) ;{ }^{13} \mathrm{C}-\mathrm{NMR}\left(101 \mathrm{MHz}, \mathrm{CDCl}_{3}\right): \delta=168.5,161.1,160.2,154.5,147.4,124.8,114.9,103.5$, 100.7, 65.5, 61.6, 55.6, 14.2; IR: $\tilde{v}=3062$, 2908, 1762, 1582, 1412, 1210, 1145, 852, 829, 675; UV-VIS $\left(\mathrm{CH}_{3} \mathrm{CN}+0.5 \%\right.$ piperidine $): \lambda_{\max }(\varepsilon)=345 \mathrm{~nm}\left(201001 \cdot \mathrm{mol}^{-1} \cdot \mathrm{cm}^{-1}\right) ; \mathrm{HRMS}(\mathrm{ESI}): \mathrm{m} / z$ calcd for $\mathrm{C}_{18} \mathrm{H}_{21} \mathrm{~N}_{2} \mathrm{O}_{5}: 345.1445[\mathrm{M}+\mathrm{H}]^{+}$; found: 345.1450 .

Ethyl-2-(4'-((3"',4"-dihydro-2"H-benzo[b][1",4"']oxazin-7"'-yl)diazenyl)phenoxy) acetate 28<smiles>CCOC(=O)COc1ccc(/N=N/c2ccc3c(c2)OCCN3)cc1</smiles>

Standard Procedure B with azobenzene 15 (100 mg, $0.39 \mathrm{mmol}, 1.0$ equiv.), and ethyl bromoacetate (46 $\mu \mathrm{L}, 0.41 \mathrm{mmol}, 1.05$ equiv.) gave azobenzene 28 (124 mg, $93 \%)$ as an orange solid after purification by silica-gel chromatography (EtOAc/petroleum ether, 1:4 $\rightarrow$ 1:1). $R_{f}=0.35$ (EtOAc/petroleum ether, 1:2); m.p. $76{ }^{\circ} \mathrm{C}$; ${ }^{1} \mathrm{H}-\mathrm{NMR}\left(400 \mathrm{MHz}, \mathrm{DMSO}-d_{6}\right): \delta=7.73(\mathrm{~d}, J=9.1 \mathrm{~Hz}, 2 \mathrm{H}), 7.34$ (dd, $J=2.0,8.5 \mathrm{~Hz}, 1$ H), $7.18(\mathrm{~d}, J=2.0 \mathrm{~Hz}, 1 \mathrm{H}), 7.06(\mathrm{~d}, J=9.1 \mathrm{~Hz}, 2 \mathrm{H}), 6.80-6.76(\mathrm{~m}, 1 \mathrm{H}), 6.68(\mathrm{~d}, J=8.5 \mathrm{~Hz}, 1 \mathrm{H})$, 4.87 (s, $2 \mathrm{H}), 4.19$ (q, $J=7.1 \mathrm{~Hz}, 2 \mathrm{H}), 4.16(\mathrm{t}, J=4.4 \mathrm{~Hz}, 2 \mathrm{H}), 3.43-3.37(\mathrm{~m}, J=2.3 \mathrm{~Hz}, 2 \mathrm{H}), 1.23$ (t, $J=7.2 \mathrm{~Hz}, 3 \mathrm{H}) ;{ }^{13} \mathrm{C}-\mathrm{NMR}\left(101 \mathrm{MHz}, \mathrm{DMSO}-d_{6}\right): \delta=168.5,158.8,146.9,143.0,142.7,138.8,123.4$, 120.2, 115.0, 113.2, 107.8, 64.8, 64.1, 60.7, 40.0, 14.0; IR: $\tilde{v}=3356,2970,2928,2870,1751,1597$, 1500, 1315, 1261, 1080, 837, 810: UV-VIS $\left(\mathrm{CH}_{3} \mathrm{CN}+0.5 \%\right.$ piperidine $): \lambda_{\max }(\varepsilon)=408 \mathrm{~nm}\left(240001 \cdot \mathrm{mol}^{-}\right.$ ${ }^{1} \cdot \mathrm{cm}^{-1}$ ); HRMS (ESI): $\mathrm{m} / z$ calcd for $\mathrm{C}_{18} \mathrm{H}_{20} \mathrm{~N}_{3} \mathrm{O}_{4}: 342.1448[\mathrm{M}+\mathrm{H}]^{+}$; found: 342.1452 . 
Ethyl-2-(4"-((3",4" ,5"'-trimethoxyphenyl)diazenyl)phenoxy)acetate 29a<smiles>CCOC(=O)COc1ccc(/N=N/c2cc(OC)c(OC)c(OC)c2)cc1</smiles>

Standard Procedure B with azobenzene 21a (1.17 g, $4.05 \mathrm{mmol}, 1.0$ equiv.) and ethyl bromoacetate (1,48 mL, $13.4 \mathrm{mmol}, 3.3$ equiv.) gave azobenzene 29a $(1.39 \mathrm{~g}, 91 \%)$ as an orange solid after purification by silica-gel chromatography (EtOAc/petroleum ether, 1:4). $R_{f}=0.52$ (EtOAc/petroleum ether, 1:2); m.p. $51^{\circ} \mathrm{C} ;{ }^{1} \mathrm{H}-\mathrm{NMR}\left(400 \mathrm{MHz}, \mathrm{CDCl}_{3}\right): \delta=7.92(\mathrm{~d}, J=8.5 \mathrm{~Hz}, 2 \mathrm{H}), 7.23(\mathrm{~s}, 2 \mathrm{H}), 7.04(\mathrm{~d}$, $J=8.8 \mathrm{~Hz}, 2 \mathrm{H}), 4.72(\mathrm{~s}, 2 \mathrm{H}), 4.31(\mathrm{q}, J=7.2 \mathrm{~Hz}, 2 \mathrm{H}), 3.98(\mathrm{~s}, 6 \mathrm{H}), 3.95(\mathrm{~s}, 3 \mathrm{H}), 1.33$ (t, $J=7.9 \mathrm{~Hz}, 3$ $\mathrm{H}) ;{ }^{13} \mathrm{C}-\mathrm{NMR}\left(101 \mathrm{MHz}, \mathrm{CDCl}_{3}\right): \delta=168.4,159.9,153.5,148.5,147.4,140.2,124.5,114.9,100.1,65.4$, 61.5, 61.0, 56.1, 14.1; IR: $\tilde{v}=2940,2913,2839,1759,1578,1215,1123,1003,845,829$; UV-VIS $\left(\mathrm{CH}_{3} \mathrm{CN}+0.5 \%\right.$ piperidine $): \lambda_{\max }(\varepsilon)=360 \mathrm{~nm}\left(221001 \cdot \mathrm{mol}^{-1} \cdot \mathrm{cm}^{-1}\right) ; \mathrm{HRMS}(\mathrm{ESI}): \mathrm{m} / z$ calcd for $\mathrm{C}_{19} \mathrm{H}_{23} \mathrm{~N}_{2} \mathrm{O}_{6}: 375.1551[\mathrm{M}+\mathrm{H}]^{+}$; found: 375.1563 .

Ethyl-2-(2'-(tert-butyldimethylsilyl)oxy-4-((3"',4",5"'-trimethoxyphenyl)diazenyl)phenoxy) acetate 29b<smiles>CCOC(=O)COc1ccc(/N=N/c2cc(OC)c(OC)c(OC)c2)cc1OC</smiles>

A solution of phenol $\mathbf{2 1 b}$ (100 mg, $0.24 \mathrm{mmol}, 1.0$ equiv.) in dry THF (10 mL) was cooled to $-20{ }^{\circ} \mathrm{C}$ under an atmosphere of argon. Solid $\mathrm{NaH}(8.6 \mathrm{mg}, 0.36 \mathrm{mmol}, 1.5$ equiv.) was added and stirred for 10 min followed by addition of ethyl bromoacetate $(133 \mu \mathrm{L}, 1.19 \mathrm{mmol}, 5.0$ equiv.). The reaction mixture was stirred at $0^{\circ} \mathrm{C}$ for $1.5 \mathrm{~h}$. After stirring for additional $1 \mathrm{~h}$ at $20^{\circ} \mathrm{C}$ the $\mathrm{pH}$ was adjusted to 7 with phosphate buffer, and $\mathrm{CHCl}_{3}(20 \mathrm{~mL})$ was added. The aqueous layer was separated and extracted with $\mathrm{CHCl}_{3}(3 \times 10 \mathrm{~mL})$. The combined organic extracts were dried with $\mathrm{Na}_{2} \mathrm{SO}_{4}$, filtered, and concentrated under reduced pressure. Purification by silica-gel chromatography (EtOAc/petroleum ether, 1:4) provided 
azobenzene 29b (75 mg, $62 \%)$ as a yellow solid. $R_{f}=0.42$ (EtOAc/petroleum ether, 1:4); m.p. $101{ }^{\circ} \mathrm{C}$; ${ }^{1} \mathrm{H}-\mathrm{NMR}\left(400 \mathrm{MHz}, \mathrm{CDCl}_{3}\right): \delta=7.54(\mathrm{dd}, J=2.3,8.5 \mathrm{~Hz}, 1 \mathrm{H}), 7.47(\mathrm{~d}, J=2.3 \mathrm{~Hz}, 1 \mathrm{H}), 7.21(\mathrm{~s}, 2 \mathrm{H})$, $6.90(\mathrm{~d}, J=8.5 \mathrm{~Hz}, 1 \mathrm{H}), 4.70(\mathrm{~s}, 2 \mathrm{H}), 4.29$ (q, $J=7.1 \mathrm{~Hz}, 2 \mathrm{H}), 3.98(\mathrm{~s}, 6 \mathrm{H}), 3.93(\mathrm{~s}, 3 \mathrm{H}), 1.31(\mathrm{t}, J=$ $7.2 \mathrm{~Hz}, 3 \mathrm{H}), 1.09-1.03$ (m, $9 \mathrm{H}), 0.26$ (s, $6 \mathrm{H})$; ${ }^{13} \mathrm{C}-\mathrm{NMR}\left(101 \mathrm{MHz}, \mathrm{CDCl}_{3}\right): \delta=168.4,153.5,151.9$, 148.5, 147.8, 145.9, 140.2, 118.6, 114.2, 113.3, 100.2, 66.1, 61.4, 61.0, 56.2, 25.7, 18.4, 14.2, -4.6; IR: $\tilde{v}$ $=2932,1759,1593,1493,1416,1277,1192,1122,837,783$; UV-VIS $\left(\mathrm{CH}_{3} \mathrm{CN}+0.5 \%\right.$ piperidine $): \lambda_{\max }$ $(\varepsilon)=369 \mathrm{~nm}\left(21300 \mathrm{l} \cdot \mathrm{mol}^{-1} \cdot \mathrm{cm}^{-1}\right)$; HRMS (ESI): $\mathrm{m} / z$ calcd for $\mathrm{C}_{25} \mathrm{H}_{37} \mathrm{~N}_{2} \mathrm{O}_{7} \mathrm{Si}: 505.2365[\mathrm{M}+\mathrm{H}]^{+}$; found: 505.2359.

Ethyl-2-(3'-hydroxy-4"-((3",4"',5"-trimethoxyphenyl)diazenyl)phenoxy)acetate 29c<smiles>CCOC(=O)COc1ccc(/N=N/c2cc(OC)c(OC)c(OC)c2)c(O)c1</smiles>

Standard Procedure B with azobenzene 21c (115 mg, $0.38 \mathrm{mmol}, 1.0$ equiv.) and ethyl bromoacetate (42 $\mu \mathrm{L}, 0.38 \mathrm{mmol}, 1.0$ equiv.) gave azobenzene $29 \mathrm{c}(110 \mathrm{mg}, 75 \%)$ as an orange solid after purification by silica-gel chromatography (EtOAc/petroleum ether, 1:4 $\rightarrow 1: 2$ ). $R_{f}=0.64$ (EtOAc/petroleum ether, 1:2); m.p. $121{ }^{\circ} \mathrm{C} ;{ }^{1} \mathrm{H}-\mathrm{NMR}\left(300 \mathrm{MHz}, \mathrm{CDCl}_{3}\right): \delta=13.57$ (br s, $\left.1 \mathrm{H}\right), 7.82(\mathrm{~d}, \mathrm{~J}=8.8 \mathrm{~Hz}, 1 \mathrm{H}), 7.11$ (s, 2 H), $6.66(\mathrm{dd}, \mathrm{J}=2.7,8.9 \mathrm{~Hz}, 1 \mathrm{H}), 6.45$ (d, J = 2.7 Hz, $1 \mathrm{H}), 4.68(\mathrm{~s}, 2 \mathrm{H}), 4.30$ (q, J = 7.1 Hz, $2 \mathrm{H}), 3.96$ $(\mathrm{s}, 6 \mathrm{H}), 3.93(\mathrm{~s}, 3 \mathrm{H}), 1.32(\mathrm{t}, \mathrm{J}=7.1 \mathrm{~Hz}, 3 \mathrm{H}) ;{ }^{13} \mathrm{C}-\mathrm{NMR}\left(101 \mathrm{MHz}, \mathrm{CDCl}_{3}\right): \delta=168.1,161.6,155.7$, 153.8, 146.1, 140.2, 134.6, 133.2, 108.2, 102.3, 99.2, 65.3, 61.6, 61.1, 56.3, 14.2; IR: $\tilde{v}=2940,2639$, 1724, 1597, 1423, 1280, 1219, 1114, 1006, 856, 825, 795; UV-VIS $\left(\mathrm{CH}_{3} \mathrm{CN}+0.5 \%\right.$ piperidine): $\lambda_{\max }(\varepsilon)$ $=390 \mathrm{~nm}\left(273001 \cdot \mathrm{mol}^{-1} \cdot \mathrm{cm}^{-1}\right)$; HRMS (ESI): $\mathrm{m} / \mathrm{z}$ calcd for $\mathrm{C}_{19} \mathrm{H}_{23} \mathrm{~N}_{2} \mathrm{O}_{7}: 391.1500[\mathrm{M}+\mathrm{H}]^{+}$; found: 391.1503 .

Ethyl-2-(2'-bromo-4"-((3",4",,5"'-trimethoxyphenyl)diazenyl)phenoxy)acetate 29d 
<smiles>CCOC(=O)COc1ccc(/N=N/c2cc(OC)c(OC)c(OC)c2)cc1Br</smiles>

Standard Procedure B with azobenzene 21d (305 mg, $0.83 \mathrm{mmol}, 1.0$ equiv.), and ethyl bromoacetate ( $0.37 \mathrm{~mL}, 3.33 \mathrm{mmol}, 4.0$ equiv.) gave azobenzene $29 \mathrm{~d}(338 \mathrm{mg}, 89 \%)$ as a yellow solid after silica-gel chromatography (EtOAc/petroleum ether, 1:4 $\rightarrow$ 1:2). $R_{f}=0.60$ (EtOAc/petroleum ether, 1:2); m.p. 112 ${ }^{\circ} \mathrm{C} ;{ }^{1} \mathrm{H}-\mathrm{NMR}\left(250 \mathrm{MHz}, \mathrm{CDCl}_{3}\right): \delta=8.19(\mathrm{~d}, J=2.4 \mathrm{~Hz}, 1 \mathrm{H}), 7.88(\mathrm{dd}, J=2.4,8.8 \mathrm{~Hz}, 1 \mathrm{H}), 7.23(\mathrm{~s}, 2$ H), $6.92(\mathrm{~d}, J=8.8 \mathrm{~Hz}, 1 \mathrm{H}), 4.80(\mathrm{~s}, 2 \mathrm{H}), 4.31(\mathrm{q}, J=7.1 \mathrm{~Hz}, 2 \mathrm{H}), 3.97(\mathrm{~s}, 6 \mathrm{H}), 3.94(\mathrm{~s}, 3 \mathrm{H}), 1.32(\mathrm{t}, J$ $=7.1 \mathrm{~Hz}, 3 \mathrm{H}) ;{ }^{13} \mathrm{C}-\mathrm{NMR}\left(63 \mathrm{MHz}, \mathrm{CDCl}_{3}\right): \delta=167.9$, 156.2, 153.5, 148.2, 147.6, 140.7, 126.5, 125.0, 113.2, 112.7, 100.4, 66.3, 61.7, 61.0, 56.2, 14.1; IR: $\tilde{v}=2943,2832,1763,1593,1485,1411,1200,1119$, 1011, 849; UV-VIS $\left(\mathrm{CH}_{3} \mathrm{CN}+0.5 \%\right.$ piperidine $): \lambda_{\max }(\varepsilon)=364 \mathrm{~nm}\left(257001 \cdot \mathrm{mol}^{-1} \cdot \mathrm{cm}^{-1}\right)$; HRMS $($ ESI): $m / z$ calcd for $\mathrm{C}_{19} \mathrm{H}_{22} \mathrm{BrN}_{2} \mathrm{O}_{6}: 453.0656 / 455.0635[\mathrm{M}+\mathrm{H}]^{+}$; found: 453.0662/455.0642.

Ethyl-2-(3'-bromo-4"-((3",4",5"'-trimethoxyphenyl)diazenyl)phenoxy)acetate 29e<smiles>CCOC(=O)COc1ccc(/N=N/c2cc(OC)c(OC)c(OC)c2)c(Br)c1</smiles>

Standard Procedure B with azobenzene 21e (453 mg, $1.24 \mathrm{mmol}, 1.0$ equiv.) and ethyl bromoacetate ( $0.55 \mathrm{~mL}, 4.94 \mathrm{mmol}, 4.0$ equiv.) gave azobenzene $29 \mathrm{e}(471 \mathrm{mg}, 84 \%)$ as an orange solid after silica-gel chromatography (EtOAc/petroleum ether, 1:4 $\rightarrow$ 1:2). $R_{f}=0.57$ (EtOAc/petroleum ether, 1:2); m.p. 93 ${ }^{\circ} \mathrm{C} ;{ }^{1} \mathrm{H}-\mathrm{NMR}\left(250 \mathrm{MHz}, \mathrm{CDCl}_{3}\right): \delta=7.75(\mathrm{~d}, J=9.0 \mathrm{~Hz}, 1 \mathrm{H}), 7.30(\mathrm{~d}, J=3.1 \mathrm{~Hz}, 3 \mathrm{H}), 6.98(\mathrm{dd}, J=$ 2.7, $9.0 \mathrm{~Hz}, 1 \mathrm{H}), 4.71(\mathrm{~s}, 2 \mathrm{H}), 4.33(\mathrm{q}, J=7.1 \mathrm{~Hz}, 2 \mathrm{H}), 3.99$ (s, $6 \mathrm{H}), 3.96$ (s, $3 \mathrm{H}), 1.35$ (t, J = 7.1 Hz, 3 $\mathrm{H}) ;{ }^{13} \mathrm{C}-\mathrm{NMR}\left(63 \mathrm{MHz}, \mathrm{CDCl}_{3}\right): \delta=168.0,160.0,153.5,148.6,144.2,140.8,127.4,118.9,118.6,114.8$, 100.7, 65.5, 61.7, 61.0, 56.1, 14.1; IR: $\tilde{v}=2982,2839,1763,1589,1416,1200,1126,1076,991,841$; UV-VIS $\left(\mathrm{CH}_{3} \mathrm{CN}+0.5 \%\right.$ piperidine $): \lambda_{\max }(\varepsilon)=369 \mathrm{~nm}\left(230001 \cdot \mathrm{mol}^{-1} \cdot \mathrm{cm}^{-1}\right) ; \mathrm{HRMS}(\mathrm{ESI}): \mathrm{m} / z$ calcd for $\mathrm{C}_{19} \mathrm{H}_{22} \mathrm{BrN}_{2} \mathrm{O}_{6}: 453.0656 / 455.0635[\mathrm{M}+\mathrm{H}]^{+}$; found: 453.0660/455.0639. 
Ethyl-2-(2'-morpholino-4'-((3", $4^{\prime \prime}, 5^{\prime \prime}$-trimethoxyphenyl)diazenyl)phenoxy)acetate 29f<smiles>CCOC(=O)COc1ccc(N=Nc2cc(OC)c(OC)c(OC)c2)cc1N1CCOCC1</smiles>

Standard Procedure D with azobenzene 29d (30.0 mg, $0.66 \mathrm{mmol}, 1.0$ equiv.) and morpholine (13.1 $\mu \mathrm{L}$, $0.132 \mathrm{mmol}, 2.0$ equiv.) gave azobenzene $29 \mathrm{f}(14.0 \mathrm{mg}, 45 \%)$ as an orange wax after silica-gel chromatography $\left(\mathrm{CH}_{2} \mathrm{Cl}_{2} / \mathrm{MeOH}, 9: 1\right) . R_{f}=0.40\left(\mathrm{CH}_{2} \mathrm{Cl}_{2} / \mathrm{MeOH}, 9: 1\right) ;{ }^{1} \mathrm{H}-\mathrm{NMR}\left(300 \mathrm{MHz}, \mathrm{CDCl}_{3}\right): \delta=$ $7.62(\mathrm{dd}, J=2.3,8.5 \mathrm{~Hz}, 1 \mathrm{H}), 7.57(\mathrm{~d}, J=2.2 \mathrm{~Hz}, 1 \mathrm{H}), 7.23(\mathrm{~s}, 2 \mathrm{H}), 6.90$ (d, $J=8.6 \mathrm{~Hz}, 1 \mathrm{H}), 4.78(\mathrm{~s}, 2$ H), 4.31 (q, J= $7.1 \mathrm{~Hz}, 2 \mathrm{H}), 3.98$ (s, $6 \mathrm{H}), 3.94(\mathrm{~s}, 3 \mathrm{H}), 3.99-3.92(\mathrm{~m}, 4 \mathrm{H}), 3.25$ (t, $J=4.4 \mathrm{~Hz}, 4 \mathrm{H})$, $1.33(\mathrm{t}, J=7.2 \mathrm{~Hz}, 3 \mathrm{H}) ;{ }^{13} \mathrm{C}-\mathrm{NMR}\left(75 \mathrm{MHz}, \mathrm{CDCl}_{3}\right): \delta=168.3,153.5,152.6,148.5,147.7,142.2,140.3$, 120.1, 112.4, 111.2, 100.1, 67.2, 65.6, 61.6, 61.0, 56.2, 51.1, 14.2; IR: $\tilde{v}=3460,2963,2842,1752,1581$, 1345, 1241, 1163, 911, 840; UV-VIS $\left(\mathrm{CH}_{3} \mathrm{CN}+0.5 \%\right.$ piperidine $): \lambda_{\max }(\varepsilon)=368 \mathrm{~nm}\left(22000 \mathrm{l} \cdot \mathrm{mol}^{-1} \cdot \mathrm{cm}^{-}\right.$ ${ }^{1}$ ); $\mathrm{HRMS}$ (ESI): $m / z$ calcd for $\mathrm{C}_{23} \mathrm{H}_{30} \mathrm{~N}_{3} \mathrm{O}_{7}: 460.2079[\mathrm{M}+\mathrm{H}]^{+}$; found: 460.2090.

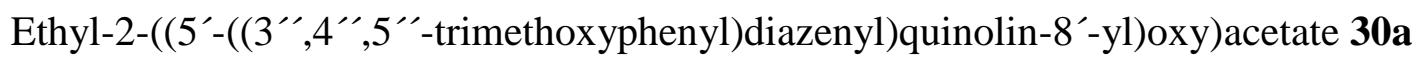<smiles>CCOC(=O)COc1ccc(N=Nc2cc(OC)c(OC)c(OC)c2)c2cccnc12</smiles>

Standard Procedure B with azobenzene 22a (359 mg, $1.06 \mathrm{mmol}, 1.0$ equiv.) and ethyl bromoacetate ( $0.47 \mathrm{~mL}, 4.23 \mathrm{mmol}, 4.0$ equiv.) gave azobenzene $\mathbf{3 0 a}(362 \mathrm{mg}, 80 \%)$ as an orange solid after silica-gel chromatography $\left(\mathrm{CH}_{2} \mathrm{Cl}_{2} / \mathrm{MeOH}, 9: 1\right) . R_{f}=0.41\left(\mathrm{CH}_{2} \mathrm{Cl}_{2} / \mathrm{MeOH}, 95: 5\right) ;$ m.p. $114{ }^{\circ} \mathrm{C} ;{ }^{1} \mathrm{H}-\mathrm{NMR}$ $\left(250 \mathrm{MHz}, \mathrm{CDCl}_{3}\right): \delta=9.27(\mathrm{~d}, J=8.2 \mathrm{~Hz}, 1 \mathrm{H}), 9.07(\mathrm{~d}, J=2.4 \mathrm{~Hz}, 1 \mathrm{H}), 7.91(\mathrm{~d}, J=8.6 \mathrm{~Hz}, 1 \mathrm{H}), 7.64$ $(\mathrm{dd}, J=3.8,8.3 \mathrm{~Hz}, 1 \mathrm{H}), 7.32(\mathrm{~s}, 2 \mathrm{H}), 7.05(\mathrm{~d}, J=8.6 \mathrm{~Hz}, 1 \mathrm{H}), 5.06(\mathrm{~s}, 2 \mathrm{H}), 4.30(\mathrm{q}, J=7.1 \mathrm{~Hz}, 2 \mathrm{H})$, 
$4.01(\mathrm{~s}, 6 \mathrm{H}), 3.96(\mathrm{~s}, 3 \mathrm{H}), 1.29(\mathrm{t}, J=7.1 \mathrm{~Hz}, 3 \mathrm{H}) ;{ }^{13} \mathrm{C}-\mathrm{NMR}\left(63 \mathrm{MHz}, \mathrm{CDCl}_{3}\right): \delta=168.3,156.1$, 153.6, 150.1, 148.9, 141.6, 140.7, 139.8, 132.1, 127.9, 122.5, 113.1, 108.9, 100.5, 66.2, 61.6, 61.1, 56.2, 14.1; IR: $\tilde{v}=2970,2835,1732,1566,1493,1308,1199,1126,999,840 ;$ UV-VIS $\left(\mathrm{CH}_{3} \mathrm{CN}+0.5 \%\right.$ piperidine): $\lambda_{\max }(\varepsilon)=389 \mathrm{~nm}\left(20900 \mathrm{l} \cdot \mathrm{mol}^{-1} \cdot \mathrm{cm}^{-1}\right)$; HRMS (ESI): $\mathrm{m} / z$ calcd for $\mathrm{C}_{22} \mathrm{H}_{24} \mathrm{~N}_{3} \mathrm{O}_{6}: 426.1660$ $[\mathrm{M}+\mathrm{H}]^{+}$; found: 426.1666 .

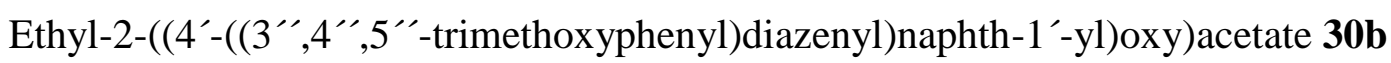<smiles>CCOC(=O)COc1ccc(N=Nc2cc(OC)c(OC)c(OC)c2)c2ccccc12</smiles>

Standard Procedure B with azobenzene 22b (363 mg, $1.07 \mathrm{mmol}, 1.0$ equiv.) and ethyl bromoacetate $(0.48 \mathrm{~mL}, 4.30 \mathrm{mmol}, 4.0$ equiv.) gave azobenzene $\mathbf{3 0 b}(436 \mathrm{mg}, 96 \%)$ as an orange solid after silica-gel chromatography (EtOAc/petroleum ether, 1:4 $\rightarrow$ 1:2). $R_{f}=0.23$ (EtOAc/petroleum ether, 1:4); m.p. 99 ${ }^{\circ} \mathrm{C} ;{ }^{1} \mathrm{H}-\mathrm{NMR}\left(250 \mathrm{MHz}, \mathrm{CDCl}_{3}\right): \delta=8.93(\mathrm{~d}, J=8.1 \mathrm{~Hz}, 1 \mathrm{H}), 8.47(\mathrm{~d}, J=7.9 \mathrm{~Hz}, 1 \mathrm{H}), 7.84(\mathrm{~d}, J=8.4$ $\mathrm{Hz}, 1 \mathrm{H}), 7.73$ (ddd, $J=1.3,6.8,8.1 \mathrm{~Hz}, 1 \mathrm{H}), 7.65$ (ddd, $J=1.3,7.0,8.2 \mathrm{~Hz}, 1 \mathrm{H}), 7.37$ (s, $2 \mathrm{H}), 6.83$ (d, $J=8.4 \mathrm{~Hz}, 1 \mathrm{H}), 4.92(\mathrm{~s}, 2 \mathrm{H}), 4.35(\mathrm{q}, J=7.1 \mathrm{~Hz}, 2 \mathrm{H}), 4.04(\mathrm{~s}, 6 \mathrm{H}), 3.98(\mathrm{~s}, 3 \mathrm{H}), 1.35(\mathrm{t}, J=7.1 \mathrm{~Hz}, 3$ $\mathrm{H}) ;{ }^{13} \mathrm{C}-\mathrm{NMR}\left(101 \mathrm{MHz}, \mathrm{CDCl}_{3}\right): \delta=168.4,156.3,153.6,149.2,142.5,140.4,132.5,127.7,126.2$, 125.7, 123.0, 122.4, 112.5, 104.8, 100.4, 65.8, 61.6, 61.1, 56.3, 14.2; IR: $\tilde{v}=2940,2835,1755,1578$, 1470, 1207, 1107, 991, 845, 768; UV-VIS $\left(\mathrm{CH}_{3} \mathrm{CN}+0.5 \%\right.$ piperidine $): \lambda_{\max }(\varepsilon)=397 \mathrm{~nm}$ (20100 $1 \cdot \mathrm{mol}^{-1} \cdot \mathrm{cm}^{-1}$ ); HRMS (ESI): $\mathrm{m} / z$ calcd for $\mathrm{C}_{23} \mathrm{H}_{25} \mathrm{~N}_{2} \mathrm{O}_{6}: 425.1707[\mathrm{M}+\mathrm{H}]^{+}$; found: 425.1711 .

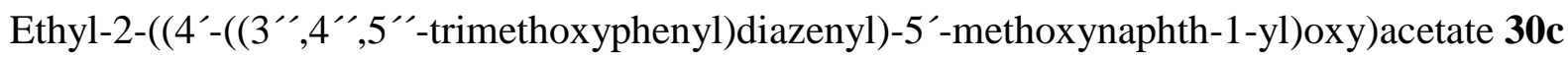


<smiles>CCOC(=O)COc1ccc(/N=N/c2cc(OC)c(OC)c(OC)c2)c2c(OC)cccc12</smiles>

Standard Procedure A1 with 3,4,5-trimethoxyaniline (183 mg, $1.0 \mathrm{mmol}, 1.0$ equiv.) and naphthol 11c (183 mg, $1.05 \mathrm{mmol}, 1.05$ equiv.) gave crude azobenzene 22c (185 mg) after evaporation of the solvent. The crude material was directly converted according to Standard Procedure B with ethyl bromoacetate $(89 \mu \mathrm{L}, 2.0 \mathrm{mmol}, 4.0$ equiv.). Azobenzene 30c $(61 \mathrm{mg}, 13 \%)$ was isolated as an orange solid after silicagel chromatography (EtOAc/petroleum ether, 1:2). $R_{f}=0.64$ (EtOAc/petroleum ether, 1:2); m.p. $143{ }^{\circ} \mathrm{C}$; ${ }^{1} \mathrm{H}-\mathrm{NMR}\left(250 \mathrm{MHz}, \mathrm{CDCl}_{3}\right): \delta=8.10(\mathrm{~d}, J=7.9 \mathrm{~Hz}, 1 \mathrm{H}), 7.53(\mathrm{t}, J=8.1 \mathrm{~Hz}, 1 \mathrm{H}), 7.36(\mathrm{~s}, 2 \mathrm{H}), 7.27$ $(\mathrm{d}, J=8.4 \mathrm{~Hz}, 1 \mathrm{H}), 7.08(\mathrm{~d}, J=7.7 \mathrm{~Hz}, 1 \mathrm{H}), 6.79$ (d, $J=8.4 \mathrm{~Hz}, 1 \mathrm{H}), 4.87(\mathrm{~s}, 2 \mathrm{H}), 4.34(\mathrm{q}, J=7.1 \mathrm{~Hz}$, $2 \mathrm{H}), 4.02(\mathrm{~s}, 6 \mathrm{H}), 3.98(\mathrm{~s}, 3 \mathrm{H}), 3.97(\mathrm{~s}, 3 \mathrm{H}), 1.34(\mathrm{t}, J=7.1 \mathrm{~Hz}, 3 \mathrm{H}) ;{ }^{13} \mathrm{C}-\mathrm{NMR}\left(63 \mathrm{MHz}, \mathrm{CDCl}_{3}\right): \delta=$ 169.4, 157.6, 155.7, 154.5, 150.0, 147.1, 141.0, 128.8, 127.4, 122.6, 116.1, 113.8, 109.7, 106.2, 101.4, 66.8, 62.4, 62.0, 57.4, 57.1, 15.1; IR: $\tilde{v}=2909,2839,1759,1593,1412,1207,1084,1002,760 ;$ UV-VIS $\left(\mathrm{CH}_{3} \mathrm{CN}+0.5 \%\right.$ piperidine $): \lambda_{\max }(\varepsilon)=388 \mathrm{~nm}\left(203001 \cdot \mathrm{mol}^{-1} \cdot \mathrm{cm}^{-1}\right) ; \mathrm{HRMS}(\mathrm{ESI}): \mathrm{m} / z$ calcd for $\mathrm{C}_{24} \mathrm{H}_{27} \mathrm{~N}_{2} \mathrm{O}_{7}$ : $455.1813[\mathrm{M}+\mathrm{H}]^{+}$; found: 455.1824.

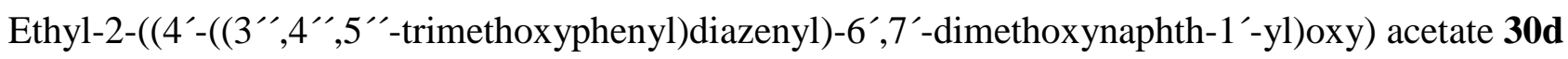<smiles>CCOC(=O)COc1ccc(N=Nc2cc(OC)c(OC)c(OC)c2)c2cc(OC)c(OC)cc12</smiles>

Standard Procedure B with azobenzene 22d (422 mg, $1.06 \mathrm{mmol}, 1.0$ equiv.) and ethyl bromoacetate ( $0.47 \mathrm{~mL}, 4.24 \mathrm{mmol}, 4.0$ equiv.) gave azobenzene 30d (424 mg, $83 \%$ ) as an orange solid after silica-gel chromatography (EtOAc/petroleum ether, 1:2 $\rightarrow$ 1:1). $R_{f}=0.53$ (EtOAc/petroleum ether, 1:2); m.p. 123 ${ }^{\circ} \mathrm{C} ;{ }^{1} \mathrm{H}-\mathrm{NMR}\left(250 \mathrm{MHz}, \mathrm{CDCl}_{3}\right): \delta=8.25(\mathrm{~s}, 1 \mathrm{H}), 7.70(\mathrm{~s}, 1 \mathrm{H}), 7.71(\mathrm{~d}, J=8.4 \mathrm{~Hz}, 1 \mathrm{H}), 7.32(\mathrm{~s}, 2 \mathrm{H})$, $6.70(\mathrm{~d}, J=8.6 \mathrm{~Hz}, 1 \mathrm{H}), 4.89$ (s, $2 \mathrm{H}), 4.32(\mathrm{q}, J=7.1 \mathrm{~Hz}, 2 \mathrm{H}), 4.09$ (s, $3 \mathrm{H}), 4.09$ (s, $3 \mathrm{H}), 4.00$ (s, $6 \mathrm{H})$, 
$3.96(\mathrm{~s}, 3 \mathrm{H}), 1.33(\mathrm{t}, J=7.1 \mathrm{~Hz}, 3 \mathrm{H}) ;{ }^{13} \mathrm{C}-\mathrm{NMR}\left(101 \mathrm{MHz}, \mathrm{CDCl}_{3}\right): \delta=168.5,155.4,153.5,150.5$, 149.6, 149.3, 141.5, 140.3, 129.0, 121.2, 111.5, 104.0, 101.9, 101.2, 100.3, 65.8, 61.5, 61.1, 56.1, 55.9, 55.7, 14.2; IR: $\tilde{v}=2940,2828,1732,1585,1485,1211,1118,1038,1011,818$; UV-VIS $\left(\mathrm{CH}_{3} \mathrm{CN}+\right.$ $0.5 \%$ piperidine): $\lambda_{\max }(\varepsilon)=408 \mathrm{~nm}\left(213001 \cdot \mathrm{mol}^{-1} \cdot \mathrm{cm}^{-1}\right)$; HRM (ESI): $\mathrm{m} / z$ calcd for $\mathrm{C}_{25} \mathrm{H}_{29} \mathrm{~N}_{2} \mathrm{O}_{8}$ : $485.1918[\mathrm{M}+\mathrm{H}]^{+}$; found: 485.1927.

Ethyl-2-((1'-((3”, $4^{\prime \prime}, 5^{\prime \prime}-$ trimethoxyphenyl)diazenyl)naphth-2'-yl)oxy)acetate 31<smiles>CCOC(=O)COc1ccc2ccccc2c1/N=N/c1cc(OC)c(OC)c(OC)c1</smiles>

Standard Procedure B with azobenzene 23 (502 mg, $1.48 \mathrm{mmol}, 1.0$ equiv.) and ethyl bromoacetate (0.66 mL, $5.92 \mathrm{mmol}, 4.0$ equiv.) gave azobenzene 31 (449 mg, $71 \%)$ as an orange solid after silica-gel chromatography (EtOAc/petroleum ether, 1:4 $\rightarrow$ 1:2). $R_{f}=0.30$ (EtOAc/petroleum ether, 1:4); m.p. $126{ }^{\circ} \mathrm{C} ;{ }^{1} \mathrm{H}-\mathrm{NMR}\left(300 \mathrm{MHz}, \mathrm{CDCl}_{3}\right): \delta=8.33(\mathrm{~d}, J=8.6 \mathrm{~Hz}, 1 \mathrm{H}), 7.85(\mathrm{~d}, J=8.7 \mathrm{~Hz}, 2 \mathrm{H}), 7.54(\mathrm{ddd}, J$ $=1.4,6.8,8.4 \mathrm{~Hz}, 1 \mathrm{H}), 7.47(\mathrm{ddd}, J=1.3,6.7,8.1 \mathrm{~Hz}, 1 \mathrm{H}), 7.37(\mathrm{~s}, 2 \mathrm{H}), 7.36(\mathrm{~d}, J=8.8 \mathrm{~Hz}, 1 \mathrm{H}), 4.79$ $(\mathrm{s}, 2 \mathrm{H}), 4.23$ (q, $J=7.1 \mathrm{~Hz}, 2 \mathrm{H}), 4.00(\mathrm{~s}, 6 \mathrm{H}), 3.97(\mathrm{~s}, 3 \mathrm{H}), 1.25(\mathrm{t}, J=7.1 \mathrm{~Hz}, 3 \mathrm{H}) ;{ }^{13} \mathrm{C}-\mathrm{NMR}$ $\left(75 \mathrm{MHz}, \mathrm{CDCl}_{3}\right): \delta=168.8,153.6,149.3,146.8,140.8,137.8,130.6,130.2,128.2,127.9,127.7,125.1$, 123.4, 117.6, 100.4, 68.7, 61.3, 61.1, 56.3, 14.1; IR: $\tilde{v}=2940,2835,1755,1593,1415,1311,1199,1103$, 991, 810; UV-VIS $\left(\mathrm{CH}_{3} \mathrm{CN}+0.5 \%\right.$ piperidine $): \lambda_{\max }(\varepsilon)=370 \mathrm{~nm}\left(268001 \cdot \mathrm{mol}^{-1} \cdot \mathrm{cm}^{-1}\right)$; HRMS (ESI): $m / z$ calcd for $\mathrm{C}_{23} \mathrm{H}_{25} \mathrm{~N}_{2} \mathrm{O}_{6}: 425.1707[\mathrm{M}+\mathrm{H}]^{+}$; found: 425.1713 .

Methyl-2-(4'-((4"'-methoxyphenyl)diazenyl)phenoxy)acetate 32a 


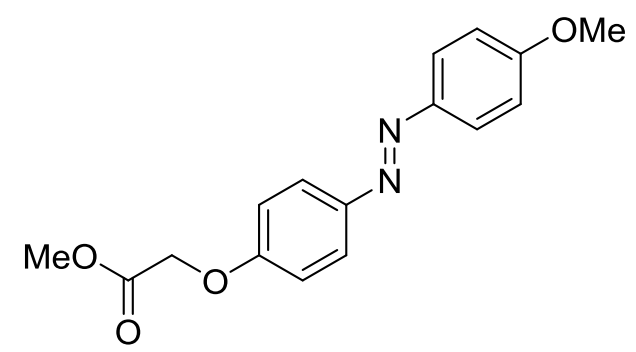

Standard Procedure B with azobenzene 16a (30 mg, $0.10 \mathrm{mmol}, 1.0$ equiv.) and MeI (104 mg, $0.73 \mathrm{mmol}, 7.0$ equiv.) gave azobenzene 32a (26 $\mathrm{mg}, 83 \%$ ) as a yellow solid after purification by silicagel chromatography (EtOAc/petroleum ether, 1:2). $R_{f}=0.58$ (EtOAc/petroleum ether, 1:2); m.p. $149{ }^{\circ} \mathrm{C}$; ${ }^{1} \mathrm{H}-\mathrm{NMR}\left(300 \mathrm{MHz}, \mathrm{CDCl}_{3}\right): \delta=7.86(\mathrm{dd}, J=1.1,9.0 \mathrm{~Hz}, 4 \mathrm{H}), 6.99(\mathrm{dd}, J=1.9,8.9 \mathrm{~Hz}, 4 \mathrm{H}), 4.70(\mathrm{~s}, 2$ $\mathrm{H}), 3.87(\mathrm{~s}, 3 \mathrm{H}), 3.81(\mathrm{~s}, 3 \mathrm{H}) ;{ }^{13} \mathrm{C}-\mathrm{NMR}\left(101 \mathrm{MHz}, \mathrm{CDCl}_{3}\right): \delta=169.0,161.7,159.5,147.8,147.0$, 124.5, 124.4, 114.9, 114.2, 65.4, 55.6, 52.4; IR: $\tilde{v}=2955,2322,1766,1578,1493,1211,1026,840$; UVVIS $\left(\mathrm{CH}_{3} \mathrm{CN}+0.5 \%\right.$ piperidine $): \lambda_{\max }(\varepsilon)=354 \mathrm{~nm}\left(260001 \cdot \mathrm{mol}^{-1} \cdot \mathrm{cm}^{-1}\right) ; \mathrm{HRMS}(\mathrm{ESI}): \mathrm{m} / z$ calcd for $\mathrm{C}_{16} \mathrm{H}_{17} \mathrm{~N}_{2} \mathrm{O}_{4}: 301.1183[\mathrm{M}+\mathrm{H}]^{+}$; found: 301.1183 .

Methyl-2-(4'-((3",4"'-dimethoxyphenyl)diazenyl)phenoxy)acetate 32b<smiles>COC(=O)COc1ccc(/N=N/c2ccc(OC)c(OC)c2)cc1</smiles>

Standard Procedure B with azobenzene 16b (100 mg, $0.32 \mathrm{mmol}, 1.0$ equiv.), and MeI (94 mg, $0.66 \mathrm{mmol}, 2.1$ equiv.) gave azobenzene $32 \mathrm{~b}(83 \mathrm{mg}, 80 \%)$ as a yellow solid after purification by silicagel chromatography (EtOAc/petroleum ether, 1:2). $R_{f}=0.62$ (EtOAc/petroleum ether, 1:2); m.p. $141{ }^{\circ} \mathrm{C}$; ${ }^{1} \mathrm{H}-\mathrm{NMR}\left(250 \mathrm{MHz}, \mathrm{CDCl}_{3}\right): \delta=7.92(\mathrm{~d}, J=9.0 \mathrm{~Hz}, 2 \mathrm{H}), 7.62(\mathrm{dd}, J=2.2,8.4 \mathrm{~Hz}, 1 \mathrm{H}), 7.52(\mathrm{~d}, J=$ $2.0 \mathrm{~Hz}, 1 \mathrm{H}), 7.09$ - $6.98(\mathrm{~m}, 3 \mathrm{H}), 4.75$ (s, $2 \mathrm{H}), 4.01(\mathrm{~s}, 3 \mathrm{H}), 4.00$ (s, $3 \mathrm{H}), 3.86(\mathrm{~s}, 3 \mathrm{H}) ;{ }^{13} \mathrm{C}-\mathrm{NMR}$ $\left(63 \mathrm{MHz}, \mathrm{CDCl}_{3}\right): \delta=169.9,160.5,152.5,150.5,148.5,147.8,125.3,121.4,115.8,111.3,102.8,66.3$, 57.0, 56.9, 53.3; IR: $\tilde{v}=2959,2612,1751,1578,1497,1439,1188,1111,1018,856,817$; UV-VIS $\left(\mathrm{CH}_{3} \mathrm{CN}+0.5 \%\right.$ piperidine $): \lambda_{\max }(\varepsilon)=376 \mathrm{~nm}\left(190001 \cdot \mathrm{mol}^{-1} \cdot \mathrm{cm}^{-1}\right) ; \mathrm{HRMS}(\mathrm{ESI}): \mathrm{m} / z$ calcd for $\mathrm{C}_{17} \mathrm{H}_{19} \mathrm{~N}_{2} \mathrm{O}_{5}: 331.1288[\mathrm{M}+\mathrm{H}]^{+}$; found: 311.1295 .

Methyl-2-(4"-((3",4" ,5"'-trimethoxyphenyl)diazenyl)phenoxy)acetate 32c 


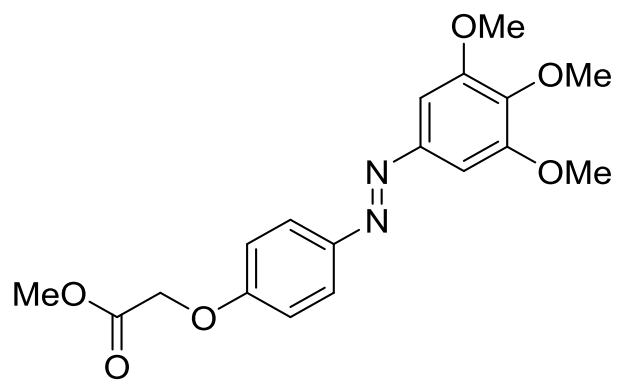

Standard Procedure B with azobenzene 16c (222 mg, $0.64 \mathrm{mmol}, 1.0$ equiv.), and MeI (188 mg, $1.32 \mathrm{mmol}, 2.1$ equiv.) gave azobenzene $32 \mathrm{c}$ (206 $\mathrm{mg}, 89 \%)$ as a yellow solid after purification by silicagel chromatography (EtOAc/petroleum ether, 1:2). $R_{f}=0.62$ (EtOAc/petroleum ether, 1:2); m.p. $51{ }^{\circ} \mathrm{C}$; ${ }^{1} \mathrm{H}-\mathrm{NMR}\left(400 \mathrm{MHz}, \mathrm{CDCl}_{3}\right.$ ): $\delta=7.92(\mathrm{~d}, J=8.5 \mathrm{~Hz}, 2 \mathrm{H}), 7.23(\mathrm{~s}, 2 \mathrm{H}), 7.04(\mathrm{~d}, J=8.8 \mathrm{~Hz}, 2 \mathrm{H}), 4.72$ $(\mathrm{s}, 2 \mathrm{H}), 4.31$ (q, $J=7.2 \mathrm{~Hz}, 2 \mathrm{H}), 3.98(\mathrm{~s}, 6 \mathrm{H}), 3.95(\mathrm{~s}, 3 \mathrm{H}), 1.33(\mathrm{t}, J=7.9 \mathrm{~Hz}, 3 \mathrm{H}) ;{ }^{13} \mathrm{C}-\mathrm{NMR}$ $\left(101 \mathrm{MHz}, \mathrm{CDCl}_{3}\right): \delta=168.4,159.9,153.5,148.5,147.4,140.2,124.5,114.9,100.1,65.4,61.5,61.0$, 56.1, 14.1; IR: $\tilde{v}=2940,2913,2839,1759,1578,1215,1123,1003,845,829 ; \mathrm{UV}-\mathrm{VIS}\left(\mathrm{CH}_{3} \mathrm{CN}+0.5 \%\right.$ piperidine): $\lambda_{\max }(\varepsilon)=360 \mathrm{~nm}\left(22100 \mathrm{l} \cdot \mathrm{mol}^{-1} \cdot \mathrm{cm}^{-1}\right)$; HRM (ESI): $\mathrm{m} / z$ calcd for $\mathrm{C}_{19} \mathrm{H}_{23} \mathrm{~N}_{2} \mathrm{O}_{6}: 375.1551$ $[\mathrm{M}+\mathrm{H}]^{+}$; found: 375.1563 .

Methyl-2-(4'-((4"'-(2"'-hydroxyethoxy)-3"',5"-dimethoxyphenyl)diazenyl)phenoxy) acetate 32d<smiles>COC(=O)COc1ccc(/N=N/c2cc(OC)c(OCCO)c(OC)c2)cc1</smiles>

Standard Procedure B with azobenzene 16c (100 mg, $0.28 \mathrm{mmol}, 1.0$ equiv.) and 2-bromoethanol (59 $\mu \mathrm{L}$, $0.83 \mathrm{mmol}, 1.0$ equiv.) gave azobenzene $32 \mathrm{~d}(82 \mathrm{mg}, 76 \%)$ as a yellow solid after purification by silicagel chromatography (EtOAc/petroleum ether, 1:1). $R_{f}=0.38$ (EtOAc/petroleum ether, 1:1); m.p. $91{ }^{\circ} \mathrm{C}$; ${ }^{1} \mathrm{H}-\mathrm{NMR}\left(250 \mathrm{MHz}, \mathrm{CDCl}_{3}\right): \delta=7.91(\mathrm{~d}, J=8.6 \mathrm{~Hz}, 2 \mathrm{H}), 7.24(\mathrm{~s}, 2 \mathrm{H}), 7.03$ (d, $\left.J=8.6 \mathrm{~Hz}, 2 \mathrm{H}\right), 4.73$ $(\mathrm{s}, 2 \mathrm{H}), 4.22(\mathrm{t}, J=3.9 \mathrm{~Hz}, 2 \mathrm{H}), 3.98(\mathrm{~s}, 6 \mathrm{H}), 3.84(\mathrm{~s}, 3 \mathrm{H}), 3.76(\mathrm{t}, J=4.0 \mathrm{~Hz}, 2 \mathrm{H}) ;{ }^{13} \mathrm{C}-\mathrm{NMR}$ $\left(63 \mathrm{MHz}, \mathrm{CDCl}_{3}\right): \delta=169.8,160.9,154.5,149.7,148.3,139.3,125.6,115.8,101.0,76.5,66.3,62.3$, 57.2, 53.3; IR: $\tilde{v}=3510,2951,2322,1751,1582,1497,1207,1126,1072,841 ; \mathrm{UV}-\mathrm{VIS}\left(\mathrm{CH}_{3} \mathrm{CN}+\right.$ $0.5 \%$ piperidine): $\lambda_{\max }(\varepsilon)=360 \mathrm{~nm}\left(198001 \cdot \mathrm{mol}^{-1} \cdot \mathrm{cm}^{-1}\right)$; HRMS (ESI): $\mathrm{m} / z$ calcd for $\mathrm{C}_{19} \mathrm{H}_{23} \mathrm{~N}_{2} \mathrm{O}_{7}$ : $391.1500[\mathrm{M}+\mathrm{H}]^{+}$; found: 391.1503 . 
Methyl-2-(4'-((4"-(2"'-bromoethoxy)-3"',5"-dimethoxyphenyl)diazenyl)phenoxy) acetate 32e<smiles>COC(=O)COc1ccc(/N=N/c2cc(OC)c(OCCBr)c(OC)c2)cc1</smiles>

Standard Procedure B with azobenzene 16c (100 mg, $0.28 \mathrm{mmol}, 1.0$ equiv.) and 1,2-dibromoethane (249 $\mu \mathrm{L}, 2.89 \mathrm{mmol}, 10.0$ equiv.) in anhydrous acetone $(20 \mathrm{~mL})$ gave azobenzene 32e $(119 \mathrm{mg}, 91 \%)$ as a chartreuse solid after purification by silica-gel chromatography (EtOAc/petroleum ether, 1:4). $R_{f}=0.57$ (EtOAc/petroleum ether, 1:4); m.p. $179{ }^{\circ} \mathrm{C} ;{ }^{1} \mathrm{H}-\mathrm{NMR}\left(300 \mathrm{MHz}, \mathrm{CDCl}_{3}\right): \delta=7.90(\mathrm{~d}, J=8.7 \mathrm{~Hz}, 2 \mathrm{H})$, $7.21(\mathrm{~s}, 2 \mathrm{H}), 7.03$ (d, $J=8.8 \mathrm{~Hz}, 2 \mathrm{H}), 4.73(\mathrm{~s}, 2 \mathrm{H}), 4.34$ (t, J= 7.0 Hz, 2 H), $3.96(\mathrm{~s}, 6 \mathrm{H}), 3.84(\mathrm{~s}, 3 \mathrm{H})$, $3.64(\mathrm{t}, J=7.0 \mathrm{~Hz}, 2 \mathrm{H}) ;{ }^{13} \mathrm{C}-\mathrm{NMR}\left(75 \mathrm{MHz}, \mathrm{CDCl}_{3}\right): \delta=168.9,160.0,153.5,148.9,147.5,138.4$, 124.7, 114.9, 100.0, 72.7, 65.3, 56.2, 52.4, 29.6; IR: $\tilde{v}=2062,2835,1759,1597,1501,1439,1408,1211$, 1130, 1076, 991, 810; UV-VIS $\left(\mathrm{CH}_{3} \mathrm{CN}+0.5 \%\right.$ piperidine $): \lambda_{\max }(\varepsilon)=360 \mathrm{~nm}\left(272001 \cdot \mathrm{mol}^{-1} \cdot \mathrm{cm}^{-1}\right)$; HRMS (ESI): $m / z$ calcd for $\mathrm{C}_{19} \mathrm{H}_{22} \mathrm{BrN}_{2} \mathrm{O}_{6}: 453.0656 / 455.0638$ [M+H] $]^{+}$; found: 453.0658/455.0640.

Methyl-2-(4'-((3"',5"'-dimethoxy-4"'-(2"'”-(4"'”'-methylpiperazin-1"'”'-yl)ethoxy)phenyl) diazenyl)phenoxy)acetate $\mathbf{3 2 f}$<smiles>COC(=O)COc1ccc(/N=N/c2cc(OC)c(OCCN3CCN(C)CC3)c(OC)c2)cc1</smiles>

A microwave vial charged with azobenzene 32e (60 mg, $0.13 \mathrm{mmol}, 1.0$ equiv.), 1-methylpiperazine (19 $\mu \mathrm{L}, 0.17$ mmol, 1.3 equiv.), $\mathrm{K}_{2} \mathrm{CO}_{3}$ (24 mg, $0.17 \mathrm{mmol}, 1.3$ equiv.), and $\mathrm{CH}_{3} \mathrm{CN}$ (4 mL) was heated to $140{ }^{\circ} \mathrm{C}$ for $10 \mathrm{~min}$. in a microwave. After cooling the solvent was removed under reduced pressure, and the residue was redissolved in $\mathrm{CHCl}_{3}(30 \mathrm{~mL})$. The organic phase was washed with water $(20 \mathrm{~mL})$, and brine $(20 \mathrm{~mL})$, dried with $\mathrm{Na}_{2} \mathrm{SO}_{4}$, filtered, and concentrated under reduced pressure. Azobenzene $32 \mathrm{f}$ (45 mg, $72 \%$ ) was obtained as an orange oil without further purification. $R_{f}=0.31\left(\mathrm{CH}_{2} \mathrm{Cl}_{2} / \mathrm{MeOH}, 9: 1\right)$; ${ }^{1} \mathrm{H}-\mathrm{NMR}\left(300 \mathrm{MHz}, \mathrm{CDCl}_{3}\right): \delta=7.87(\mathrm{~d}, J=8.9 \mathrm{~Hz}, 2 \mathrm{H}), 7.18(\mathrm{~s}, 2 \mathrm{H}), 7.00(\mathrm{~d}, J=8.9 \mathrm{~Hz}, 2 \mathrm{H}), 4.70$ 
(s, $2 \mathrm{H}), 4.16(\mathrm{t}, J=5.8 \mathrm{~Hz}, 2 \mathrm{H}), 3.91(\mathrm{~s}, 6 \mathrm{H}), 3.81(\mathrm{~s}, 3 \mathrm{H}), 2.79(\mathrm{t}, J=6.1 \mathrm{~Hz}, 2 \mathrm{H}), 2.63(\mathrm{br} \mathrm{s}, 4 \mathrm{H})$, 2.49 (br s, $4 \mathrm{H}$ ), 2.27 (s, $3 \mathrm{H}) ;{ }^{13} \mathrm{C}-\mathrm{NMR}\left(75 \mathrm{MHz}, \mathrm{CDCl}_{3}\right.$ ): $\delta=168.8,159.8,153.6,148.5,147.4,139.3$, 124.5, 114.8, 100.0, 70.6, 65.3, 57.9, 56.0, 55.0, 53.4, 52.3, 46.0; IR: $\tilde{v}=2940,2797,2361,1740,1597$, 1497, 1204, 1123, 1003, 841; UV-VIS $\left(\mathrm{CH}_{3} \mathrm{CN}+0.5 \%\right.$ piperidine $): \lambda_{\max }(\varepsilon)=362 \mathrm{~nm}\left(216001 \cdot \mathrm{mol}^{-1} \cdot \mathrm{cm}^{-}\right.$ ${ }^{1}$ ); HRMS (ESI): $m / z$ calcd for $\mathrm{C}_{24} \mathrm{H}_{33} \mathrm{~N}_{4} \mathrm{O}_{6}: 473.2395[\mathrm{M}+\mathrm{H}]^{+}$; found: 473.2398 .

Methyl- $N-\left(5^{\prime}-\right.$ methoxy-2'-((4"'-methoxyphenyl)diazenyl)phenyl)succinamate 33a<smiles>COC(=O)CCC(=O)Nc1cc(OC)ccc1/N=N/c1ccc(OC)cc1</smiles>

Standard Procedure B with azobenzene 17a (70.0 mg, $0.20 \mathrm{mmol}, 1.0$ equiv.) and MeI (24.4 $\mu \mathrm{L}$, $0.39 \mathrm{mmol}, 2.0$ equiv.) gave azobenzene 33a (66 mg, $91 \%$ ) as an ocher solid after purification by silicagel chromatography (EtOAc/petroleum ether, 1:4 $\rightarrow$ 1:2). $R_{f}=0.46$ (EtOAc/petroleum ether, 1:2); m.p. $126{ }^{\circ} \mathrm{C} ;{ }^{1} \mathrm{H}-\mathrm{NMR}\left(300 \mathrm{MHz}, \mathrm{CDCl}_{3}\right): \delta=10.74$ (br s, $\left.1 \mathrm{H}\right), 8.36(\mathrm{~d}, J=2.7 \mathrm{~Hz}, 1 \mathrm{H}), 7.90(\mathrm{~d}, J=8.9 \mathrm{~Hz}$, $2 \mathrm{H}), 7.87$ (d, $J=9.0 \mathrm{~Hz}, 1 \mathrm{H}), 7.09$ (d, $J=9.0 \mathrm{~Hz}, 2 \mathrm{H}), 6.76(\mathrm{dd}, J=2.7,9.0 \mathrm{~Hz}, 1 \mathrm{H}), 3.96(\mathrm{~s}, 3 \mathrm{H})$, 3.95 (s, $3 \mathrm{H}), 3.78$ (s, $3 \mathrm{H}), 2.87$ (br. s, $4 \mathrm{H}$ ); ${ }^{13} \mathrm{C}-\mathrm{NMR}\left(101 \mathrm{MHz}, \mathrm{CDCl}_{3}\right): \delta=173.2,170.1,162.8$, $161.7,146.7,136.9,133.4,124.1,123.7,114.4,110.9,103.5,55.7,55.6,52.0,32.8,29.1$; IR: $\tilde{v}=3337$, 2932, 2835, 1744, 1670, 1582, 1470, 1227, 1168, 1034, 837; UV-VIS ( $\mathrm{CH}_{3} \mathrm{CN}+0.5 \%$ piperidine): $\lambda_{\max }$ $(\varepsilon)=384 \mathrm{~nm}\left(245001 \cdot \mathrm{mol}^{-1} \cdot \mathrm{cm}^{-1}\right)$; HRMS (ESI): $\mathrm{m} / z$ calcd for $\mathrm{C}_{19} \mathrm{H}_{22} \mathrm{~N}_{3} \mathrm{O}_{5}: 372.1554[\mathrm{M}+\mathrm{H}]^{+}$; found: 372.1563 .

Methyl- $N-\left(2^{\prime}-\left(\left(4^{\prime \prime}-\right.\right.\right.$ bromophenyl $)$ diazenyl $)-5^{\prime}-$ methoxyphenyl $)$ succinamate 33b<smiles>COC(=O)CCC(=O)Nc1cc(OC)ccc1/N=N/c1ccc(Br)cc1</smiles> 
Standard Procedure B with azobenzene 17b $(555 \mathrm{mg}, 1.37 \mathrm{mmol}, 1.0$ equiv.) and MeI (170 $\mu \mathrm{L}$, $2.73 \mathrm{mmol}, 2.0$ equiv.) gave azobenzene $33 \mathrm{~b}(520 \mathrm{mg}, 91 \%)$ as a yellow solid after purification by silicagel chromatography $\left(\mathrm{CH}_{2} \mathrm{Cl}_{2} / \mathrm{MeOH}, 98: 2\right) . \mathrm{R}_{\mathrm{f}}=0.31\left(\mathrm{CH}_{2} \mathrm{Cl}_{2} / \mathrm{MeOH}, 98: 2\right) ;$ m.p. $140{ }^{\circ} \mathrm{C} ;{ }^{1} \mathrm{H}-\mathrm{NMR}$ $\left(300 \mathrm{MHz} \mathrm{CDCl}_{3}\right): \delta=10.66($ br s, $1 \mathrm{H}), 8.32(\mathrm{~d}, J=2.7 \mathrm{~Hz}, 1 \mathrm{H}), 7.84(\mathrm{~d}, J=9.0 \mathrm{~Hz}, 1 \mathrm{H}), 7.74(\mathrm{~d}, J=$ $8.8 \mathrm{~Hz}, 2 \mathrm{H}), 7.65(\mathrm{~d}, J=8.8 \mathrm{~Hz}, 2 \mathrm{H}), 6.72(\mathrm{dd}, J=2.7,9.1 \mathrm{~Hz}, 1 \mathrm{H}), 3.91(\mathrm{~s}, 3 \mathrm{H}), 3.72(\mathrm{~s}, 3 \mathrm{H}), 2.81$ (s, $4 \mathrm{H}) ;{ }^{13} \mathrm{C}-\mathrm{NMR}\left(75 \mathrm{MHz}, \mathrm{CDCl}_{3}\right): \delta=173.2,170.3,163.8,151.2,137.5,133.4,132.4,124.7,124.4$, 123.8, 111.1, 103.5, 55.8, 52.0, 32.8, 29.0; IR: $\tilde{v}=3329,2943,2322,1732,1604,1527,1474,1219$, 1157, 833; UV-VIS $\left(\mathrm{CH}_{3} \mathrm{CN}+0.5 \%\right.$ piperidine $): \lambda_{\max }(\varepsilon)=383 \mathrm{~nm}\left(19100 \mathrm{l} \cdot \mathrm{mol}^{-1} \cdot \mathrm{cm}^{-1}\right) ; \mathrm{HRMS}(\mathrm{ESI})$ : $m / z$ calcd for $\mathrm{C}_{18} \mathrm{H}_{19} \mathrm{BrN}_{3} \mathrm{O}_{4}: 420.0553 / 422.0535[\mathrm{M}+\mathrm{H}]^{+}$; found: 420.0555/422.0537.

$N-\left(2^{\prime}-\left(\left(3^{\prime \prime}, 4^{\prime \prime}-\right.\right.\right.$ Dimethoxyphenyl)diazenyl)-5'-methoxyphenyl)succinimide 34b

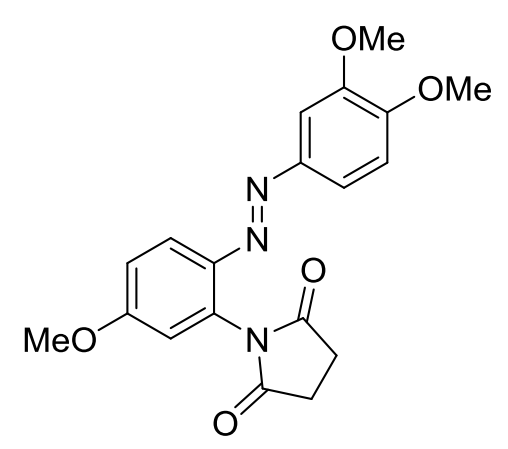

Standard Procedure B with azobenzene 19b $(69.4 \mathrm{mg}, 0.19 \mathrm{mmol}, 1.0$ equiv.) and MeI (25.4 $\mu \mathrm{L}$, $0.41 \mathrm{mmol}, 2.1$ equiv.) gave azobenzene $\mathbf{3 4 b}(47 \mathrm{mg}, 68 \%)$ as an orange solid after purification by silicagel chromatography (EtOAc/petroleum ether, 1:1 $\rightarrow$ EtOAc). $R_{f}=0.50$ (EtOAc); m.p. $89{ }^{\circ} \mathrm{C} ;{ }^{1} \mathrm{H}-\mathrm{NMR}$ $\left(300 \mathrm{MHz}, \mathrm{CDCl}_{3}\right): \delta=7.91(\mathrm{~d}, J=9.0 \mathrm{~Hz}, 1 \mathrm{H}), 7.46(\mathrm{dd}, J=2.2,8.5 \mathrm{~Hz}, 1 \mathrm{H}), 7.29(\mathrm{~d}, J=2.1 \mathrm{~Hz}, 1$ H), $7.07(\mathrm{dd}, J=2.8,9.1 \mathrm{~Hz}, 1 \mathrm{H}), 6.97(\mathrm{~d}, J=8.6 \mathrm{~Hz}, 1 \mathrm{H}), 6.86(\mathrm{~d}, J=2.8 \mathrm{~Hz}, 1 \mathrm{H}), 3.97(\mathrm{~s}, 3 \mathrm{H}), 3.93$ $(\mathrm{s}, 3 \mathrm{H}), 3.90$ (s, $3 \mathrm{H}), 3.03$ - $2.90(\mathrm{~m}, 4 \mathrm{H}) ;{ }^{13} \mathrm{C}-\mathrm{NMR}\left(101 \mathrm{MHz}, \mathrm{CDCl}_{3}\right): \delta=176.2,162.0,151.8,149.4$, 147.3, 141.2, 132.2, 119.4, 119.1, 116.3, 113.5, 110.5, 102.9, 56.2, 55.8, 55.7, 28.8; IR: $\tilde{v}=2943,2322$, 1713, 1504, 1373, 1234, 1177, 1015, 814; UV-VIS $\left(\mathrm{CH}_{3} \mathrm{CN}+0.5 \%\right.$ piperidine $): \lambda_{\max }(\varepsilon)=374 \mathrm{~nm}$ (20600 $1 \cdot \mathrm{mol}^{-1} \cdot \mathrm{cm}^{-1}$ ); HRMS (ESI): $\mathrm{m} / z$ calcd for $\mathrm{C}_{19} \mathrm{H}_{20} \mathrm{~N}_{3} \mathrm{O}_{5}: 370.1403[\mathrm{M}+\mathrm{H}]^{+}$; found: 370.1404 .

$N-\left(2^{\prime}-\left(\left(4^{\prime \prime}-\left(2^{\prime \prime \prime}-B r o m o e t h o x y\right)-3{ }^{\prime \prime}, 5^{\prime \prime}\right.\right.\right.$-dimethoxyphenyl)diazenyl)-5'-methoxyphenyl) succinimide 34d 


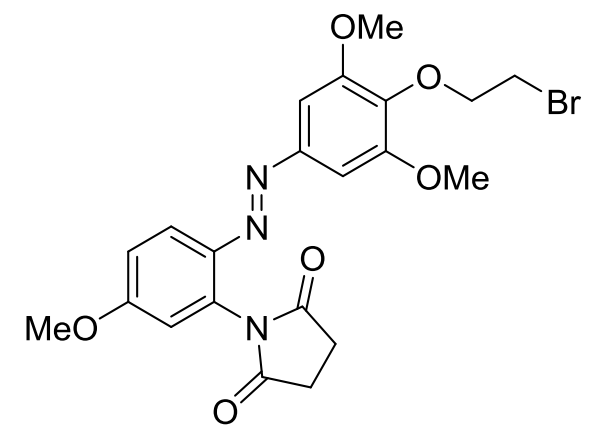

Standard Procedure B with azobenzene 19c (200 mg, $0.52 \mathrm{mmol}, 1.0$ equiv.) and 1,2-dibromoethane (447 $\mu \mathrm{L}, 5.19 \mathrm{mmol}, 10.0$ equiv.) in anhydrous acetone $(50 \mathrm{~mL})$ gave azobenzene $\mathbf{3 4 d}(108 \mathrm{mg}, 43 \%)$ as a red solid after purification by silica-gel chromatography (EtOAc/petroleum ether, 1:2 $\rightarrow 2: 1$ ). $R_{f}=0.23$ (EtOAc/petroleum ether, 1:1); m.p. $126{ }^{\circ} \mathrm{C} ;{ }^{1} \mathrm{H}-\mathrm{NMR}\left(400 \mathrm{MHz}, \mathrm{CDCl}_{3}\right): \delta=7.91(\mathrm{~d}, J=9.1 \mathrm{~Hz}, 1 \mathrm{H})$, $7.09(\mathrm{dd}, J=2.6,9.1 \mathrm{~Hz}, 1 \mathrm{H}), 7.06$ (s, $2 \mathrm{H}), 6.89$ (d, $J=2.6 \mathrm{~Hz}, 1 \mathrm{H}), 4.35$ (t, J = 7.2 Hz, $2 \mathrm{H}), 3.93$ (s, 6 $\mathrm{H}), 3.92(\mathrm{~s}, 3 \mathrm{H}), 3.65(\mathrm{t}, J=7.3 \mathrm{~Hz}, 2 \mathrm{H}), 3.06-2.87(\mathrm{~m}, 4 \mathrm{H}) ;{ }^{13} \mathrm{C}-\mathrm{NMR}\left(101 \mathrm{MHz}, \mathrm{CDCl}_{3}\right): \delta=176.1$, $162.5,153.4,149.2,141.0,138.7,132.8,118.8,116.3,113.5,100.1,72.8,56.1,55.9,29.6,28.8 ;$ IR: $\tilde{v}=$ 2936, 2835, 2318, 1709, 1377, 1234, 1177, 1123, 856, 822; UV-VIS ( $\mathrm{CH}_{3} \mathrm{CN}+0.5 \%$ piperidine): $\lambda_{\max }$ $(\varepsilon)=368 \mathrm{~nm}\left(242001 \cdot \mathrm{mol}^{-1} \cdot \mathrm{cm}^{-1}\right)$; HRMS (ESI): $\mathrm{m} / \mathrm{z}$ calcd for $\mathrm{C}_{21} \mathrm{H}_{23} \mathrm{BrN}_{3} \mathrm{O}_{6}: 492.0765 / 494.0747$ $[\mathrm{M}+\mathrm{H}]^{+}$; found: 492.0769/494.0750.

$N-\left(2^{\prime}-\left(\left(3^{\prime \prime}, 5^{\prime \prime}-D i m e t h o x y-4{ }^{\prime \prime}-\left(2^{\prime \prime \prime}-\left(4^{\prime \prime \prime \prime \prime}-m e t h y l p i p e r a z i n-1{ }^{\prime \prime \prime \prime}-y l\right) e t h o x y\right) p h e n y l\right) d i a z e n y l\right)-5{ }^{\prime}-\right.$ methoxyphenyl)succinimide 34e<smiles>COc1ccc(N=Nc2cc(OC)c(OCCN3CCN(C)CC3)c(OC)c2)c(N2C(=O)CCC2=O)c1</smiles>

A microwave vial charged with azobenzene 34d (64 mg, $0.13 \mathrm{mmol}, 1.0$ equiv.), 1-methylpiperazine (20 $\mu \mathrm{L}, 0.18 \mathrm{mmol}, 1.3$ equiv.), $\mathrm{K}_{2} \mathrm{CO}_{3}$ ( $25 \mathrm{mg}, 0.18 \mathrm{mmol}, 1.3$ equiv.), and $\mathrm{CH}_{3} \mathrm{CN}$ (4 mL) was heated to $140{ }^{\circ} \mathrm{C}$ for $10 \mathrm{~min}$. in a microwave. After cooling the solvent was removed under reduced pressure, and the residue was redissolved in $\mathrm{CHCl}_{3}(10 \mathrm{~mL})$. The organic phase was washed with water $(5 \mathrm{~mL})$, brine $(5 \mathrm{~mL})$, dried with $\mathrm{Na}_{2} \mathrm{SO}_{4}$, filtered, and concentrated under reduced pressure. Azobenzene 34e (43 mg, $65 \%)$ was obtained as an orange oil after purification by silica-gel chromatography $\left(\mathrm{CH}_{2} \mathrm{Cl}_{2} / \mathrm{MeOH}, 95: 5\right.$ $\rightarrow$ 90:10). $R_{f}=0.35\left(\mathrm{CH}_{2} \mathrm{Cl}_{2} / \mathrm{MeOH}, 9: 1\right) ;{ }^{1} \mathrm{H}-\mathrm{NMR}\left(300 \mathrm{MHz}, \mathrm{CDCl}_{3}\right): \delta=7.89(\mathrm{~d}, J=9.0 \mathrm{~Hz}, 1 \mathrm{H})$, 
$7.07(\mathrm{dd}, J=2.7,9.1 \mathrm{~Hz}, 1 \mathrm{H}), 7.03(\mathrm{~s}, 2 \mathrm{H}), 6.87(\mathrm{~d}, J=2.7 \mathrm{~Hz}, 1 \mathrm{H}), 4.17(\mathrm{t}, J=5.8 \mathrm{~Hz}, 2 \mathrm{H}), 3.90(\mathrm{~s}, 3$ H), 3.90 (s, 6 H), 3.03 - 2.87 (m, 4 H), 2.82 (t, J=5.8 Hz, 2 H), 2.69 (br s, 4 H), 2.56 (br s, 4 H), 2.34 (s, $3 \mathrm{H}) ;{ }^{13} \mathrm{C}-\mathrm{NMR}\left(75 \mathrm{MHz}, \mathrm{CDCl}_{3}\right): \delta=176.1,162.3,153.6,148.9,141.0,139.7,132.7,118.8,116.3$, 113.5, 100.2, 70.6, 57.9, 56.0, 55.8, 55.0, 53.3, 46.0, 28.8; IR: $\tilde{v}=2936,2797,1713,1601,1454,1223$, 1123, 1003, 822; UV-VIS $\left(\mathrm{CH}_{3} \mathrm{CN}+0.5 \%\right.$ piperidine $): \lambda_{\max }(\varepsilon)=371 \mathrm{~nm}\left(264001 \cdot \mathrm{mol}^{-1} \cdot \mathrm{cm}^{-1}\right) ; \mathrm{HRMS}$ (ESI): $m / z$ calcd for $\mathrm{C}_{26} \mathrm{H}_{34} \mathrm{~N}_{5} \mathrm{O}_{6}: 512.2504[\mathrm{M}+\mathrm{H}]^{+}$; found: 512.2510 .

Methyl- $N-\left(4^{\prime}-\right.$ methoxy-2'-((3", $4^{\prime \prime}, 5^{\prime \prime}-$ trimethoxyphenyl)diazenyl $)$ phenyl)succinamate 35<smiles>COC(=O)CCC(=O)Nc1ccc(OC)cc1/N=N/c1cc(OC)c(OC)c(OC)c1</smiles>

Standard Procedure B with azobenzene 20 (160 mg, $0.38 \mathrm{mmol}, 1.0$ equiv.) and MeI (47.7 $\mu \mathrm{L}$, $0.77 \mathrm{mmol}, 2.0$ equiv.) gave azobenzene 35 (147 $\mathrm{mg}, 89 \%)$ as a yellow solid after purification by silicagel chromatography (acetone/petroleum ether, 1:4). $\mathrm{R}_{\mathrm{f}}=0.38$ (acetone/petroleum ether, 1:3); m.p. $129{ }^{\circ} \mathrm{C} ;{ }^{1} \mathrm{H}-\mathrm{NMR}\left(300 \mathrm{MHz}, \mathrm{CDCl}_{3}\right): \delta=9.66($ br s, $1 \mathrm{H}), 8.53(\mathrm{~d}, J=9.1 \mathrm{~Hz}, 1 \mathrm{H}), 7.31(\mathrm{~d}, J=3.0 \mathrm{~Hz}, 1$ H), 7.25 (s, $2 \mathrm{H}), 7.04(\mathrm{dd}, J=3.0,9.2 \mathrm{~Hz}, 1 \mathrm{H}), 3.97$ (s, $6 \mathrm{H}), 3.94(\mathrm{~s}, 3 \mathrm{H}), 3.84(\mathrm{~s}, 3 \mathrm{H}), 3.65(\mathrm{~s}, 3 \mathrm{H})$, 2.81 - 2.68 (m, $4 \mathrm{H}) ;{ }^{13} \mathrm{C}-\mathrm{NMR}\left(101 \mathrm{MHz}, \mathrm{CDCl}_{3}\right): \delta=173.4,169.4,155.8,153.7,148.4,141.1,139.9$, 130.7, 121.7, 119.9, 101.5, 100.6, 61.1, 56.3, 55.6, 52.0, 32.8, 29.5; IR: $\tilde{v}=3306,2940,2835,1744$, 1597, 1508, 1215, 1119, 1007, 845, 818; UV-VIS $\left(\mathrm{CH}_{3} \mathrm{CN}+0.5 \%\right.$ piperidine $): \lambda_{\max }(\varepsilon)=409 \mathrm{~nm}$ (23300 $1 \cdot \mathrm{mol}^{-1} \cdot \mathrm{cm}^{-1}$ ); HRMS (ESI): $\mathrm{m} / z$ calcd for $\mathrm{C}_{21} \mathrm{H}_{26} \mathrm{~N}_{3} \mathrm{O}_{7}: 432.1765[\mathrm{M}+\mathrm{H}]^{+}$; found: 432.1773 .

Ethyl -2-(4'-((4"'-methyl-3",4"'-dihydro-2"'H-benzo[b][1"',4"]oxazin-7-yl)diazenyl) phenoxy)acetate 36a

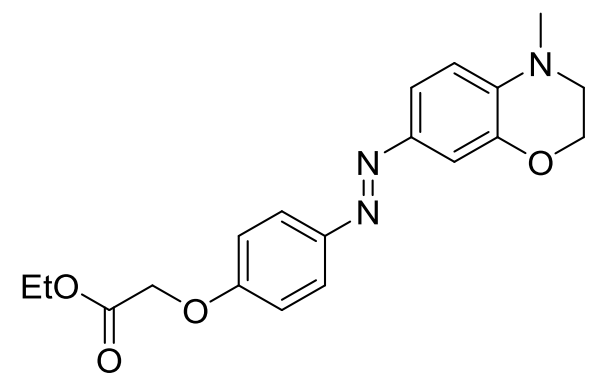

Standard Procedure E with azobenzene 28 (66 mg, 0.19 mmol, 1.0 equiv.) and paraformaldehyde (17 mg, $0.58 \mathrm{mmol}, 3.0$ equiv.) gave azobenzene $\mathbf{3 6 a}(57 \mathrm{mg}, 83 \%)$ as an orange solid after purification by silica- 
gel chromatography $\left(\mathrm{CHCl}_{3}\right) . R_{f}=0.31\left(\mathrm{CH}_{2} \mathrm{Cl}_{2}\right)$; m.p. $112{ }^{\circ} \mathrm{C} ;{ }^{1} \mathrm{H}-\mathrm{NMR}\left(300 \mathrm{MHz}, \mathrm{CDCl}_{3}\right): \delta=7.83(\mathrm{~d}$, $J=9.0 \mathrm{~Hz}, 2 \mathrm{H}), 7.52(\mathrm{dd}, J=2.2,8.6 \mathrm{~Hz}, 1 \mathrm{H}), 7.40(\mathrm{~d}, J=2.1 \mathrm{~Hz}, 1 \mathrm{H}), 7.00(\mathrm{~d}, J=9.0 \mathrm{~Hz}, 2 \mathrm{H}), 6.72$ $(\mathrm{d}, J=8.7 \mathrm{~Hz}, 1 \mathrm{H}), 4.69$ (s, $2 \mathrm{H}), 4.36-4.24$ (m, $4 \mathrm{H}), 3.43-3.37$ (m, $2 \mathrm{H}), 3.02$ (s, $3 \mathrm{H}), 1.32$ (t, $J=7.2$ $\mathrm{Hz}, 3 \mathrm{H}) ;{ }^{13} \mathrm{C}-\mathrm{NMR}\left(101 \mathrm{MHz}, \mathrm{CDCl}_{3}\right): \delta=168.6,159.0,148.0,144.8,144.0,139.1,124.0,120.6,114.8$, 110.8, 107.7, 65.6, 64.4, 61.5, 48.9, 38.5, 14.2; IR: $\tilde{v}=3460,2075,2972,1739,1582,1520,1377,1203$, 810; UV-VIS $\left(\mathrm{CH}_{3} \mathrm{CN}+0.5 \%\right.$ piperidine): $\lambda_{\max }(\varepsilon)=419 \mathrm{~nm}\left(268001 \cdot \mathrm{mol}^{-1} \cdot \mathrm{cm}^{-1}\right) ;$ HRMS $(\mathrm{ESI}): \mathrm{m} / z$ calcd for $\mathrm{C}_{19} \mathrm{H}_{22} \mathrm{~N}_{3} \mathrm{O}_{4}: 356.1605[\mathrm{M}+\mathrm{H}]^{+}$; found: 356.1606 .

Ethyl-2-(4'-((4"-benzyl-3"',4"'-dihydro-2"H-benzo[b][1",4"']oxazin-7-yl)diazenyl) phenoxy)acetate $36 \mathbf{b}$

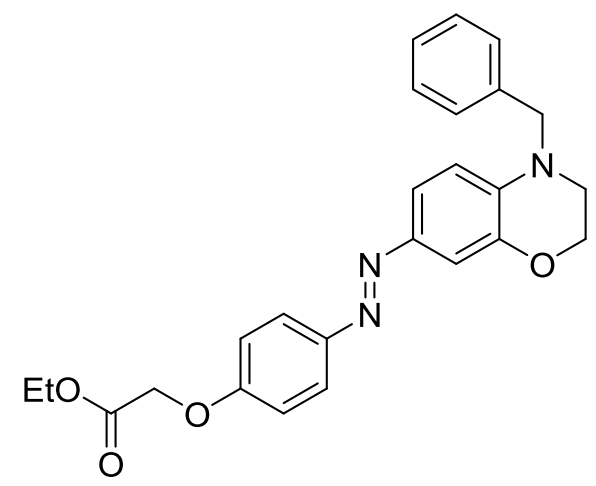

Standard Procedure E with azobenzene 28 (124 mg, $0.36 \mathrm{mmol}, 1.0$ equiv.) and benzaldehyde (116 mg, $1.09 \mathrm{mmol}, 3.0$ equiv.) gave azobenzene $\mathbf{3 6 b}(105 \mathrm{mg}, 68 \%)$ as an orange solid after purification with silica-gel chromatography $\left(\mathrm{CH}_{2} \mathrm{Cl}_{2}\right.$ /petroleum ether, $\left.1: 1 \rightarrow 2: 1\right) . R_{f}=0.14\left(\mathrm{CH}_{2} \mathrm{Cl}_{2}\right) ;$ m.p. $193{ }^{\circ} \mathrm{C} ;{ }^{1} \mathrm{H}-$ NMR (300 MHz, $\left.\mathrm{CDCl}_{3}\right): \delta=7.83(\mathrm{~d}, J=9.0 \mathrm{~Hz}, 2 \mathrm{H}), 7.46(\mathrm{qd}, J=2.3,4.6 \mathrm{~Hz}, 2 \mathrm{H}), 7.40-7.26(\mathrm{~m}, 5$ H), $7.00(\mathrm{~d}, J=9.0 \mathrm{~Hz}, 2 \mathrm{H}), 6.74(\mathrm{~d}, J=9.2 \mathrm{~Hz}, 1 \mathrm{H}), 4.69(\mathrm{~s}, 2 \mathrm{H}), 4.59(\mathrm{~s}, 2 \mathrm{H}), 4.36$ - $4.25(\mathrm{~m}, 4 \mathrm{H})$, $3.54-3.46(\mathrm{~m}, 3 \mathrm{H}), 1.32(\mathrm{t}, J=7.1 \mathrm{~Hz}, 3 \mathrm{H}) ;{ }^{13} \mathrm{C}-\mathrm{NMR}\left(75 \mathrm{MHz}, \mathrm{CDCl}_{3}\right): \delta=168.6,159.0,148.0$, 144.6, 143.8, 138.3, 137.1, 128.8, 127.4, 126.9, 124.0, 120.6, 114.8, 111.1, 108.5, 65.6, 64.3, 61.5, 54.5, 47.4, 14.2; IR: $\tilde{v}=3460,2909,2870,2326,1763,1582,1246,1080,880,810 ;$ UV-VIS $\left(\mathrm{CH}_{3} \mathrm{CN}+0.5 \%\right.$ piperidine): $\lambda_{\max }(\varepsilon)=420 \mathrm{~nm}\left(253001 \cdot \mathrm{mol}^{-1} \cdot \mathrm{cm}^{-1}\right)$; HRMS (ESI): $\mathrm{m} / z$ calcd for $\mathrm{C}_{25} \mathrm{H}_{26} \mathrm{~N}_{3} \mathrm{O}_{4}: 432.1918$ $[\mathrm{M}+\mathrm{H}]^{+}$; found: 432.1921 .

$N-\left(2^{\prime}-\left(\left(4^{\prime \prime}-(\right.\right.\right.$ Dimethylamino)phenyl)diazenyl)-5'-methoxyphenyl)succinimide 37c 


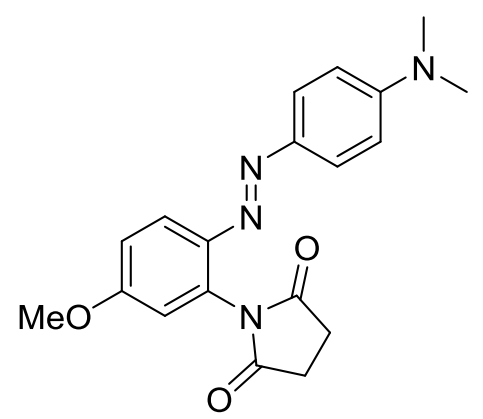

Standard Procedure D with azobenzene $33 \mathrm{~b}(100.0 \mathrm{mg}, 0.24 \mathrm{mmol}, 1.0$ equiv.) and dimethylamine $(92 \mu \mathrm{L}, 5.2 \mathrm{M}$ in $\mathrm{EtOH}, 0.48 \mathrm{mmol}, 2.0$ equiv.) gave azobenzene 37c $(73 \mathrm{mg}, 88 \%)$ as a yellow solid after silica-gel chromatography (EtOAc/petroleum ether, 1:4 $\rightarrow$ EtOAc). $R_{f}=0.29$ (EtOAc/petroleum ether, 2:1); m.p. $94{ }^{\circ} \mathrm{C} ;{ }^{1} \mathrm{H}-\mathrm{NMR}\left(300 \mathrm{MHz}, \mathrm{CDCl}_{3}\right): \delta=7.89(\mathrm{~d}, J=9.1 \mathrm{~Hz}, 1 \mathrm{H}), 7.69(\mathrm{~d}, J=9.0 \mathrm{~Hz}, 2$ H), $7.05(\mathrm{dd}, J=2.7,9.1 \mathrm{~Hz}, 1 \mathrm{H}), 6.82(\mathrm{~d}, J=2.7 \mathrm{~Hz}, 1 \mathrm{H}), 6.72(\mathrm{~d}, J=9.1 \mathrm{~Hz}, 2 \mathrm{H}), 3.88(\mathrm{~s}, 3 \mathrm{H}), 3.08$ (s, $6 \mathrm{H}), 2.97$ (s, $4 \mathrm{H}) ;{ }^{13} \mathrm{C}-\mathrm{NMR}\left(101 \mathrm{MHz}, \mathrm{CDCl}_{3}\right): \delta=176.4,161.1,152.3,144.0,141.8,131.5,124.7$, 118.8, 116.3, 113.3, 111.5, 55.7, 40.3, 28.8; IR: $\tilde{v}=2920$, 2361, 1717, 1601, 1369, 1146, 826; UV-VIS $\left(\mathrm{CH}_{3} \mathrm{CN}+0.5 \%\right.$ piperidine $): \lambda_{\max }(\varepsilon)=421 \mathrm{~nm}\left(224001 \cdot \mathrm{mol}^{-1} \cdot \mathrm{cm}^{-1}\right) ; \mathrm{HRMS}(\mathrm{ESI}): \mathrm{m} / z$ calcd for $\mathrm{C}_{19} \mathrm{H}_{21} \mathrm{~N}_{4} \mathrm{O}_{3}: 353.1608[\mathrm{M}+\mathrm{H}]^{+}$; found: 353.1610 .

$N$-(5'-Methoxy-2'-((4"'-morpholinophenyl)diazenyl)phenyl)succinimide 37d

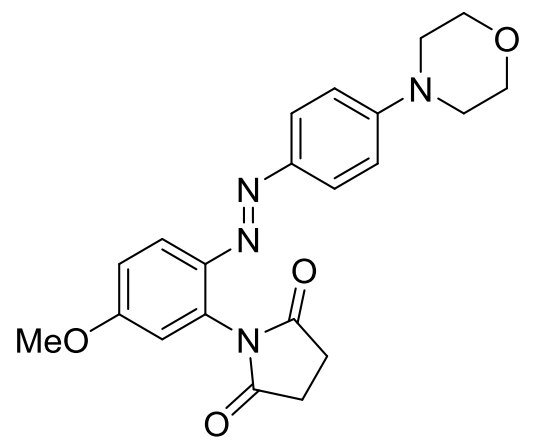

Standard Procedure D with azobenzene $33 b(80.0 \mathrm{mg}, 0.19 \mathrm{mmol}, 1.0$ equiv.) and morpholine $(33 \mu \mathrm{L}$, $0.38 \mathrm{mmol}, 2.0$ equiv.) gave azobenzene $37 \mathrm{~d}(76 \mathrm{mg}, 94 \%)$ as a dark red solid after silica-gel chromatography $\left(\mathrm{CH}_{2} \mathrm{Cl}_{2} / \mathrm{MeOH}, 98: 2\right) . \quad R_{f}=0.46\left(\mathrm{CH}_{2} \mathrm{Cl}_{2} / \mathrm{MeOH}, 98: 2\right) ;$ m.p. $85{ }^{\circ} \mathrm{C} ;{ }^{1} \mathrm{H}-\mathrm{NMR}$ $\left(300 \mathrm{MHz}, \mathrm{CDCl}_{3}\right): \delta=7.90(\mathrm{~d}, J=9.1 \mathrm{~Hz}, 1 \mathrm{H}), 7.69(\mathrm{~d}, J=9.0 \mathrm{~Hz}, 2 \mathrm{H}), 7.06(\mathrm{dd}, J=2.7,9.1 \mathrm{~Hz}, 1$ H), $6.92(\mathrm{~d}, J=9.1 \mathrm{~Hz}, 2 \mathrm{H}), 6.83(\mathrm{~d}, J=2.7 \mathrm{~Hz}, 1 \mathrm{H}), 3.91-3.84(\mathrm{~m}, 8 \mathrm{H}), 3.33$ - 3.26 (m, $4 \mathrm{H}), 2.97$ (s, $4 \mathrm{H}) ;{ }^{13} \mathrm{C}-\mathrm{NMR}\left(75 \mathrm{MHz}, \mathrm{CDCl}_{3}\right): \delta=176.3,161.6,153.0,146.0,141.6,132.0,124.4,118.9,116.3$, 114.4, 113.4, 66.6, 55.8, 48.1, 28.8; IR: $\tilde{v}=2928,2851,2322,1708,1593,1377,1146,926$, 826; UVVIS $\left(\mathrm{CH}_{3} \mathrm{CN}+0.5 \%\right.$ piperidine $): \lambda_{\max }(\varepsilon)=404 \mathrm{~nm}\left(243001 \cdot \mathrm{mol}^{-1} \cdot \mathrm{cm}^{-1}\right) ; \mathrm{HRMS}(\mathrm{ESI}): \mathrm{m} / z$ calcd for $\mathrm{C}_{21} \mathrm{H}_{23} \mathrm{~N}_{4} \mathrm{O}_{4}$ : 395.1714 [M+H] $]^{+}$; found: 395.1714. 
$N$-(5'-Methoxy-2'-((4"'-(4"')-methylpiperazin-1"'"-yl)phenyl)diazenyl)phenyl) succinimide 37e<smiles>COc1ccc(N=Nc2ccc(N3CCN(C)CC3)cc2)c(N2C(=O)CCC2=O)c1</smiles>

Standard Procedure D with azobenzene 33b (100.0 mg, $0.24 \mathrm{mmol}, 1.0$ equiv.) and 1-methylpiperazine (53 $\mu \mathrm{L}, 0.48 \mathrm{mmol}, 2.0$ equiv.) gave azobenzene 37e $(94 \mathrm{mg}, 97 \%)$ as an ocher solid after silica-gel chromatography $\left(\mathrm{CH}_{2} \mathrm{Cl}_{2} / \mathrm{MeOH}, 98: 2 \rightarrow\right.$ 93:7). $R_{f}=0.32\left(\mathrm{CH}_{2} \mathrm{Cl}_{2} / \mathrm{MeOH}, 95: 5\right) ;$ m.p. $86{ }^{\circ} \mathrm{C} ;{ }^{1} \mathrm{H}-\mathrm{NMR}$ (400 MHz, MeOD): $\delta=7.85(\mathrm{~d}, J=9.1 \mathrm{~Hz}, 1 \mathrm{H}), 7.69$ (d, $J=9.1 \mathrm{~Hz}, 2 \mathrm{H}), 7.12$ (dd, $J=2.6,9.1 \mathrm{~Hz}, 1$ H), $7.03(\mathrm{~d}, J=9.1 \mathrm{~Hz}, 2 \mathrm{H}), 6.96(\mathrm{~d}, J=2.9 \mathrm{~Hz}, 1 \mathrm{H}), 3.89(\mathrm{~s}, 3 \mathrm{H}), 3.46-3.39(\mathrm{~m}, 4 \mathrm{H}), 2.96(\mathrm{~s}, 4 \mathrm{H})$, $2.79(\mathrm{t}, J=4.4 \mathrm{~Hz}, 4 \mathrm{H}), 2.48(\mathrm{~s}, 3 \mathrm{H}) ;{ }^{13} \mathrm{C}-\mathrm{NMR}(101 \mathrm{MHz}, \mathrm{MeOD}): \delta=177.7,161.8,152.8,145.7$, 141.7, 132.4, 124.0, 117.9, 115.7, 114.7, 113.5, 55.0, 53.9, 46.7, 44.0, 28.3; IR: $\tilde{v}=2932,2839,1709$, 1597, 1373, 1234, 1138, 1007, 822; UV-VIS $\left(\mathrm{CH}_{3} \mathrm{CN}+0.5 \%\right.$ piperidine $): \lambda_{\max }(\varepsilon)=409 \mathrm{~nm}$ (21000 $1 \cdot \mathrm{mol}^{-1} \cdot \mathrm{cm}^{-1}$ ); HRMS (ESI): $\mathrm{m} / z$ calcd for $\mathrm{C}_{22} \mathrm{H}_{26} \mathrm{~N}_{5} \mathrm{O}_{3}: 408.2030[\mathrm{M}+\mathrm{H}]^{+}$; found: 408.2034 .

2-(4'-((4"'-(dimethylamino)phenyl)diazenyl)phenoxy)acetic acid $\mathbf{3 8 b}$<smiles>CN(C)c1ccc(/N=N/c2ccc(OCC(=O)O)cc2)cc1</smiles>

Standard Procedure C with ester 26b $(50.0 \mathrm{mg}, 0.15 \mathrm{mmol}, 1.0$ equiv.) gave carboxylic acid $\mathbf{3 8 b}$ (35.6 mg, $78 \%)$ as an ocher solid after evaporation of the solvent. $R_{f}=0.19(\mathrm{MeOH}) ;$ m.p. $279{ }^{\circ} \mathrm{C} ;{ }^{1} \mathrm{H}-$ NMR (500 MHz, CD $\left.{ }_{3} \mathrm{OD}\right): \delta=7.77(\mathrm{t}, J=7.6 \mathrm{~Hz}, 4 \mathrm{H}), 7.03(\mathrm{~d}, J=8.2 \mathrm{~Hz}, 2 \mathrm{H}), 6.82(\mathrm{~d}, J=8.5 \mathrm{~Hz}, 2$ $\mathrm{H}), 4.45$ (s, $2 \mathrm{H}), 3.07$ (s, $6 \mathrm{H}) ;{ }^{13} \mathrm{C}-\mathrm{NMR}\left(126 \mathrm{MHz}, \mathrm{CD}_{3} \mathrm{OD}\right): \delta=176.3,161.8,154.0,148.8,145.0$, 
125.6, 124.7, 116.1, 112.9, 68.7, 40.6; IR: $\tilde{v}=3460,2943,2789,1740,1597,1420,1366,1231,1034$, 826; UV-VIS $\left(\mathrm{CH}_{3} \mathrm{CN}+0.5 \%\right.$ piperidine): $\lambda_{\max }(\varepsilon)=408 \mathrm{~nm}\left(244001 \cdot \mathrm{mol}^{-1} \cdot \mathrm{cm}^{-1}\right) ;$ HRMS $(\mathrm{ESI}): \mathrm{m} / z$ calcd for $\mathrm{C}_{16} \mathrm{H}_{18} \mathrm{~N}_{3} \mathrm{O}_{3}: 300.1343[\mathrm{M}+\mathrm{H}]^{+}$; found: 300.1342 .

2-(4'-((4"'-morpholinophenyl)diazenyl)phenoxy)acetic acid 38c

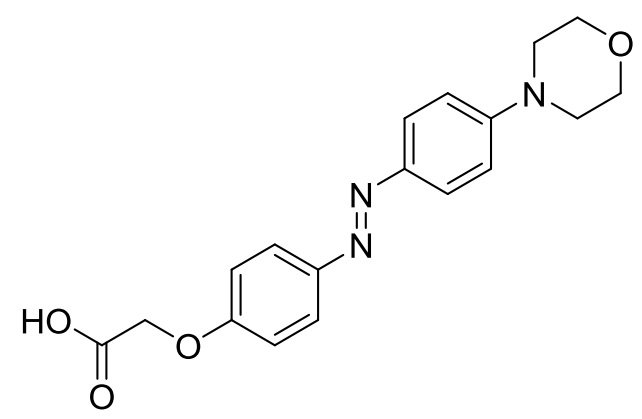

Standard Procedure C with ester 26c (70.0 mg, $0.19 \mathrm{mmol}, 1.0$ equiv.) gave carboxylic acid 38c $(54.2 \mathrm{mg}, 84 \%)$ as a yellow solid after evaporation of the solvent. $R_{f}=0.20(\mathrm{MeOH}) ;$ m.p. $312{ }^{\circ} \mathrm{C} ;{ }^{1} \mathrm{H}-$ NMR (500 MHz, DMSO-d $\left.)_{6}\right): \delta=7.76(\mathrm{~d}, J=9.2 \mathrm{~Hz}, 2 \mathrm{H}), 7.73(\mathrm{~d}, J=9.2 \mathrm{~Hz}, 2 \mathrm{H}), 7.08(\mathrm{~d}, J=9.2 \mathrm{~Hz}$, $2 \mathrm{H}), 6.90(\mathrm{~d}, J=8.8 \mathrm{~Hz}, 2 \mathrm{H}), 4.14(\mathrm{~s}, 2 \mathrm{H}), 3.78-3.74(\mathrm{~m}, 4 \mathrm{H}), 3.30-3.27(\mathrm{~m}, 4 \mathrm{H}) ;{ }^{13} \mathrm{C}-\mathrm{NMR}$ $\left(126 \mathrm{MHz}, \mathrm{DMSO}-d_{6}\right): \delta=169.1,161.9,153.0,146.1,145.0,124.2,123.9,115.4,114.6,68.9,66.4,47.8$; IR: $\tilde{v}=3464,3205,2970,2851,1740,1574,1423,1377,1346,1227,1123,926,841$; UV-VIS $\left(\mathrm{CH}_{3} \mathrm{CN}\right.$ $+0.5 \%$ piperidine): $\lambda_{\max }(\varepsilon)=392 \mathrm{~nm}\left(26200 \mathrm{l} \cdot \mathrm{mol}^{-1} \cdot \mathrm{cm}^{-1}\right) ; \mathrm{HRMS}(\mathrm{ESI}): \mathrm{m} / z$ calcd for $\mathrm{C}_{18} \mathrm{H}_{20} \mathrm{~N}_{3} \mathrm{O}_{4}$ : $342.1448[\mathrm{M}+\mathrm{H}]^{+}$; found: 342.1454 .

2-(4'-((4"'-(4"'-methylpiperazin-1"'-yl)phenyl)diazenyl)phenoxy)acetic acid 38d<smiles>CN1CCN(c2ccc(/N=N/c3ccc(OCC(=O)O)cc3)cc2)CC1</smiles>

Standard Procedure C with ester 26d (70.0 mg, $0.18 \mathrm{mmol}, 1.0$ equiv.) gave carboxylic acid 38d $(38.2 \mathrm{mg}, 59 \%)$ as a yellow solid after evaporation of the solvent. $R_{f}=0.20(\mathrm{MeOH})$; m.p. $255{ }^{\circ} \mathrm{C}$ (decomp.); ${ }^{1} \mathrm{H}-\mathrm{NMR}\left(500 \mathrm{MHz}, \mathrm{D}_{2} \mathrm{O}\right): \delta=7.67$ (d, $\left.J=8.5 \mathrm{~Hz}, 2 \mathrm{H}\right), 7.64(\mathrm{~d}, J=8.8 \mathrm{~Hz}, 2 \mathrm{H}), 7.04$ (d, $J$ $=8.5 \mathrm{~Hz}, 2 \mathrm{H}), 6.99(\mathrm{~d}, J=8.2 \mathrm{~Hz}, 2 \mathrm{H}), 4.47$ (s, $2 \mathrm{H}), 3.22($ br s, $4 \mathrm{H}), 2.55$ (br s, $4 \mathrm{H}), 2.24$ (s, $3 \mathrm{H})$; ${ }^{13} \mathrm{C}-\mathrm{NMR}\left(126 \mathrm{MHz}, \mathrm{D}_{2} \mathrm{O}\right): \delta=176.2,160.0,152.9,146.5,145.4,124.0,123.9,116.4,115.0,66.8,53.4$, 
48.0, 44.4; IR: $\tilde{v}=3460,2947,2843,2681,2596,1744,1597,1234,1080,840$; UV-VIS $\left(\mathrm{CH}_{3} \mathrm{CN}+\right.$ $0.5 \%$ piperidine): $\lambda_{\max }(\varepsilon)=395 \mathrm{~nm}\left(262001 \cdot \mathrm{mol}^{-1} \cdot \mathrm{cm}^{-1}\right)$; HRMS (ESI): $\mathrm{m} / z$ calcd for $\mathrm{C}_{19} \mathrm{H}_{23} \mathrm{~N}_{4} \mathrm{O}_{3}$ : $355.1765[\mathrm{M}+\mathrm{H}]^{+}$; found: 355.1769 .

2-(4'-((4"'-methyl-3"',4"'-dihydro-2"H-benzo[b][1",4"']oxazin-7"'-yl)diazenyl)phenoxy) acetic acid 39a<smiles>CN1CCOc2cc(/N=N/c3ccc(OCC(=O)O)cc3)ccc21</smiles>

Standard Procedure $\mathbf{C}$ with ester 36a (45.0 mg, $0.13 \mathrm{mmol}, 1.0$ equiv.) gave carboxylic acid 39a (34 mg, $76 \%)$ as a red solid after purification by silica-gel chromatography $\left(\mathrm{CH}_{2} \mathrm{Cl}_{2} / \mathrm{MeOH}, 10: 1\right) . R_{f}=0.10$ $\left(\mathrm{CH}_{2} \mathrm{Cl}_{2} / \mathrm{MeOH}, 10: 1\right)$; m.p. $172{ }^{\circ} \mathrm{C} ;{ }^{1} \mathrm{H}-\mathrm{NMR}\left(400 \mathrm{MHz}, \mathrm{DMSO}-d_{6}\right): \delta=13.11$ (br s, $\left.1 \mathrm{H}\right), 7.75(\mathrm{~d}, J=$ $9.1 \mathrm{~Hz}, 2 \mathrm{H}), 7.43(\mathrm{dd}, J=2.3,8.5 \mathrm{~Hz}, 1 \mathrm{H}), 7.19(\mathrm{~d}, J=2.0 \mathrm{~Hz}, 1 \mathrm{H}), 7.04$ (d, $J=8.8 \mathrm{~Hz}, 2 \mathrm{H}), 6.80(\mathrm{~d}, J$ $=8.8 \mathrm{~Hz}, 1 \mathrm{H}), 4.76(\mathrm{~s}, 2 \mathrm{H}), 4.29-4.19(\mathrm{~m}, 2 \mathrm{H}), 3.42-3.37(\mathrm{~m}, 2 \mathrm{H}), 2.98(\mathrm{~s}, 3 \mathrm{H}) ;{ }^{13} \mathrm{C}-\mathrm{NMR}$ (101 MHz, DMSO- $\left.d_{6}\right): \delta=170.4,159.7,147.2,144.1,143.9,140.0,124.0,121.3,115.4,111.4,106.7$, 65.2, 64.5, 48.5, 38.4; IR: $\tilde{v}=2916,2851,2326,1736,1520,1215,1080,829,795$; UV-VIS $\left(\mathrm{CH}_{3} \mathrm{CN}+\right.$ $0.5 \%$ piperidine): $\lambda_{\max }(\varepsilon)=417 \mathrm{~nm}\left(266001 \cdot \mathrm{mol}^{-1} \cdot \mathrm{cm}^{-1}\right)$; HRMS $(\mathrm{ESI}): \mathrm{m} / z$ calcd for $\mathrm{C}_{17} \mathrm{H}_{18} \mathrm{~N}_{3} \mathrm{O}_{4}$ : $328.1292[\mathrm{M}+\mathrm{H}]^{+}$; found: 328.1296 .

2-(4'-((4"'-benzyl-3"',4"-dihydro-2"H-benzo[b][1",4"']oxazin-7"'-yl)diazenyl)phenoxy) acetic acid 39b<smiles>O=C(O)COc1ccc(/N=N/c2ccc3c(c2)OCCN3Cc2ccccc2)cc1</smiles>

Standard Procedure C with ester $\mathbf{3 6 b}(80.0 \mathrm{mg}, 0.19 \mathrm{mmol}, 1.0$ equiv.) gave carboxylic acid $\mathbf{3 9 b}(67 \mathrm{mg}$, $89 \%)$ as an orange solid after purification by silica-gel chromatography $\left(\mathrm{CH}_{2} \mathrm{Cl}_{2} / \mathrm{MeOH}, 10: 1\right) . R_{f}=0.31$ $\left(\mathrm{CH}_{2} \mathrm{Cl}_{2} / \mathrm{MeOH}, 10: 1\right)$; m.p. $87{ }^{\circ} \mathrm{C} ;{ }^{1} \mathrm{H}-\mathrm{NMR}\left(400 \mathrm{MHz}, \mathrm{DMSO}-d_{6}\right): \delta=13.11$ (br s, $\left.1 \mathrm{H}\right), 7.74(\mathrm{~d}, J=$ $9.1 \mathrm{~Hz}, 2 \mathrm{H}), 7.40-7.25$ (m, $6 \mathrm{H}), 7.24$ (d, $J=2.3 \mathrm{~Hz}, 1 \mathrm{H}), 7.04(\mathrm{~d}, J=9.1 \mathrm{~Hz}, 2 \mathrm{H}), 6.80$ (d, $J=8.8 \mathrm{~Hz}$, $1 \mathrm{H}), 4.76(\mathrm{~s}, 2 \mathrm{H}), 4.66(\mathrm{~s}, 2 \mathrm{H}), 4.28(\mathrm{t}, J=4.4 \mathrm{~Hz}, 2 \mathrm{H}), 3.55(\mathrm{t}, J=4.4 \mathrm{~Hz}, 2 \mathrm{H}) ;{ }^{13} \mathrm{C}-\mathrm{NMR}(101 \mathrm{MHz}$, 
DMSO- $\left.d_{6}\right): \delta=170.4,159.6,147.2,143.9,143.6,138.8,138.0,129.1,127.5,127.4,124.0,121.3,115.4$, 111.5, 107.3, 65.2, 64.3, 54.0, 47.5; IR: $\tilde{v}=3460,2920,2866,2578,1736,1597,1315,1234,844,725$; UV-VIS $\left(\mathrm{CH}_{3} \mathrm{CN}+0.5 \%\right.$ piperidine $): \lambda_{\max }(\varepsilon)=418 \mathrm{~nm}\left(227001 \cdot \mathrm{mol}^{-1} \cdot \mathrm{cm}^{-1}\right) ; \mathrm{HRMS}(\mathrm{ESI}): \mathrm{m} / z$ calcd for $\mathrm{C}_{23} \mathrm{H}_{22} \mathrm{~N}_{3} \mathrm{O}_{4}$ : $404.1605[\mathrm{M}+\mathrm{H}]^{+}$; found: 404.1608 .

2-(4'-((4"'-methoxyphenyl)diazenyl)phenoxy)acetic acid 40a<smiles>COc1ccc(/N=N/c2ccc(OCC(=O)O)cc2)cc1</smiles>

Standard Procedure C with ester 32a (24 mg, $0.08 \mathrm{mmol}, 1.0$ equiv.) gave carboxylic acid 40a (22 mg, $96 \%$ ) as a yellow solid after evaporation. $R_{f}=0.22$ (EtOAc/petroleum ether, 3:7 $+0.5 \%$ formic acid); m.p. $104{ }^{\circ} \mathrm{C} ;{ }^{1} \mathrm{H}-\mathrm{NMR}\left(400 \mathrm{MHz}, \mathrm{DMSO}-d_{6}\right): \delta=13.10$ (br s, $\left.1 \mathrm{H}\right), 7.84(\mathrm{t}, J=7.9 \mathrm{~Hz}, 4 \mathrm{H}), 7.11(\mathrm{t}, J=$ $9.8 \mathrm{~Hz}, 4 \mathrm{H}), 4.80(\mathrm{~s}, 2 \mathrm{H}), 3.86(\mathrm{~s}, 3 \mathrm{H}) ;{ }^{13} \mathrm{C}-\mathrm{NMR}\left(101 \mathrm{MHz}, \mathrm{DMSO}-d_{6}\right): \delta=170.3,162.0,160.4$, 146.9, 146.6, 124.7, 124.5, 115.5, 115.0, 65.2, 56.1; IR: $\tilde{v}=2916,2569,1736,1705,1597,1578,1497$, 1234, 1146, 841; UV-VIS $\left(\mathrm{CH}_{3} \mathrm{CN}+0.5 \%\right.$ piperidine $): \lambda_{\max }(\varepsilon)=360 \mathrm{~nm}\left(250001 \cdot \mathrm{mol}^{-1} \cdot \mathrm{cm}^{-1}\right) ; \mathrm{HRMS}$ (ESI): $m / z$ calcd for $\mathrm{C}_{15} \mathrm{H}_{15} \mathrm{~N}_{2} \mathrm{O}_{4}: 287.1026[\mathrm{M}+\mathrm{H}]^{+}$; found: 287.1027 .

2-(4'-((3",4"'-dimethoxyphenyl)diazenyl)phenoxy)acetic acid 40b<smiles>COc1ccc(/N=N/c2ccc(OCC(=O)O)cc2)cc1OC</smiles>

Standard Procedure $\mathbf{C}$ with ester 32b (60 mg, $0.18 \mathrm{mmol}, 1.0$ equiv.) gave carboxylic acid $40 \mathrm{~b}$ (56 mg, $98 \%$ ) as a yellow solid after evaporation. $R_{f}=0.22$ (EtOAc/petroleum ether, $3: 7+0.5 \%$ formic acid); m.p. $190{ }^{\circ} \mathrm{C} ;{ }^{1} \mathrm{H}-\mathrm{NMR}\left(300 \mathrm{MHz}, \mathrm{DMSO}-d_{6}\right): \delta=7.83(\mathrm{~d}, J=9.0 \mathrm{~Hz}, 2 \mathrm{H}), 7.53(\mathrm{dd}, J=2.2,8.5 \mathrm{~Hz}, 1$ H), $7.42(\mathrm{~d}, J=2.2 \mathrm{~Hz}, 1 \mathrm{H}), 7.14(\mathrm{~d}, J=8.7 \mathrm{~Hz}, 1 \mathrm{H}), 7.07(\mathrm{~d}, J=8.8 \mathrm{~Hz}, 2 \mathrm{H}), 4.77$ (s, $2 \mathrm{H}), 3.85$ (s, 3 $\mathrm{H}), 3.84(\mathrm{~s}, 3 \mathrm{H}) ;{ }^{13} \mathrm{C}-\mathrm{NMR}\left(101 \mathrm{MHz}, \mathrm{CDCl}_{3}\right): \delta=170.4,160.3,152.0,149.9,146.9,146.5,124.5$, 120.2, 115.5, 111.7, 102.3, 65.2, 56.2, 55.9; IR: $\tilde{v}=2916,2851,2577,1736,1705,1578,1497,1231$, 
1018, 845; UV-VIS $\left(\mathrm{CH}_{3} \mathrm{CN}+0.5 \%\right.$ piperidine $): \lambda_{\max }(\varepsilon)=372 \mathrm{~nm}\left(25300 \mathrm{l} \cdot \mathrm{mol}^{-1} \cdot \mathrm{cm}^{-1}\right)$; HRMS $($ ESI): $m / z$ calcd for $\mathrm{C}_{16} \mathrm{H}_{17} \mathrm{~N}_{2} \mathrm{O}_{5}: 317.1132[\mathrm{M}+\mathrm{H}]^{+}$; found: 317.1134 .

2-(4'-((3"',4",5"'-trimethoxyphenyl)diazenyl)phenoxy)acetic acid 40c<smiles>COc1cc(/N=N/c2ccc(OCC(=O)O)cc2)cc(OC)c1OC</smiles>

Standard Procedure C with ester 32c (135 mg, 0.37 mmol, 1.0 equiv.) gave carboxylic acid 40c (106 mg, $82 \%$ ) as an orange solid after purification by silica-gel chromatography (EtOAc/petroleum ether, 3:7 + $0.5 \%$ formic acid). $R_{f}=0.22$ (EtOAc/petroleum ether, $3: 7+0.5 \%$ formic acid); m.p. $110{ }^{\circ} \mathrm{C} ;{ }^{1} \mathrm{H}-\mathrm{NMR}$ $\left(300 \mathrm{MHz}, \mathrm{CDCl}_{3}\right): \delta=7.93(\mathrm{~d}, J=9.0 \mathrm{~Hz}, 2 \mathrm{H}), 7.23(\mathrm{~s}, 2 \mathrm{H}), 7.06(\mathrm{~d}, J=9.0 \mathrm{~Hz}, 2 \mathrm{H}), 4.79(\mathrm{~s}, 2 \mathrm{H})$, $3.98(\mathrm{~s}, 6 \mathrm{H}), 3.94(\mathrm{~s}, 3 \mathrm{H}) ;{ }^{13} \mathrm{C}-\mathrm{NMR}\left(101 \mathrm{MHz}, \mathrm{CDCl}_{3}\right): \delta=172.6,159.4,153.5,148.5,147.7,140.4$, 124.6, 114.9, 100.2, 64.8, 61.0, 56.2; IR: $\tilde{v}=2943,2839,2573,1732,1585,1416,1219,1126,991,845$; UV-VIS $\left(\mathrm{CH}_{3} \mathrm{CN}+0.5 \%\right.$ piperidine $): \lambda_{\max }(\varepsilon)=368 \mathrm{~nm}\left(221001 \cdot \mathrm{mol}^{-1} \cdot \mathrm{cm}^{-1}\right) ; \mathrm{HRMS}(\mathrm{ESI}): \mathrm{m} / z$ calcd for $\mathrm{C}_{17} \mathrm{H}_{19} \mathrm{~N}_{2} \mathrm{O}_{6}: 347.1238[\mathrm{M}+\mathrm{H}]^{+}$; found: 347.1241 .

2-(4'-((4"'-(2"'”-hydroxyethoxy)-3"',5"'-dimethoxyphenyl)diazenyl)phenoxy)acetic acid 40d<smiles>COc1cc(/N=N/c2ccc(OCC(=O)O)cc2)cc(OC)c1OCCO</smiles>

Standard Procedure C with ester 32d (68 mg, $0.17 \mathrm{mmol}, 1.0$ equiv.) gave carboxylic acid $40 d$ (36 mg, $57 \%)$ as a yellow solid after purification by preparative $\mathrm{HPLC}\left(\mathrm{H}_{2} \mathrm{O} / \mathrm{CH}_{3} \mathrm{CN}, 70: 30 \rightarrow 0: 100\right) . R_{f}=0.31$ $\left(\mathrm{CH}_{2} \mathrm{Cl}_{2} / \mathrm{MeOH}, 8: 2\right)$; m.p. $183{ }^{\circ} \mathrm{C} ;{ }^{1} \mathrm{H}-\mathrm{NMR}\left(300 \mathrm{MHz}, \mathrm{DMSO}-d_{6}\right): \delta=7.86(\mathrm{~d}, \mathrm{~J}=9.0 \mathrm{~Hz}, 2 \mathrm{H}), 7.21$ (s, 2 H), 7.09 (d, J = 8.9 Hz, 2 H), $4.79(\mathrm{~s}, 2 \mathrm{H}), 3.96$ (t, J = 5.6 Hz, 2 H), 3.87 (s, $6 \mathrm{H}), 3.64$ (t, J = 5.6 Hz, $2 \mathrm{H}) ;{ }^{13} \mathrm{C}-\mathrm{NMR}\left(126 \mathrm{MHz}, \mathrm{DMSO}-d_{6}\right): \delta=170.4,160.8,153.8,148.2,146.8,139.6,124.8,115.6,100.5$, 74.8, 65.3, 60.7, 56.5; IR: $\tilde{v}=3410,2932,1593,1493,1258,1126,984,826 ; \mathrm{UV}-\mathrm{VIS}\left(\mathrm{CH}_{3} \mathrm{CN}+0.5 \%\right.$ 
piperidine): $\lambda_{\max }(\varepsilon)=369 \mathrm{~nm}\left(220001 \cdot \mathrm{mol}^{-1} \cdot \mathrm{cm}^{-1}\right)$; HRMS (ESI): $\mathrm{m} / z$ calcd for $\mathrm{C}_{18} \mathrm{H}_{21} \mathrm{~N}_{2} \mathrm{O}_{7}: 377.1343$ $[\mathrm{M}+\mathrm{H}]^{+}$; found: 377.1347 .

2-(4'-((3"',5"'-dimethoxy-4"'-(2"'-(4"'”-methylpiperazin-1"'”'-yl)ethoxy)phenyl)diazenyl)phenoxy)acetic acid 40f<smiles>COc1cc(/N=N/c2ccc(OCC(=O)O)cc2)cc(OC)c1OCCN1CCN(C)CC1</smiles>

Standard Procedure C with ester 32f (35 mg, $0.074 \mathrm{mmol}, 1.0$ equiv.) gave carboxylic acid $40 \mathrm{f}$ (26 mg, $77 \%)$ as a yellow solid after purification by preparative $\operatorname{HPLC}\left(\mathrm{H}_{2} \mathrm{O} / \mathrm{CH}_{3} \mathrm{CN}, 90: 10 \rightarrow 0: 100\right) . R_{f}=0.22$ $\left(\mathrm{CH}_{2} \mathrm{Cl}_{2} / \mathrm{MeOH}, 8: 2\right)$; m.p. $128{ }^{\circ} \mathrm{C} ;{ }^{1} \mathrm{H}-\mathrm{NMR}\left(300 \mathrm{MHz}, \mathrm{D}_{2} \mathrm{O}\right): \delta=7.26(\mathrm{~d}, J=7.9 \mathrm{~Hz}, 2 \mathrm{H}), 6.70(\mathrm{~d}, J=$ $8.0 \mathrm{~Hz}, 2 \mathrm{H}), 6.39$ (s, $2 \mathrm{H}), 4.31$ (s, $2 \mathrm{H}), 3.70$ (s, $2 \mathrm{H}), 3.45$ (s, $6 \mathrm{H}), 3.15$ (br s, $4 \mathrm{H}), 2.77$ (s, $3 \mathrm{H}), 2.88$ 2.66 (br s, $4 \mathrm{H}$ ); ${ }^{13} \mathrm{C}-\mathrm{NMR}\left(101 \mathrm{MHz}, \mathrm{D}_{2} \mathrm{O}\right): \delta=175.7,160.4,152.4,147.8,145.7,137.3,124.3,114.7$, 99.6, 68.8, 66.7, 56.1, 55.6, 52.4, 49.6, 42.9; IR: $\tilde{v}=3422$, 2997, 2634, 2361, 1597, 1412, 1219, 1126, 1042, 941, 840; UV-VIS $\left(\mathrm{CH}_{3} \mathrm{CN}+0.5 \%\right.$ piperidine $): \lambda_{\max }(\varepsilon)=368 \mathrm{~nm}\left(22900 \mathrm{l} \cdot \mathrm{mol}^{-1} \cdot \mathrm{cm}^{-1}\right) ; \mathrm{HRMS}$ (ESI): $m / z$ calcd for $\mathrm{C}_{23} \mathrm{H}_{31} \mathrm{~N}_{4} \mathrm{O}_{6}: 459.2238[\mathrm{M}+\mathrm{H}]^{+}$; found: 459.2243 .

$N-\left(2^{\prime}-\left(\left(4^{\prime \prime}-(\right.\right.\right.$ Dimethylamino)phenyl)diazenyl)-5'-methoxyphenyl)succinamic acid 41c<smiles>COc1ccc(/N=N/c2ccc(N(C)C)cc2)c(NC(=O)CCC(=O)O)c1</smiles>

Standard Procedure C with ester 37c (38 mg, $0.11 \mathrm{mmol}, 1.0$ equiv.) gave carboxylic acid $41 \mathrm{c}$ (33 $\mathrm{mg}$, $83 \%)$ as an orange solid after purification by preparative $\mathrm{HPLC}\left(\mathrm{H}_{2} \mathrm{O} / \mathrm{CH}_{3} \mathrm{CN}, 70: 30 \rightarrow 0: 100\right) . R_{f}=0.18$ $\left(\mathrm{CH}_{2} \mathrm{Cl}_{2} / \mathrm{MeOH}, 8: 2\right)$; m.p. $286{ }^{\circ} \mathrm{C} ;{ }^{1} \mathrm{H}-\mathrm{NMR}\left(300 \mathrm{MHz}, \mathrm{DMSO}-d_{6}\right): \delta=11.75$ (s, $\left.1 \mathrm{H}\right), 8.16(\mathrm{~d}, J=2.7$ $\mathrm{Hz}, 1 \mathrm{H}), 8.10(\mathrm{~d}, J=9.0 \mathrm{~Hz}, 2 \mathrm{H}), 7.66(\mathrm{~d}, J=9.0 \mathrm{~Hz}, 1 \mathrm{H}), 6.79(\mathrm{~d}, J=9.1 \mathrm{~Hz}, 2 \mathrm{H}), 6.66(\mathrm{dd}, J=2.8$, $9.0 \mathrm{~Hz}, 1 \mathrm{H}), 3.79$ (s, $3 \mathrm{H}), 3.05$ (s, $6 \mathrm{H}), 2.50$ - 2.48 (m, $2 \mathrm{H}), 2.25$ - 2.19 (m, $2 \mathrm{H}) ;{ }^{13} \mathrm{C}-\mathrm{NMR}(101 \mathrm{MHz}$, 
DMSO- $\left.d_{6}\right): \delta=174.6,173.8,161.4,152.3,143.9,139.2,135.3,126.0,117.1,112.0,109.4,105.5,55.8$, 39.4, 36.2, 34.8; IR: $\tilde{v}=3345,2920,1654,1589,1362,1280,1141,1030,822$; UV-VIS $\left(\mathrm{CH}_{3} \mathrm{CN}+0.5 \%\right.$ piperidine): $\lambda_{\max }(\varepsilon)=450 \mathrm{~nm}\left(21300 \mathrm{l} \cdot \mathrm{mol}^{-1} \cdot \mathrm{cm}^{-1}\right)$; HRS (ESI): $\mathrm{m} / z$ calcd for $\mathrm{C}_{19} \mathrm{H}_{23} \mathrm{~N}_{4} \mathrm{O}_{4}: 371.1714$ $[\mathrm{M}+\mathrm{H}]^{+}$; found: 371.1718 .

$N$-(5'-Methoxy-2'-((4"'-morpholinophenyl)diazenyl)phenyl)succinamic acid 41d<smiles>COc1ccc(/N=N/c2ccc(N3CCOCC3)cc2)c(NC(=O)CCC(=O)O)c1</smiles>

Standard Procedure C with ester 37d (25 mg, $0.06 \mathrm{mmol}, 1.0$ equiv.) gave carboxylic acid $41 \mathrm{~d}$ (18 mg, $69 \%)$ as a yellow solid after purification by preparative $\mathrm{HPLC}\left(\mathrm{H}_{2} \mathrm{O} / \mathrm{CH}_{3} \mathrm{CN}, 70: 30 \rightarrow 0: 100\right) . R_{f}=0.22$ $\left(\mathrm{CH}_{2} \mathrm{Cl}_{2} / \mathrm{MeOH}, 8: 2\right)$; m.p. $207{ }^{\circ} \mathrm{C} ;{ }^{1} \mathrm{H}-\mathrm{NMR}\left(300 \mathrm{MHz}, \mathrm{DMSO}-d_{6}\right): \delta=10.23(\mathrm{~s}, 1 \mathrm{H}), 8.02(\mathrm{~d}, J=2.6$ $\mathrm{Hz}, 1 \mathrm{H}), 7.90$ (d, $J=8.9 \mathrm{~Hz}, 2 \mathrm{H}), 7.70(\mathrm{~d}, J=9.0 \mathrm{~Hz}, 1 \mathrm{H}), 7.09$ (d, $J=9.0 \mathrm{~Hz}, 2 \mathrm{H}), 6.76$ (dd, $J=2.6$, $9.0 \mathrm{~Hz}, 1 \mathrm{H}), 3.82(\mathrm{~s}, 3 \mathrm{H}), 3.79-3.72(\mathrm{~m}, 4 \mathrm{H}), 3.30-3.27(\mathrm{~m}, 4 \mathrm{H}), 2.76(\mathrm{t}, J=6.2 \mathrm{~Hz}, 2 \mathrm{H}), 2.56(\mathrm{t}, J=$ $6.8 \mathrm{~Hz}, 2 \mathrm{H}) ;{ }^{13} \mathrm{C}-\mathrm{NMR}\left(101 \mathrm{MHz}, \mathrm{DMSO}-d_{6}\right): \delta=173.8,170.7,161.5,152.7,144.6,137.6,134.5$, 124.5, 118.8, 114.0, 109.8, 105.3, 65.9, 55.5, 47.2, 31.7, 28.9; IR: $\tilde{v}=3375,2970,1701,1589,1234$, 1111, 1038, 918, 818; UV-VIS $\left(\mathrm{CH}_{3} \mathrm{CN}+0.5 \%\right.$ piperidine $): \lambda_{\max }(\varepsilon)=414 \mathrm{~nm}\left(280001 \cdot \mathrm{mol}^{-1} \cdot \mathrm{cm}^{-1}\right)$; HRMS (ESI): $m / z$ calcd for $\mathrm{C}_{21} \mathrm{H}_{25} \mathrm{~N}_{4} \mathrm{O}_{5}: 413.1819[\mathrm{M}+\mathrm{H}]^{+}$; found: 413.1825 .

$N$-(5'-Methoxy-2'-((4"'-(4"'--methylpiperazin-1"'"-yl)phenyl)diazenyl)phenyl)succinamic acid 41e<smiles>COc1ccc(/N=N/c2ccc(N3CCN(C)CC3)cc2)c(NC(=O)CCC(=O)O)c1</smiles>

Standard Procedure C with ester 37e (25 mg, $0.06 \mathrm{mmol}, 1.0$ equiv.) gave carboxylic acid $41 \mathrm{e}(13 \mathrm{mg}$, $38 \%)$ as a yellow solid after purification by preparative $\operatorname{HPLC}\left(\mathrm{H}_{2} \mathrm{O} / \mathrm{CH}_{3} \mathrm{CN}, 70: 30 \rightarrow 0: 100\right) . R_{f}=0.12$ 
$\left(\mathrm{CH}_{2} \mathrm{Cl}_{2} / \mathrm{MeOH}, 8: 2\right)$; m.p. $160{ }^{\circ} \mathrm{C} ;{ }^{1} \mathrm{H}-\mathrm{NMR}$ (300 MHz, DMSO- $\left.d_{6}\right): \delta=10.26$ (s, $\left.1 \mathrm{H}\right), 8.03(\mathrm{~d}, J=2.6$ $\mathrm{Hz}, 1 \mathrm{H}), 7.88(\mathrm{~d}, J=8.9 \mathrm{~Hz}, 2 \mathrm{H}), 7.70(\mathrm{~d}, J=9.0 \mathrm{~Hz}, 1 \mathrm{H}), 7.07$ (d, $J=9.0 \mathrm{~Hz}, 2 \mathrm{H}), 6.75(\mathrm{dd}, J=2.7$, $9.0 \mathrm{~Hz}, 1 \mathrm{H}), 3.81(\mathrm{~s}, 3 \mathrm{H}), 3.40-3.27(\mathrm{~m}, 4 \mathrm{H}), 2.75(\mathrm{t}, J=6.6 \mathrm{~Hz}, 2 \mathrm{H}), 2.56(\mathrm{t}, J=6.9 \mathrm{~Hz}, 2 \mathrm{H}), 2.48$ $2.41(\mathrm{~m}, 4 \mathrm{H}), 2.23(\mathrm{~s}, 3 \mathrm{H}) ;{ }^{13} \mathrm{C}-\mathrm{NMR}\left(101 \mathrm{MHz}, \mathrm{DMSO}-d_{6}\right): \delta=173.9,170.8,161.4,152.6,144.3$, 137.5, 134.5, 124.6, 118.7, 114.1, 109.7, 105.3, 55.5, 54.3, 46.9, 45.7, 31.8, 29.0; IR: $\tilde{v}=3368,2967$, 2839, 1682, 1585, 1508, 1285, 1238, 1150, 1033, 829; UV-VIS $\left(\mathrm{CH}_{3} \mathrm{CN}+0.5 \%\right.$ piperidine $): \lambda_{\max }(\varepsilon)=$ $417 \mathrm{~nm}\left(19300 \mathrm{l} \cdot \mathrm{mol}^{-1} \cdot \mathrm{cm}^{-1}\right)$; HRMS (ESI): $\mathrm{m} / z$ calcd for $\mathrm{C}_{22} \mathrm{H}_{28} \mathrm{~N}_{5} \mathrm{O}_{4}: 426.2136[\mathrm{M}+\mathrm{H}]^{+}$; found: 426.2139 .

$N-\left(5^{\prime}-\right.$ Methoxy-2'-((4"'-methoxyphenyl)diazenyl)phenyl)succinamic acid 42a<smiles>COc1ccc(/N=N/c2ccc(OC)cc2NC(=O)CCC(=O)O)cc1</smiles>

Standard Procedure C with ester 33a (60.0 mg, 0.16 mmol, 1.0 equiv.) gave carboxylic acid $42 \mathbf{a}(57 \mathrm{mg}$, $99 \%)$ as a chartreuse solid after evaporation of the solvent. $R_{f}=0.23\left(\mathrm{CH}_{2} \mathrm{Cl}_{2} / \mathrm{MeOH}, 95: 5\right)$; m.p. $167^{\circ} \mathrm{C}$; ${ }^{1} \mathrm{H}-\mathrm{NMR}\left(300 \mathrm{MHz}, \mathrm{DMSO}-d_{6}\right): \delta=12.19(\mathrm{~s}, 1 \mathrm{H}), 10.22(\mathrm{~s}, 1 \mathrm{H}), 8.04(\mathrm{~d}, J=2.8 \mathrm{~Hz}, 1 \mathrm{H}), 8.00(\mathrm{~d}, J=$ $8.9 \mathrm{~Hz}, 2 \mathrm{H}), 7.74(\mathrm{~d}, J=9.0 \mathrm{~Hz}, 1 \mathrm{H}), 7.13$ (d, $J=9.0 \mathrm{~Hz}, 2 \mathrm{H}), 6.78(\mathrm{dd}, J=2.8,9.1 \mathrm{~Hz}, 1 \mathrm{H}), 3.87$ (s, 3 $\mathrm{H}), 3.84(\mathrm{~s}, 3 \mathrm{H}), 2.79(\mathrm{t}, J=6.5 \mathrm{~Hz}, 2 \mathrm{H}), 2.58(\mathrm{t}, J=6.7 \mathrm{~Hz}, 2 \mathrm{H}) ;{ }^{13} \mathrm{C}-\mathrm{NMR}\left(101 \mathrm{MHz}, \mathrm{DMSO}-d_{6}\right): \delta=$ 174.3, 171.3, 162.5, 162.0, 146.9, 138.6, 134.9, 125.2, 119.1, 114.9, 110.4, 105.9, 56.1, 56.0, 32.1, 29.3; IR: $\tilde{v}=3368,2924,2592,1713,1578,1470,1231,1146,1026,833 ;$ UV-VIS $\left(\mathrm{CH}_{3} \mathrm{CN}+0.5 \%\right.$ piperidine): $\lambda_{\max }(\varepsilon)=386 \mathrm{~nm}\left(214001 \cdot \mathrm{mol}^{-1} \cdot \mathrm{cm}^{-1}\right)$; HRMS (ESI): $\mathrm{m} / z$ calcd for $\mathrm{C}_{18} \mathrm{H}_{20} \mathrm{~N}_{3} \mathrm{O}_{5}: 358.1397$ $[\mathrm{M}+\mathrm{H}]^{+}$; found: 358.1402 .

$N-\left(2^{\prime}-\left(\left(3^{\prime \prime}, 4^{\prime \prime}-\right.\right.\right.$ Dimethoxyphenyl $)$ diazenyl $)-5^{\prime}-$ methoxyphenyl)succinamic acid 42b 
<smiles>COc1ccc(/N=N/c2ccc(OC)c(OC)c2)c(NC(=O)CCC(=O)O)c1</smiles>

Standard Procedure $\mathbf{C}$ with ester 34b (40.0 mg, 0.11 mmol, 1.0 equiv.) gave carboxylic acid $42 \mathbf{b}$ (41 mg, $98 \%)$ as a yellow solid after purification by silica-gel chromatography $\left(\mathrm{CH}_{2} \mathrm{Cl}_{2} / \mathrm{MeOH}, 98: 2 \rightarrow\right.$ 95:5). $R_{f}$ $=0.13\left(\mathrm{CH}_{2} \mathrm{Cl}_{2} / \mathrm{MeOH}, 95: 5\right)$; m.p. $158{ }^{\circ} \mathrm{C} ;{ }^{1} \mathrm{H}-\mathrm{NMR}\left(300 \mathrm{MHz}, \mathrm{CDCl}_{3}\right): \delta=11.00(\mathrm{~s}, 1 \mathrm{H}), 8.32(\mathrm{~d}, J=$ $2.6 \mathrm{~Hz}, 1 \mathrm{H}), 7.85(\mathrm{~d}, J=9.0 \mathrm{~Hz}, 1 \mathrm{H}), 7.51(\mathrm{dd}, J=2.3,8.5 \mathrm{~Hz}, 1 \mathrm{H}), 7.47(\mathrm{~d}, J=2.1 \mathrm{~Hz}, 1 \mathrm{H}), 7.03(\mathrm{~d}, J$ $=8.5 \mathrm{~Hz}, 1 \mathrm{H}), 6.76(\mathrm{dd}, J=2.7,9.0 \mathrm{~Hz}, 1 \mathrm{H}), 4.01(\mathrm{~s}, 3 \mathrm{H}), 4.01(\mathrm{~s}, 3 \mathrm{H}), 3.93(\mathrm{~s}, 3 \mathrm{H}), 2.91-2.79(\mathrm{~m}, 4$ $\mathrm{H}) ;{ }^{13} \mathrm{C}-\mathrm{NMR}\left(101 \mathrm{MHz}, \mathrm{CDCl}_{3}\right): \delta=175.8,170.3,162.7,151.6,149.8,146.6,136.2,133.3,125.3$, 119.4, 111.0, 110.6, 103.8, 102.0, 56.2, 56.0, 55.7, 32.5, 29.0; IR: $\tilde{v}=2940,2839,2322,1717,1585$, 1504, 1420, 1231, 1107, 1022, 856, 810; UV-VIS $\left(\mathrm{CH}_{3} \mathrm{CN}+0.5 \%\right.$ piperidine $): \lambda_{\max }(\varepsilon)=395 \mathrm{~nm}$ $\left(247001 \cdot \mathrm{mol}^{-1} \cdot \mathrm{cm}^{-1}\right)$; HRMS (ESI): $\mathrm{m} / z$ calcd for $\mathrm{C}_{19} \mathrm{H}_{22} \mathrm{~N}_{3} \mathrm{O}_{6}: 388.1503[\mathrm{M}+\mathrm{H}]^{+}$; found: 388.1512 .

$N$-(5'-Methoxy-2'-((3", $4^{\prime \prime}, 5^{\prime \prime}$-trimethoxyphenyl)diazenyl)phenyl)succinamic acid 42c<smiles>COc1ccc(/N=N/c2cc(OC)c(OC)c(OC)c2)c(NC(=O)CCC(=O)O)c1</smiles>

Standard Procedure B with azobenzene 19c (100 mg, $0.26 \mathrm{mmol}, 1.0$ equiv.) and MeI (32.3 $\mu \mathrm{L}$, $0.52 \mathrm{mmol}, 2.0$ equiv.) gave crude azobenzene $34 \mathrm{c}(99 \mathrm{mg})$ after evaporation of the solvent. The crude material was directly converted according to Standard Procedure $\mathbf{C}$ to give carboxylic acid $\mathbf{4 2 c}(77 \mathrm{mg}$, $71 \%$ ) as a yellow solid after purification by silica-gel chromatography (EtOAc/petroleum ether, 3:7 + $0.5 \%$ formic acid). $R_{f}=0.19$ (EtOAc/petroleum ether, $3: 7+0.5 \%$ formic acid); m.p. $165{ }^{\circ} \mathrm{C} ;{ }^{1} \mathrm{H}-\mathrm{NMR}$ $\left(300 \mathrm{MHz}, \mathrm{DMSO}-d_{6}\right): \delta=8.02(\mathrm{~d}, J=2.7 \mathrm{~Hz}, 1 \mathrm{H}), 7.75(\mathrm{~d}, J=9.1 \mathrm{~Hz}, 1 \mathrm{H}), 7.38(\mathrm{~s}, 2 \mathrm{H}), 6.79(\mathrm{dd}, J=$ 2.8, $9.1 \mathrm{~Hz}, 1 \mathrm{H}), 3.91(\mathrm{~s}, 6 \mathrm{H}), 3.84(\mathrm{~s}, 3 \mathrm{H}), 3.75(\mathrm{~s}, 3 \mathrm{H}), 2.75(\mathrm{t}, J=6.6 \mathrm{~Hz}, 2 \mathrm{H}), 2.56(\mathrm{t}, J=6.6 \mathrm{~Hz}, 2$ $\mathrm{H}) ;{ }^{13} \mathrm{C}-\mathrm{NMR}\left(101 \mathrm{MHz}, \mathrm{DMSO}-d_{6}\right): \delta=174.4,171.5,163.0,153.7,148.6,140.2,139.3,134.9,118.5$, 110.6, 106.1, 101.1, 60.7, 56.5, 56.1, 32.3, 29.7; IR: $\tilde{v}=3291,2940,2361,1732,1597,1458,1231,1119$, 
1006, 821; UV-VIS $\left(\mathrm{CH}_{3} \mathrm{CN}+0.5 \%\right.$ piperidine $): \lambda_{\max }(\varepsilon)=393 \mathrm{~nm}\left(206001 \cdot \mathrm{mol}^{-1} \cdot \mathrm{cm}^{-1}\right)$; $\mathrm{HRMS}(\mathrm{ESI})$ : $m / z$ calcd for $\mathrm{C}_{20} \mathrm{H}_{24} \mathrm{~N}_{3} \mathrm{O}_{7}: 418.1609[\mathrm{M}+\mathrm{H}]^{+}$; found: 418.1617 .

$N-\left(2^{\prime}-\left(\left(3^{\prime \prime}, 5^{\prime \prime}-D i m e t h o x y-4{ }^{\prime \prime}-\left(2^{\prime \prime \prime}-\left(4^{\prime \prime \prime \prime}-\right.\right.\right.\right.\right.$ methylpiperazin-1"'”'-yl)ethoxy)phenyl)diazenyl)-5'methoxyphenyl)succinamic acid 42e<smiles>COc1ccc(/N=N/c2cc(OC)c(OCCN3CCN(C)CC3)c(OC)c2)c(NC(=O)CCC(=O)O)c1</smiles>

Standard Procedure $\mathbf{C}$ with ester 34e (36 mg, $0.07 \mathrm{mmol}, 1.0$ equiv.) gave carboxylic acid $42 \mathrm{e}$ (35 mg, $94 \%)$ as an orange solid after purification by preparative HPLC $\left(\mathrm{H}_{2} \mathrm{O} / \mathrm{CH}_{3} \mathrm{CN}, 90: 10 \rightarrow 0: 100\right) . R_{f}=0.24$ $\left(\mathrm{CH}_{2} \mathrm{Cl}_{2} / \mathrm{MeOH}, 8: 2\right)$; m.p. $208{ }^{\circ} \mathrm{C} ;{ }^{1} \mathrm{H}-\mathrm{NMR}\left(500 \mathrm{MHz}, \mathrm{DMSO}-d_{6}\right): \delta=10.37$ (s, $\left.1 \mathrm{H}\right), 8.02(\mathrm{~d}, J=2.7$ $\mathrm{Hz}, 1 \mathrm{H}), 7.75(\mathrm{~d}, J=9.2 \mathrm{~Hz}, 1 \mathrm{H}), 7.38(\mathrm{~s}, 2 \mathrm{H}), 6.79(\mathrm{dd}, J=2.7,9.2 \mathrm{~Hz}, 1 \mathrm{H}), 4.04(\mathrm{t}, J=6.0 \mathrm{~Hz}, 2 \mathrm{H})$, $3.90(\mathrm{~s}, 6 \mathrm{H}), 3.84(\mathrm{~s}, 3 \mathrm{H}), 2.75(\mathrm{t}, J=6.6 \mathrm{~Hz}, 2 \mathrm{H}), 2.64(\mathrm{t}, J=6.0 \mathrm{~Hz}, 2 \mathrm{H}), 2.56(\mathrm{t}, J=6.6 \mathrm{~Hz}, 2 \mathrm{H})$, 2.46 (br. s, 4 H), 2.33 (br s, 4 H), 2.16 (s, 3 H); ${ }^{13} \mathrm{C}-\mathrm{NMR}\left(126 \mathrm{MHz}\right.$, DMSO- $d_{6}$ ): $\delta=174.0,171.1,162.6$, 153.4, 148.1, 138.9, 138.8, 134.5, 118.1, 110.1, 105.6, 100.7, 70.5, 57.4, 56.1, 55.6, 54.6, 52.8, 45.6, 32.0, 29.3; IR: $\tilde{v}=3379,2940,2835,1736,1694,1593,1385,1215,1126,1030,845$; UV-VIS $\left(\mathrm{CH}_{3} \mathrm{CN}+\right.$ $0.5 \%$ piperidine): $\lambda_{\max }(\varepsilon)=393 \mathrm{~nm}\left(241001 \cdot \mathrm{mol}^{-1} \cdot \mathrm{cm}^{-1}\right) ; \mathrm{HRMS}(\mathrm{ESI}): \mathrm{m} / z$ calcd for $\mathrm{C}_{26} \mathrm{H}_{36} \mathrm{~N}_{5} \mathrm{O}_{7}$ : $530.2609[\mathrm{M}+\mathrm{H}]^{+}$; found: 530.2613 .

$N$-(4'-Methoxy-2'-((3",4"',5"'-trimethoxyphenyl)diazenyl)phenyl)succinamic acid $\mathbf{4 3}$<smiles>COc1ccc(NC(=O)CCC(=O)O)c(/N=N/c2cc(OC)c(OC)c(OC)c2)c1</smiles>

Standard Procedure C with ester 35 (80 mg, 0.19 mmol, 1.0 equiv.) gave carboxylic acid 43 (58 mg, $75 \%)$ as a yellow solid after purification by silica-gel chromatography $\left(\mathrm{CH}_{2} \mathrm{Cl}_{2} / \mathrm{MeOH}, 98: 2 \rightarrow\right.$ 95:5). $R_{f}$ $=0.11\left(\mathrm{CH}_{2} \mathrm{Cl}_{2} / \mathrm{MeOH}, 95: 5\right)$; m.p. $163{ }^{\circ} \mathrm{C} ;{ }^{1} \mathrm{H}-\mathrm{NMR}$ (300 MHz, DMSO-d $\left.)_{6}\right): \delta=10.03$ (br s, $\left.1 \mathrm{H}\right), 8.05$ 
$(\mathrm{d}, J=9.0 \mathrm{~Hz}, 1 \mathrm{H}), 7.40(\mathrm{~s}, 2 \mathrm{H}), 7.20(\mathrm{~d}, J=3.0 \mathrm{~Hz}, 1 \mathrm{H}), 7.10(\mathrm{dd}, J=3.0,9.0 \mathrm{~Hz}, 1 \mathrm{H}), 3.88(\mathrm{~s}, 6 \mathrm{H})$, $3.77(\mathrm{~s}, 3 \mathrm{H}), 3.74(\mathrm{~s}, 3 \mathrm{H}), 2.66(\mathrm{t}, J=6.7 \mathrm{~Hz}, 2 \mathrm{H}), 2.51(\mathrm{t}, J=6.5 \mathrm{~Hz}, 2 \mathrm{H}) ;{ }^{13} \mathrm{C}-\mathrm{NMR}(101 \mathrm{MHz}$, DMSO- $\left.d_{6}\right): \delta=175.0,170.9,156.2,153.7,148.5,142.3,140.9,131.8,124.9,119.7,101.6,98.9,60.7$, 56.5, 55.9, 29.8, 28.3; IR: $\tilde{v}=3310,2936,2832$, 1721, 1655, 1531, 1404, 1304, 1215, 1007, 833; UVVIS $\left(\mathrm{CH}_{3} \mathrm{CN}+0.5 \%\right.$ piperidine $): \lambda_{\max }(\varepsilon)=393 \mathrm{~nm}\left(255001 \cdot \mathrm{mol}^{-1} \cdot \mathrm{cm}^{-1}\right) ; \mathrm{HRMS}(\mathrm{ESI}): \mathrm{m} / z$ calcd for $\mathrm{C}_{20} \mathrm{H}_{24} \mathrm{~N}_{3} \mathrm{O}_{7}$ : $418.1609[\mathrm{M}+\mathrm{H}]^{+}$; found: 418.1620.

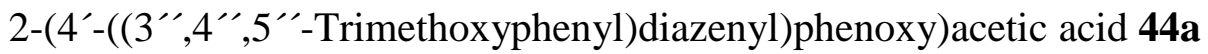<smiles>COc1cc(/N=N/c2ccc(OCC(=O)O)cc2)cc(OC)c1OC</smiles>

Standard Procedure $\mathbf{C}$ with ester 29a (151 mg, $0.41 \mathrm{mmol}, 1.0$ equiv.) gave carboxylic acid 44a (123 mg, $85 \%$ ) as an orange solid after purification by silica-gel chromatography (EtOAc/petroleum ether, 3:7 + $0.5 \%$ formic acid). $R_{f}=0.22$ (EtOAc/petroleum ether, $3: 7+0.5 \%$ formic acid); m.p. $110{ }^{\circ} \mathrm{C} ;{ }^{1} \mathrm{H}-\mathrm{NMR}$ $\left(300 \mathrm{MHz}, \mathrm{CDCl}_{3}\right): \delta=7.93(\mathrm{~d}, J=9.0 \mathrm{~Hz}, 2 \mathrm{H}), 7.23(\mathrm{~s}, 2 \mathrm{H}), 7.06(\mathrm{~d}, J=9.0 \mathrm{~Hz}, 2 \mathrm{H}), 4.79(\mathrm{~s}, 2 \mathrm{H})$, $3.98(\mathrm{~s}, 6 \mathrm{H}), 3.94$ (s, $3 \mathrm{H}) ;{ }^{13} \mathrm{C}-\mathrm{NMR}\left(101 \mathrm{MHz}, \mathrm{CDCl}_{3}\right): \delta=172.6,159.4,153.5,148.5,147.7,140.4$, 124.6, 114.9, 100.2, 64.8, 61.0, 56.2; IR: $\tilde{v}=2943,2839,2573,1732,1585,1416,1219$, 1126, 991, 845; UV-VIS $\left(\mathrm{CH}_{3} \mathrm{CN}+0.5 \%\right.$ piperidine $): \lambda_{\max }(\varepsilon)=368 \mathrm{~nm}\left(221001 \cdot \mathrm{mol}^{-1} \cdot \mathrm{cm}^{-1}\right) ; \mathrm{HRMS}(\mathrm{ESI}): \mathrm{m} / z$ calcd for $\mathrm{C}_{17} \mathrm{H}_{19} \mathrm{~N}_{2} \mathrm{O}_{6}: 347.1238[\mathrm{M}+\mathrm{H}]^{+}$; found: 347.1241 .

2-(3'-Hydroxy-4'-((3", $4^{\prime \prime}, 5^{\prime \prime}-$ trimethoxyphenyl)diazenyl)phenoxy)acetic acid 44c<smiles>COc1cc(/N=N/c2ccc(OCC(=O)O)cc2O)cc(OC)c1OC</smiles> 
Standard Procedure $\mathbf{C}$ with ester 29c (100 mg, $0.26 \mathrm{mmol}, 1.0$ equiv.) gave carboxylic acid 44c $(80 \mathrm{mg}$, $86 \%$ ) as a red solid after purification by silica-gel chromatography (EtOAc/petroleum ether, 3:7 $\rightarrow$ EtOAc $+0.5 \%$ formic acid). $R_{f}=0.17$ (EtOAc/petroleum ether, 3:7 + 0.5\% formic acid); m.p. $204{ }^{\circ} \mathrm{C}$; ${ }^{1} \mathrm{H}-\mathrm{NMR}\left(300 \mathrm{MHz}, \mathrm{DMSO}-d_{6}\right): \delta=11.88$ (br s, $\left.1 \mathrm{H}\right), 7.73(\mathrm{~d}, J=9.0 \mathrm{~Hz}, 1 \mathrm{H}), 7.31(\mathrm{~s}, 2 \mathrm{H}), 6.60$ (dd, $J$ $=2.7,9.0 \mathrm{~Hz}, 1 \mathrm{H}), 6.50(\mathrm{~d}, J=2.6 \mathrm{~Hz}, 1 \mathrm{H}), 4.71(\mathrm{~s}, 2 \mathrm{H}), 3.88(\mathrm{~s}, 6 \mathrm{H}), 3.74(\mathrm{~s}, 3 \mathrm{H}) ;{ }^{13} \mathrm{C}-\mathrm{NMR}$ (75 MHz, DMSO- $\left.d_{6}\right): \delta=169.7,162.2,156.0,153.4,146.9,139.6,132.9,126.9,108.1,102.2,99.8,65.2$, 60.2, 56.1; IR: $\tilde{v}=3545,2943,2839,2530,1751,1600,1226,1130,1060,825$; UV-VIS $\left(\mathrm{CH}_{3} \mathrm{CN}+\right.$ $0.5 \%$ piperidine): $\lambda_{\max }(\varepsilon)=390 \mathrm{~nm}\left(273001 \cdot \mathrm{mol}^{-1} \cdot \mathrm{cm}^{-1}\right) ; \mathrm{HRMS}(\mathrm{ESI}): \mathrm{m} / z$ calcd for $\mathrm{C}_{17} \mathrm{H}_{19} \mathrm{~N}_{2} \mathrm{O}_{7}$ : $363.1187[\mathrm{M}+\mathrm{H}]^{+}$; found: 363.1184 .

2-(2'-Hydroxy-4'-((3"',4",5"'-trimethoxyphenyl)diazenyl)phenoxy)acetic acid 44g<smiles>COc1cc(/N=N/c2ccc(OCC(=O)O)c(O)c2)cc(OC)c1OC</smiles>

Standard Procedure C with ester 29b (60 mg, $0.12 \mathrm{mmol}, 1.0$ equiv.) gave carboxylic acid $\mathbf{4 4 g}$ (40 mg, $94 \%$ ) as an orange solid after purification by silica-gel chromatography (EtOAc/petroleum ether, 3:7 $\rightarrow$ EtOAc $+0.5 \%$ formic acid). $R_{f}=0.15$ (EtOAc/petroleum ether, $3: 7+0.5 \%$ formic acid); m.p. $132{ }^{\circ} \mathrm{C}$; ${ }^{1} \mathrm{H}-\mathrm{NMR}\left(400 \mathrm{MHz}, \mathrm{DMSO}-d_{6}\right): \delta=(\mathrm{br} \mathrm{s}, 1 \mathrm{H}), 7.41(\mathrm{dd}, J=2.3,8.5 \mathrm{~Hz}, 1 \mathrm{H}), 7.34(\mathrm{~d}, J=2.0 \mathrm{~Hz}, 1 \mathrm{H})$, $7.18(\mathrm{~s}, 2 \mathrm{H}), 7.00(\mathrm{~d}, J=8.5 \mathrm{~Hz}, 1 \mathrm{H}), 4.77(\mathrm{~s}, 2 \mathrm{H}), 3.88(\mathrm{~s}, 6 \mathrm{H}), 3.75(\mathrm{~s}, 3 \mathrm{H}) ;{ }^{13} \mathrm{C}-\mathrm{NMR}(101 \mathrm{MHz}$, DMSO- $\left.d_{6}\right): \delta=170.1,153.3,149.5,147.8,147.5,146.7,139.8,118.3,113.4,106.2,99.9,65.5,60.2$, 56.0; IR: $\tilde{v}=3507,3005,2360,1748,1597,1493,1207,1114,995,806 ;$ UV-VIS $\left(\mathrm{CH}_{3} \mathrm{CN}+0.5 \%\right.$ piperidine): $\lambda_{\max }(\varepsilon)=367 \mathrm{~nm}\left(211001 \cdot \mathrm{mol}^{-1} \cdot \mathrm{cm}^{-1}\right)$; HRMS (ESI): $\mathrm{m} / z$ calcd for $\mathrm{C}_{17} \mathrm{H}_{19} \mathrm{~N}_{2} \mathrm{O}_{7}: 363.1187$ $[\mathrm{M}+\mathrm{H}]^{+}$; found: 363.1194 .

2-((5'-((3"',4",5"'-Trimethoxyphenyl)diazenyl)quinolin-8'-yl)oxy)acetic acid 45a 


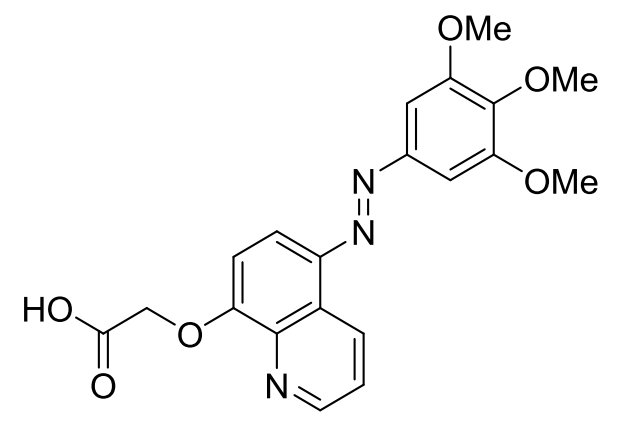

Standard Procedure C with ester 30a (190 mg, $0.46 \mathrm{mmol}, 1.0$ equiv.) gave carboxylic acid 45a (176 mg, $97 \%$ ) as a yellow solid after evaporation of the solvent. $R_{f}=0.20$ (EtOAc/petroleum ether, 3:7 $+0.5 \%$ formic acid); m.p. $310{ }^{\circ} \mathrm{C}$ decomp.; ${ }^{1} \mathrm{H}-\mathrm{NMR}\left(300 \mathrm{MHz}, \mathrm{DMSO}-d_{6}\right): \delta=9.33(\mathrm{dd}, J=1.7,8.6 \mathrm{~Hz}, 1 \mathrm{H})$, $9.01(\mathrm{dd}, J=1.7,4.1 \mathrm{~Hz}, 1 \mathrm{H}), 7.93(\mathrm{~d}, J=8.7 \mathrm{~Hz}, 1 \mathrm{H}), 7.77(\mathrm{dd}, J=4.1,8.6 \mathrm{~Hz}, 1 \mathrm{H}), 7.37(\mathrm{~s}, 2 \mathrm{H})$, $7.29(\mathrm{~d}, J=8.8 \mathrm{~Hz}, 1 \mathrm{H}), 5.05(\mathrm{~s}, 2 \mathrm{H}), 3.94(\mathrm{~s}, 6 \mathrm{H}), 3.78(\mathrm{~s}, 3 \mathrm{H}) ;{ }^{13} \mathrm{C}-\mathrm{NMR}\left(126 \mathrm{MHz}\right.$, DMSO- $\left.d_{6}\right): \delta=$ 171.5, 157.1, 153.8, 150.0, 148.9, 140.4, 139.8, 138.9, 132.8, 127.7, 123.7, 114.5, 109.6, 100.7, 68.5, 60.9, 56.5; IR: $\tilde{v}=2936,2835,1624,1408,1312,1107,991,783$; UV-VIS $\left(\mathrm{CH}_{3} \mathrm{CN}+0.5 \%\right.$ piperidine $)$ : $\lambda_{\max }(\varepsilon)=393 \mathrm{~nm}\left(255001 \cdot \mathrm{mol}^{-1} \cdot \mathrm{cm}^{-1}\right)$; HRMS (ESI): $\mathrm{m} / z$ calcd for $\mathrm{C}_{20} \mathrm{H}_{20} \mathrm{~N}_{3} \mathrm{O}_{6}: 398.1347[\mathrm{M}+\mathrm{H}]^{+}$; found: 398.1357 .

2-((4"-((3", $44^{\prime \prime}, 5^{\prime \prime}-$ Trimethoxyphenyl)diazenyl)naphth-1'-yl)oxy)acetic acid 45b<smiles>COc1cc(/N=N/c2ccc(OCC(=O)O)c3ccccc23)cc(OC)c1OC</smiles>

Standard Procedure $\mathbf{C}$ with ester $\mathbf{3 0 b}$ (166 mg, 0.39 mmol, 1.0 equiv.) gave carboxylic acid $45 \mathbf{b}$ (150 mg, $97 \%$ ) as an orange solid after evaporation of the solvent. $R_{f}=0.24$ (EtOAc/petroleum ether, 3:7 $+0.5 \%$ formic acid); m.p. $295{ }^{\circ} \mathrm{C}$; ${ }^{1} \mathrm{H}-\mathrm{NMR}\left(300 \mathrm{MHz}, \mathrm{DMSO}-d_{6}\right): \delta=8.91(\mathrm{~d}, J=8.3 \mathrm{~Hz}, 1 \mathrm{H}), 8.32(\mathrm{~d}, J=8.2$ $\mathrm{Hz}, 1 \mathrm{H}), 7.84(\mathrm{~d}, J=8.6 \mathrm{~Hz}, 1 \mathrm{H}), 7.71(\mathrm{ddd}, J=0.9,7.0,8.4 \mathrm{~Hz}, 1 \mathrm{H}), 7.61(\mathrm{ddd}, J=0.9,7.0,8.4 \mathrm{~Hz}, 1$ H), 7.33 (s, $2 \mathrm{H}), 6.87$ (d, J = 8.7 Hz, $1 \mathrm{H}), 4.42$ (s, $2 \mathrm{H}), 3.93$ (s, $6 \mathrm{H}), 3.76(\mathrm{~s}, 3 \mathrm{H})$ ) ${ }^{13} \mathrm{C}-\mathrm{NMR}(75 \mathrm{MHz}$, DMSO- $\left.d_{6}\right): \delta=169.1,158.8,153.8,149.1,140.1,140.1,132.4,128.0,126.0,125.6,123.1,122.9,113.8$, 106.0, 100.5, 69.2, 60.7, 56.5; IR: $\tilde{v}=3449$, 2936, 2835, 1609, 1404, 1219, 1130, 984, 814, 760; UV-VIS $\left(\mathrm{CH}_{3} \mathrm{CN}+0.5 \%\right.$ piperidine $): \lambda_{\max }(\varepsilon)=409 \mathrm{~nm}(233001 \cdot \mathrm{mol}-1 \cdot \mathrm{cm}-1) ; \mathrm{HRMS}(\mathrm{ESI}): \mathrm{m} / z$ calcd for $\mathrm{C}_{21} \mathrm{H}_{21} \mathrm{~N}_{2} \mathrm{O}_{6}: 397.1394[\mathrm{M}+\mathrm{H}]^{+}$; found: 397.1398. 
2-((4'-((3"',4",5"'-Trimethoxyphenyl)diazenyl)-5'-methoxynaphthalen-1'-yl)oxy)acetic acid 45c<smiles>COc1cc(/N=N/c2ccc(OCC(=O)O)c3cccc(OC)c23)cc(OC)c1OC</smiles>

Standard Procedure C with ester 30c (45 mg, $0.10 \mathrm{mmol}, 1.0$ equiv.) gave carboxylic acid 45c (38 mg, $90 \%)$ as an orange solid after evaporation of the solvent. $R_{f}=0.22$ (EtOAc/petroleum ether, 3:7 $+0.5 \%$ formic acid); m.p. $196{ }^{\circ} \mathrm{C}$; ${ }^{1} \mathrm{H}-\mathrm{NMR}\left(300 \mathrm{MHz}\right.$, DMSO- $\left.d_{6}\right): \delta=7.94(\mathrm{~d}, J=8.4 \mathrm{~Hz}, 1 \mathrm{H}), 7.55(\mathrm{t}, J=8.1$ $\mathrm{Hz}, 1 \mathrm{H}), 7.30(\mathrm{~s}, 2 \mathrm{H}), 7.29(\mathrm{~d}, J=7.7 \mathrm{~Hz}, 1 \mathrm{H}), 7.21(\mathrm{~d}, J=7.7 \mathrm{~Hz}, 1 \mathrm{H}), 6.99(\mathrm{~d}, J=8.5 \mathrm{~Hz}, 1 \mathrm{H}), 4.93$ $(\mathrm{s}, 2 \mathrm{H}), 3.92(\mathrm{~s}, 3 \mathrm{H}), 3.91(\mathrm{~s}, 6 \mathrm{H}), 3.77(\mathrm{~s}, 3 \mathrm{H}) ;{ }^{13} \mathrm{C}-\mathrm{NMR}\left(75 \mathrm{MHz}, \mathrm{DMSO}-d_{6}\right): \delta=169.8,156.4$, 154.7, 153.3, 148.5, 144.3, 139.5, 127.0, 126.6, 121.0, 114.4, 113.0, 109.1, 105.6, 100.1, 65.4, 60.2, 56.4, 55.9; IR: $\tilde{v}=2970,2835,1751,1593,1408,1215,1123,1084,748 ; \mathrm{UV}-\mathrm{VIS}\left(\mathrm{CH}_{3} \mathrm{CN}+0.5 \%\right.$ piperidine): $\lambda_{\max }(\varepsilon)=398 \mathrm{~nm}\left(22000 \mathrm{l} \cdot \mathrm{mol}^{-1} \cdot \mathrm{cm}^{-1}\right) ; \mathrm{HRMS}(\mathrm{ESI}): \mathrm{m} / z$ calcd for $\mathrm{C}_{22} \mathrm{H}_{23} \mathrm{~N}_{2} \mathrm{O}_{7}: 427.1500$ $[\mathrm{M}+\mathrm{H}]^{+}$; found: 427.1505 .

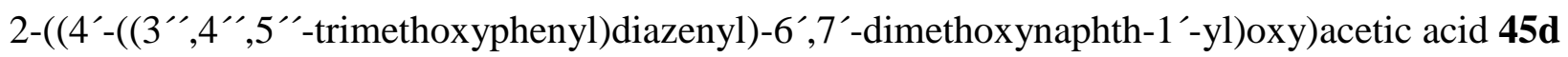<smiles>COc1cc2c(/N=N/c3cc(OC)c(OC)c(OC)c3)ccc(OCC(=O)O)c2cc1OC</smiles>

Standard Procedure C with ester 30d (160 mg, 0.33 mmol, 1.0 equiv.) gave carboxylic acid 45d (149 mg, $99 \%$ ) as an ocher solid after evaporation of the solvent. $R_{f}=0.23$ (EtOAc/petroleum ether, 3:7 $+0.5 \%$ formic acid); m.p. $235{ }^{\circ} \mathrm{C}$; ${ }^{1} \mathrm{H}-\mathrm{NMR}\left(250 \mathrm{MHz}, \mathrm{DMSO}-d_{6}\right): \delta=8.26(\mathrm{~s}, 1 \mathrm{H}), 7.73(\mathrm{~d}, J=8.6 \mathrm{~Hz}, 1 \mathrm{H})$, 7.58 (s, $1 \mathrm{H}), 7.35$ (s, $2 \mathrm{H}), 6.91(\mathrm{~d}, J=8.6 \mathrm{~Hz}, 1 \mathrm{H}), 4.99$ (s, $2 \mathrm{H}), 4.00$ (s, $3 \mathrm{H}), 3.95$ (s, $3 \mathrm{H}), 3.94$ (s, 6 $\mathrm{H}), 3.78(\mathrm{~s}, 3 \mathrm{H}) ;{ }^{13} \mathrm{C}-\mathrm{NMR}\left(63 \mathrm{MHz}, \mathrm{DMSO}-d_{6}\right): \delta=171.3,156.8,154.7,151.8,150.7,150.0,141.1$, 
129.8, 121.6, 112.7, 105.8, 103.0, 102.1, 101.4, 66.5, 61.6, 57.3, 56.7, 56.6; IR: $\tilde{v}=2932,2832,2361$, 1732, 1481, 1312, 1211, 1168, 1111, 1002, 810; UV-VIS $\left(\mathrm{CH}_{3} \mathrm{CN}+0.5 \%\right.$ piperidine $): \lambda_{\max }(\varepsilon)=419 \mathrm{~nm}$ (26000 $1 \cdot \mathrm{mol}^{-1} \cdot \mathrm{cm}^{-1}$ ); HRMS (ESI): $\mathrm{m} / z$ calcd for $\mathrm{C}_{23} \mathrm{H}_{25} \mathrm{~N}_{2} \mathrm{O}_{8}: 457.1605[\mathrm{M}+\mathrm{H}]^{+}$; found: 457.1611 . 
Supplementary Note 1. 

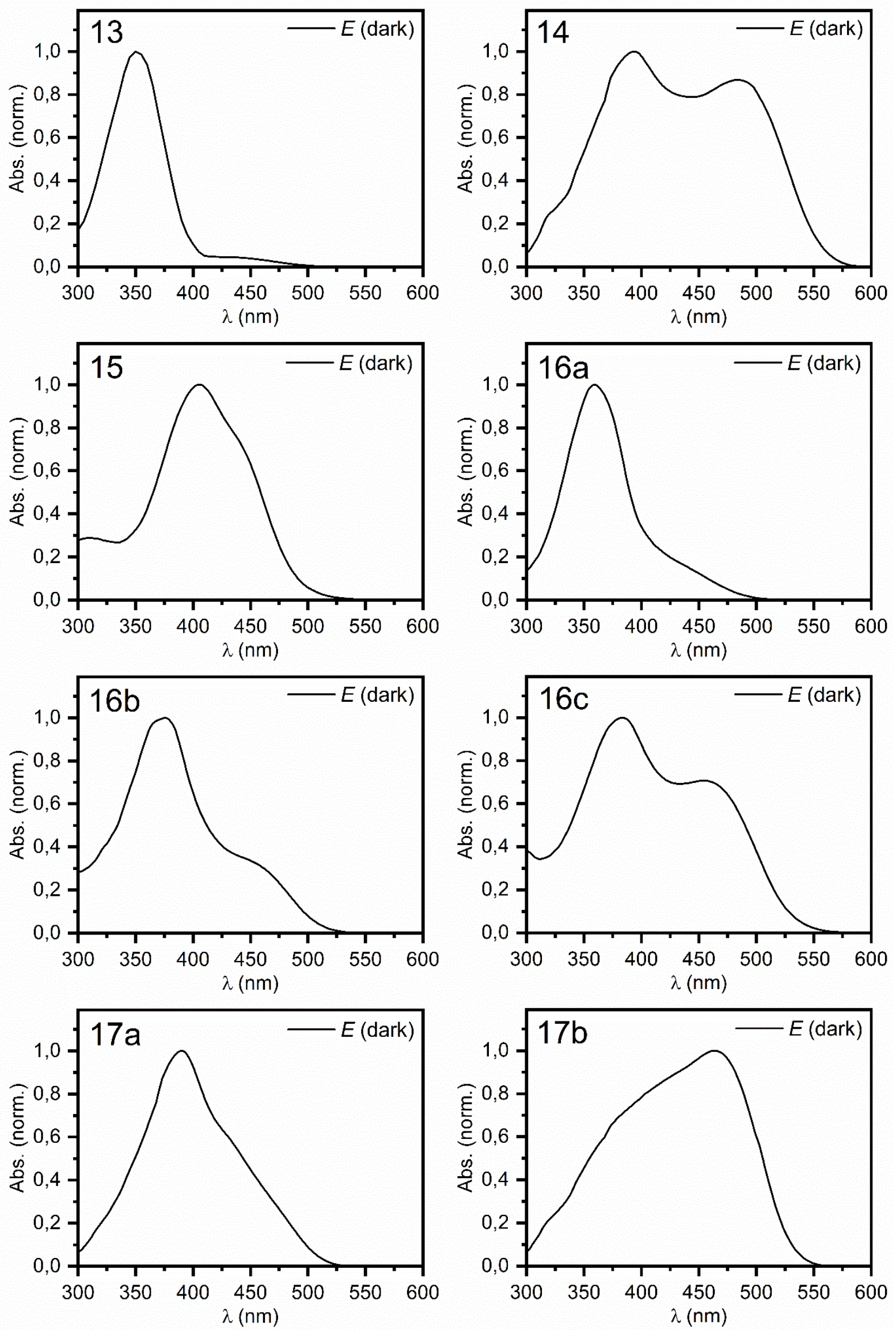

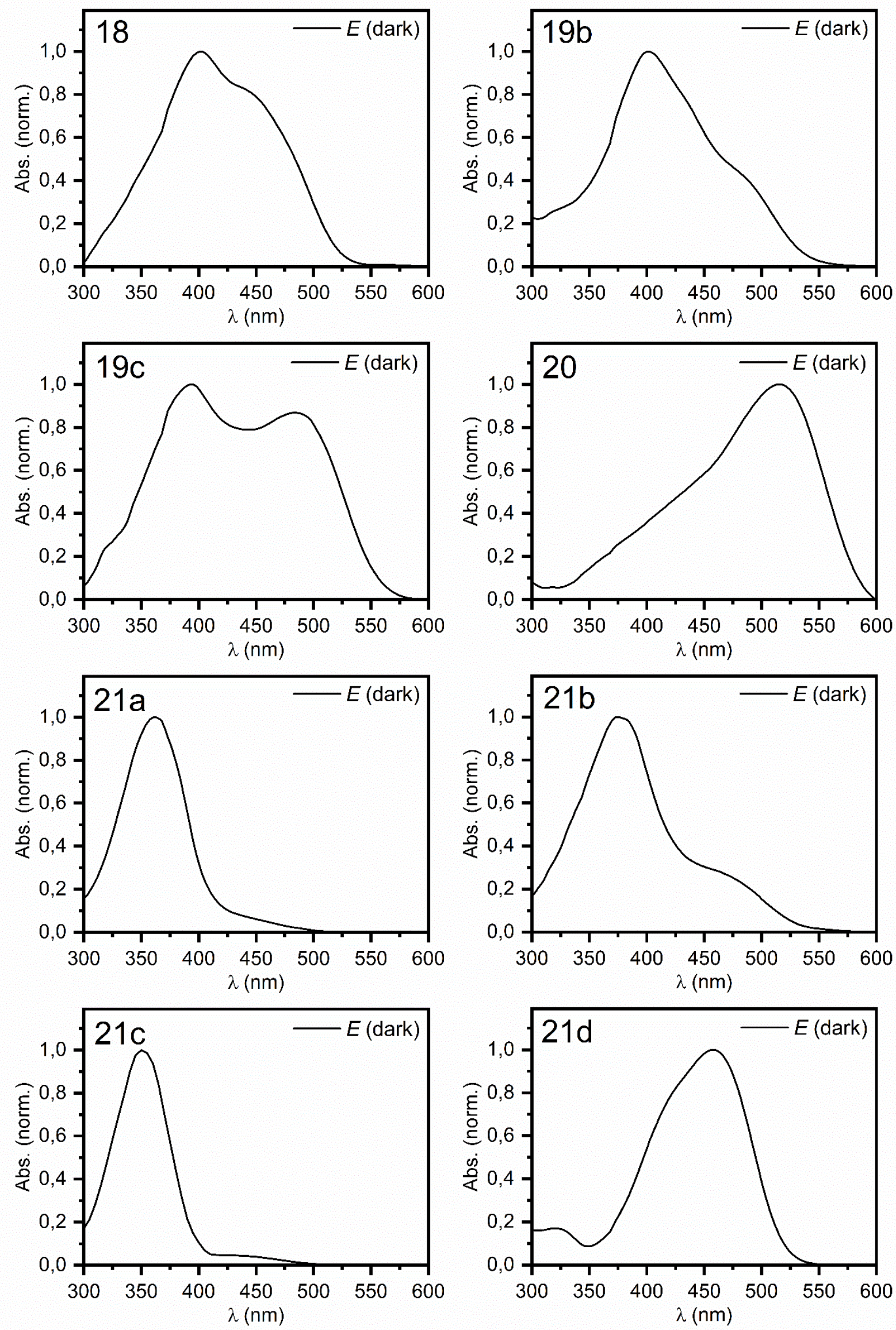

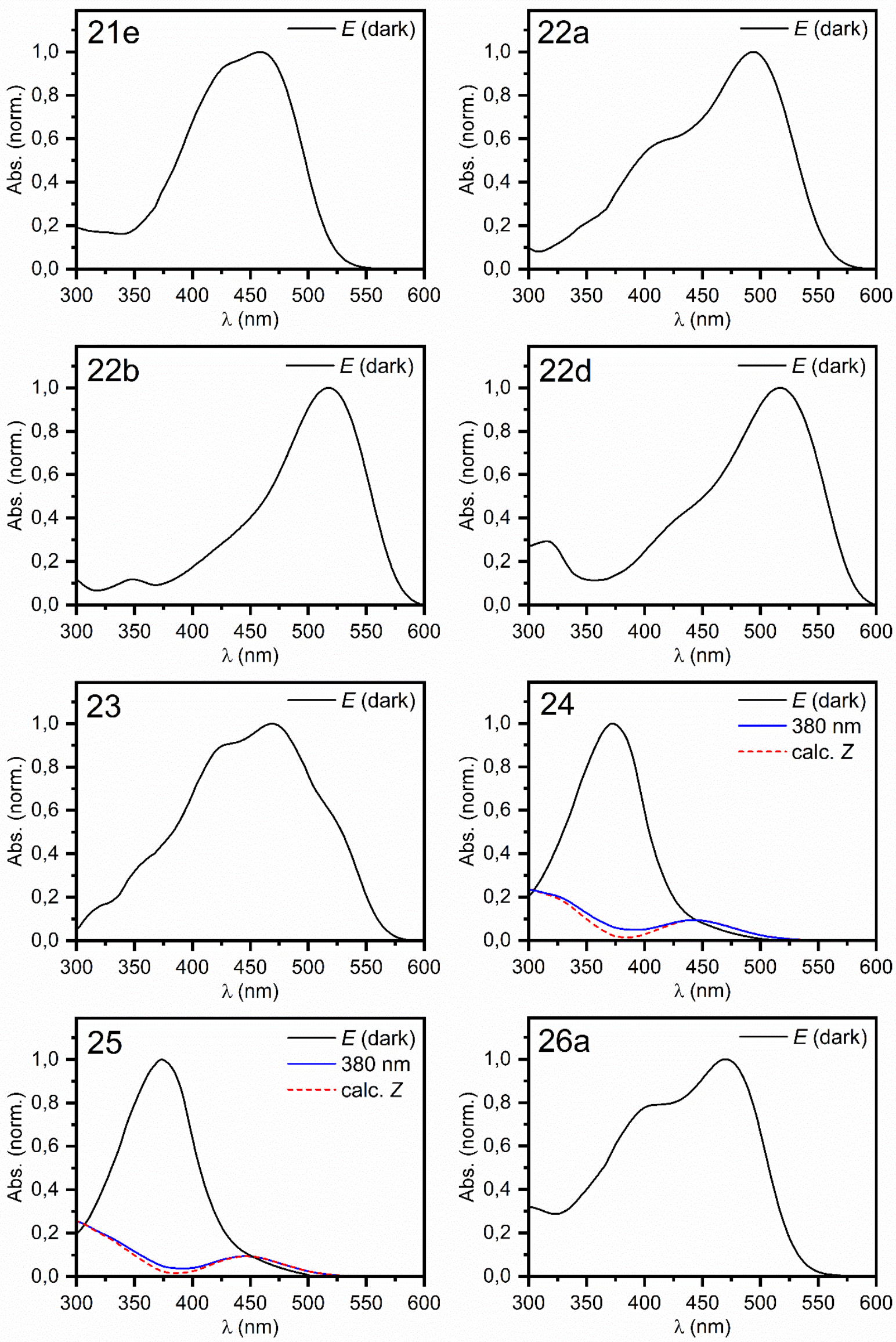

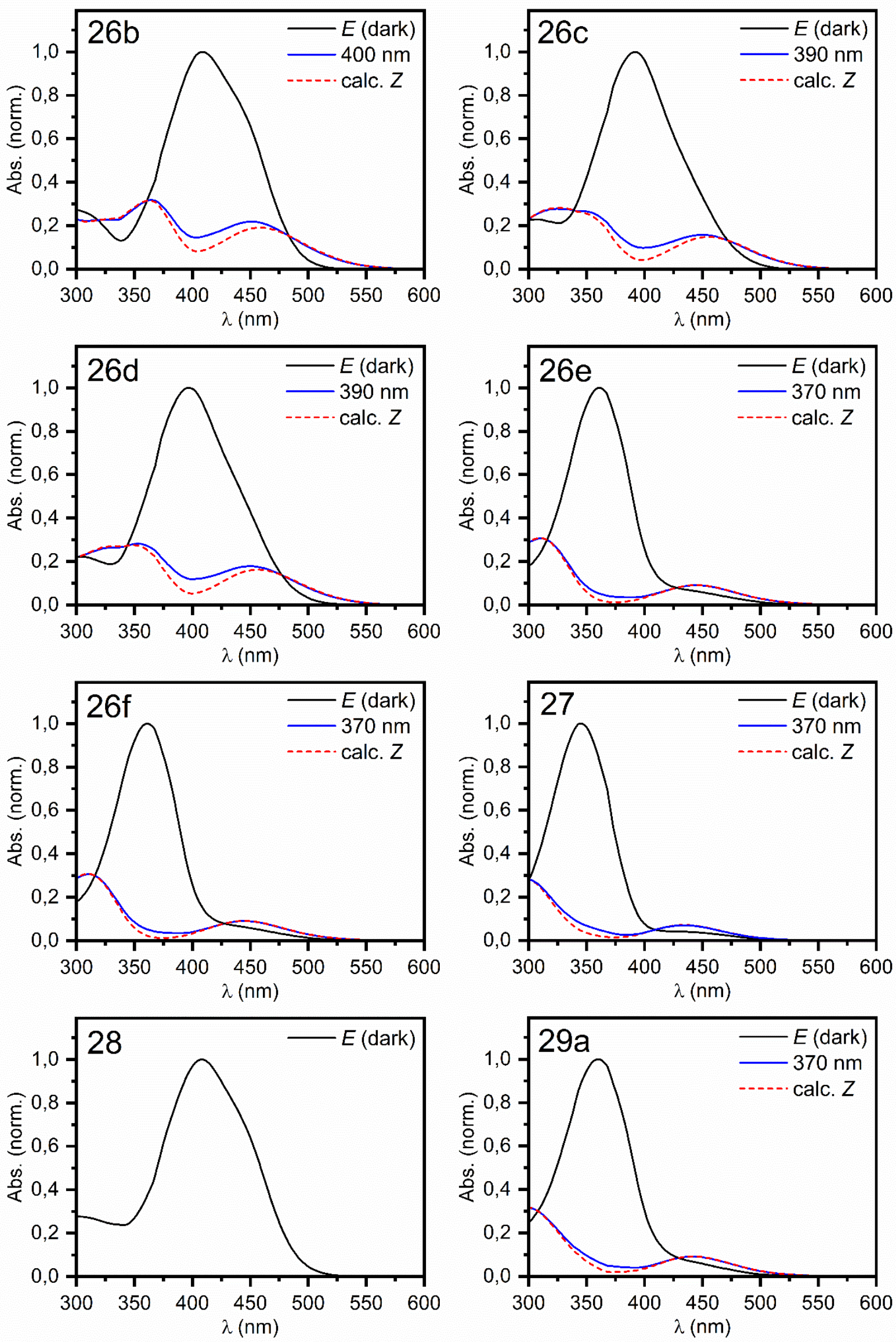

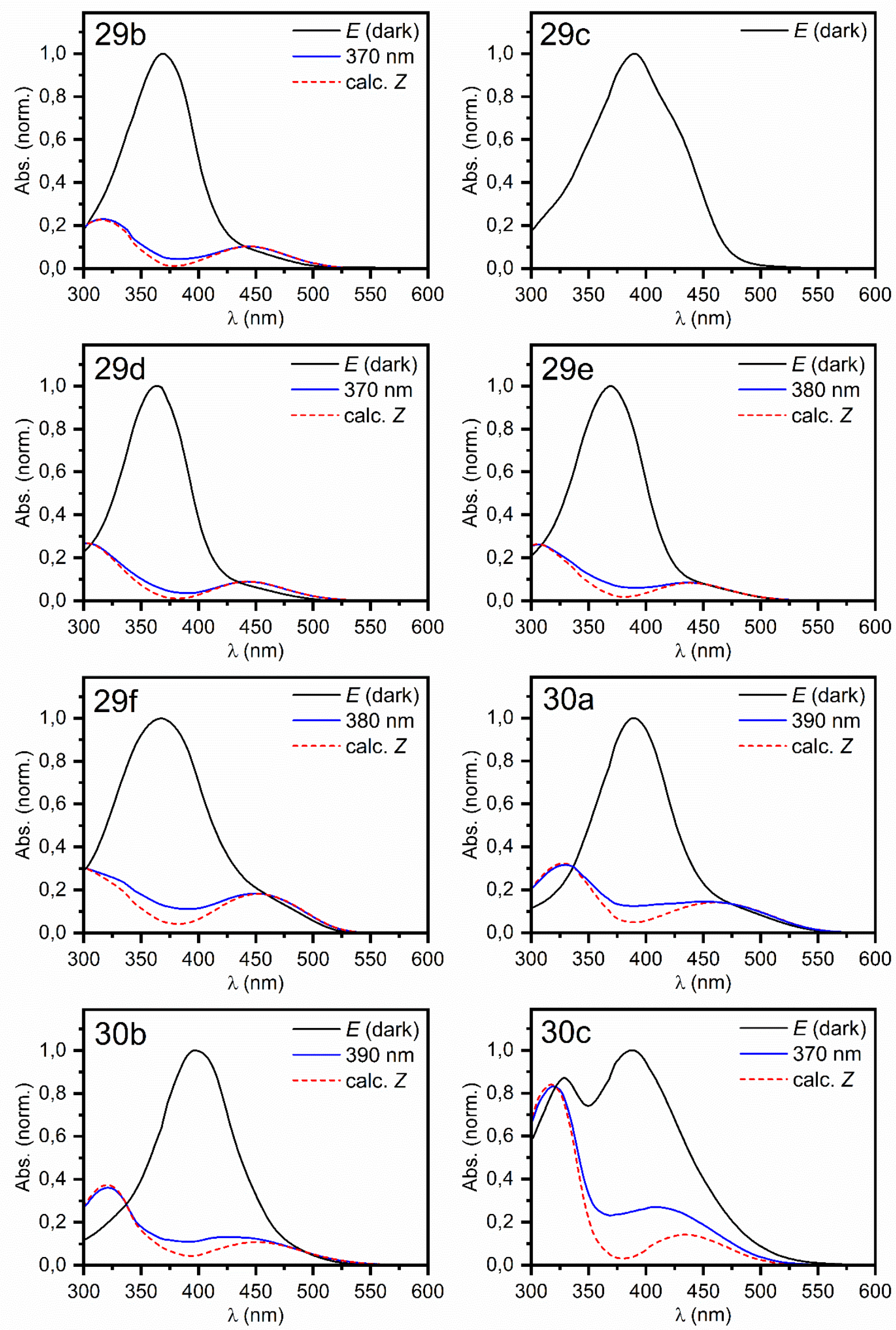

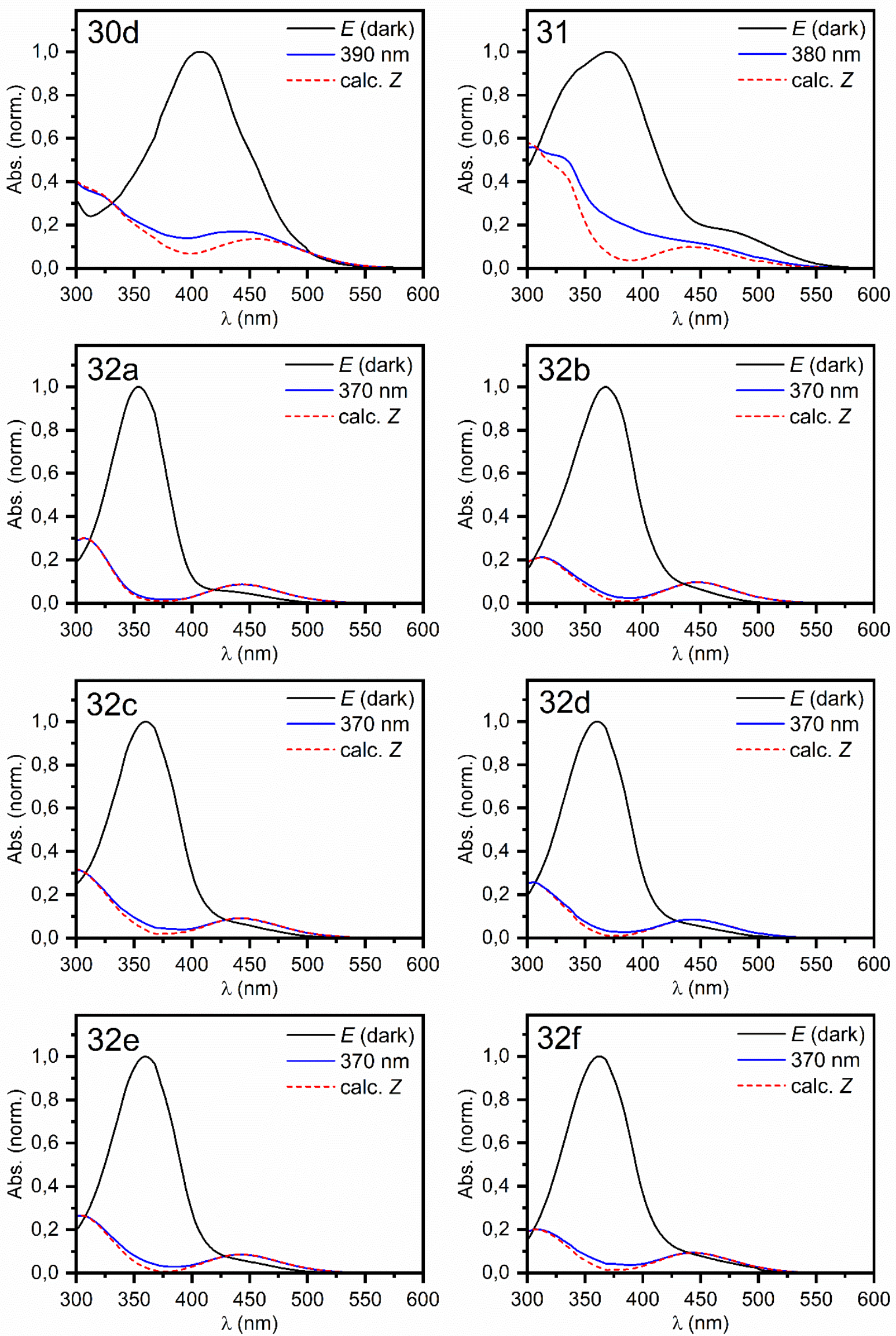

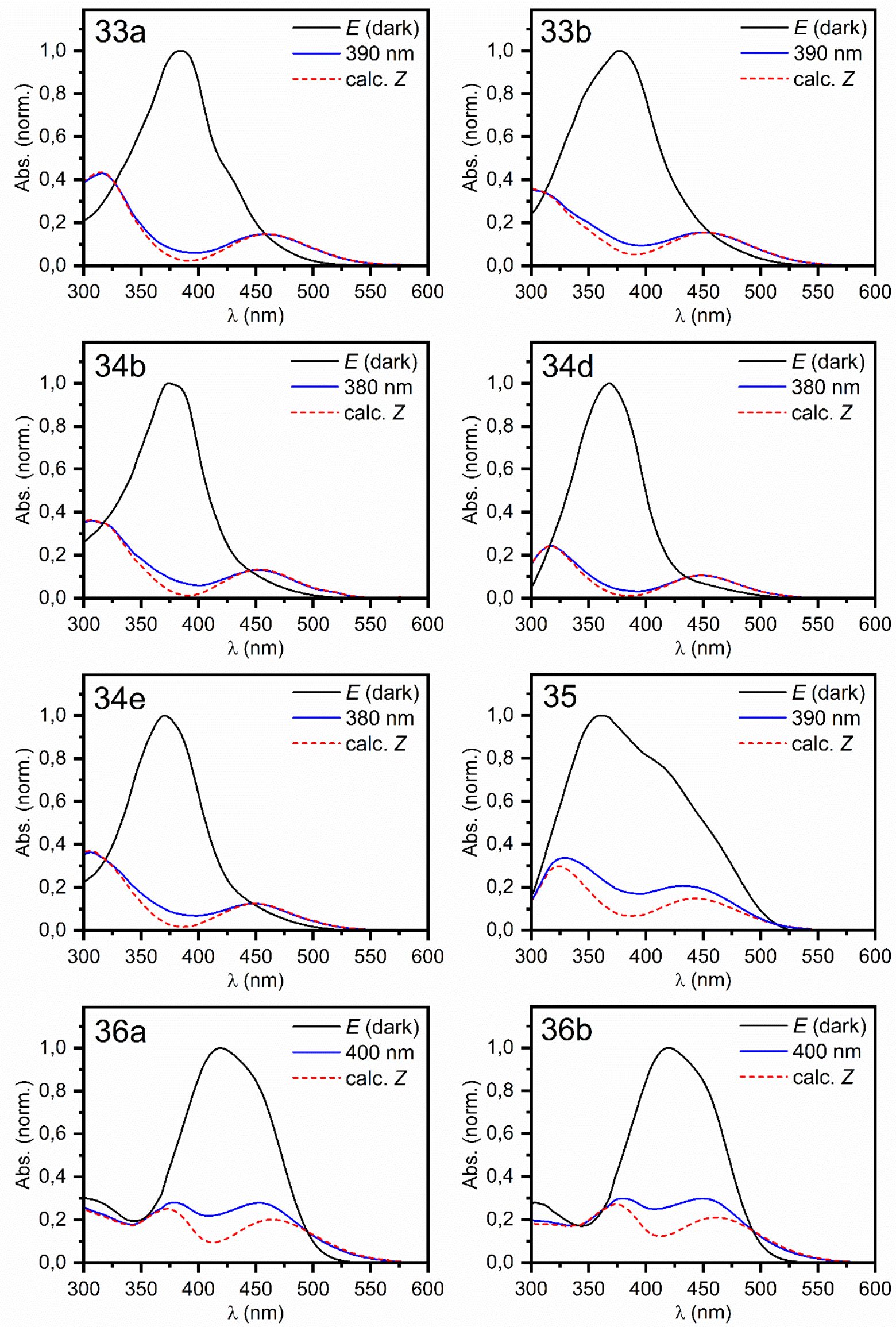

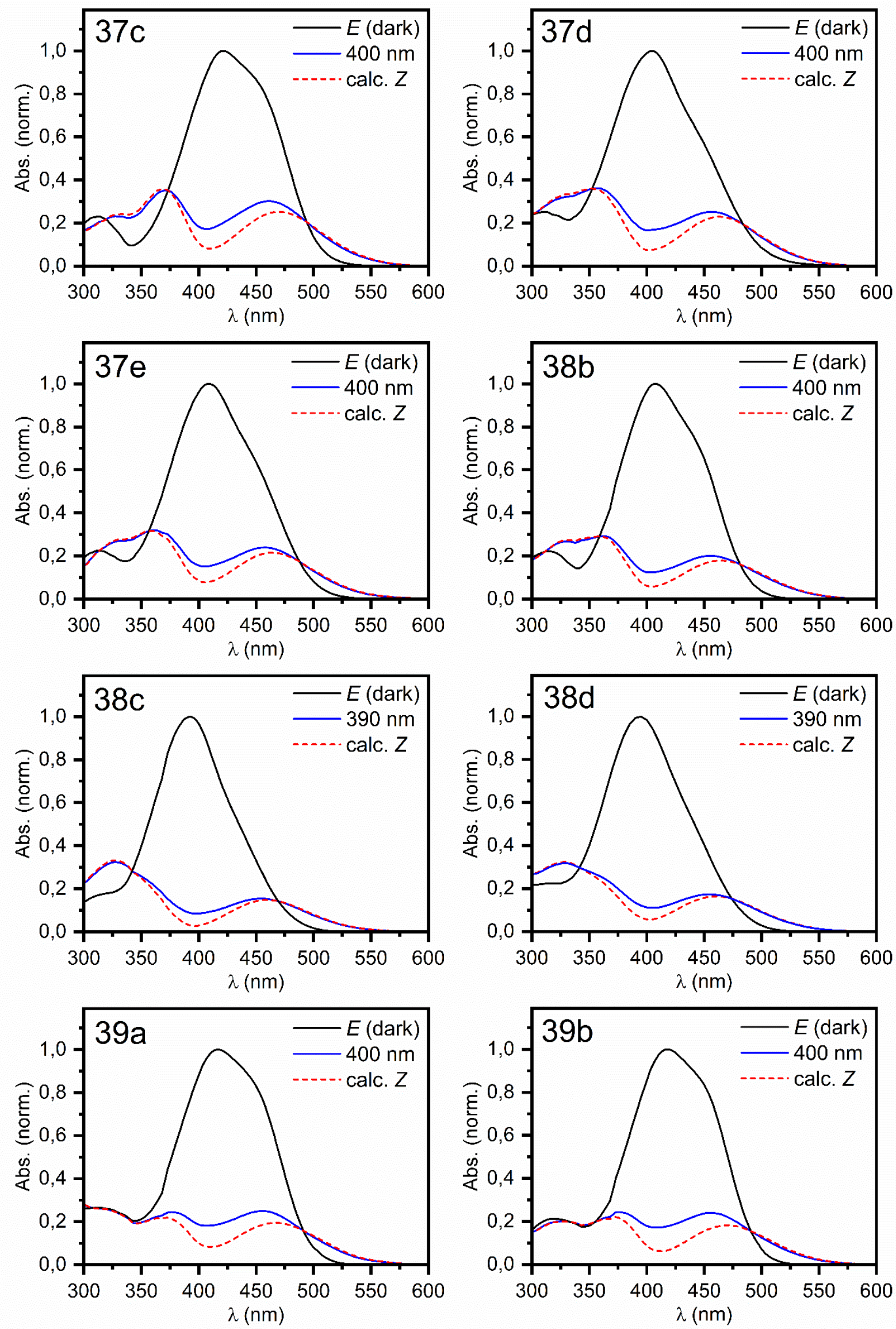

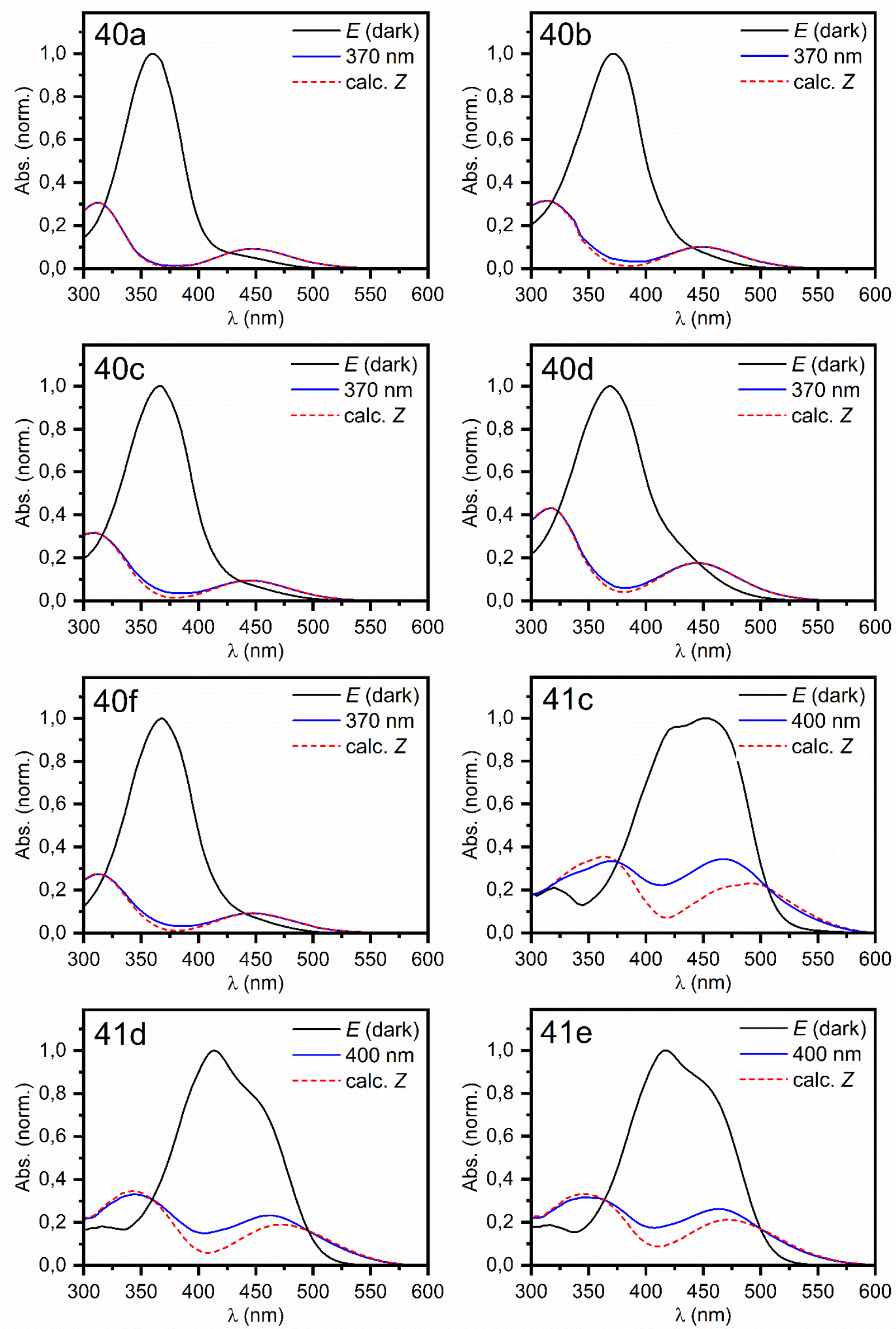

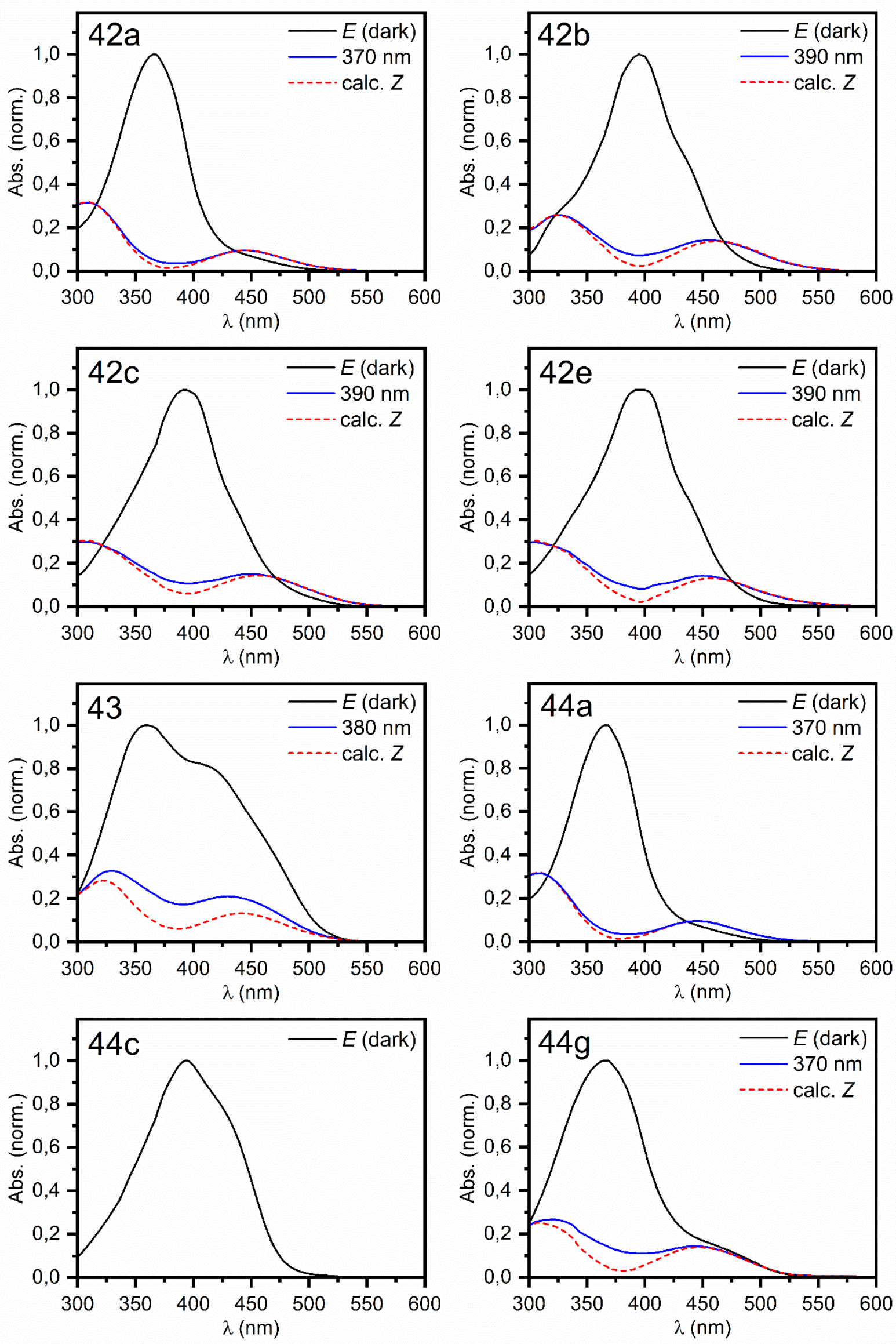

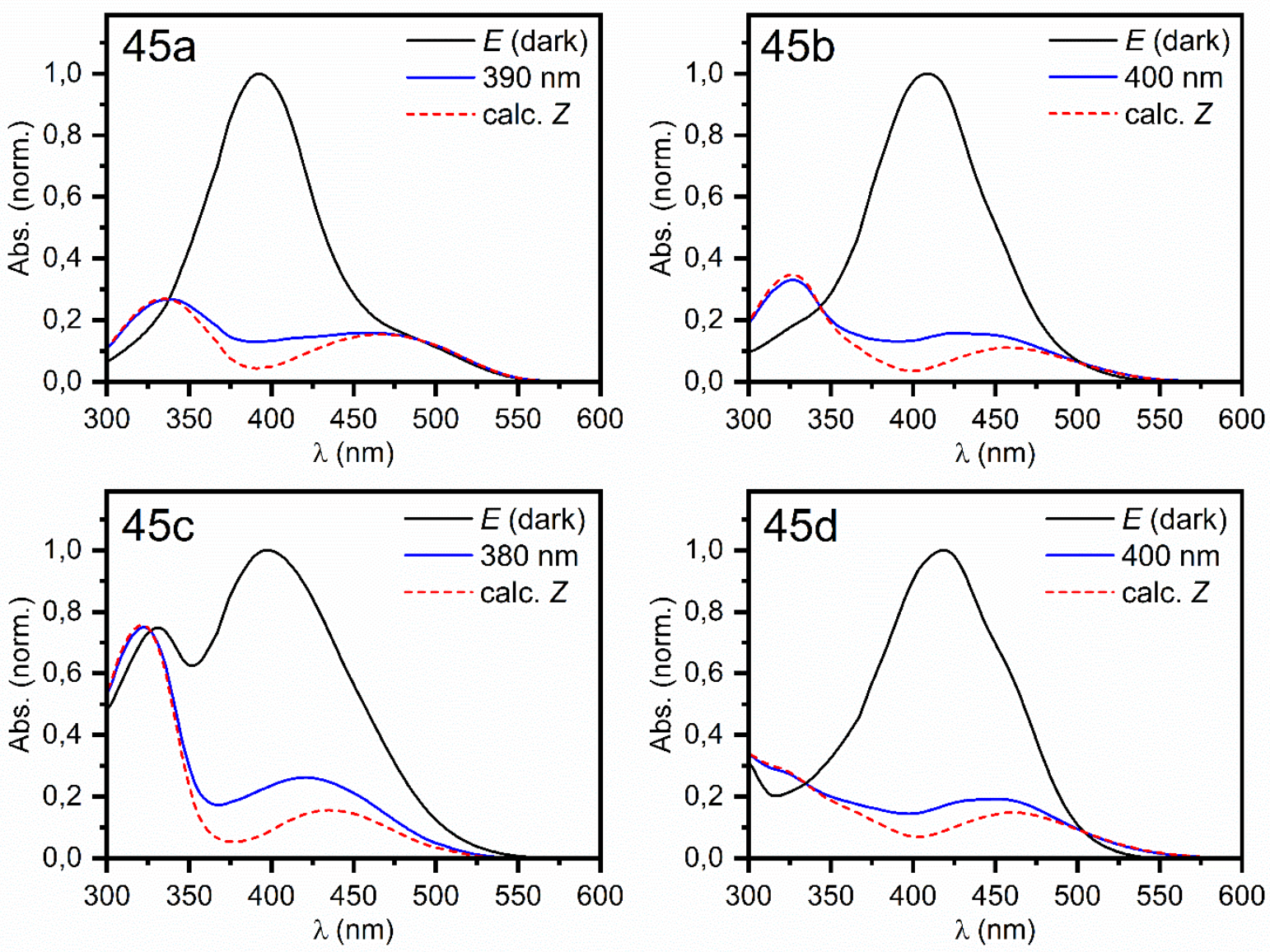


\section{Supplementary Note 2.}
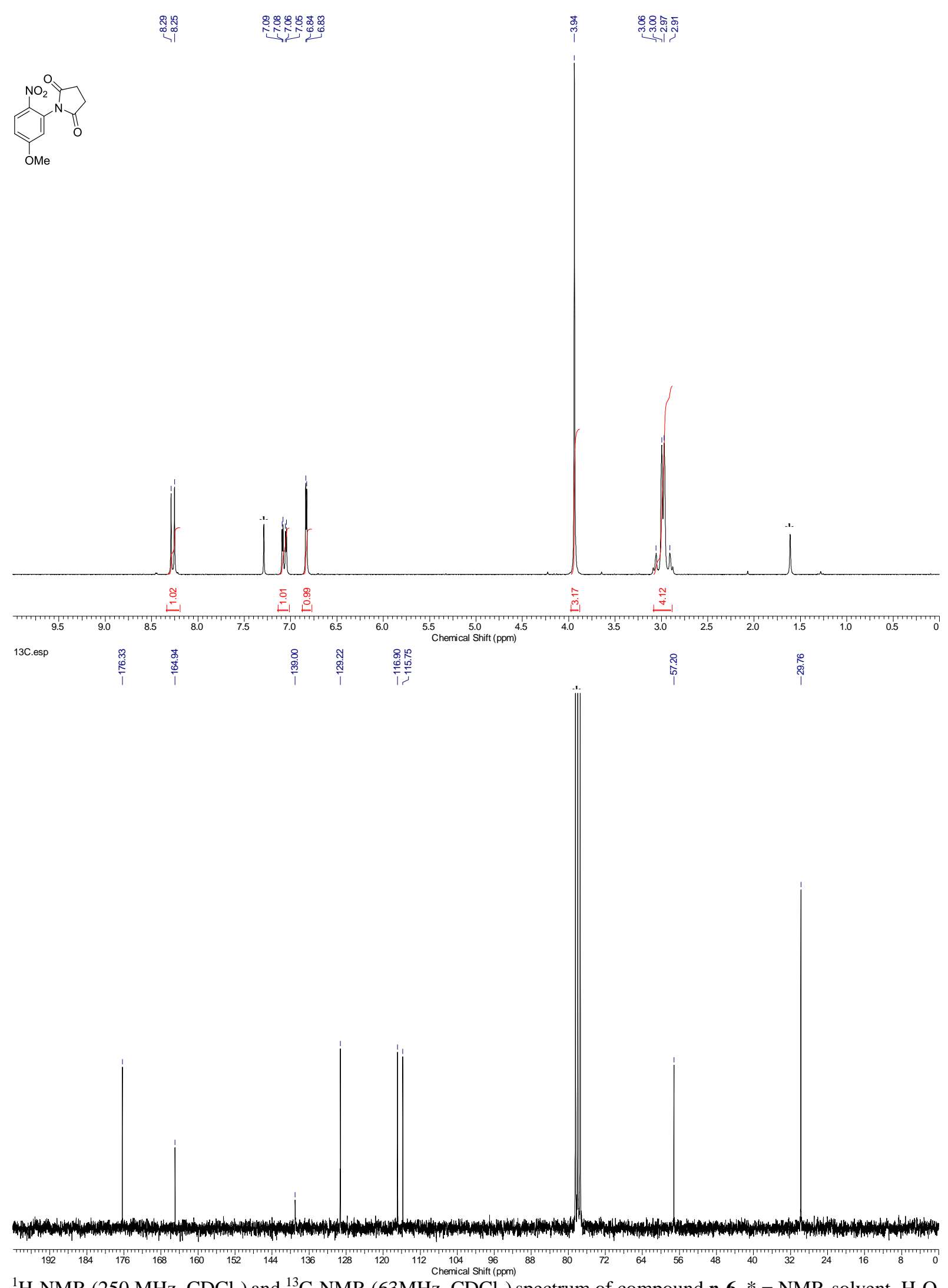

${ }^{1} \mathrm{H}-\mathrm{NMR}\left(250 \mathrm{MHz}, \mathrm{CDCl}_{3}\right)$ and ${ }^{13} \mathrm{C}-\mathrm{NMR}\left(63 \mathrm{MHz}, \mathrm{CDCl}_{3}\right)$ spectrum of compound $\boldsymbol{p}$-6. * = NMR-solvent, $\mathrm{H}_{2} \mathrm{O}$ 


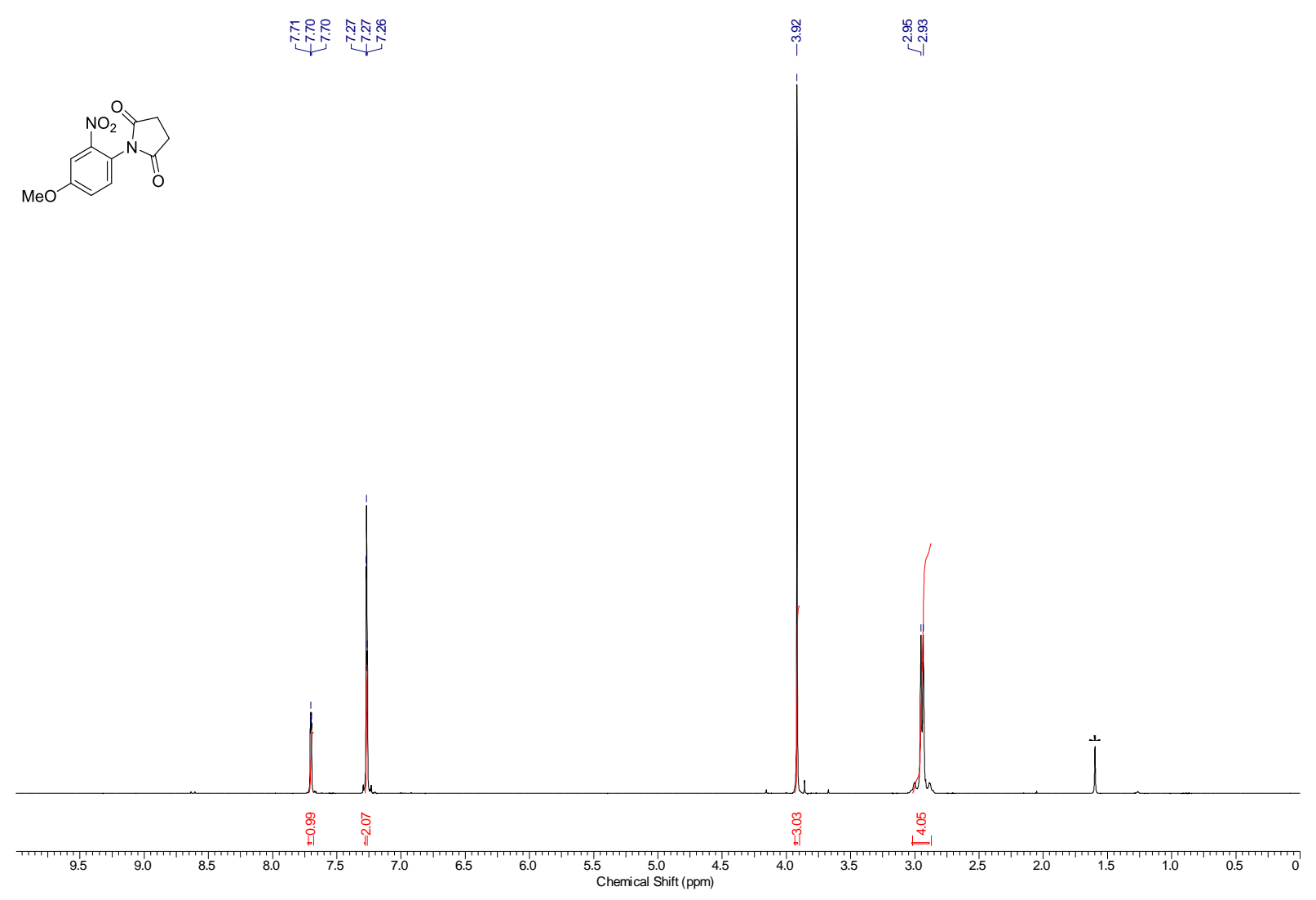

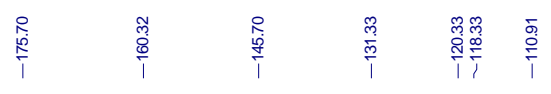
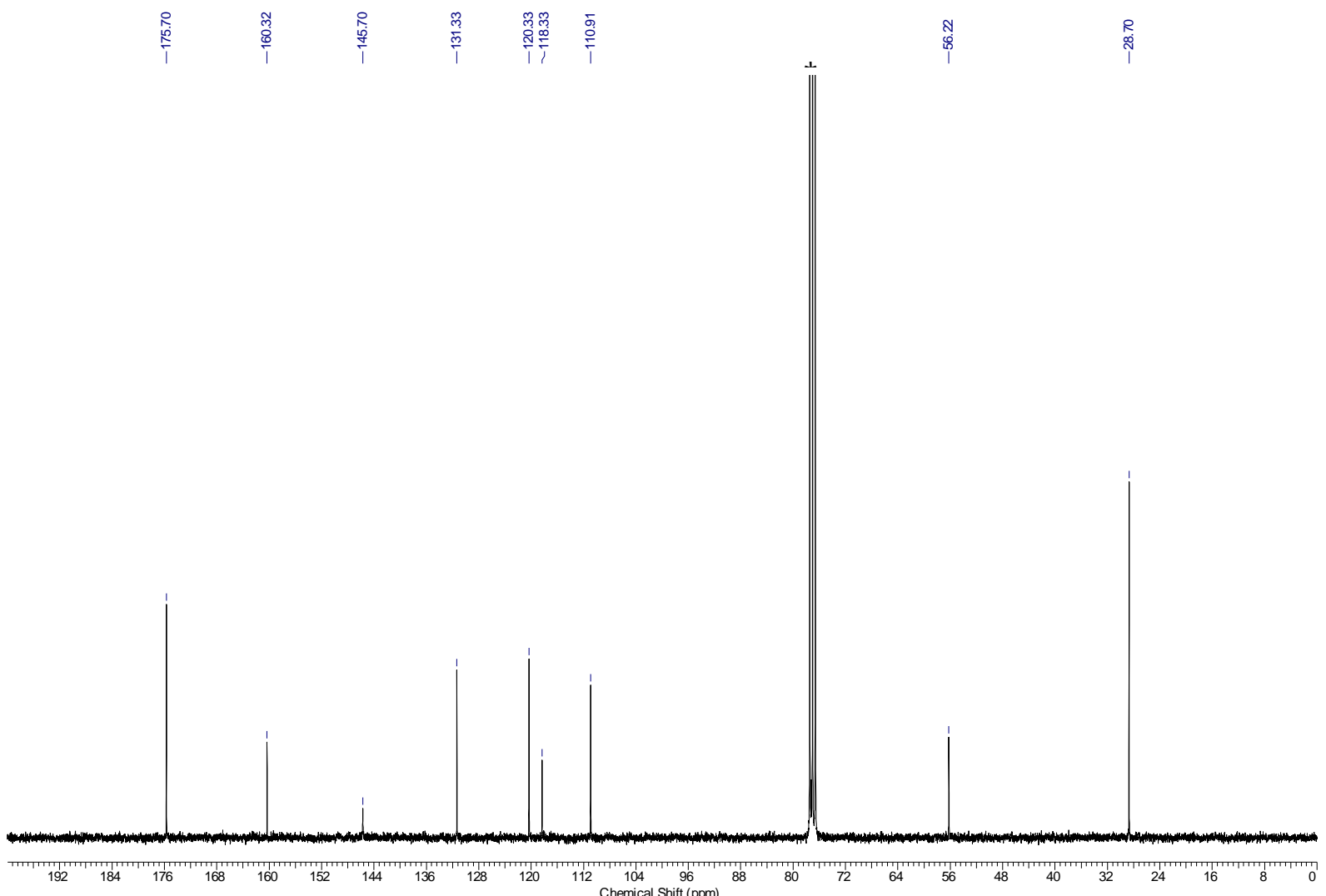

${ }^{1} \mathrm{H}-\mathrm{NMR}\left(300 \mathrm{MHz}, \mathrm{CDCl}_{3}\right.$ ) and ${ }^{13} \mathrm{C}-\mathrm{NMR}\left(75 \mathrm{MHz}, \mathrm{CDCl}_{3}\right.$ ) spectrum of compound $\boldsymbol{m}$-6. * = NMR-solvent, $\mathrm{H}_{2} \mathrm{O}$ 


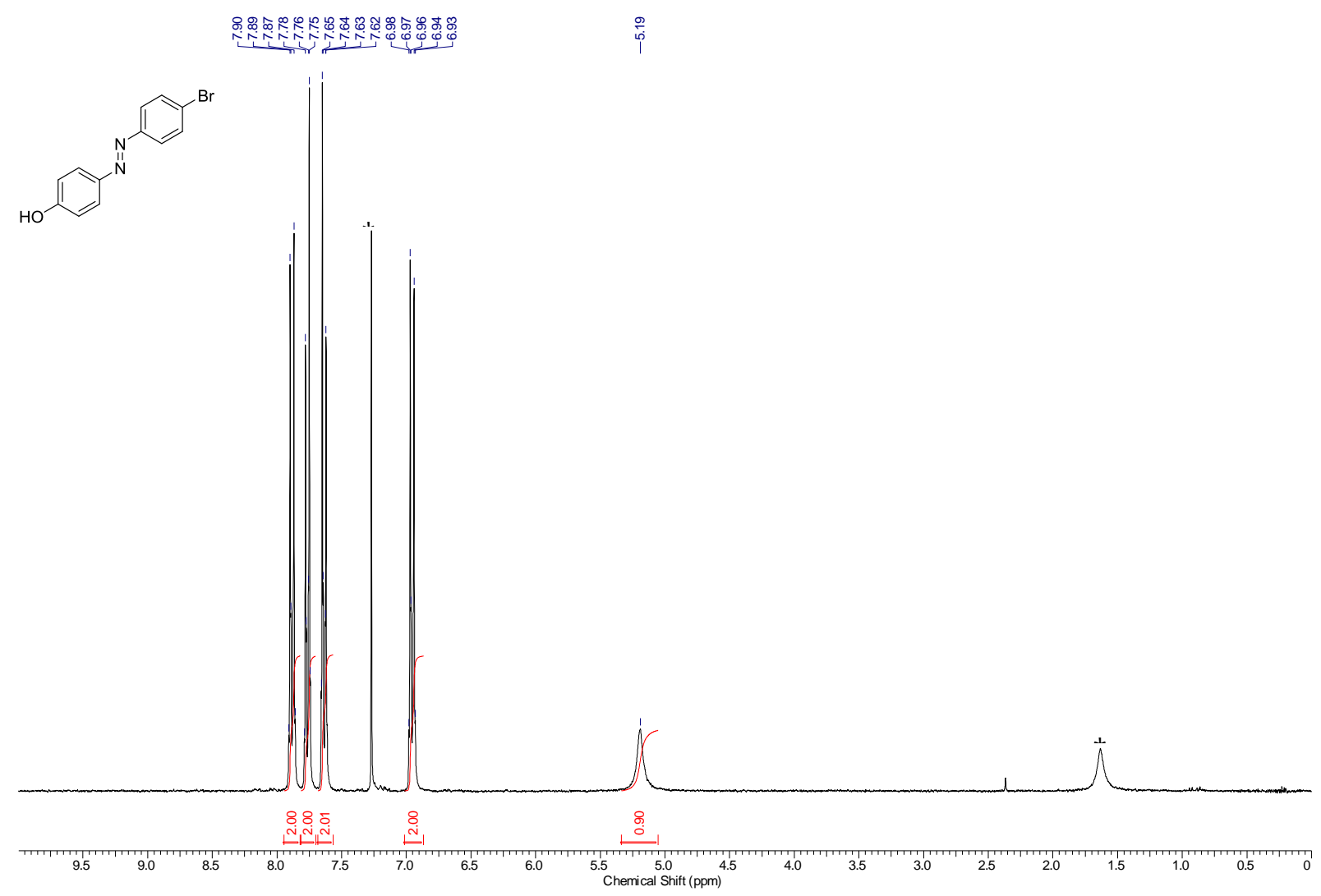

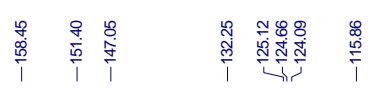

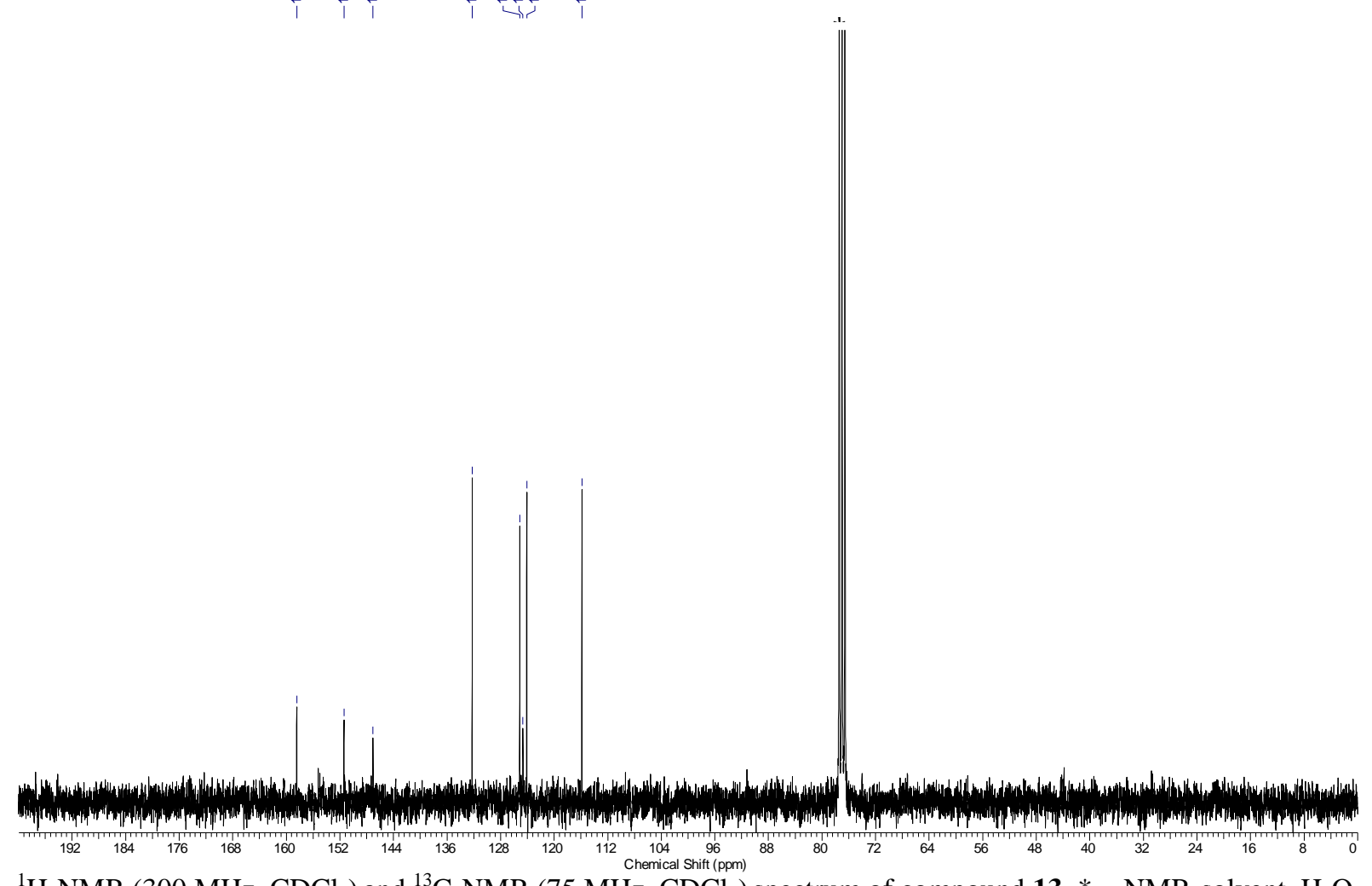

${ }^{1} \mathrm{H}-\mathrm{NMR}\left(300 \mathrm{MHz}, \mathrm{CDCl}_{3}\right.$ ) and ${ }^{13} \mathrm{C}-\mathrm{NMR}\left(75 \mathrm{MHz}, \mathrm{CDCl}_{3}\right)$ spectrum of compound 13. * = NMR-solvent, $\mathrm{H}_{2} \mathrm{O}$ 

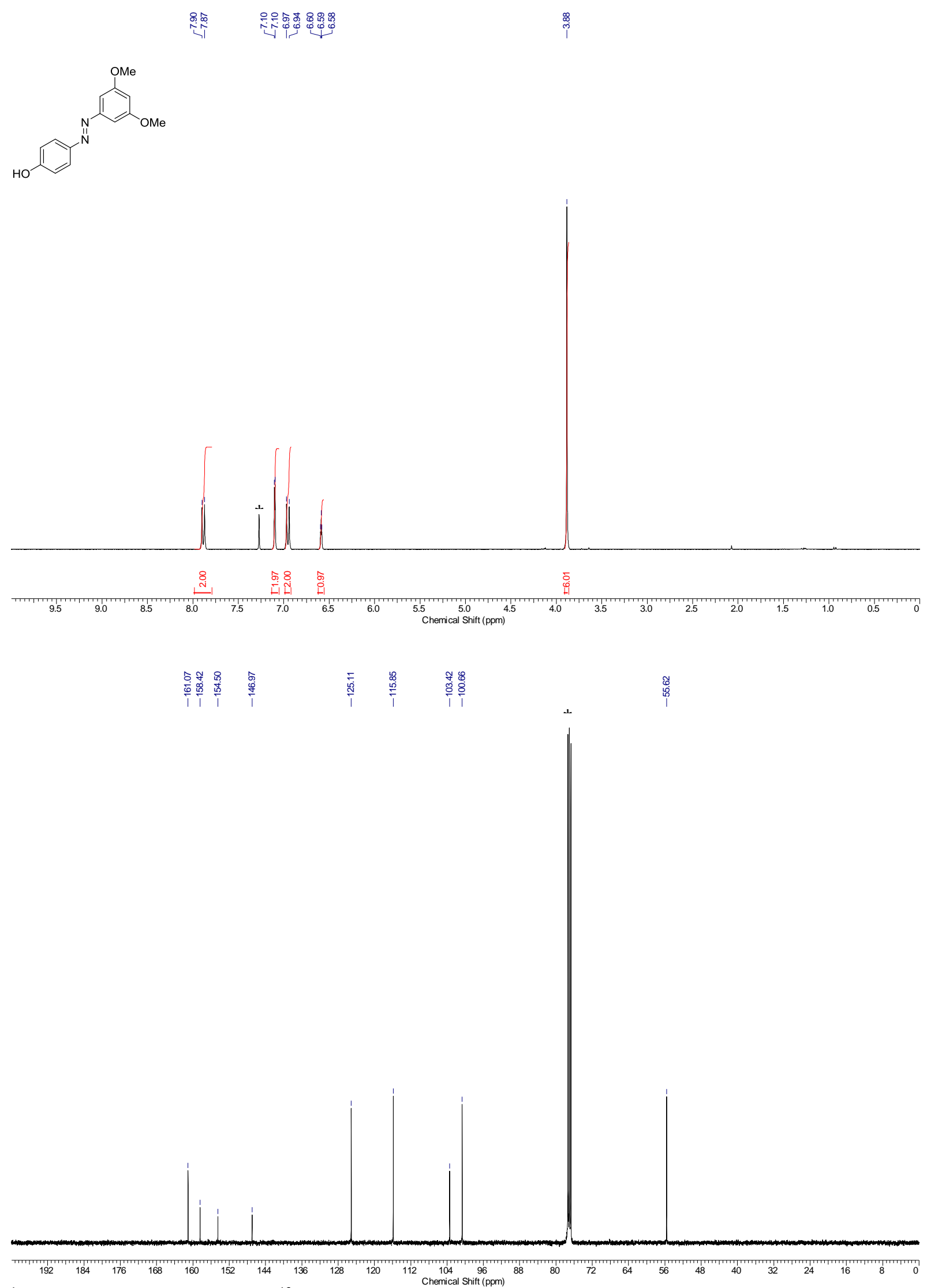

${ }^{1} \mathrm{H}-\mathrm{NMR}\left(300 \mathrm{MHz}, \mathrm{CDCl}_{3}\right)$ and ${ }^{13} \mathrm{C}-\mathrm{NMR}\left(101 \mathrm{MHz}^{\text {Chencial }} \mathrm{CDCl}_{3}\right.$ ) spectrum of compound 14. * = NMR-solvent 


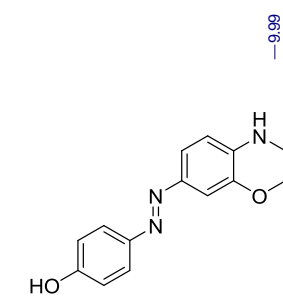

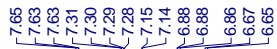

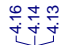

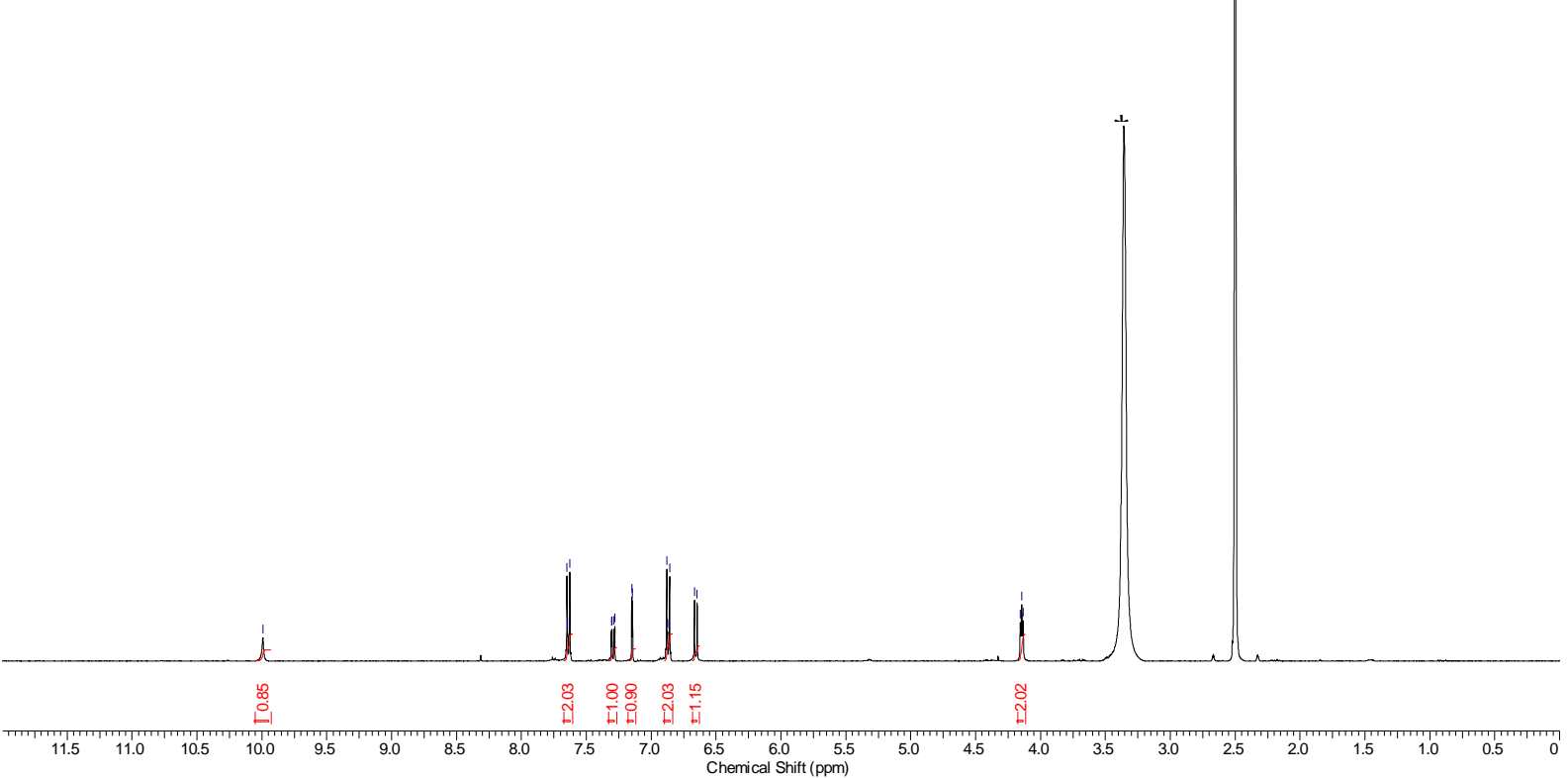

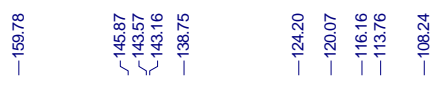
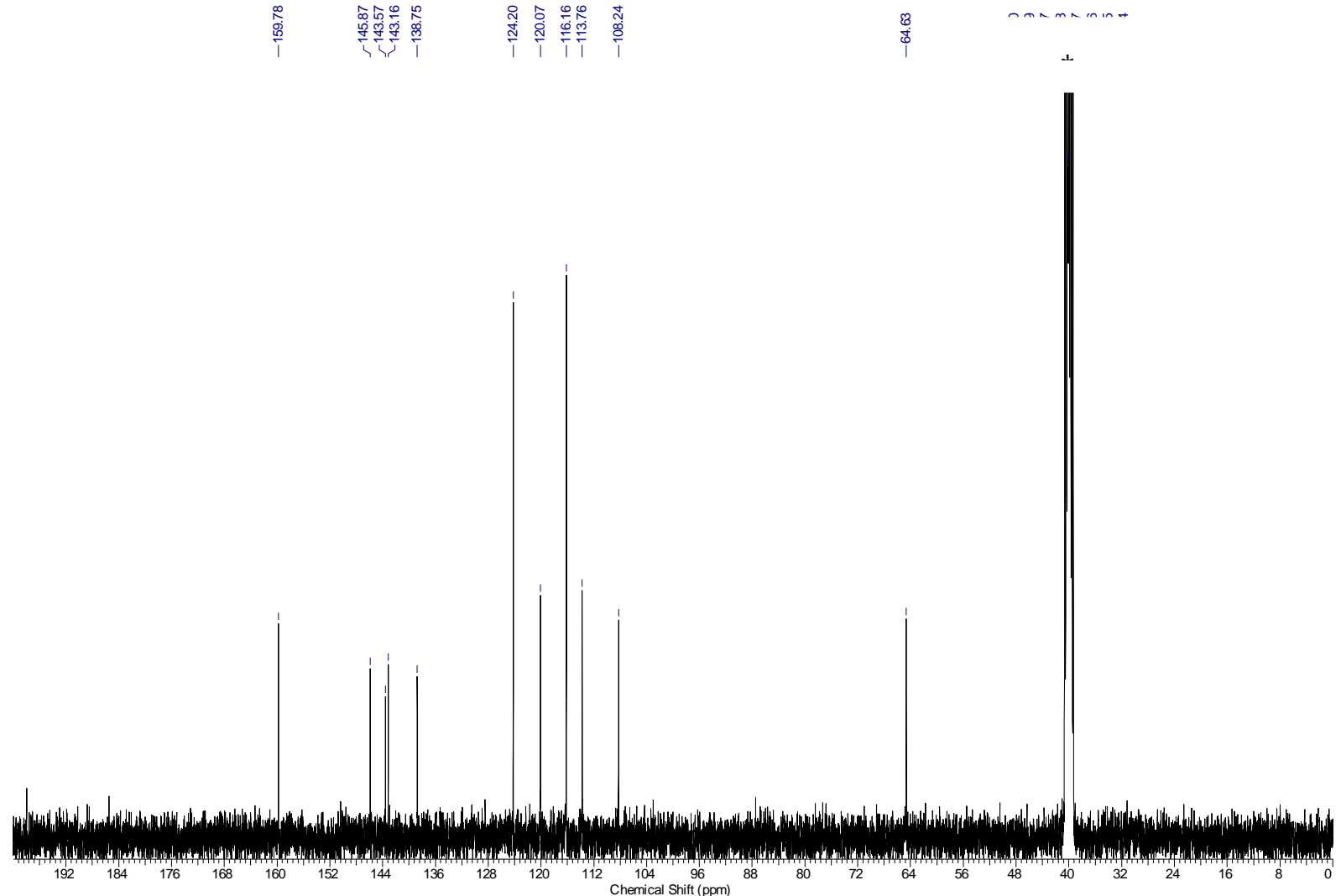

${ }^{1} \mathrm{H}-\mathrm{NMR}\left(400 \mathrm{MHz}\right.$, DMSO- $d_{6}$ ) and ${ }^{13} \mathrm{C}-\mathrm{NMR}\left(101 \mathrm{MHz}\right.$, DMSO- $\left.d_{6}\right)$ spectrum of compound 15. $*=\mathrm{H}_{2} \mathrm{O}$ 


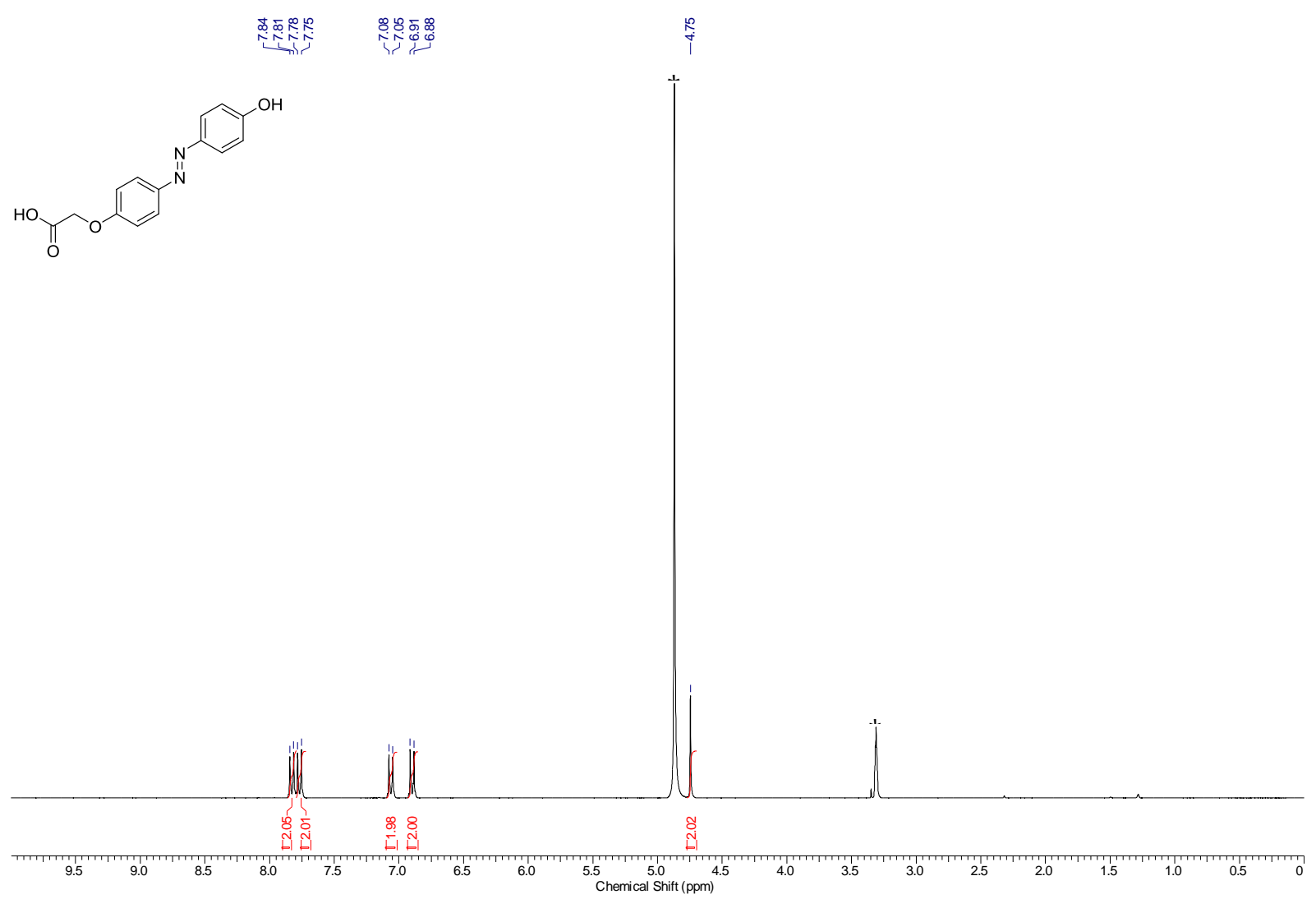

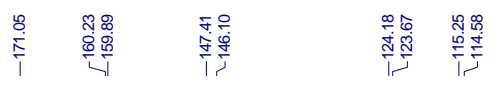

6
$\dot{0}$
1

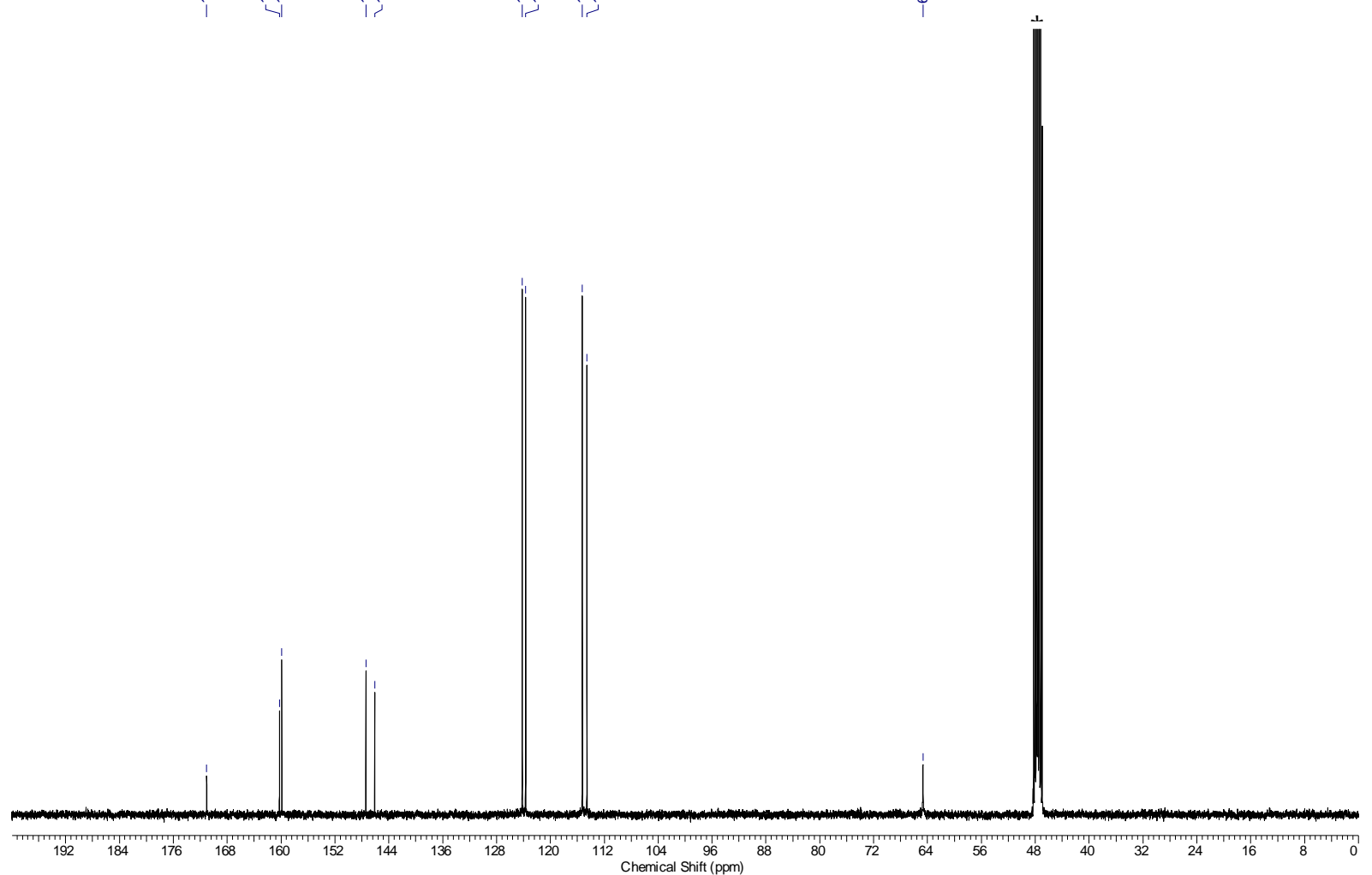

${ }^{1} \mathrm{H}-\mathrm{NMR}\left(300 \mathrm{MHz}, \mathrm{CD}_{3} \mathrm{OD}\right)$ and ${ }^{13} \mathrm{C}-\mathrm{NMR}\left(101 \mathrm{MHz}, \mathrm{CD}_{3} \mathrm{OD}\right)$ spectrum of compound 16a. * = NMR-solvent, $\mathrm{H}_{2} \mathrm{O}$ 

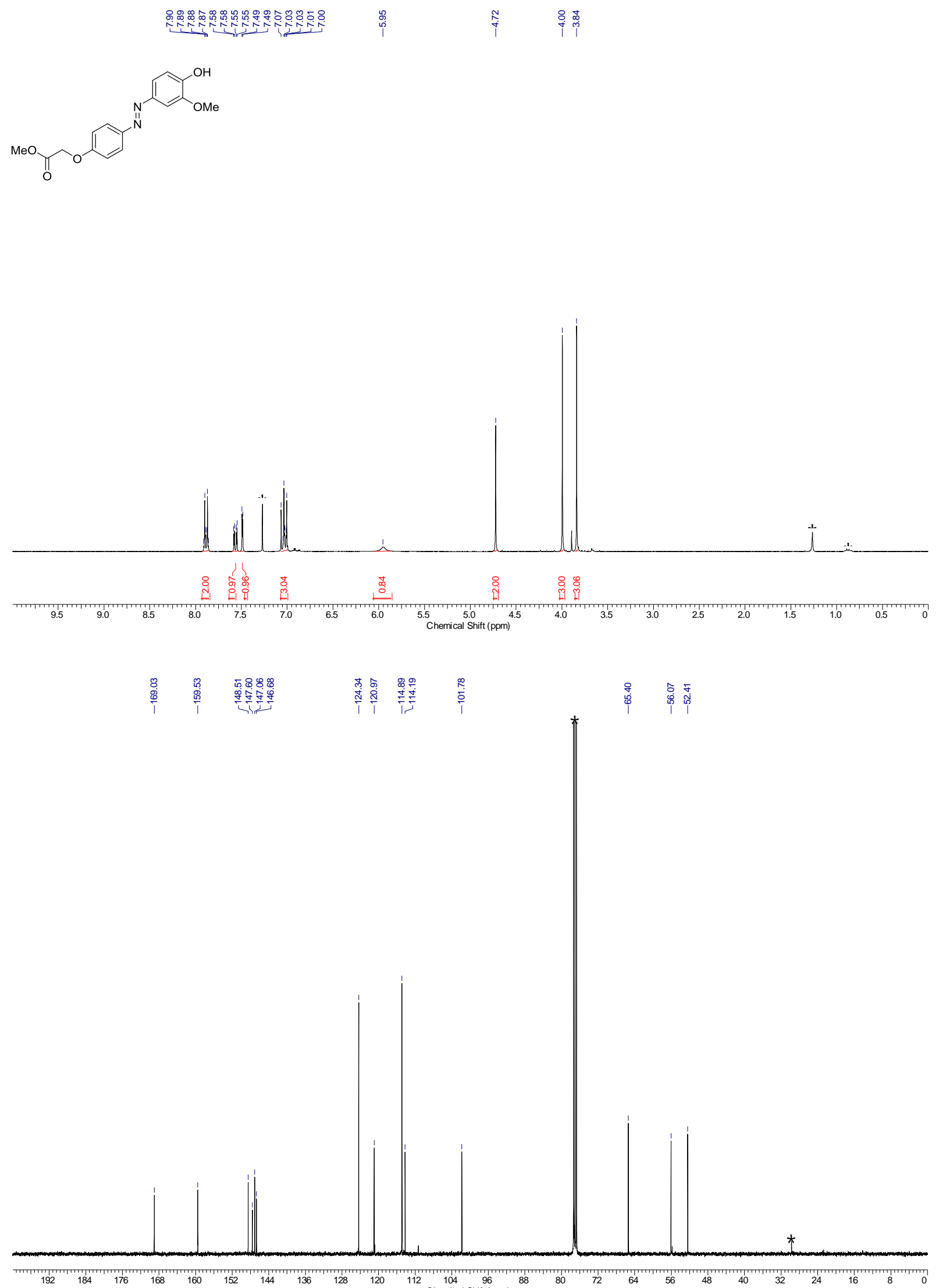

${ }^{1} \mathrm{H}-\mathrm{NMR}\left(300 \mathrm{MHz}, \mathrm{CDCl}_{3}\right)$ and ${ }^{13} \mathrm{C}-\mathrm{NMR}\left(101 \mathrm{MHz}, \mathrm{CDCl}_{3}\right)$ spectrum of compound $\mathbf{1 6 b} . *$ = NMR-solvent, grease 

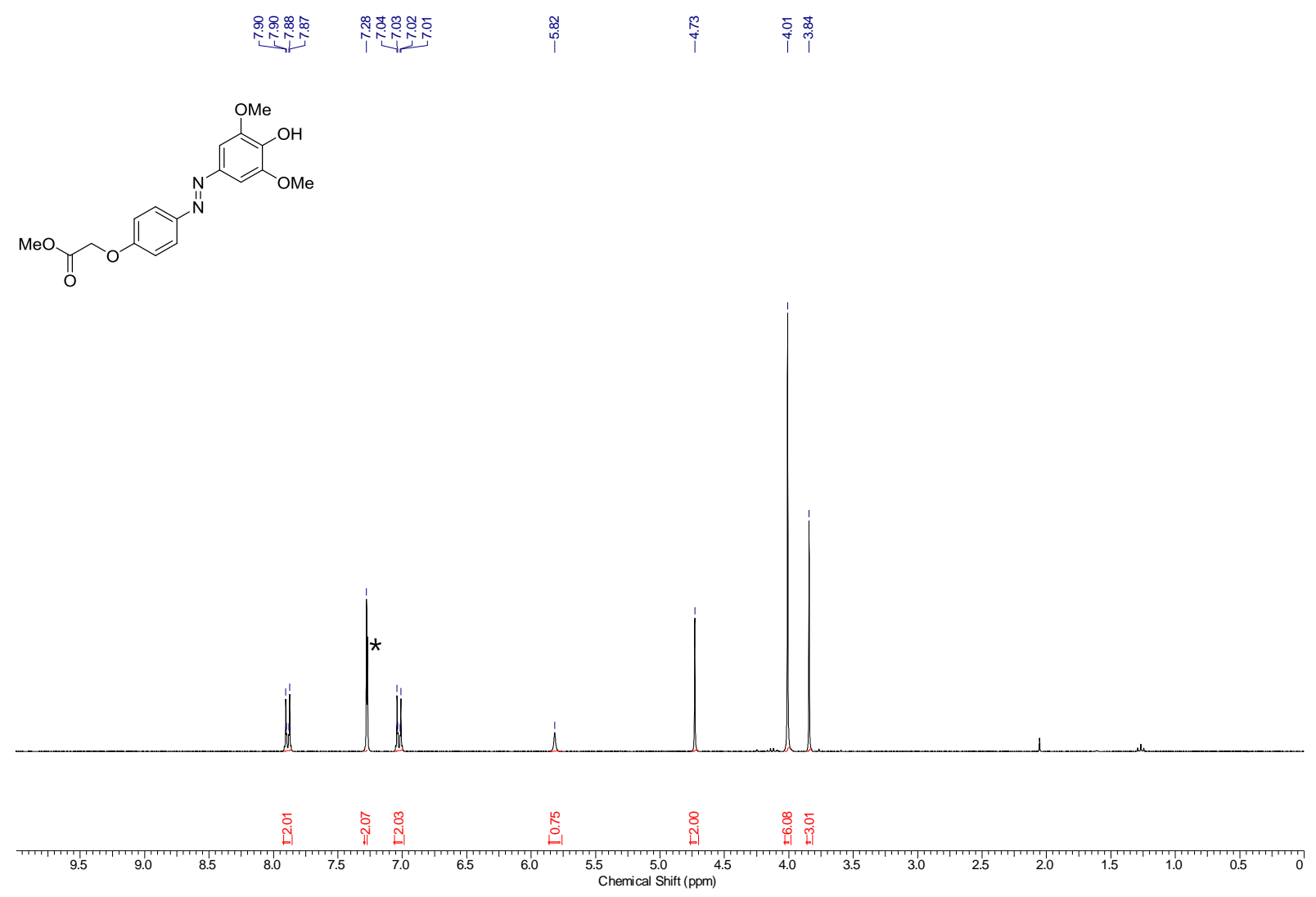

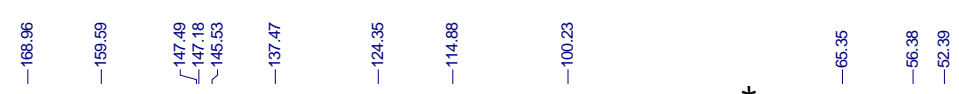

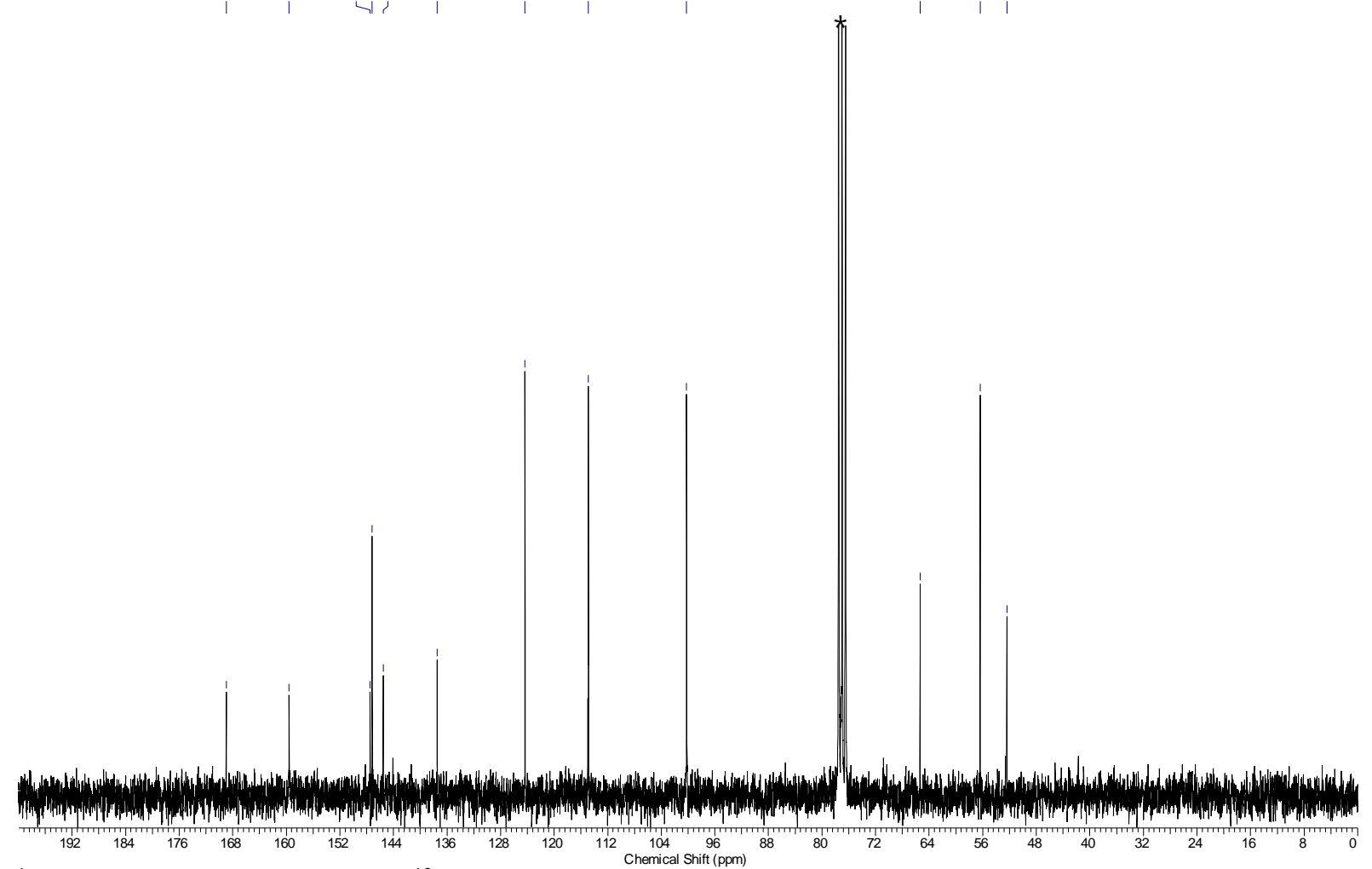

${ }^{1} \mathrm{H}-\mathrm{NMR}\left(300 \mathrm{MHz}, \mathrm{CDCl}_{3}\right.$ ) and ${ }^{13} \mathrm{C}-\mathrm{NMR}\left(63 \mathrm{MHz}^{\mathrm{CDCl}} \mathrm{CDCl}_{3}\right.$ ) spectrum of compound 16c. * = NMR-solvent 

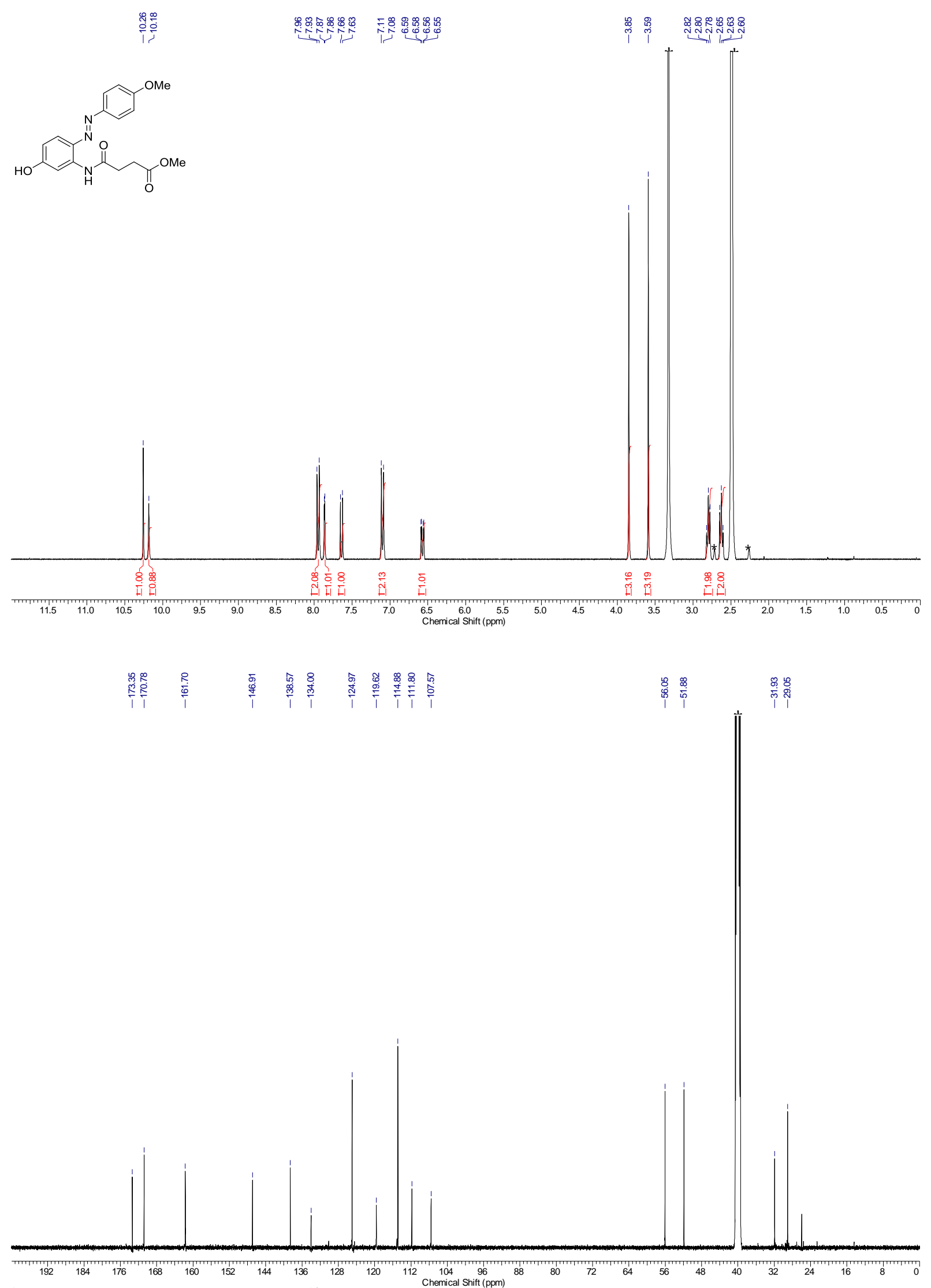

${ }^{1} \mathrm{H}-\mathrm{NMR}\left(300 \mathrm{MHz}\right.$, DMSO- $d_{6}$ ) and ${ }^{13} \mathrm{C}-\mathrm{NMR}\left(126 \mathrm{MHz}\right.$, DMSO- $d_{6}$ ) spectrum of compound 17a. * = NMR-solvent, $\mathrm{H}_{2} \mathrm{O}$ 


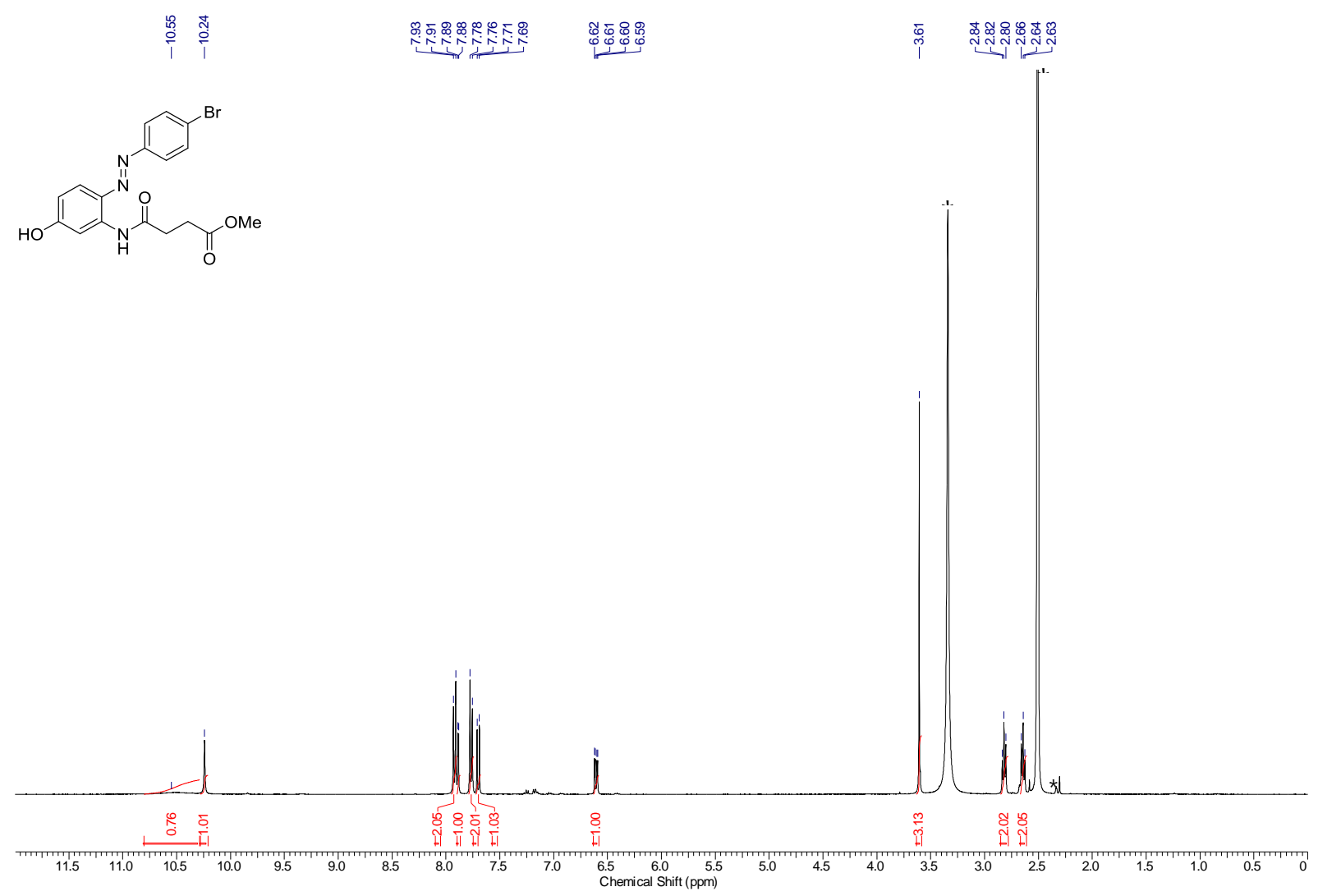

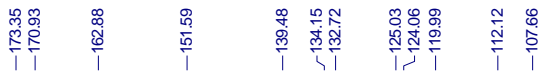
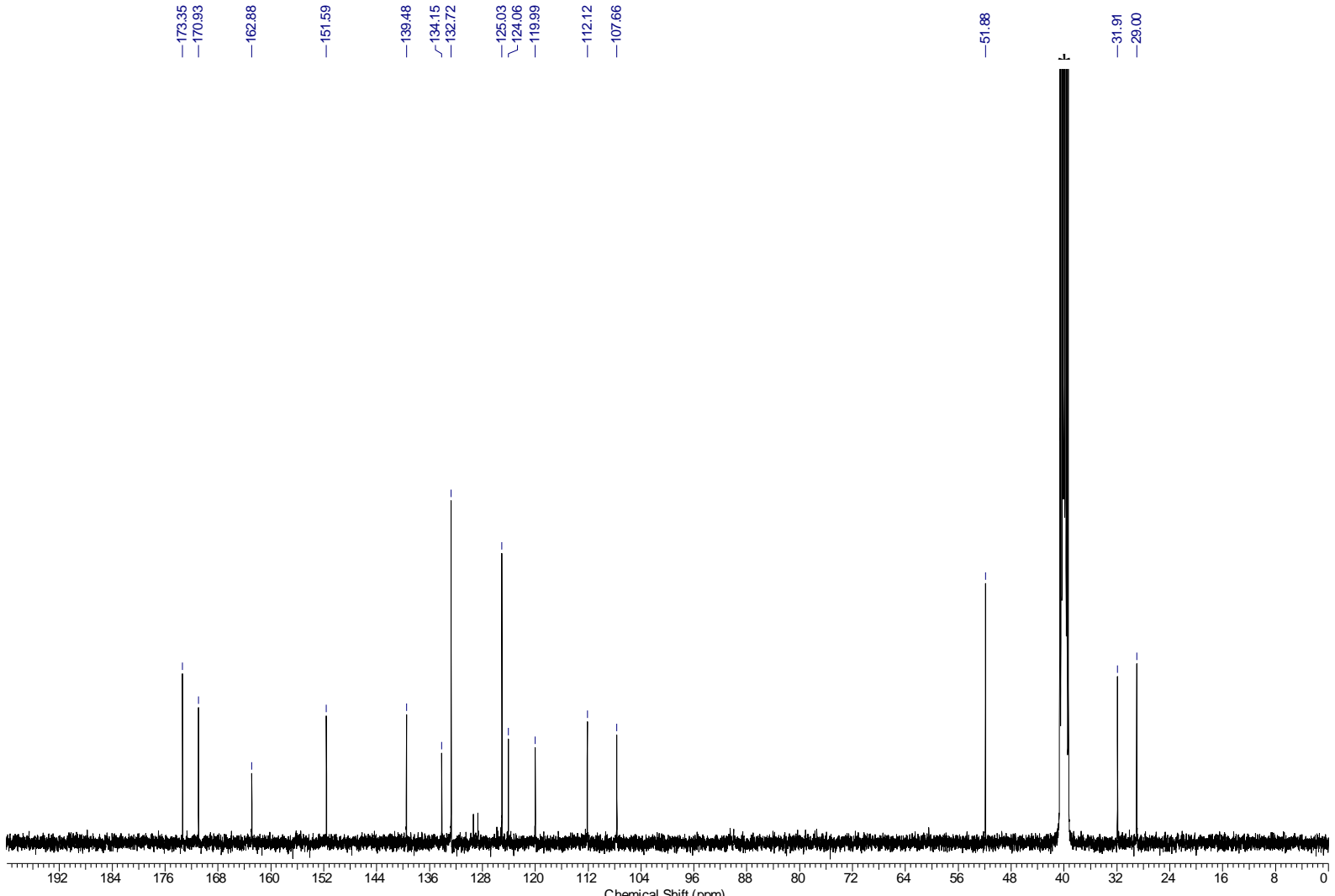

${ }^{1} \mathrm{H}-\mathrm{NMR}\left(400 \mathrm{MHz}\right.$, DMSO- $d_{6}$ ) and ${ }^{13} \mathrm{C}-\mathrm{NMR}\left(101 \mathrm{MHz}\right.$, DMSO- $\left.d_{6}\right)$ spectrum of compound $\mathbf{1 7 \mathbf { b }}$. ${ }^{*}=$ NMR-solvent, $\mathrm{H}_{2} \mathrm{O}$ 

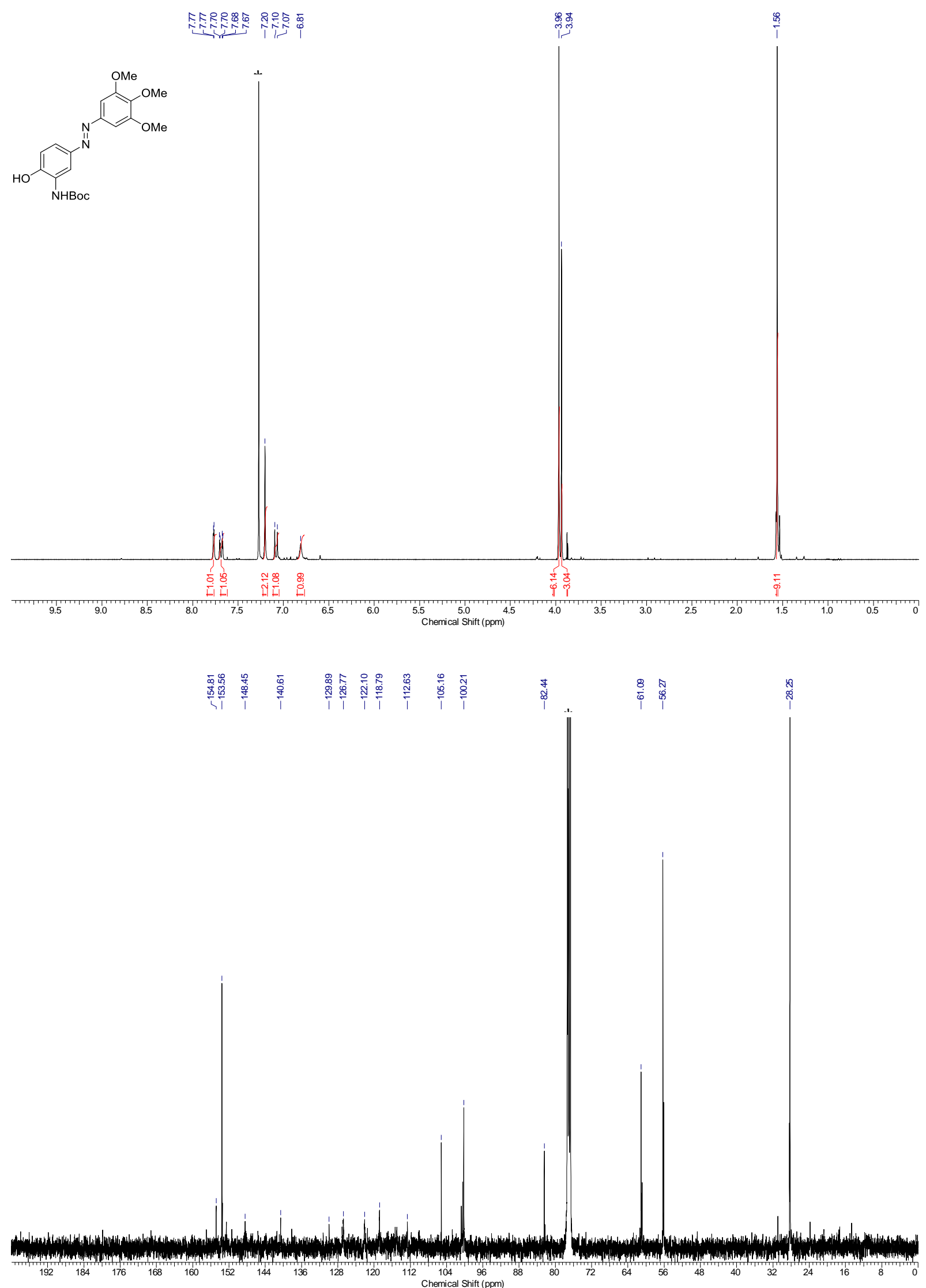

${ }^{1} \mathrm{H}-\mathrm{NMR}\left(300 \mathrm{MHz}, \mathrm{CDCl}_{3}\right.$ ) and ${ }^{13} \mathrm{C}-\mathrm{NMR}\left(101 \mathrm{MHz}, \mathrm{CDCl}_{3}\right)$ spectrum of compound 18. * = NMR-solvent 


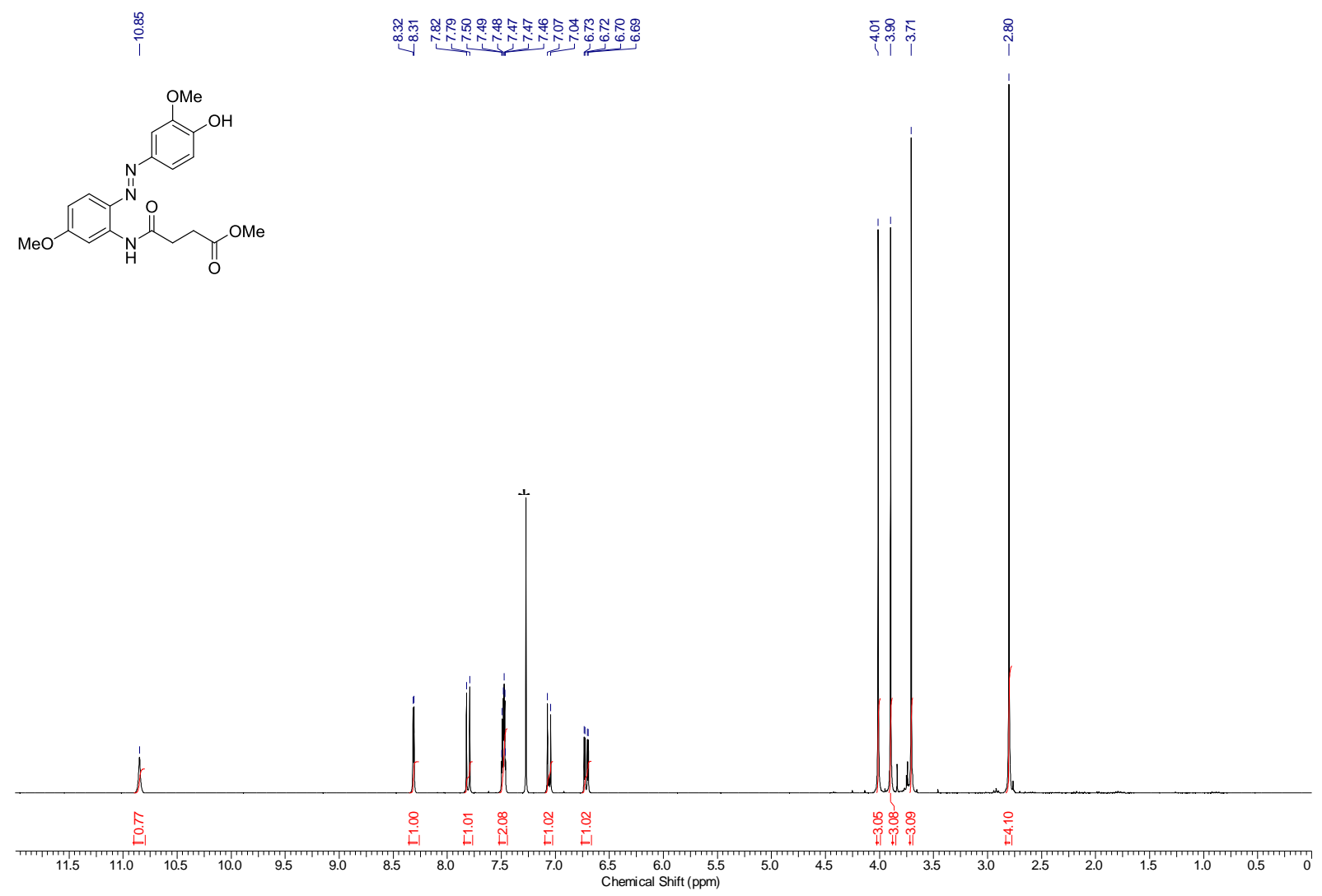

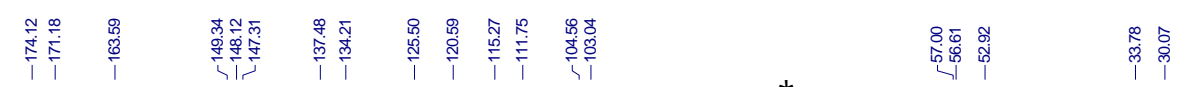

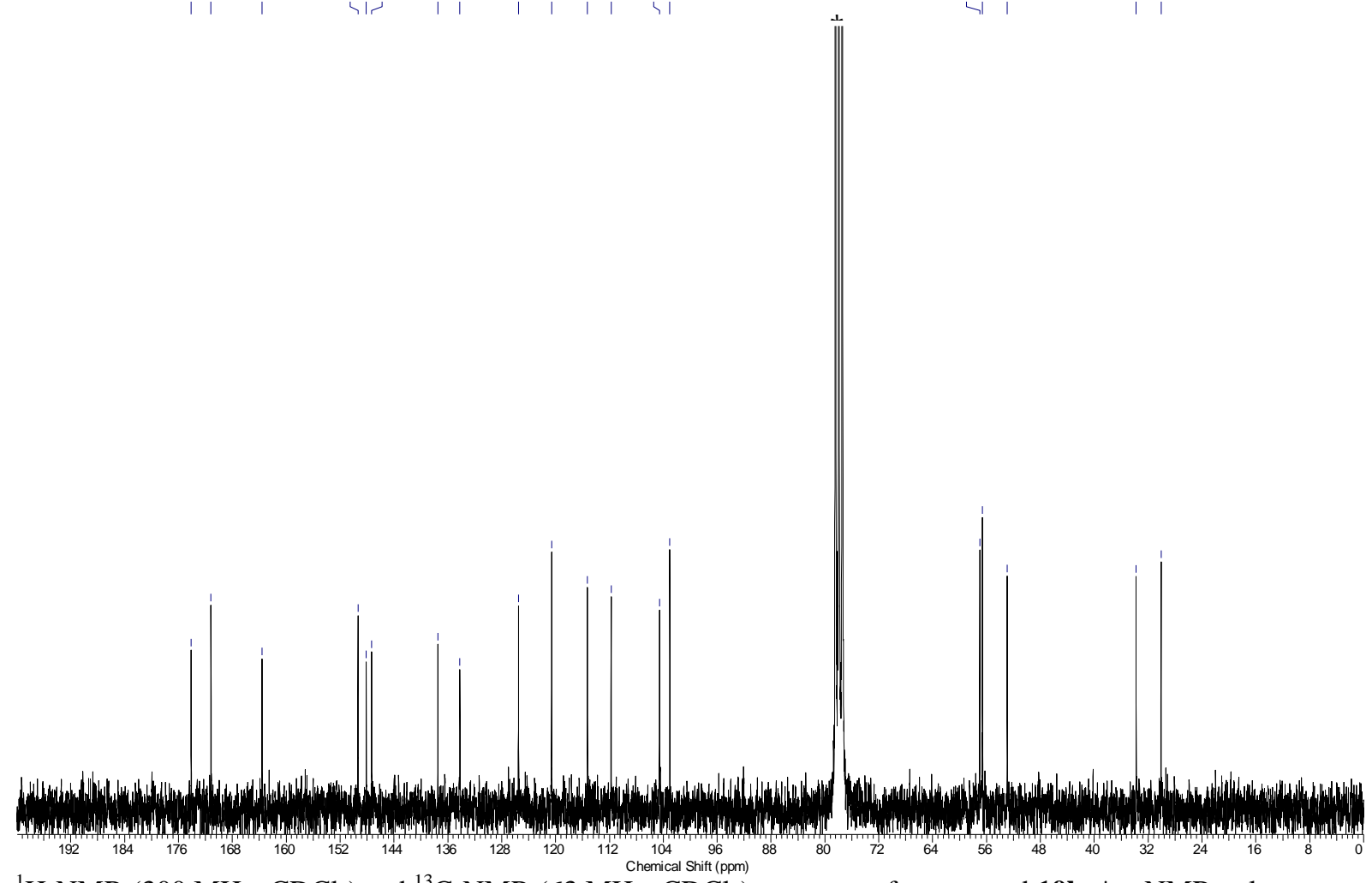

${ }^{1} \mathrm{H}-\mathrm{NMR}\left(300 \mathrm{MHz}, \mathrm{CDCl}_{3}\right.$ ) and ${ }^{13} \mathrm{C}-\mathrm{NMR}\left(63 \mathrm{MHz}^{\mathrm{C}} \mathrm{CDCl}_{3}\right)$ spectrum of compound 19b. * = NMR-solvent 


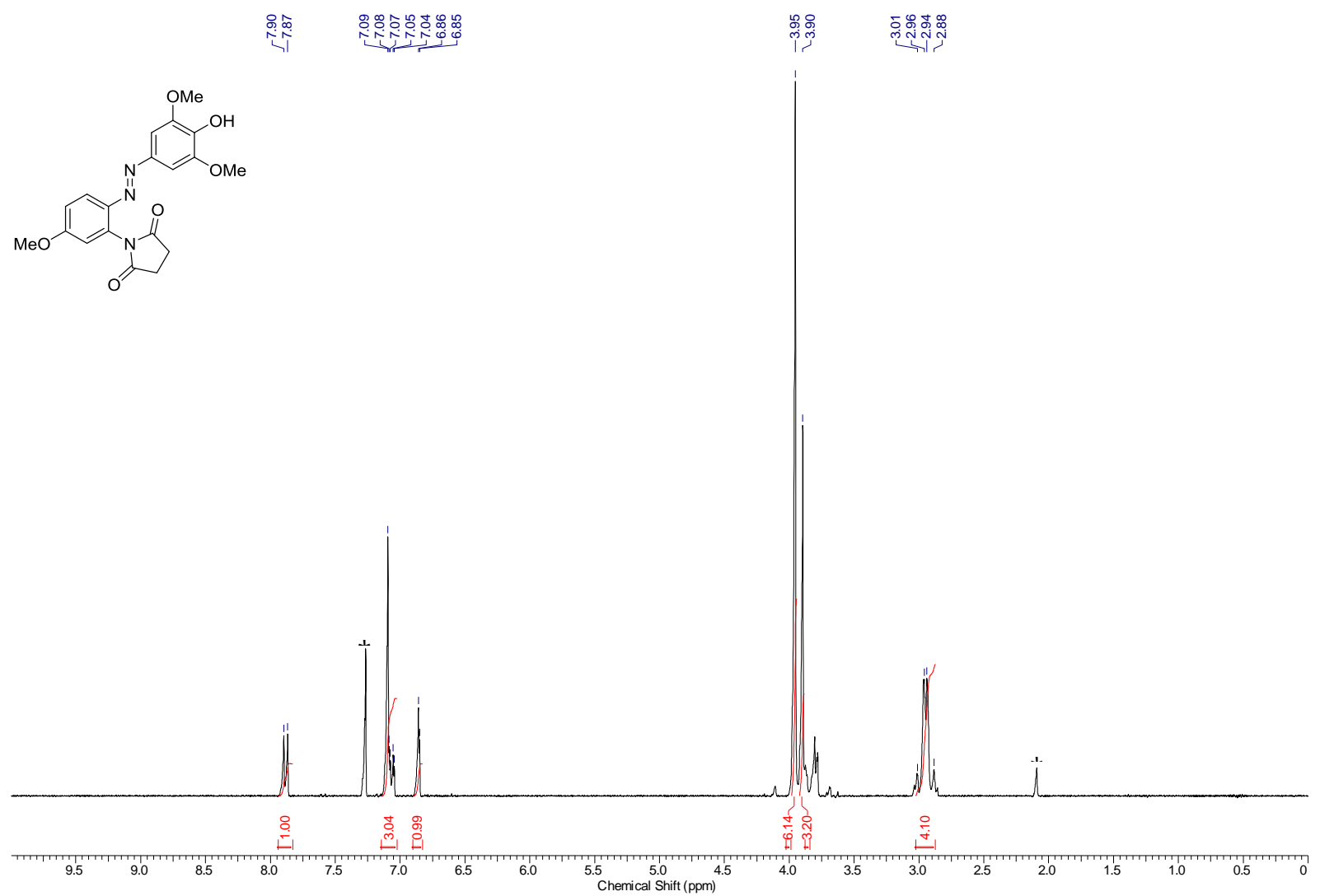

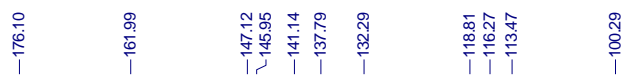
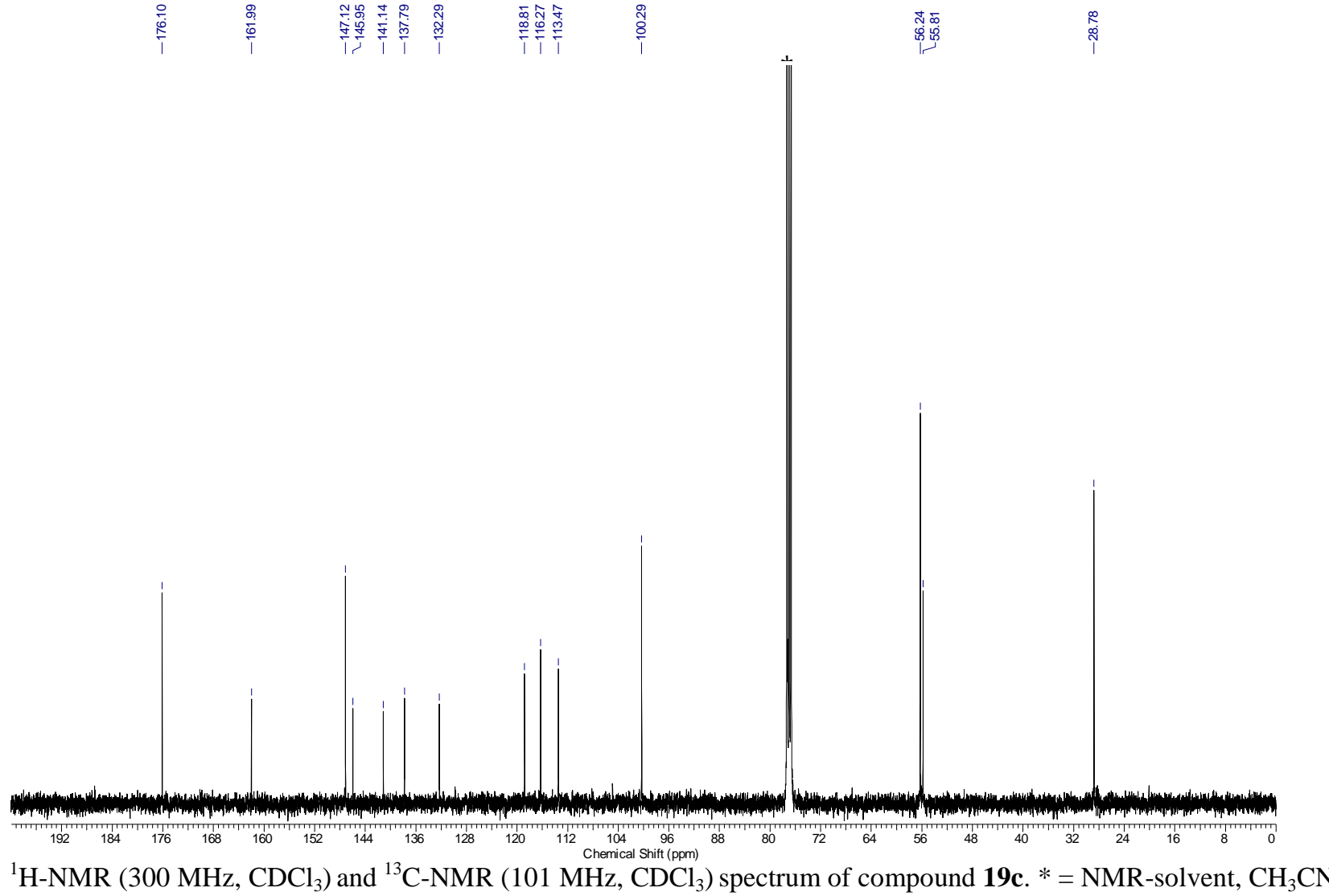


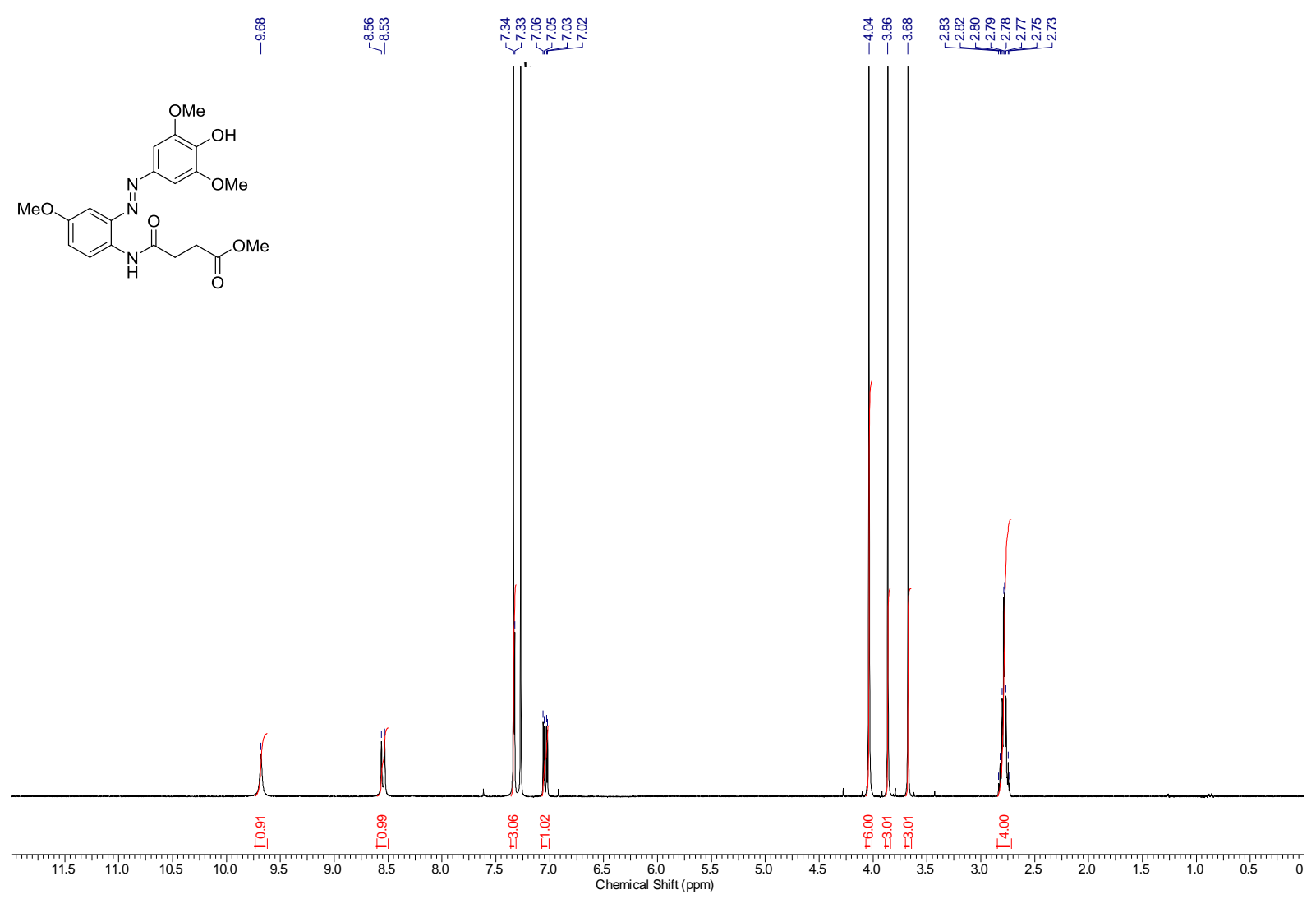

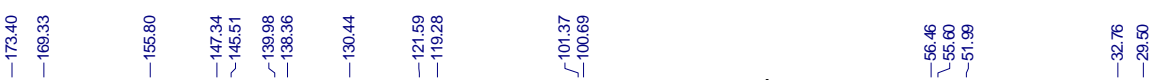

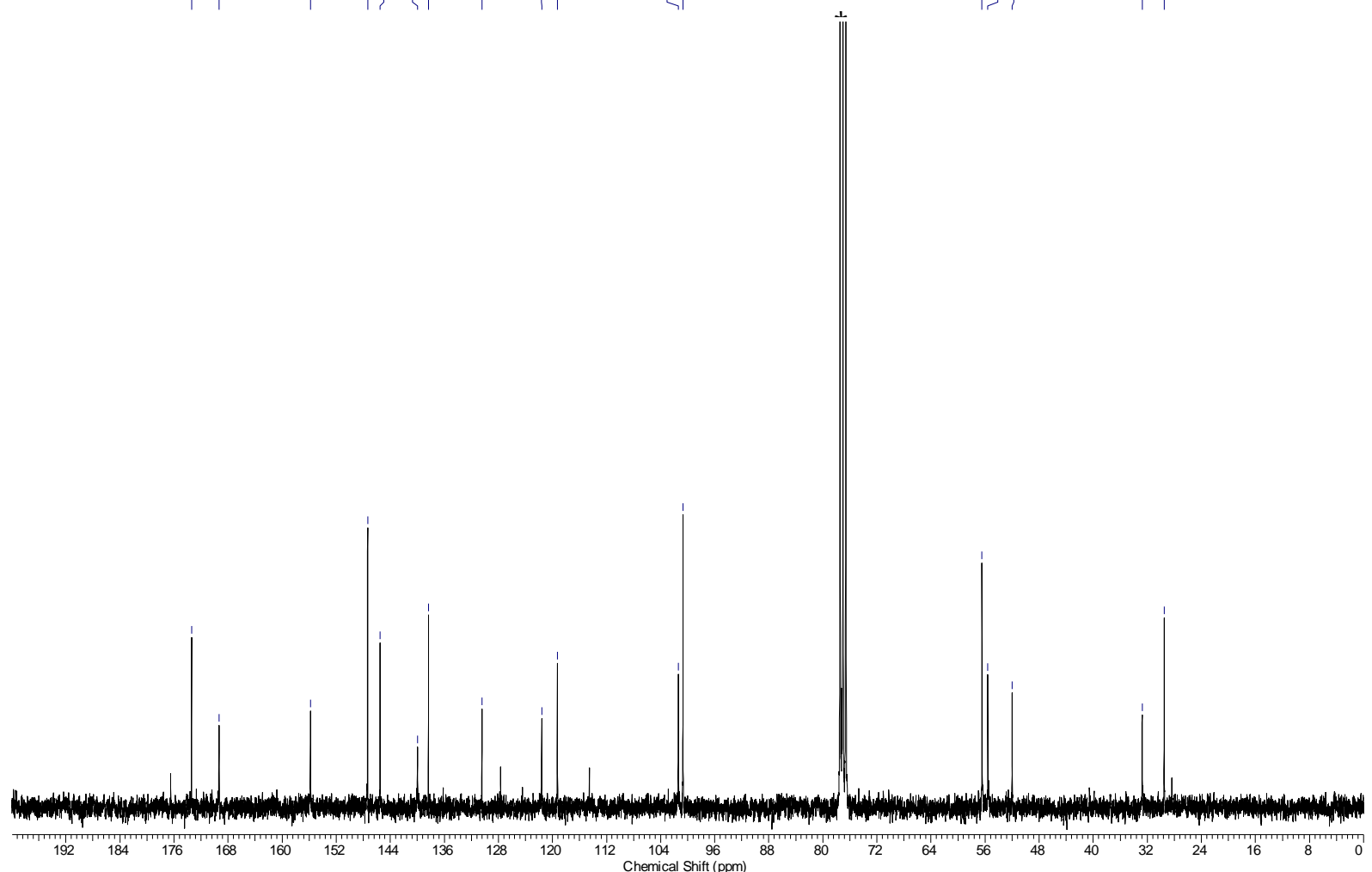

${ }^{1} \mathrm{H}-\mathrm{NMR}\left(300 \mathrm{MHz}, \mathrm{CDCl}_{3}\right)$ and ${ }^{13} \mathrm{C}-\mathrm{NMR}\left(75 \mathrm{MHz}, \mathrm{CDCl}_{3}\right)$ spectrum of compound $\mathbf{2 0} . *$ = NMR-solvent 


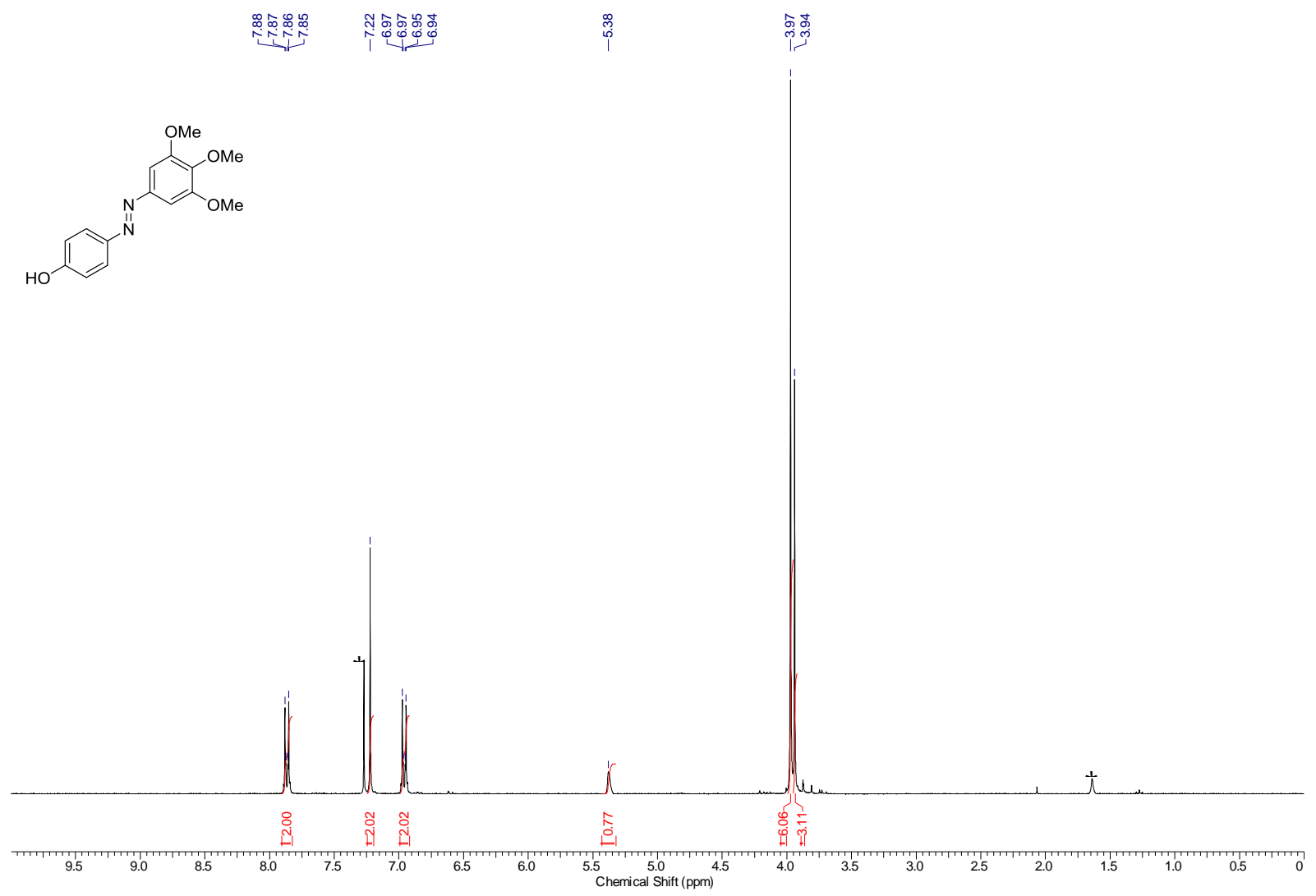

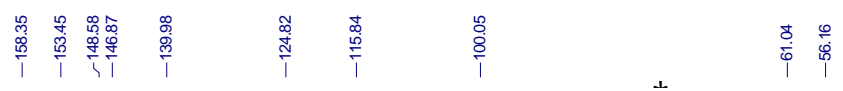

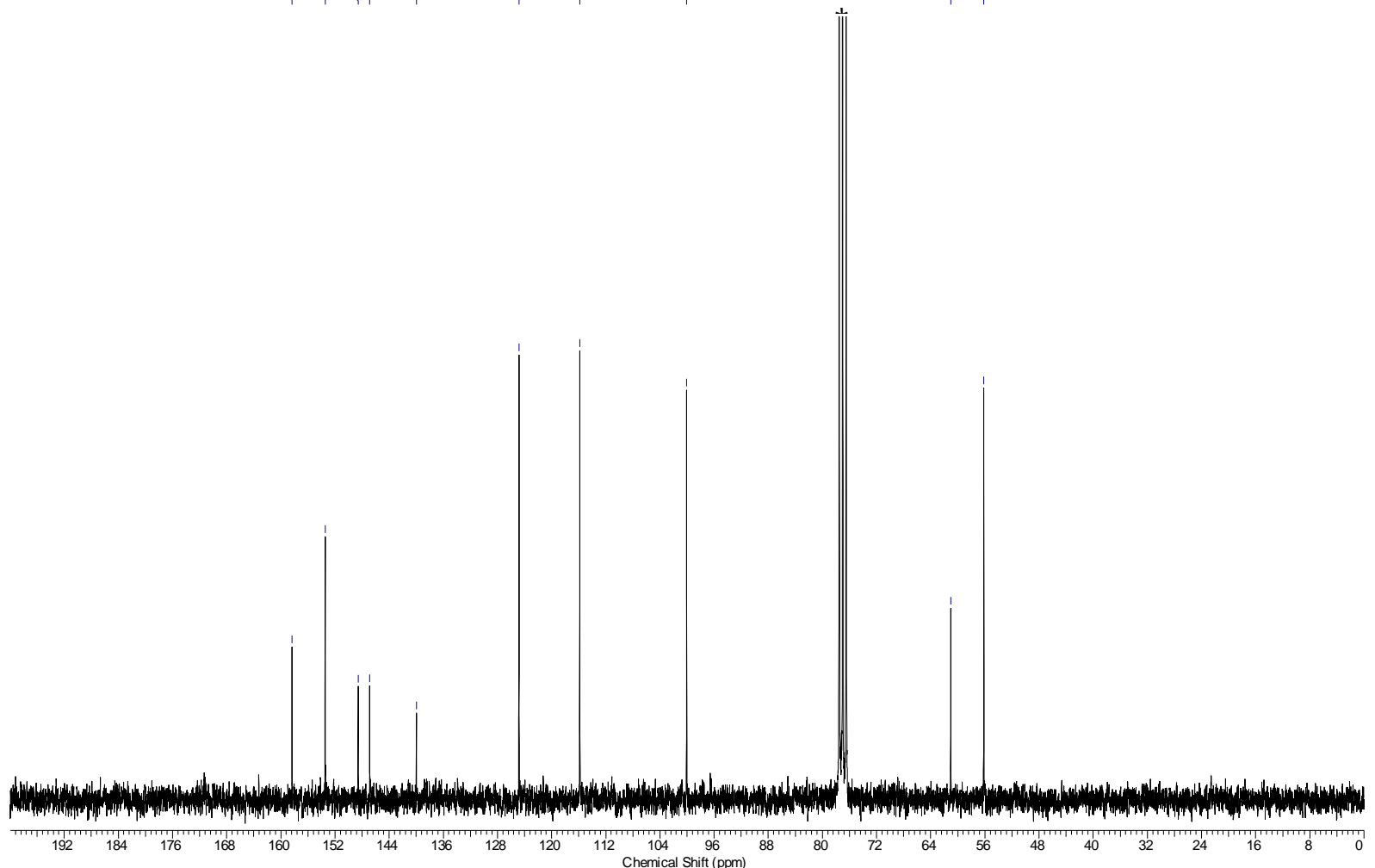

${ }^{1} \mathrm{H}-\mathrm{NMR}\left(300 \mathrm{MHz}, \mathrm{CDCl}_{3}\right.$ ) and ${ }^{13} \mathrm{C}-\mathrm{NMR}\left(63 \mathrm{MHz}, \mathrm{CDCl}_{3}\right.$ ) spectrum of compound 21a. * = NMR-solvent, $\mathrm{H}_{2} \mathrm{O}$ 

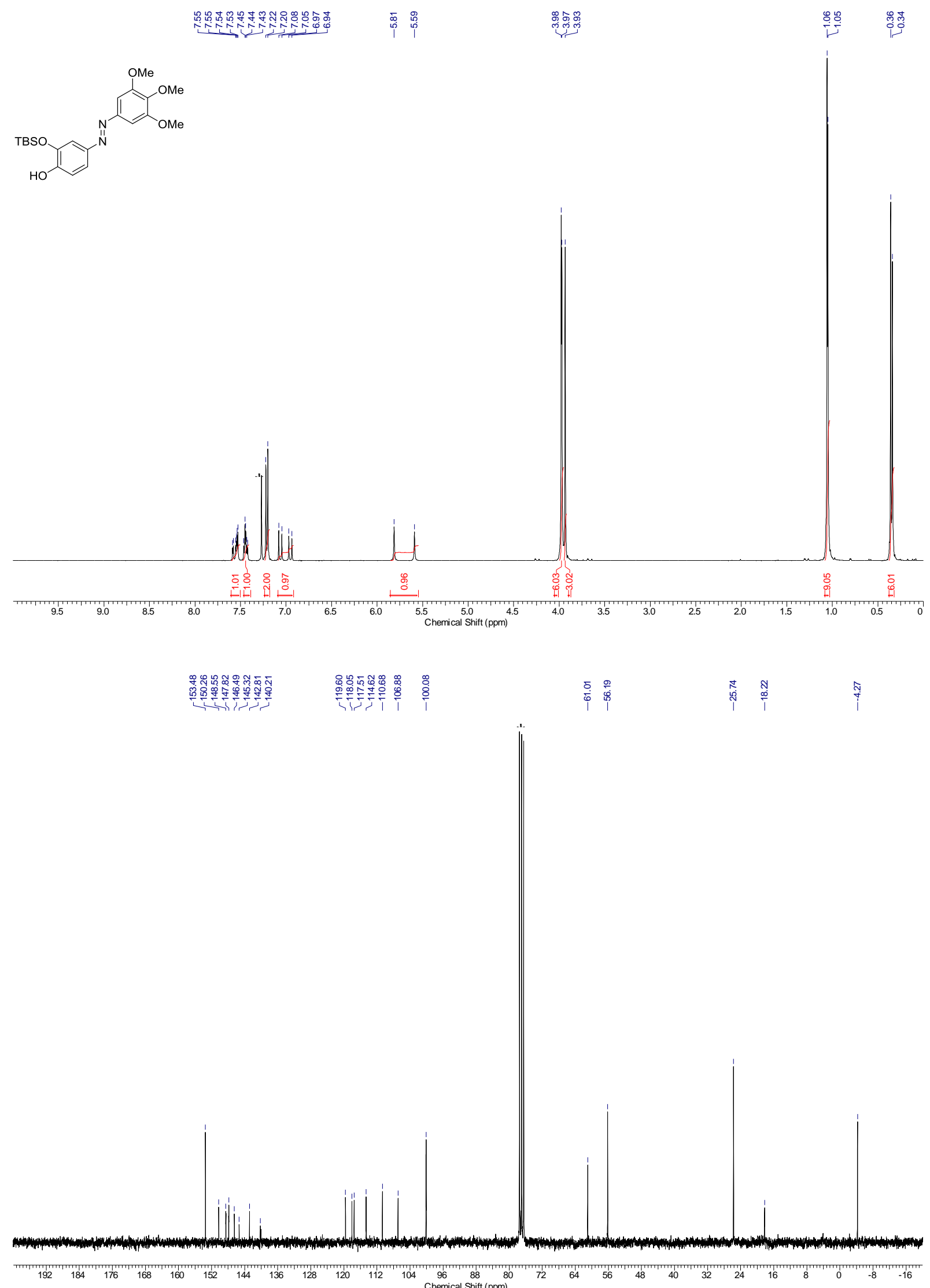

${ }^{1} \mathrm{H}-\mathrm{NMR}\left(250 \mathrm{MHz}, \mathrm{CDCl}_{3}\right)$ and ${ }^{13} \mathrm{C}-\mathrm{NMR}\left(63 \mathrm{MHz}, \mathrm{CDCl}_{3}\right)$ spectrum of compound 21b. * = NMR-solvent 

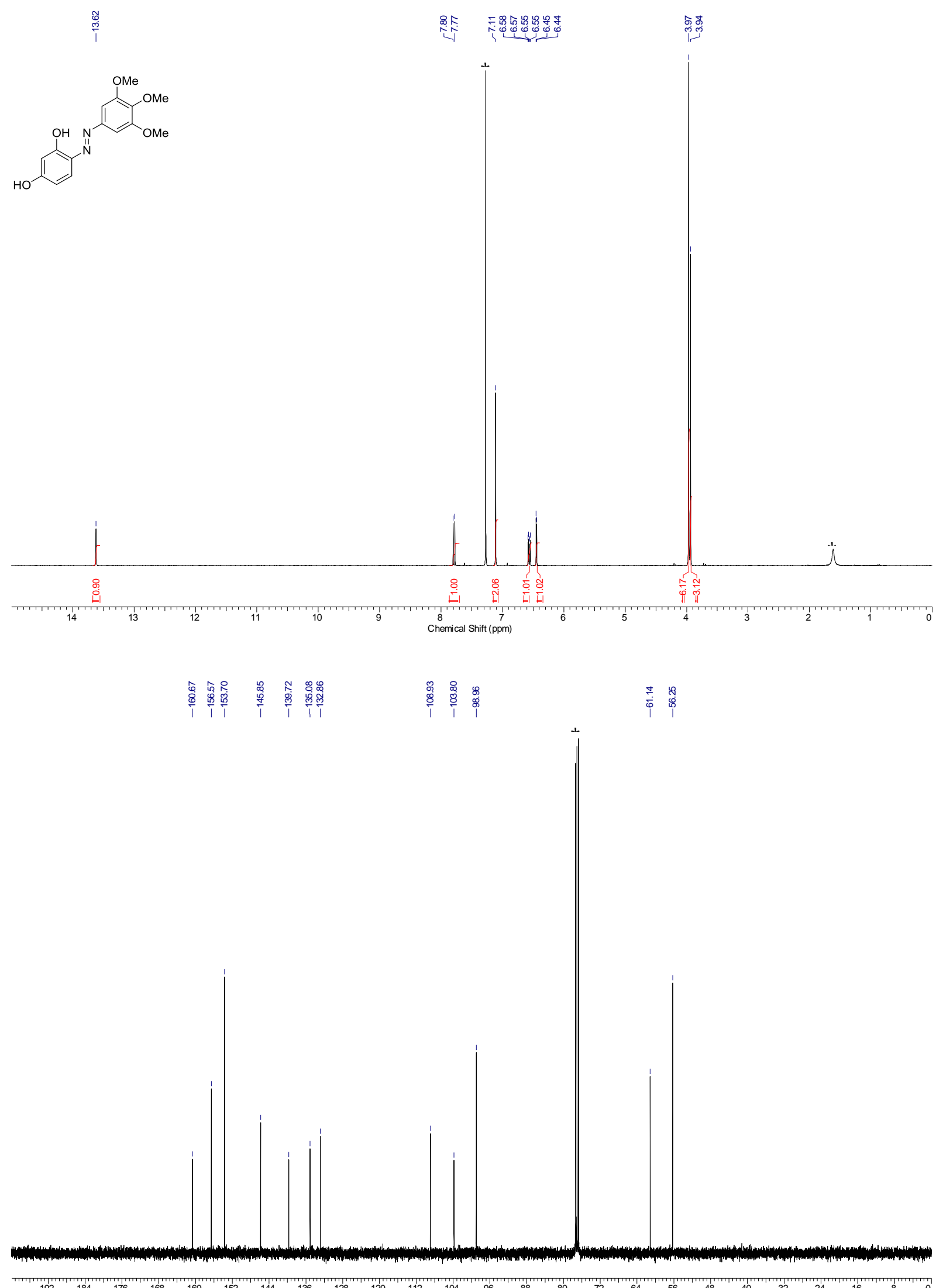

${ }^{1} \mathrm{H}-\mathrm{NMR}\left(400 \mathrm{MHz}, \mathrm{CDCl}_{3}\right.$ ) and ${ }^{13} \mathrm{C}-\mathrm{NMR}\left(101 \mathrm{MHz}^{\text {Chemical Shit (pom) }}{ }_{3}\right.$ ) spectrum of compound 21c. * = NMR-solvent, $\mathrm{H}_{2} \mathrm{O}$ 


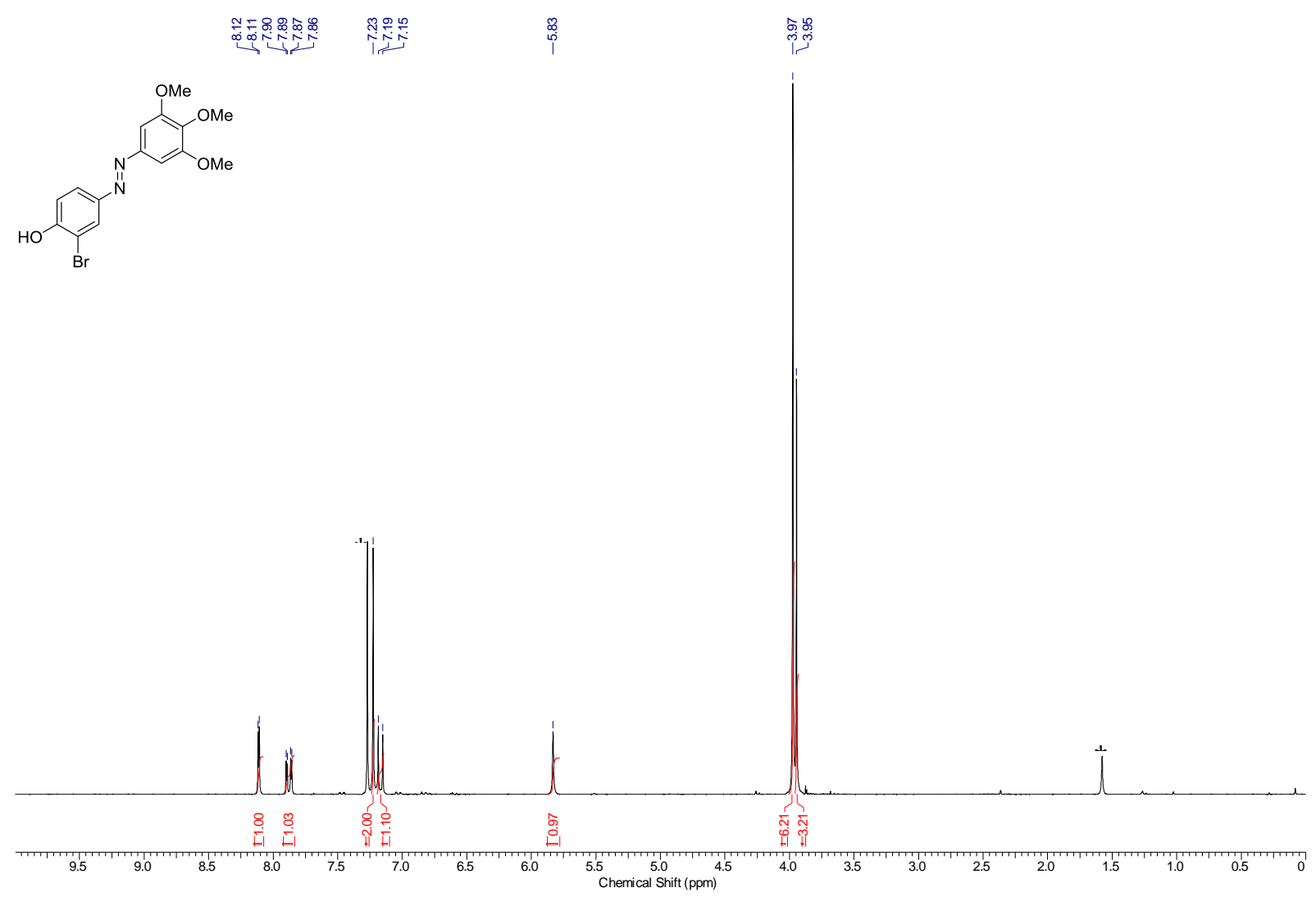

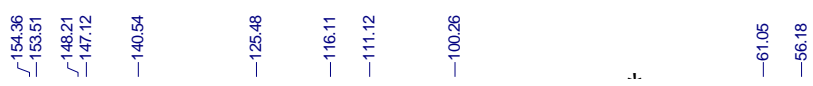
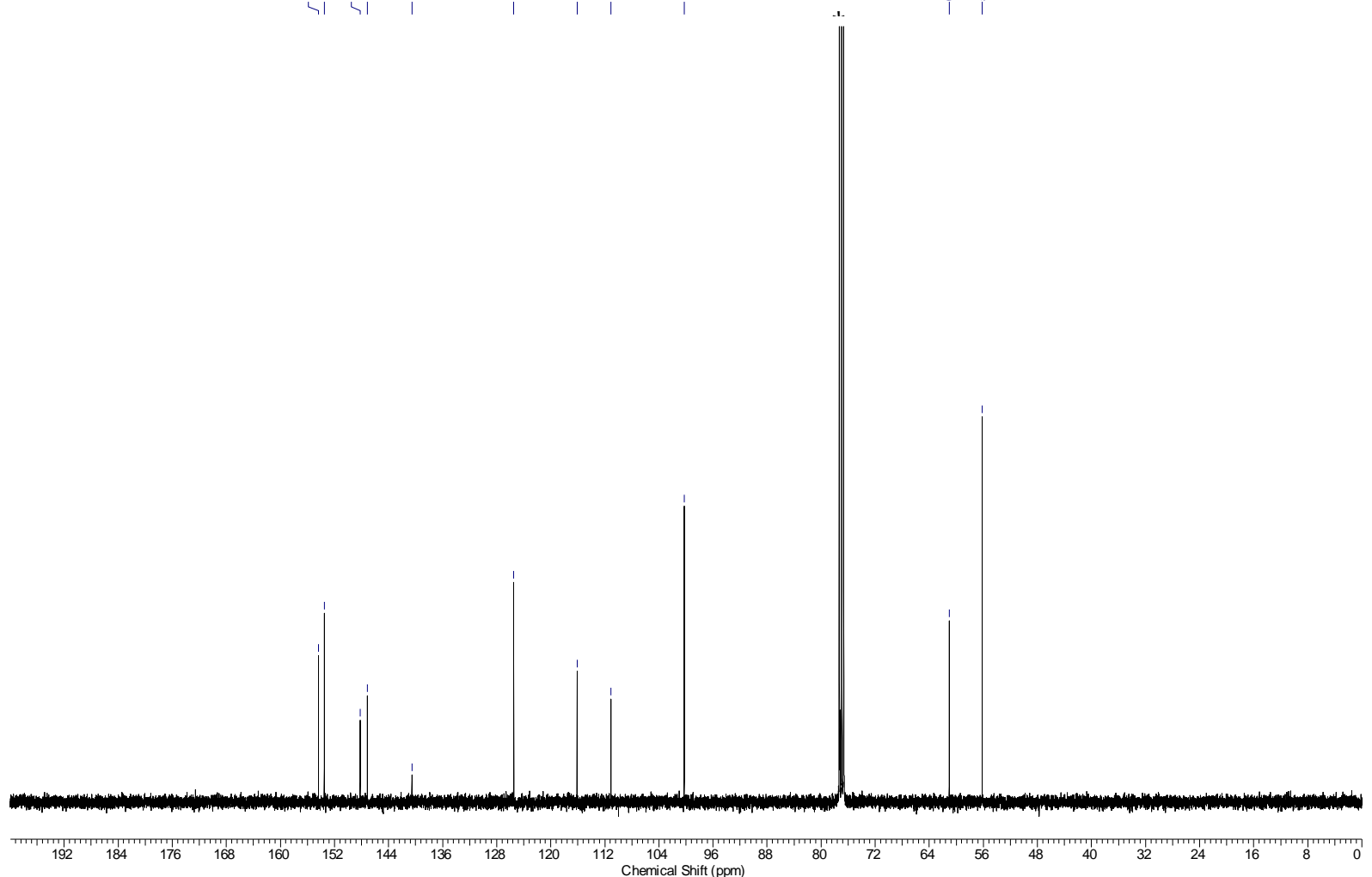

${ }^{1} \mathrm{H}-\mathrm{NMR}\left(250 \mathrm{MHz}, \mathrm{CDCl}_{3}\right)$ and ${ }^{13} \mathrm{C}-\mathrm{NMR}\left(101 \mathrm{MHz}, \mathrm{CDCl}_{3}\right)$ spectrum of compound 21d. * = NMR-solvent, $\mathrm{H}_{2} \mathrm{O}$ 


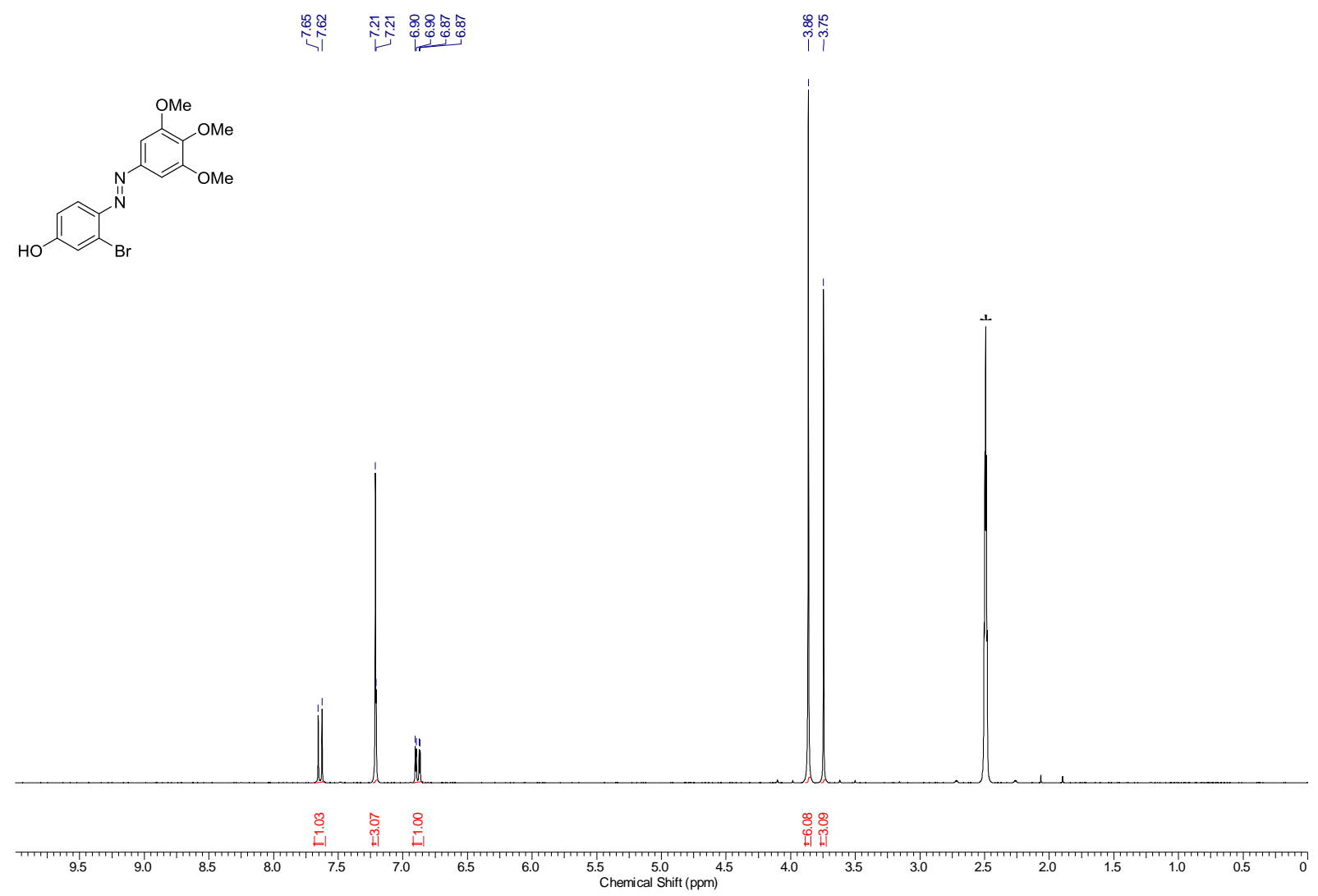

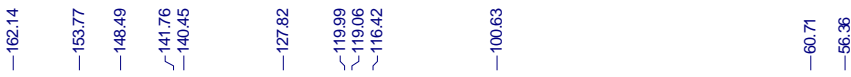

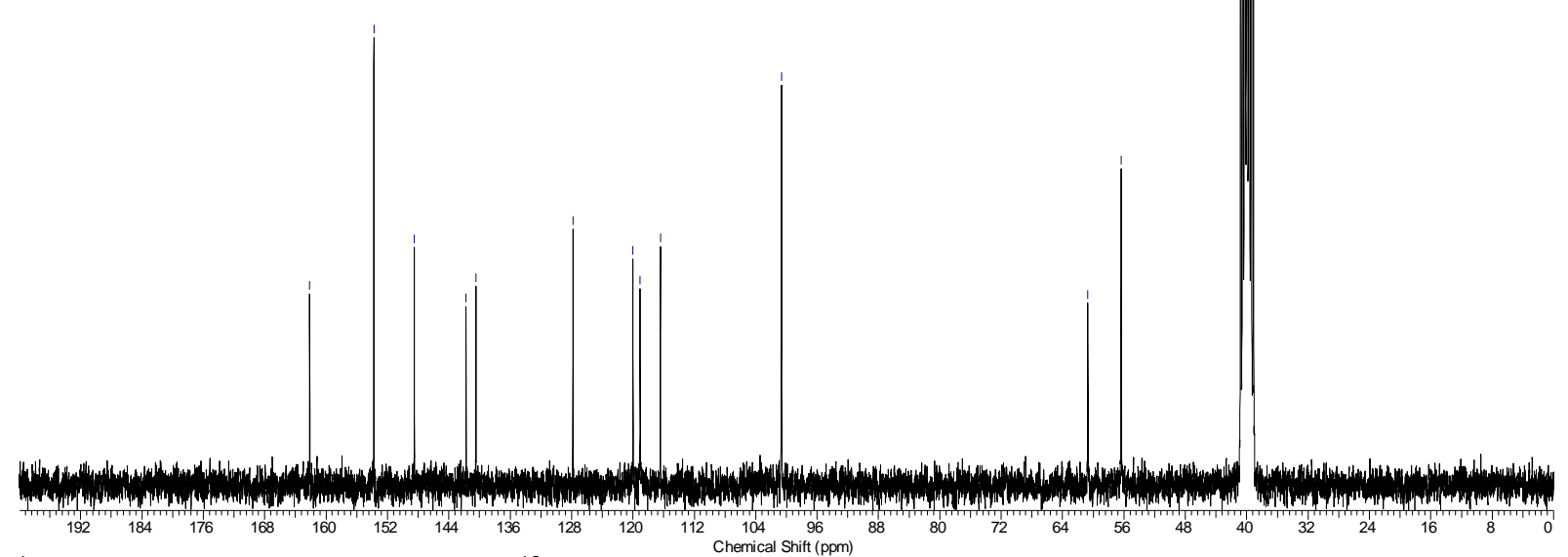

${ }^{1} \mathrm{H}-\mathrm{NMR}\left(300 \mathrm{MHz}\right.$, DMSO- $d_{6}$ ) and ${ }^{13} \mathrm{C}-\mathrm{NMR}\left(75 \mathrm{MHz}\right.$, DMSO- $d_{6}$ ) spectrum of compound 21e. 


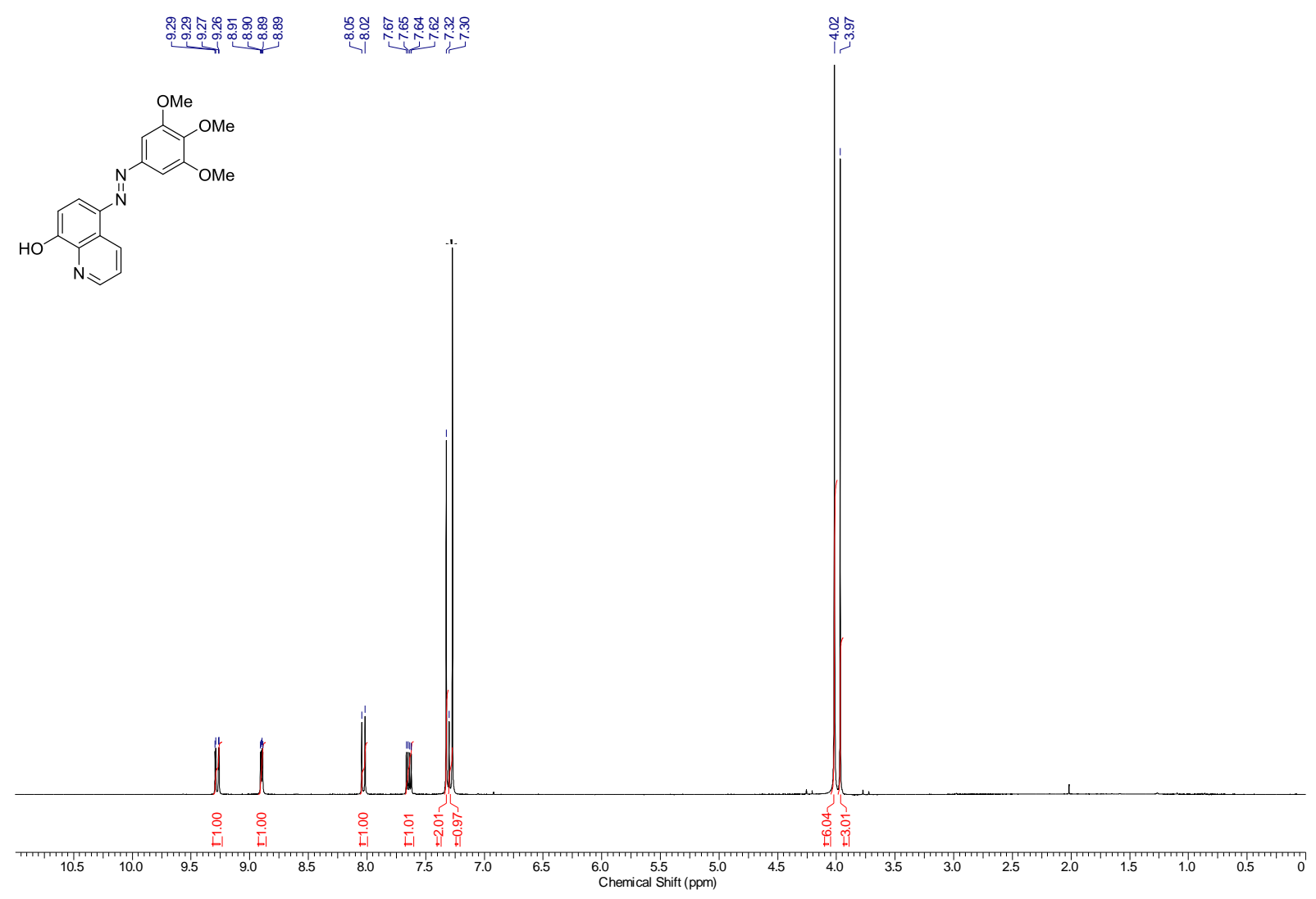

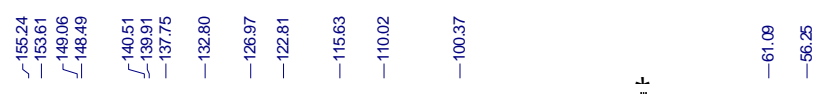

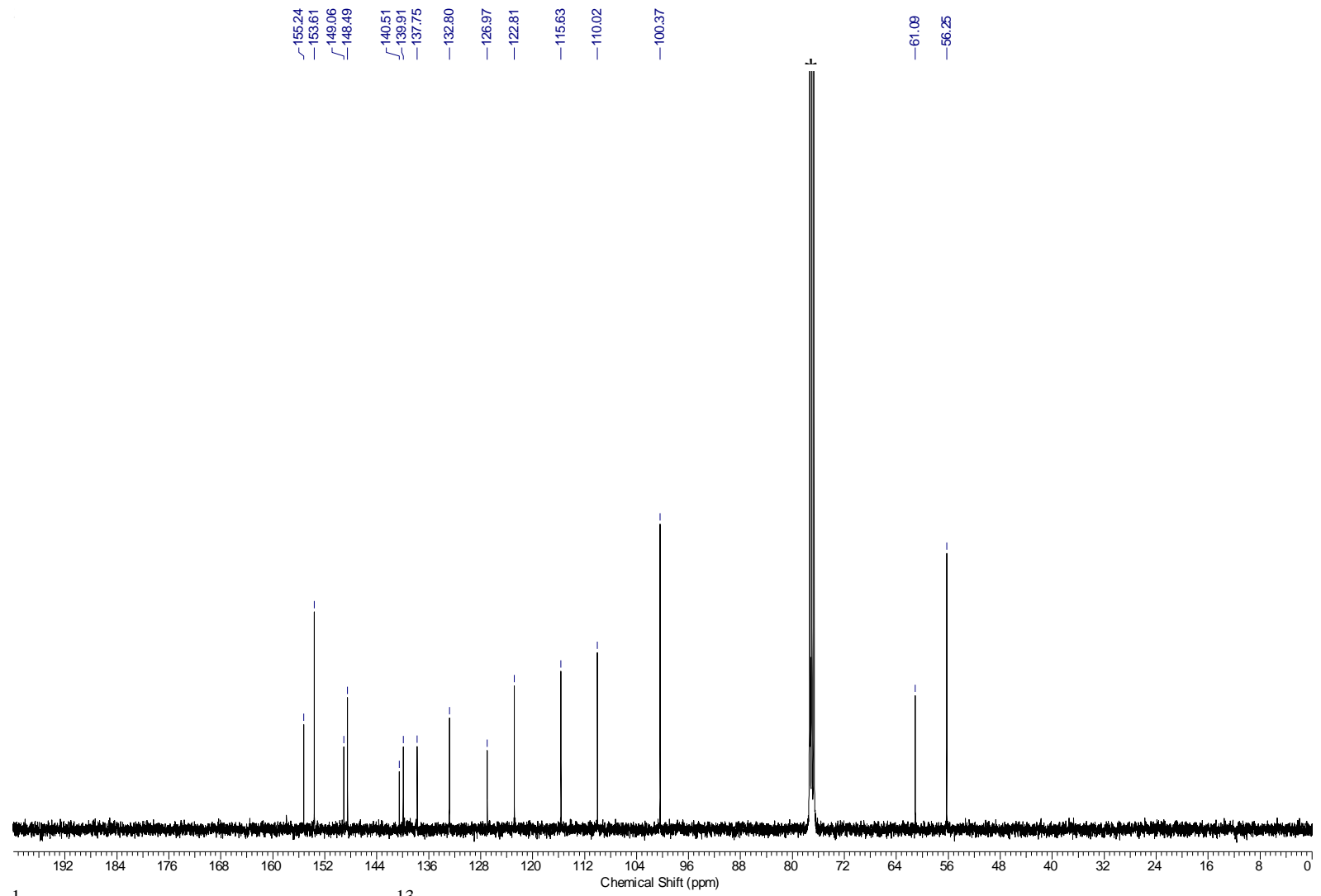

${ }^{1} \mathrm{H}-\mathrm{NMR}\left(300 \mathrm{MHz}, \mathrm{CDCl}_{3}\right)$ and ${ }^{13} \mathrm{C}-\mathrm{NMR}\left(101 \mathrm{MHz}, \mathrm{CDCl}_{3}\right)$ spectrum of compound 22a. * = NMR-solvent 

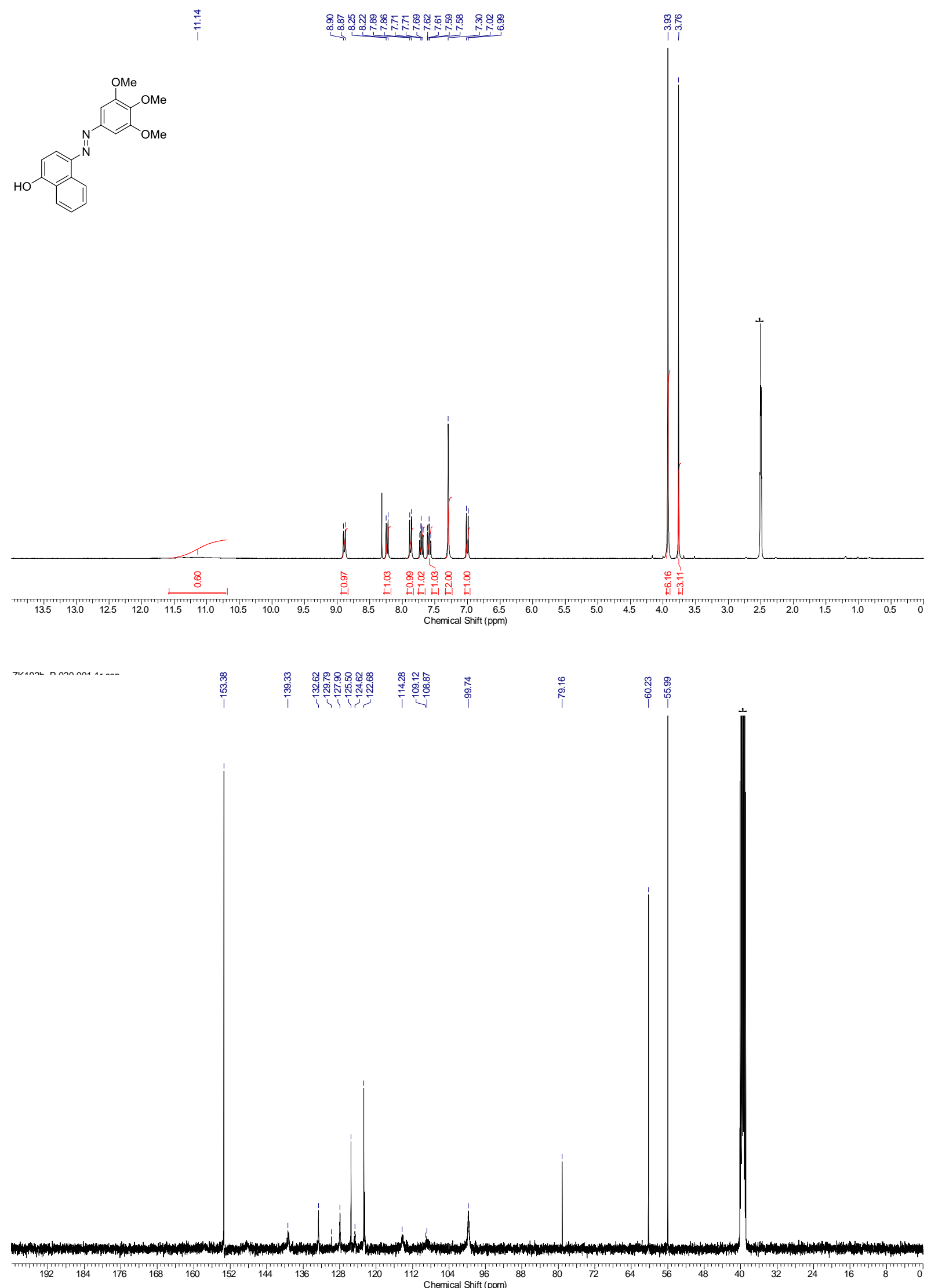

${ }^{1}$ H-NMR (300 MHz, DMSO- $d_{6}$ ) and ${ }^{13}$ C-NMR (75 MHz, DMSO- $d_{6}$ ) spectrum of compound 22b. * $=$ NMR-solvent 

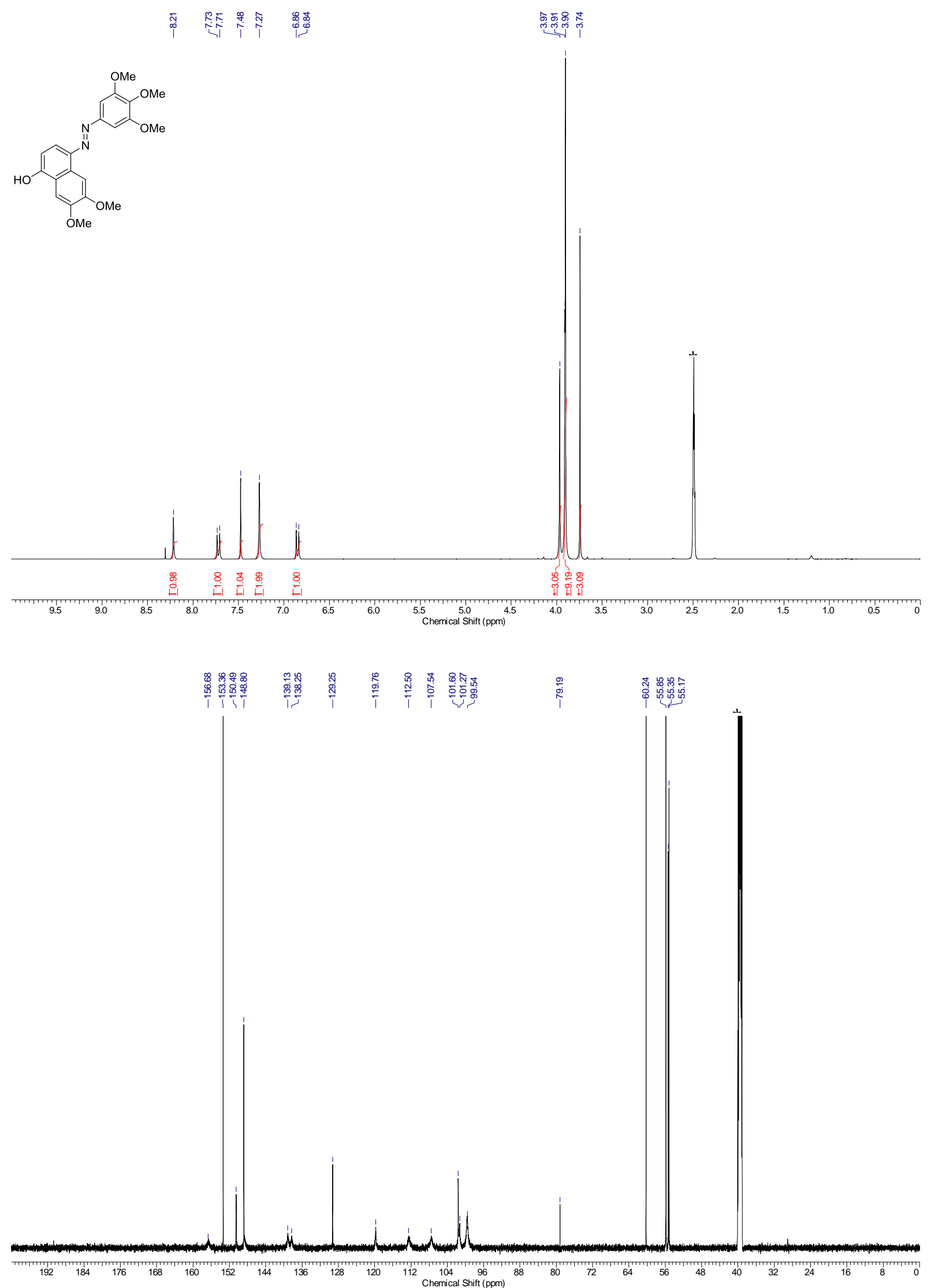

${ }^{1} \mathrm{H}-\mathrm{NMR}\left(600 \mathrm{MHz}\right.$, DMSO- $d_{6}$ ) and ${ }^{13} \mathrm{C}-\mathrm{NMR}\left(151 \mathrm{MHz}\right.$, DMSO- $\left.d_{6}\right)$ spectrum of compound 22d. * = NMR-solvent 


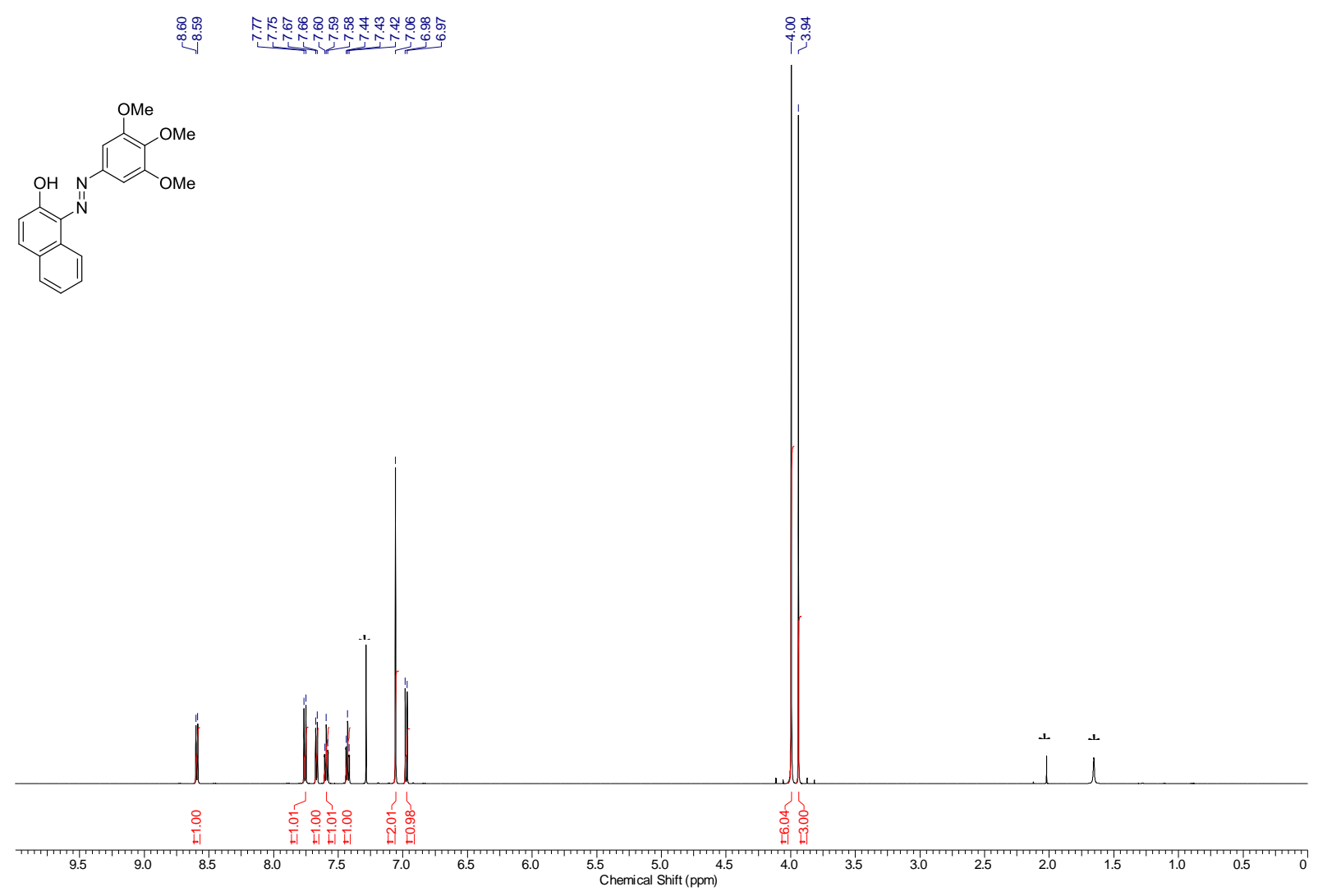

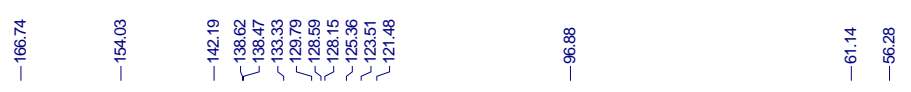

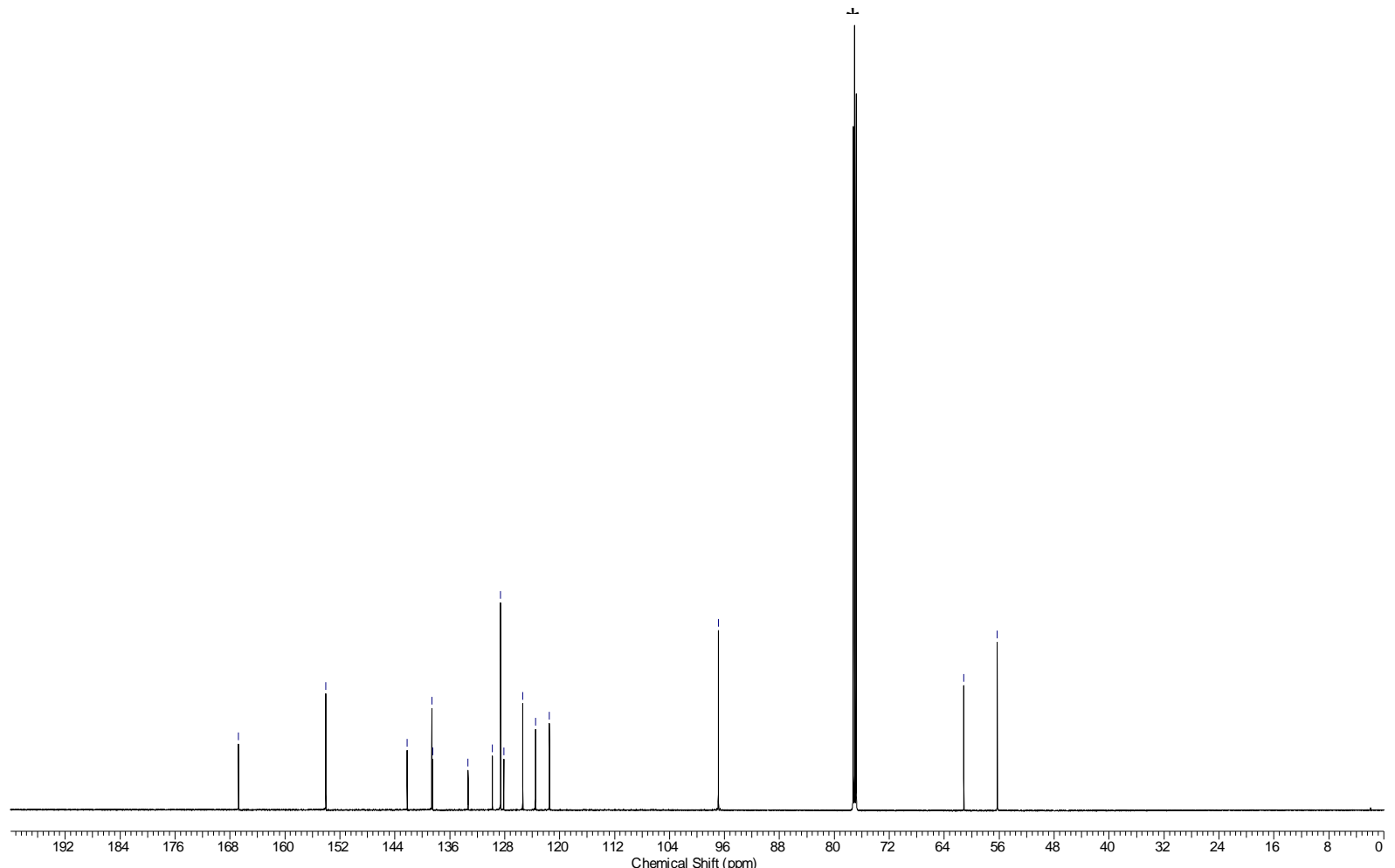

${ }^{1} \mathrm{H}-\mathrm{NMR}\left(600 \mathrm{MHz}, \mathrm{CDCl}_{3}\right.$ ) and ${ }^{13} \mathrm{C}-\mathrm{NMR}\left(151 \mathrm{MHz}, \mathrm{CDCl}_{3}\right)$ spectrum of compound 23. * = NMR-solvent, $\mathrm{CH}_{3} \mathrm{CN}_{2} \mathrm{H}_{2} \mathrm{O}$ 

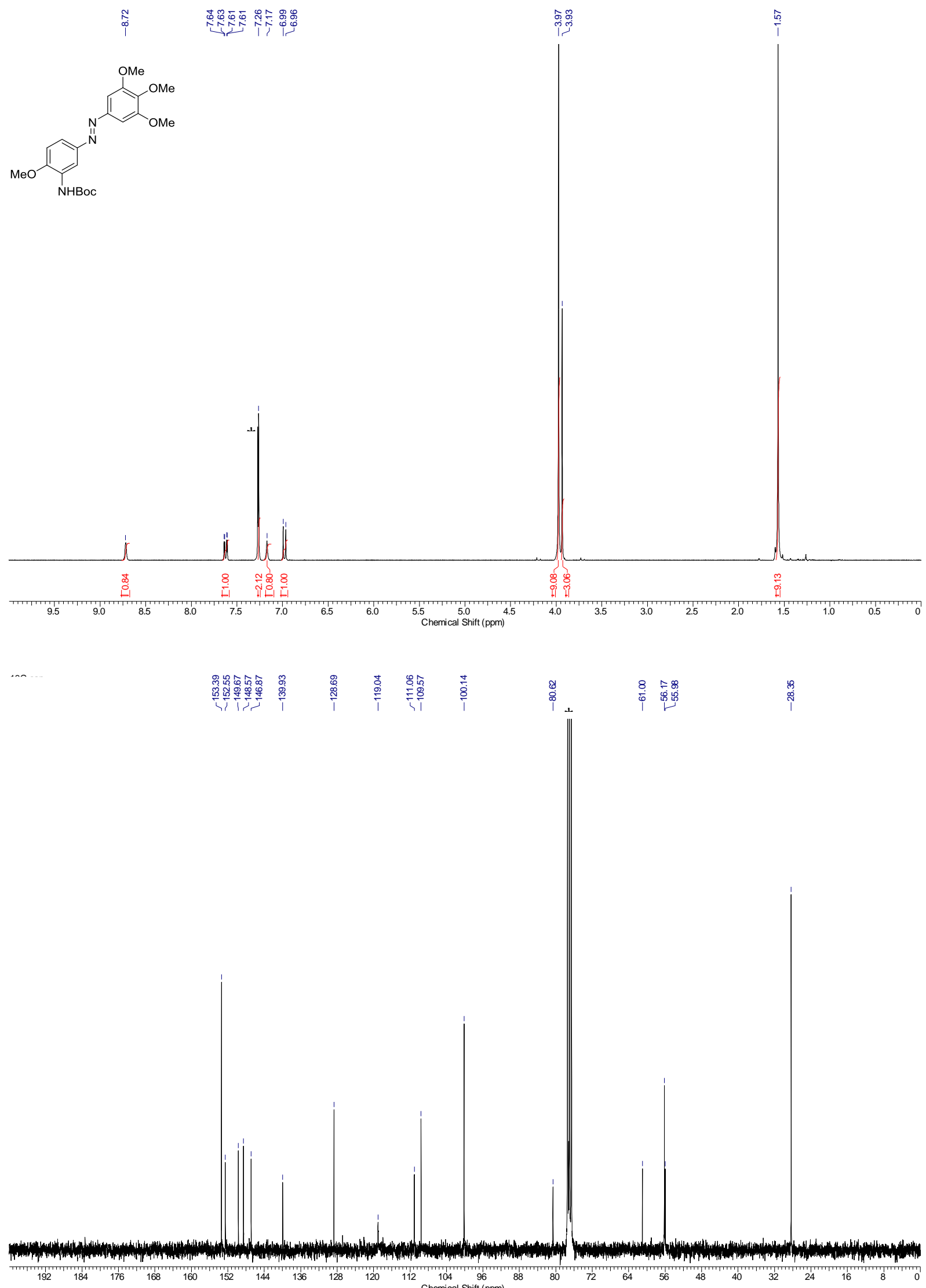

${ }^{1} \mathrm{H}-\mathrm{NMR}\left(300 \mathrm{MHz}, \mathrm{CDCl}_{3}\right)$ and ${ }^{13} \mathrm{C}-\mathrm{NMR}\left(75 \mathrm{MHz}, \mathrm{CDCl}_{3}\right.$ ) spectrum of compound 24. * = NMR-solvent 


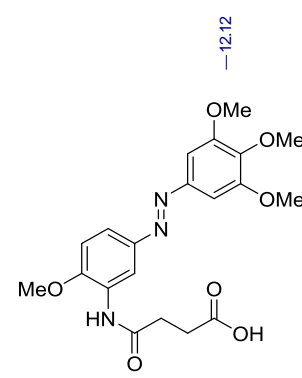

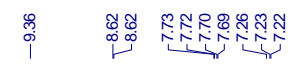

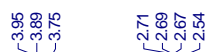

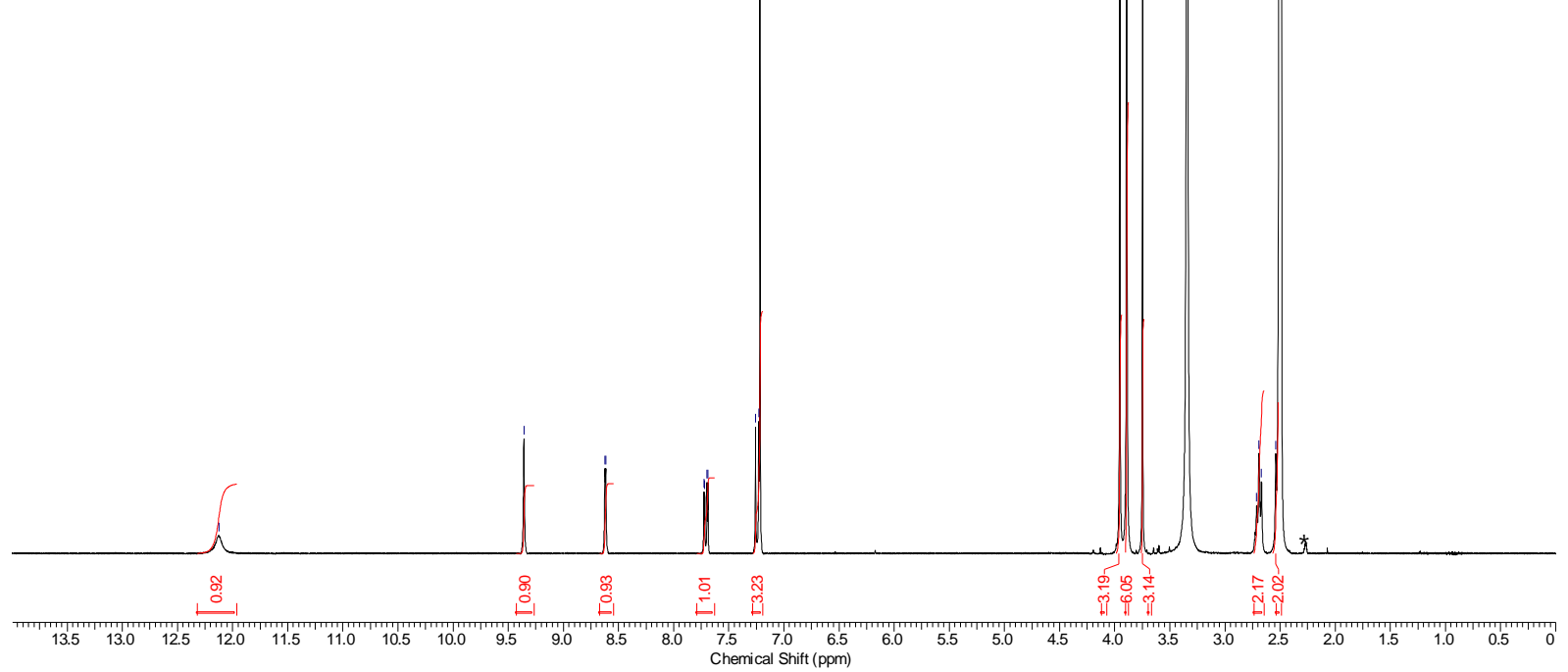

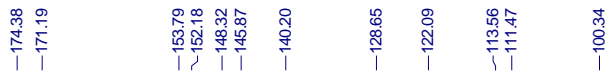

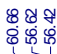

迎

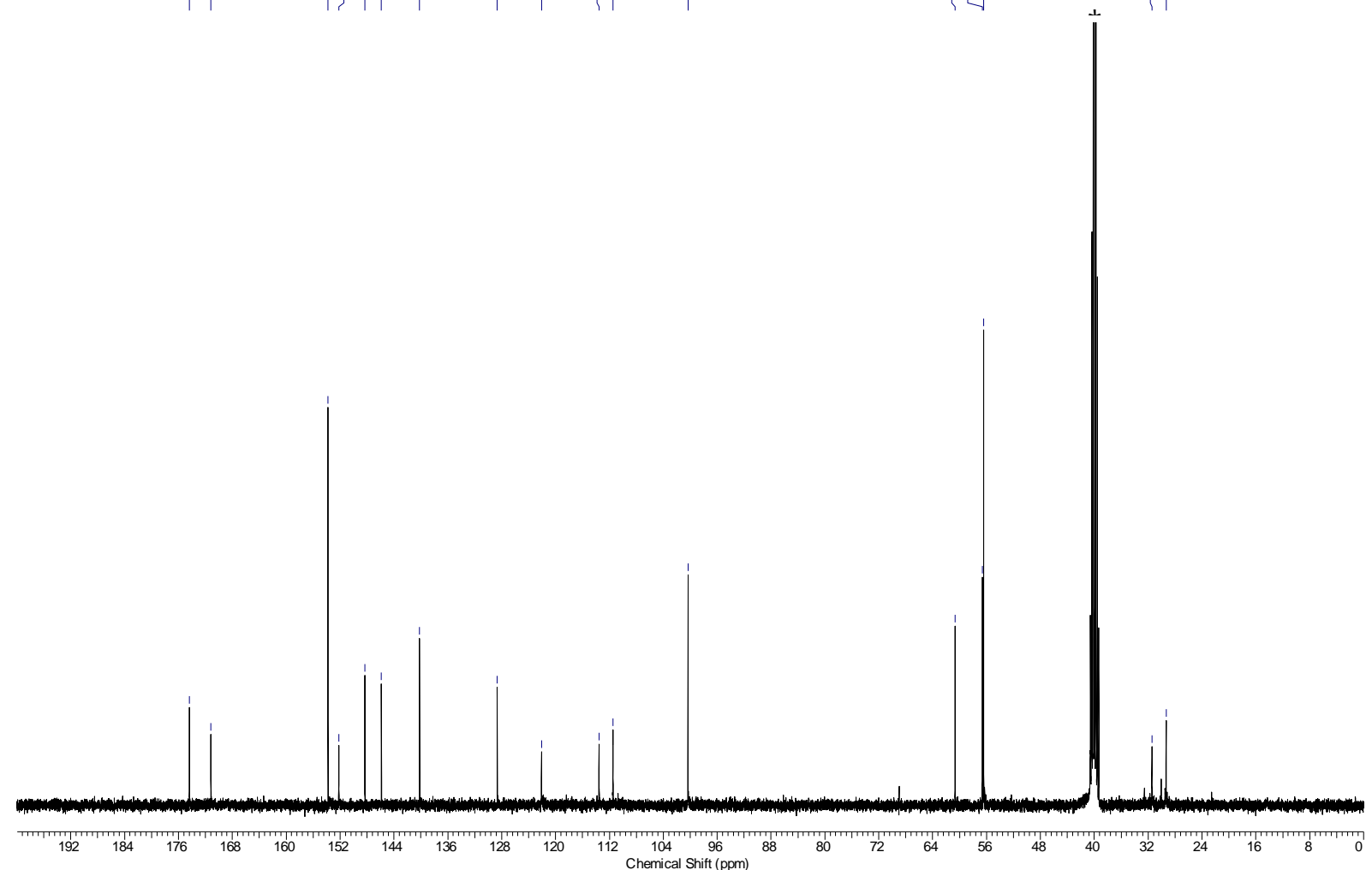

${ }^{1} \mathrm{H}-\mathrm{NMR}\left(300 \mathrm{MHz}, \mathrm{DMSO}-d_{6}\right.$ ) and ${ }^{13} \mathrm{C}-\mathrm{NMR}\left(101 \mathrm{MHz}\right.$, DMSO- $d_{6}$ ) spectrum of compound $25 . *=$ NMR-solvent, $\mathrm{H}_{2} \mathrm{O}$ 

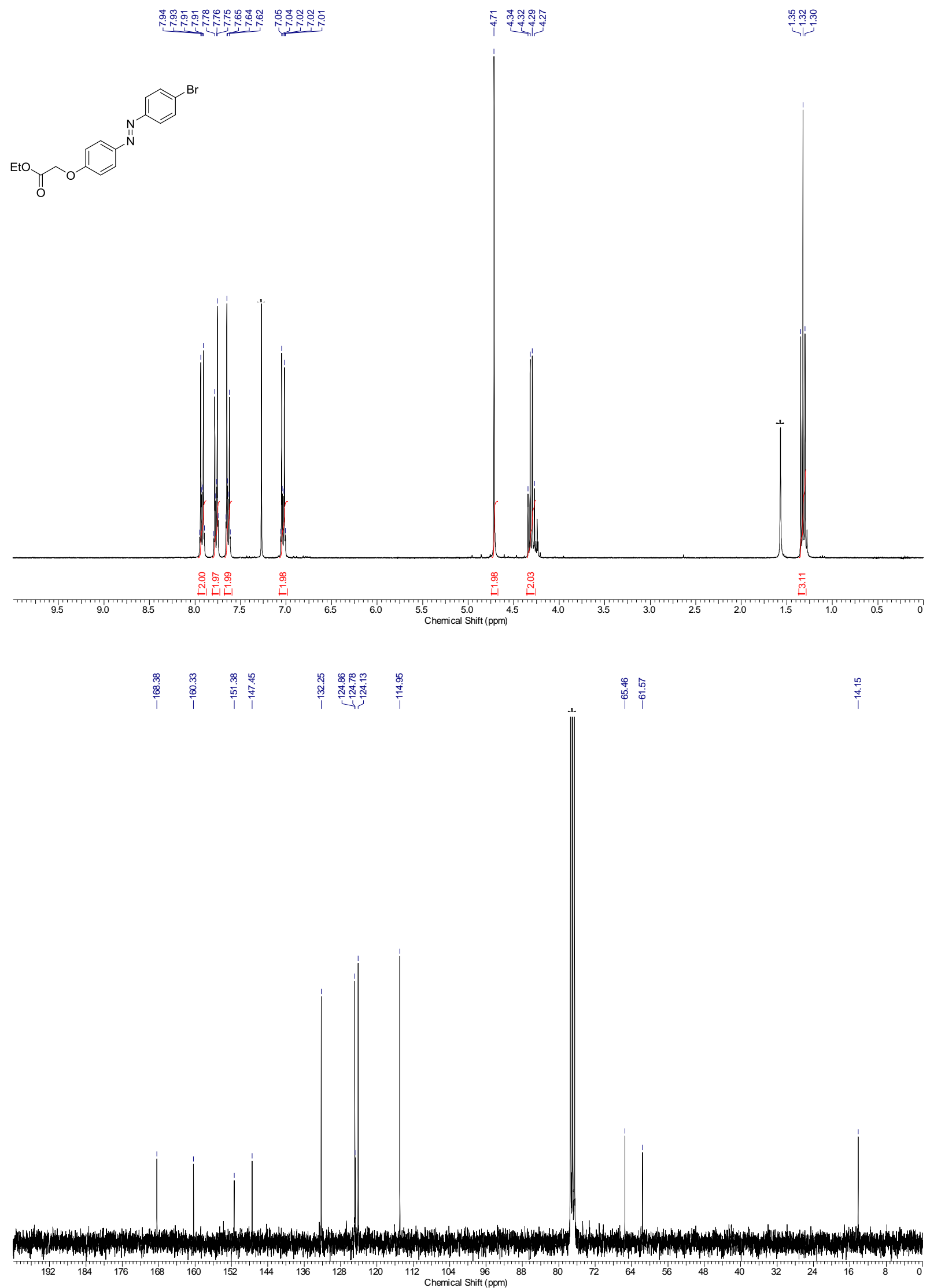

${ }^{1} \mathrm{H}-\mathrm{NMR}\left(300 \mathrm{MHz}, \mathrm{CDCl}_{3}\right.$ ) and ${ }^{13} \mathrm{C}-\mathrm{NMR}\left(75 \mathrm{MHz}^{-\mathrm{CDCl}_{3}}\right.$ ) spectrum of compound 26a. * = NMR-solvent, $\mathrm{H}_{2} \mathrm{O}$ 


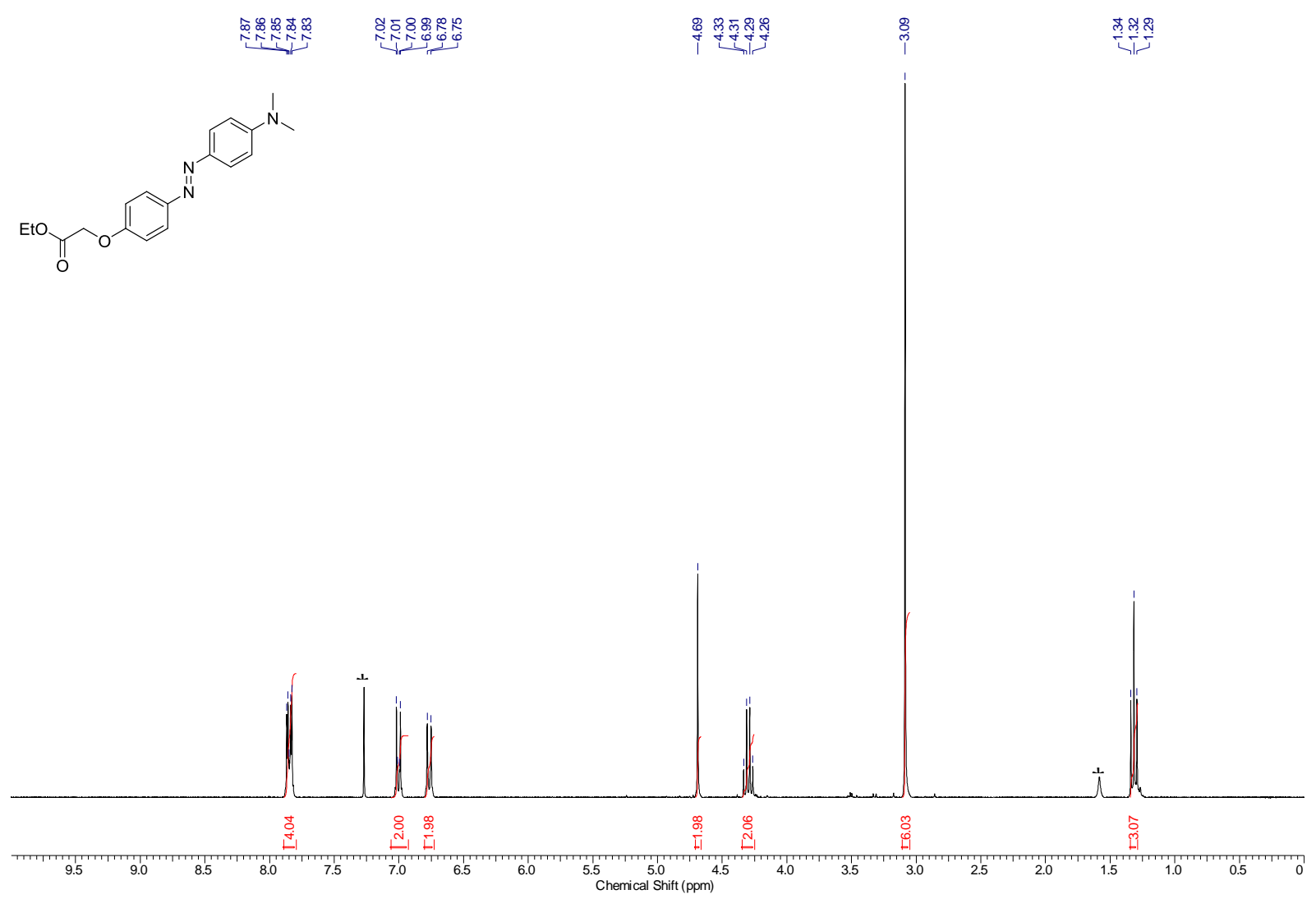

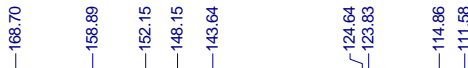

$\begin{array}{lll}8 & 8 \\ 0 & 0 \\ 1 & 0 & 1 \\ 1 & 1\end{array}$

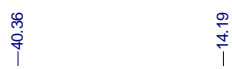

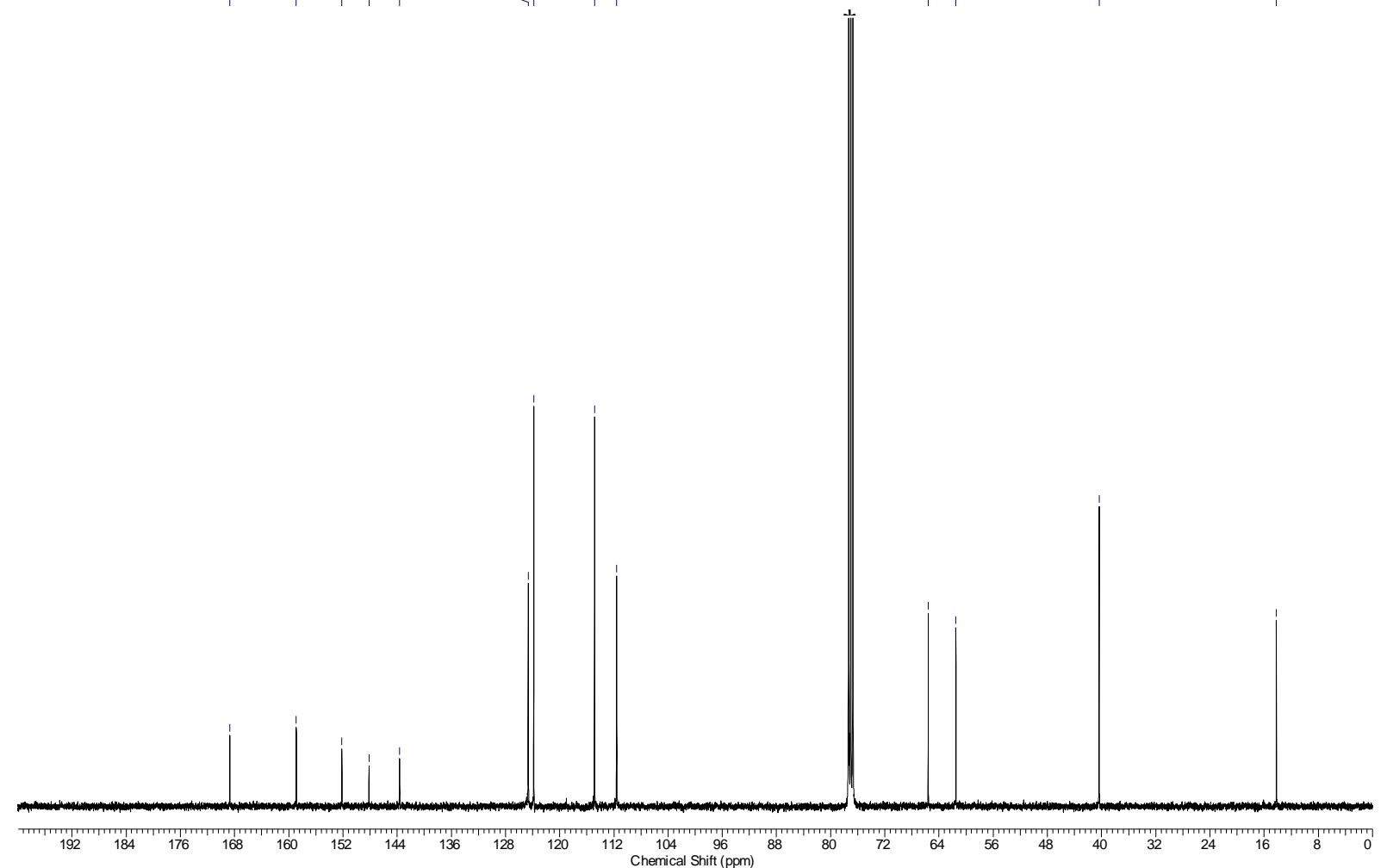

${ }^{1} \mathrm{H}-\mathrm{NMR}\left(300 \mathrm{MHz}, \mathrm{CDCl}_{3}\right.$ ) and ${ }^{13} \mathrm{C}-\mathrm{NMR}\left(101 \mathrm{MHz}^{\text {Chem }} \mathrm{CDCl}_{3}\right.$ ) spectrum of compound 26b. * = NMR-solvent, $\mathrm{H}_{2} \mathrm{O}$ 

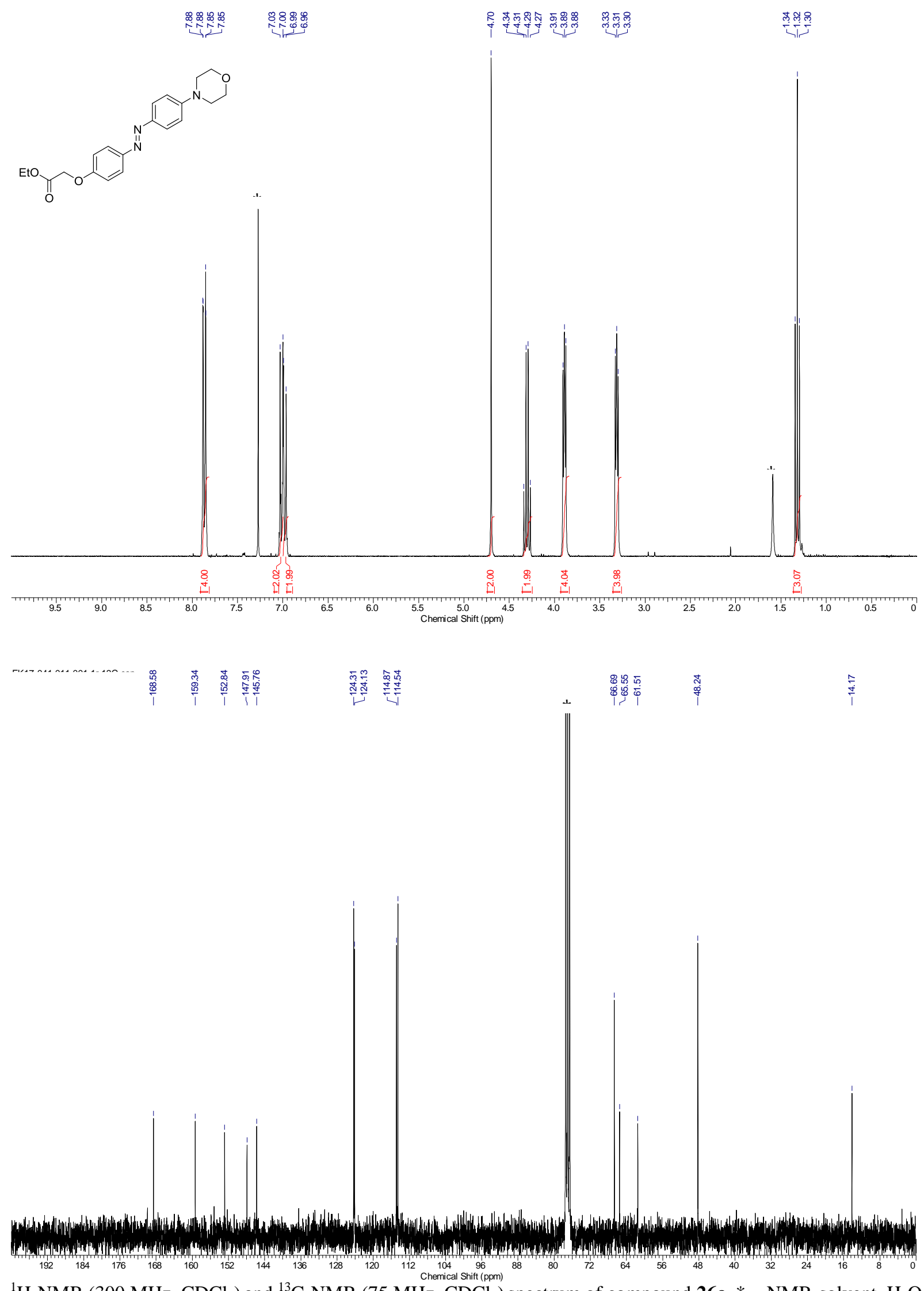

${ }^{1} \mathrm{H}-\mathrm{NMR}\left(300 \mathrm{MHz}, \mathrm{CDCl}_{3}\right)$ and ${ }^{13} \mathrm{C}-\mathrm{NMR}\left(75 \mathrm{MHz}^{\text {chencical hitt( (pom) }}\right.$ ) spectrum of compound 26c. * = NMR-solvent, $\mathrm{H}_{2} \mathrm{O}$ 


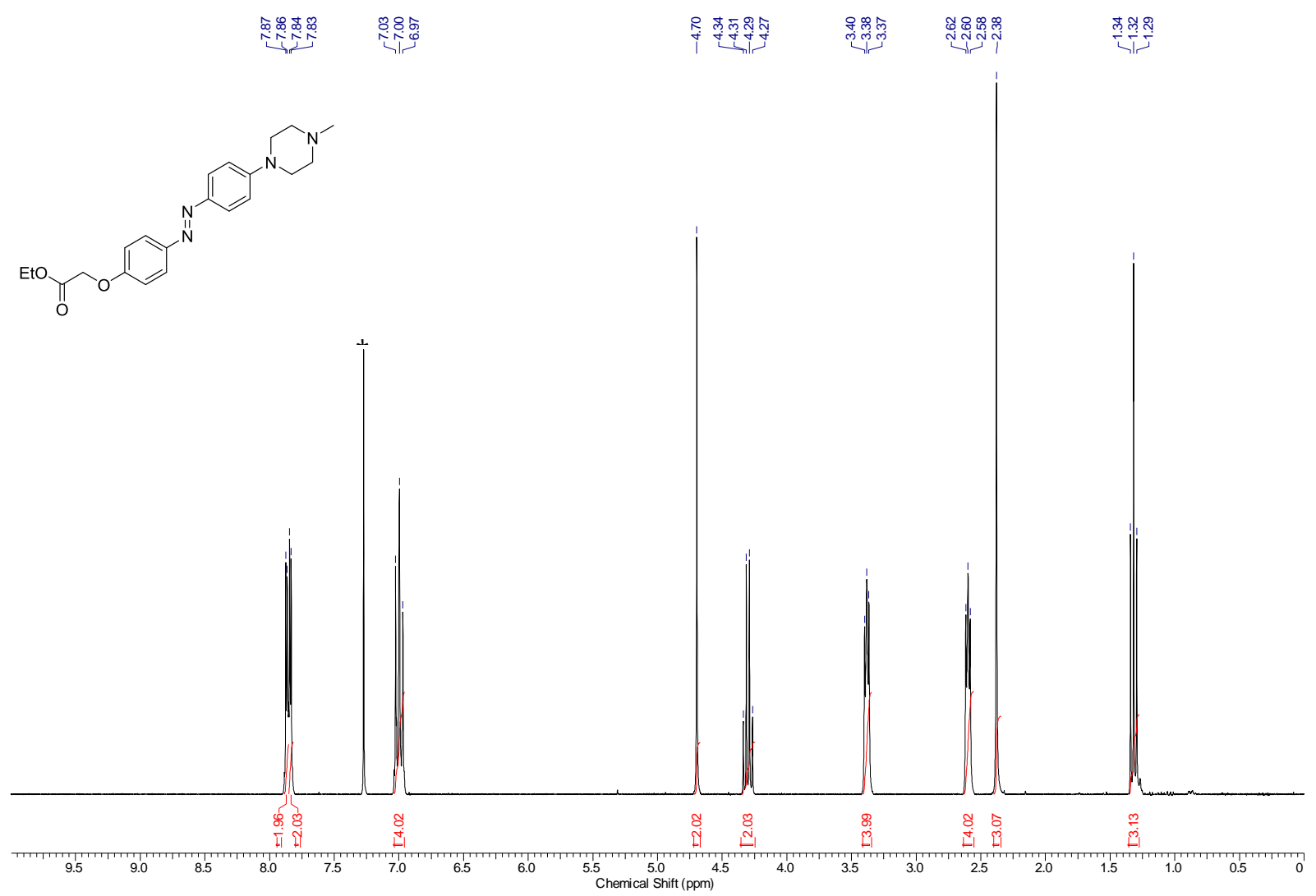

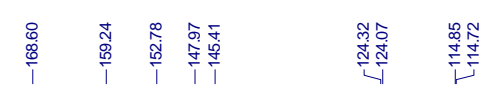

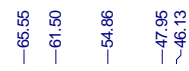

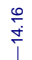

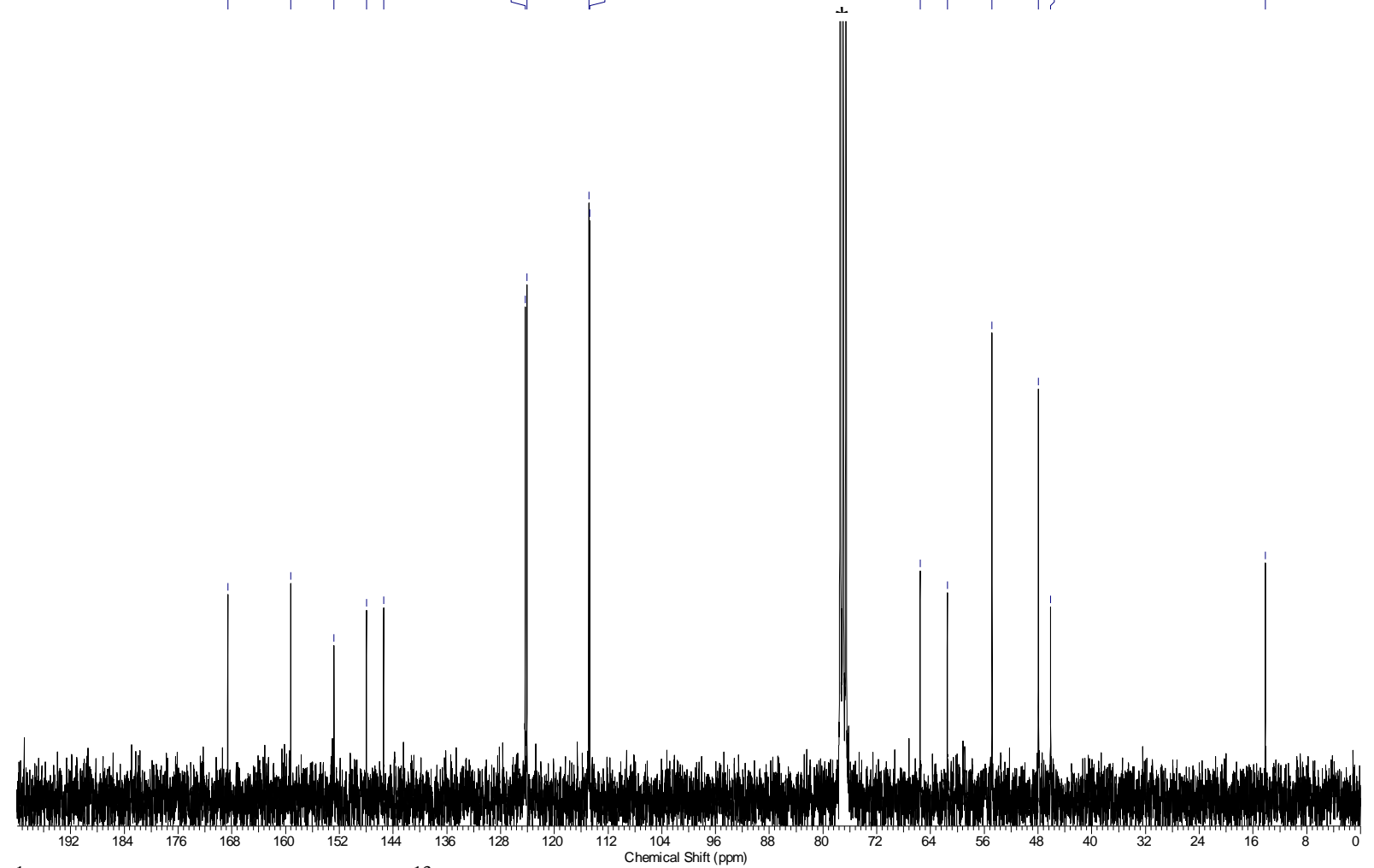

${ }^{1} \mathrm{H}-\mathrm{NMR}\left(300 \mathrm{MHz}, \mathrm{CDCl}_{3}\right.$ ) and ${ }^{13} \mathrm{C}-\mathrm{NMR}\left(75 \mathrm{MHz}, \mathrm{CDCl}_{3}\right.$ ) spectrum of compound 26d. * = NMR-solvent 


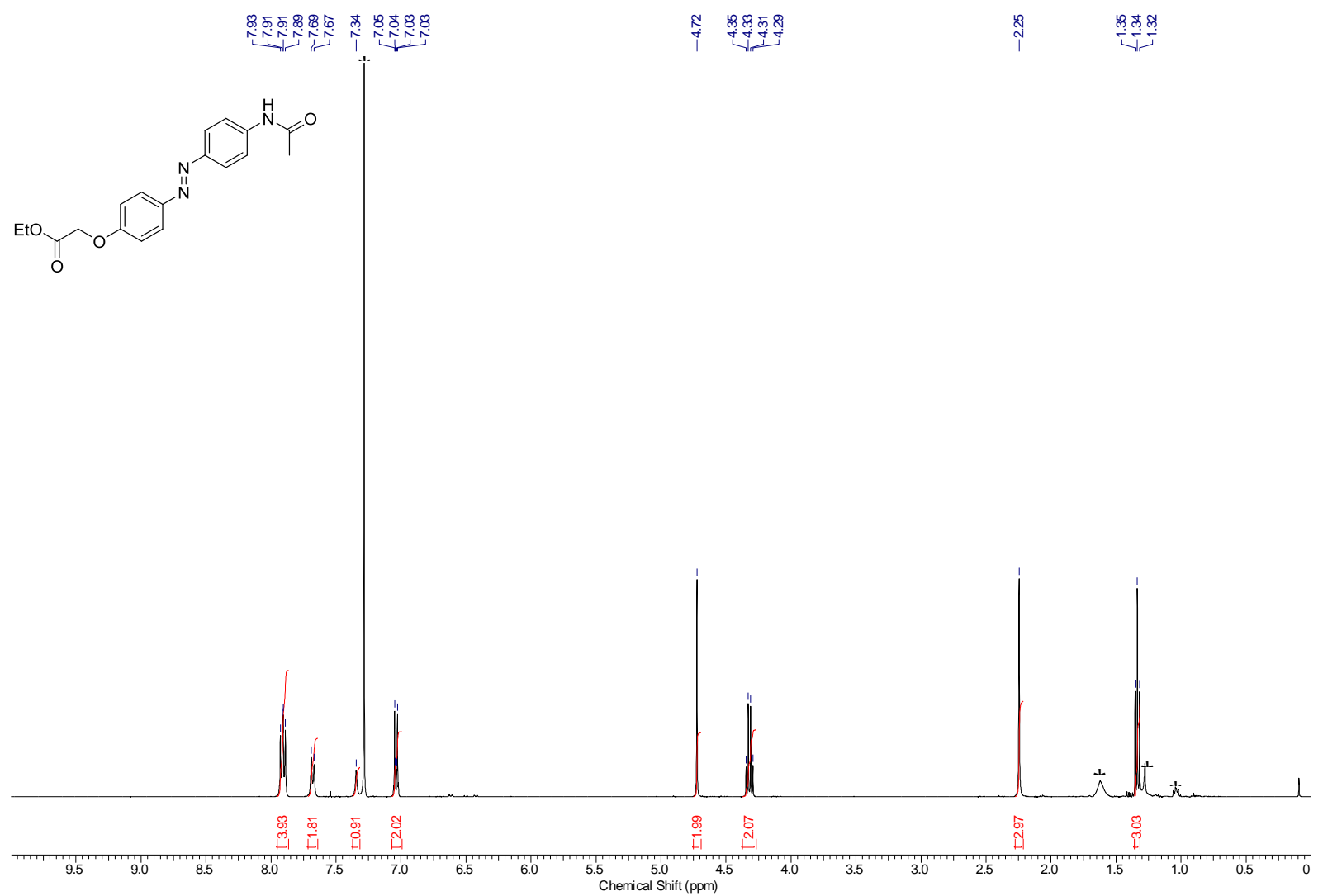

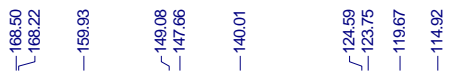

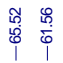
票望

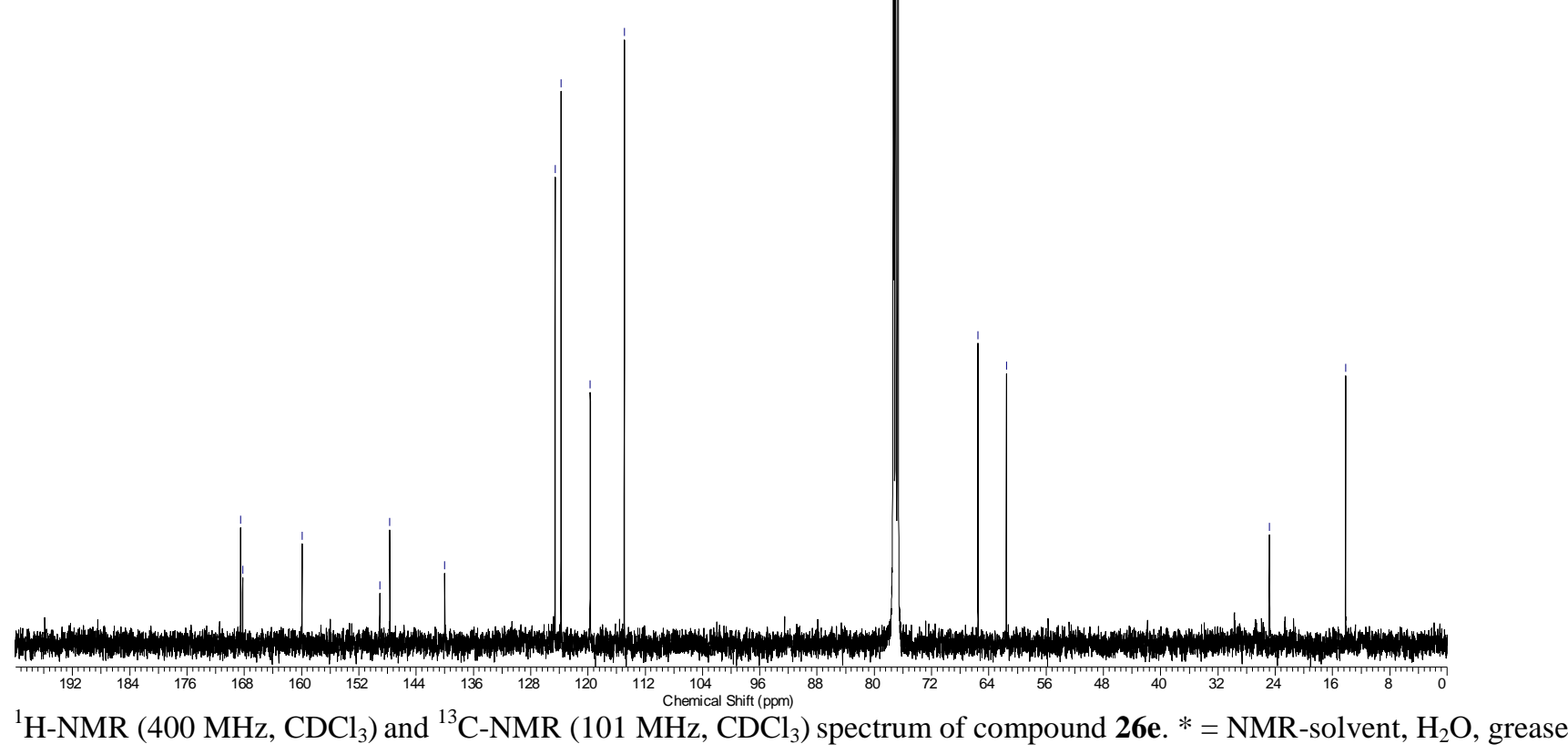



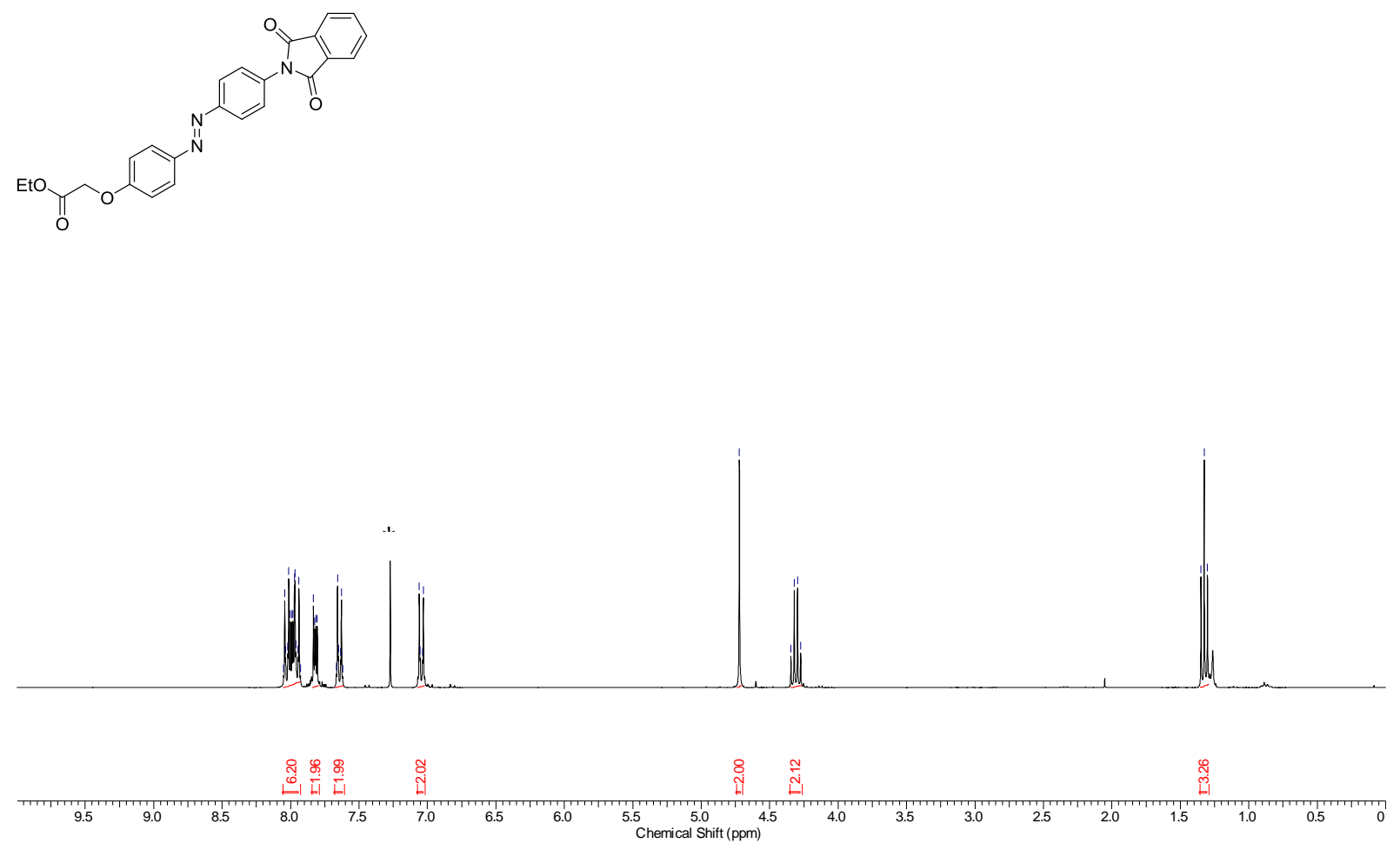

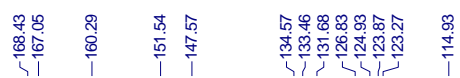

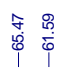

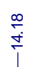

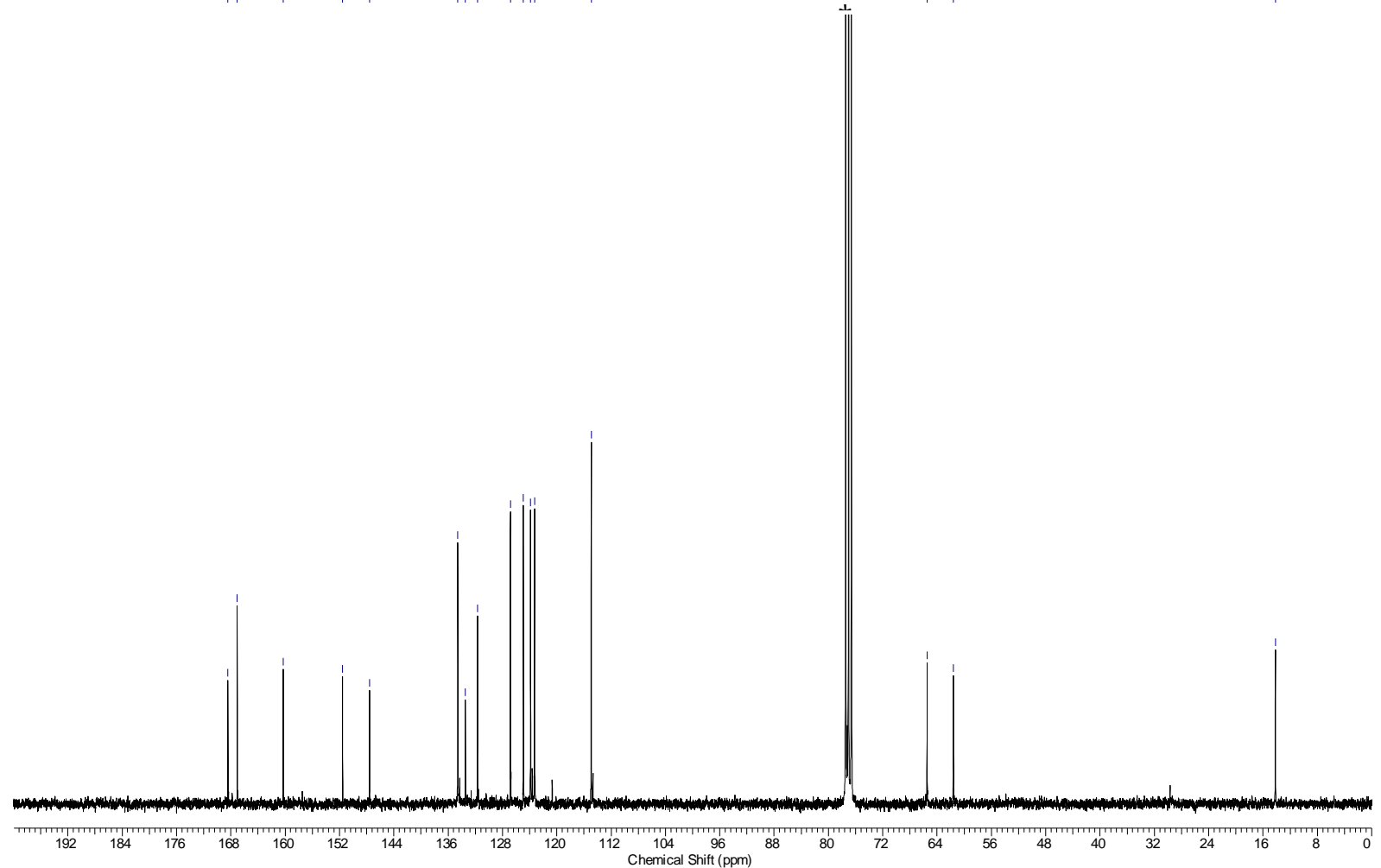

${ }^{1} \mathrm{H}-\mathrm{NMR}\left(300 \mathrm{MHz}, \mathrm{CDCl}_{3}\right.$ ) and ${ }^{13} \mathrm{C}-\mathrm{NMR}\left(75 \mathrm{MHz}, \mathrm{CDCl}_{3}\right.$ ) spectrum of compound 26f. * = NMR-solvent 
<smiles>CCOC(=O)COc1ccc(N=Nc2cc(OC)cc(OCC#N)c2)cc1</smiles>

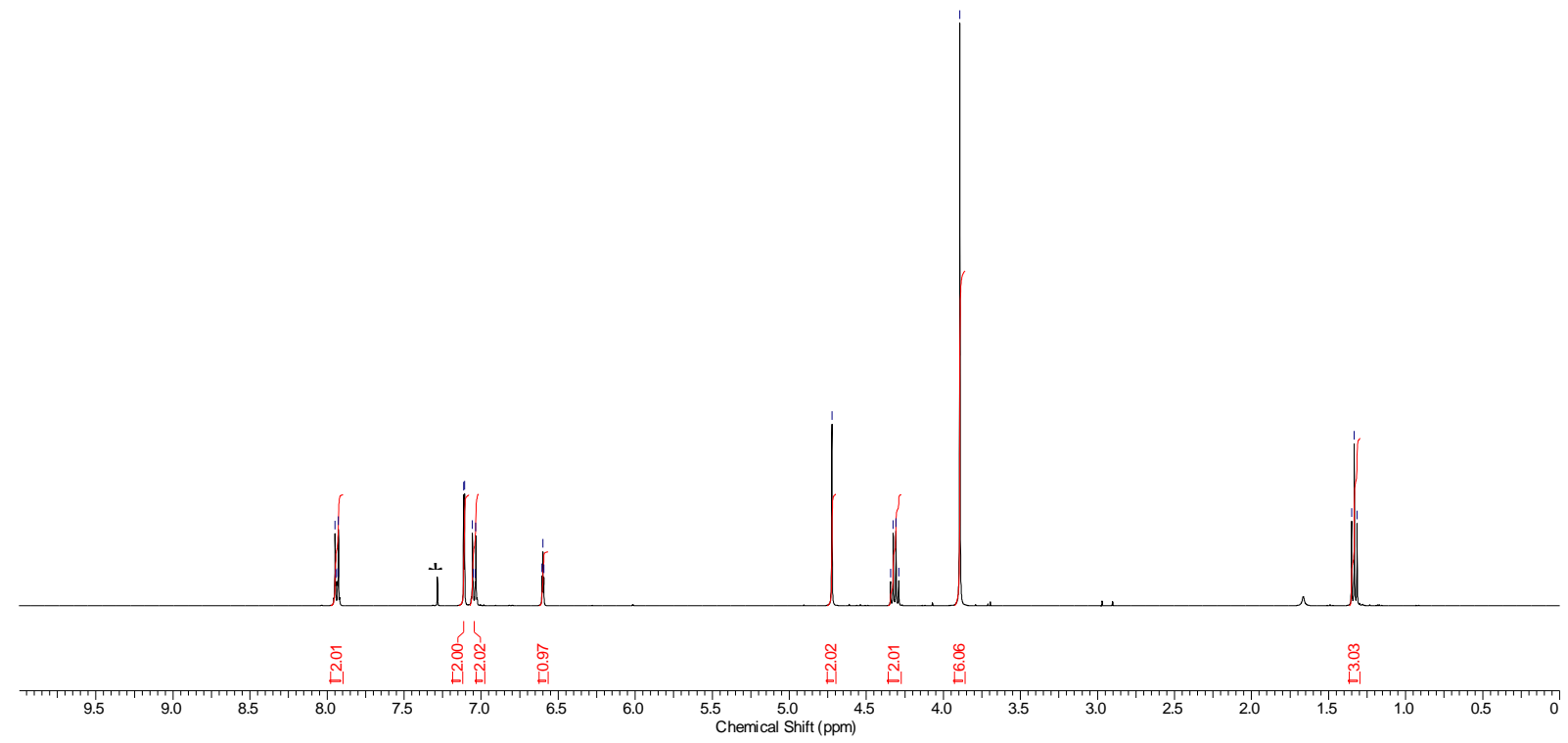

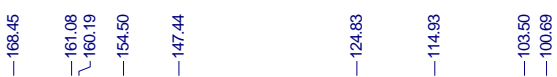

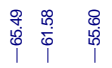

$\stackrel{\frac{\infty}{+}}{\frac{0}{+}}$ 

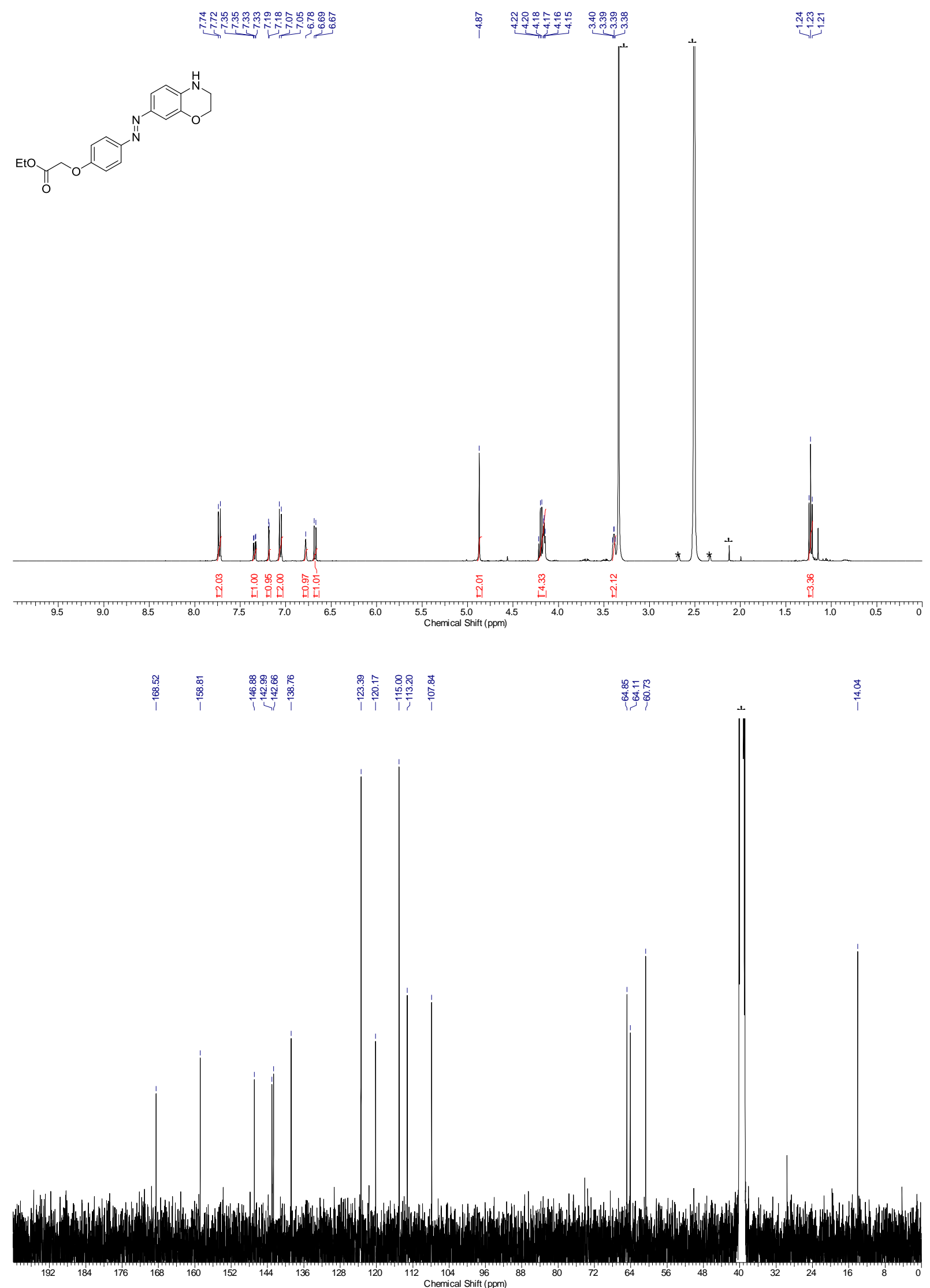

${ }^{1} \mathrm{H}-\mathrm{NMR}\left(400 \mathrm{MHz}, \mathrm{DMSO}-d_{6}\right.$ ) and ${ }^{13} \mathrm{C}-\mathrm{NMR}\left(101 \mathrm{MHz}\right.$, DMSO- $d_{6}$ ) spectrum of compound 28. $*=$ NMR-solvent, $\mathrm{H}_{2} \mathrm{O}$, $\mathrm{CH}_{3} \mathrm{CN}$ 

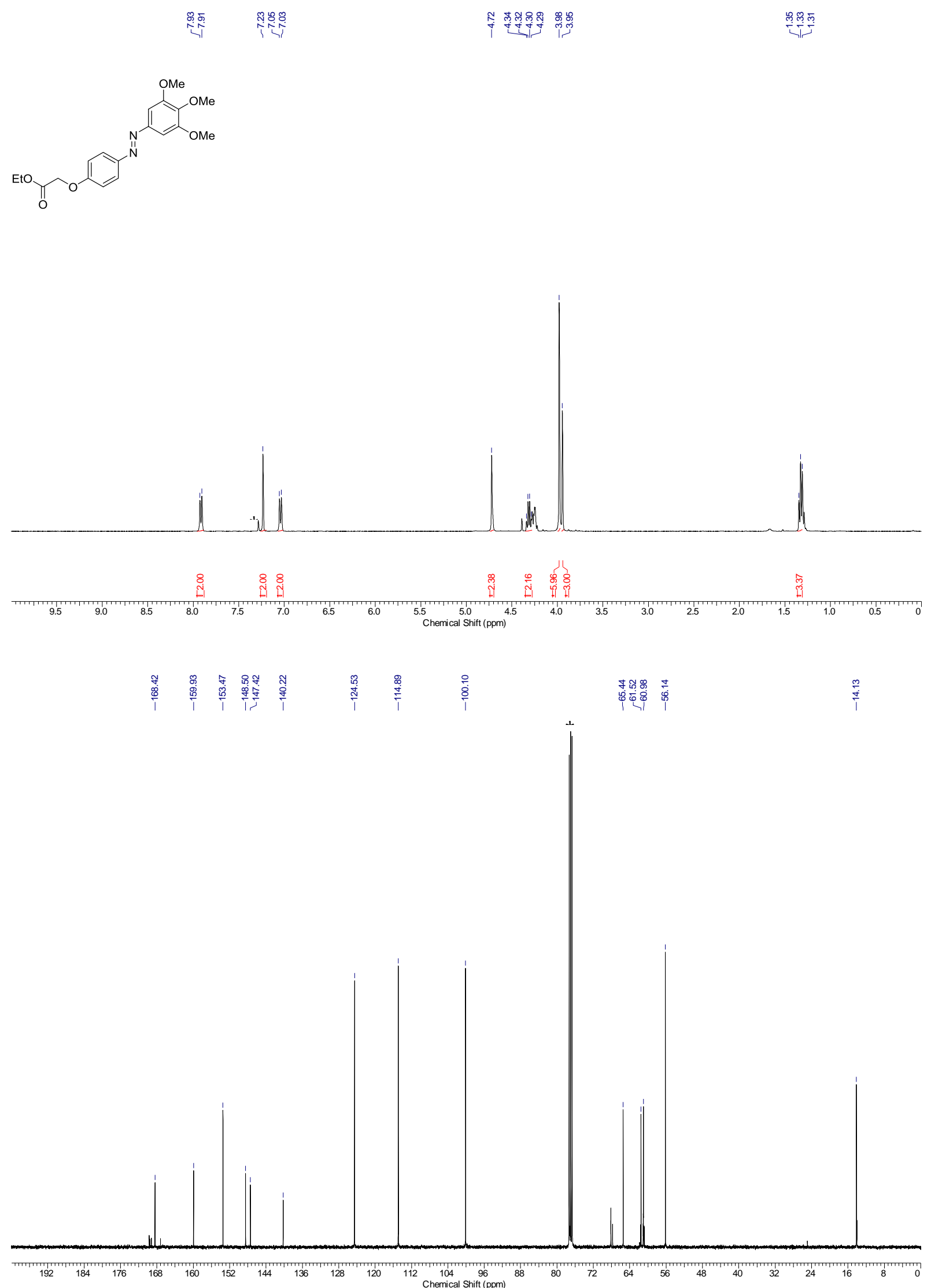

${ }^{1} \mathrm{H}-\mathrm{NMR}\left(400 \mathrm{MHz}, \mathrm{CDCl}_{3}\right)$ and ${ }^{13} \mathrm{C}-\mathrm{NMR}\left(101 \mathrm{MHz}^{\mathrm{C}} \mathrm{CDCl}_{3}\right)$ spectrum of compound 29a/32c. * = NMR-solvent 


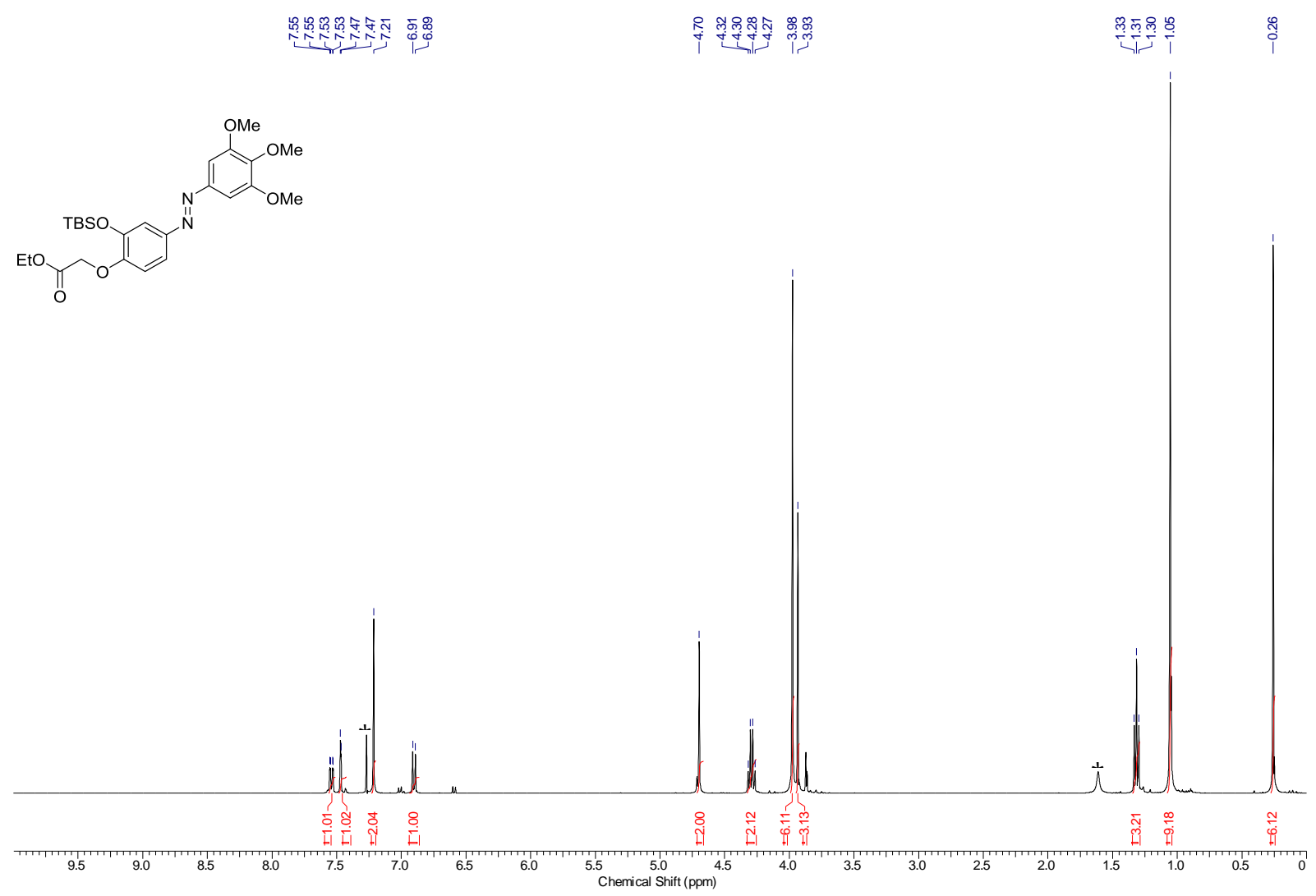

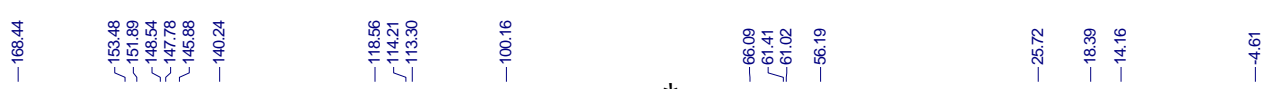

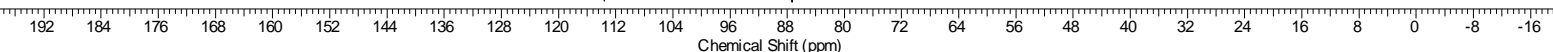

${ }^{1} \mathrm{H}-\mathrm{NMR}\left(400 \mathrm{MHz}, \mathrm{CDCl}_{3}\right.$ ) and ${ }^{13} \mathrm{C}-\mathrm{NMR}\left(101 \mathrm{MHz}, \mathrm{CDCl}_{3}\right.$ ) spectrum of compound 29b. * = NMR-solvent, $\mathrm{H}_{2} \mathrm{O}$ 

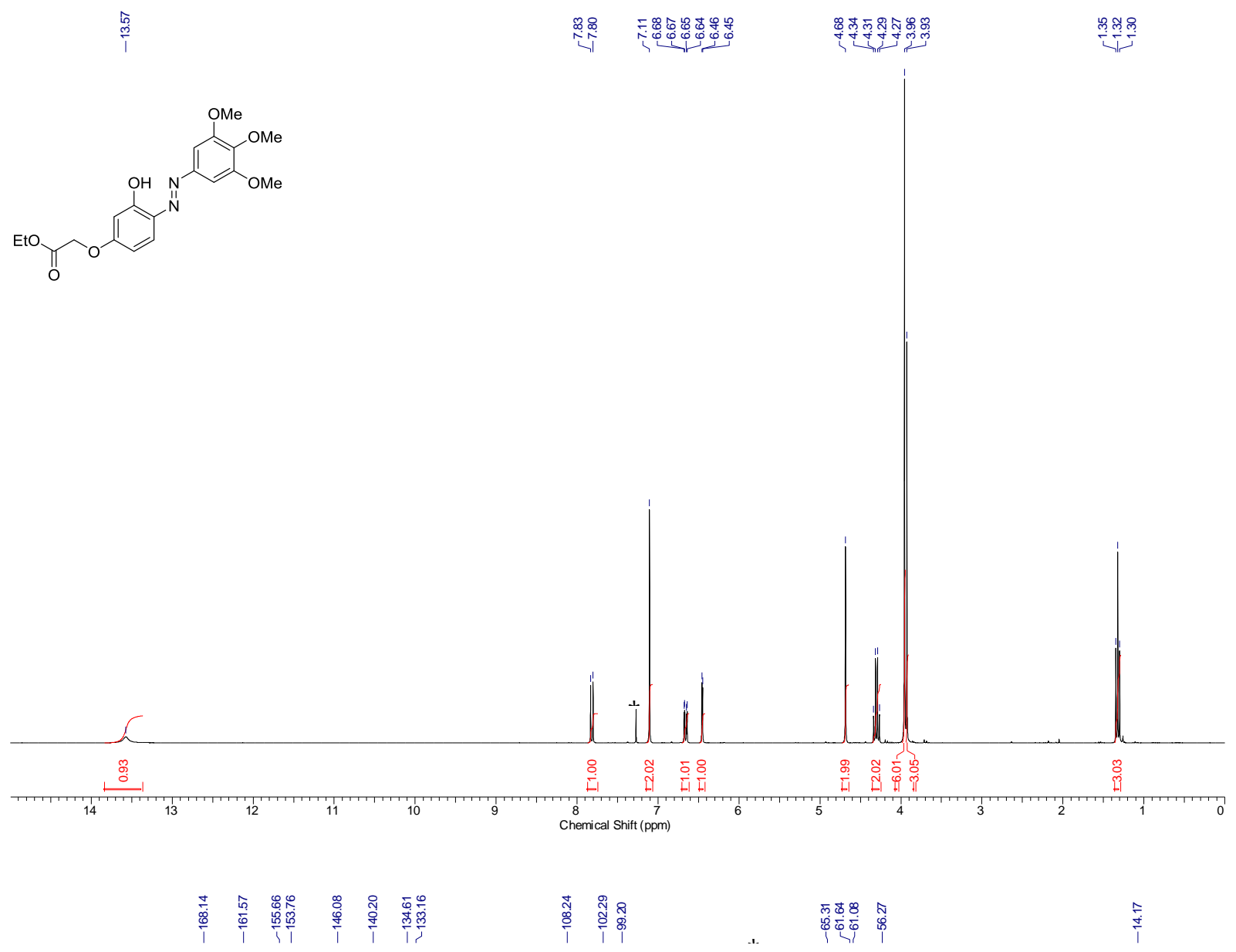

1.

${ }^{1} \mathrm{H}-\mathrm{NMR}\left(300 \mathrm{MHz}, \mathrm{CDCl}_{3}\right.$ ) and ${ }^{13} \mathrm{C}-\mathrm{NMR}\left(101 \mathrm{MHz}, \mathrm{CDCl}_{3}\right)$ spectrum of compound 29c. * = NMR-solvent 


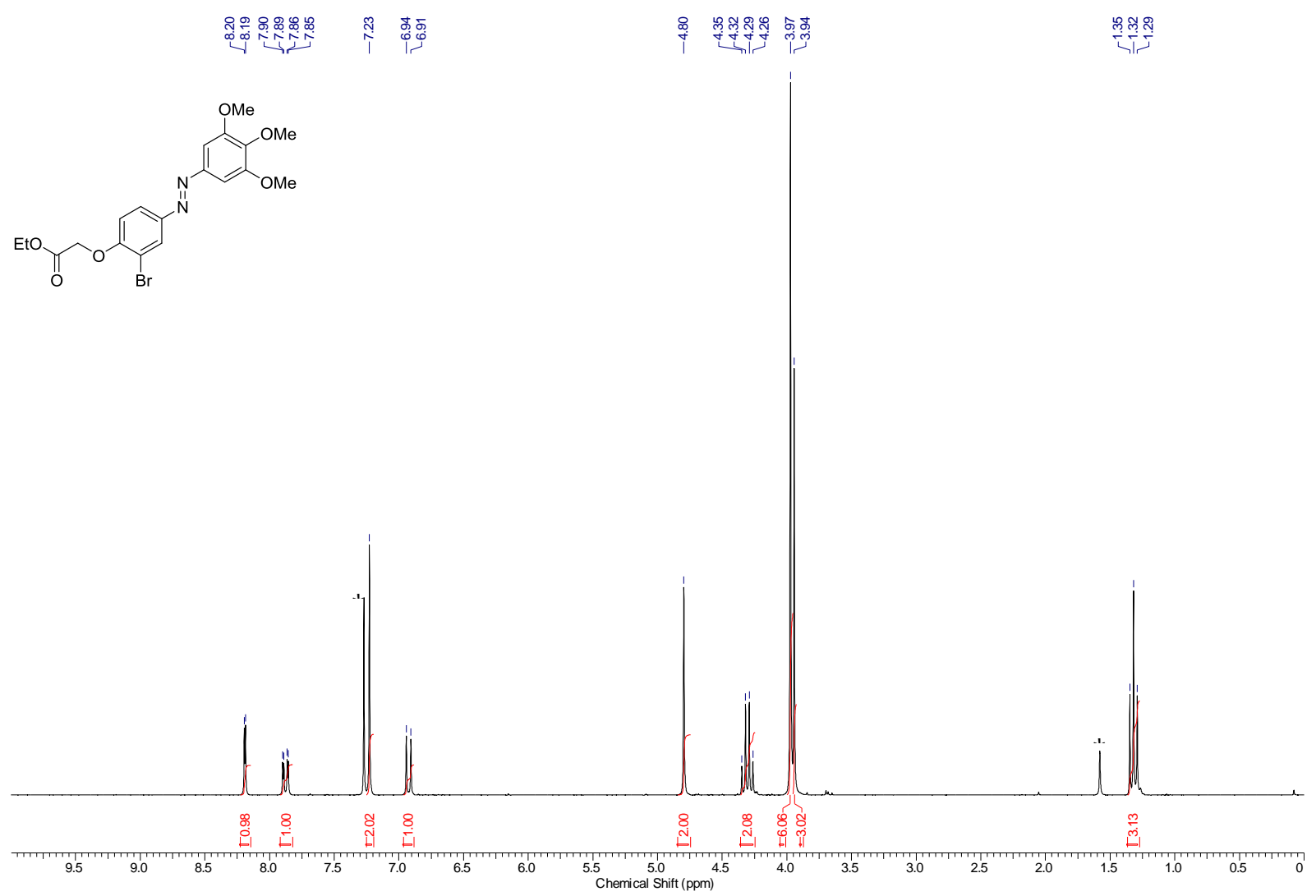

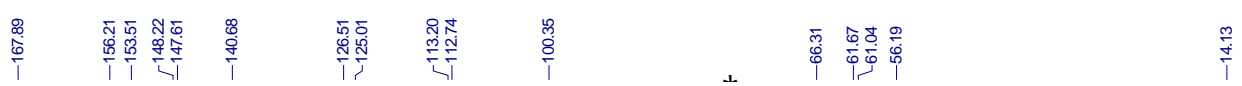

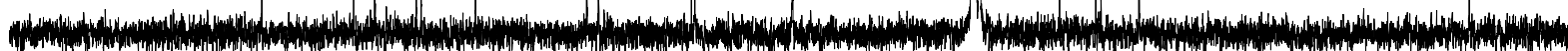

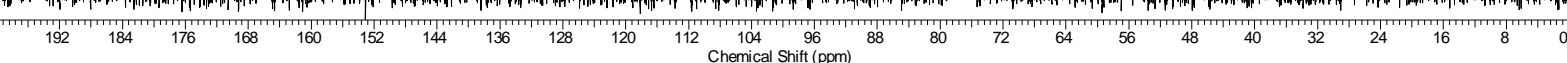

${ }^{1} \mathrm{H}-\mathrm{NMR}\left(250 \mathrm{MHz}, \mathrm{CDCl}_{3}\right)$ and ${ }^{13} \mathrm{C}-\mathrm{NMR}\left(63 \mathrm{MHz}^{-\mathrm{CDCl}_{3}}\right)$ spectrum of compound 29d. * = NMR-solvent, $\mathrm{H}_{2} \mathrm{O}$ 


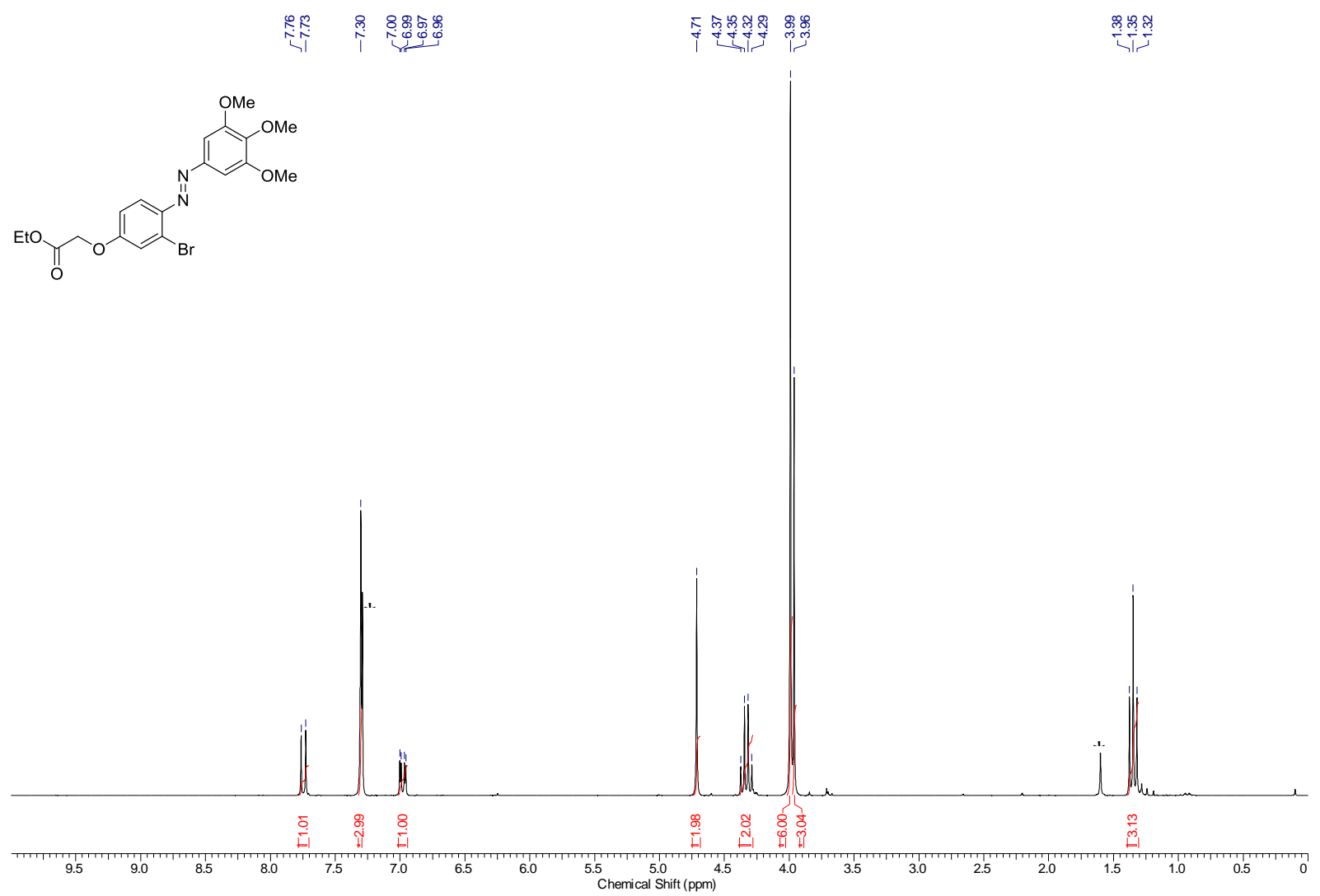

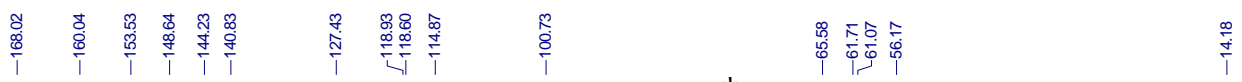

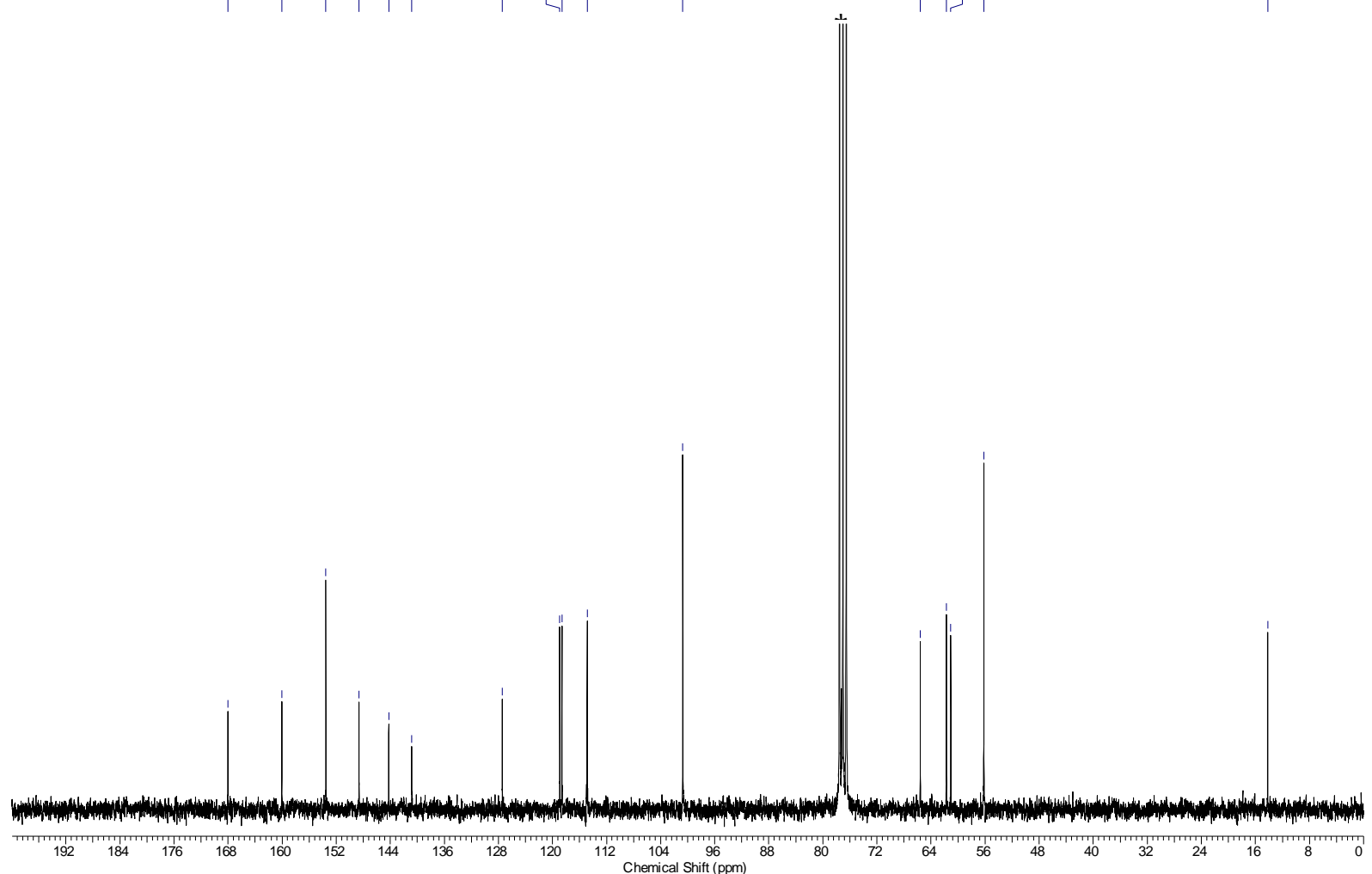

${ }^{1} \mathrm{H}-\mathrm{NMR}\left(250 \mathrm{MHz}, \mathrm{CDCl}_{3}\right)$ and ${ }^{13} \mathrm{C}-\mathrm{NMR}\left(63 \mathrm{MHz}^{\mathrm{C}} \mathrm{CDCl}_{3}\right)$ spectrum of compound 29e. * = NMR-solvent, $\mathrm{H}_{2} \mathrm{O}$ 


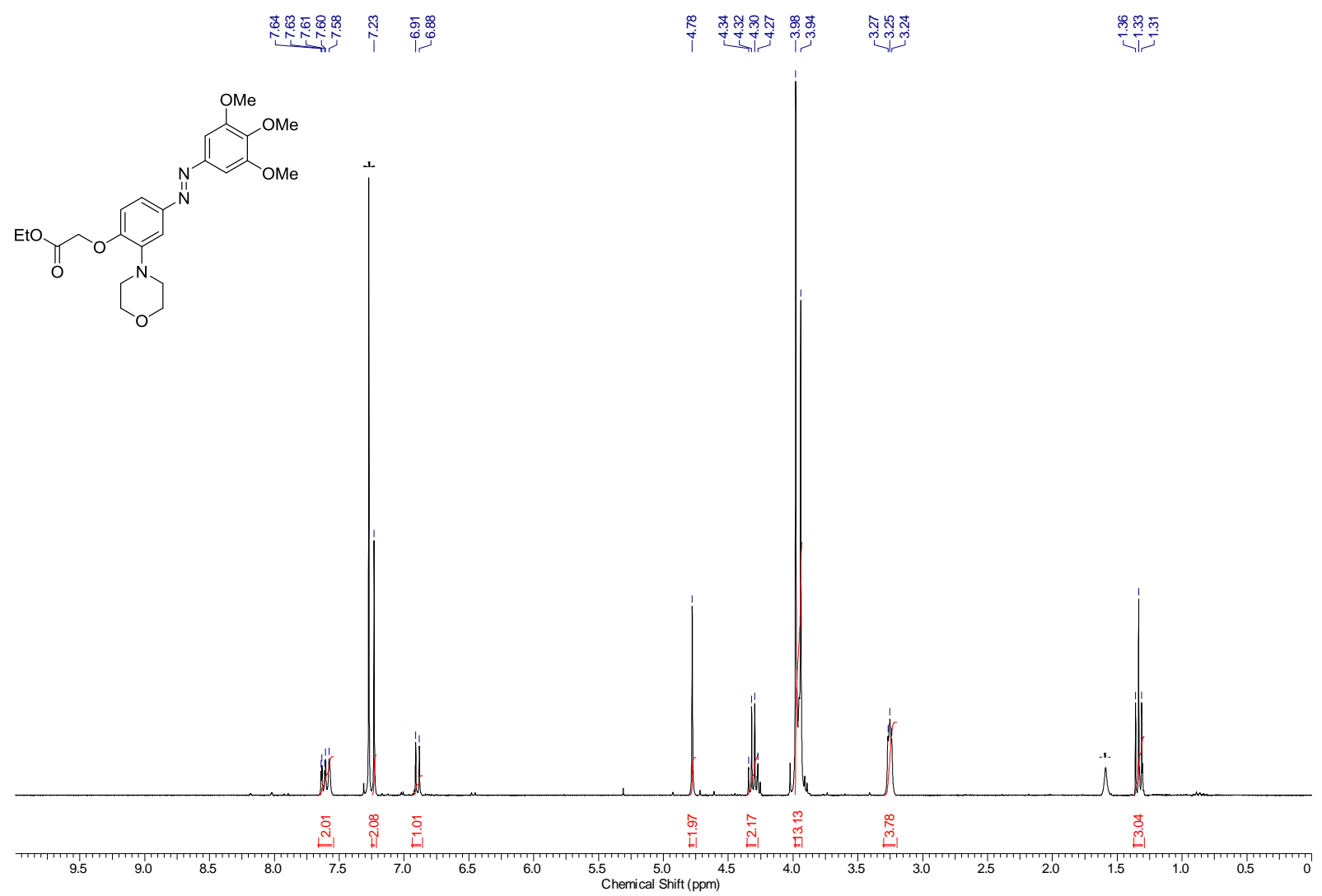

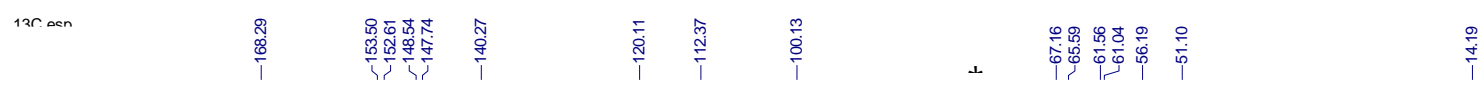

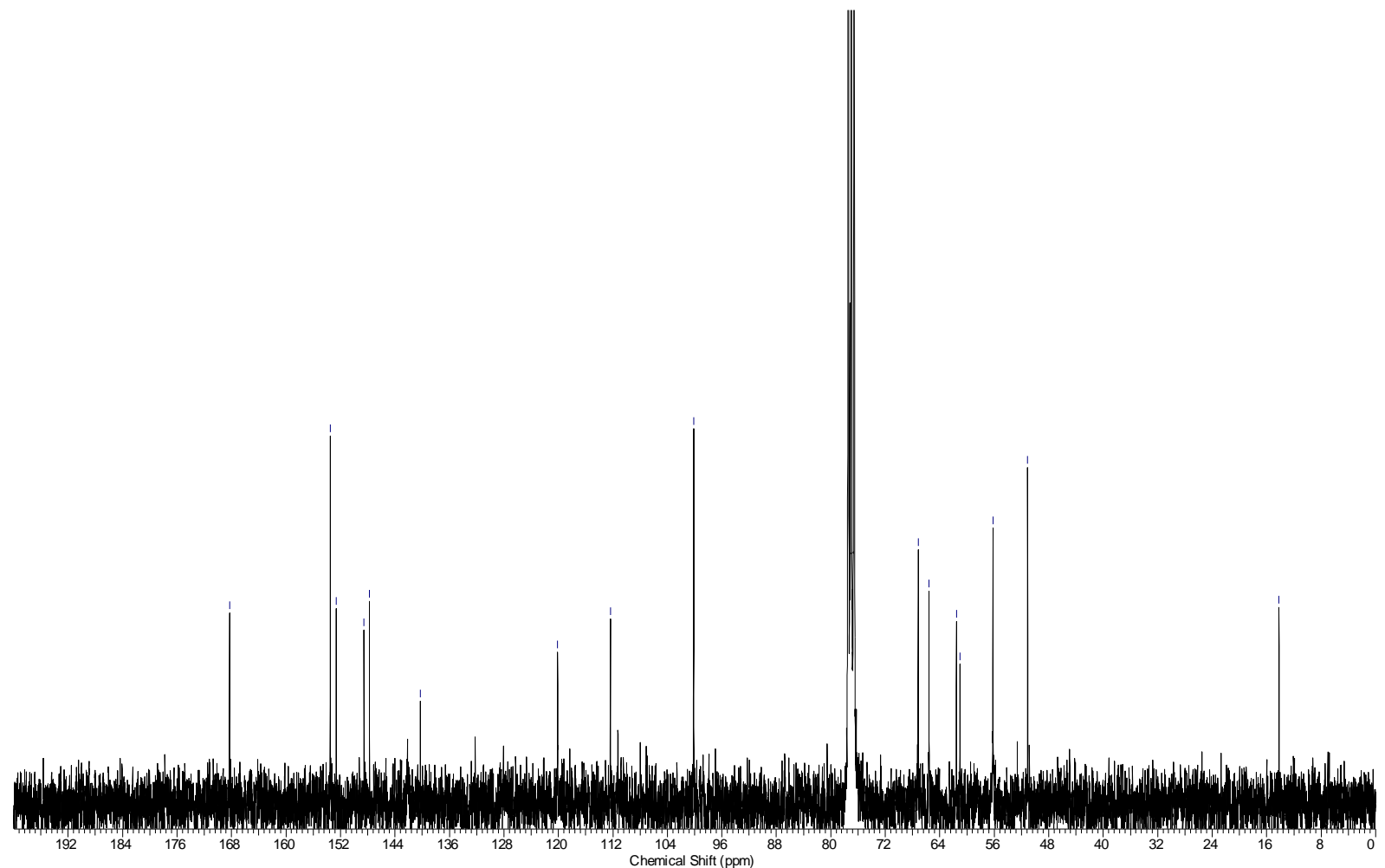

${ }^{1} \mathrm{H}-\mathrm{NMR}\left(250 \mathrm{MHz}, \mathrm{CDCl}_{3}\right.$ ) and ${ }^{13} \mathrm{C}-\mathrm{NMR}\left(63 \mathrm{MHz}, \mathrm{CDCl}_{3}\right.$ ) spectrum of compound 29f. * = NMR-solvent, $\mathrm{H}_{2} \mathrm{O}$ 


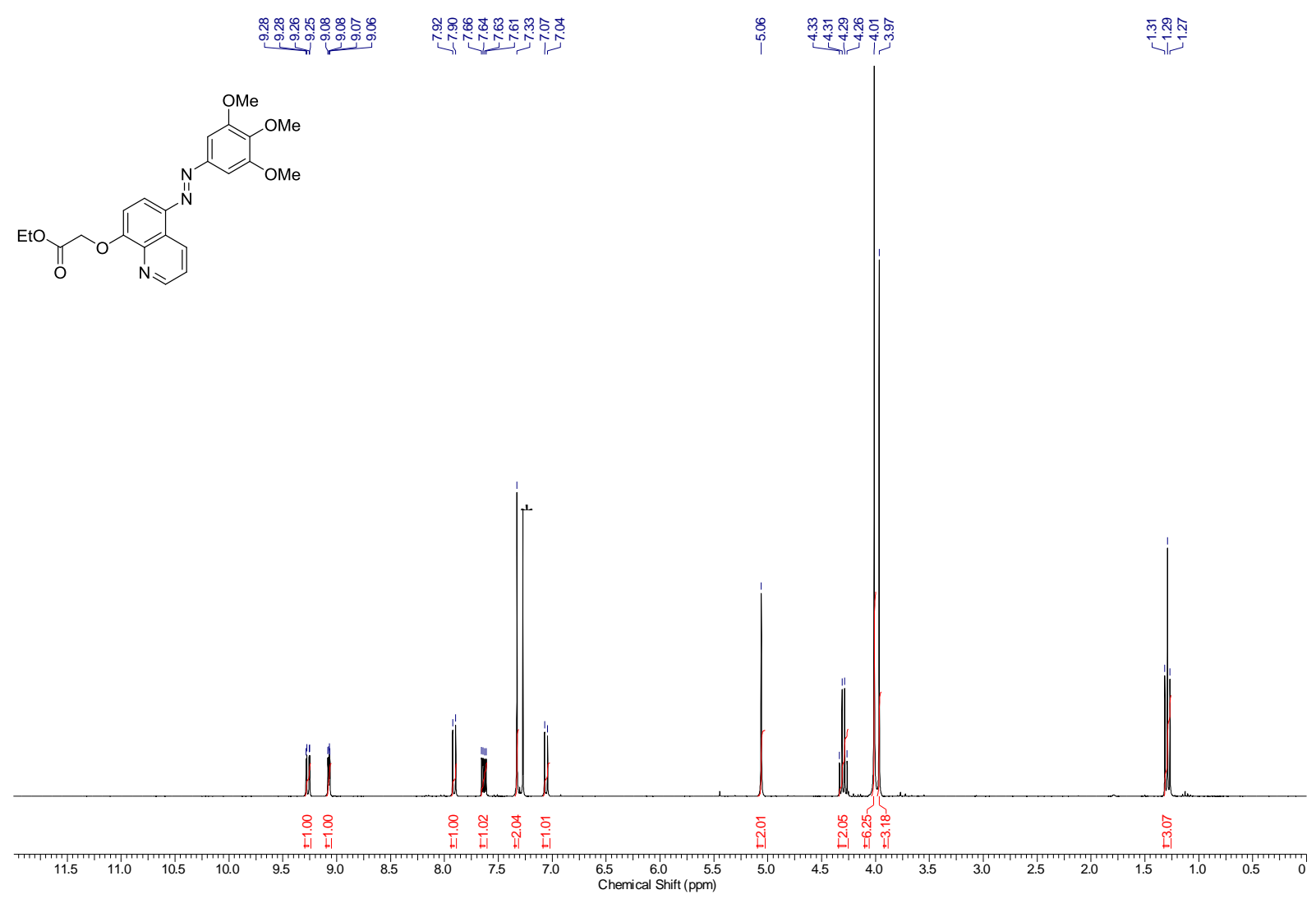

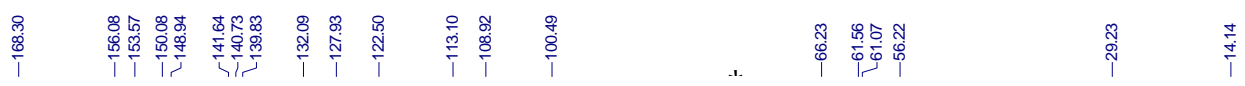

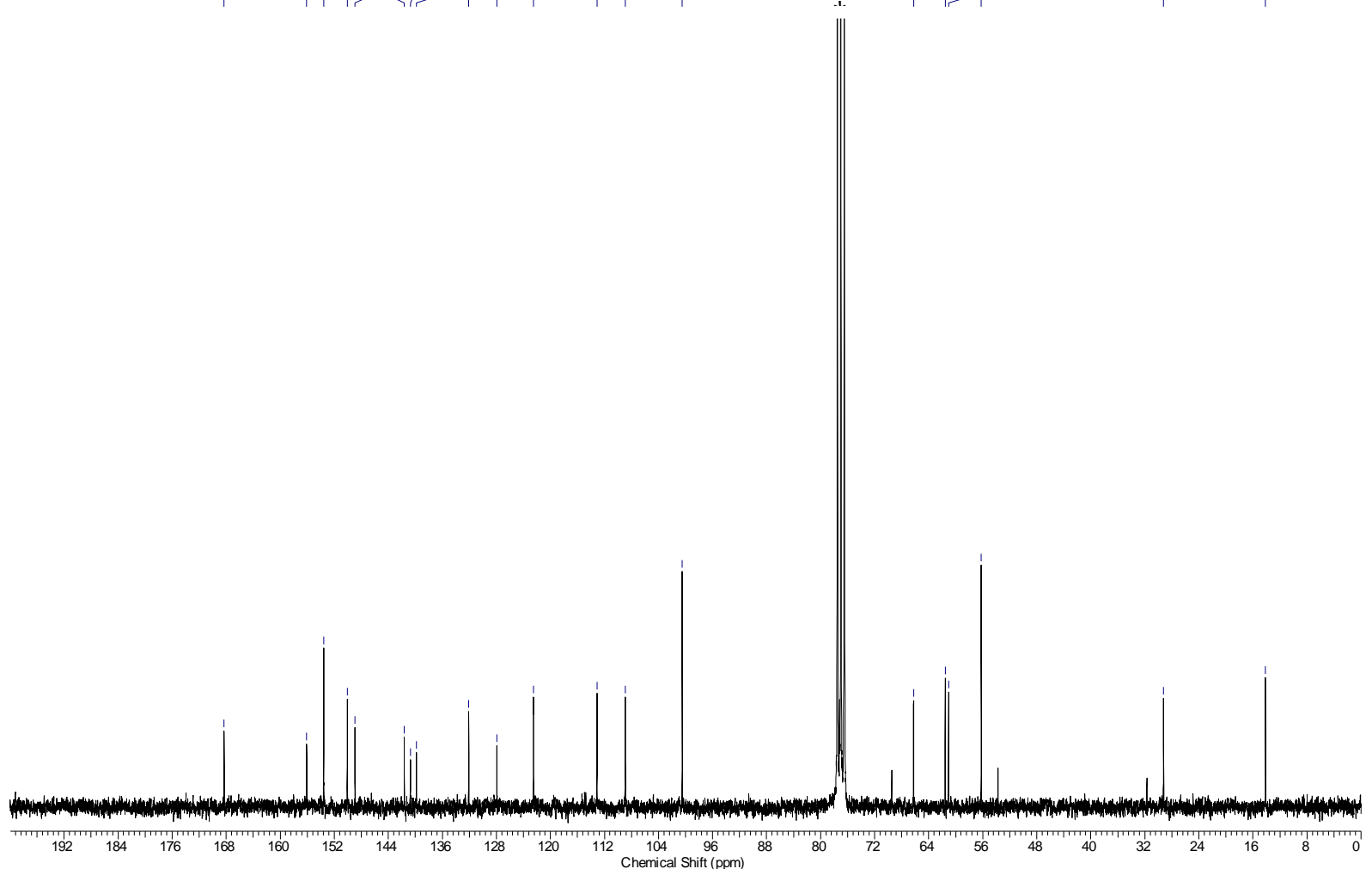

${ }^{1} \mathrm{H}-\mathrm{NMR}\left(300 \mathrm{MHz}, \mathrm{CDCl}_{3}\right.$ ) and ${ }^{13} \mathrm{C}-\mathrm{NMR}\left(63 \mathrm{MHz}^{\mathrm{CDCl} \mathrm{CDCl}_{3}}\right.$ ) spectrum of compound 30a. * = NMR-solvent 


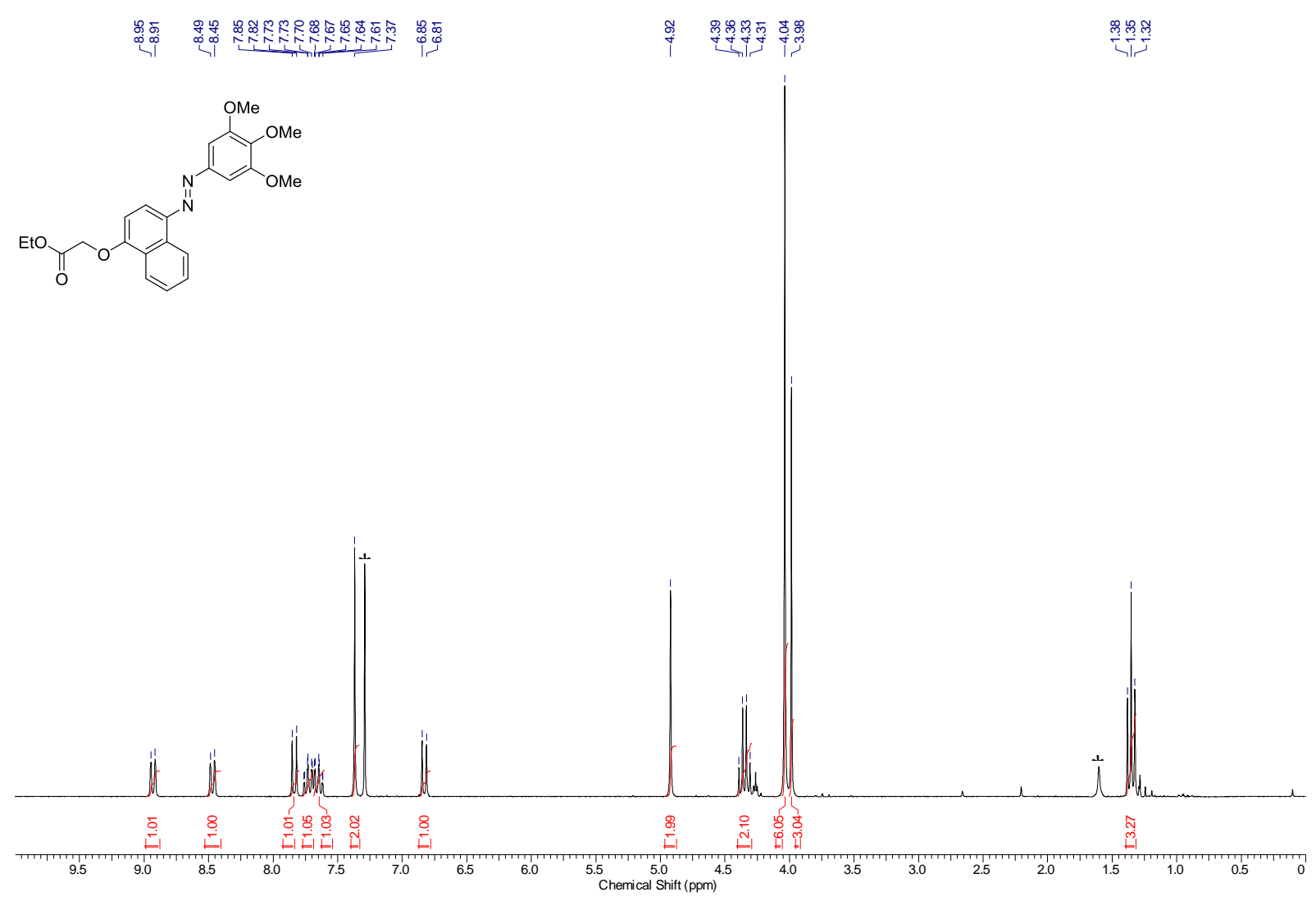

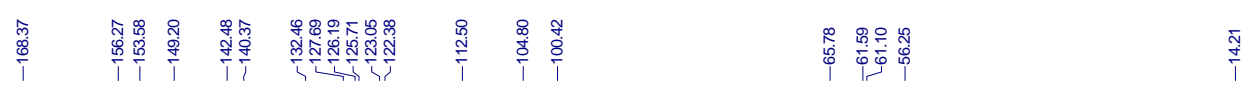

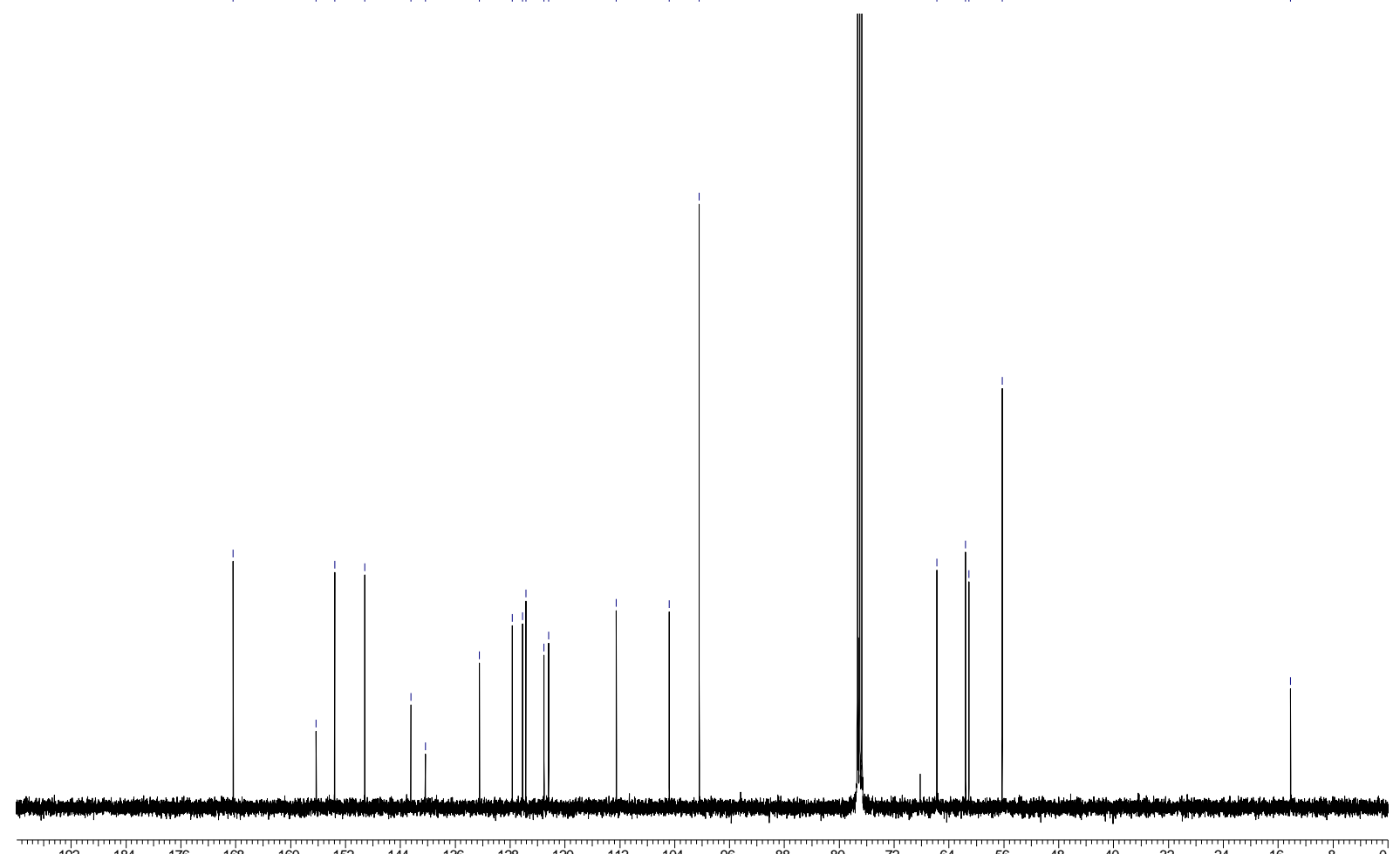

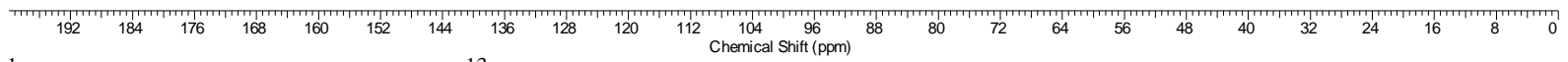

${ }^{1} \mathrm{H}-\mathrm{NMR}\left(250 \mathrm{MHz}, \mathrm{CDCl}_{3}\right)$ and ${ }^{13} \mathrm{C}-\mathrm{NMR}\left(101 \mathrm{MHz}, \mathrm{CDCl}_{3}\right)$ spectrum of compound 30b. * = NMR-solvent, $\mathrm{H}_{2} \mathrm{O}$ 


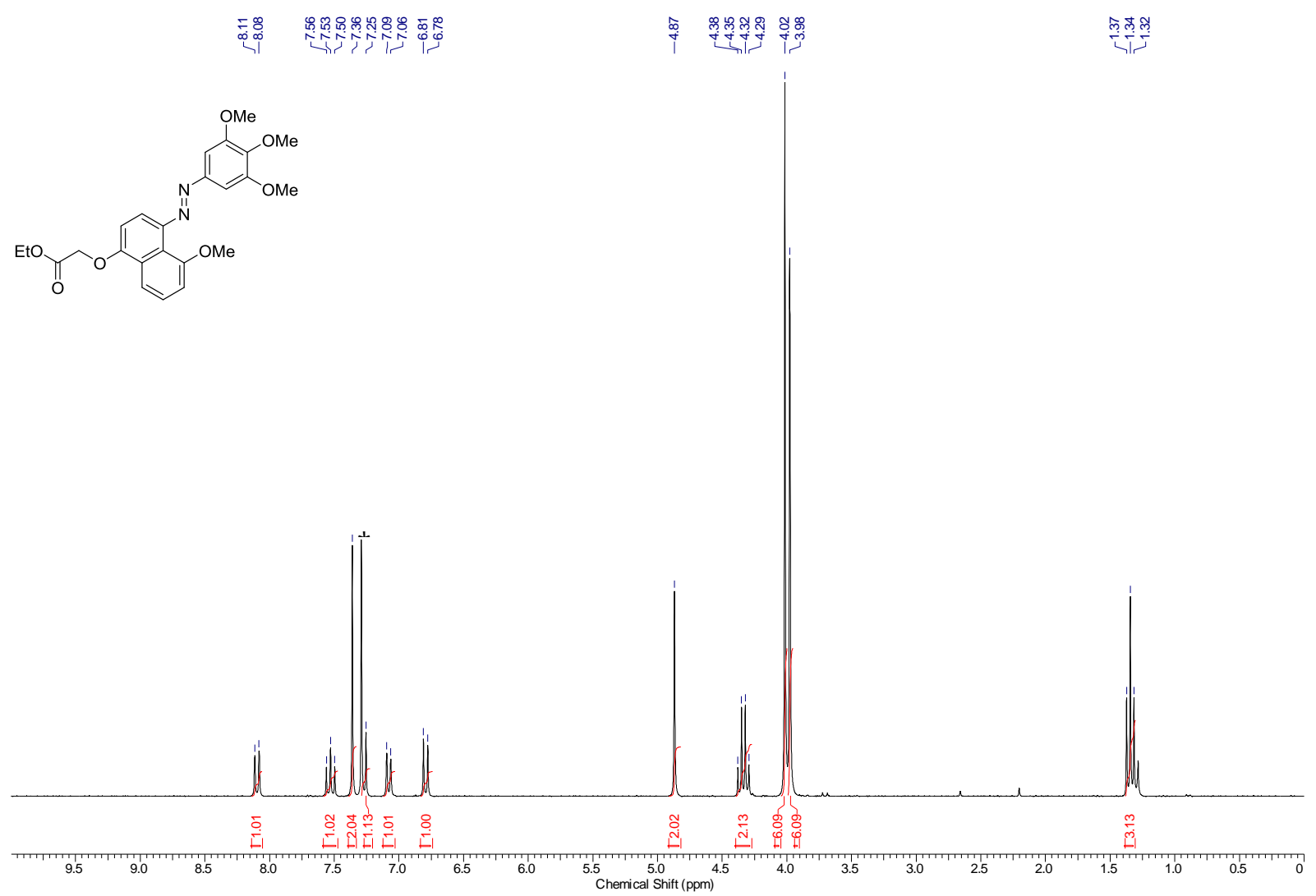

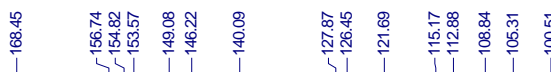

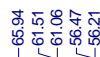

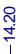

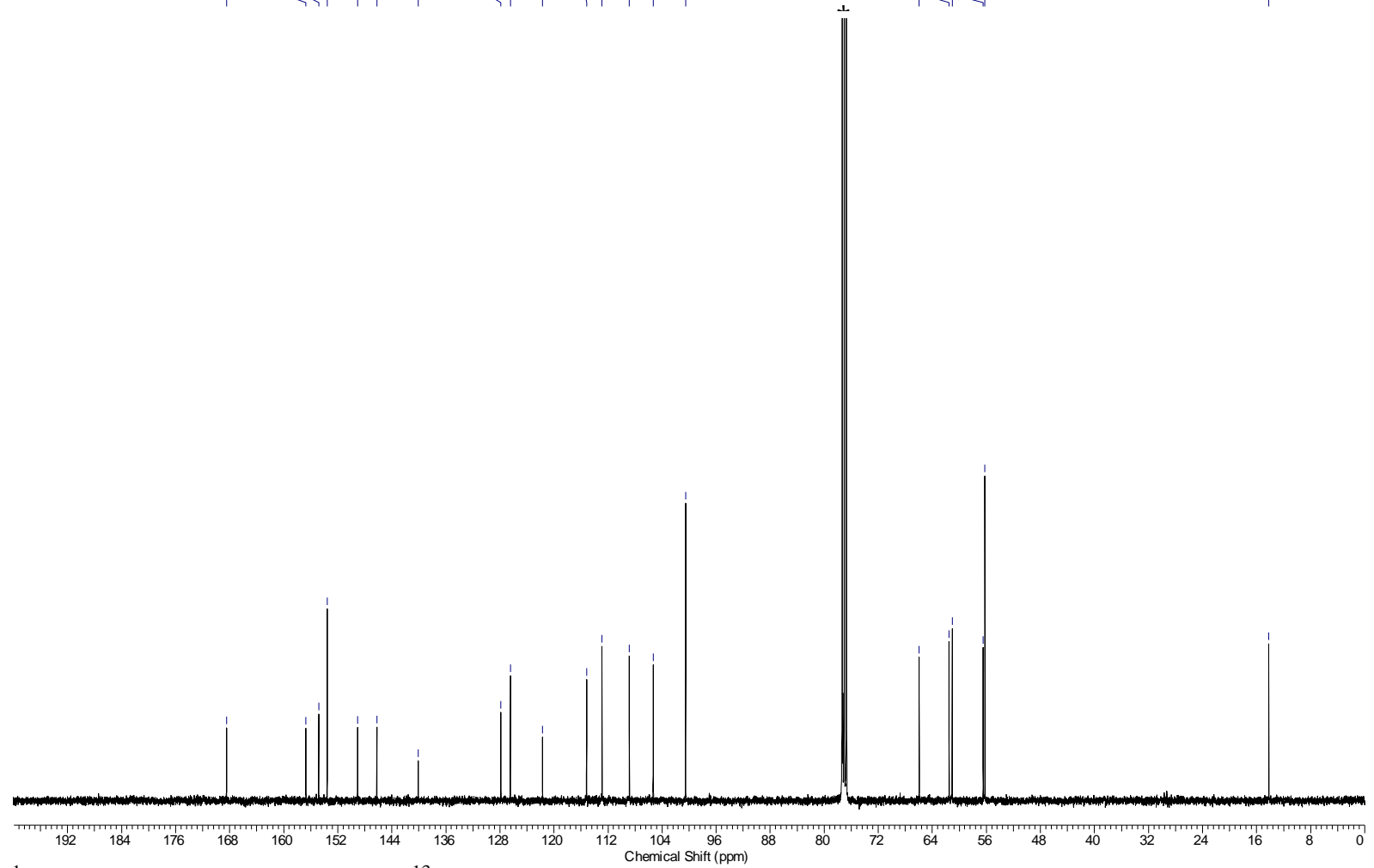

${ }^{1} \mathrm{H}-\mathrm{NMR}\left(250 \mathrm{MHz}, \mathrm{CDCl}_{3}\right)$ and ${ }^{13} \mathrm{C}-\mathrm{NMR}\left(63 \mathrm{MHz}, \mathrm{CDCl}_{3}\right)$ spectrum of compound 30c. * = NMR-solvent 

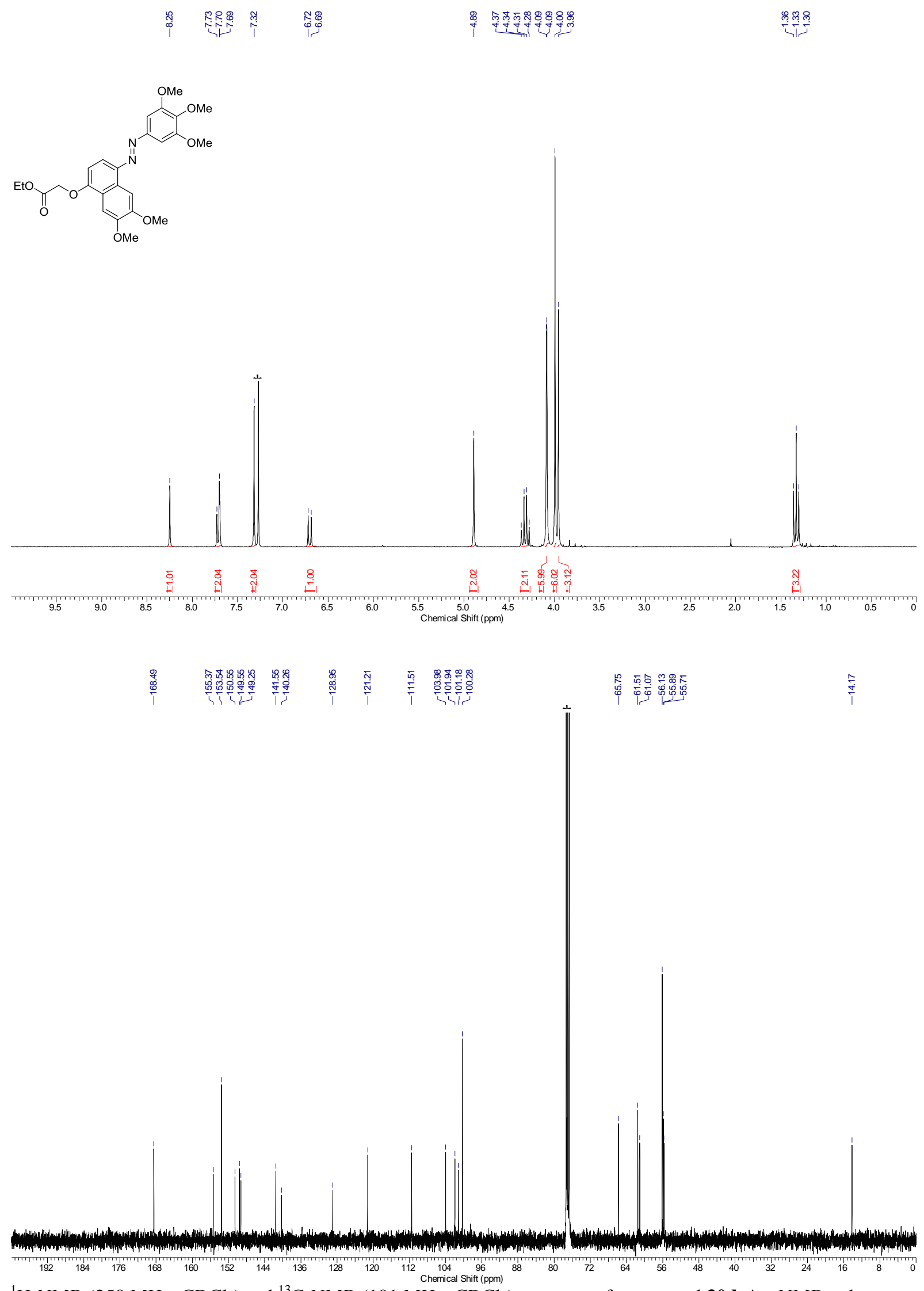

${ }^{1} \mathrm{H}-\mathrm{NMR}\left(250 \mathrm{MHz}, \mathrm{CDCl}_{3}\right)$ and ${ }^{13} \mathrm{C}-\mathrm{NMR}\left(101 \mathrm{MHz}, \mathrm{CDCl}_{3}\right)$ spectrum of compound 30d. * = NMR-solvent 

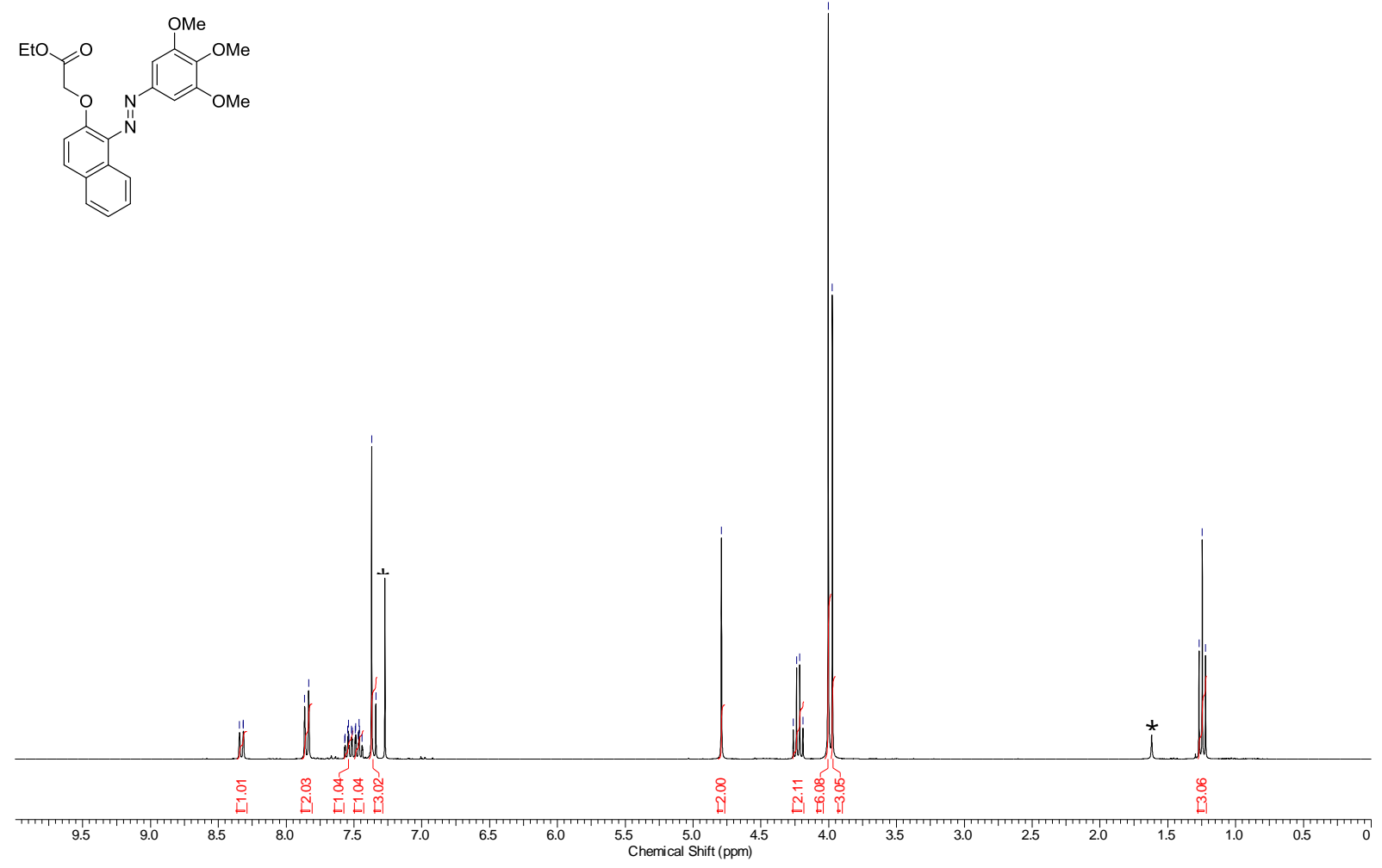

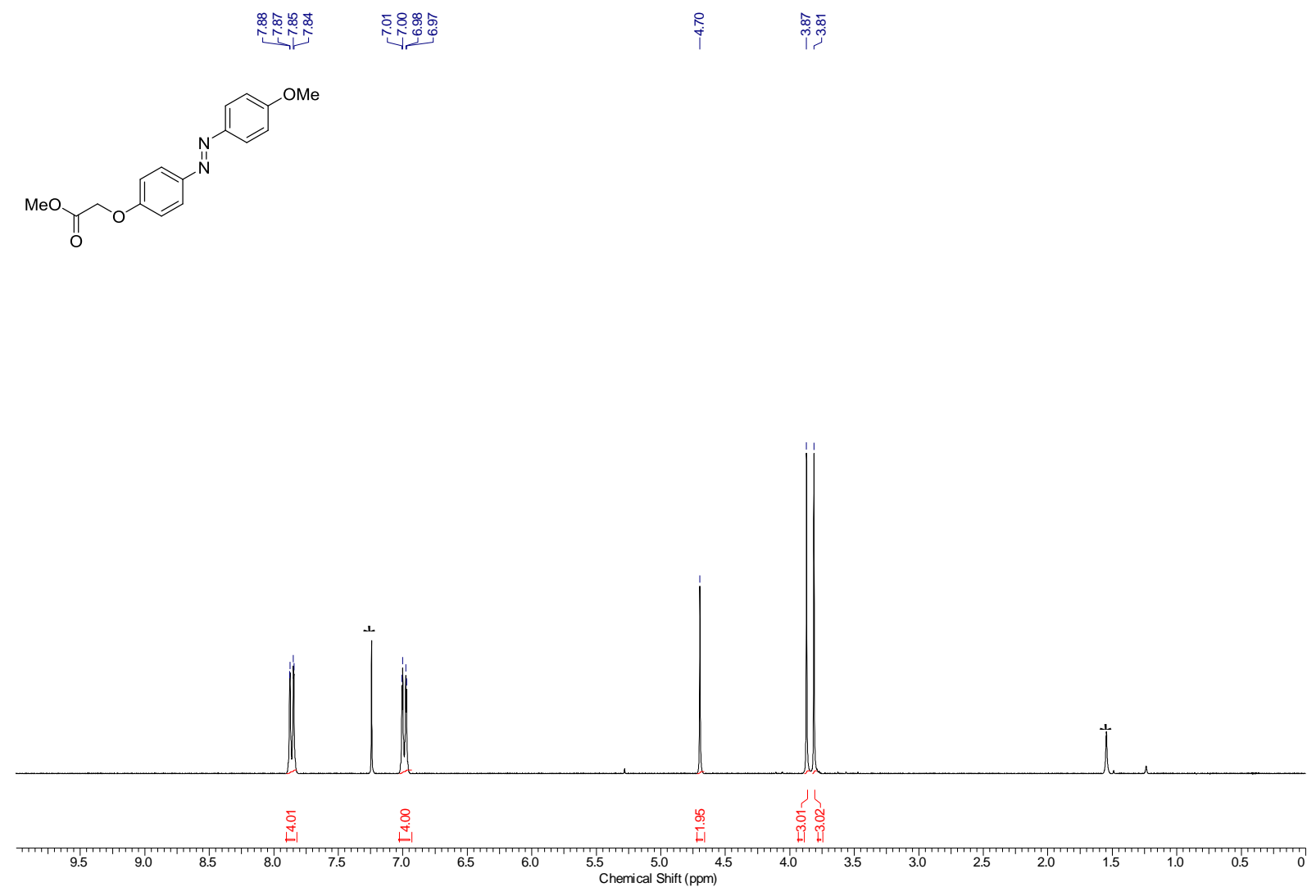

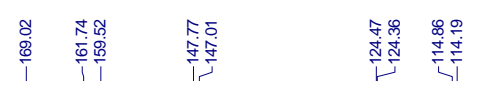

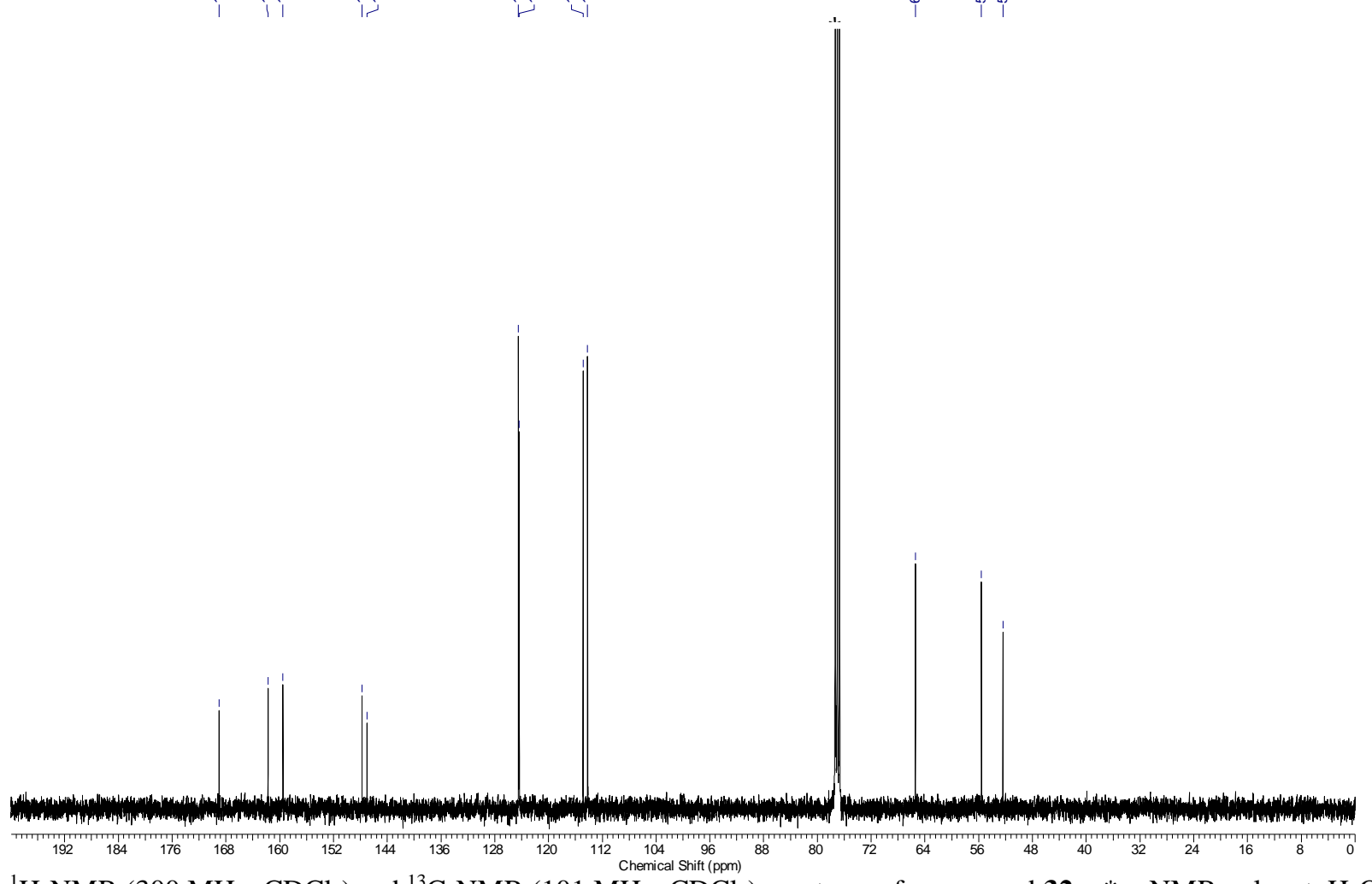

${ }^{1} \mathrm{H}-\mathrm{NMR}\left(300 \mathrm{MHz}, \mathrm{CDCl}_{3}\right.$ ) and ${ }^{13} \mathrm{C}-\mathrm{NMR}\left(101 \mathrm{MHz}, \mathrm{CDCl}_{3}\right.$ ) spectrum of compound 32a. * = NMR-solvent, $\mathrm{H}_{2} \mathrm{O}$ 

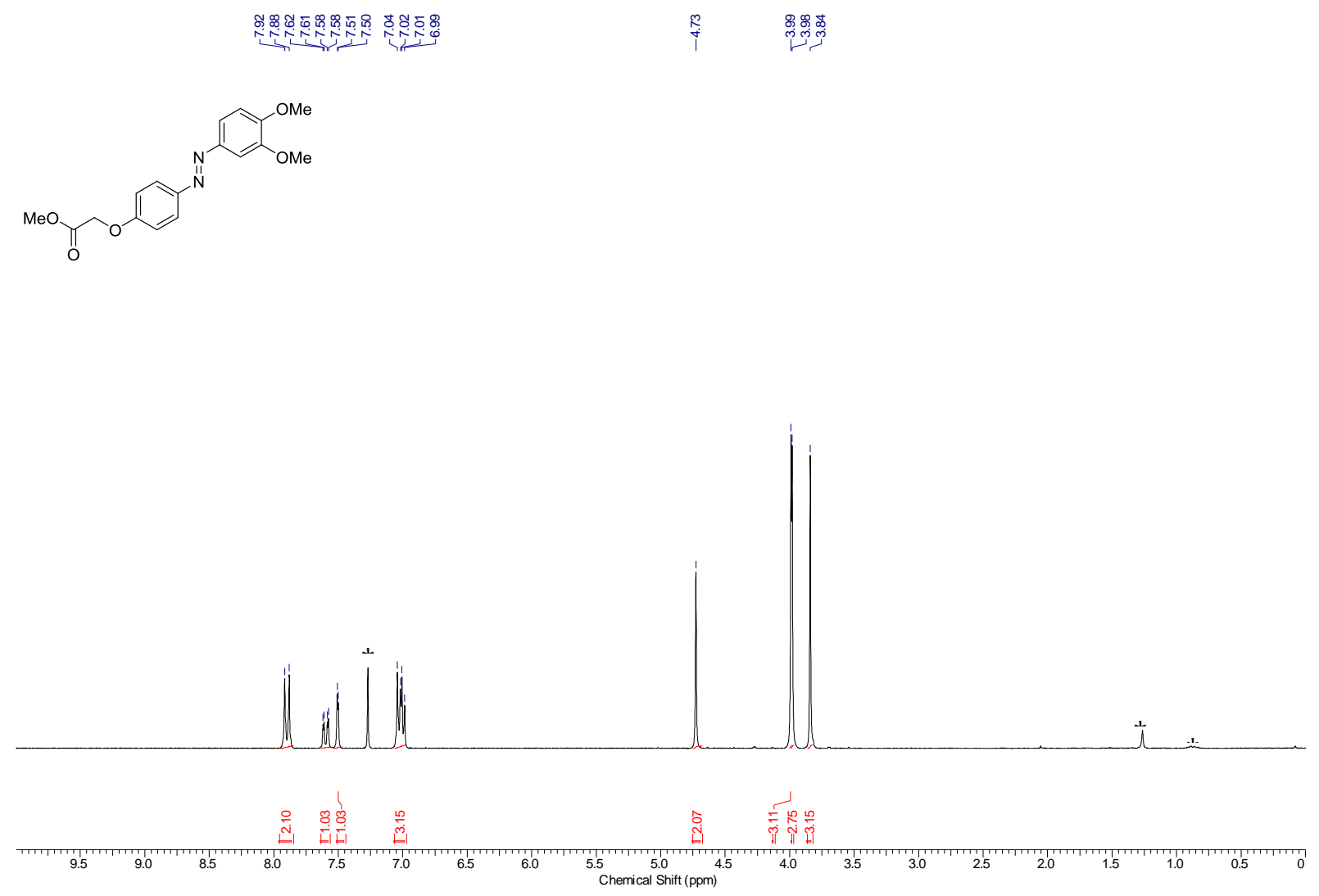

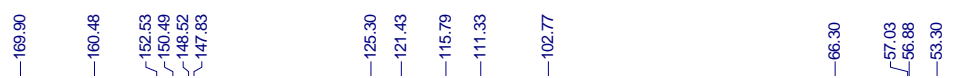

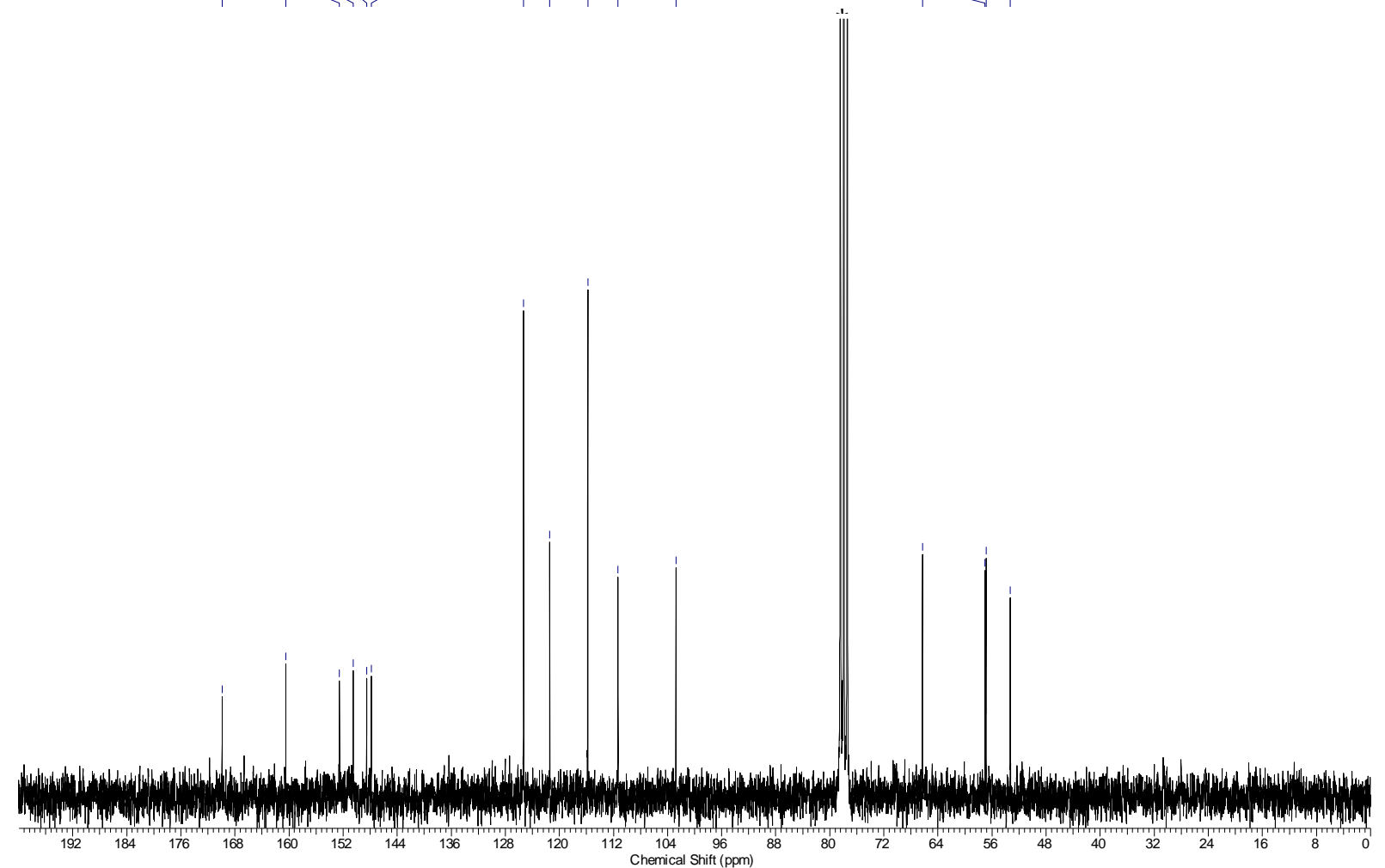

${ }^{1} \mathrm{H}-\mathrm{NMR}\left(250 \mathrm{MHz}, \mathrm{CDCl}_{3}\right)$ and ${ }^{13} \mathrm{C}-\mathrm{NMR}\left(63 \mathrm{MHz}, \mathrm{CDCl}_{3}\right)$ spectrum of compound 32b. * = NMR-solvent, grease 


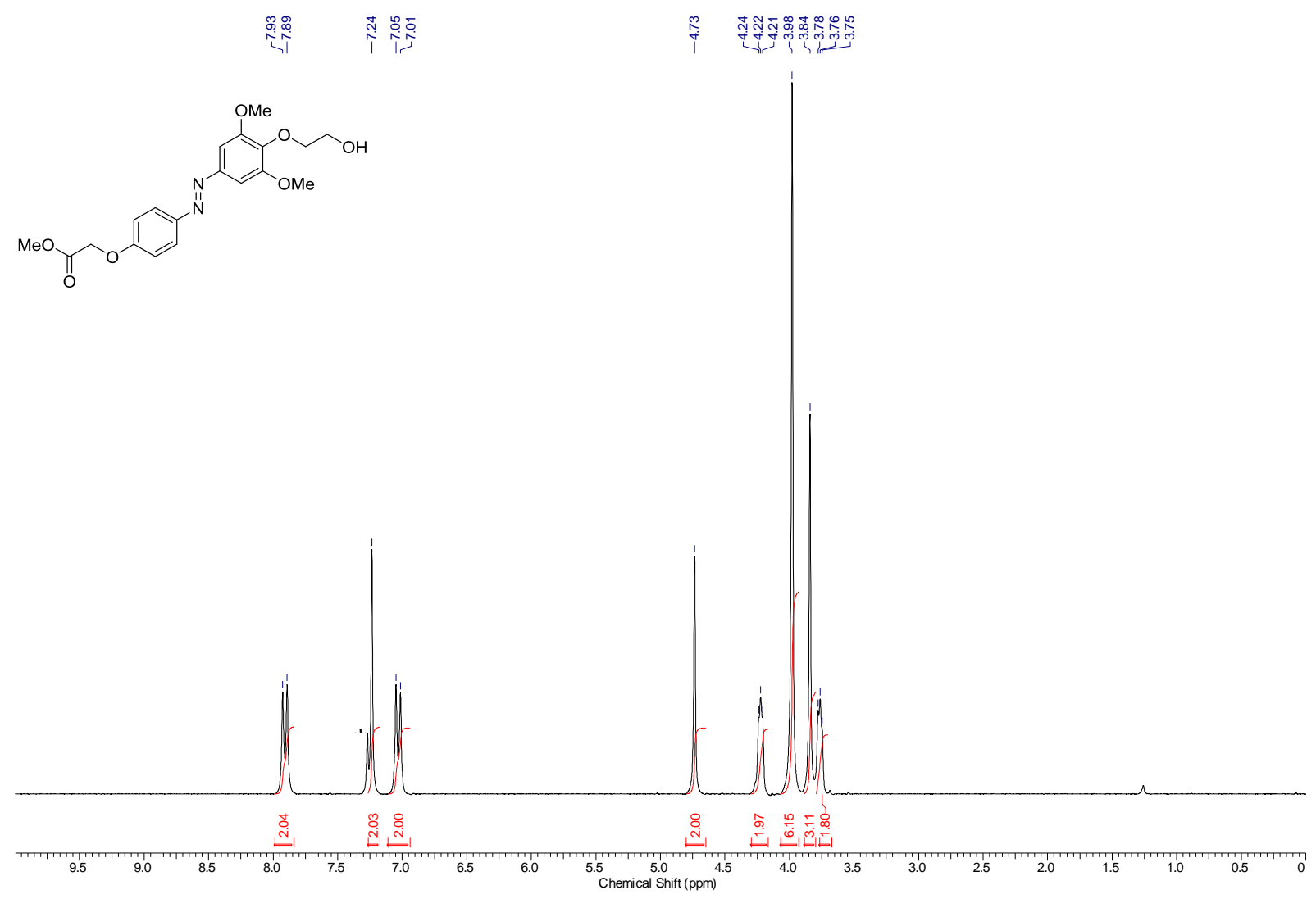

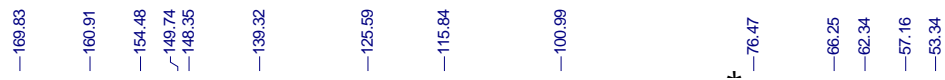




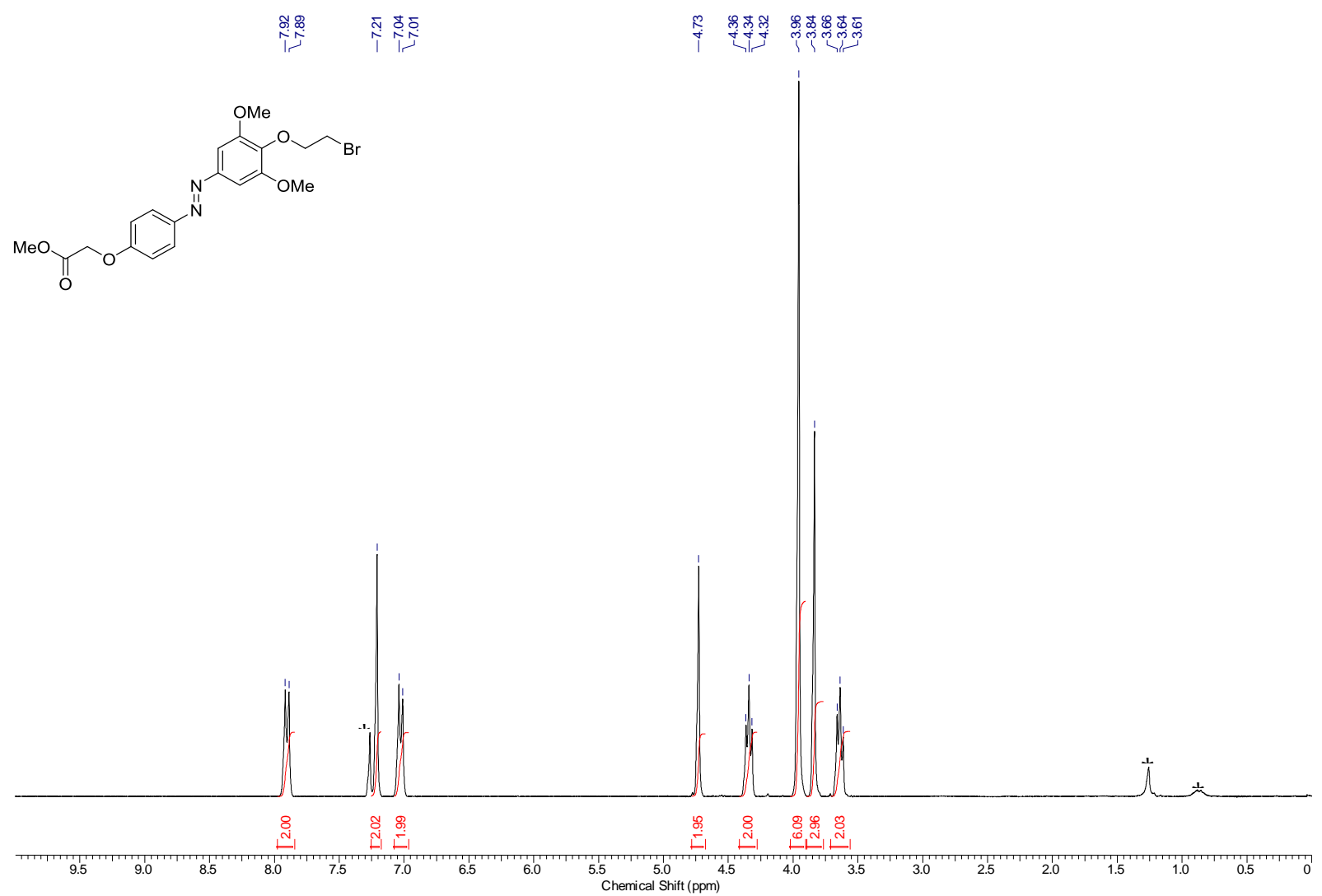

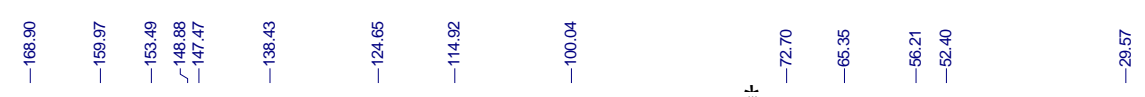
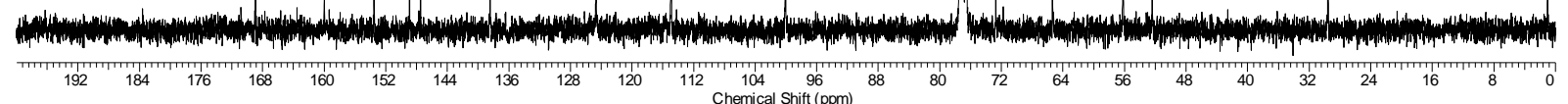

${ }^{1} \mathrm{H}-\mathrm{NMR}\left(300 \mathrm{MHz}, \mathrm{CDCl}_{3}\right.$ ) and ${ }^{13} \mathrm{C}-\mathrm{NMR}\left(75 \mathrm{MHz}, \mathrm{CDCl}_{3}\right.$ ) spectrum of compound 32e. * = NMR-solvent, grease 


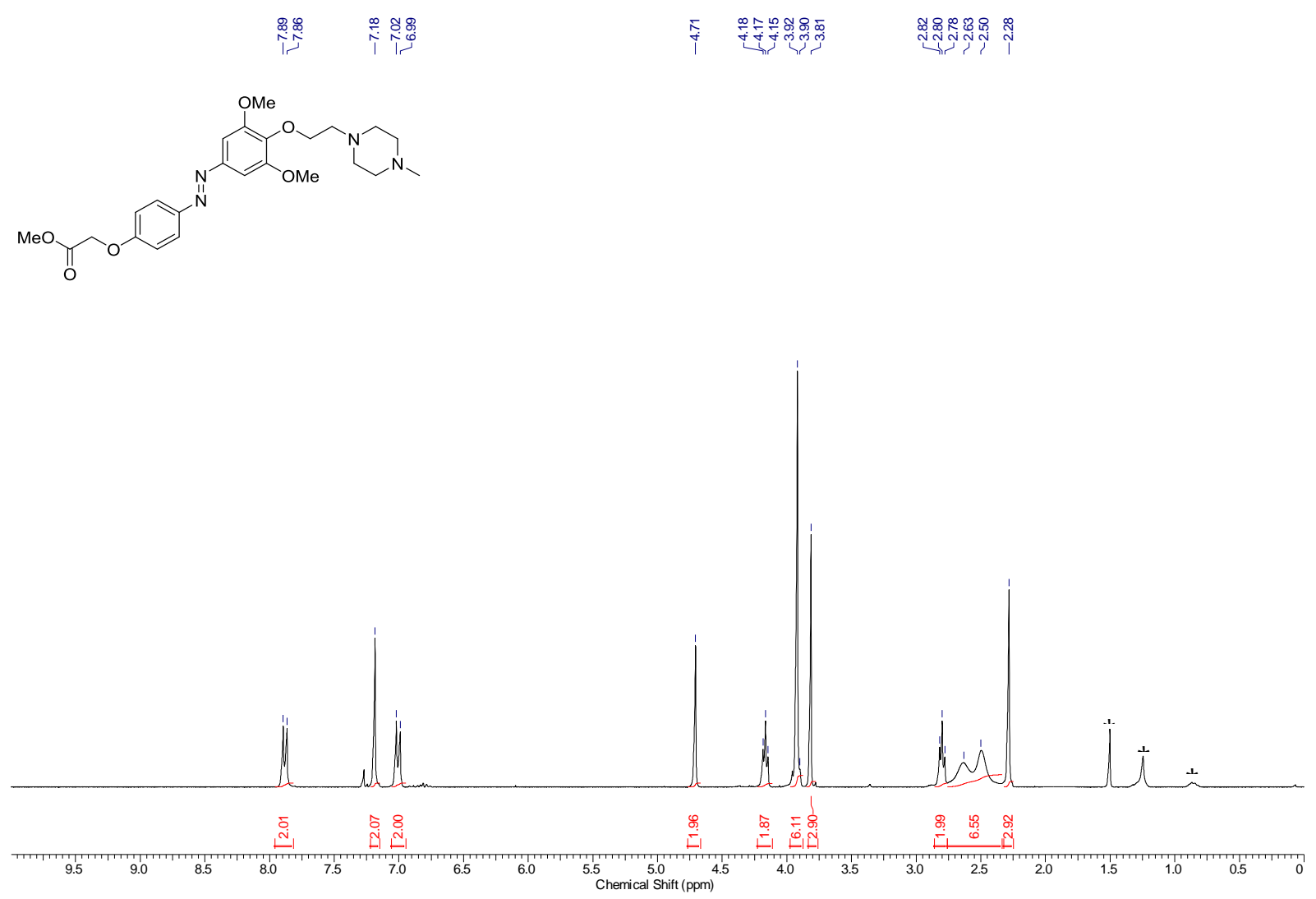

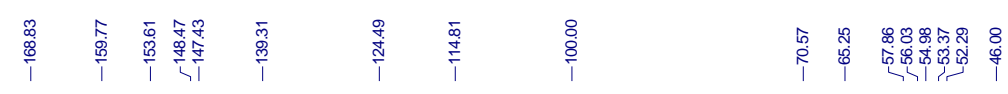

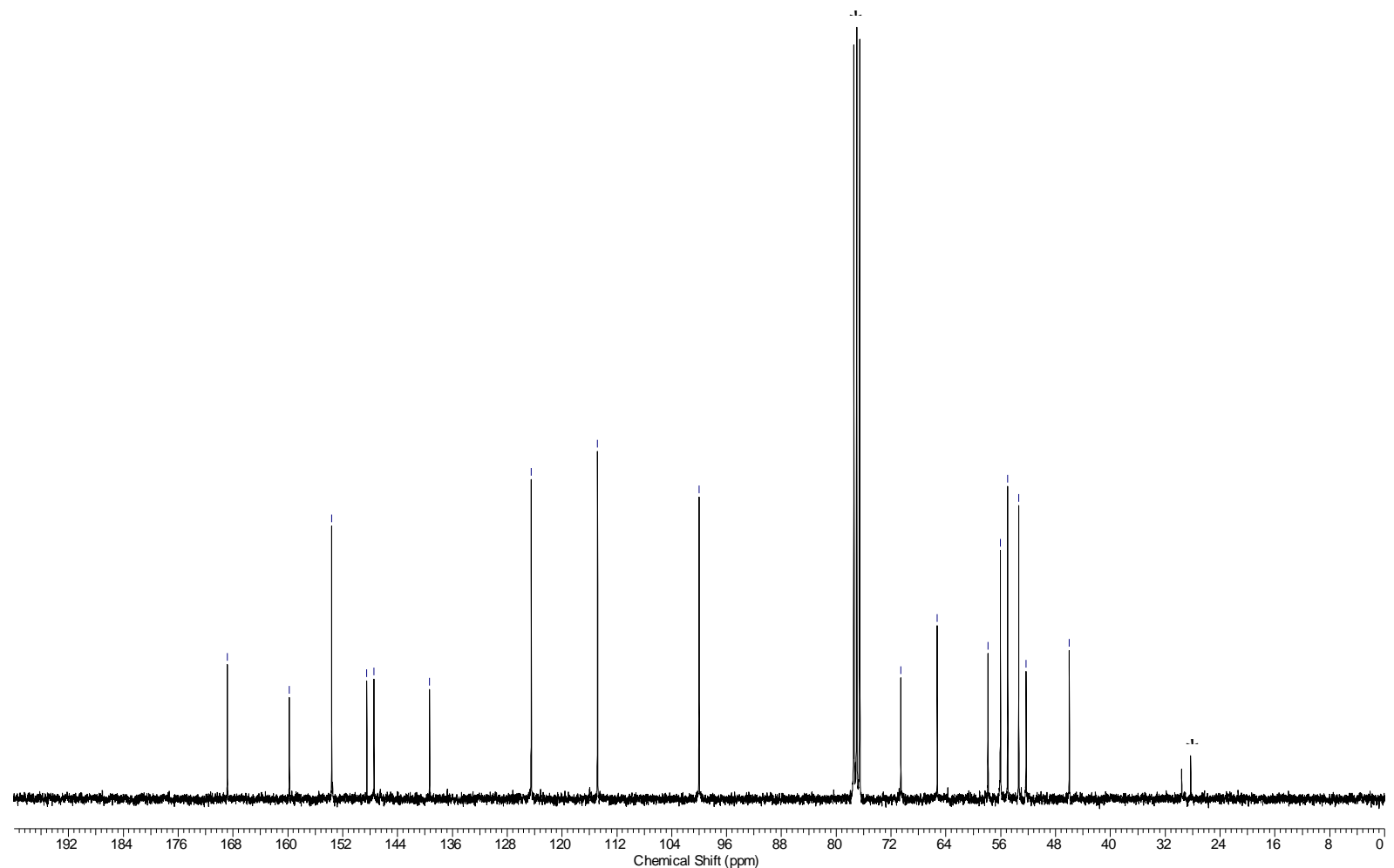

${ }^{1} \mathrm{H}-\mathrm{NMR}\left(300 \mathrm{MHz}, \mathrm{CDCl}_{3}\right.$ ) and ${ }^{13} \mathrm{C}-\mathrm{NMR}\left(75 \mathrm{MHz}, \mathrm{CDCl}_{3}\right.$ ) spectrum of compound 32f. * = NMR-solvent, $\mathrm{H}_{2} \mathrm{O}$, grease 

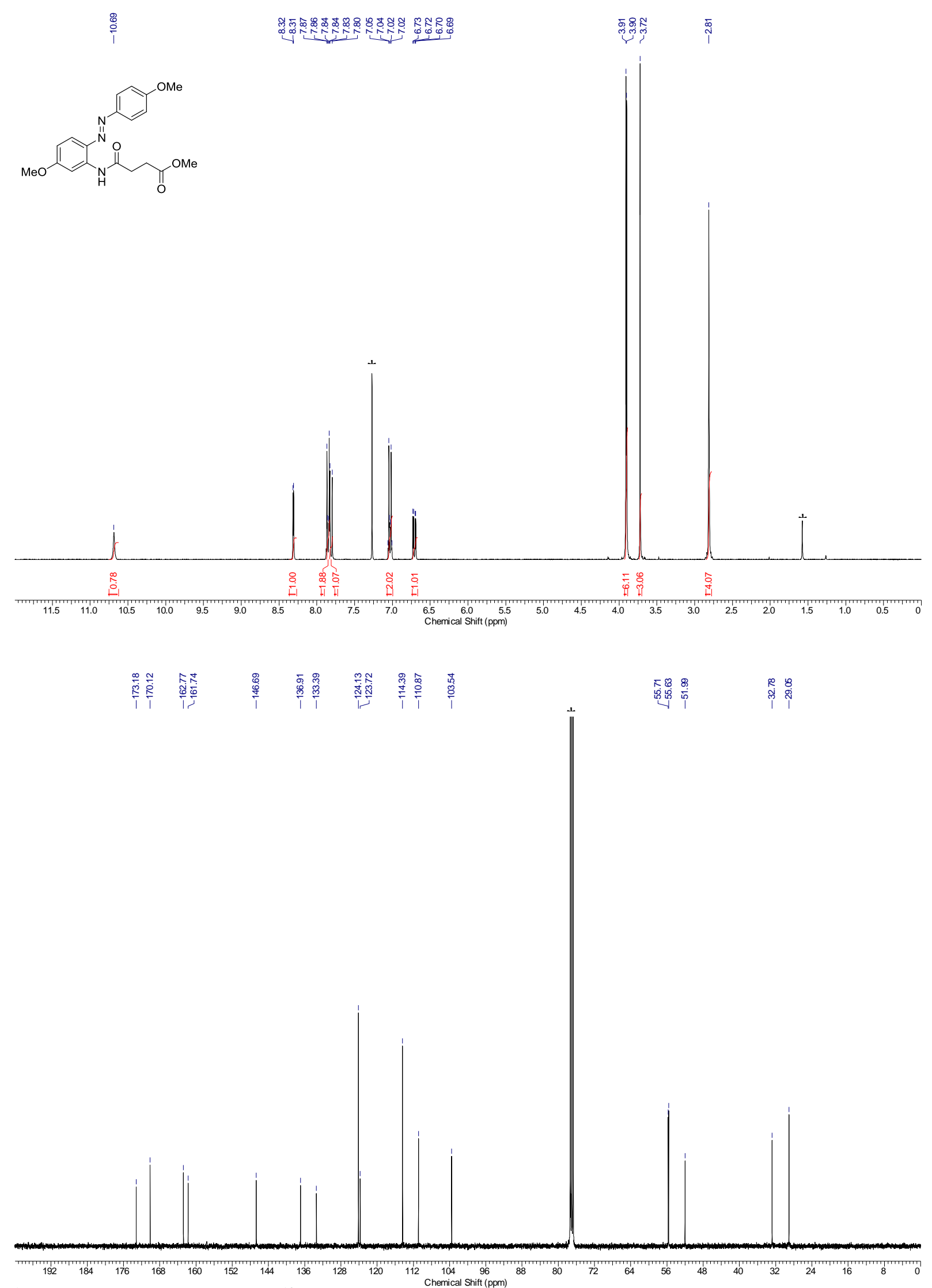

${ }^{1} \mathrm{H}-\mathrm{NMR}\left(300 \mathrm{MHz}, \mathrm{CDCl}_{3}\right.$ ) and ${ }^{13} \mathrm{C}-\mathrm{NMR}\left(101 \mathrm{MHz}, \mathrm{CDCl}_{3}\right)$ spectrum of compound 33a. * = NMR-solvent, $\mathrm{H}_{2} \mathrm{O}$ 

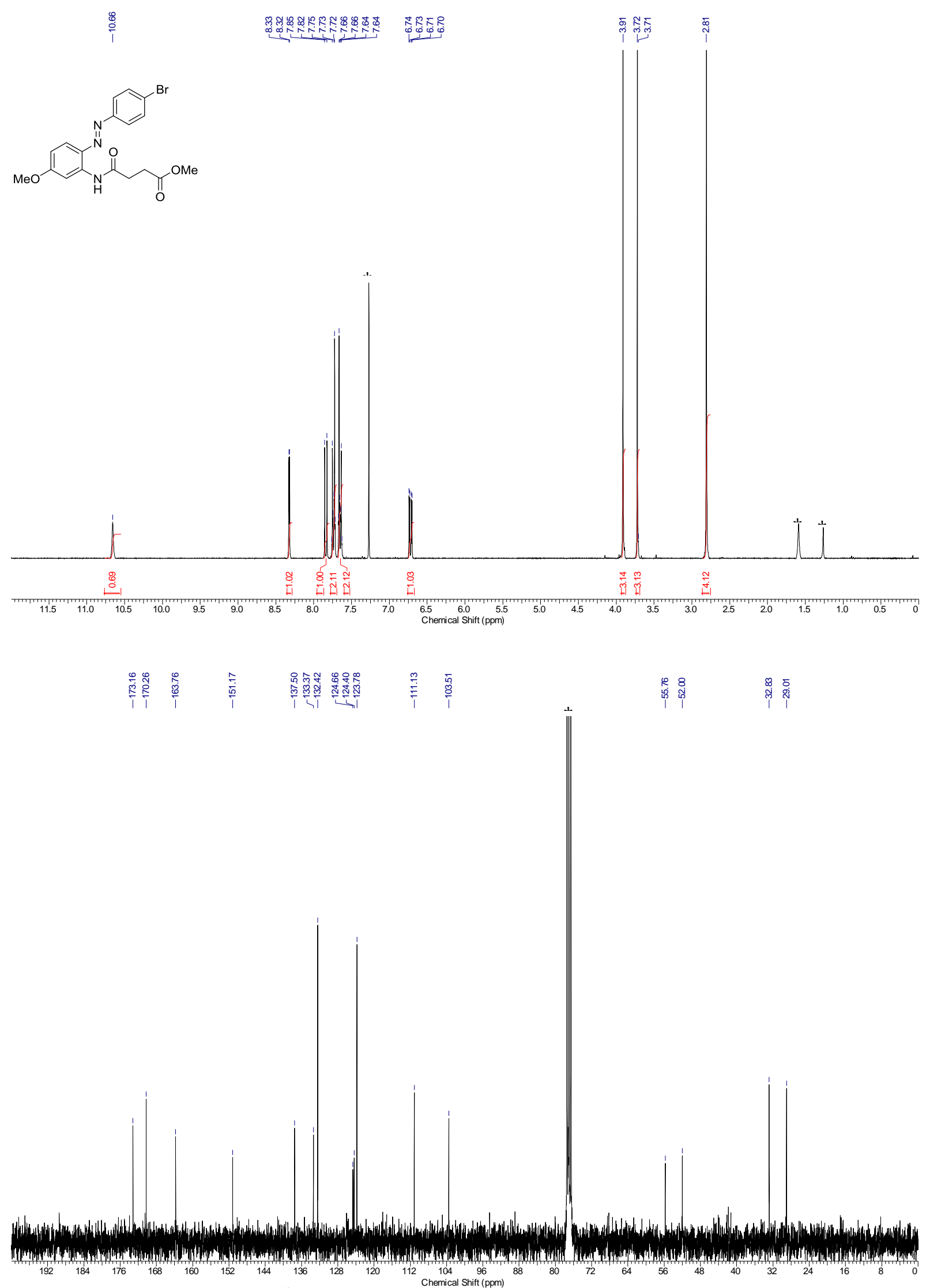

${ }^{1} \mathrm{H}-\mathrm{NMR}\left(300 \mathrm{MHz}, \mathrm{CDCl}_{3}\right.$ ) and ${ }^{13} \mathrm{C}-\mathrm{NMR}\left(75 \mathrm{MHz}, \mathrm{CDCl}_{3}\right.$ ) spectrum of compound 33b. * = NMR-solvent, $\mathrm{H}_{2} \mathrm{O}$, grease 

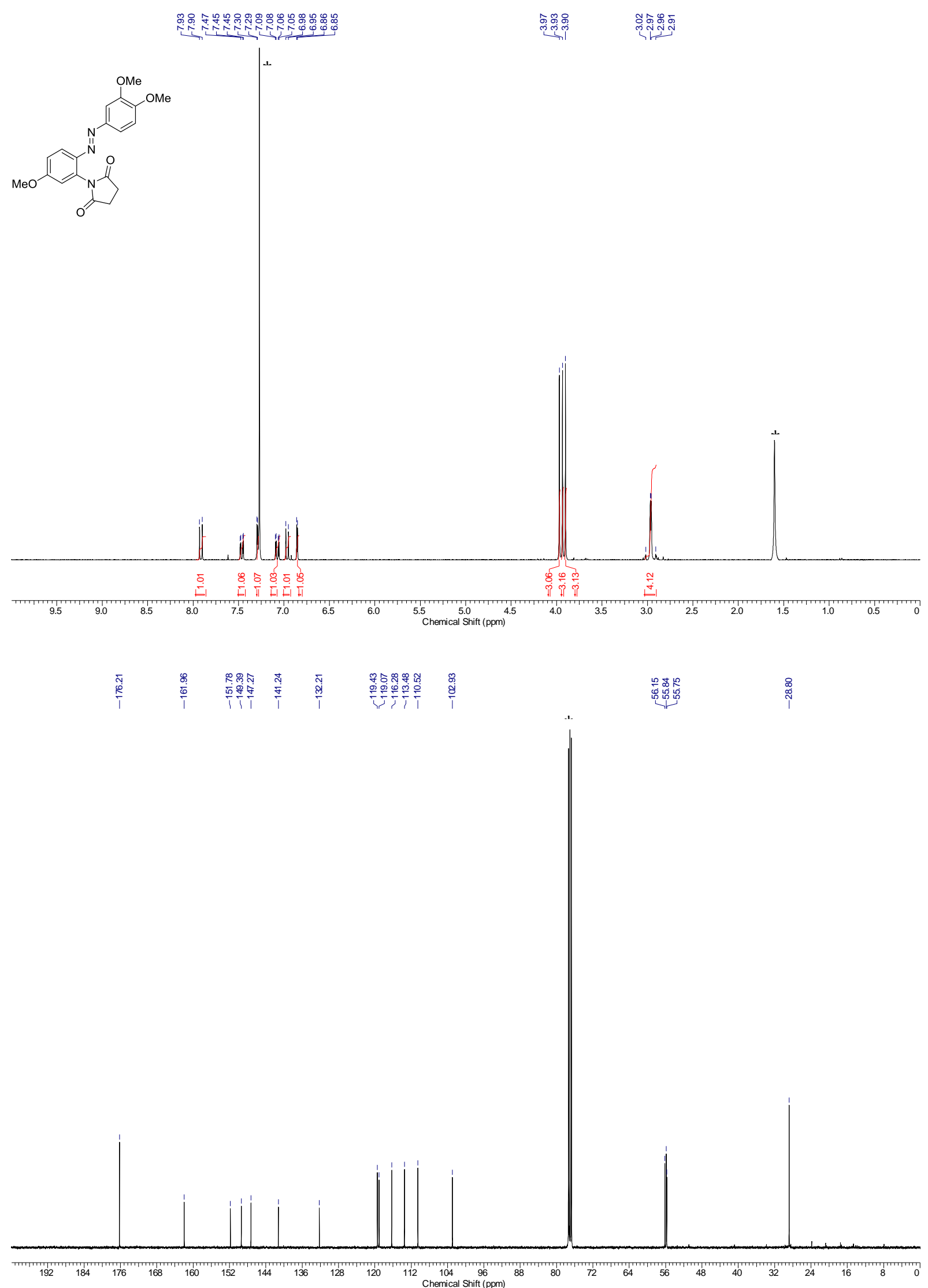

${ }^{1} \mathrm{H}-\mathrm{NMR}\left(300 \mathrm{MHz}, \mathrm{CDCl}_{3}\right.$ ) and ${ }^{13} \mathrm{C}-\mathrm{NMR}\left(101 \mathrm{MHz}^{\text {chencal Shit (pom) }} \mathrm{CDCl}_{3}\right.$ ) spectrum of compound 34b. * = NMR-solvent, $\mathrm{H}_{2} \mathrm{O}$ 


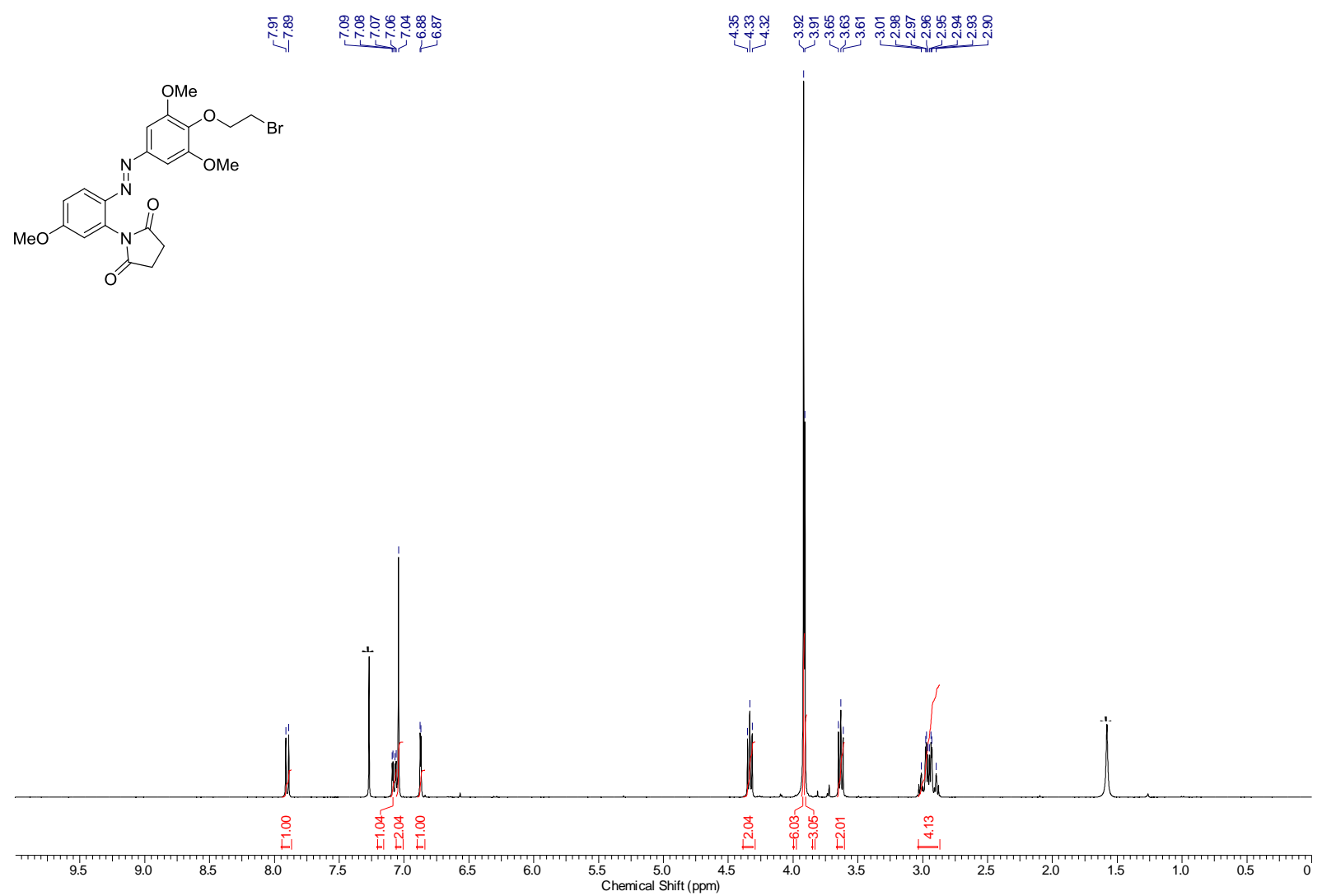

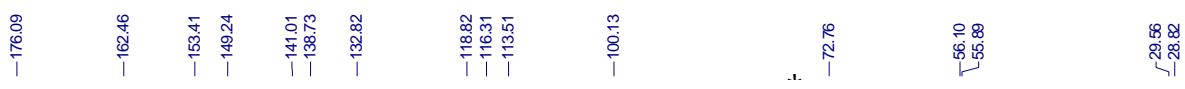

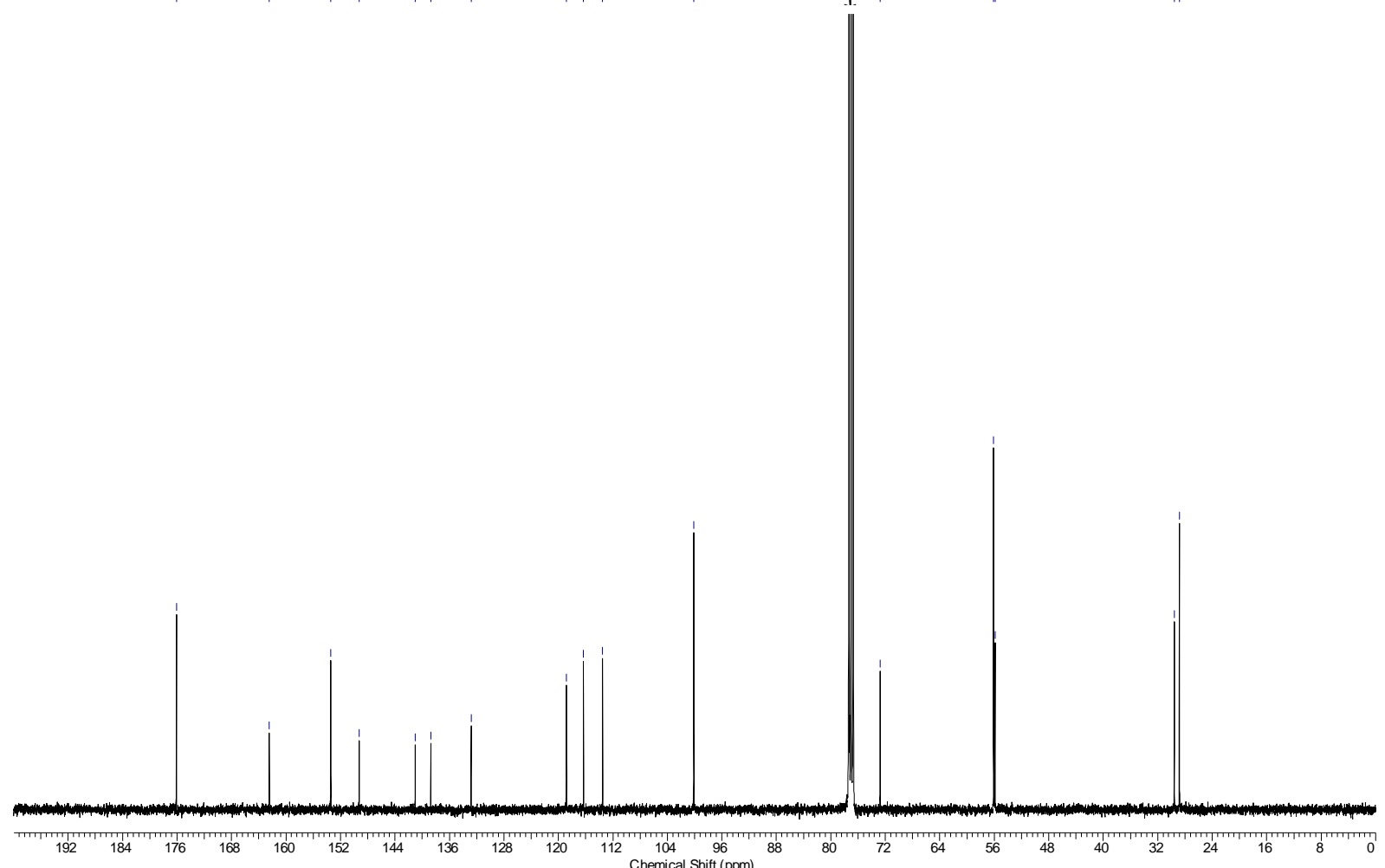

${ }^{1} \mathrm{H}-\mathrm{NMR}\left(400 \mathrm{MHz}, \mathrm{CDCl}_{3}\right)$ and ${ }^{13} \mathrm{C}-\mathrm{NMR}\left(101 \mathrm{MHz}, \mathrm{CDCl}_{3}\right)$ spectrum of compound 34d. * = NMR-solvent, $\mathrm{H}_{2} \mathrm{O}$ 

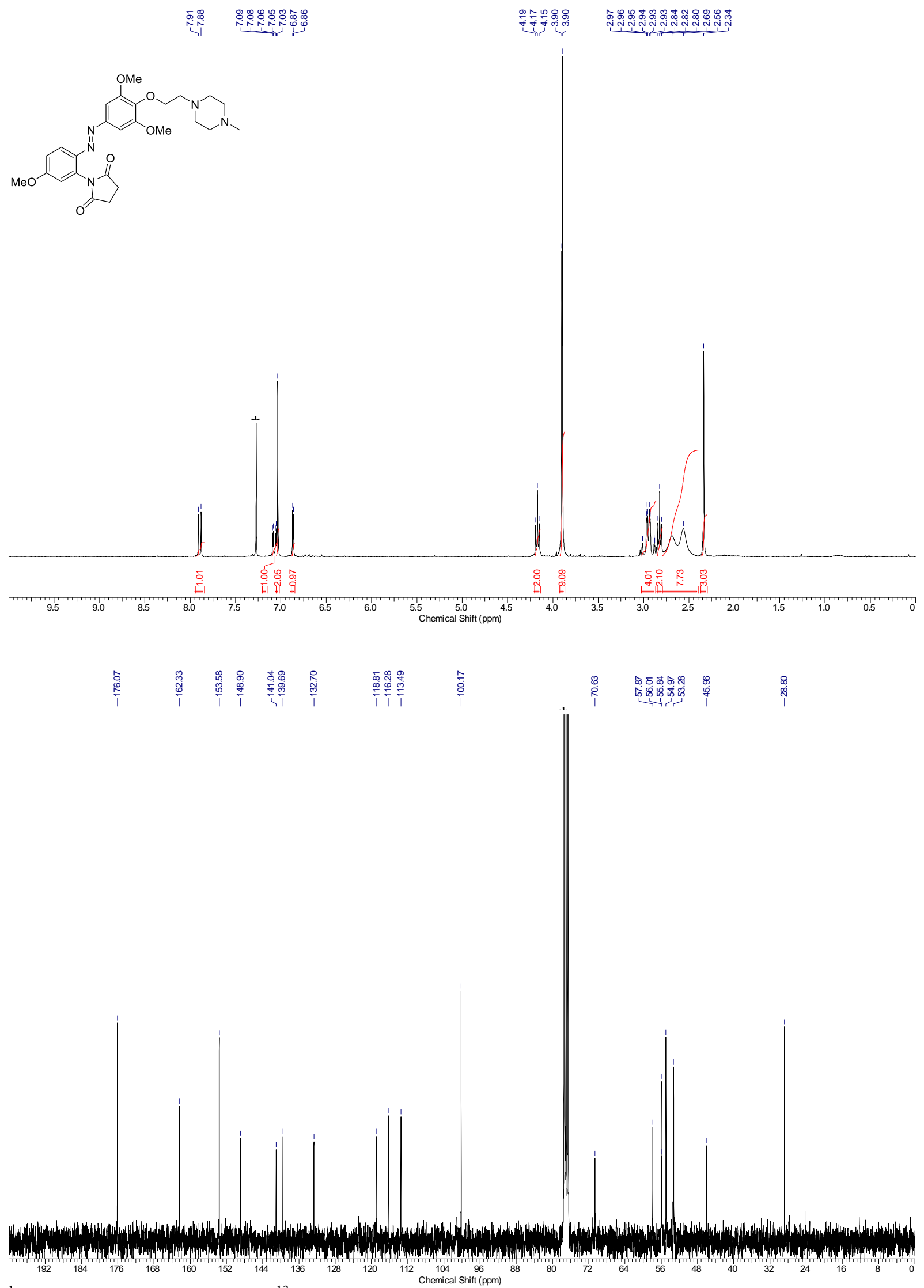

${ }^{1} \mathrm{H}-\mathrm{NMR}\left(300 \mathrm{MHz}, \mathrm{CDCl}_{3}\right.$ ) and ${ }^{13} \mathrm{C}-\mathrm{NMR}\left(75 \mathrm{MHz}, \mathrm{CDCl}_{3}\right.$ ) spectrum of compound 34e. * = NMR-solvent 


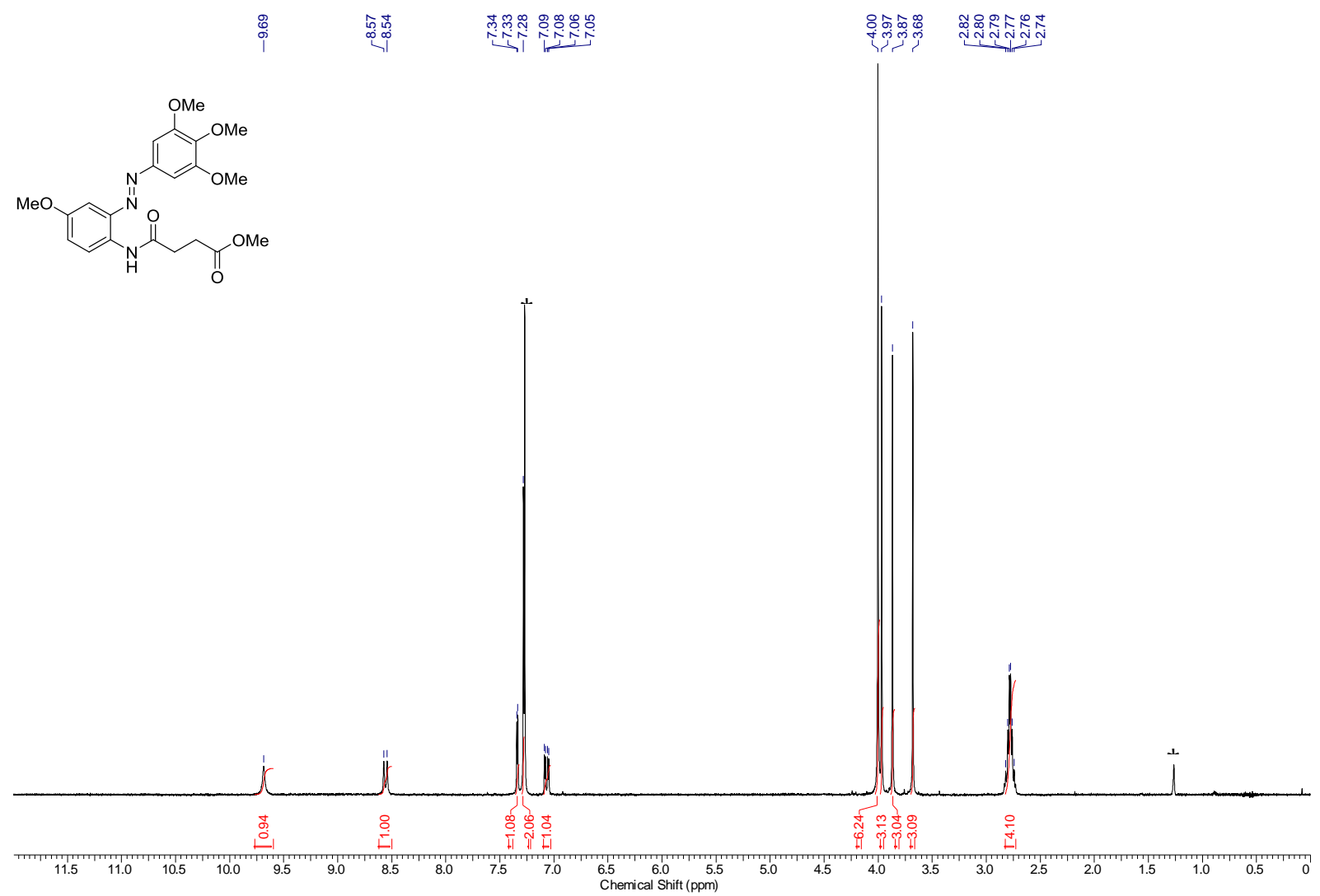

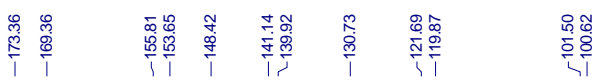

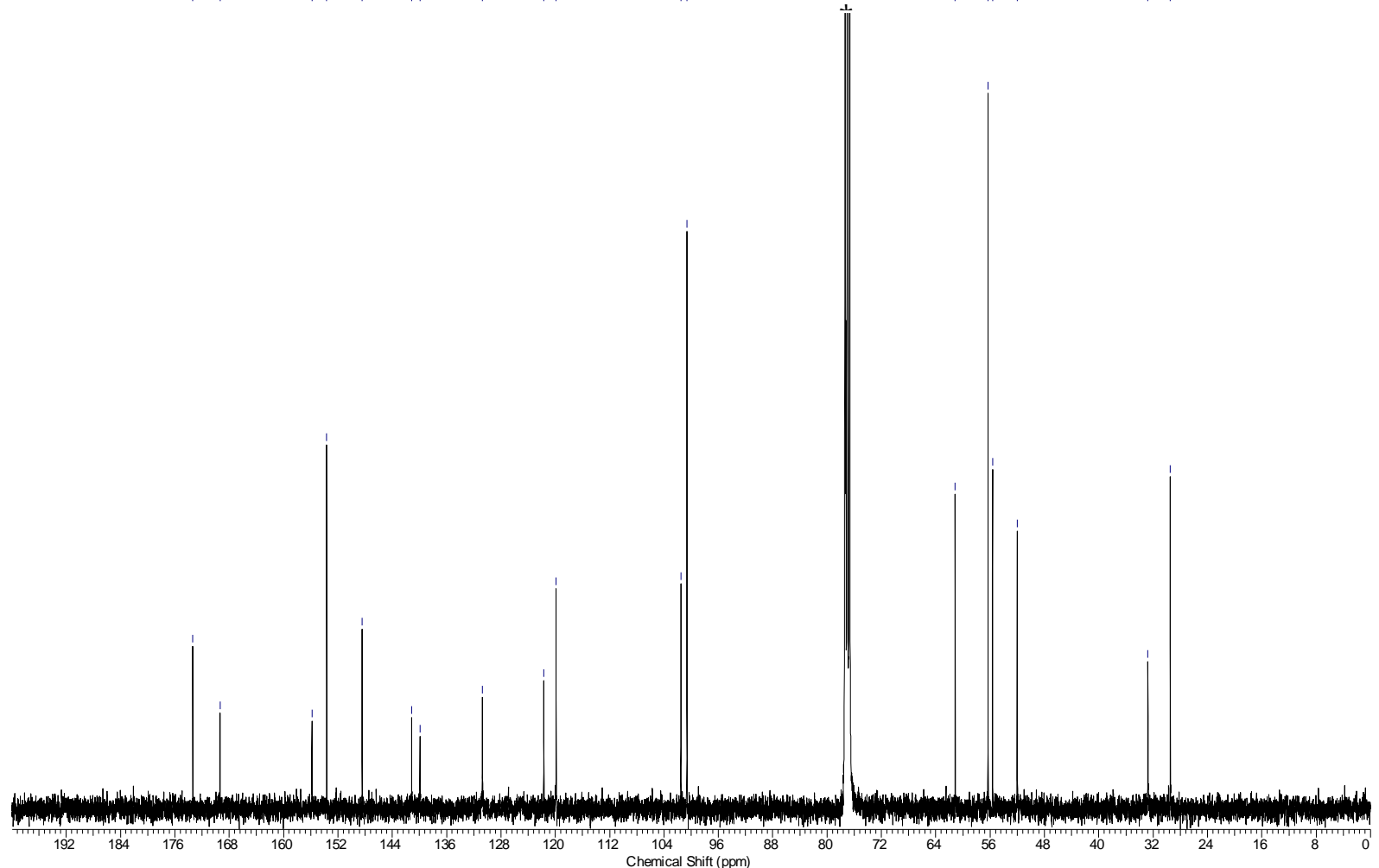

${ }^{1} \mathrm{H}-\mathrm{NMR}\left(300 \mathrm{MHz}, \mathrm{CDCl}_{3}\right.$ ) and ${ }^{13} \mathrm{C}-\mathrm{NMR}\left(101 \mathrm{MHz}, \mathrm{CDCl}_{3}\right)$ spectrum of compound 35. * = NMR-solvent, grease 


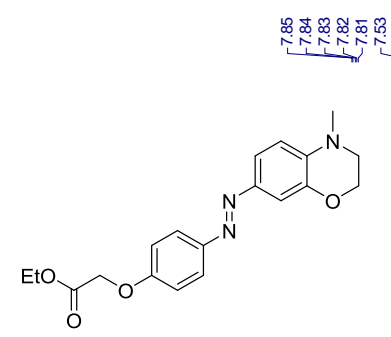

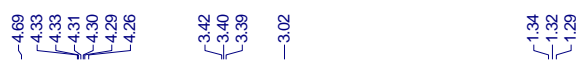

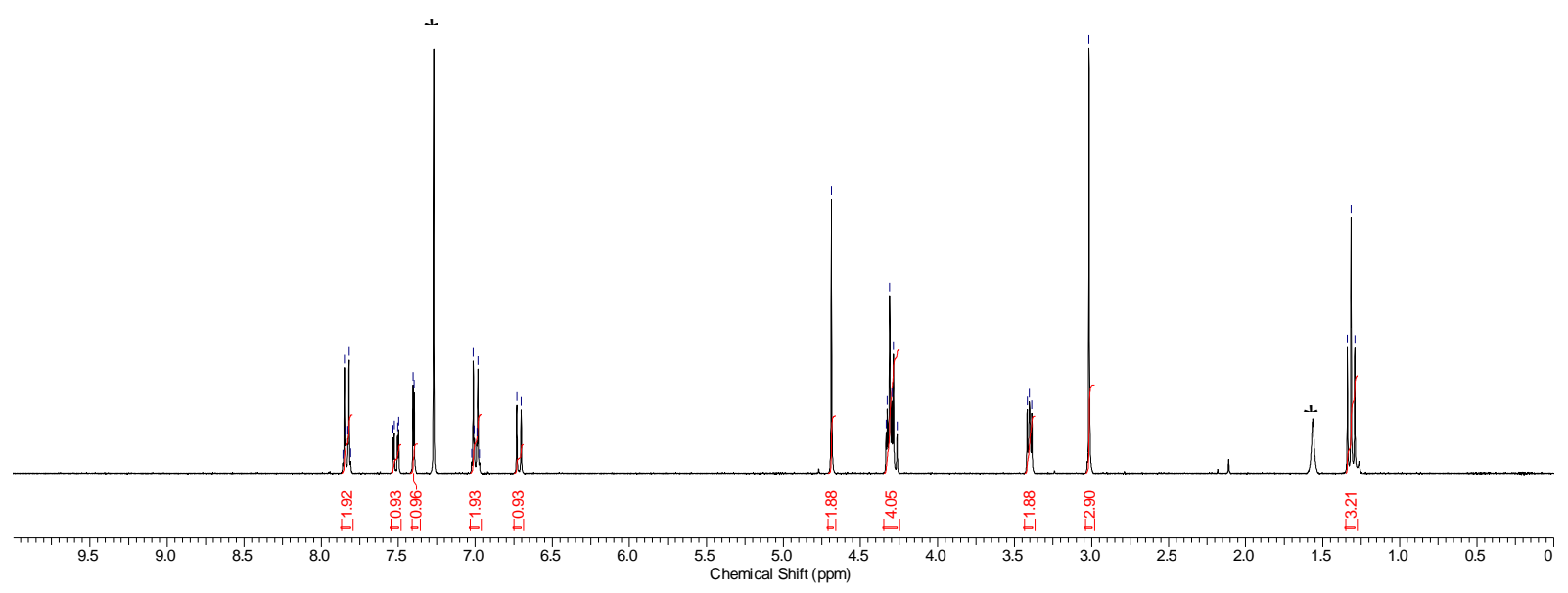

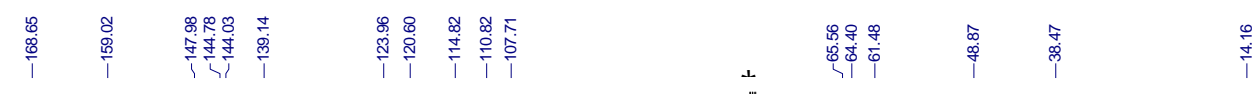

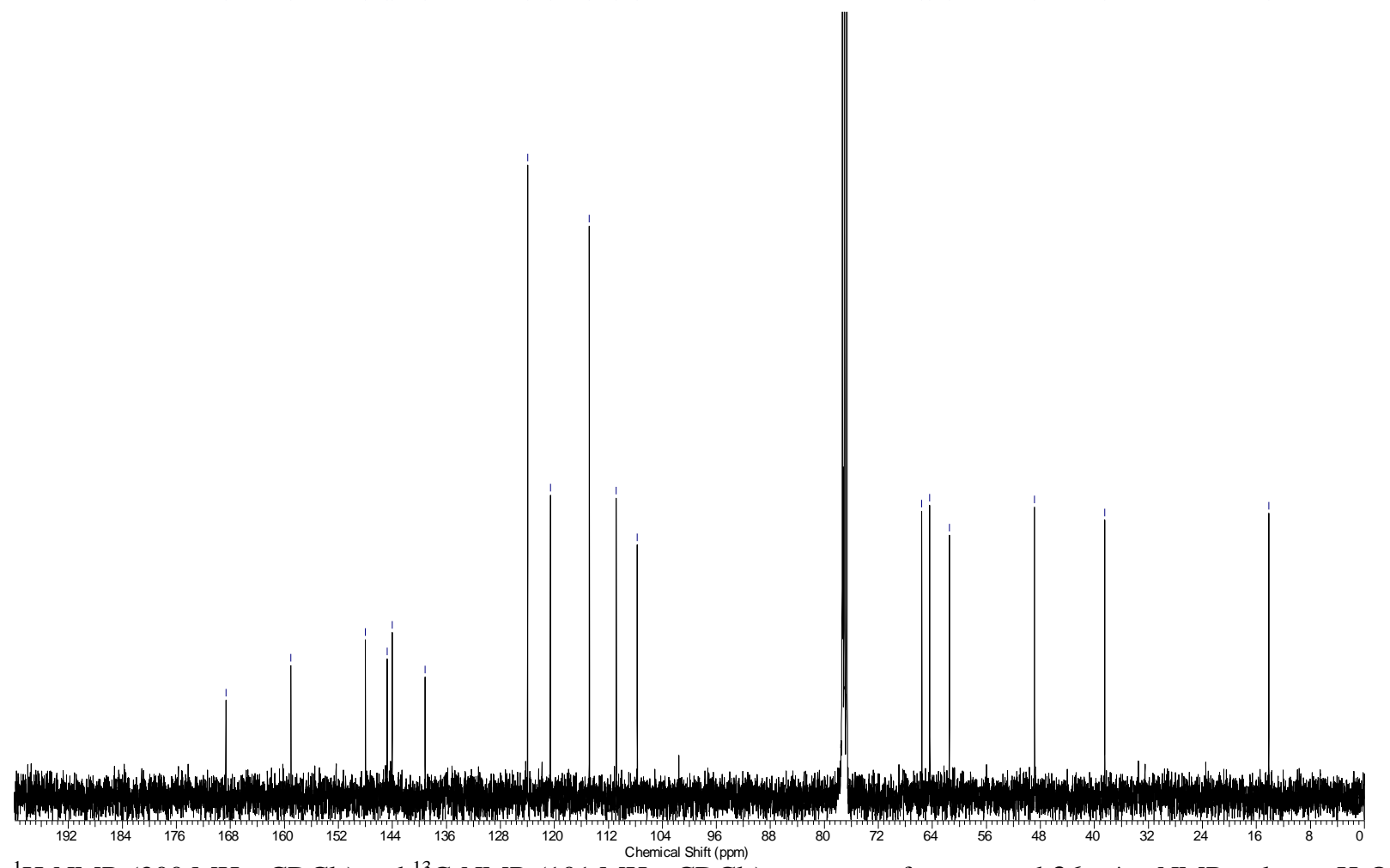

${ }^{1} \mathrm{H}-\mathrm{NMR}\left(300 \mathrm{MHz}, \mathrm{CDCl}_{3}\right.$ ) and ${ }^{13} \mathrm{C}-\mathrm{NMR}\left(101 \mathrm{MHz}, \mathrm{CDCl}_{3}\right.$ ) spectrum of compound 36a. * = NMR-solvent, $\mathrm{H}_{2} \mathrm{O}$ 

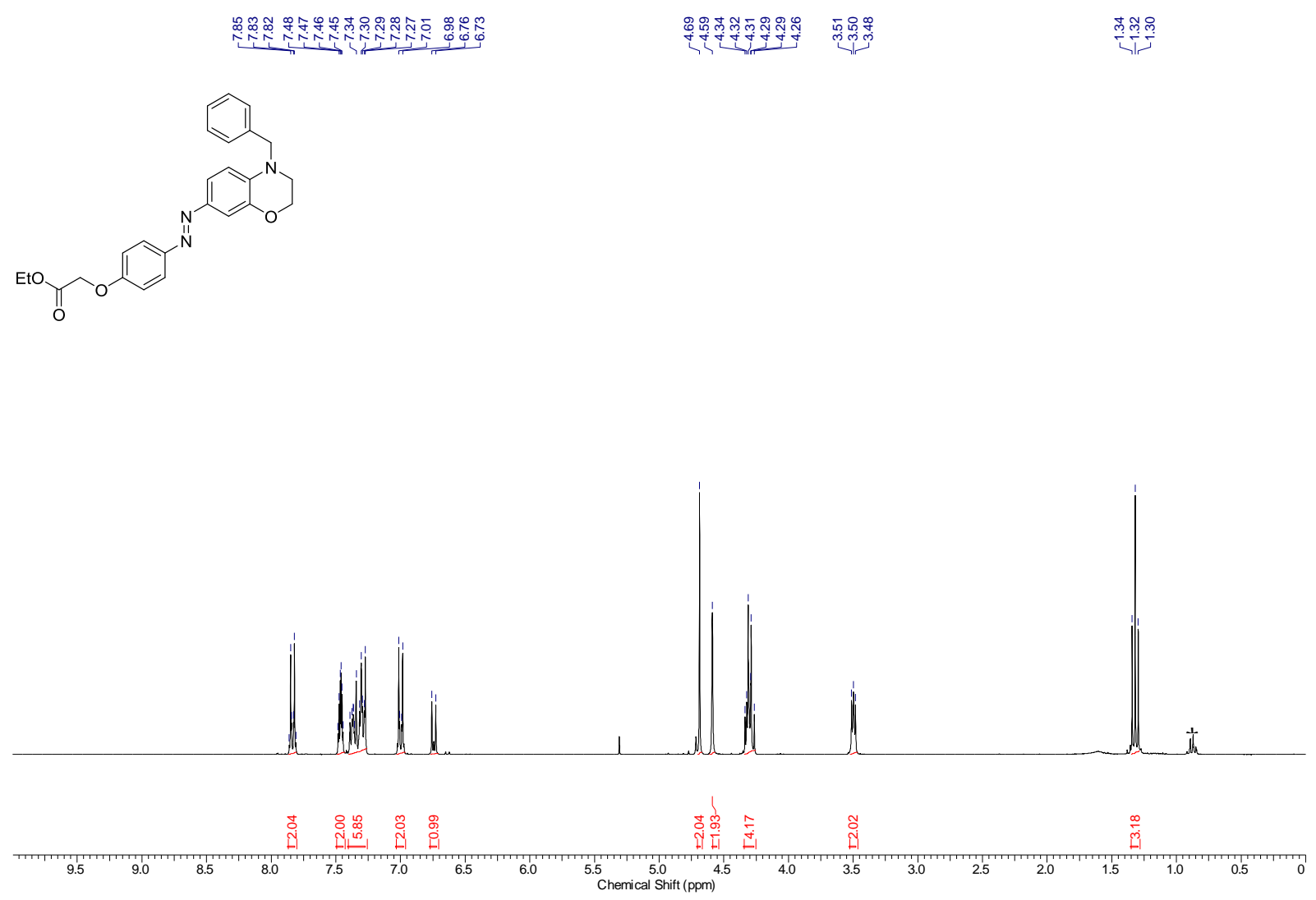

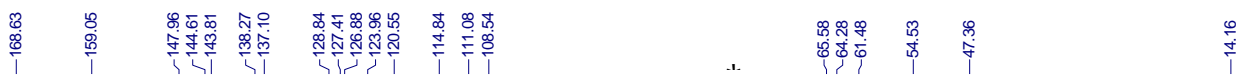

1.w.

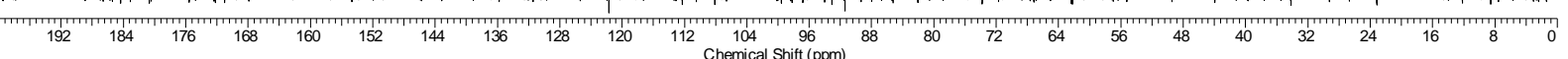

${ }^{1} \mathrm{H}-\mathrm{NMR}\left(300 \mathrm{MHz}, \mathrm{CDCl}_{3}\right)$ and ${ }^{13} \mathrm{C}-\mathrm{NMR}\left(75 \mathrm{MHz}, \mathrm{CDCl}_{3}\right)$ spectrum of compound $\mathbf{3 6} \mathbf{b} . *$ = NMR-solvent, grease 

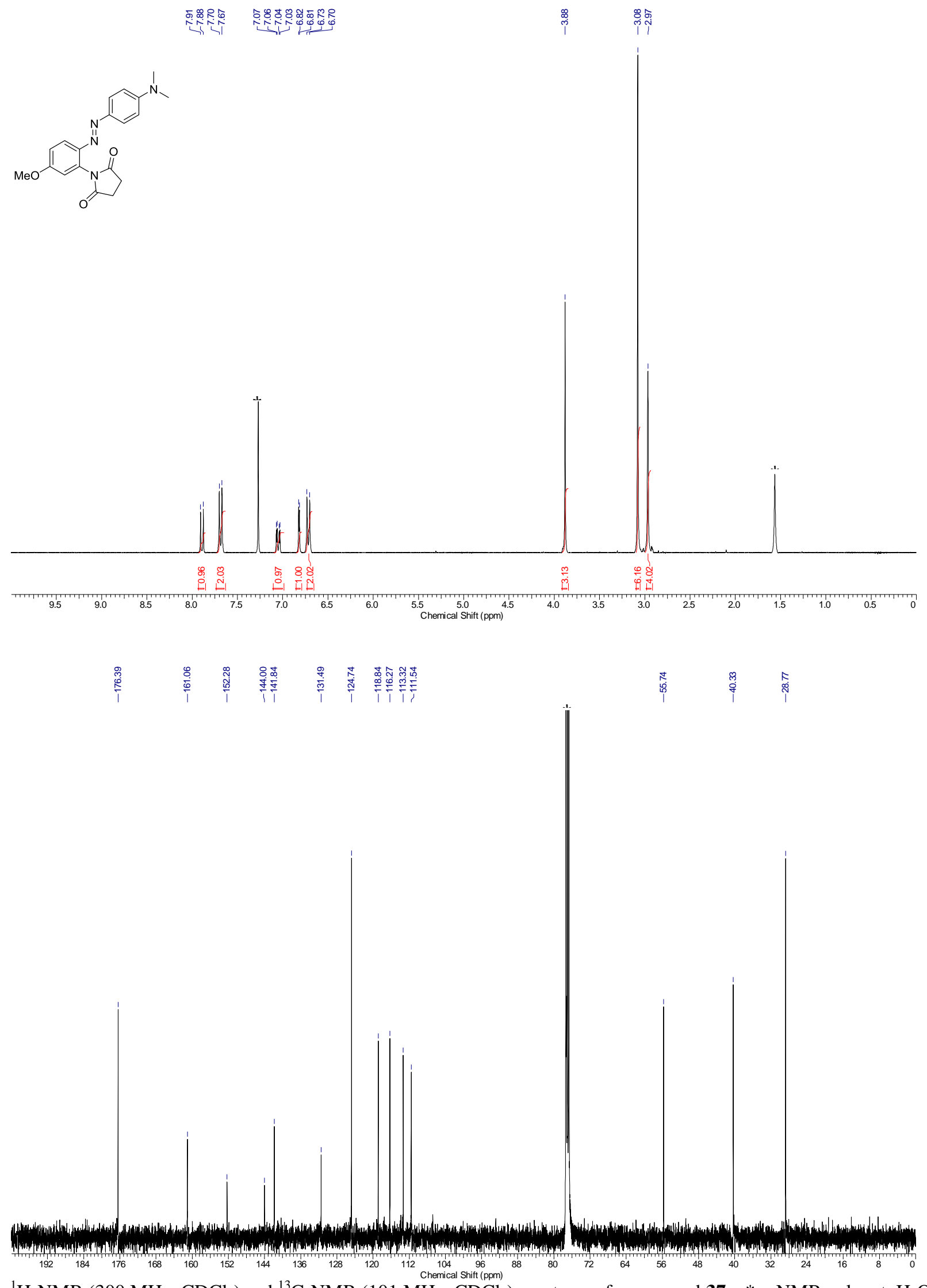

${ }^{1} \mathrm{H}-\mathrm{NMR}\left(300 \mathrm{MHz}, \mathrm{CDCl}_{3}\right.$ ) and ${ }^{13} \mathrm{C}-\mathrm{NMR}\left(101 \mathrm{MHz}^{\text {Chemical Shit (pom) }} \mathrm{CDCl}_{3}\right.$ ) spectrum of compound 37c. * = NMR-solvent, $\mathrm{H}_{2} \mathrm{O}$ 


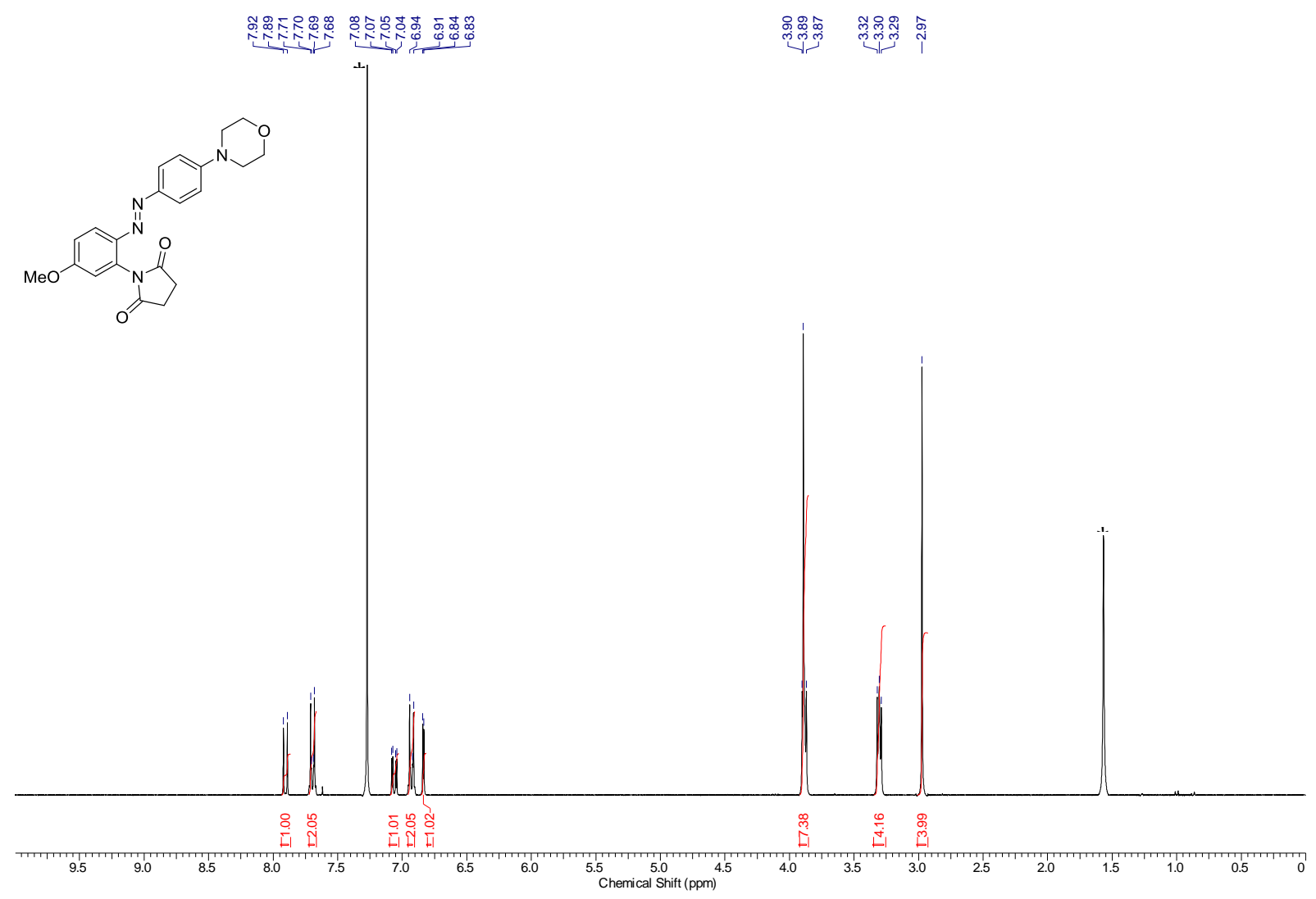

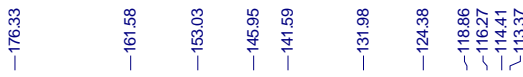
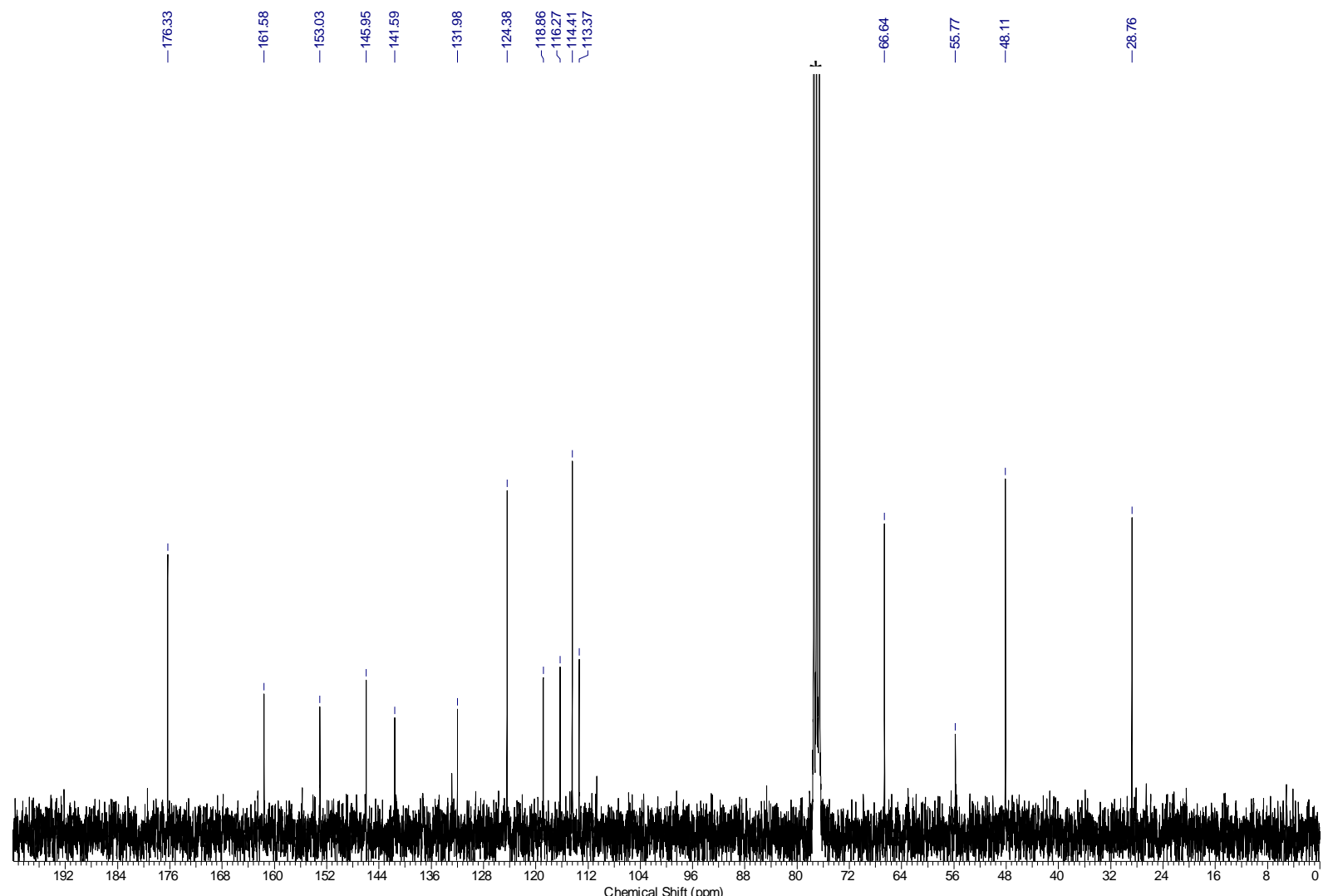

${ }^{1} \mathrm{H}-\mathrm{NMR}\left(300 \mathrm{MHz}, \mathrm{CDCl}_{3}\right)$ and ${ }^{13} \mathrm{C}-\mathrm{NMR}\left(75 \mathrm{MHz}, \mathrm{CDCl}_{3}\right.$ ) spectrum of compound 37d. * = NMR-solvent, $\mathrm{H}_{2} \mathrm{O}$ 


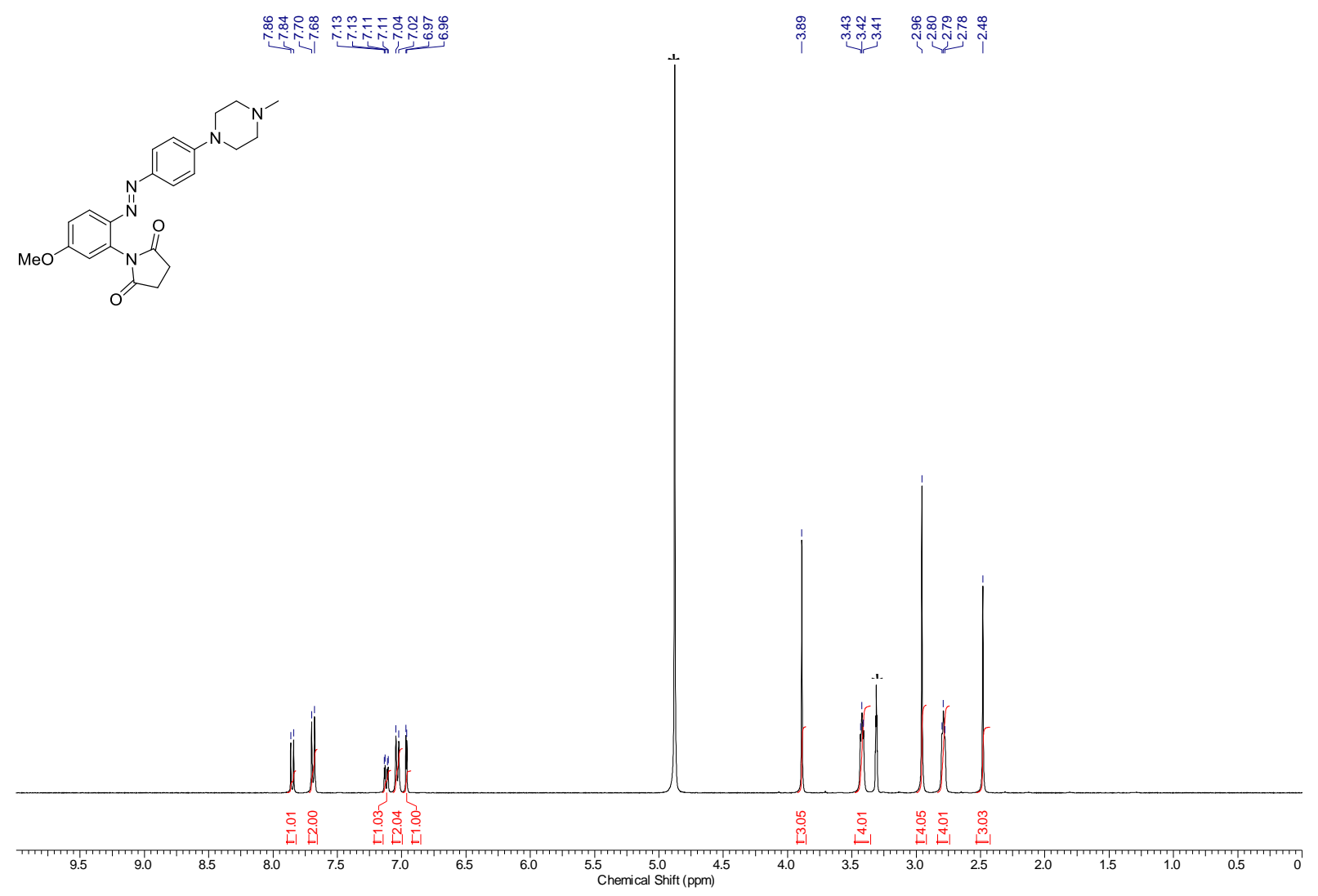

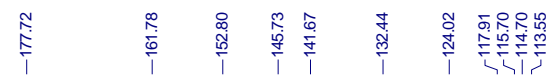
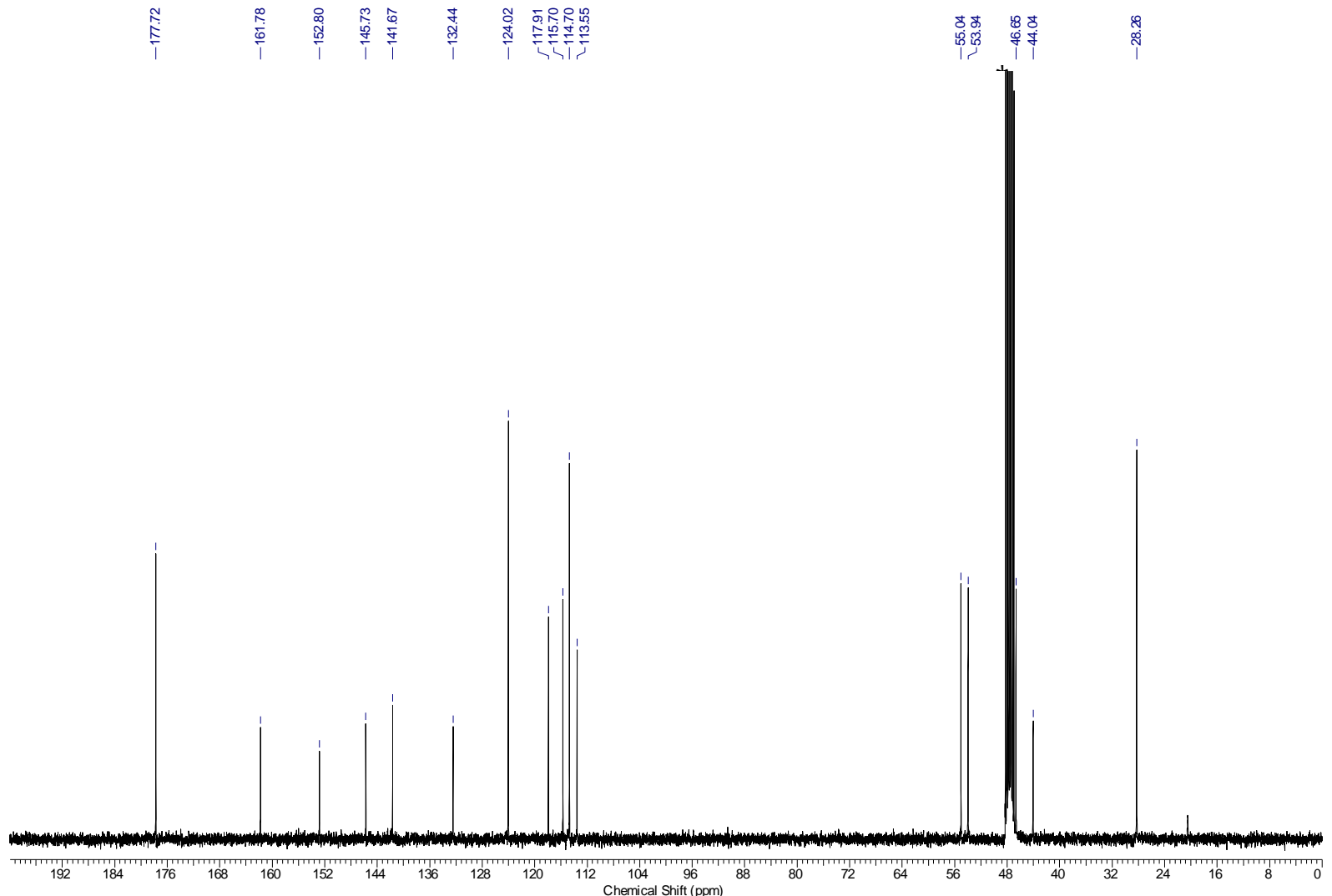

${ }^{1} \mathrm{H}-\mathrm{NMR}\left(400 \mathrm{MHz}, \mathrm{CD}_{3} \mathrm{OD}\right)$ and ${ }^{13} \mathrm{C}-\mathrm{NMR}\left(101 \mathrm{MHz}, \mathrm{CD}_{3} \mathrm{OD}\right)$ spectrum of compound 37e. * = NMR-solvent, $\mathrm{H}_{2} \mathrm{O}$ 


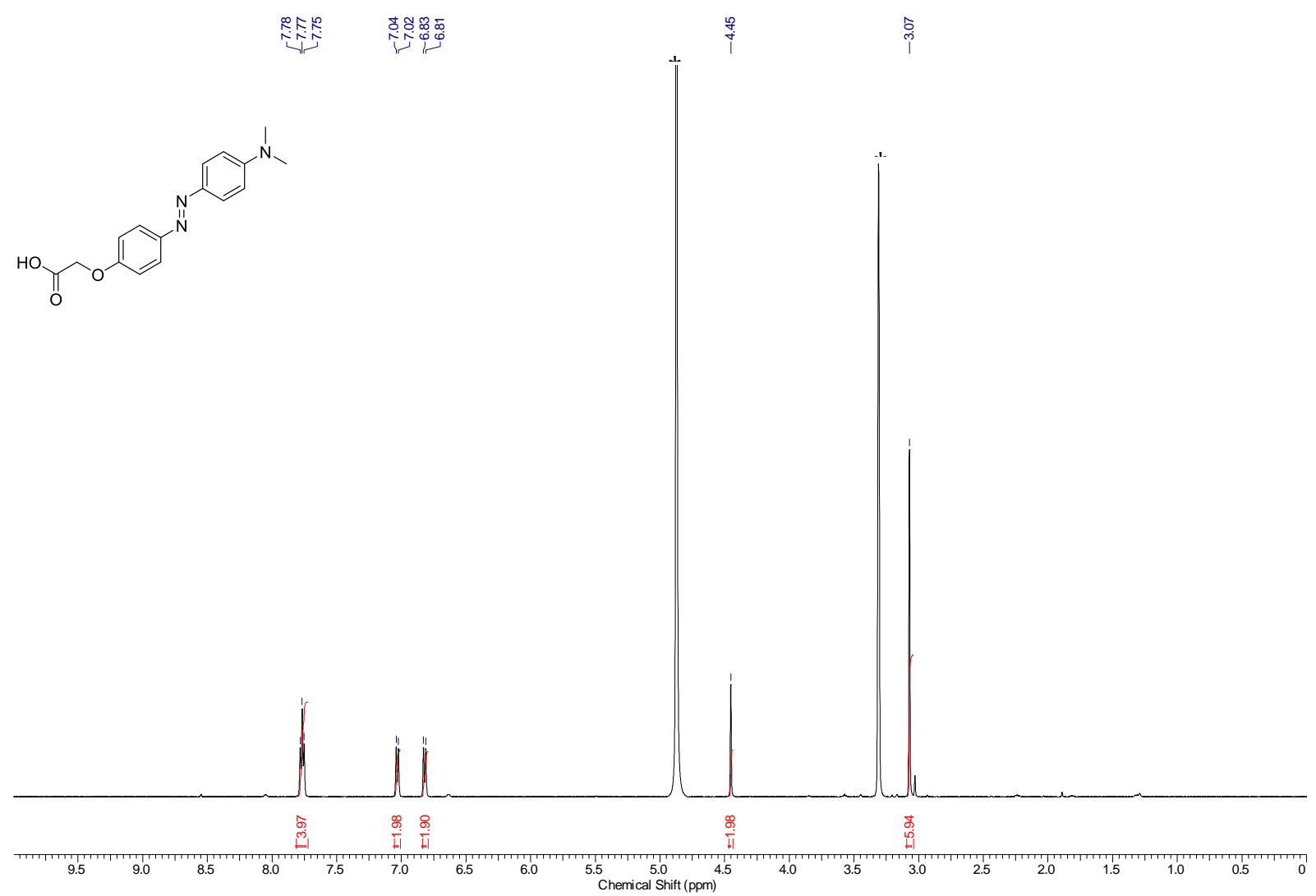

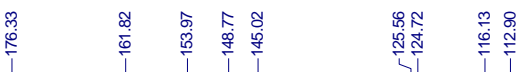

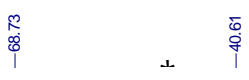

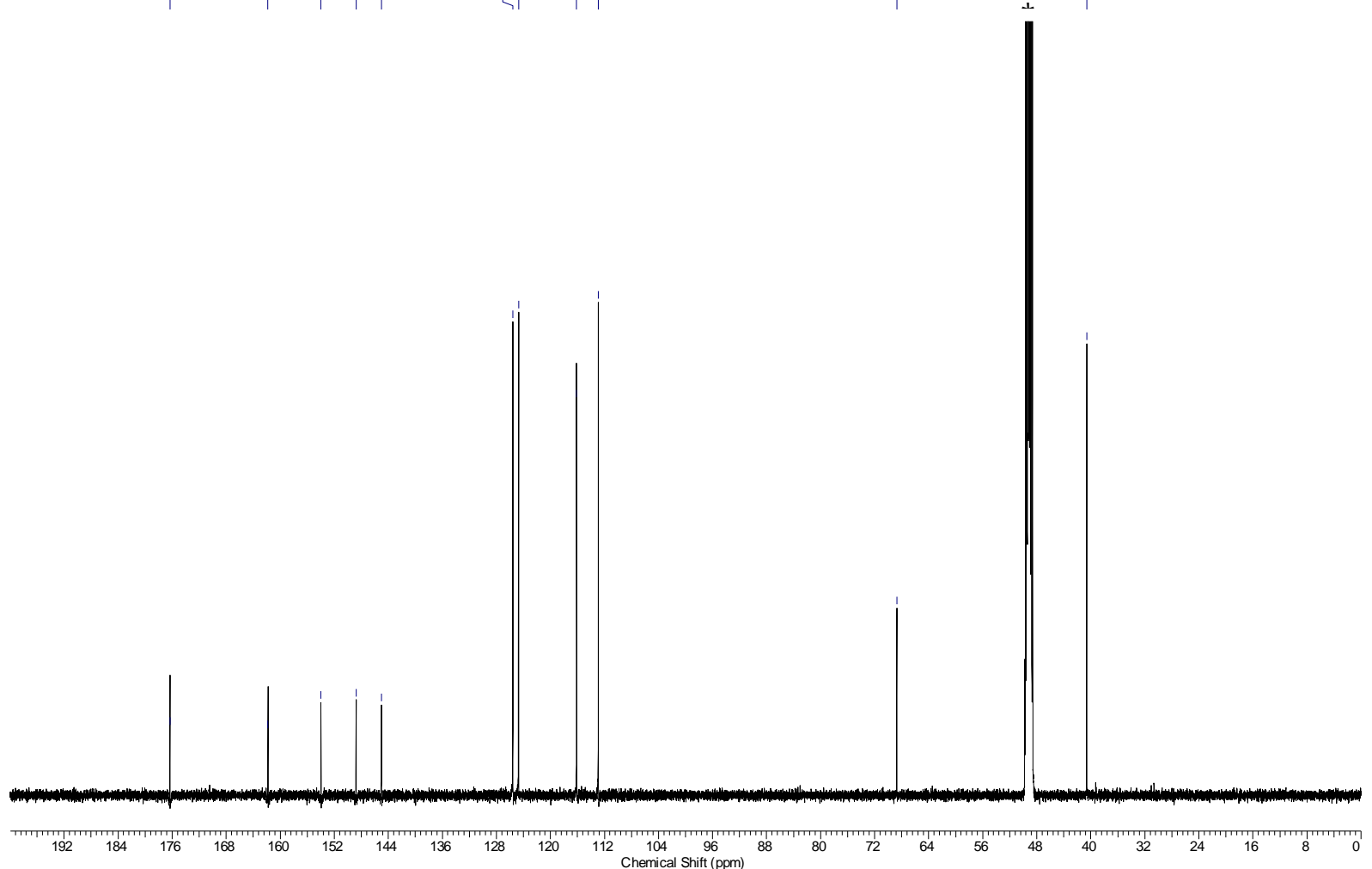

${ }^{1} \mathrm{H}-\mathrm{NMR}\left(500 \mathrm{MHz}, \mathrm{CD}_{3} \mathrm{OD}\right)$ and ${ }^{13} \mathrm{C}-\mathrm{NMR}\left(126 \mathrm{MHz}, \mathrm{CD}_{3} \mathrm{OD}\right)$ spectrum of compound $\mathbf{3 8 b}$. * = NMR-solvent, $\mathrm{H}_{2} \mathrm{O}$ 


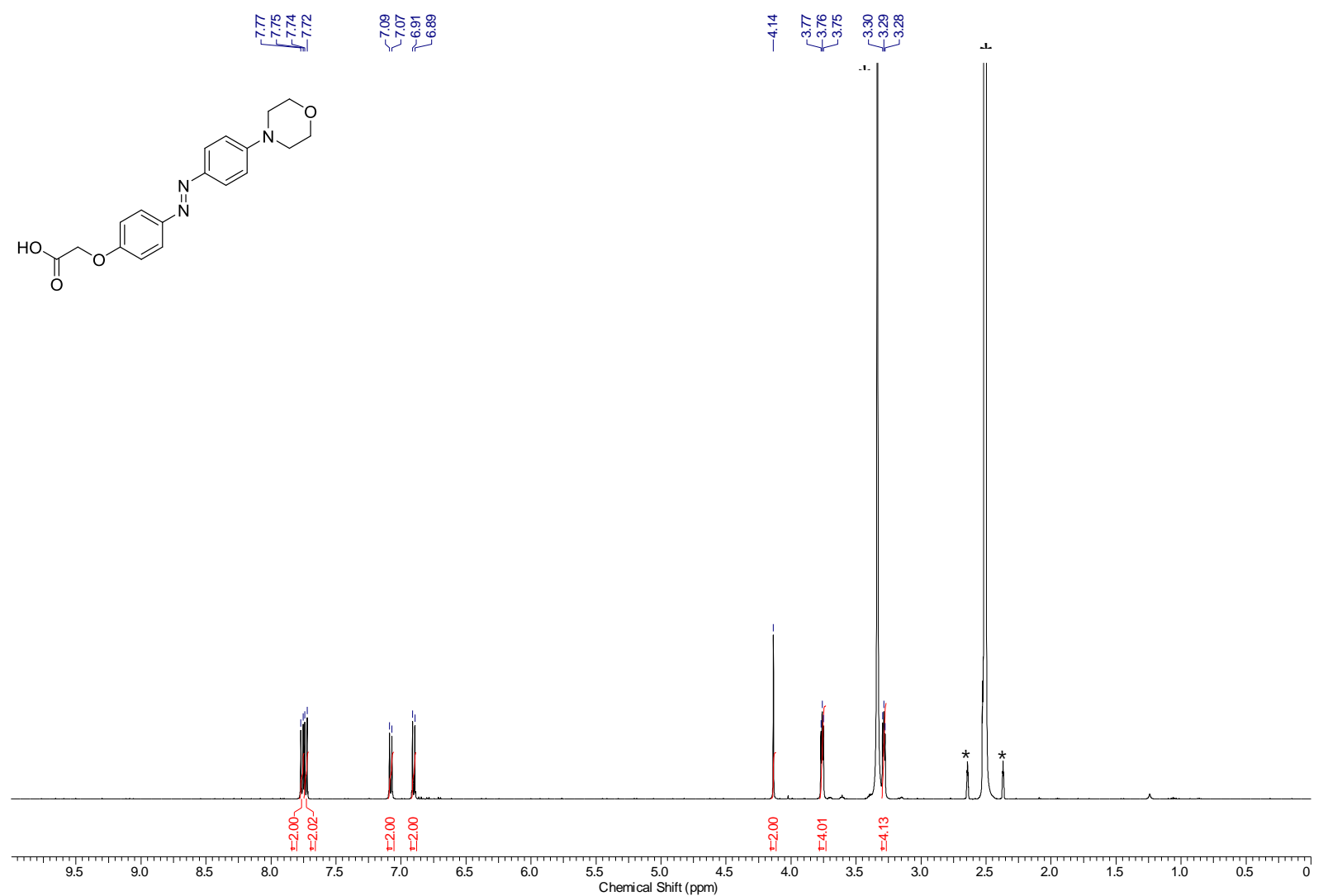

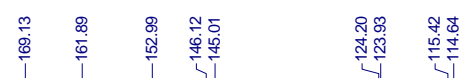

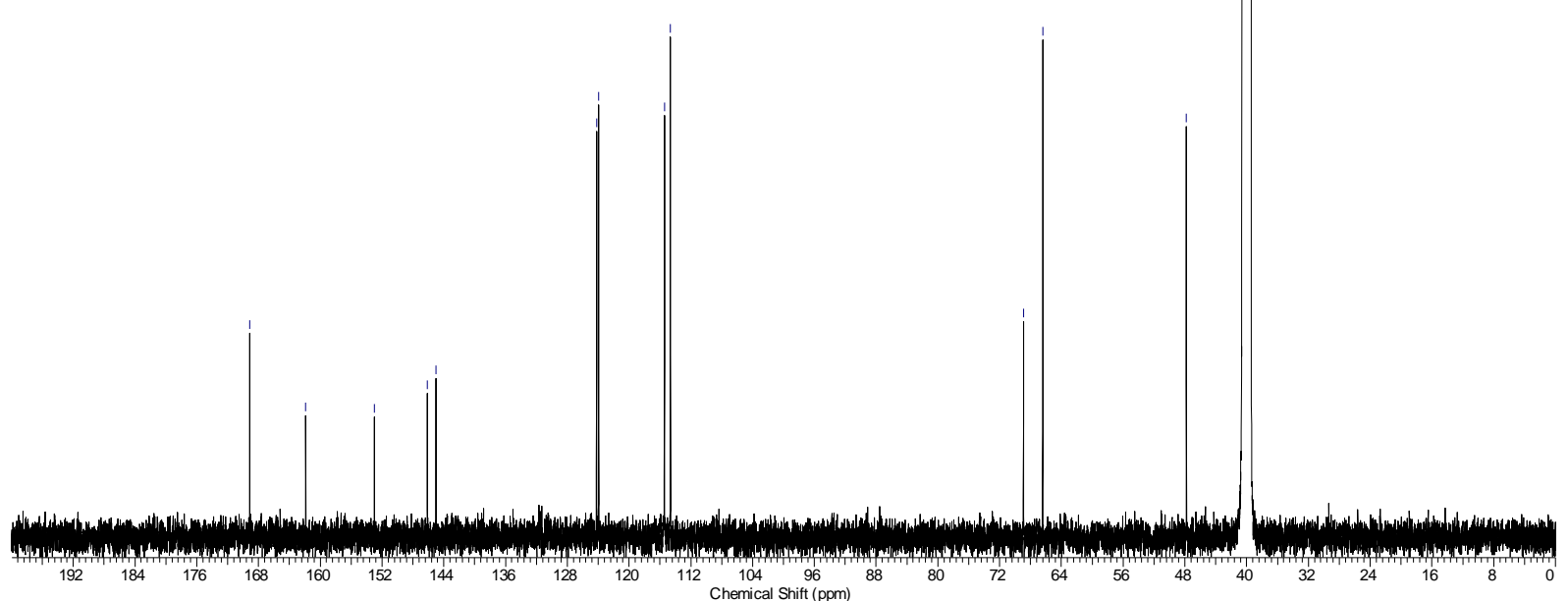

${ }^{1} \mathrm{H}-\mathrm{NMR}\left(500 \mathrm{MHz}, \mathrm{DMSO}-d_{6}\right)$ and ${ }^{13} \mathrm{C}-\mathrm{NMR}\left(126 \mathrm{MHz}\right.$, DMSO- $\left.d_{6}\right)$ spectrum of compound 38c. $*=\mathrm{NMR}$-solvent, $\mathrm{H}_{2} \mathrm{O}$ 


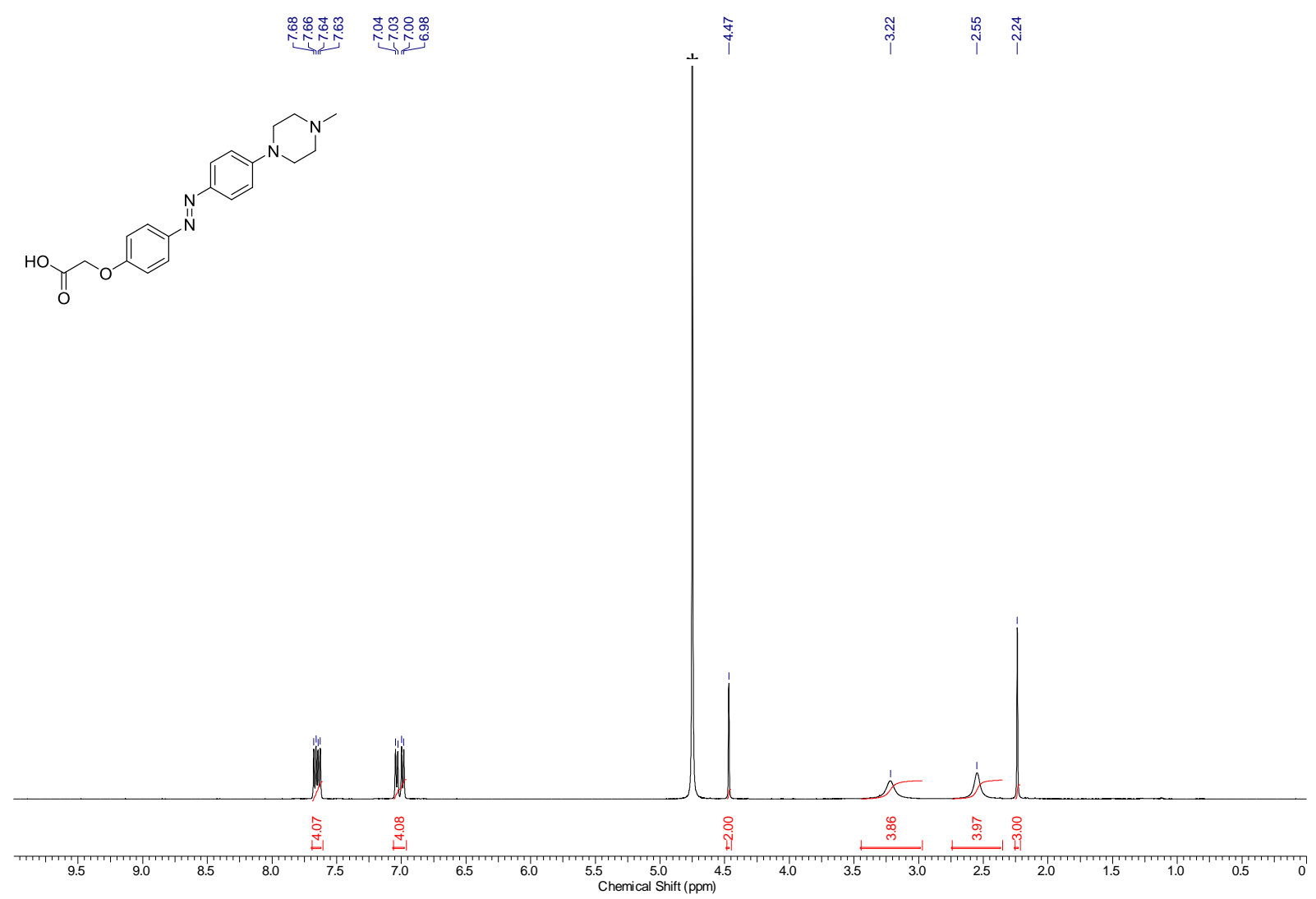

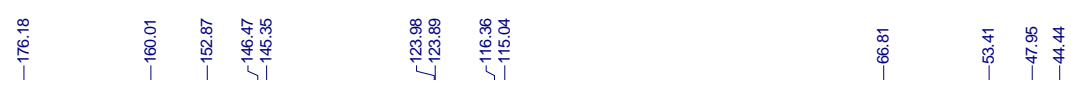

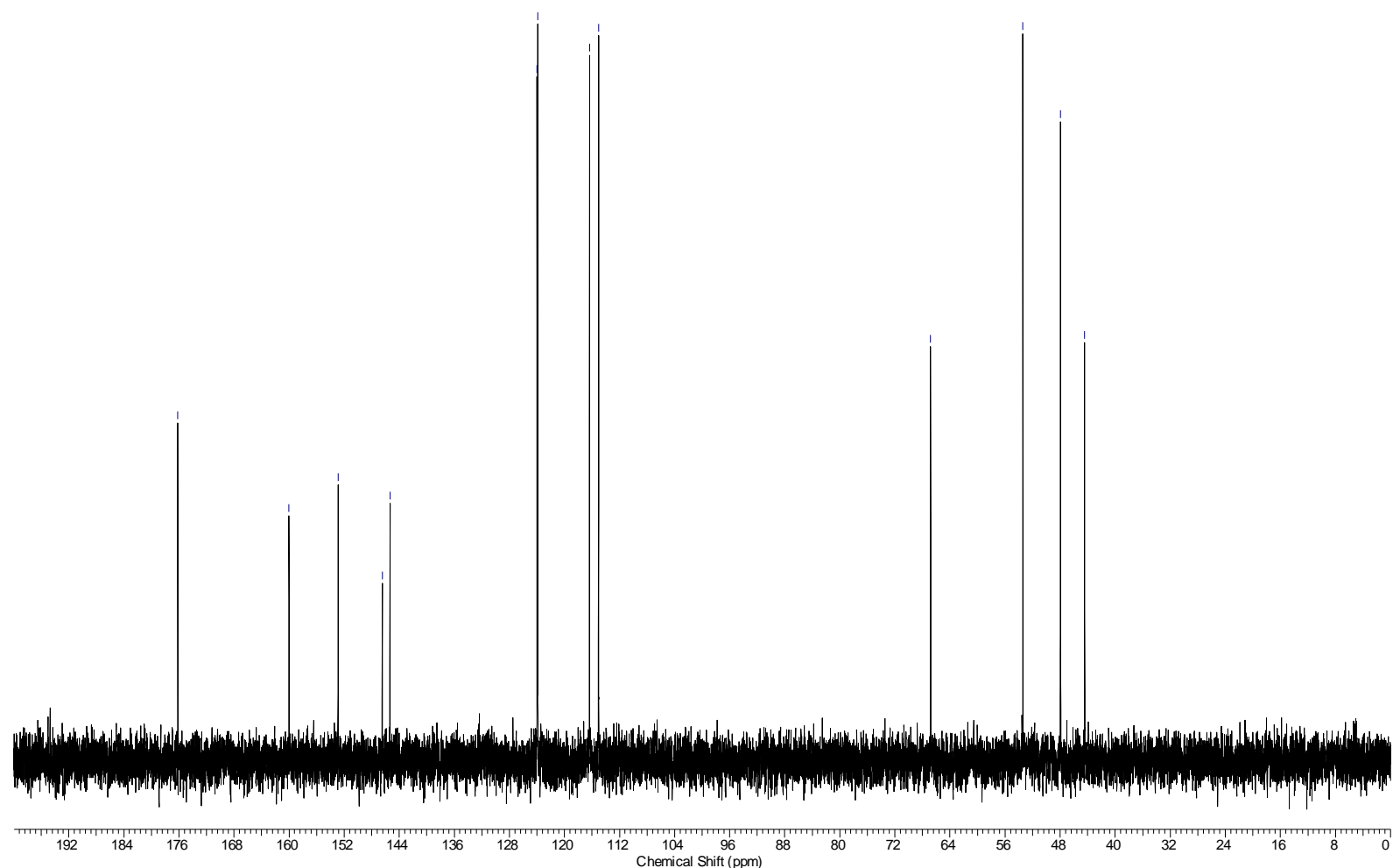

${ }^{1} \mathrm{H}-\mathrm{NMR}\left(500 \mathrm{MHz}, \mathrm{D}_{2} \mathrm{O}\right)$ and ${ }^{13} \mathrm{C}-\mathrm{NMR}\left(126 \mathrm{MHz}, \mathrm{D}_{2} \mathrm{O}\right)$ spectrum of compound 38d. * = NMR-solvent 


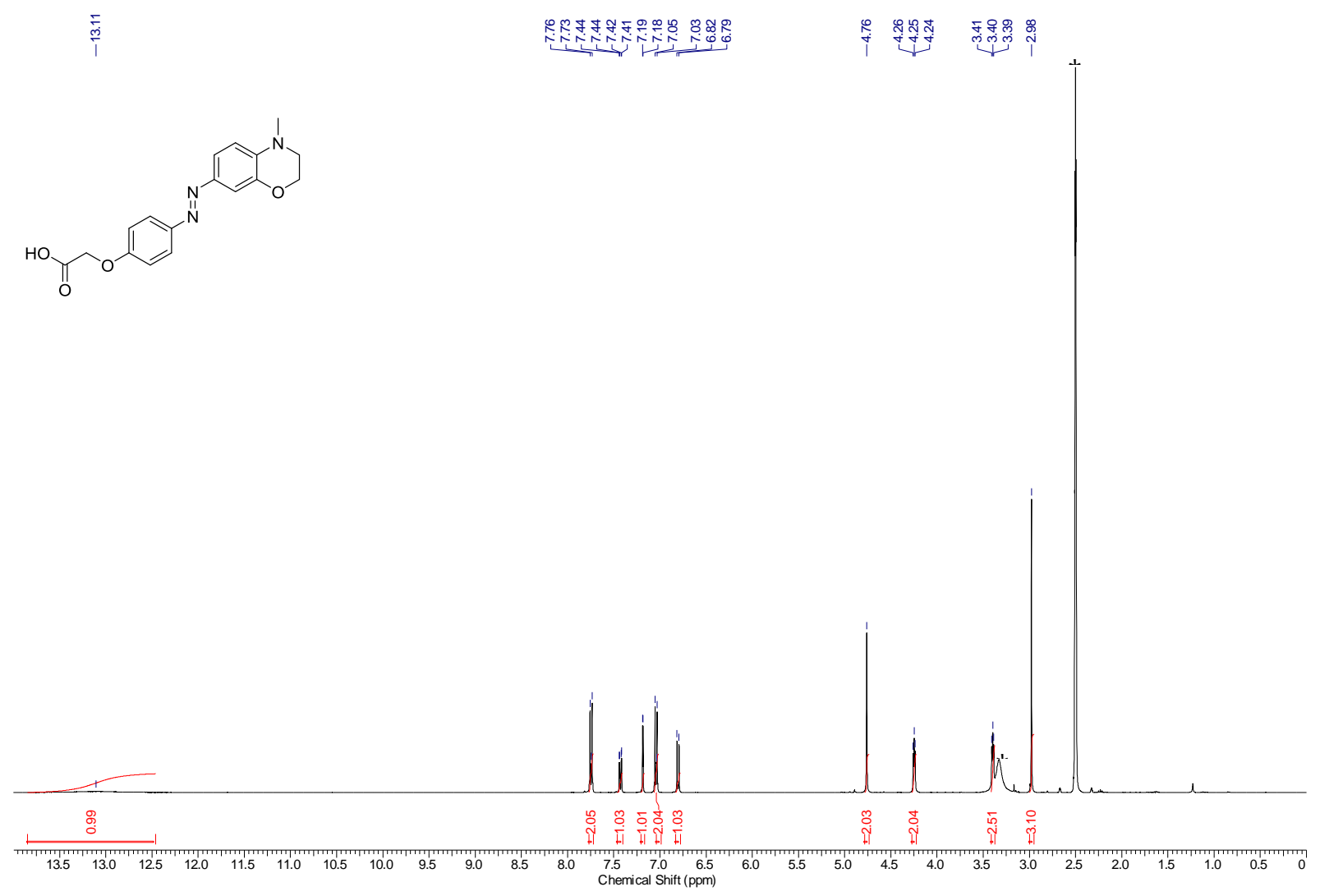

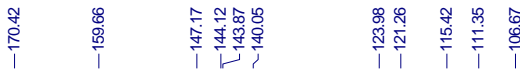

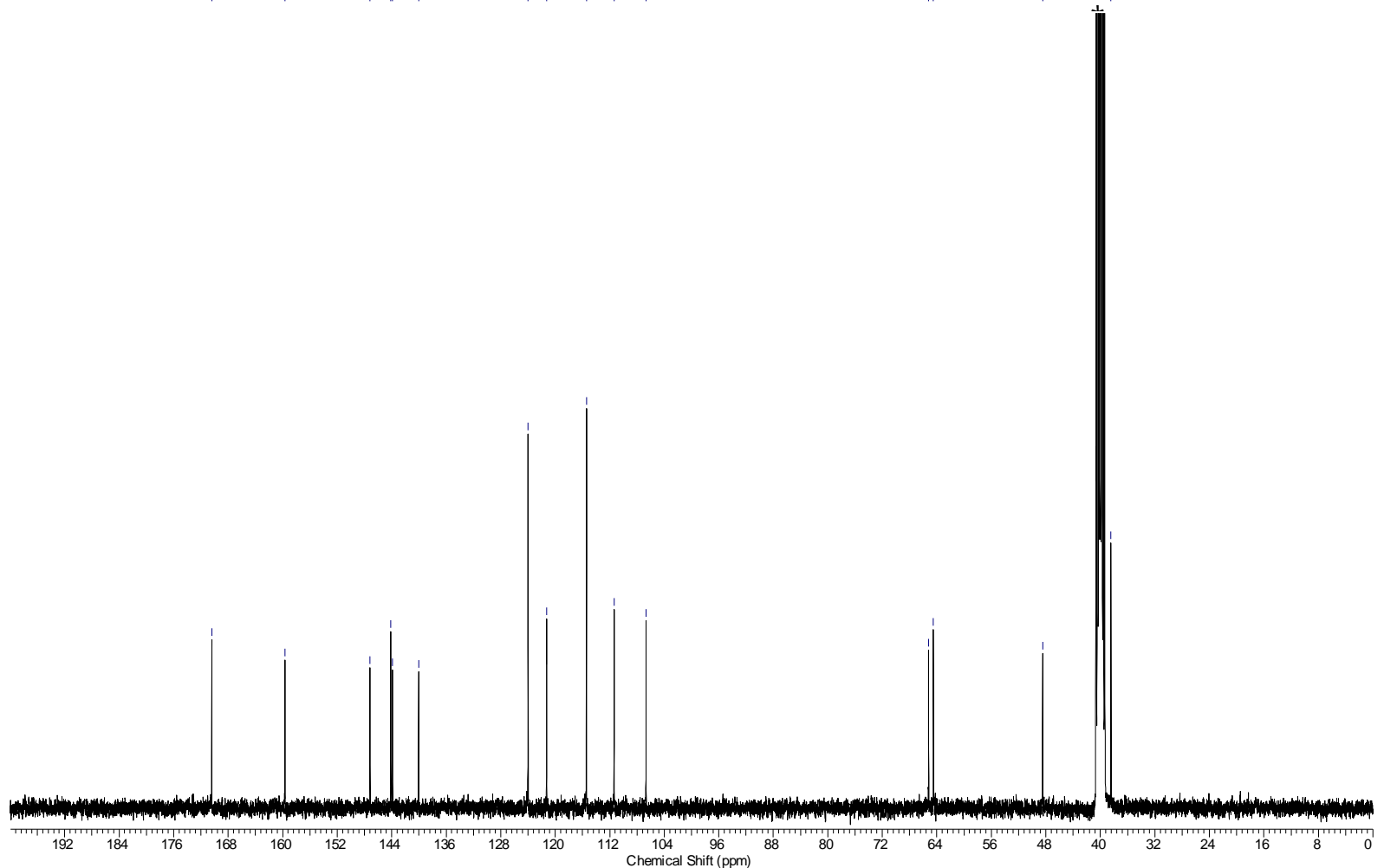

${ }^{1} \mathrm{H}-\mathrm{NMR}\left(400 \mathrm{MHz}\right.$, DMSO- $d_{6}$ ) and ${ }^{13} \mathrm{C}-\mathrm{NMR}\left(101 \mathrm{MHz}\right.$, DMSO- $d_{6}$ ) spectrum of compound 39a. * = NMR-solvent, $\mathrm{H}_{2} \mathrm{O}$ 

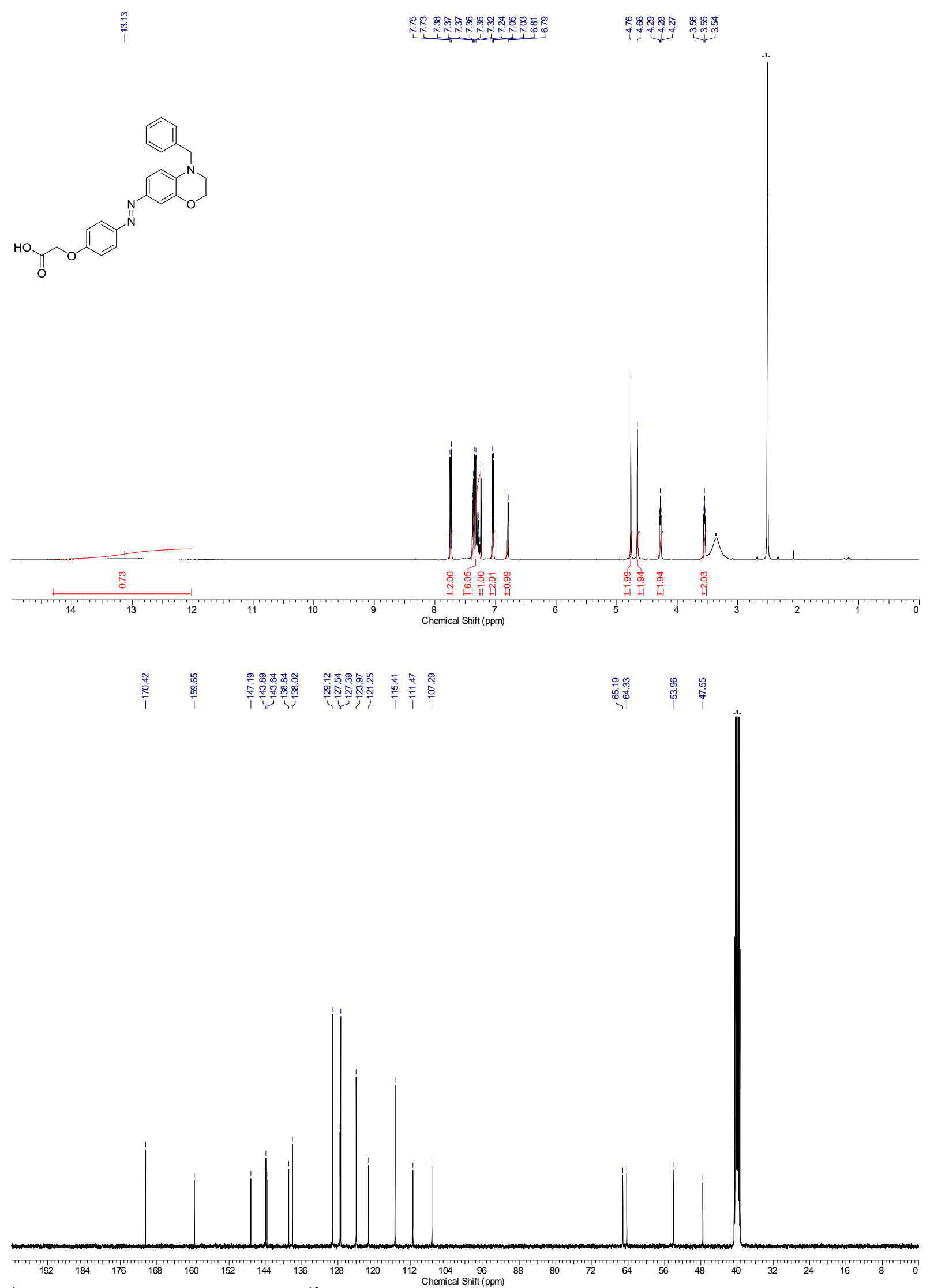

${ }^{1} \mathrm{H}-\mathrm{NMR}\left(400 \mathrm{MHz}\right.$, DMSO- $d_{6}$ ) and ${ }^{13} \mathrm{C}-\mathrm{NMR}\left(101 \mathrm{MHz}\right.$, DMSO- $d_{6}$ ) spectrum of compound 39b. * = NMR-solvent, $\mathrm{H}_{2} \mathrm{O}$ 


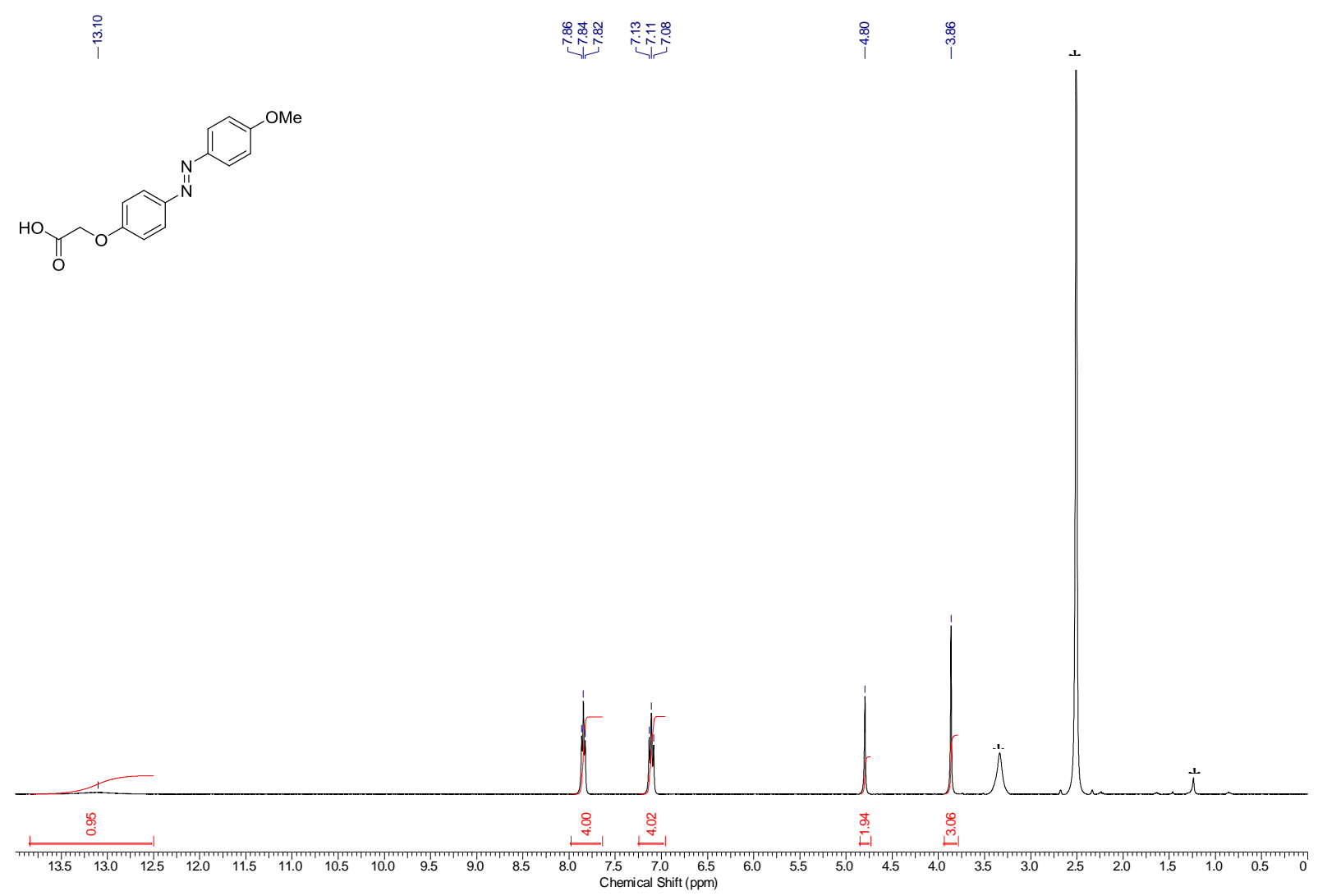

\begin{tabular}{|c|c|c|}
\hline 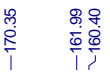 & 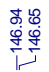 & 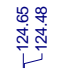 \\
\hline
\end{tabular}
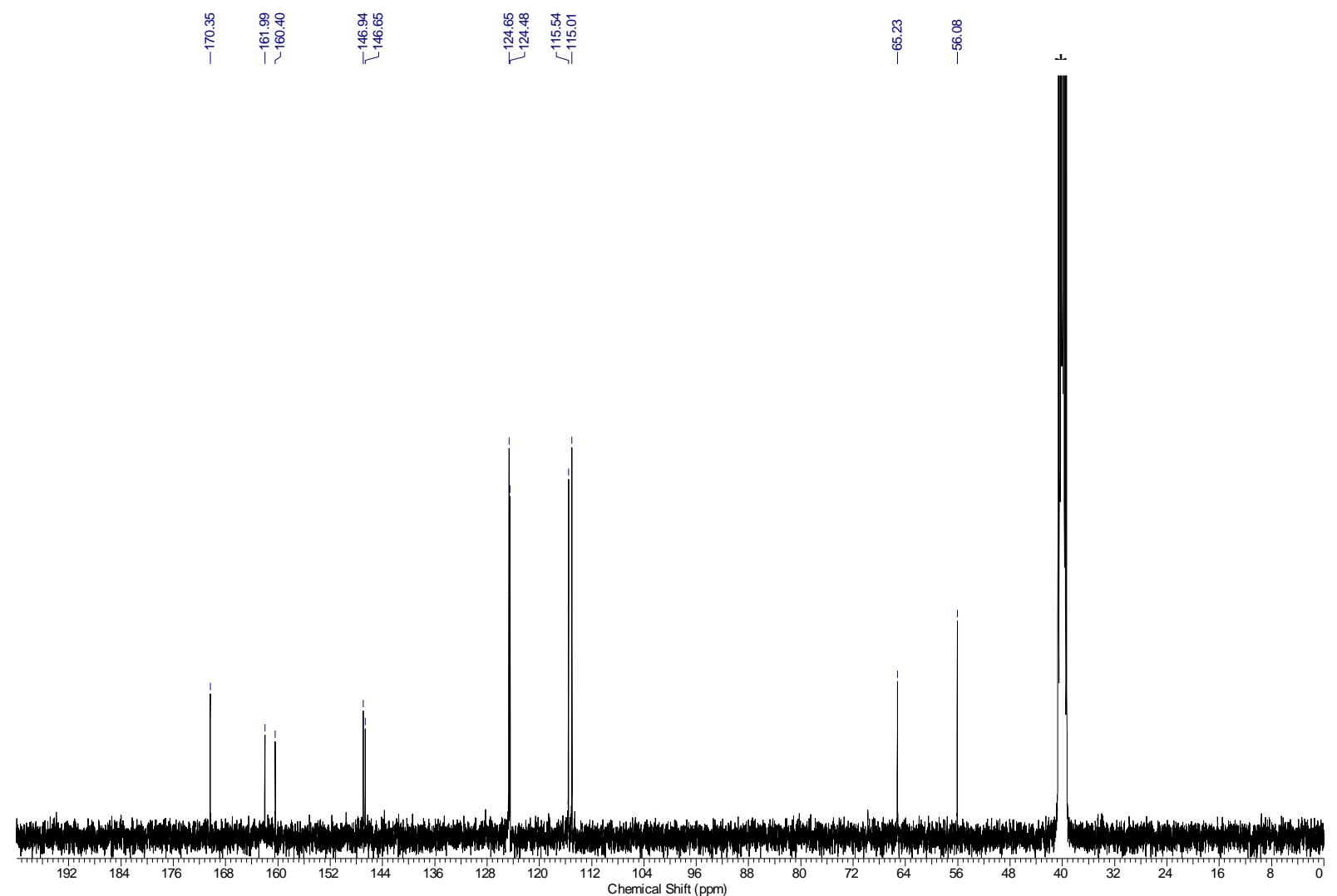

${ }^{1} \mathrm{H}-\mathrm{NMR}\left(400 \mathrm{MHz}, \mathrm{DMSO}-d_{6}\right)$ and ${ }^{13} \mathrm{C}-\mathrm{NMR}\left(101 \mathrm{MHz}, \mathrm{DMSO}-d_{6}\right)$ spectrum of compound 40a. $*$ = NMR-solvent, $\mathrm{H}_{2} \mathrm{O}$, grease 


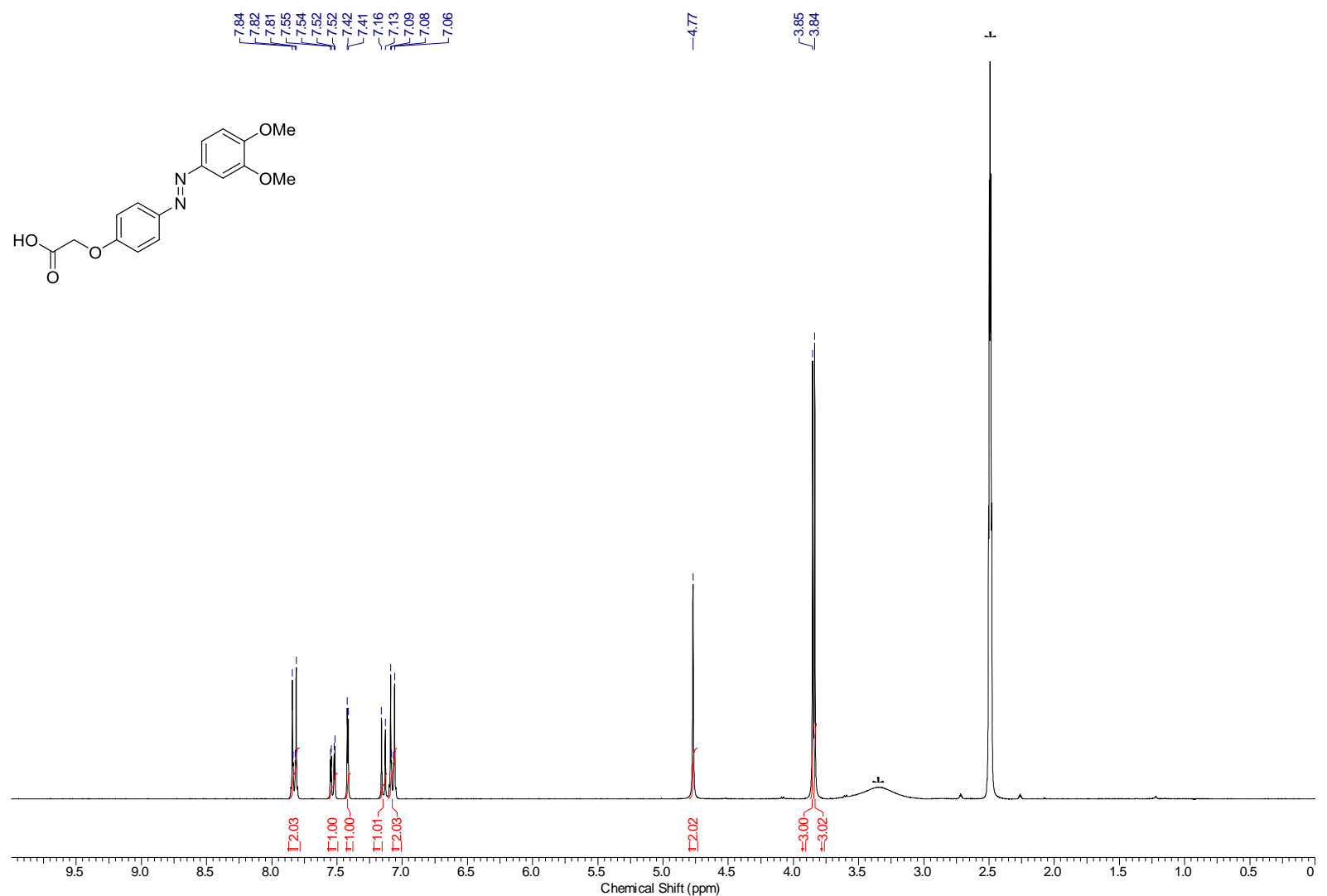

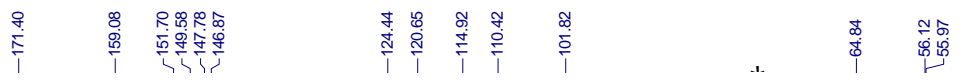

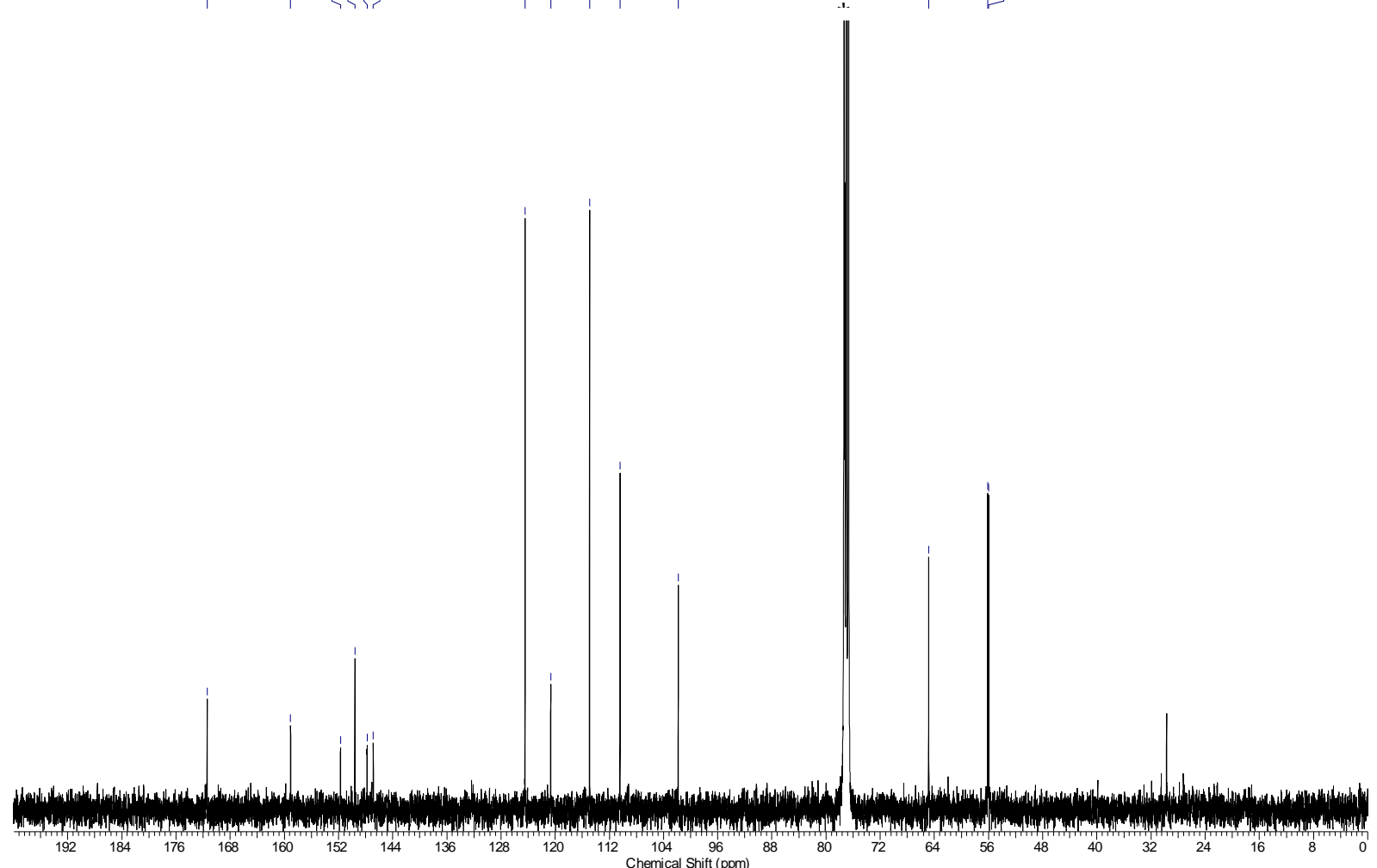

${ }^{1} \mathrm{H}-\mathrm{NMR}\left(300 \mathrm{MHz}, \mathrm{DMSO}-d_{6}\right)$ and ${ }^{13} \mathrm{C}-\mathrm{NMR}\left(101 \mathrm{MHz}, \mathrm{CDCl}_{3}\right)$ spectrum of compound 40b. * = NMR-solvent, $\mathrm{H}_{2} \mathrm{O}$ 


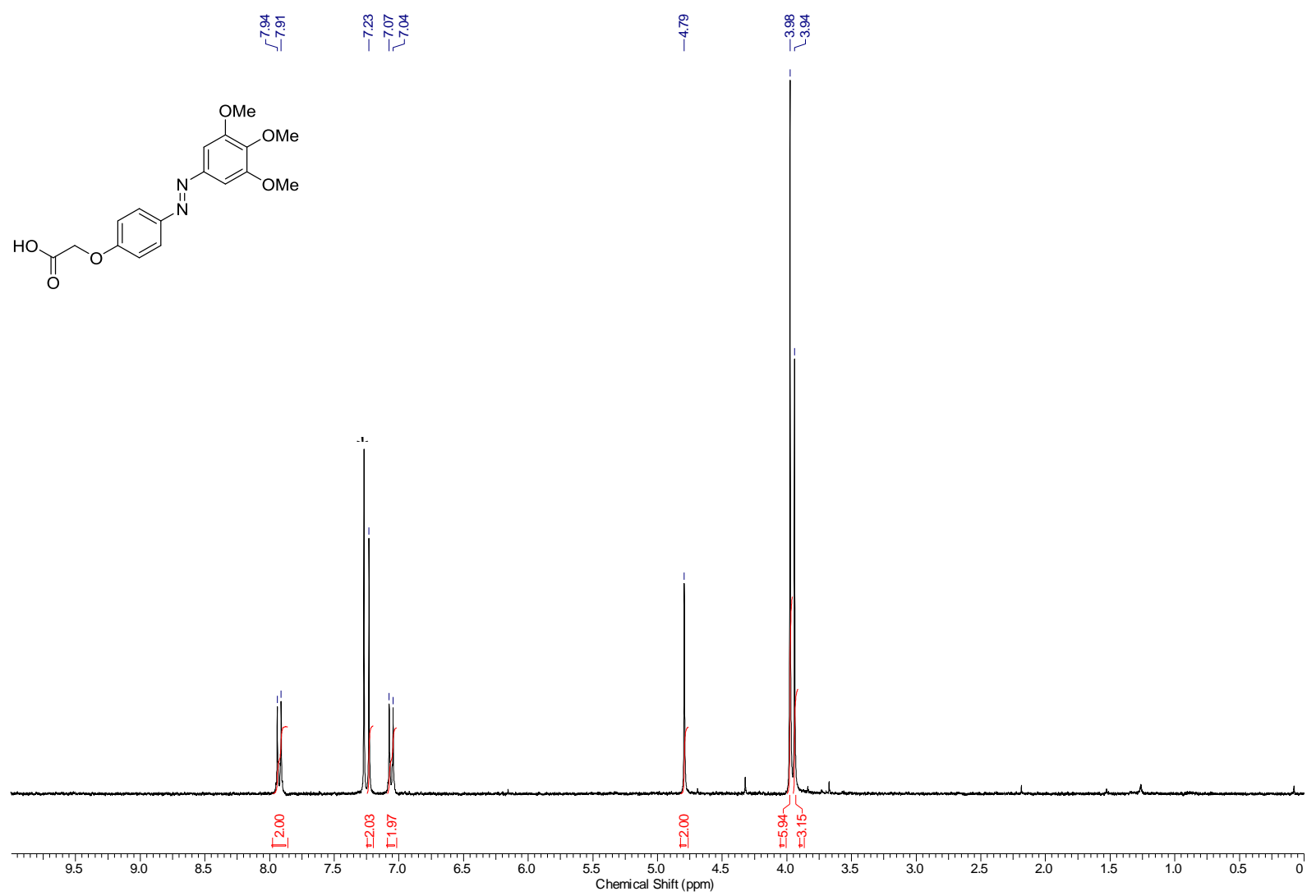

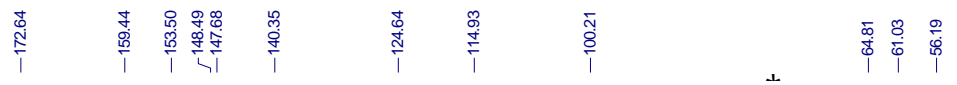

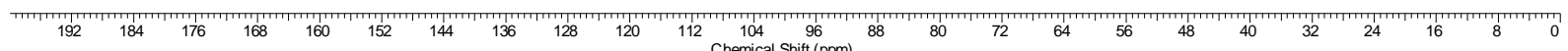
${ }^{1} \mathrm{H}-\mathrm{NMR}\left(300 \mathrm{MHz}, \mathrm{CDCl}_{3}\right)$ and ${ }^{13} \mathrm{C}-\mathrm{NMR}\left(101 \mathrm{MHz}, \mathrm{CDCl}_{3}\right.$ ) spectrum of compound 40c/44a. $*$ = NMR-solvent 


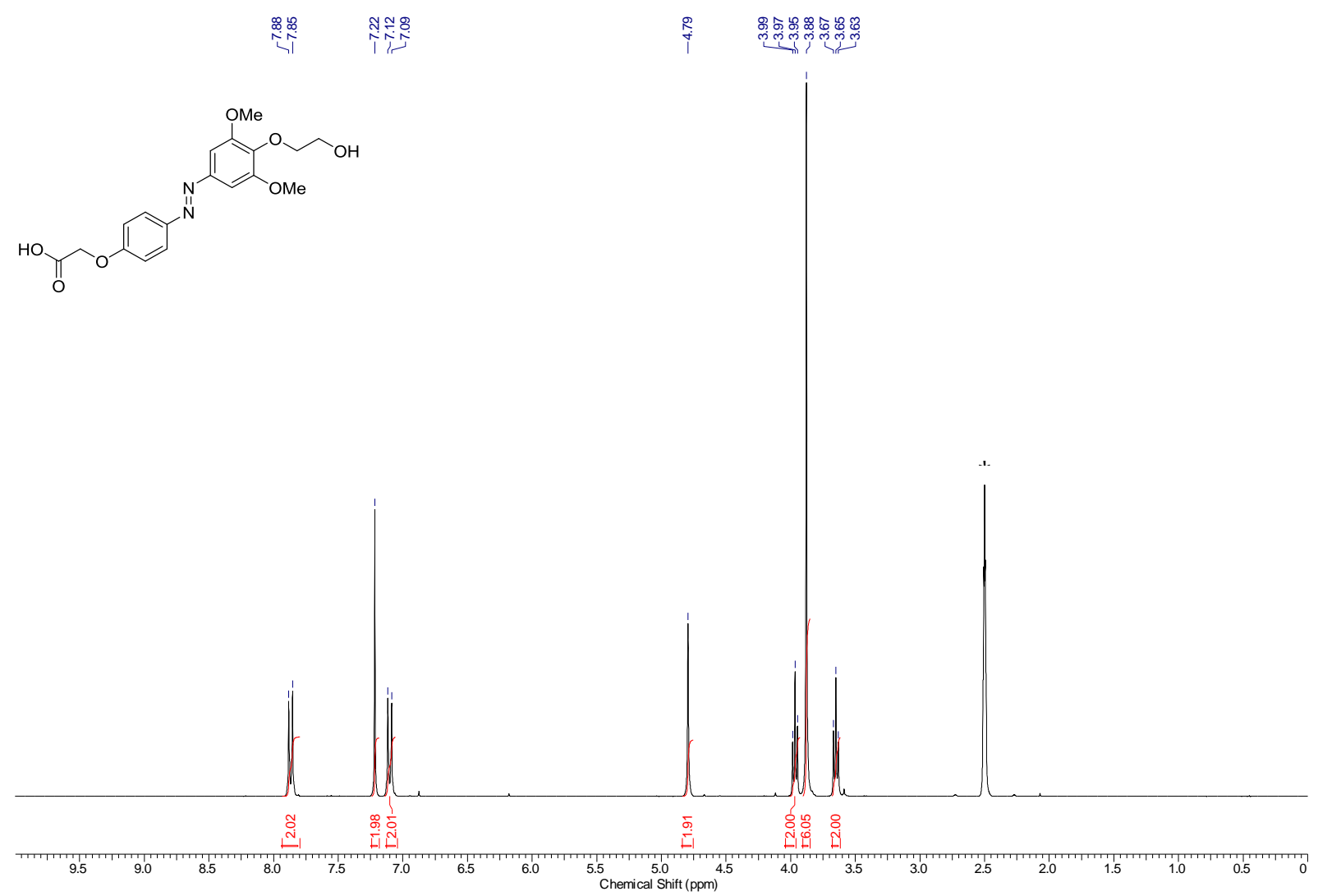

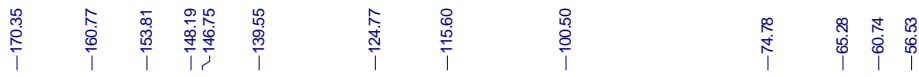

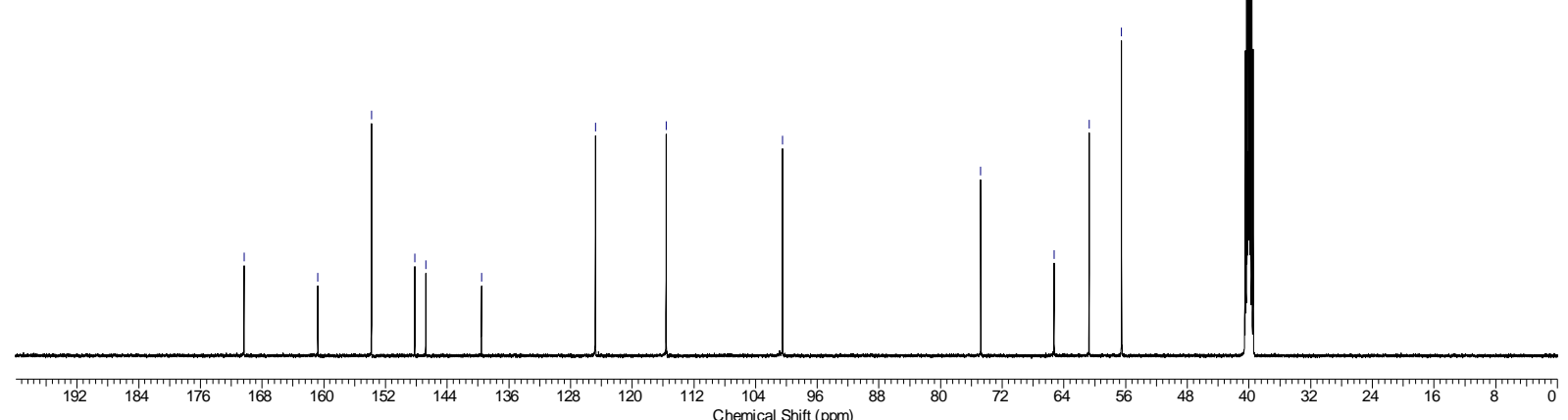

${ }^{1} \mathrm{H}-\mathrm{NMR}\left(300 \mathrm{MHz}\right.$, DMSO- $d_{6}$ ) and ${ }^{13} \mathrm{C}-\mathrm{NMR}\left(126 \mathrm{MHz}\right.$, DMSO- $\left.d_{6}\right)$ spectrum of compound 40d. * = NMR-Solvent 


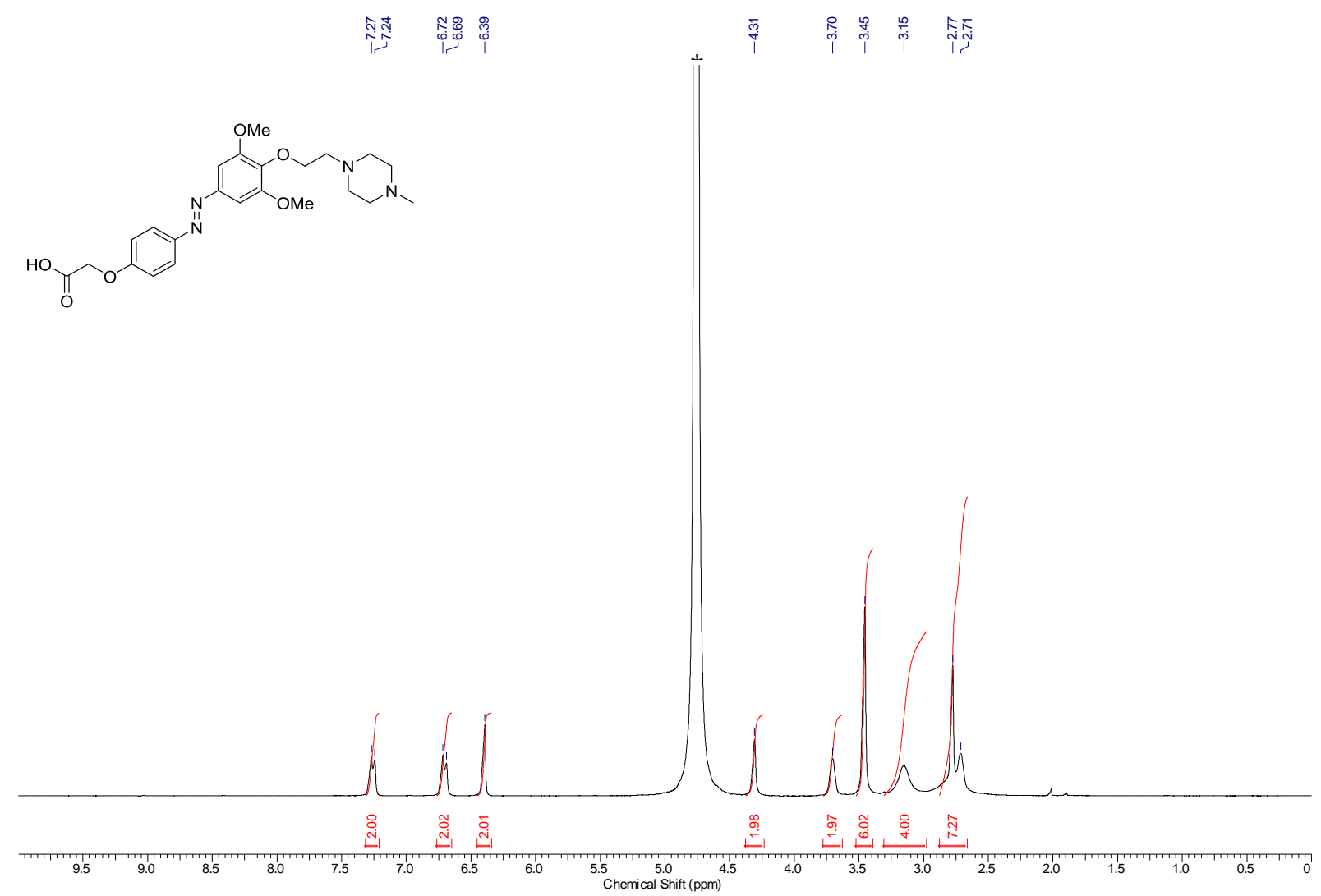

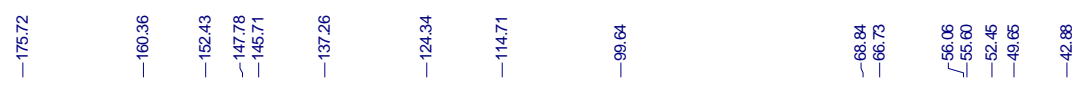

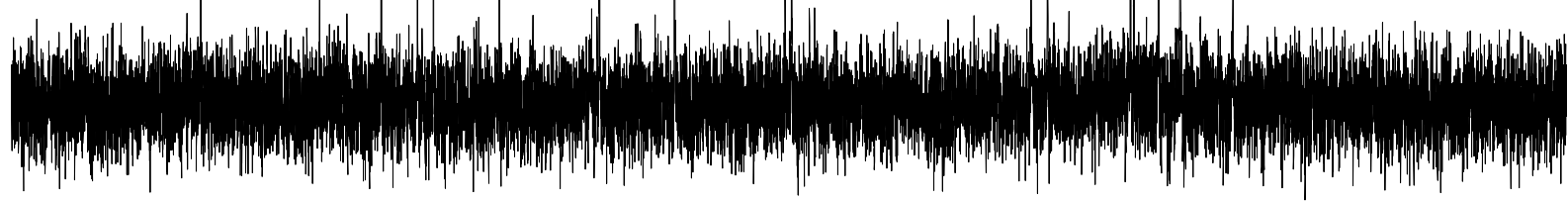

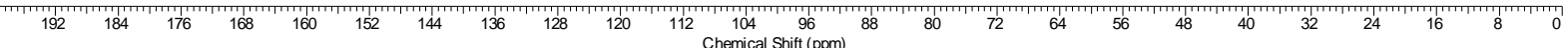

${ }^{1} \mathrm{H}-\mathrm{NMR}\left(300 \mathrm{MHz}, \mathrm{D}_{2} \mathrm{O}\right)$ and ${ }^{13} \mathrm{C}-\mathrm{NMR}\left(101 \mathrm{MHz}, \mathrm{D}_{2} \mathrm{O}\right)$ spectrum of compound 40f. * = NMR-Solvent 


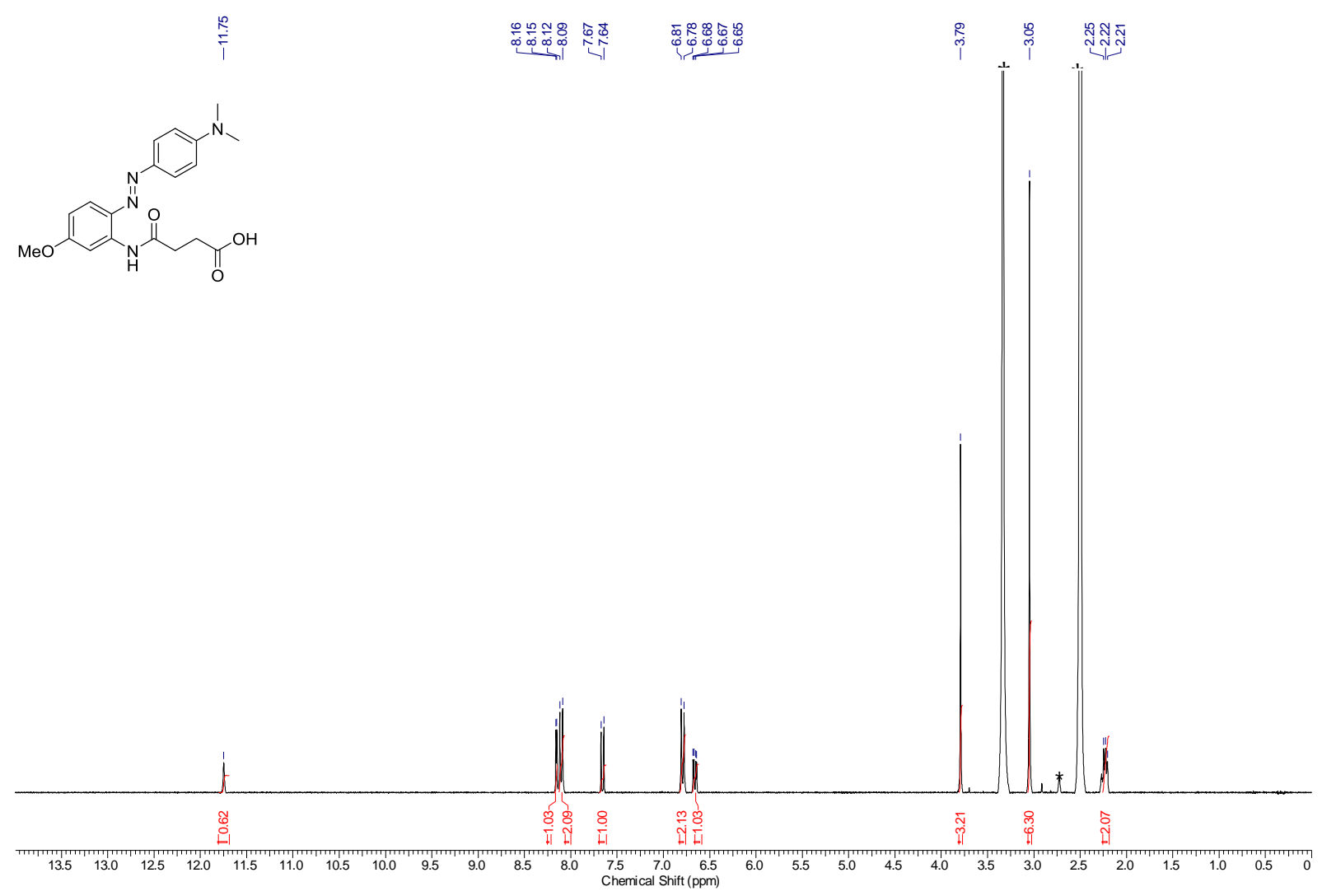

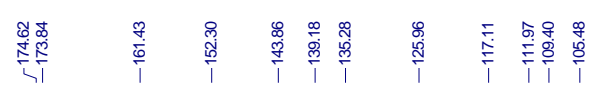

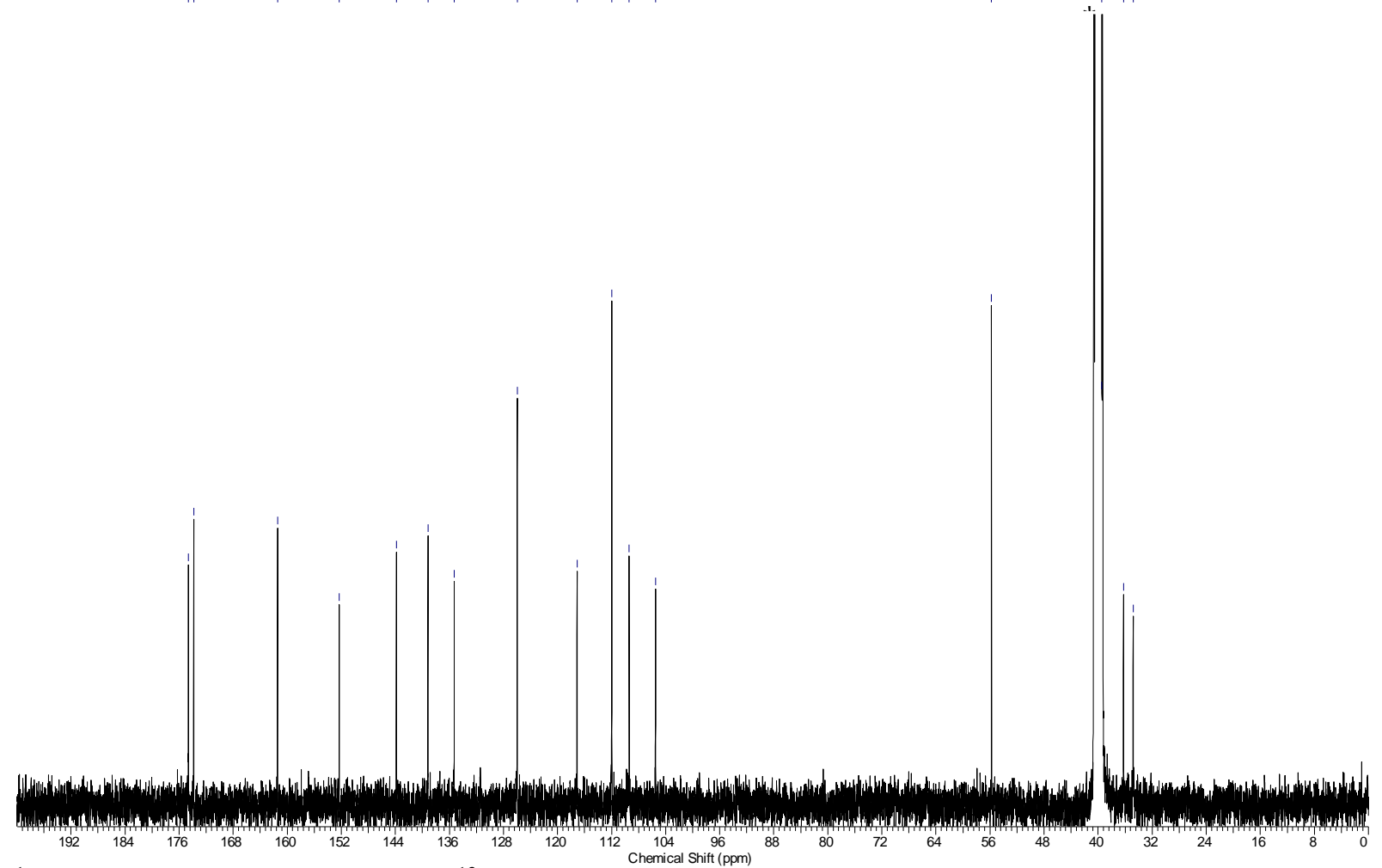

${ }^{1} \mathrm{H}-\mathrm{NMR}\left(300 \mathrm{MHz}\right.$, DMSO- $d_{6}$ ) and ${ }^{13} \mathrm{C}-\mathrm{NMR}\left(101 \mathrm{MHz}\right.$, DMSO- $d_{6}$ ) spectrum of compound 41c. * = NMR-solvent, $\mathrm{H}_{2} \mathrm{O}$ 


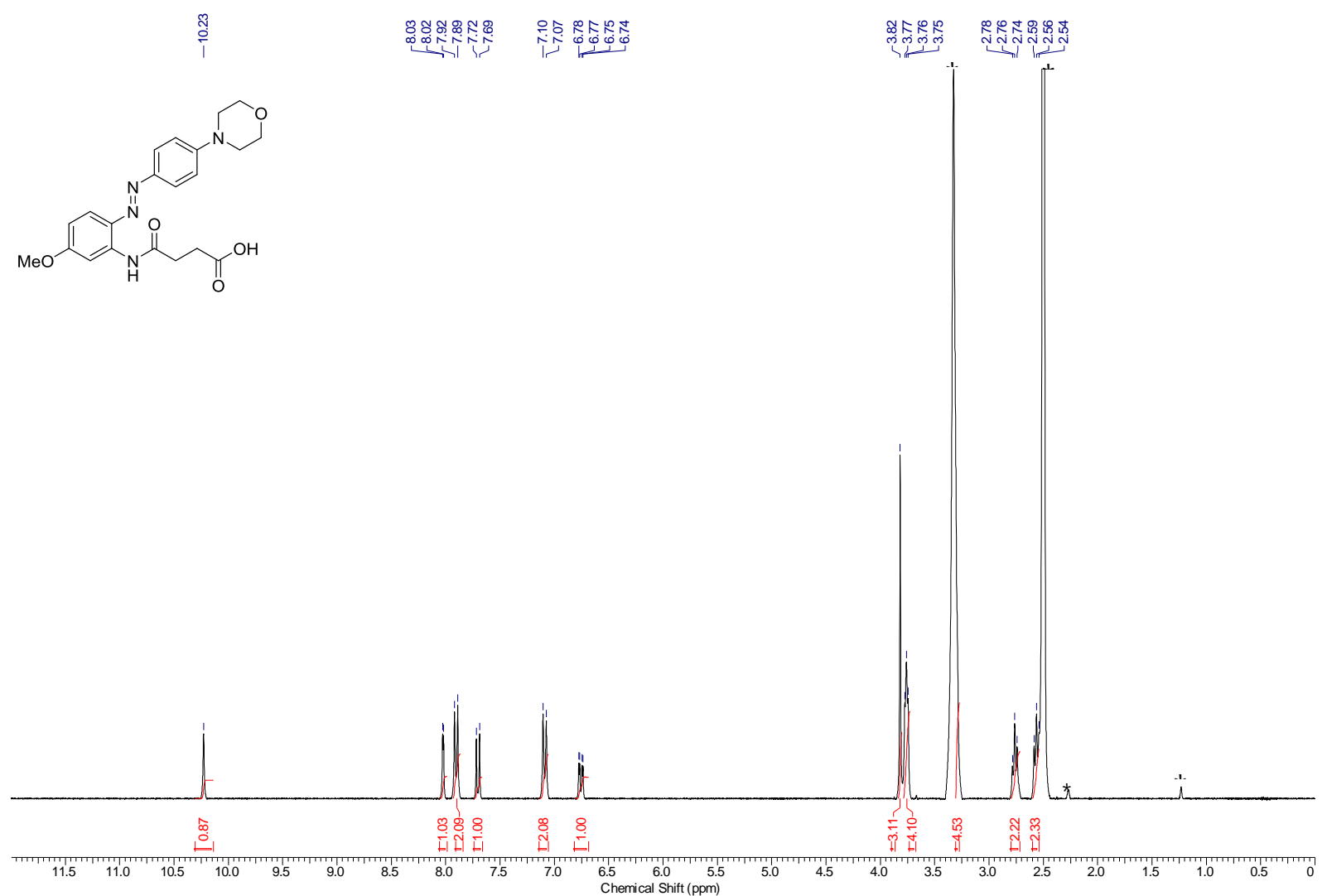

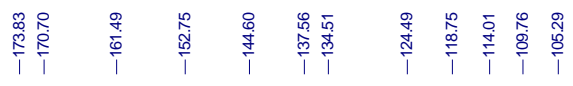

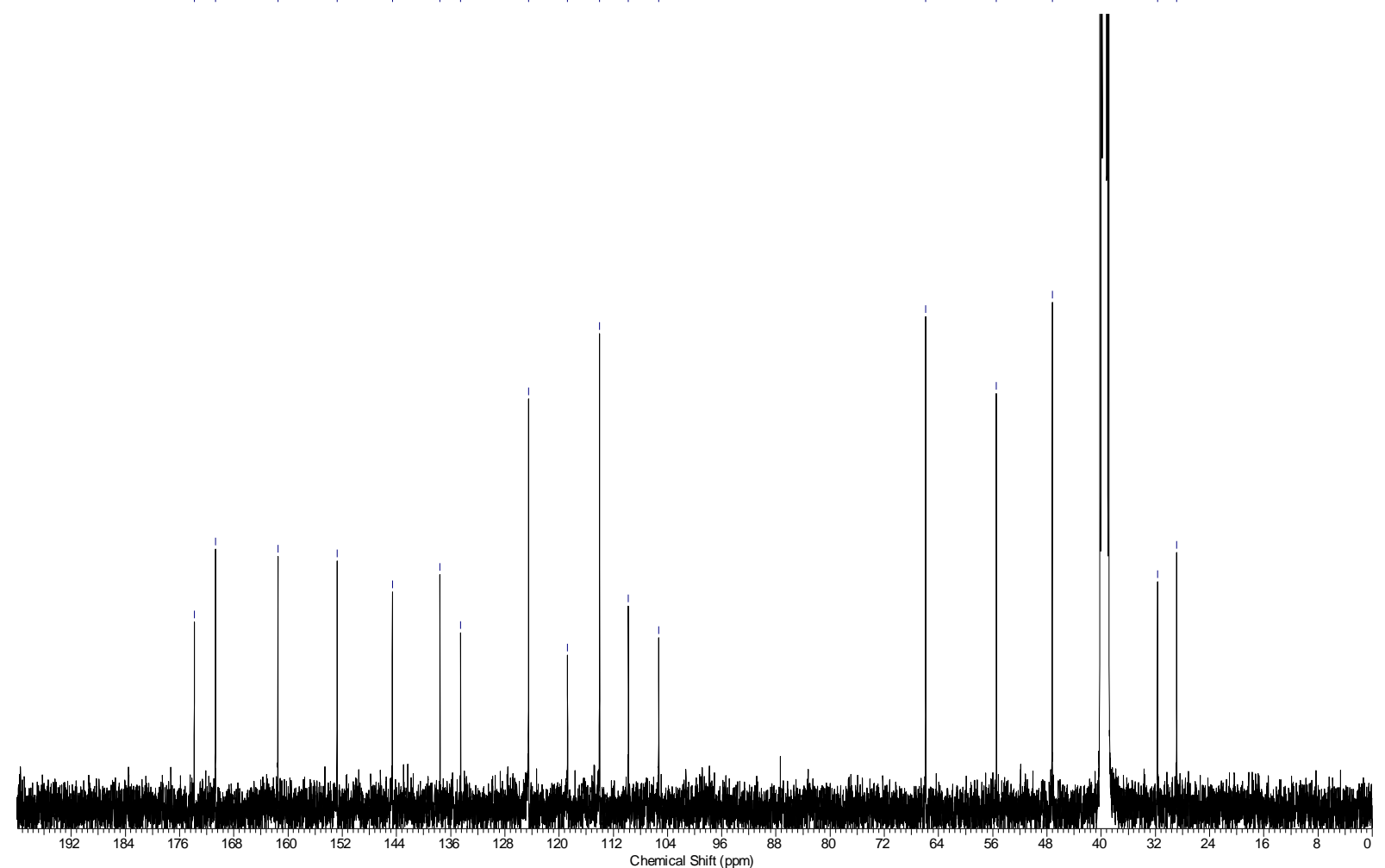

${ }^{1} \mathrm{H}-\mathrm{NMR}\left(300 \mathrm{MHz}\right.$, DMSO- $d_{6}$ ) and ${ }^{13} \mathrm{C}-\mathrm{NMR}\left(101 \mathrm{MHz}\right.$, DMSO- $d_{6}$ ) spectrum of compound 41d. * = NMR-solvent, $\mathrm{H}_{2} \mathrm{O}$, grease 


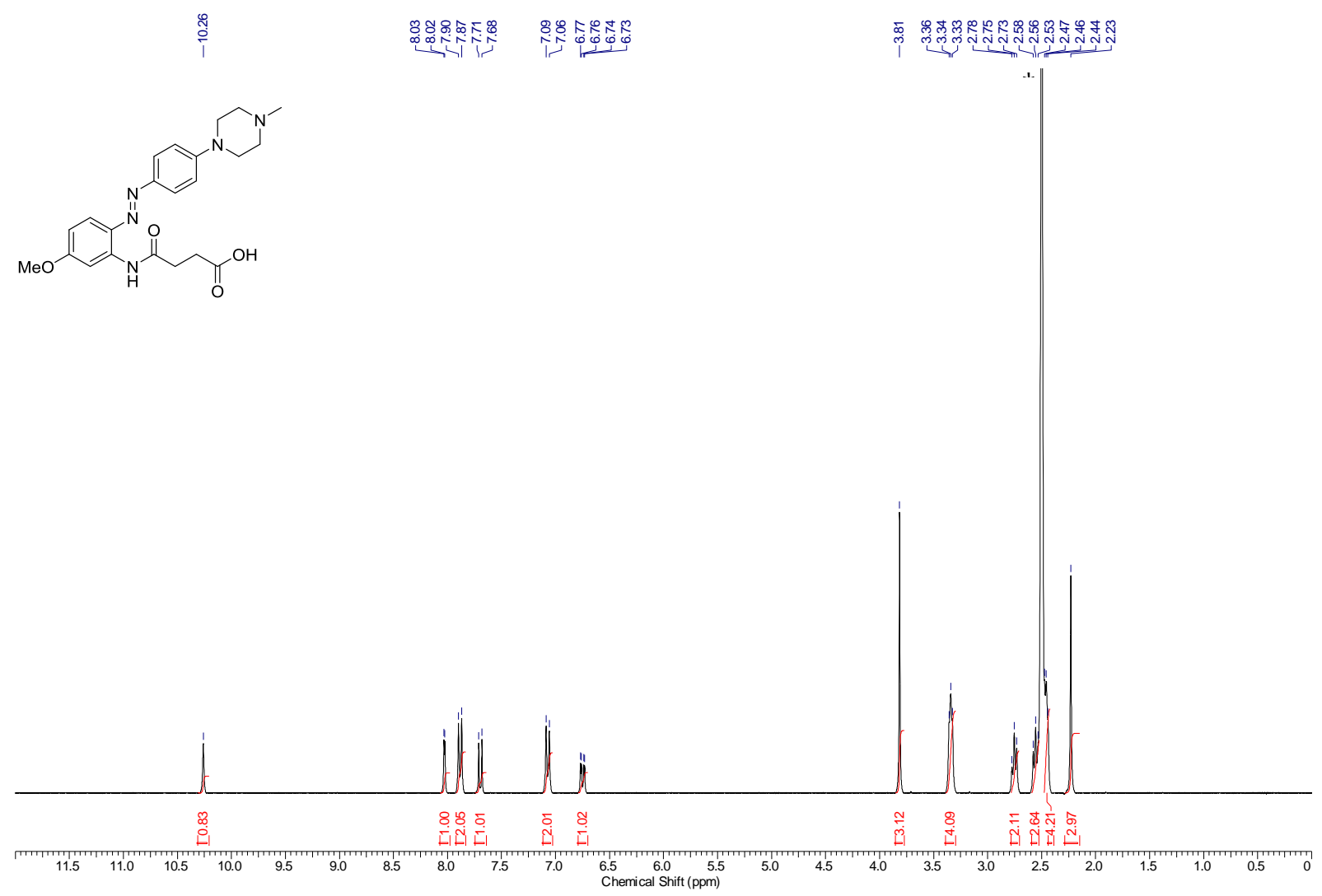

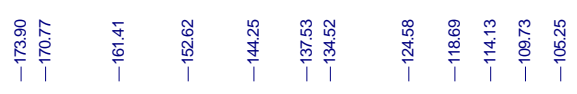




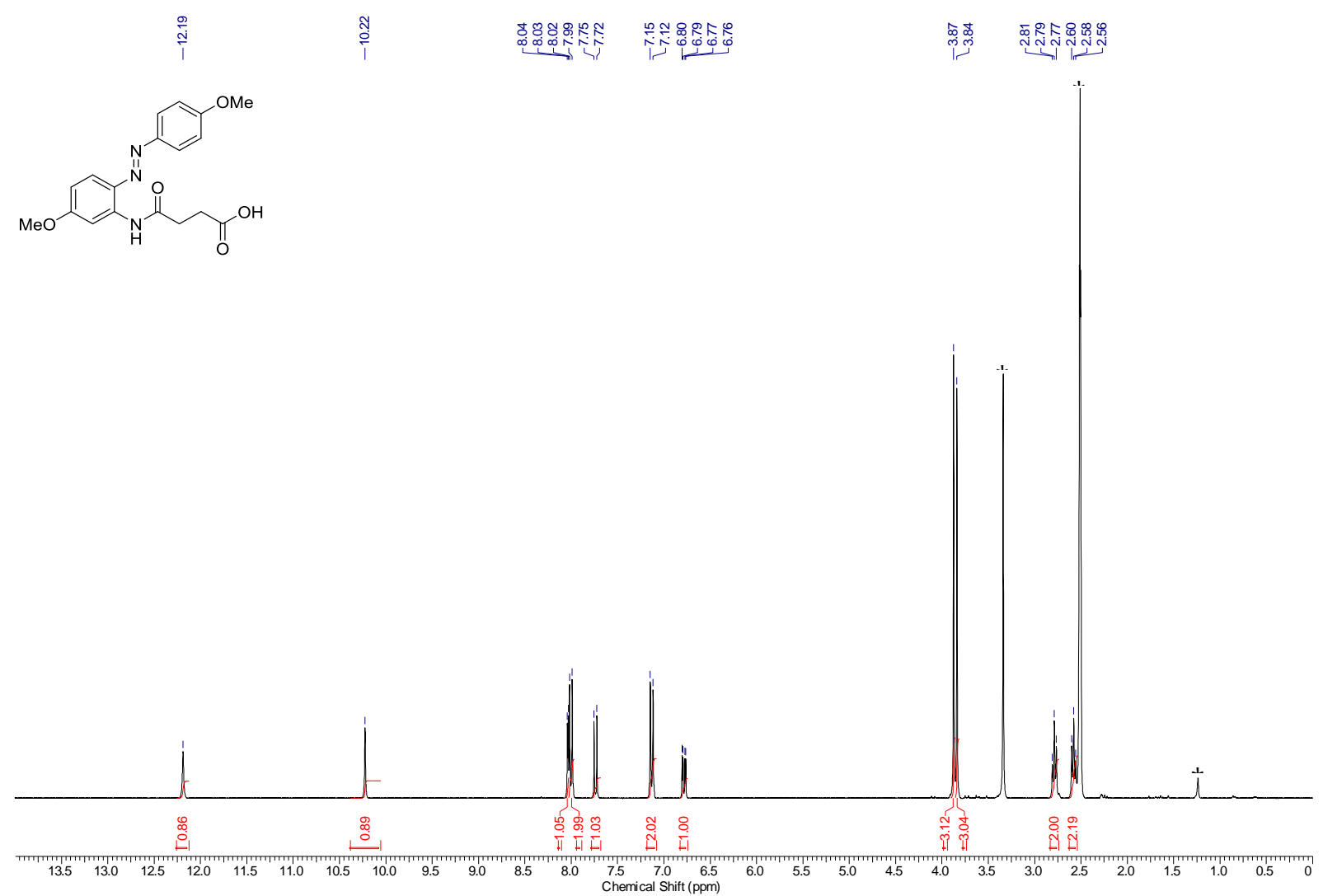

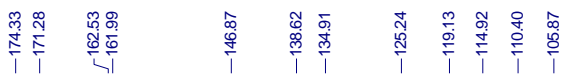

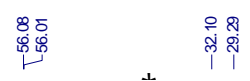

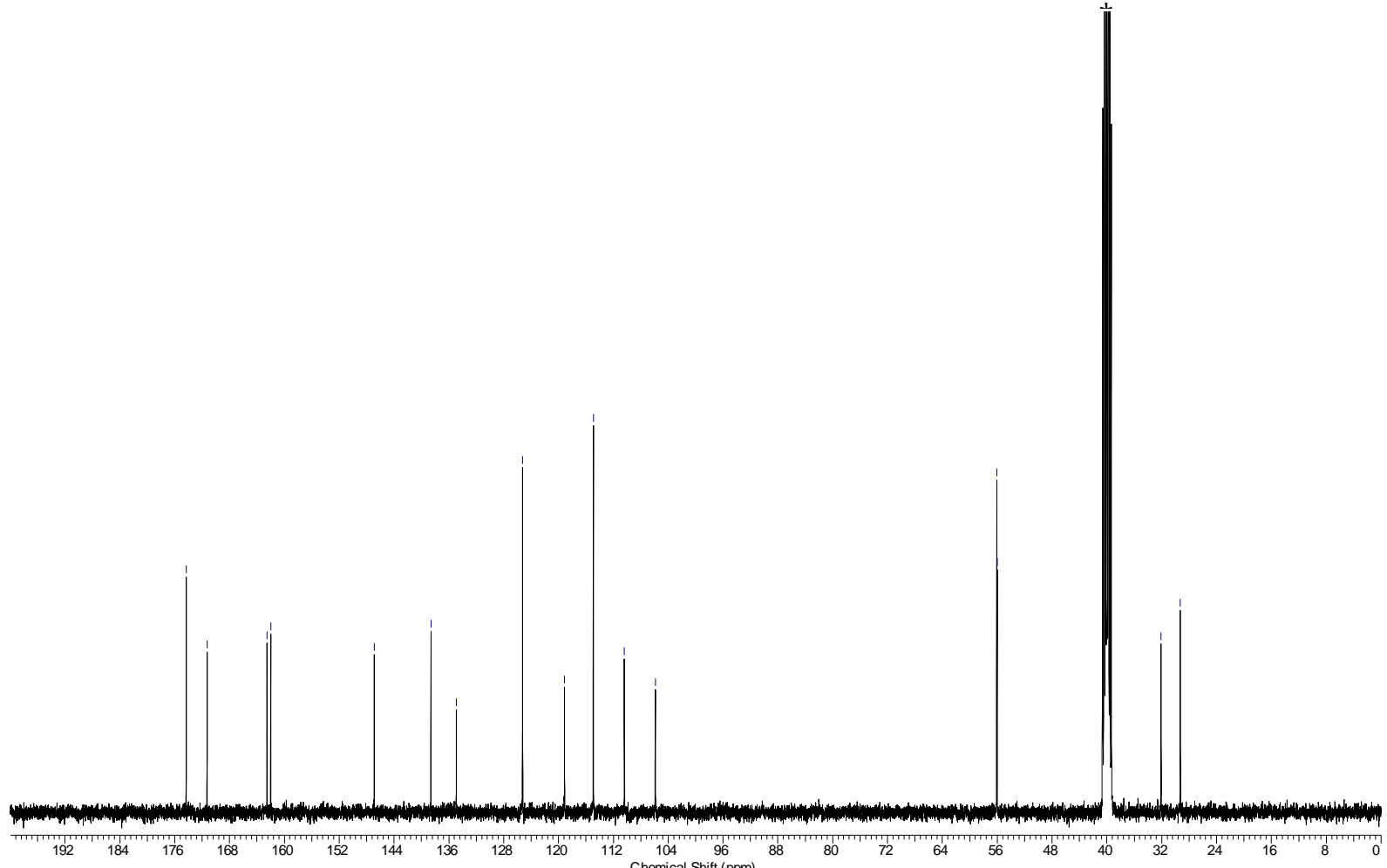

${ }^{1} \mathrm{H}-\mathrm{NMR}\left(300 \mathrm{MHz}\right.$, DMSO- $d_{6}$ ) and ${ }^{13} \mathrm{C}-\mathrm{NMR}\left(101 \mathrm{MHz}\right.$, DMSO- $d_{6}$ ) spectrum of compound 42a. $*$ = NMR-solvent, $\mathrm{H}_{2} \mathrm{O}$, grease 

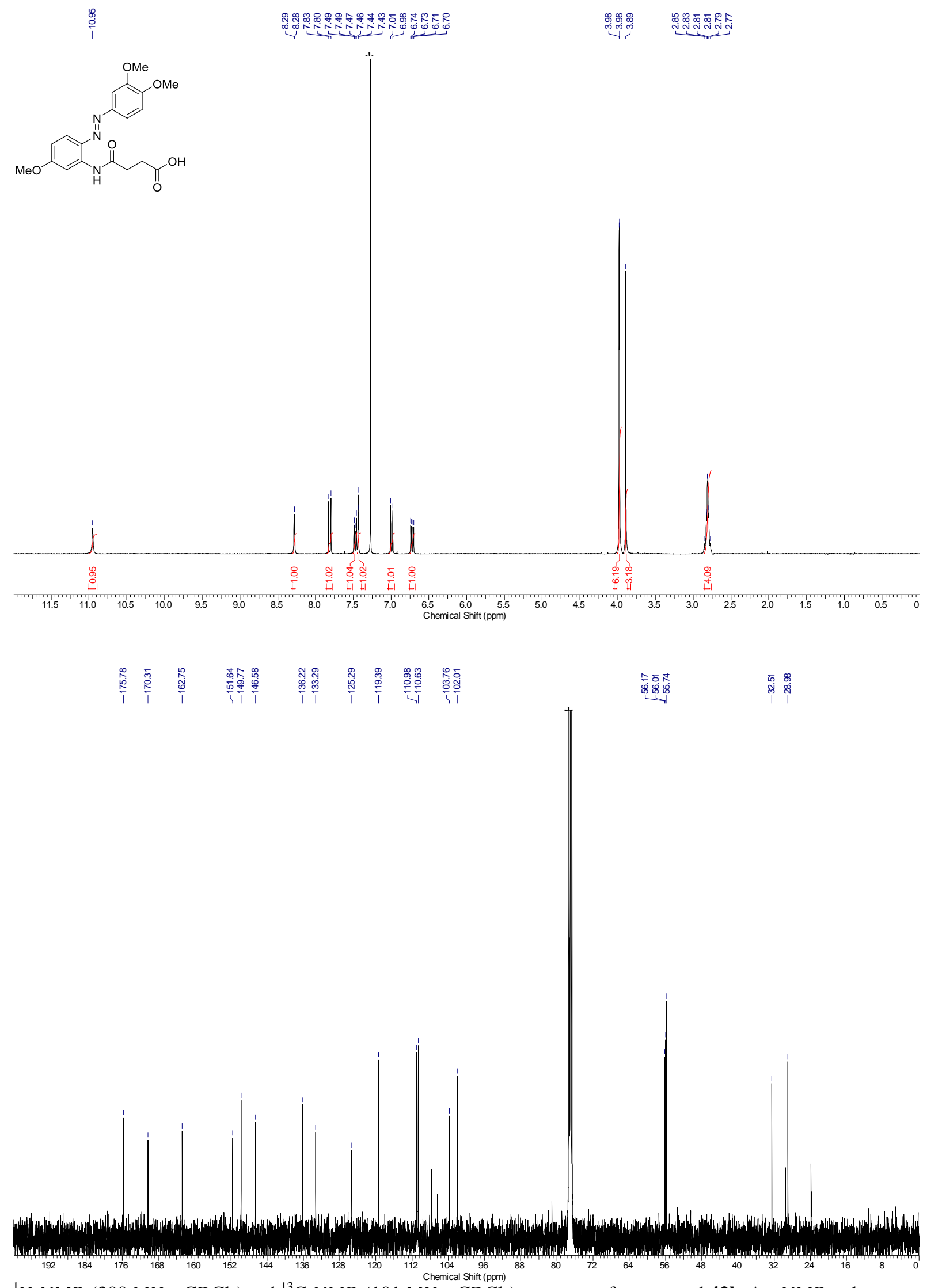

${ }^{1} \mathrm{H}-\mathrm{NMR}\left(300 \mathrm{MHz}, \mathrm{CDCl}_{3}\right)$ and ${ }^{13} \mathrm{C}-\mathrm{NMR}\left(101 \mathrm{MHz}, \mathrm{CDCl}_{3}\right)$ spectrum of compound $\mathbf{4 2 b} . *=$ NMR-solvent 


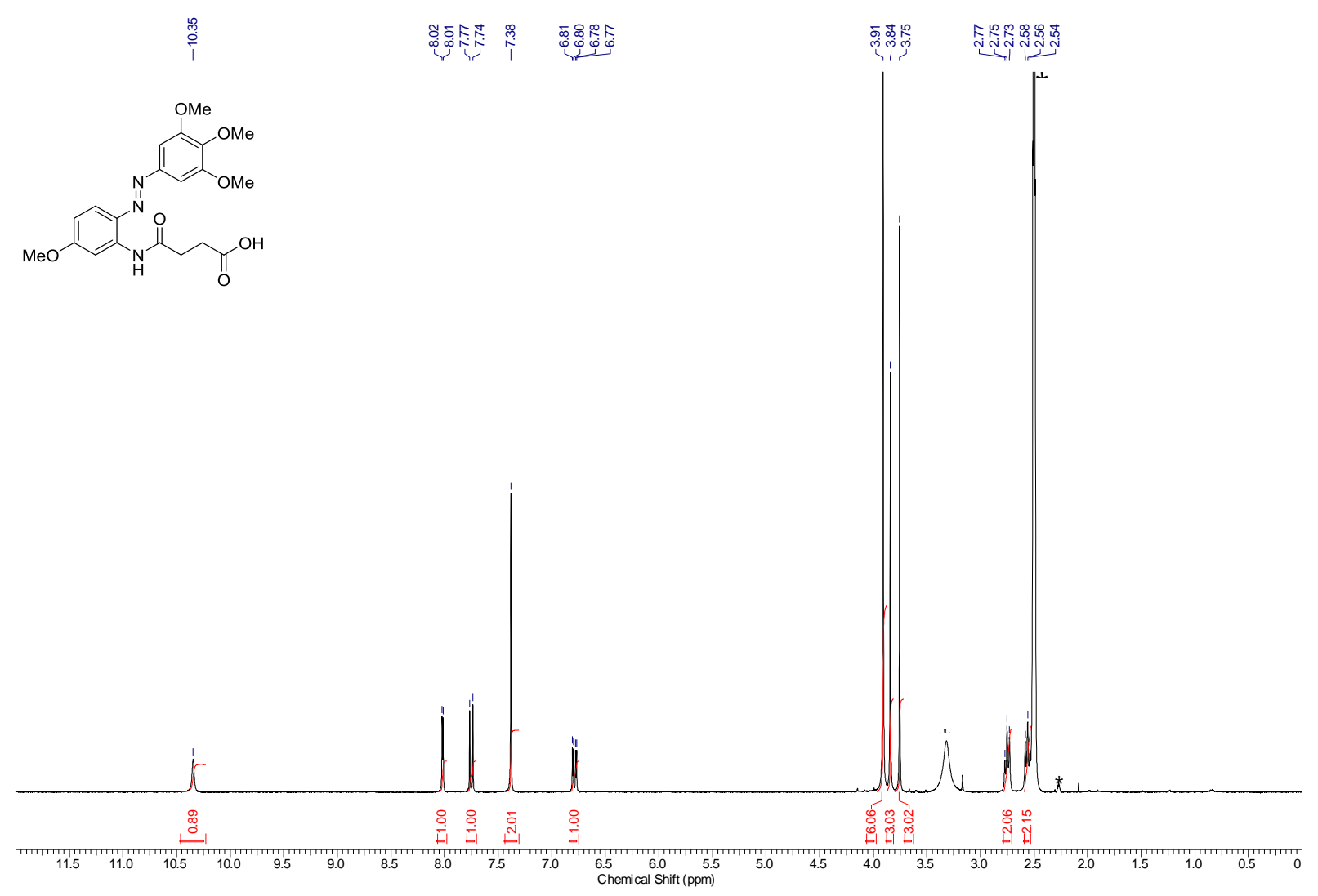

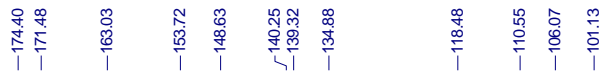

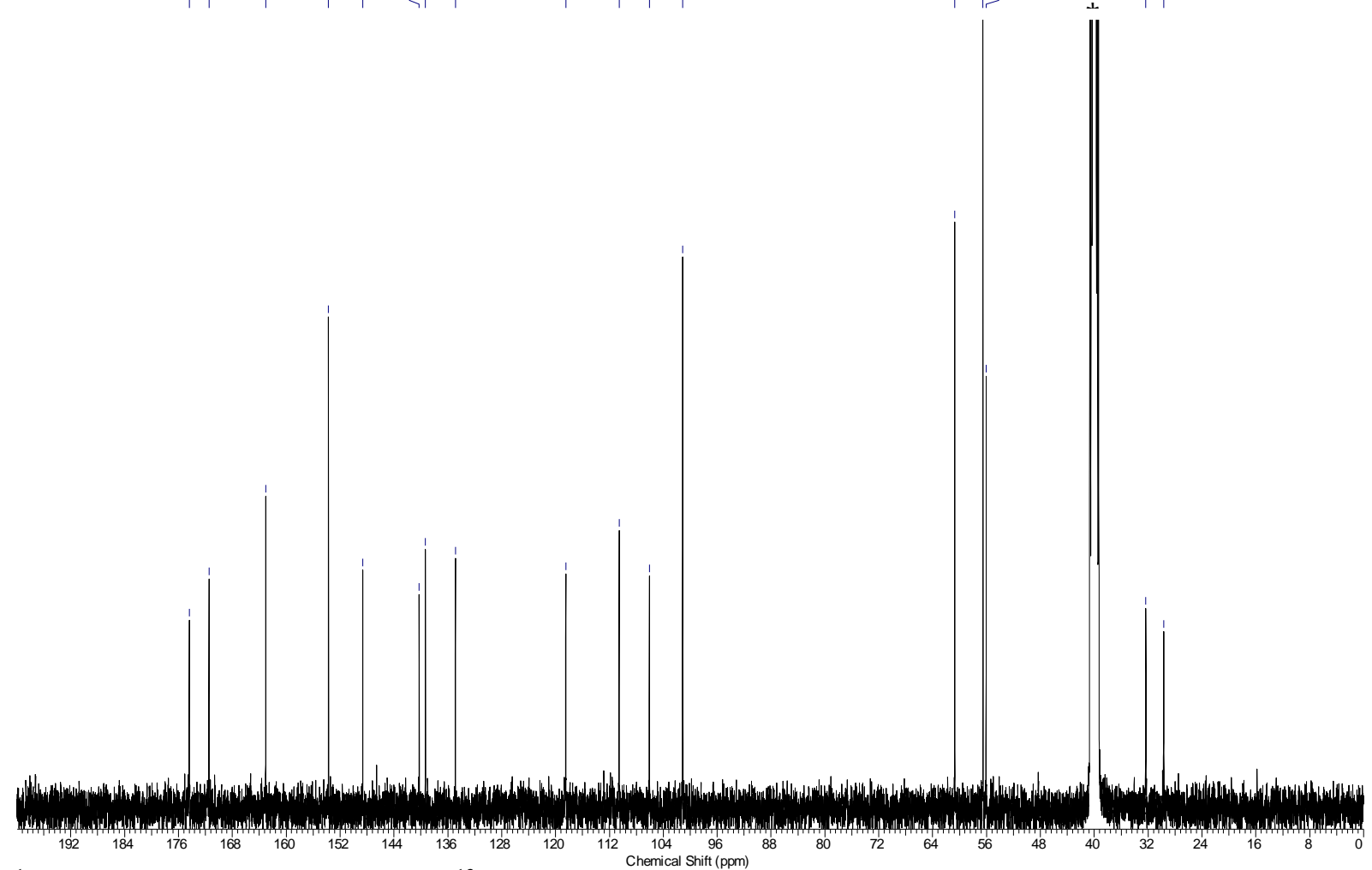

${ }^{1} \mathrm{H}-\mathrm{NMR}\left(300 \mathrm{MHz}\right.$, DMSO- $d_{6}$ ) and ${ }^{13} \mathrm{C}-\mathrm{NMR}\left(101 \mathrm{MHz}\right.$, DMSO- $\left.d_{6}\right)$ spectrum of compound 42c. * = NMR-solvent, $\mathrm{H}_{2} \mathrm{O}$ 


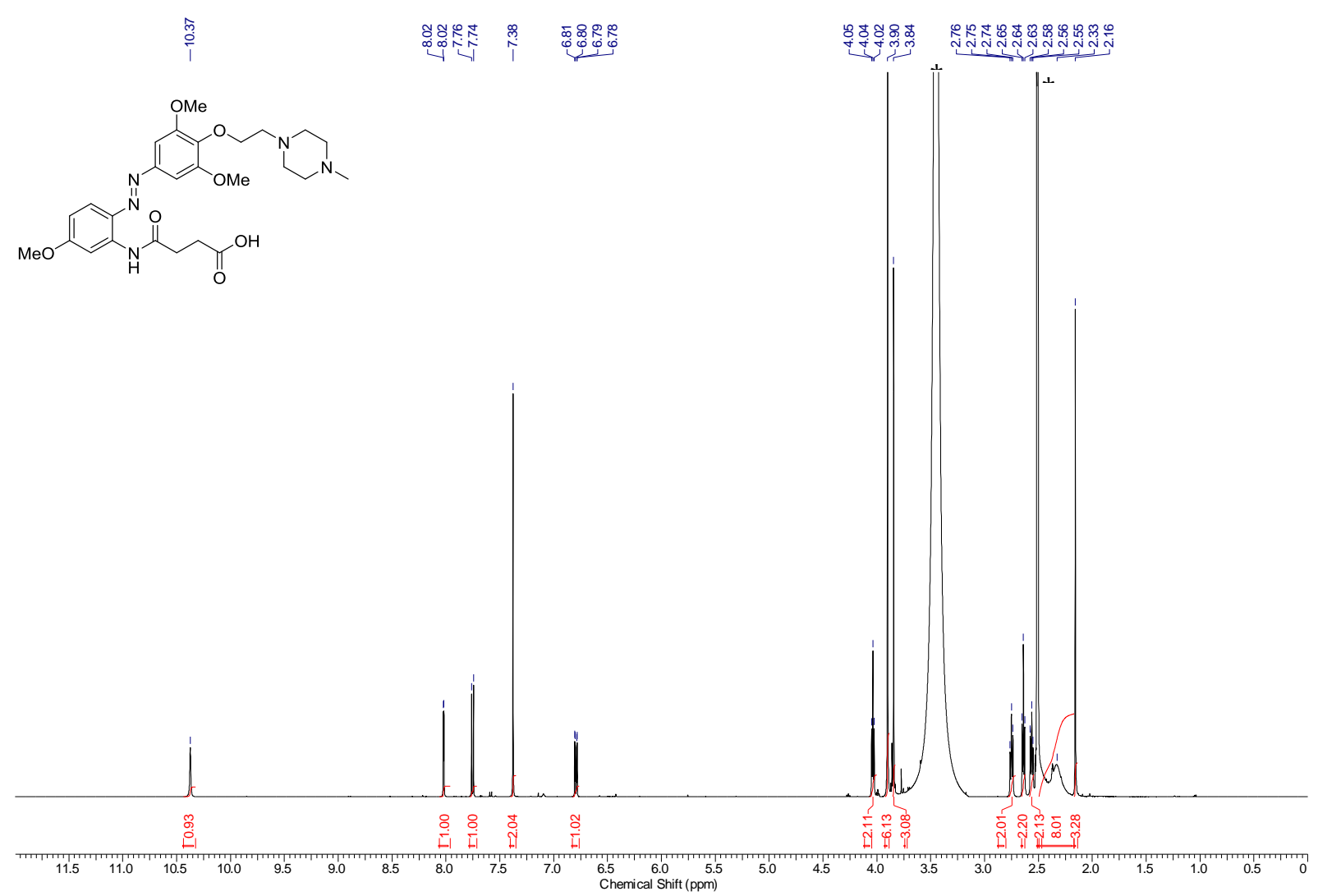

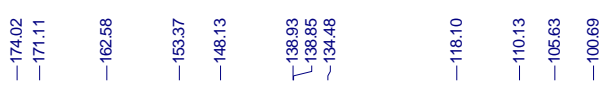

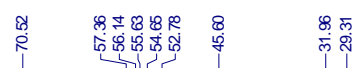

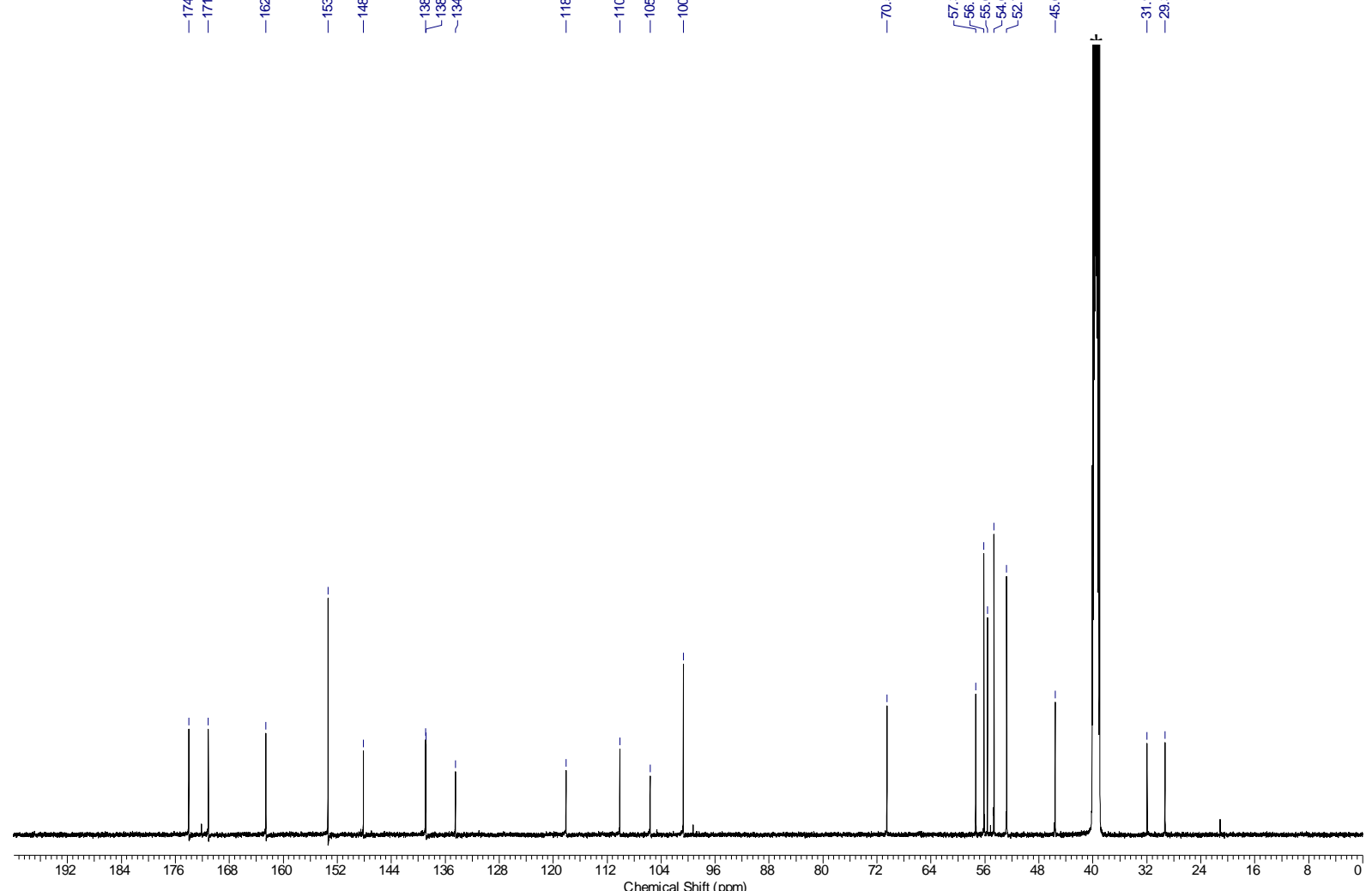

${ }^{1} \mathrm{H}-\mathrm{NMR}\left(500 \mathrm{MHz}\right.$, DMSO- $d_{6}$ ) and ${ }^{13} \mathrm{C}-\mathrm{NMR}\left(126 \mathrm{MHz}\right.$, DMSO- $\left.d_{6}\right)$ spectrum of compound 42e. * = NMR-solvent, $\mathrm{H}_{2} \mathrm{O}$ 

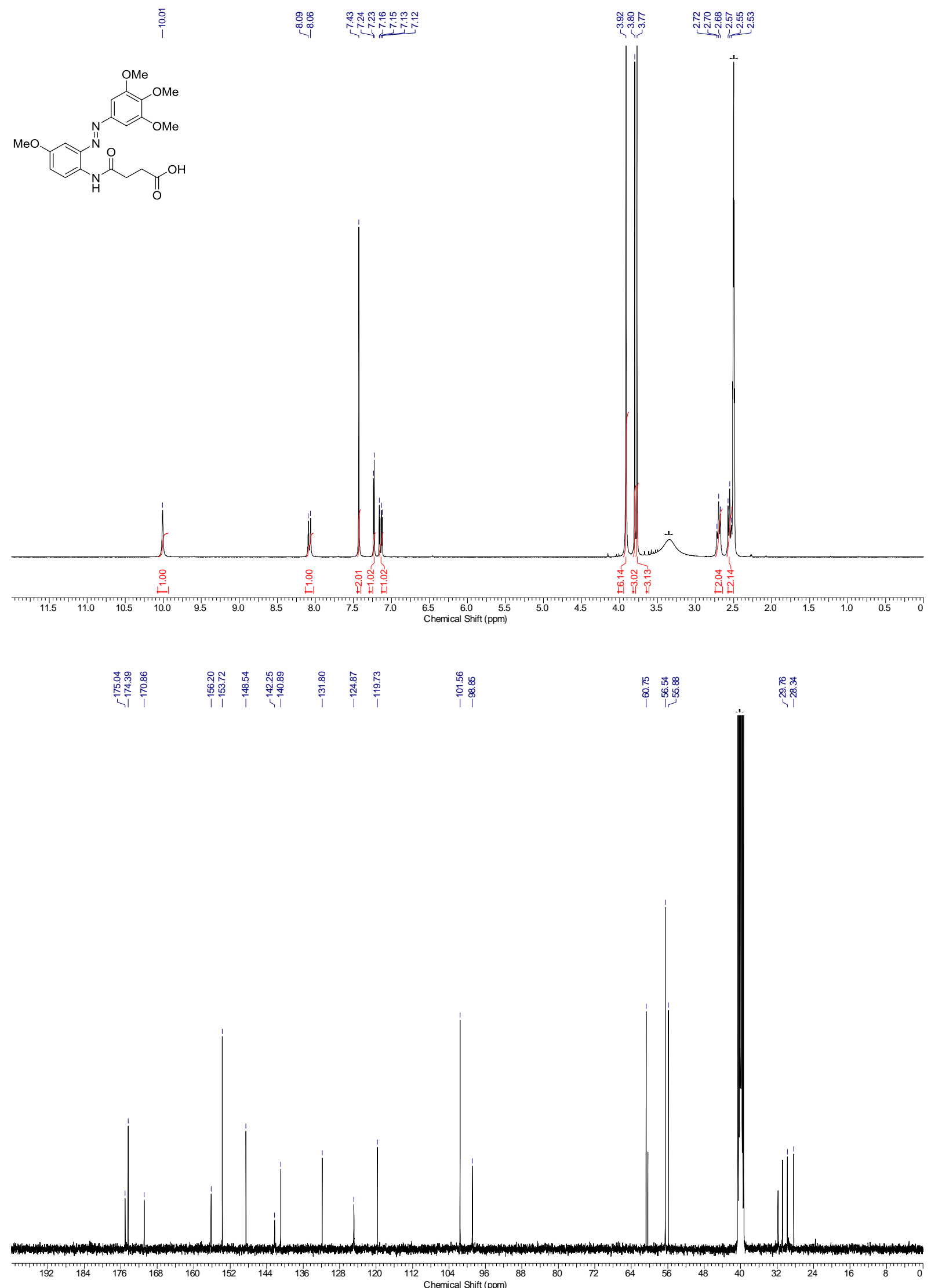

${ }^{1} \mathrm{H}-\mathrm{NMR}\left(300 \mathrm{MHz}, \mathrm{DMSO}-d_{6}\right)$ and ${ }^{13} \mathrm{C}-\mathrm{NMR}\left(101 \mathrm{MHz}\right.$, DMSO- $\left.d_{6}\right)$ spectrum of compound $43 . *=$ NMR-solvent, $\mathrm{H}_{2} \mathrm{O}$ 


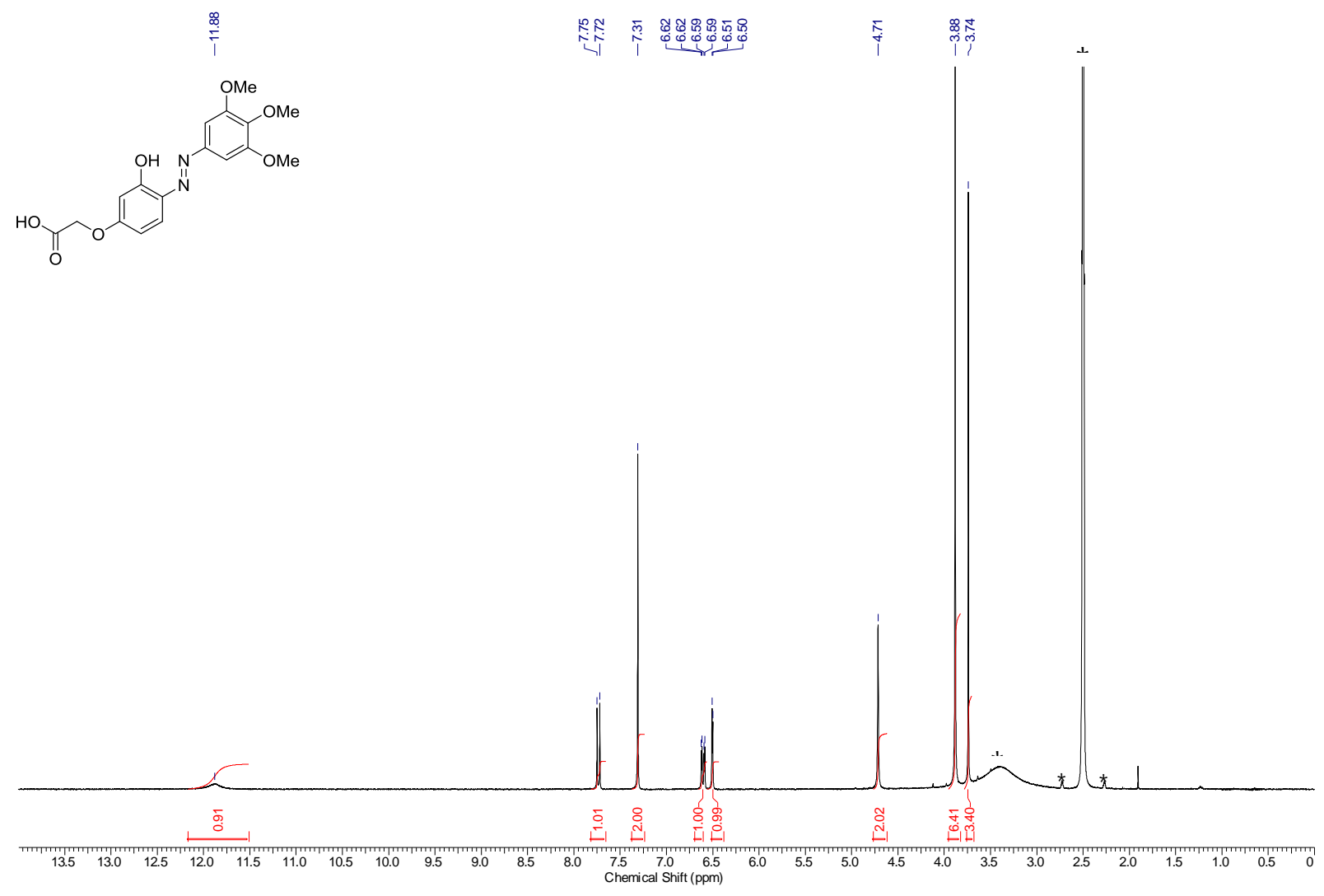

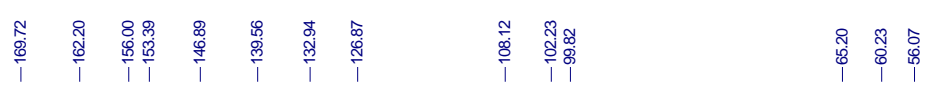

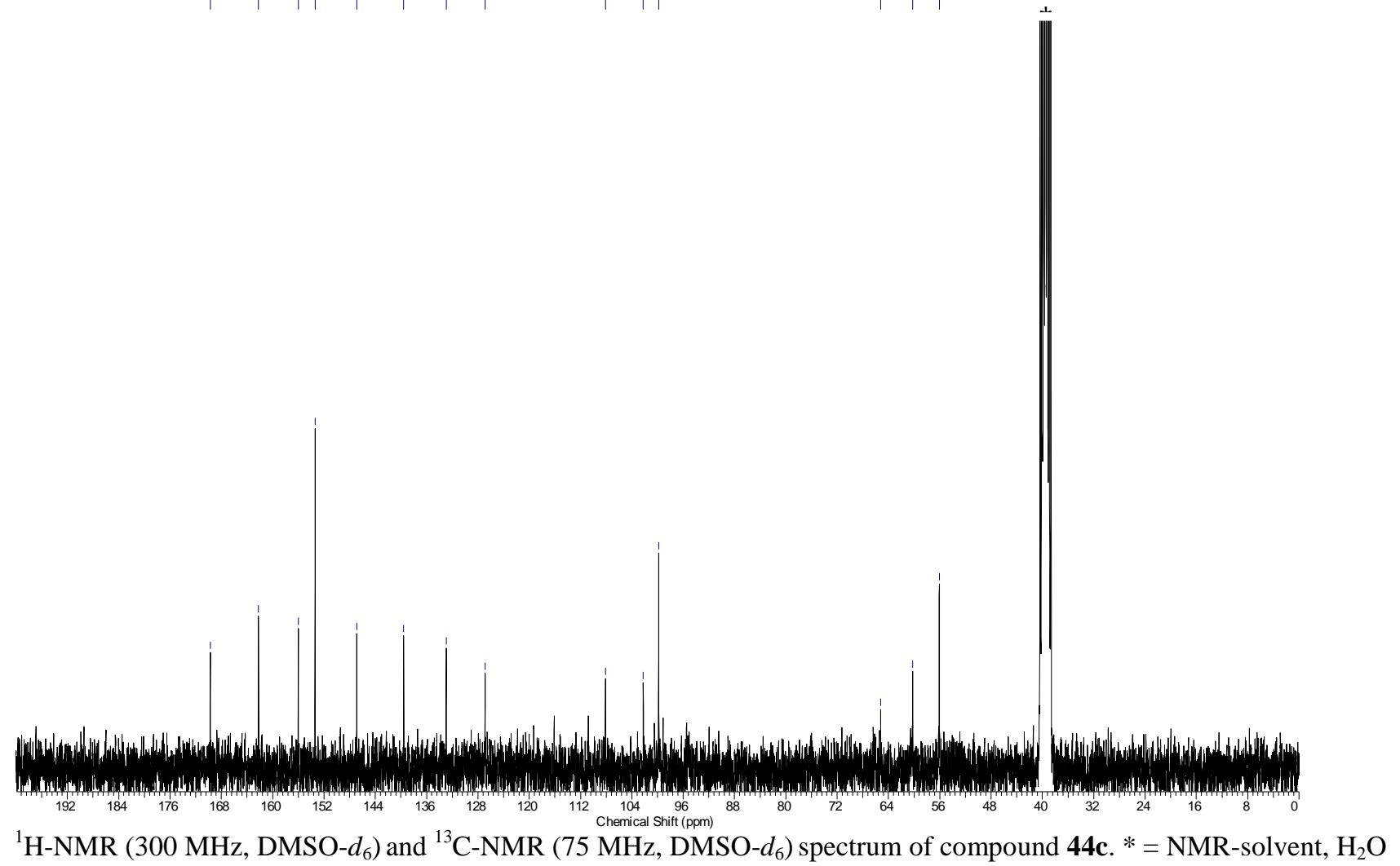




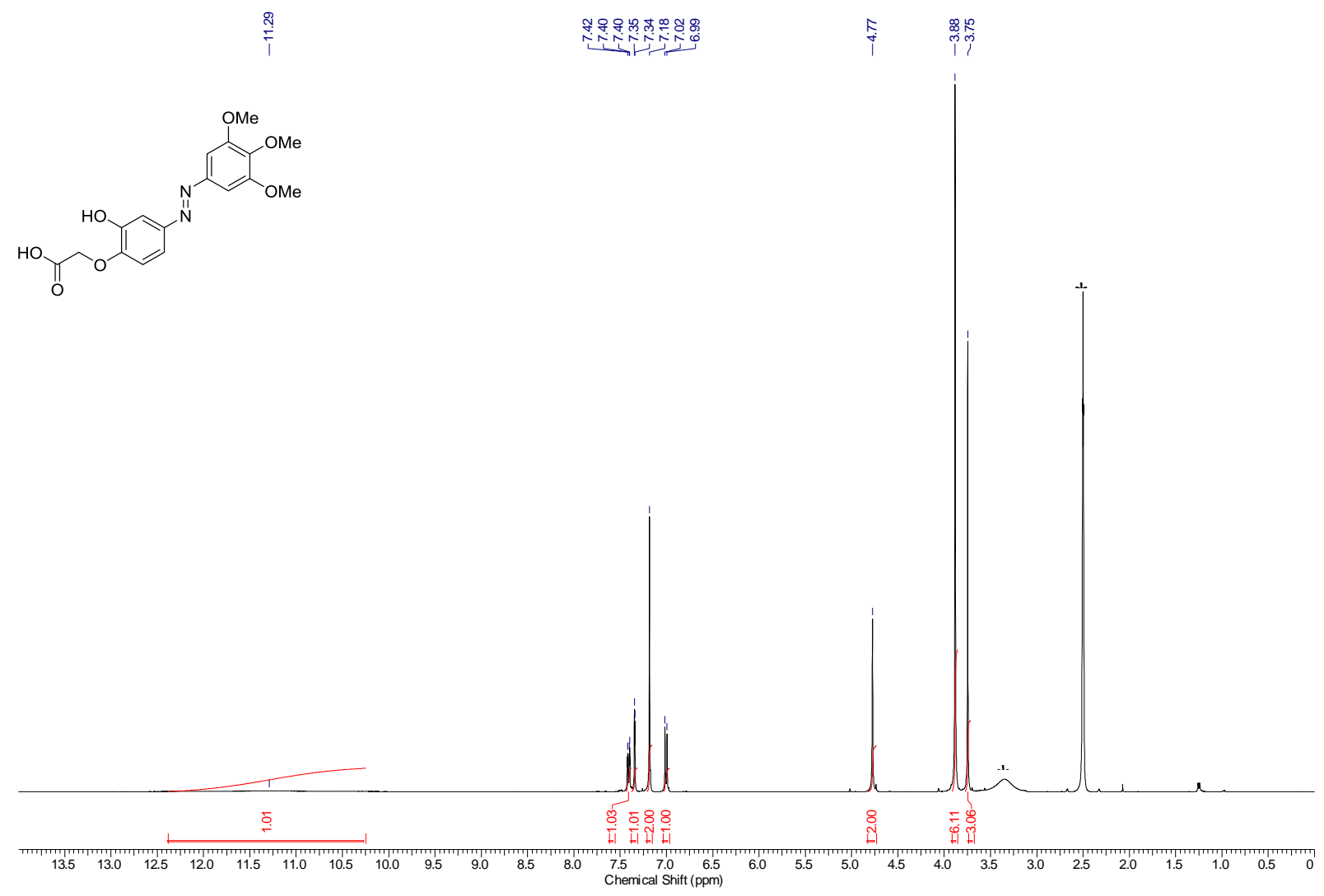

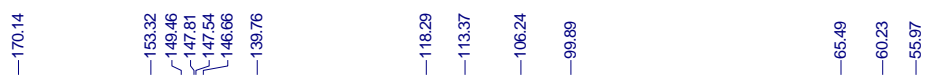

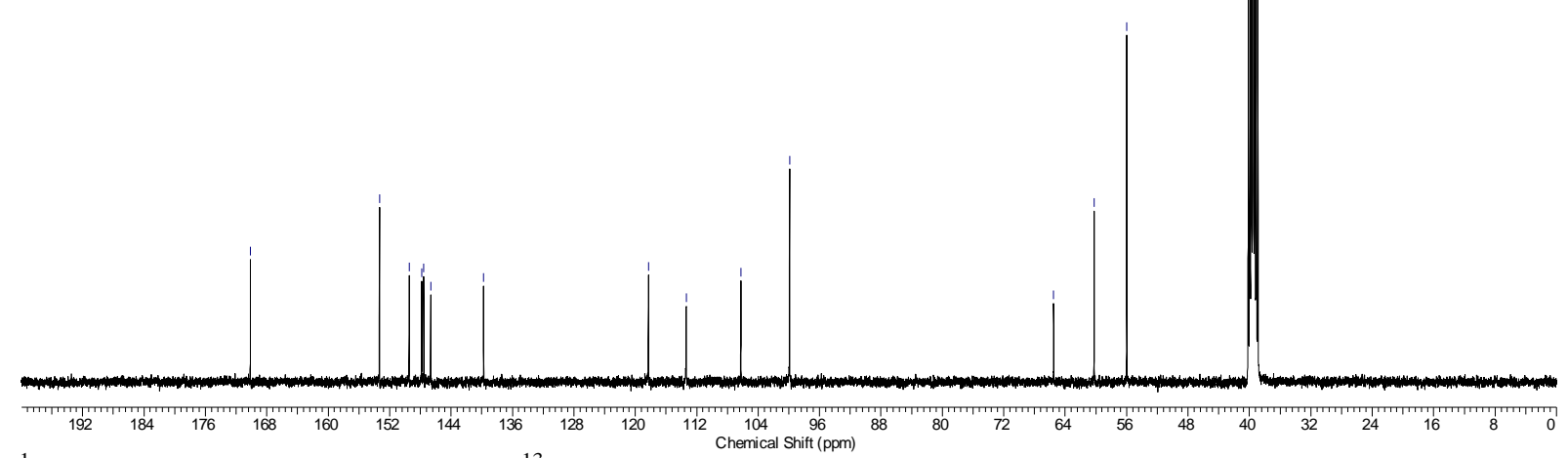

${ }^{1} \mathrm{H}-\mathrm{NMR}\left(400 \mathrm{MHz}\right.$, DMSO- $d_{6}$ ) and ${ }^{13} \mathrm{C}-\mathrm{NMR}\left(101 \mathrm{MHz}\right.$, DMSO- $\left.d_{6}\right)$ spectrum of compound 44g. * $=$ NMR-solvent, $\mathrm{H}_{2} \mathrm{O}$ 


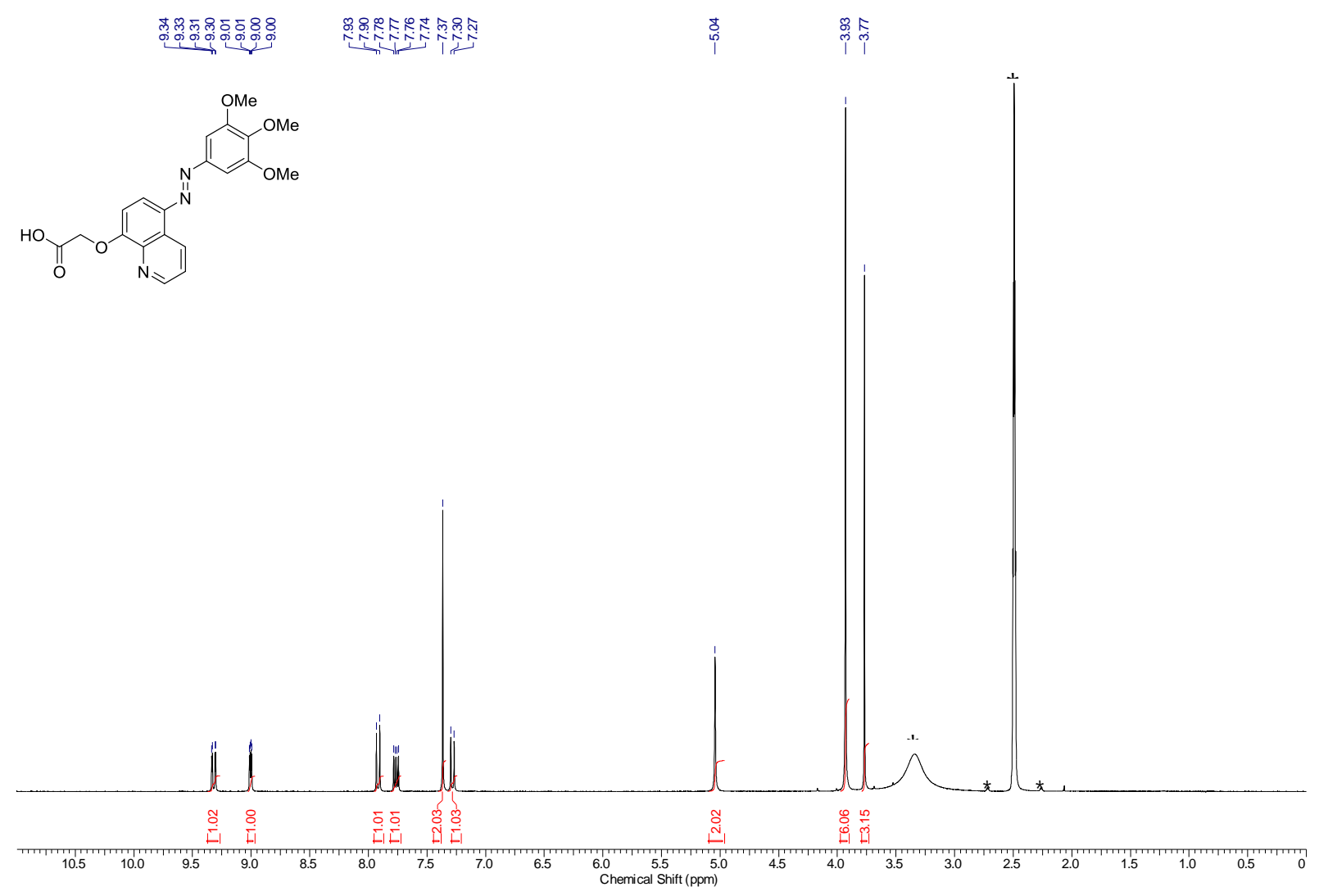

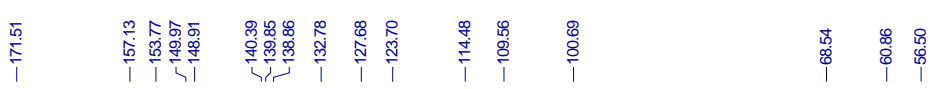

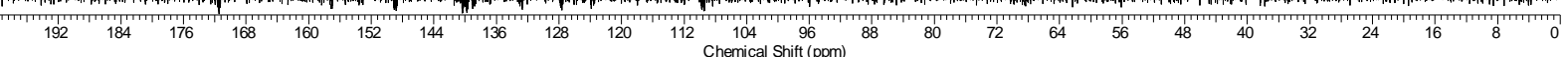

${ }^{1} \mathrm{H}-\mathrm{NMR}\left(300 \mathrm{MHz}\right.$, DMSO- $d_{6}$ ) and ${ }^{13} \mathrm{C}-\mathrm{NMR}\left(126 \mathrm{MHz}\right.$, DMSO- $d_{6}$ ) spectrum of compound 45a. * = NMR-solvent, $\mathrm{H}_{2} \mathrm{O}$ 


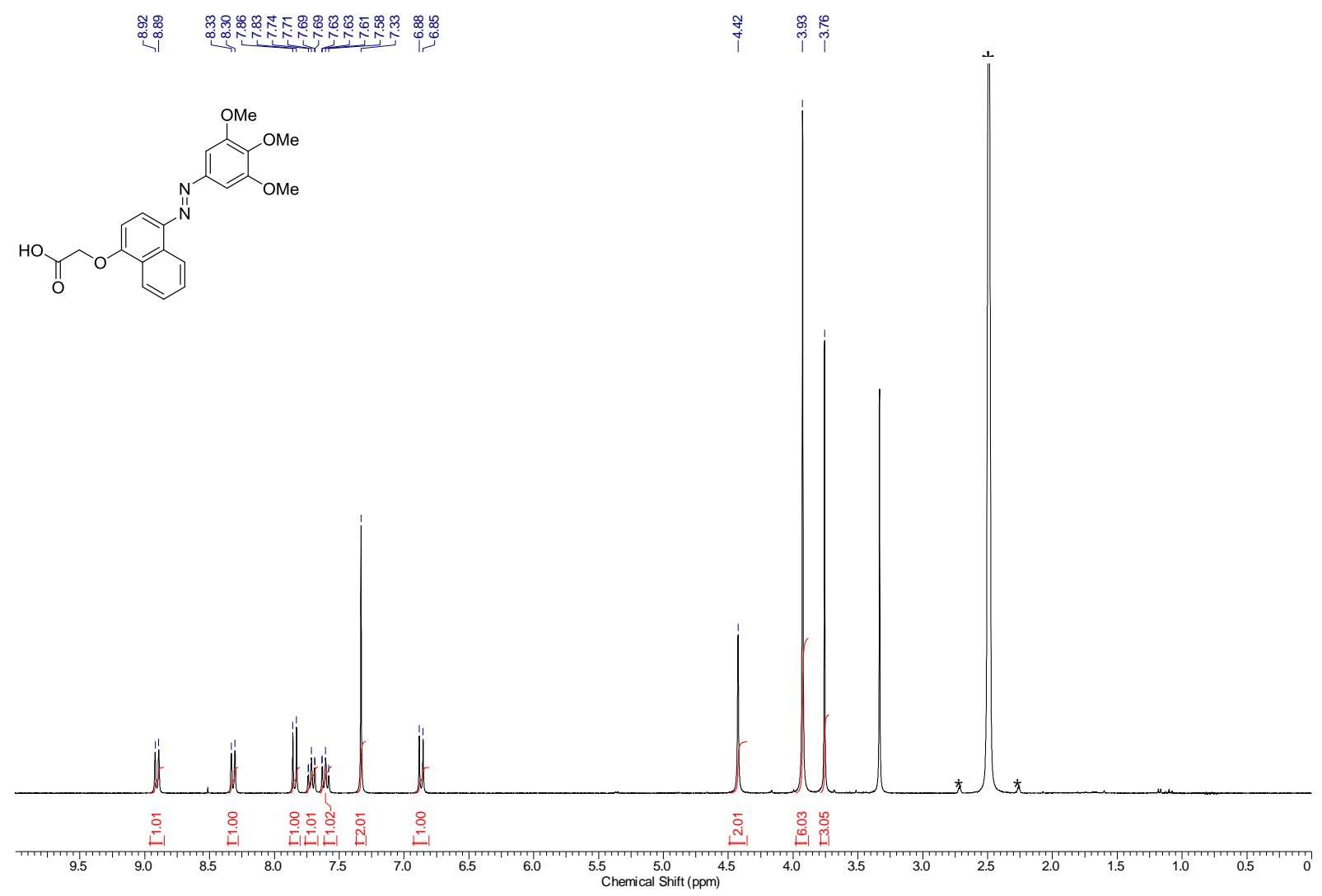

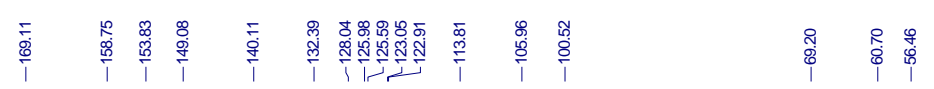

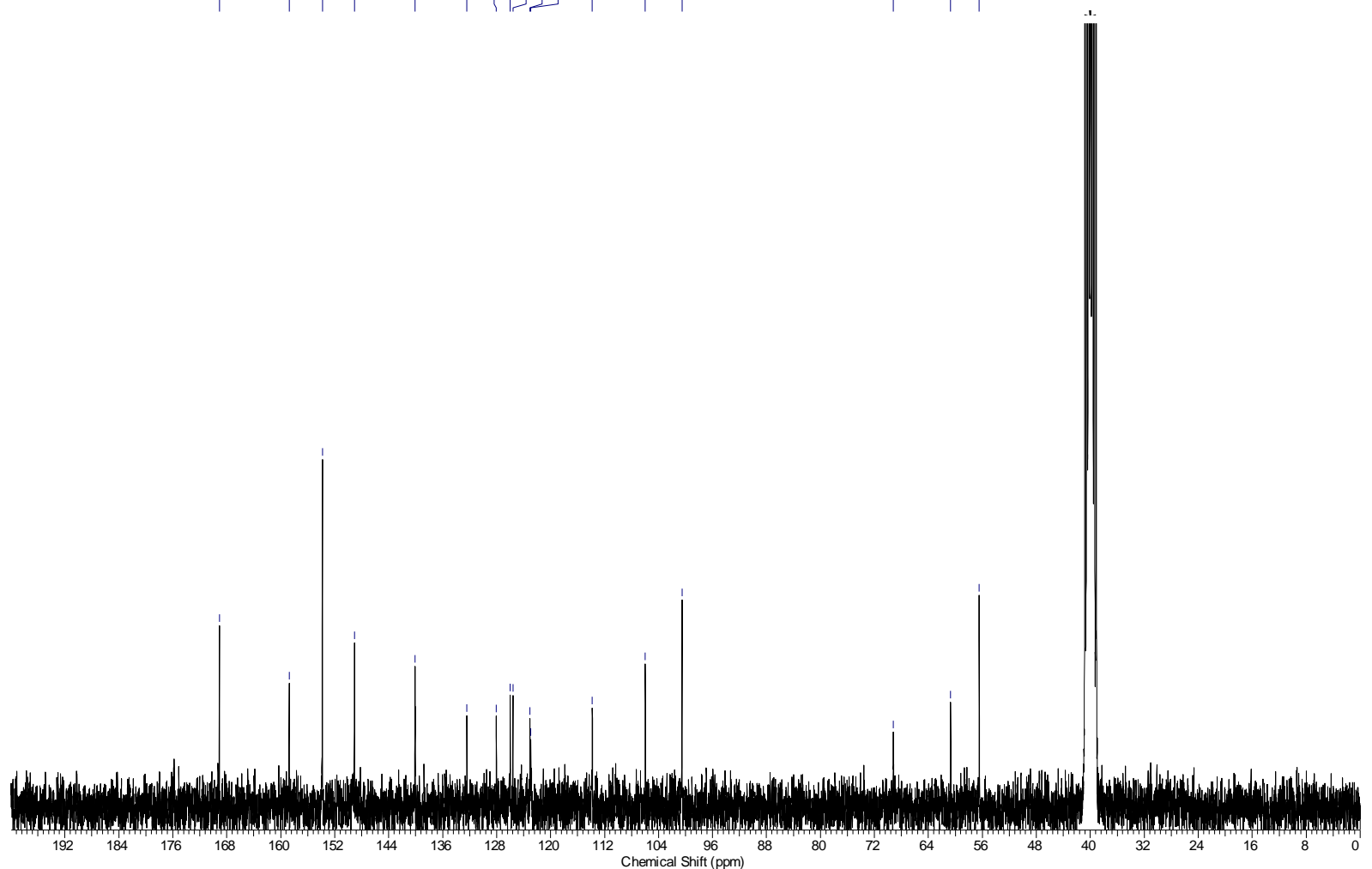

${ }^{1} \mathrm{H}-\mathrm{NMR}\left(300 \mathrm{MHz}, \mathrm{DMSO}-d_{6}\right.$ ) and ${ }^{13} \mathrm{C}-\mathrm{NMR}$ (75 MHz, DMSO- $d_{6}$ ) spectrum of compound $\mathbf{4 5 b}$. * = NMR-solvent 


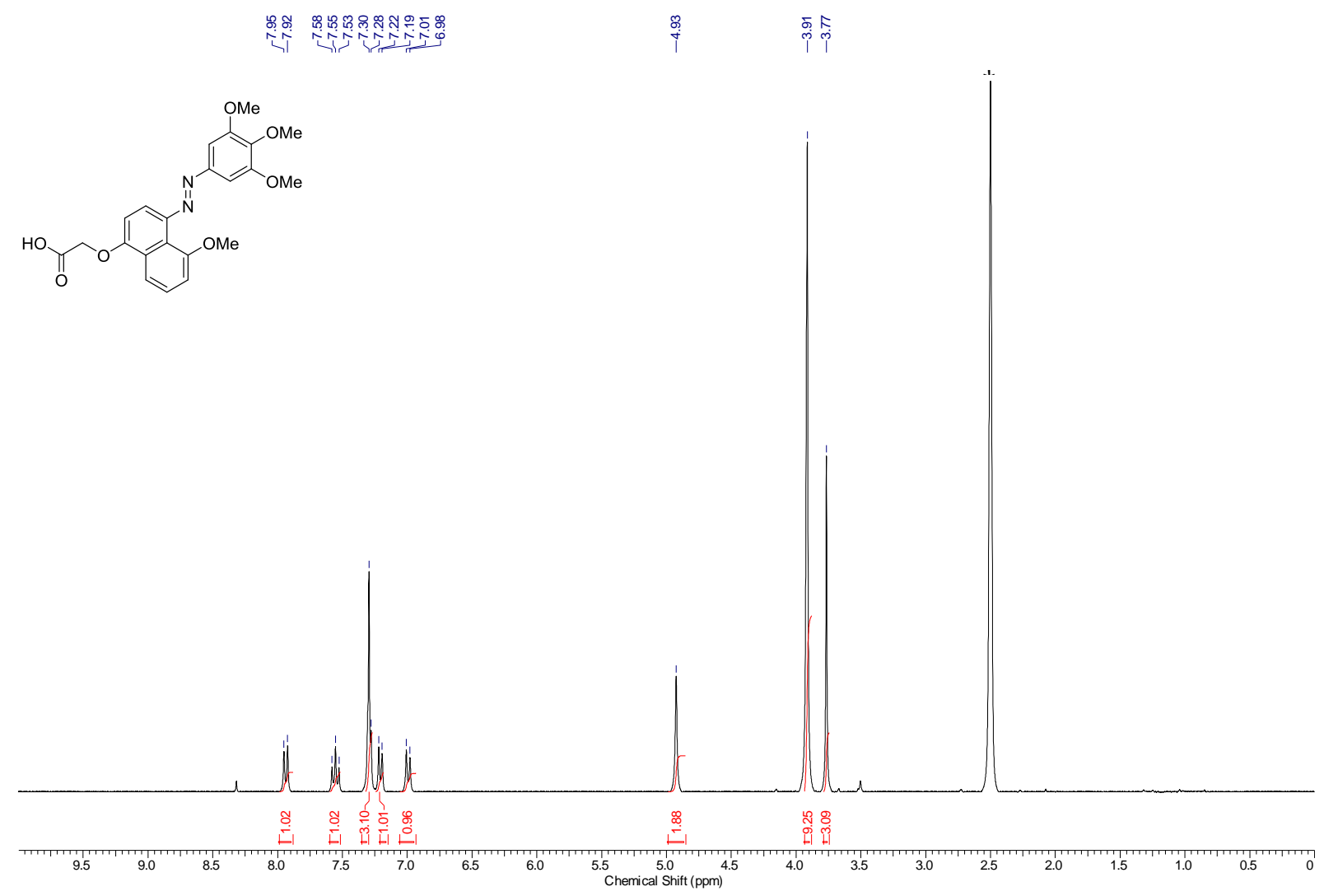

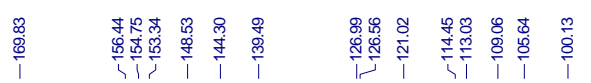

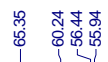

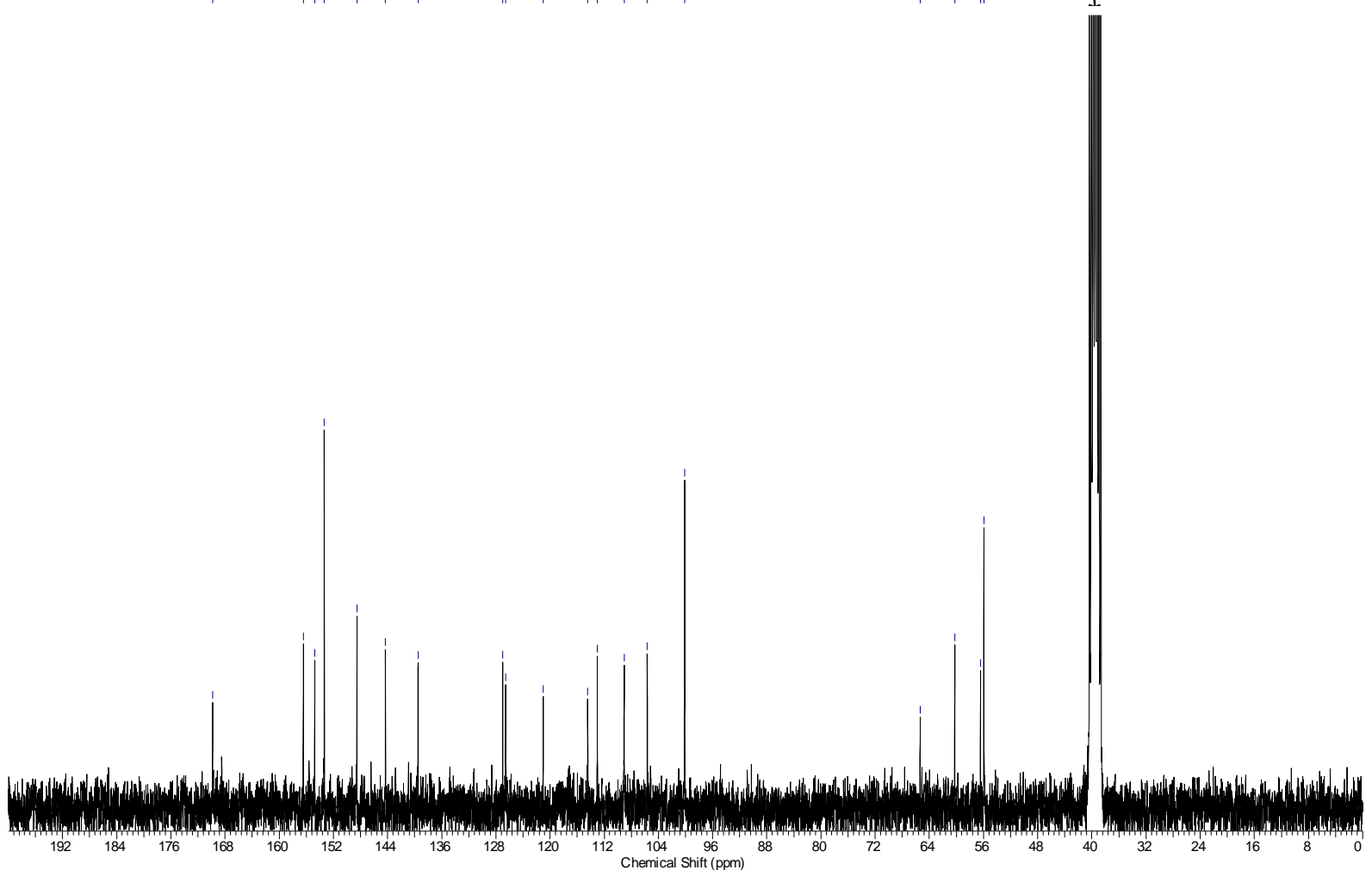

${ }^{1} \mathrm{H}-\mathrm{NMR}\left(300 \mathrm{MHz}\right.$, DMSO- $d_{6}$ ) and ${ }^{13} \mathrm{C}-\mathrm{NMR}\left(75 \mathrm{MHz}\right.$, DMSO- $d_{6}$ ) spectrum of compound 45c. $*=$ NMR-solvent 


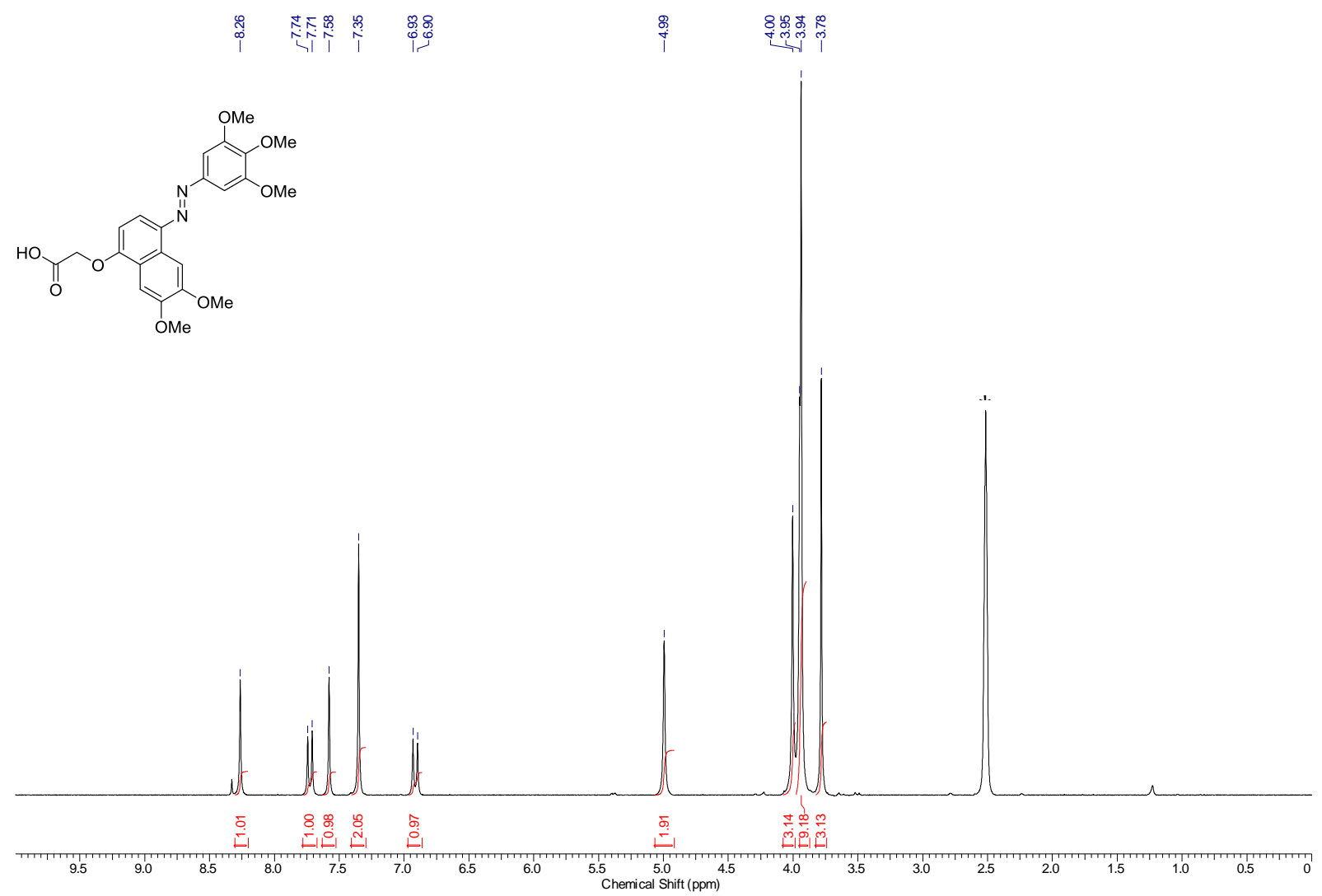

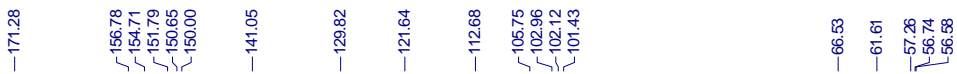

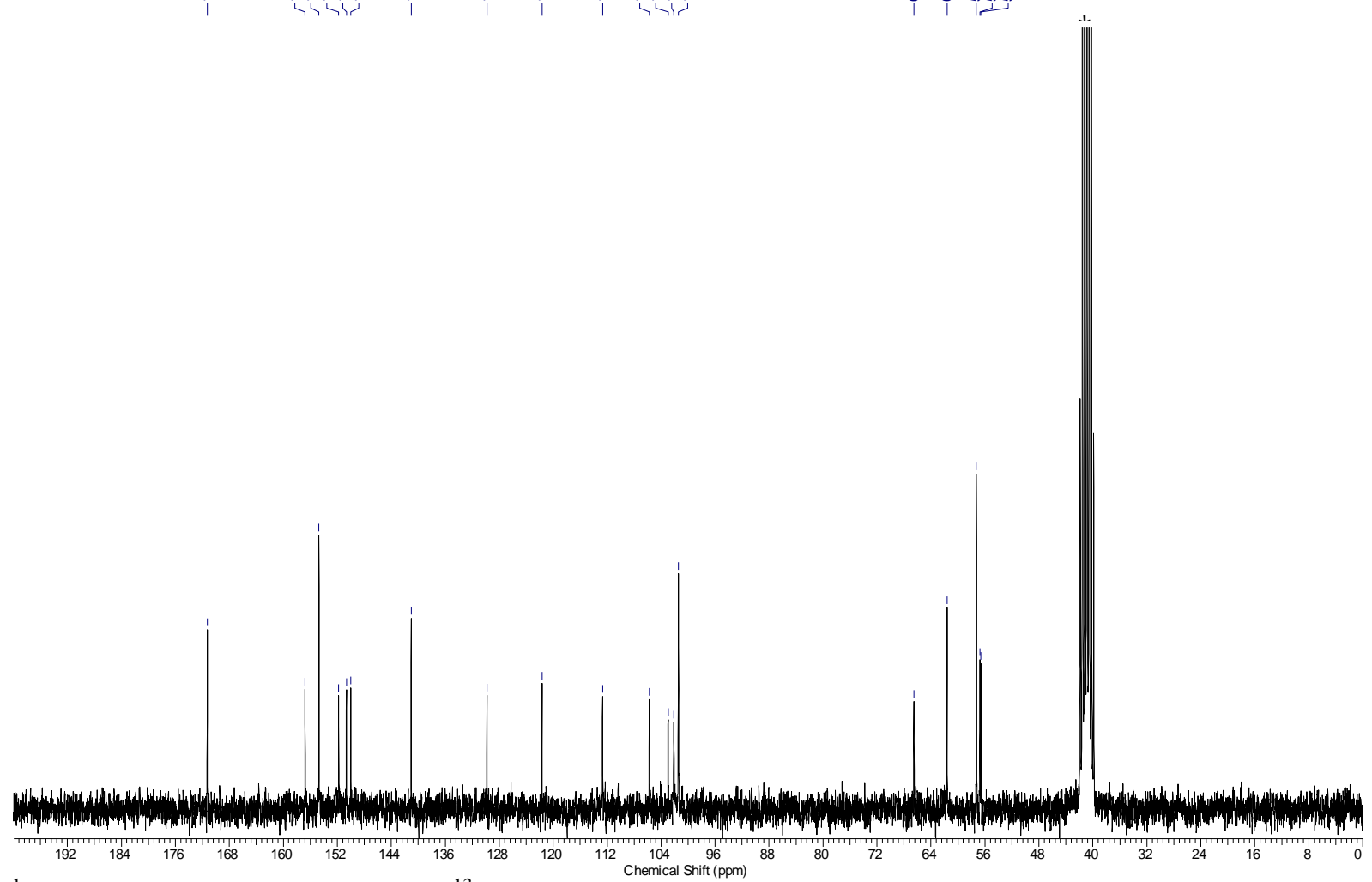

${ }^{1} \mathrm{H}-\mathrm{NMR}\left(250 \mathrm{MHz}\right.$, DMSO- $d_{6}$ ) and ${ }^{13} \mathrm{C}-\mathrm{NMR}\left(63 \mathrm{MHz}\right.$, DMSO- $d_{6}$ ) spectrum of compound 45d. * = NMR-solvent 


\section{Supplemental References}

1 P. G. Wuts and T. W. Greene, Greene's protective groups in organic synthesis, John Wiley \& Sons, 2006.

2 B. W. Stein, S. K. Cary, J. M. Berg, E. R. Birnbaum, S. A. Kozimor, V. Mocko and B. L. Scott, J. Organomet. Chem., 2018, 857, 170.

3 P.-A. Deyris, T. Cañeque, F. Gomes, V. Narbonne, G. Maestri and M. Malacria, Heterocycles, 2014, 88, 807.

4. S. G. Ramkumar and S. Ramakrishnan, Macromolecules, 2010, 43, 2307-2312.

5. D. M. Mofford, G. R. Reddy and S. C. Miller, J. Am. Chem. Soc., 2014, 136, 13277.

6. E. M. Doherty, C. Fotsch, Y. Bo, P. P. Chakrabarti, N. Chen, N. Gavva, N. Han, M. G. Kelly, J. Kincaid, L. Klionsky, Q. Liu, V. I. Ognyanov, R. Tamir, X. Wang, J. Zhu, M. H. Norman and J. J. S. Treanor, J. Med. Chem., 2005, 48, 71.

7 W. L. Armarego, Purification of laboratory chemicals, Butterworth-Heinemann, 2017.

8 E. Fischer, J. Phys. Chem., 1967, 71, 3704. 